\title{
CALTRANS: Impact of the Use of Portland-Limestone Cement on Concrete Performance as Plain or Reinforced Material
}

\author{
Final Report
}

Keshav Bharadwaj, Oregon State University, Corvallis, OR Krishna Siva Teja Chopperla, Oregon State University, Corvallis, OR

Antara Choudhary, Oregon State University, Corvallis, OR

Deborah Glosser, Oregon State University, Corvallis, OR Rita Maria Ghantous, Oregon State University, Corvallis, OR Gokul Dev Vasudevan, Oregon State University, Corvallis, OR

Dr. Jason H. Ideker, Oregon State University, Corvallis, OR

Dr. O. Burkan Isgor, Oregon State University, Corvallis, OR

Dr. David Trejo, Oregon State University, Corvallis, OR Dr. W. Jason Weiss, PI, Oregon State University, Corvallis, OR

April 30 ${ }^{\text {th }}, 2021$, Revised June $29^{\text {th }}, 2021$ 


\section{Table of Contents}

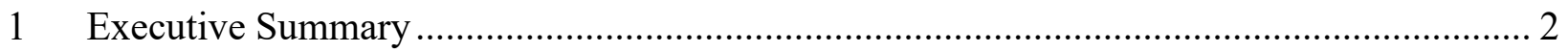

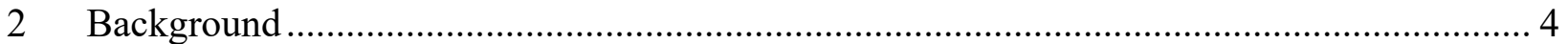

3 Constituent Materials and Mixtures Considered in the Experimental Plan ............................. 5

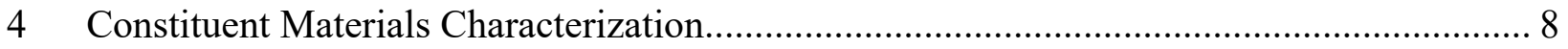

$4.1 \quad$ Research Objective ......................................................................................... 8

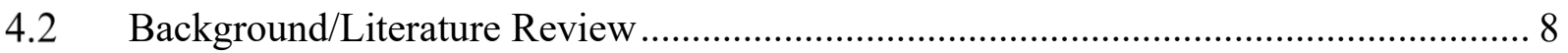

4.3 An Overview of the Experimental Characterization Performed for the Cementitious

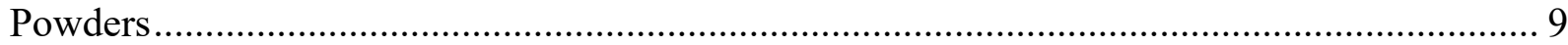

4.4 Chemical Composition and Loss on Ignition.......................................................... 10

4.4.1 Experimental Methods .................................................................................... 10

4.4.2 Typical Experimental Measurement and Interpretation ........................................... 11

4.4.3 Experimental Results and Discussion ................................................................ 12

$4.5 \quad$ Particle Size Analysis ............................................................................................. 14

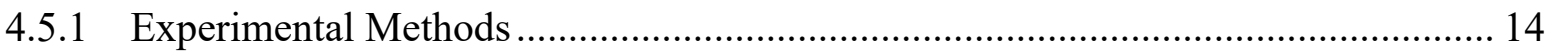

4.5.2 Typical Experimental Measurement and Interpretation ........................................ 15

4.5.3 Experimental Results and Discussion ............................................................... 17

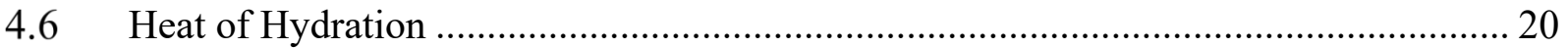

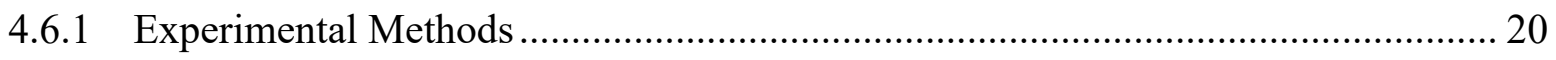

4.6.2 Typical Experimental Measurement and Interpretation ............................................ 22

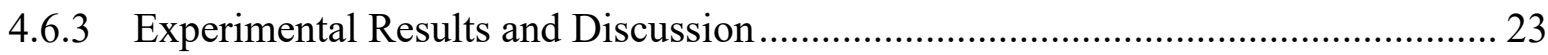

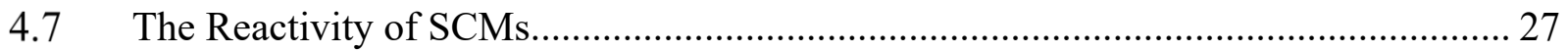

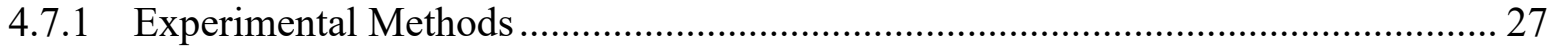

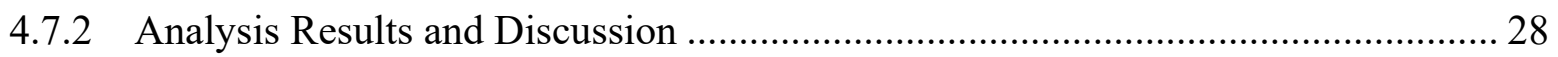

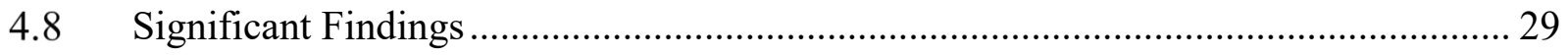

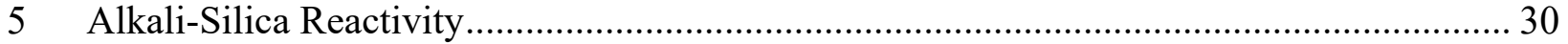

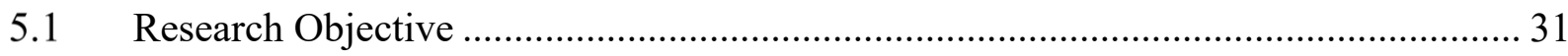

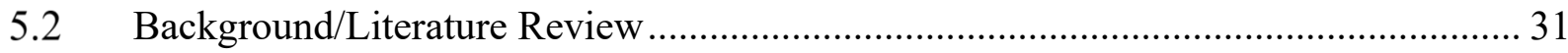

$5.3 \quad$ Experimental Test Matrix …………………………............................................. 34 


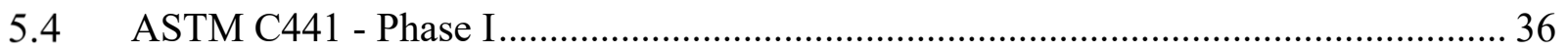

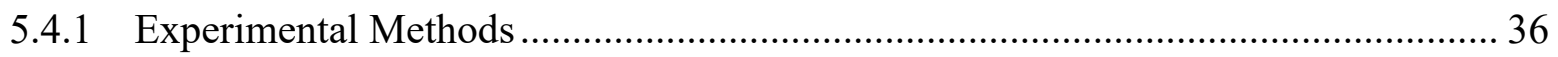

5.4.2 Typical Experimental Measurement and Interpretation .......................................... 37

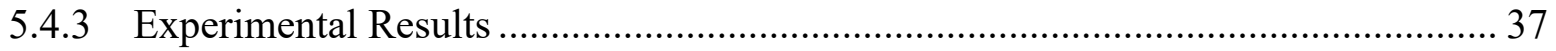

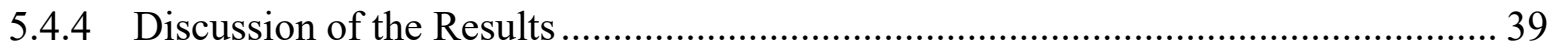

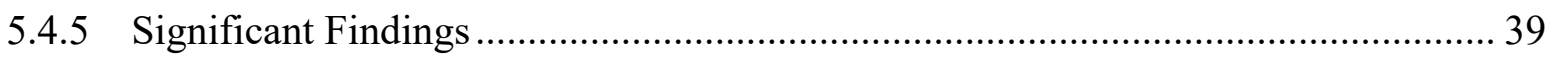

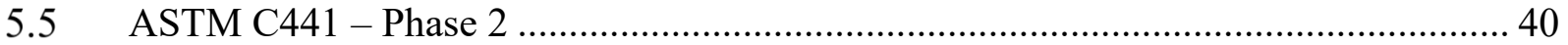

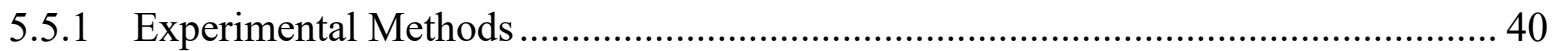

5.5.2 Typical Experimental Measurement and Interpretation ....................................... 40

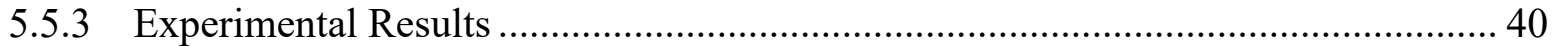

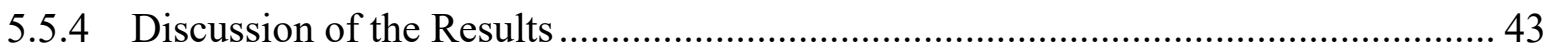

5.5.5 Significant Findings ....................................................................................... 43

5.6 ASTM C1260 - Phase 2 ……………………..................................................... 43

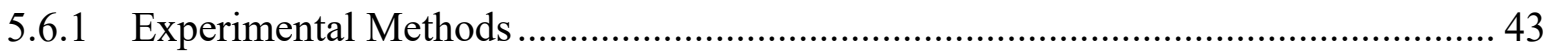

5.6.2 Typical Experimental Measurement and Interpretation .......................................... 44

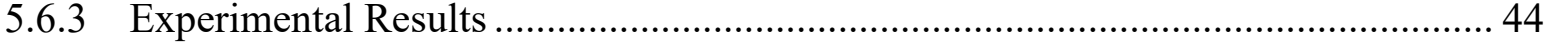

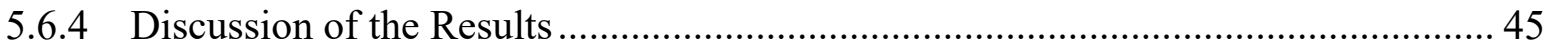

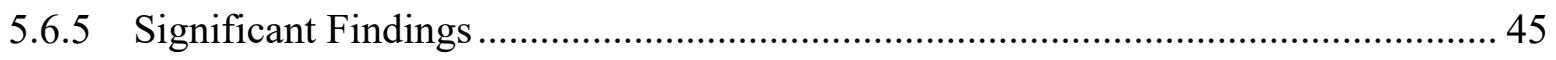

$5.7 \quad$ ASTM C1567 - Phase 2 ……………………........................................................ 45

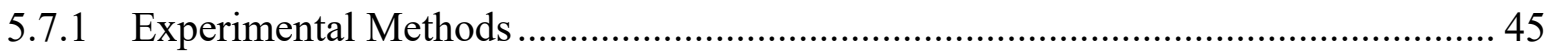

5.7.2 Typical Experimental Measurement and Interpretation ........................................... 46

5.7.3 Experimental Results ............................................................................................. 46

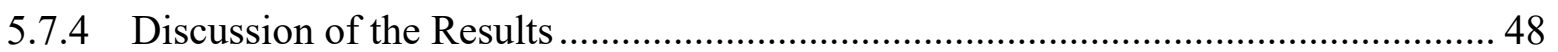

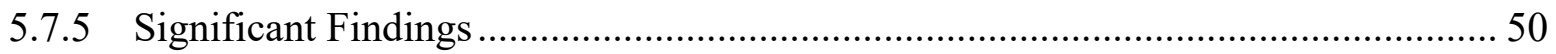

5.8 AASHTO T 380 (Miniature Concrete Prism Test) ........................................................ 50

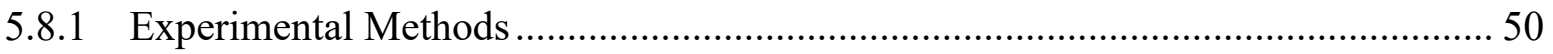

5.8.2 Typical Experimental Measurement and Interpretation .......................................... 51

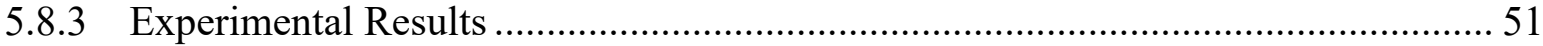

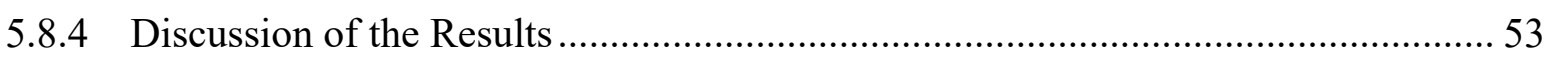

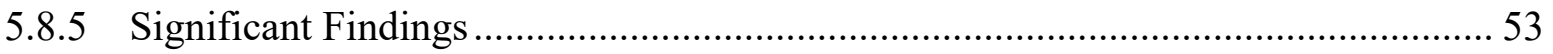




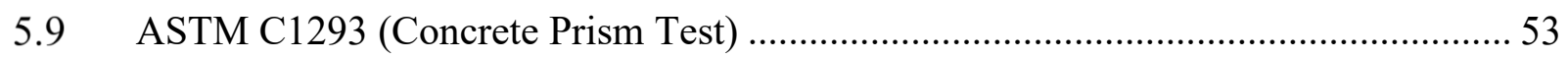

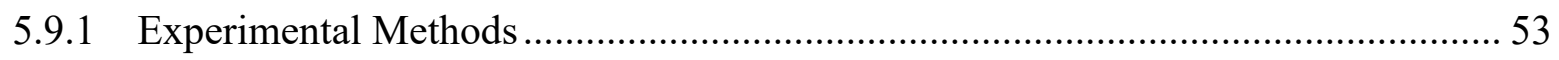

5.9.2 Typical Experimental Measurement and Interpretation ............................................ 54

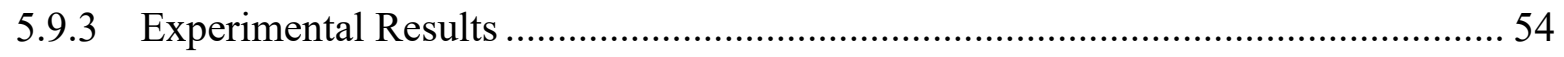

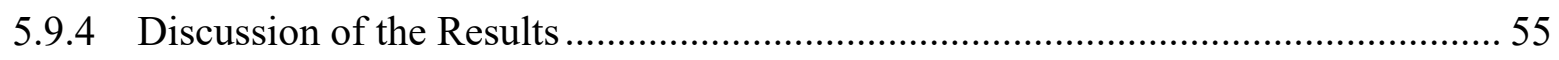

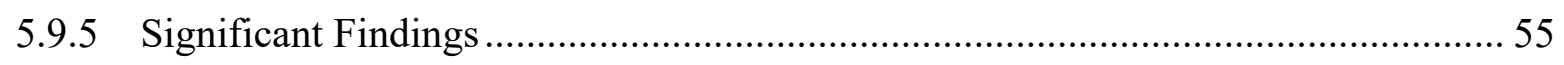

$6 \quad$ Shrinkage and Restrained Shrinkage Cracking ................................................................... 56

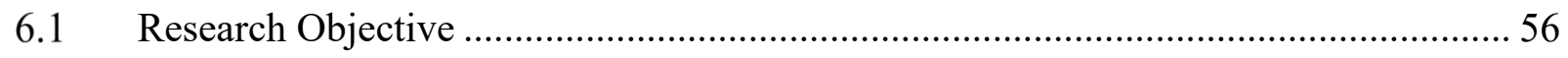

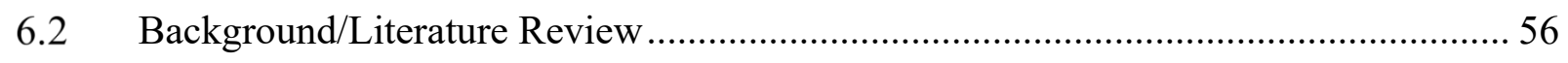

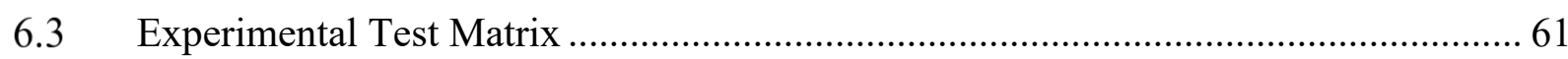

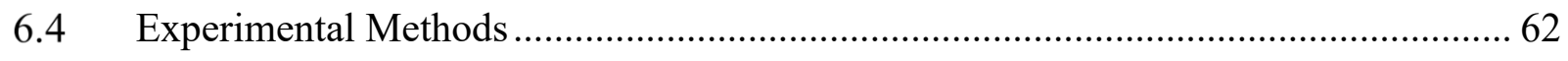

6.5 Typical Experimental Measurement and Interpretation ....................................................... 64

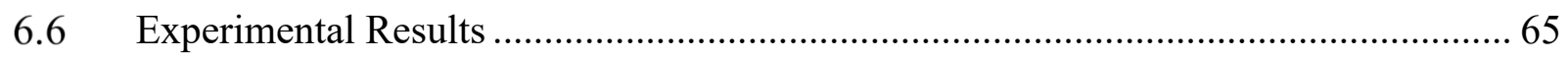

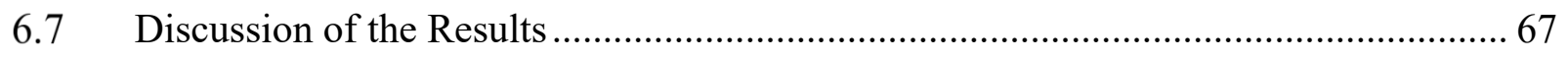

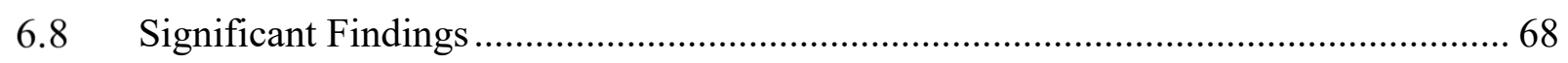

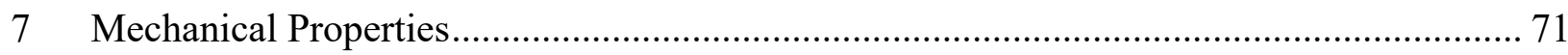

$7.1 \quad$ Research Objective …………………………...................................................... 71

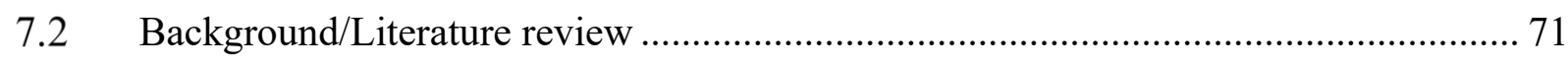

7.3 Experimental Test Matrix ................................................................................... 72

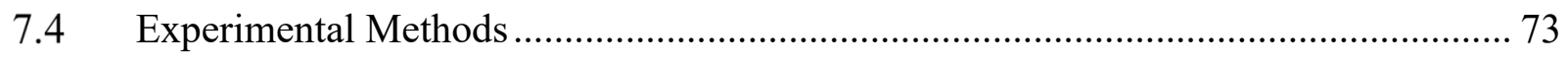

7.5 Typical Experimental Measurement and Interpretation ............................................... 75

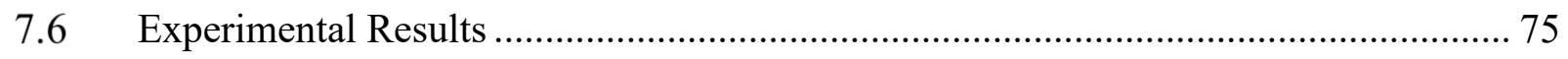

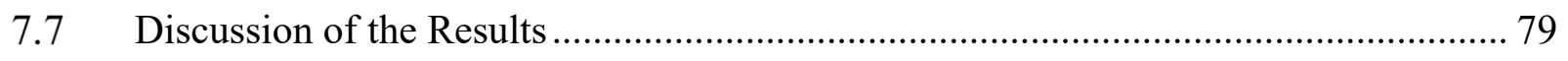

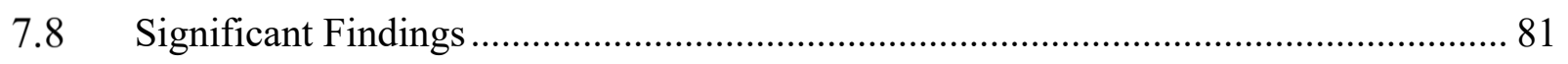

$8 \quad$ Transport Properties ........................................................................................... 82

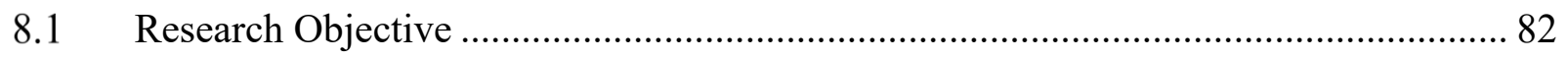

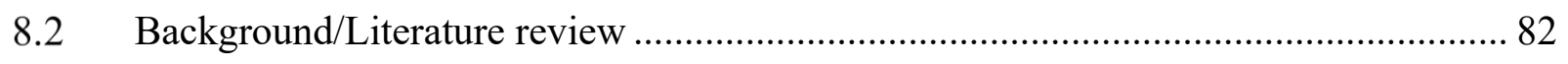

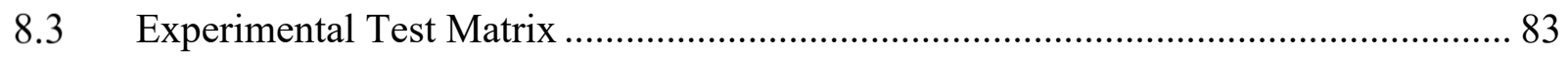

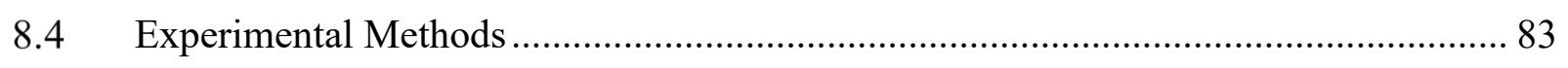

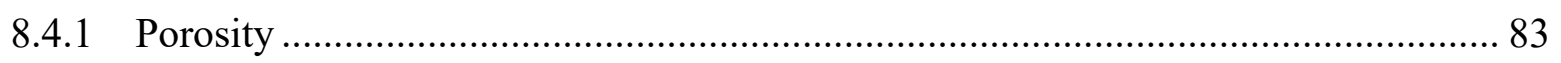




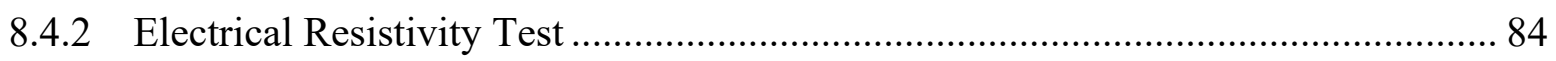

8.4.3 Formation Factor and Pore Connectivity ................................................................. 84

8.5 Typical Experimental Measurement and Interpretation ............................................ 85

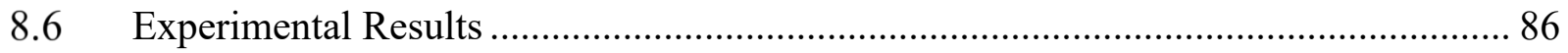

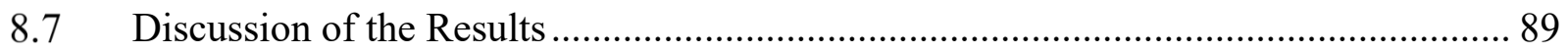

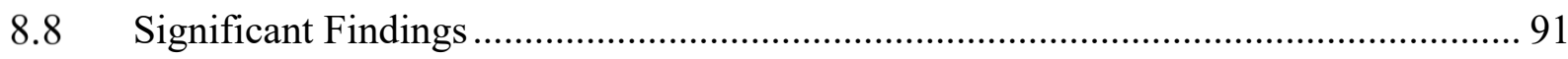

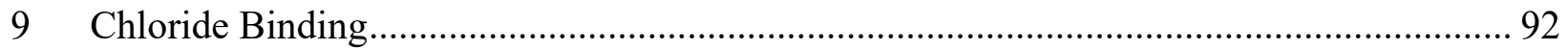

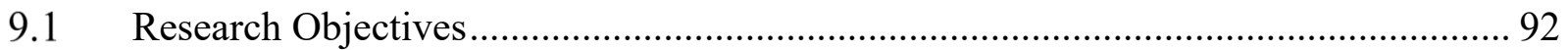

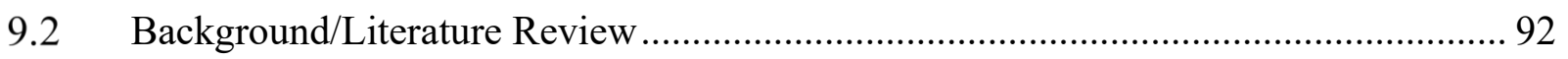

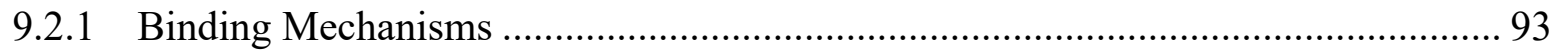

9.2.2 Limestone in Chloride Binding .............................................................................. 93

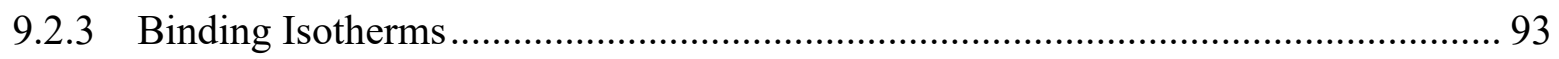

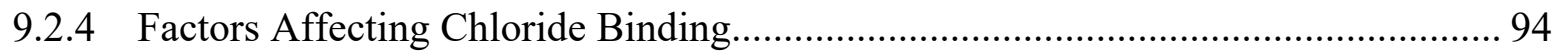

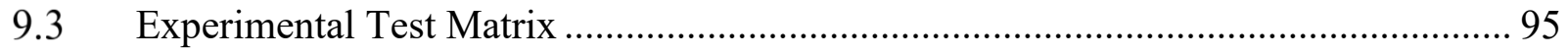

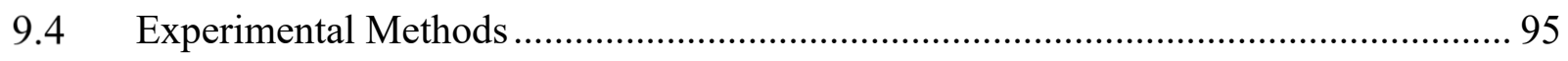

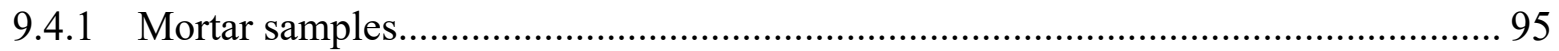

9.4.2 Simulated pore solution ...................................................................................... 96

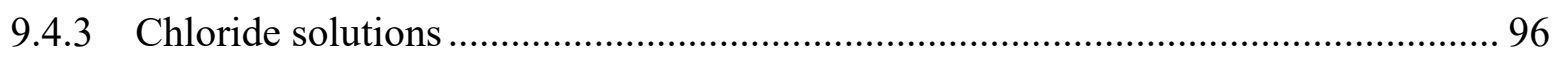

9.5 Typical Experimental Measurement and Interpretation ……………………….......... 96

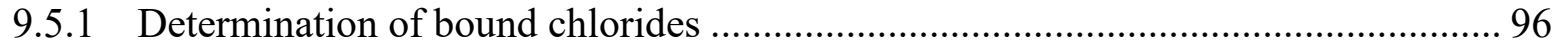

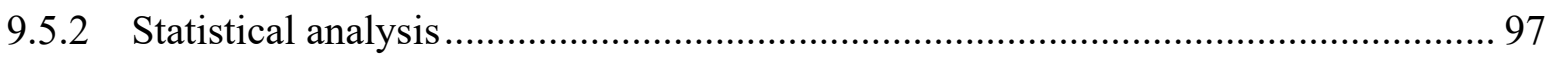

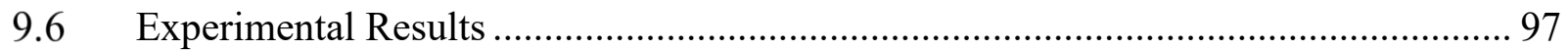

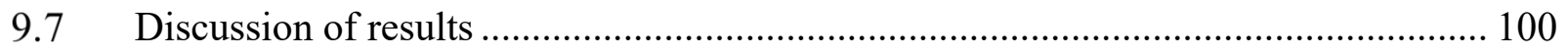

9.7.1 Difference in binding of OPC, PLC, and OPC+10LS ......................................... 100

9.7.2 Difference in binding of M0 and M1-M5 ...................................................... 100

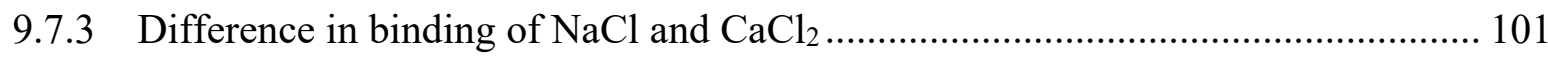

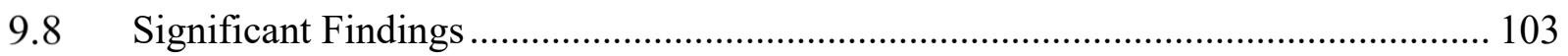

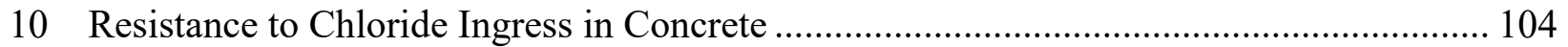

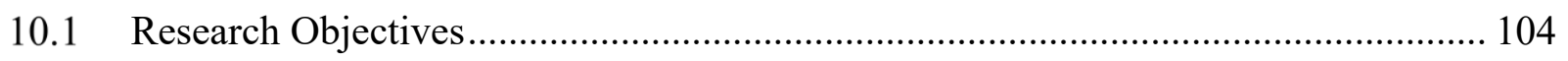

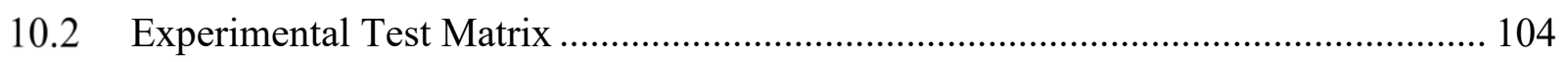




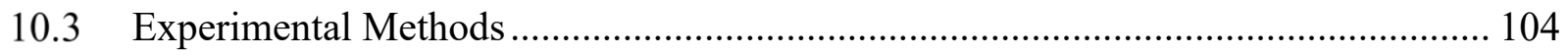

10.3.1 Porosity, Resistivity, and Apparent Formation Factor........................................ 105

10.3.2 Experimental determination of acid soluble chloride profile and chloride apparent

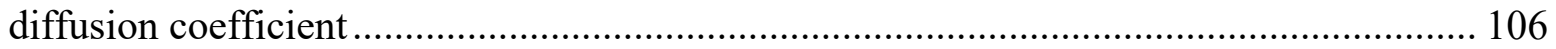

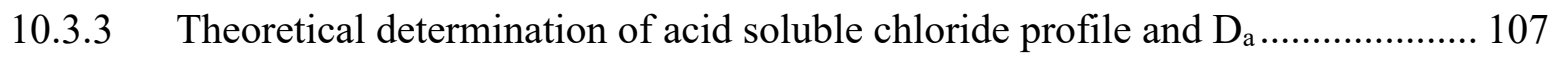

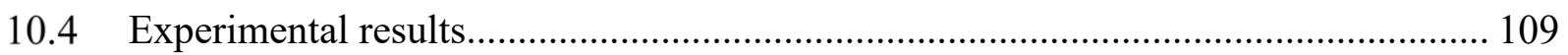

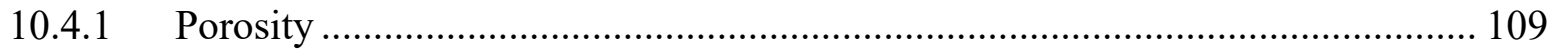

10.4.2 Experimental determination of acid soluble chloride profile and chloride apparent diffusion coefficient (ASTM C 1556).......................................................................... 110

10.4.3 Theoretical determination of acid soluble chloride profile and chloride apparent diffusion coefficient parameters ................................................................................ 114

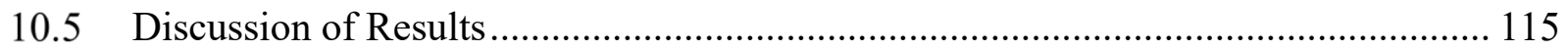

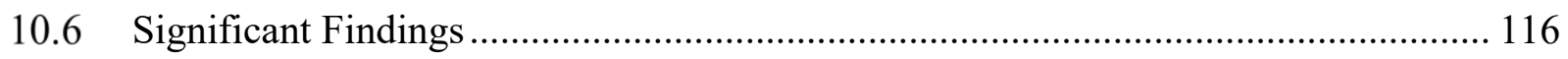

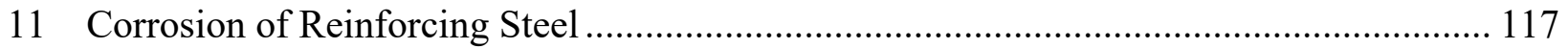

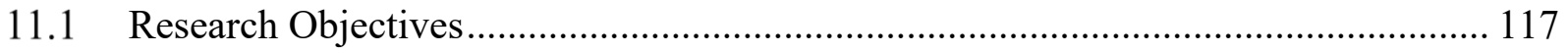

11.2 Background/Literature Review ....................................................................... 117

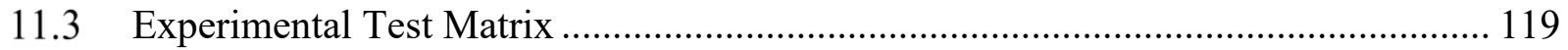

11.4 Experimental Methods ...................................................................................... 120

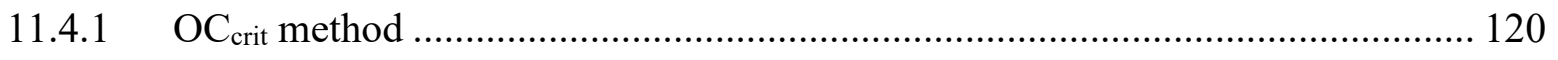

11.4.2 Modified ASTM G109 method..................................................................... 122

11.5 Experimental Results ...................................................................................... 124

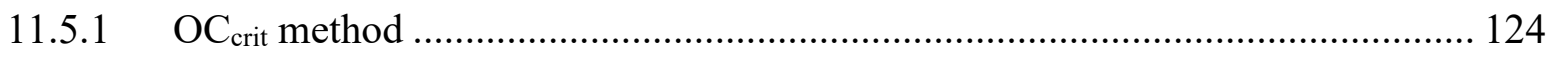

11.5.2 Modified ASTM G109 method....................................................................... 132

11.6 Significant Findings .......................................................................................... 135

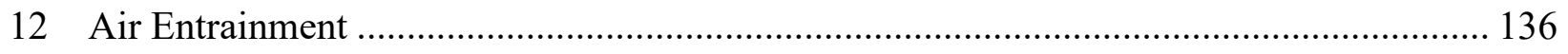

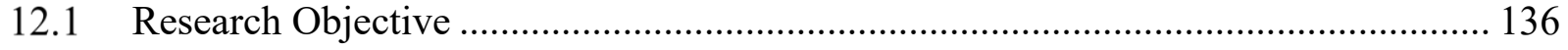

12.2 Background and Literature Review …………………....................................... 136

12.3 Experimental Test Matrix .................................................................................... 137

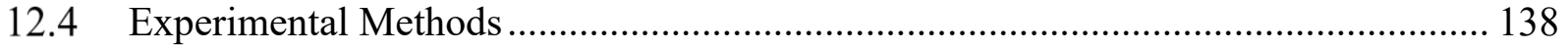

12.5 Typical Experimental Measurement and Interpretation ........................................... 138

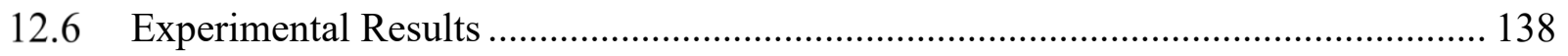




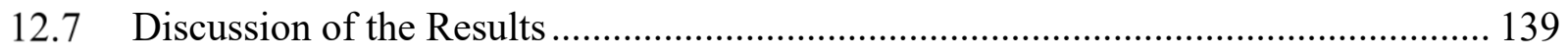

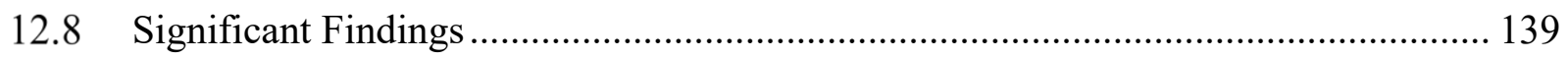

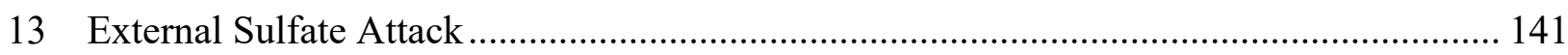

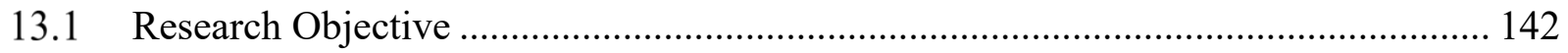

13.2 Background and Literature Review .................................................................. 142

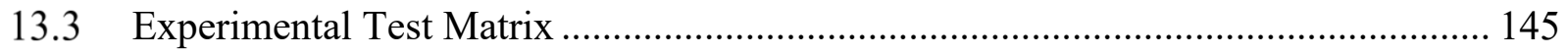

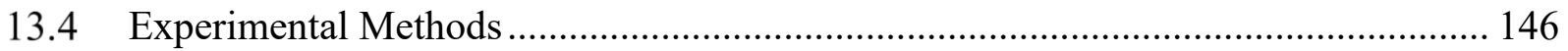

13.5 Typical Experimental Measurement and Interpretation ............................................ 147

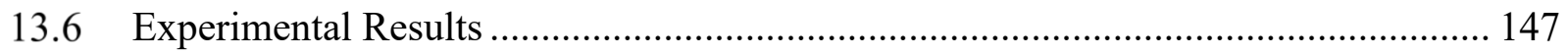

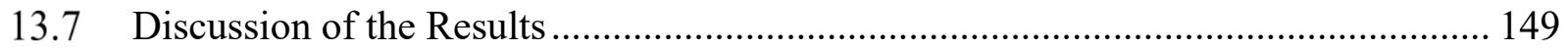

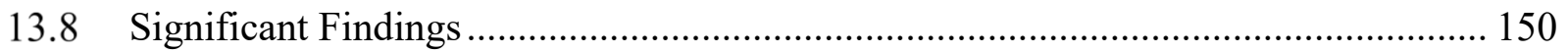

14 Construction Schedule ……………………………................................................. 151

14.1 Research Objectives ........................................................................................... 151

14.2 Background/Literature Review ..................................................................... 152

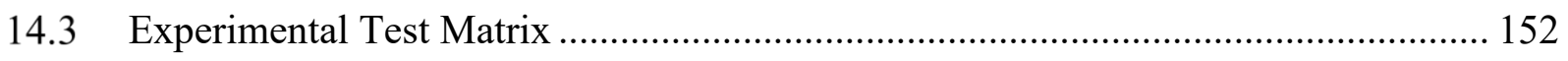

14.4 Experimental and Analytical Methods.................................................................... 152

14.5 Experimental Results ....................................................................................... 153

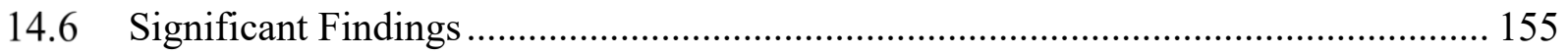

15 Impact on Greenhouse Gas Emissions............................................................................... 156

15.1 Background of Sustainability Efforts in the Cement Industry .................................... 156

15.2 Life Cycle Inventory (LCI) of PLC Concrete............................................................ 157

15.3 Significant Findings ........................................................................................... 161

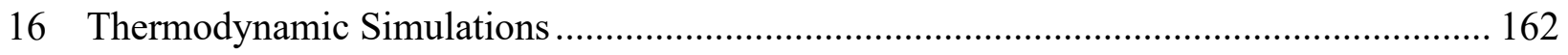

16.1 Research Objective ........................................................................................ 162

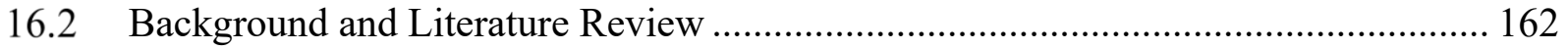

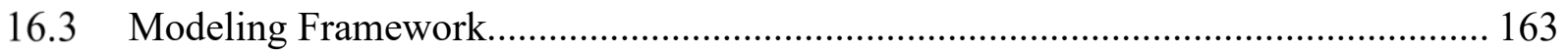

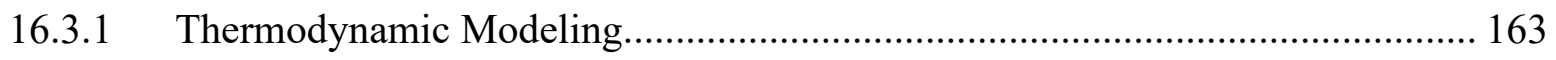

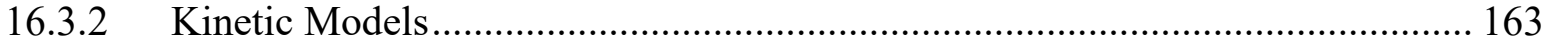

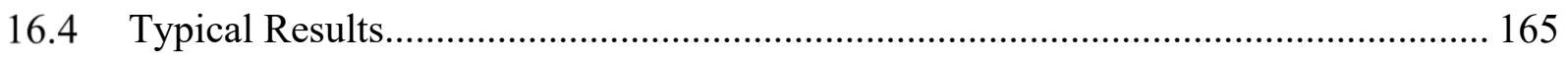

16.5 Modeling Predictions of Experimental Results ......................................................... 169 


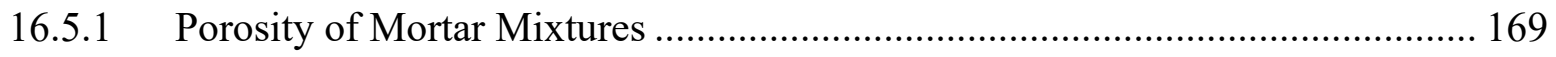

16.6 Comparison of PLC and OPC Using Modeling.................................................... 170

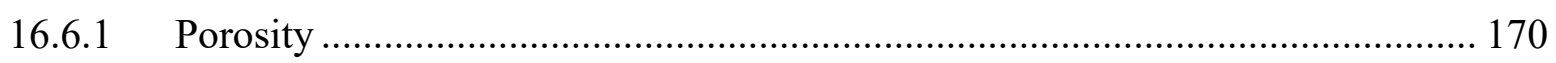

16.6.2 Reacted and Unreacted Calcite (From Limestone) ........................................... 172

16.6.3 Calcium Hydroxide (CH) Consumed.......................................................... 175

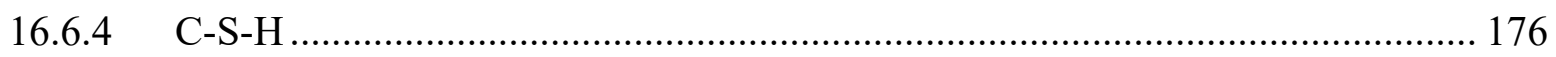

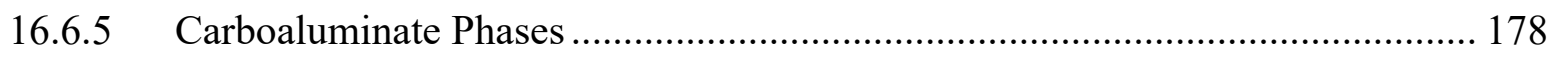

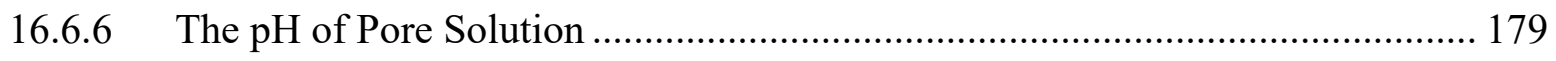

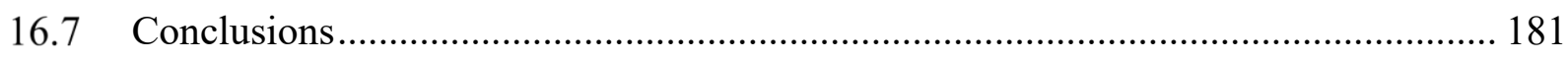

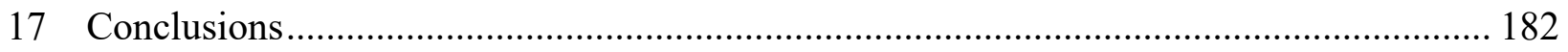

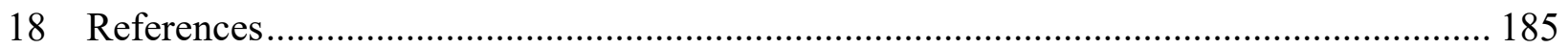

19 APPENDIX A - Determination of ultimate Degree of Reactivity of SCM ………............ 197

19.1 Scope

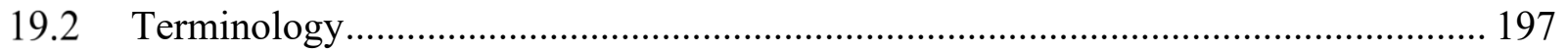

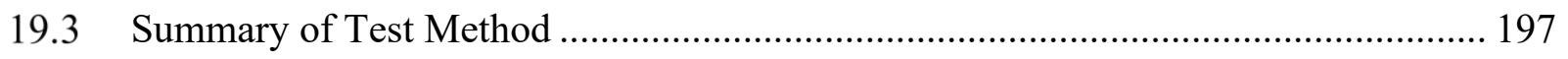

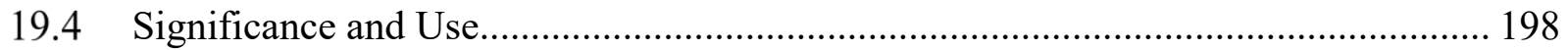

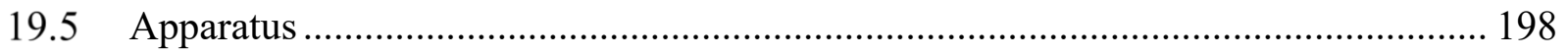

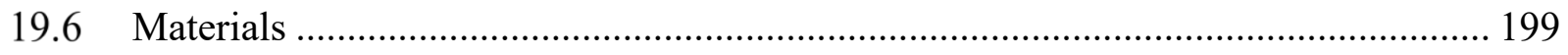

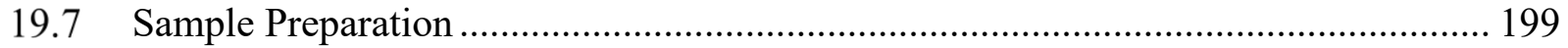

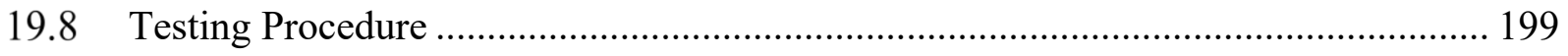

19.9 Calculation and Interpretation of Results …………............................................... 200

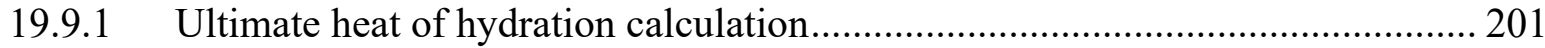

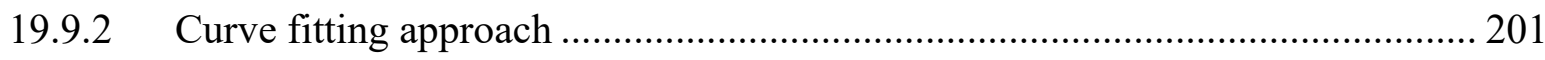

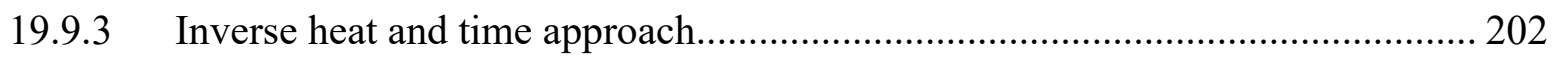

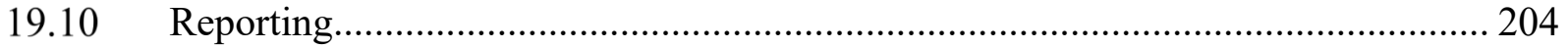

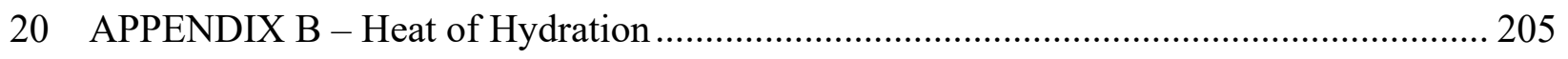

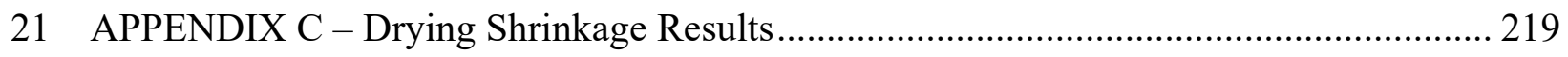

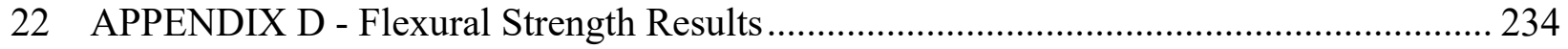

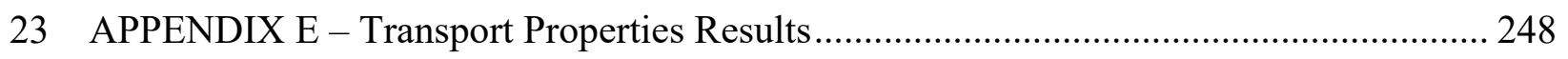

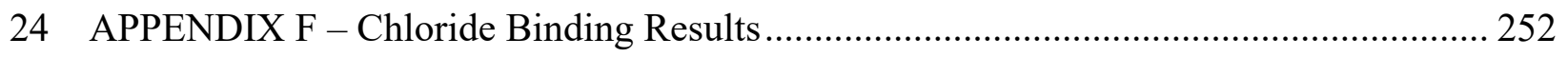


25 APPENDIX G - Transport Properties of Concrete ………………………........................ 278

26 APPENDIX H - Additional Data from Thermodynamic Modeling ………………............. 285

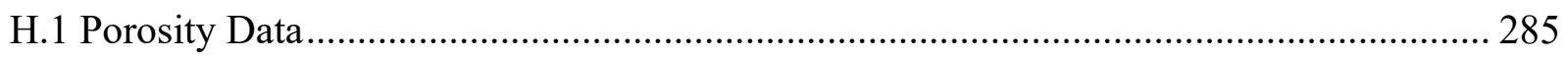

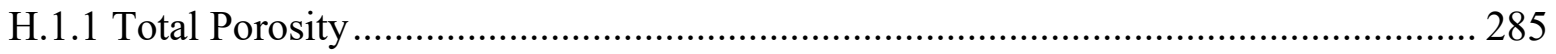

H.1.2 Phase Volumes (including Pore Volume Distribution)............................................. 286

H.2 Reacted and Unreacted Limestone Data ......................................................................... 288

H.3 CH Consumed Data ............................................................................................. 289

27 APPENDIX I - Additional Mixture Proportions for GHG Modeling.................................. 290

I.1 Jointed Plain Concrete Mix Design ........................................................................ 290

I.2 Concrete Deck containing Slag - Mix Design ................................................................ 292

I.3 Concrete Deck containing Fly Ash - Mix Design.............................................................. 294 


\section{List of Tables}

Table 3.1. Nomenclature of cements used in this study 6

Table 4.1a. Chemical composition of OPCs and PLCs from Company A and B..................... 12

Table 4.2. Chemical composition of SCMs and limestone................................................. 14

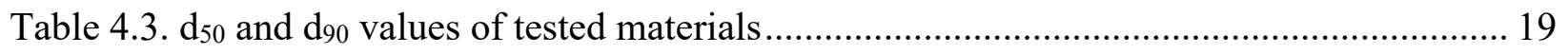

Table 4.4. Mixture proportions of cement paste mixtures .................................................... 21

Table 4.5. Experimental matrix of heat of reaction tests ................................................ 22

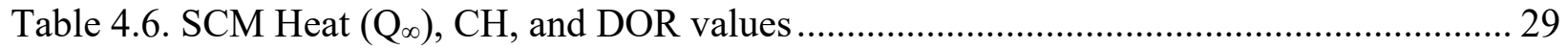

Table 5.1. Experimental matrix for ASTM C441 phase-II and ASTM C1567 testing............... 34

Table 5.2. Experimental matrix for AASHTO T 380 testing ….......................................... 36

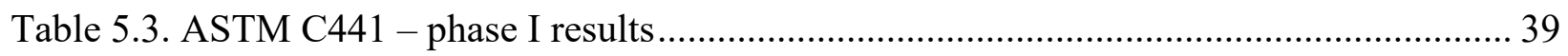

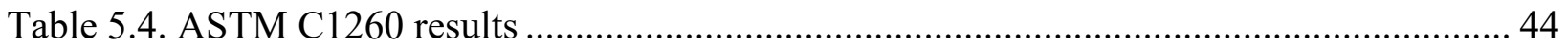

Table 5.5. Estimated major constituents in the reactive fine aggregates by petrographic

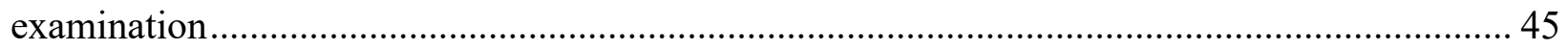

Table 6.1. Mixture proportions for mixtures evaluated for shrinkage ................................. 62

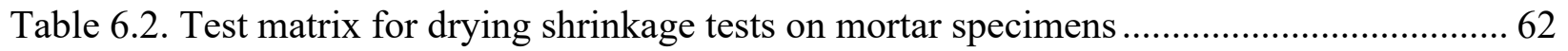

Table 10.1. Mixture proportions of concrete samples .................................................... 104

Table 10.2. Experimental characterizations performed on concrete samples......................... 104

Table 10.3. Resistivity of pore solution after 7 and 14 days of immersion ........................... 105

Table 10.4. Experimental values of $\mathrm{C}_{\mathrm{s}-\mathrm{a}}$ and $\mathrm{D}_{\mathrm{a}}$ for OPC and PLC concrete samples.............. 114

Table 10.5. Freundlich binding parameters of concrete samples........................................ 114

Table 11.1. Experimental program for OCcrit study ................................................. 121

Table 12.1. Comparison of measured air contents for OPC and PLC mixtures; note that the target

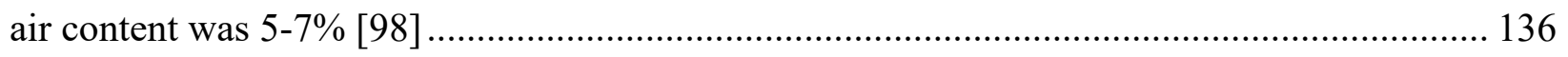


Table 12.2. The measured air contents of the mixtures with F1 fine aggregate 138

Table 12.3. The measured air contents of the mixtures with F2 fine aggregate 139

Table 13.1. Experimental matrix for ASTM C1012 testing ..... 146

Table 14.1. Statistical comparisons 153

Table 14.2. p-values for different mixtures. 154

Table 15.1. Transportation distances used to calculate greenhouse gas reduction in the LCI GHG tool (from $[176,177])$. 159

Table 15.2. Example CALTRANS concrete mixture designs evaluated using the GHG reduction

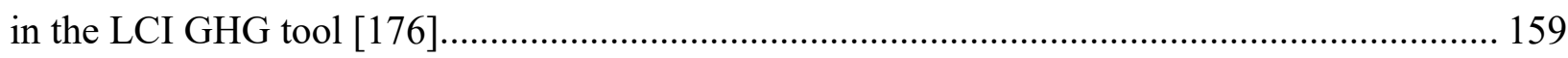

Table 16.1. Difference in porosity between OPC and PLC systems .................................... 171

Table 16.2. Reactive alumina in each PLC mixture .......................................................... 174

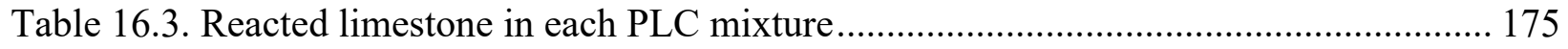

Table 16.4. Decrease in C-S-H volume when OPC is replaced with PLC ............................. 177

Table 16.5. Volume fraction of carboaluminate phases in the PLC systems ......................... 179

Table 16.6. Difference in pore solution $\mathrm{pH}$ when OPC is replaced with PLC ........................ 180

Table A.1. Comparison of different heat released correction methods ................................... 201

Table F.1. Binding Parameters of Cement A - Mixtures 0 to $5(\mathrm{NaCl})$................................. 268

Table F.2. Binding Parameters of Cement B - Mixtures 0 to $4(\mathrm{NaCl}){ }^{*} M 5$ was not cast for Cement B 269

Table F.3. Binding Parameters of Cement C - Mixtures 0 to $4(\mathrm{NaCl}) * M 5$ was not cast for Cement C 270

Table F.4. Binding Parameters of Cement D - Mixtures 0 to $5(\mathrm{NaCl})$................................ 271

Table F.5. Binding Parameters of Cement D - Mixtures 0 to $5\left(\mathrm{CaCl}_{2}\right)$................................ 272

Table F.6. Binding Parameters of Cement E - Mixtures 0 to $4(\mathrm{NaCl}){ }^{*}$ M5 was not cast for Cement E 273 
Table F.7. Bound chloride content for OPCs immersed in $\mathrm{NaCl}-$ Cements A to E, Mixtures 0 to

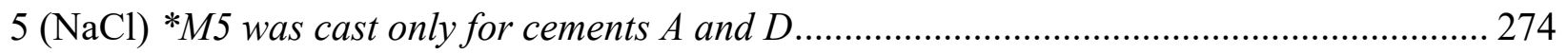

Table F.8. Bound chloride content for PLCs immersed in $\mathrm{NaCl}-\mathrm{Cements} \mathrm{A}$ to E, Mixtures 0 to 5

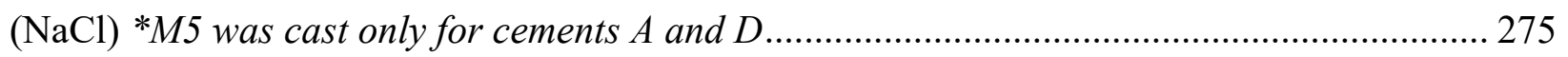

Table F.9. Bound chloride content for OPC+10LS mixtures immersed in $\mathrm{NaCl}-\mathrm{Cements} \mathrm{A}$ to $\mathrm{E}$, Mixtures 0 to $5(\mathrm{NaCl}) * M 5$ was cast only for cements $A$ and $D$........................................ 276

Table F.10. Bound chloride content for Cement D mixtures immersed in $\mathrm{CaCl}_{2}-$ Mixtures 0 to 5

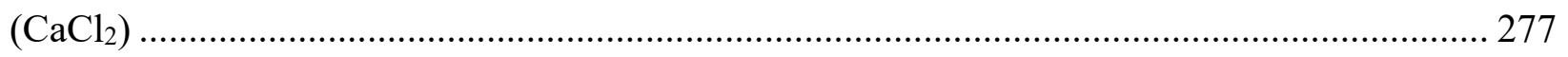

Table H.1. Total porosity of paste from thermodynamic modeling ....................................... 285

Table H.2. Total porosity of mortar calculated from the PPMC ........................................ 286

Table H.3. Phase volumes determined from thermodynamic modeling (output of the PPM)... 286

Table H.4. Reacted calcite (limestone) in the systems as determined from thermodynamic

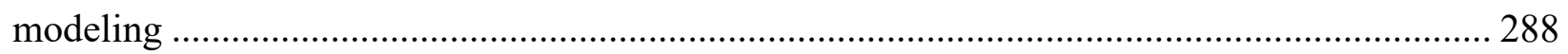

Table H.5. Unreacted calcite (limestone) that remains in the systems as determined from

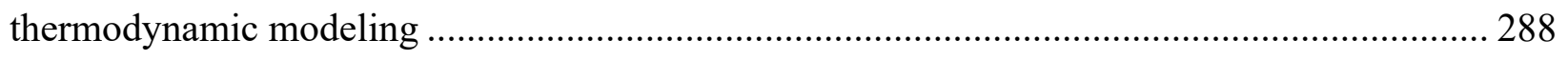

Table H.6. CH Consumed in the systems as determined from thermodynamic modeling ........ 289 


\section{List of Figures}

Figure 4-1. (a) PANalytical Epsilon 3XLE XRF spectrometer (b) a cement bead.................... 11

Figure 4-2. Horiba LA-920 Particle Size Analyzer ............................................................. 15

Figure 4-3. Typical particle size distributions of cements showing (a) volume vs. diameter (b) cumulative volume vs. diameter 16

Figure 4-4. Particle size distributions depicting volume fraction vs particle diameter of (a) Cements

(b) SCMs; cumulative volume fraction vs particle diameter of (c) Cements (d) SCMs. 19

Figure 4-5. TAM Air isothermal Calorimeter used for heat flow measurements from samples .. 20

Figure 4-6. Typical Heat of reaction plot. 23

Figure 4-7. (a-f) Total heat released per unit binder in cement paste measured for seven days using Isothermal Calorimeter for Cement $\mathrm{A}$ for $\mathrm{M0}, 1,2,3,4$ and 5 respectively .......................... 26

Figure 4-8. Maximum degree of pozzolanic reactivity (DOR*) results for CALTRANS SCMs 28

Figure 5-1. Expansion results of mortar and concrete prisms [65] ....................................... 32

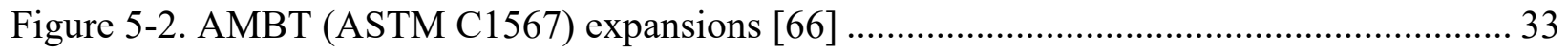

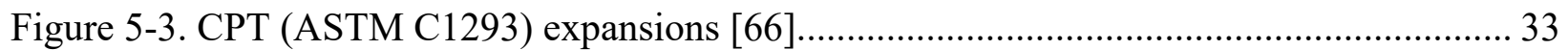

Figure 5-4. Top view of the ASTM C441 set up used........................................................ 37

Figure 5-5. ASTM C441 - phase I 14-d expansion results of received PLCs and OPCs ............ 38

Figure 5-6. ASTM C441 results for 25\% FA1 mixtures .................................................. 40

Figure 5-7. ASTM C441 results for 20\% FA1 + 5\% silica fume mixtures ............................ 41

Figure 5-8. ASTM C441 results for 50\% slag mixtures .............................................. 41

Figure 5-9. ASTM C441 results for 25\% FA1 + 25\% slag mixtures .................................. 42

Figure 5-10. ASTM C441 results for 25\% FA2 mixtures ................................................ 42 
Figure 5-11. ASTM C1260 mortar bars immersed in $1 \mathrm{~N} \mathrm{NaOH}$ solution in a test container; note that the length of each prism is $285 \mathrm{~mm}$ for scale.

Figure 5-12. ASTM C1567 results comparing the expansions of OPCs, PLCs, and OPC $+10 \%$ LS systems with F1 aggregate (very-highly reactive as per ASTM C1778).... 46

Figure 5-13. Comparison of ASTM C1567 expansions of mixtures with F1 aggregate and cements B_OIIV and B_L15 with 25\% and 35\% FA2.

Figure 5-14. ASTM C1567 results comparing the expansions of OPCs, PLCs, and OPC $+10 \%$ limestone systems with F2 aggregate (very-highly reactive as per ASTM C1778)..... 47

Figure 5-15. Correlation between available alkali (ASTM C311) and total alkali of Class F fly ashes (Data from [69] and [70]).

Figure 5-16. Sum of sodium and potassium ion concentrations in the pore solutions of hardened cement pastes that were sealed cured at $23^{\circ} \mathrm{C}$ for 28 days for the materials in this project ........ 50

Figure 5-17. Top view of the MCPT specimens immersed in $1 \mathrm{~N} \mathrm{NaOH}$ solution..................... 51

Figure 5-18. 56-day AASHTO T 380 expansion results of the mixtures with F1 aggregate and portland cement $\mathrm{B}$ 52

Figure 5-19. 56-day AASHTO T 380 expansion results of the mixtures with F2 aggregate ....... 52

Figure 5-20. ASTM C1293 expansion results (Note: the total test duration is 365 days)........... 54

Figure 6-1. (a) Autogenous, (b) unrestrained, and (c) restrained shrinkage (ASTM C1581) for

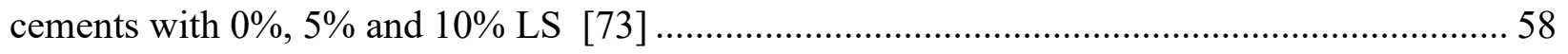

Figure 6-2. The influence of the fineness of limestone on the restrained shrinkage stress development in OPC $+10 \%$ LS systems [74] .... 59

Figure 6-3. Autogenous shrinkage measurements for OPC, PLC, and PLC-slag systems with: (a) w/b 0.39; and (b) w/b 0.34 [76] 60

Figure 6-4. (a) Free shrinkage of sealed and unsealed OPC and PLC concrete and (b) Stress Developed for OPC and PLC when restrained from shrinking freely. [76]

Figure 6-5. Drying shrinkage Comparator where a) illustrates the reference bar's measurement and b) shows the sample's measurement. 63

Figure 6-6. Typical plot of drying shrinkage results of (Cement B-Mixture 4) 64 
Figure 6-7. (a)-(f) Drying shrinkage results of Cement D for M0, 1, 2, 3, 4, and 5, respectively 67

Figure 6-8. 1:1 Shrinkage Plots OPC vs. PLC and OPC vs. OPC +LS for a) 7 days of drying, b) 14 days of drying, c) 28 days of drying, and d) ultimate drying. 70

Figure 7-1. The Experimental Setup for the B3B test: a) without the sample b) showing a mortar disk during testing and c) showing a mortar disk after testing (note the three vertical pins provide

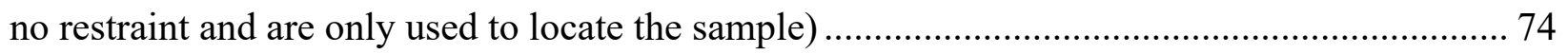

Figure 7-2. Typical plot of normalized flexural strength results of (Cement A-M3) ................... 75

Figure 7-3. (a)-(f) Flexural strength results using B3B test for Cement B for M0, 1, 2, 3, 4, and 5

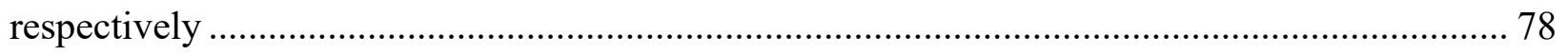

Figure 7-4. (a)-(d) Flexural strength results using B3B test for M0-M4 .................................... 81

Figure 8-1. Typical plot of normalized porosity results of Cement A for all mixtures ................. 85

Figure 8-2. Typical plot of porosity results comparing porosities of PLC mixtures with OPC

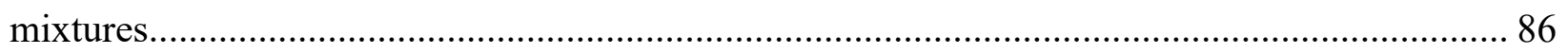

Figure 8-3. (a) Normalized porosity, and (b) Normalized electrical resistivity results for all the mixtures made using Cement $\mathrm{E}$ 87

Figure 8-4. Porosity of PLC and OPC + 10 LS mortar compared to porosity of OPC mortars after 90 days 88

Figure 8-5. Resistivity of PLC and OPC + 10 LS mortar compared to pore connectivity of OPC mortars after 90 days 88

Figure 8-6. (a) Formation factor and (b) Pore connectivity of PLC and OPC + 10 LS mortar compared to pore connectivity of OPC mortars after 90 days. .90

Figure 9-1. Automatic titration device used for determination of bound chlorides...................... 97

Figure 9-2. Binding isotherms for cement A (M0) (a) OPC, (b) PLC, (c) OPC+10LS................. 98

Figure 9-3. Binding isotherms for cement A (a) OPC, (b) PLC, (c) OPC+10LS....................... 100

Figure 9-4. Freundlich fit for all data except for slag mixtures .............................................. 101

Figure 9-5. Chloride binding isotherms for cement $\mathrm{D}$ of $\mathrm{M} 0$ and $\mathrm{M} 2$ exposed to $\mathrm{CaCl}_{2}$ salts in simulated pore solution. 103 
Figure 10-1. Acid-soluble chloride profile, experimental data to determine $\mathrm{C}_{\mathrm{s}-\mathrm{a}}$ and $\mathrm{D}_{\mathrm{a}} \ldots \ldots \ldots . . .106$

Figure 10-2. Freundlich binding isotherm: (a) determined experimentally on mortar samples, (b)

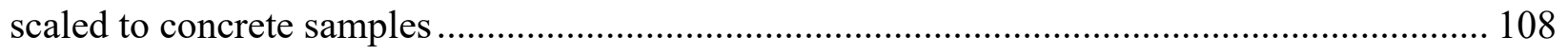

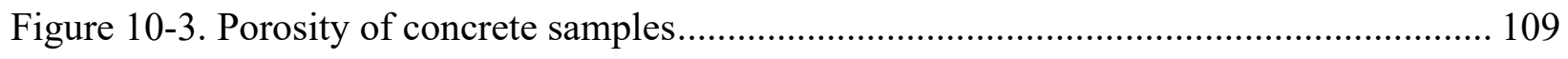

Figure 10-4. Formation factor of OPC, PLC and OPC+LS concrete samples. Circles show data

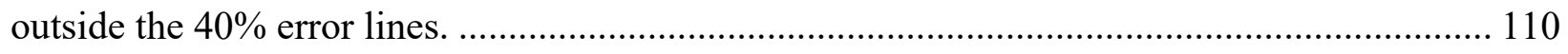

Figure 10-5. Acid-soluble chloride profiles obtained from experimental data for both OPC and

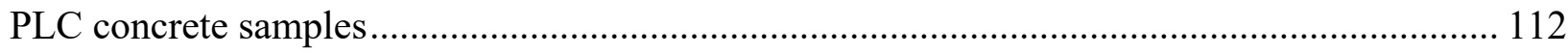

Figure 10-6. Acid-soluble chloride profiles obtained theoretically for both M0, OPC and M0, PLC

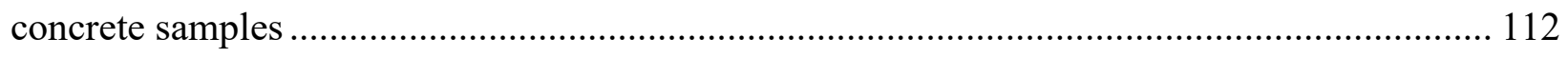

Figure 10-7. Experimental values of $\mathrm{D}_{\mathrm{a}}$ for OPC and PLC concrete samples........................ 113

Figure 10-8. Experimental values of $\mathrm{C}_{\mathrm{s}-\mathrm{a}}$ for OPC and PLC concrete samples ...................... 113

Figure 10-9. (a) Calculated values of $\mathrm{D}_{\mathrm{a}}$ based on the porosity and formation factor measurements for OPC and PLC concrete samples, (b) Calculated values of $\mathrm{C}_{\mathrm{s}-\mathrm{a}}$ based on porosity and formation factor measurements for OPC and PLC concrete samples ............................................... 115

Figure 10-10. Comparison between the chloride content of OPC and PLC concrete at $50 \mathrm{~mm}$ depth determined at an exposure period 20 years ..................................................................... 116

Figure 11-1. Setup for the $\mathrm{OC}_{\text {crit }}$ test method............................................................... 122

Figure 11-2.Setup for the modified ASTM G109 test method. .......................................... 123

Figure 11-3. Open circuit potential of anode specimens made with E_OIIV ......................... 125

Figure 11-4. (a) Open circuit potential of anode specimens made with E_OIIV-25FA1 and (b) Open circuit potential of anode specimens made with E_L11-25FA1 ................................. 126

Figure 11-5. (a) Open circuit potential of anode specimens made with E_OIIV-20FA1-5SF and (b) Open circuit potential of anode specimens made with E_L11-20FA1-5SF 127

Figure 11-6. (a) Open circuit potential of anode specimens made with E_OIIV-25FA1-25SL and (b) Open circuit potential of anode specimens made with E_L11-25FA1-25SL .................... 128 
Figure 11-7. (a) Open circuit potential of anode specimens made with E_OIIV-50SL and (b) Open circuit potential of anode specimens made with E_L11-50SL ........................................... 129

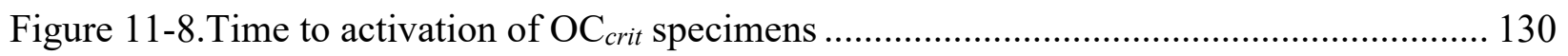

Figure 11-9. Average chloride concentration of activated specimens based on AASHTO T 260

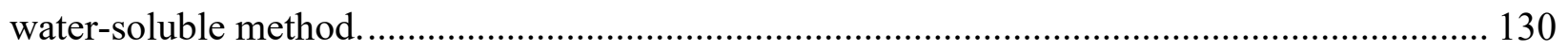

Figure 11-10. Average chloride concentration of activated specimens based on AASHTO T 260

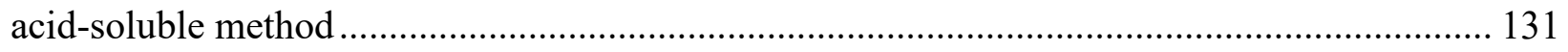

Figure 11-11. Ratio of water to acid soluble chlorides of activated specimens ...................... 131

Figure 11-12. Critical and average chloride concentration............................................. 132

Figure 11-13. Total integrated charge passed for modified ASTM G109 specimens ............... 134

Figure 11-14. Critical chloride threshold of modified ASTM G109 specimens ..................... 134

Figure 12-1. Durability factor of the mixtures tested using ASTM C666 (Procedure A) [98]... 137

Figure 13-1. External sulfate attack expansion ratio (PLC/OPC) of mortars with plain cements

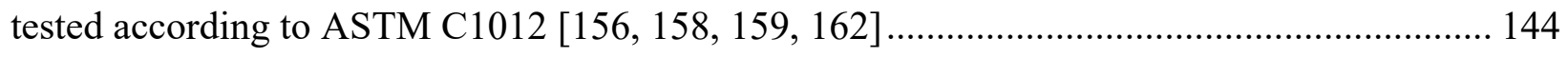

Figure 13-2. External sulfate attack expansion ratio (PLC/OPC) of mortars with SCMs tested according to ASTM C1012 [160-163] ...................................................................... 145

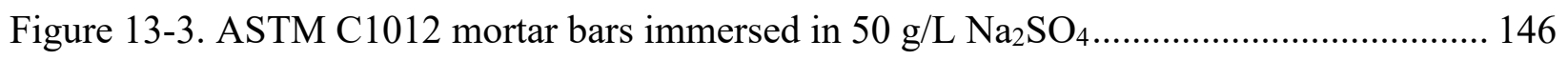

Figure 13-4. 6-month (26 weeks) expansion data of the mortar bars with clinker A ............... 147

Figure 13-5. 1-year (52 weeks) expansion data of the mortar bars with clinker A ................. 148

Figure 13-6. 6-month (26 weeks) expansion data of the mortar bars with clinker D ............... 148

Figure 13-7. 1-year (52 weeks) expansion data of the mortar bars with clinker D ................. 149

Figure 13-8. ASTM C1012 6-month expansion results: comparison of OPC and PLC mixtures 150

Figure 14-1. Slump values of fresh concrete mixtures. ................................................... 151

Figure 15-1. Process flow diagram for LCI greenhouse gas reduction tool [177] .................. 158 
Figure 15-2. Greenhouse gas reductions compared for 10 and 15\% interground limestone replacements for example CALTRANS concrete mixtures..................................................... 160

Figure 16-1. Output of the MPK model for clinker and SCM oxides ...................................... 164

Figure 16-2. Typical outputs of thermodynamic modeling showing the phase assemblage and the output of the PPM showing the different volumes of phases in the Clinker + Limestone systems 168

Figure 16-3. Porosity of the mortar mixtures of OPC and PLC (Model vs. Experiment) .......... 170

Figure 16-4. Plot of predicted porosity of PLC systems vs OPC systems.................................. 171

Figure 16-5. Plot of porosity of $\mathrm{PLC}+\mathrm{Al}_{2} \mathrm{O}_{3}$ systems showing the synergy between limestone and

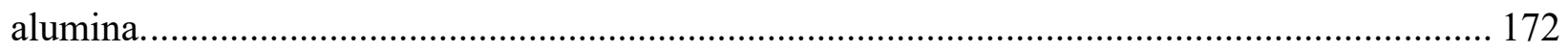

Figure 16-6. Reactive alumina in all mixtures................................................................... 173

Figure 16-7. (a) Reacted calcite, and, (b) Unreacted calcite in PLC systems........................... 174

Figure 16-8. Plot of predicted CH consumed in PLC systems vs OPC systems........................ 176

Figure 16-9. Plot of predicted C-S-H volume in PLC systems vs OPC systems........................ 177

Figure 16-10. Plot of predicted volume of hemi-/monocarbonates in PLC systems vs OPC systems. 178

Figure 16-11. Plot of predicted $\mathrm{pH}$ of pore solution of PLC systems vs OPC systems.............. 180

Figure A-19-1. Reference reaction lines of pure silica and pure alumina under simulated

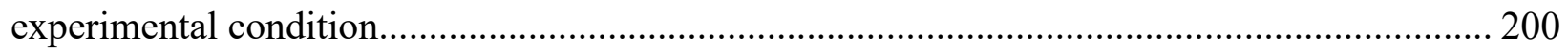

Figure A-19-2. Heat release curves determined by isothermal calorimetry for SCM1 and SCM2. The heat release data for SCM2 data was extrapolated to equilibrium to calculate the heat release correction. SCM1 did not require any extrapolation. ............................................................ 202

Figure A-19-3. 1/Q vs 1/t curve for SCM1 and SCM2 with their corresponding linear trendline 203

Figure A-19-4. Typical TGA plot of reacted SCM paste ……………........................................ 204

Figure B-20-1. Heat of Hydration of Cement A - M0 .......................................................... 205

Figure B-20-2. Heat of Hydration of Cement A - M1 .......................................................... 205 
Figure B-20-3. Heat of Hydration of Cement A - M2 ........................................................... 206

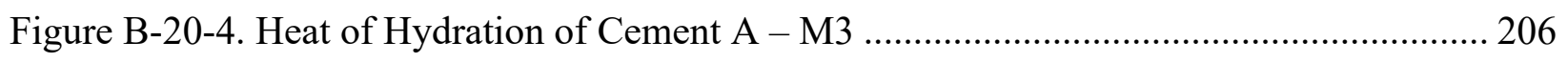

Figure B-20-5. Heat of Hydration of Cement A - M4 _.......................................................... 207

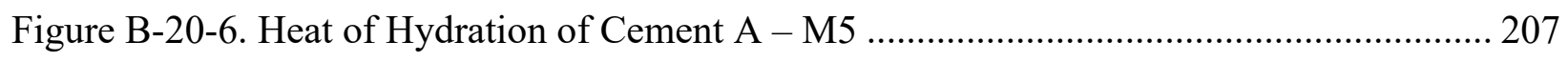

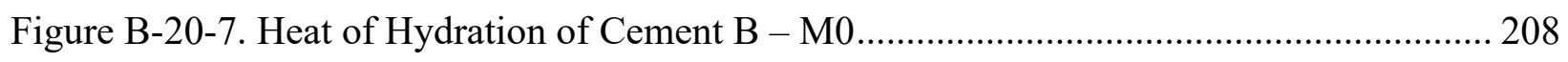

Figure B-20-8. Heat of Hydration of Cement B - M1 ........................................................... 208

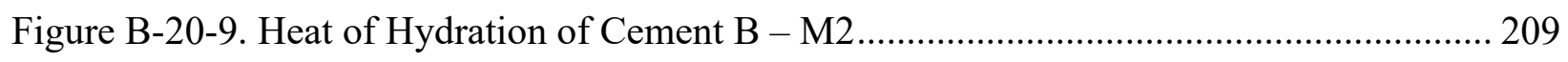

Figure B-20-10. Heat of Hydration of Cement B - M3 ………............................................... 209

Figure B-20-11. Heat of Hydration of Cement B - M4 ......................................................... 210

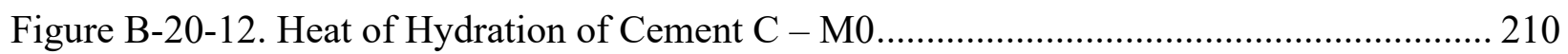

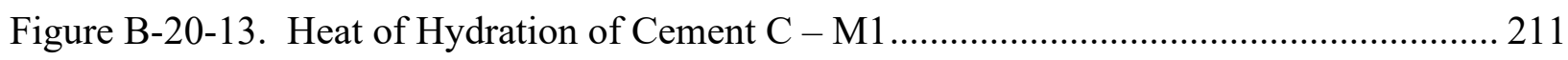

Figure B-20-14. Heat of Hydration of Cement C - M2 ......................................................... 211

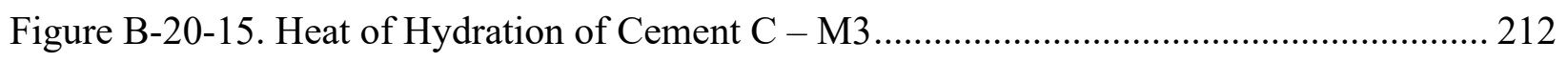

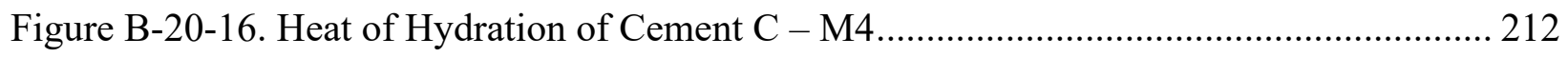

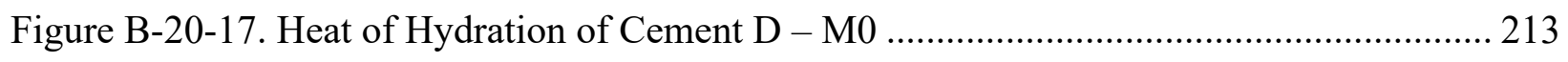

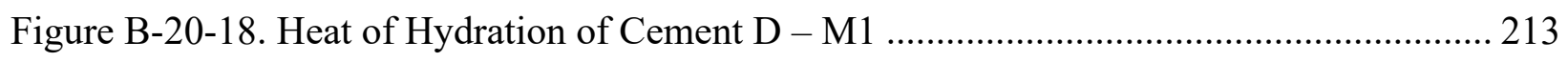

Figure B-20-19. Heat of Hydration of Cement D - M2 …................................................... 214

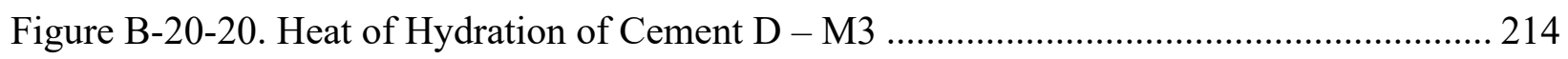

Figure B-20-21. Heat of Hydration of Cement D - M4 ...................................................... 215

Figure B-20-22. Heat of Hydration of Cement D - M5 ………………………………..... 215

Figure B-20-23. Heat of Hydration of Cement E - M0 …………………………................. 216

Figure B-20-24. Heat of Hydration of Cement E - M1 ......................................................... 216

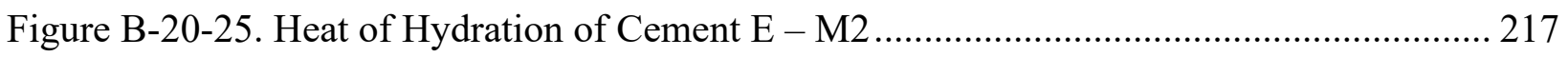


Figure B-20-26. Heat of Hydration of Cement E - M3 ……................................................... 217

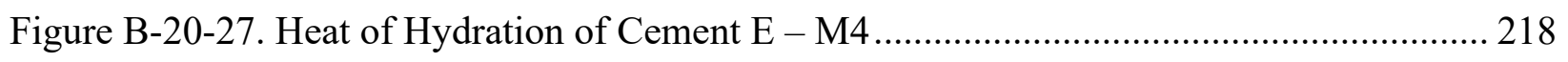

Figure C-21-1. Drying Shrinkage of Cement A - M0 _............................................................ 219

Figure C-21-2. Drying Shrinkage of Cement A - M1 ......................................................... 220

Figure C-21-3. Drying Shrinkage of Cement A - M2 ………………….............................. 220

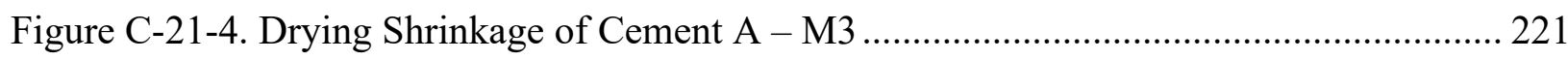

Figure C21-5. Drying Shrinkage of Cement A - M4 .......................................................... 221

Figure C-21-6. Drying Shrinkage of Cement A - M5 …………........................................... 222

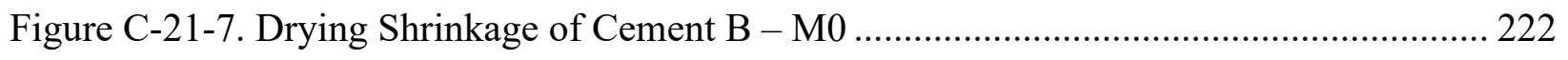

Figure C-21-8. Drying Shrinkage of Cement B - M1 ......................................................... 223

Figure C-21-9. Drying Shrinkage of Cement B - M2 ……………….................................... 223

Figure C-21-10. Drying Shrinkage of Cement B - M3 …………………………………........ 224

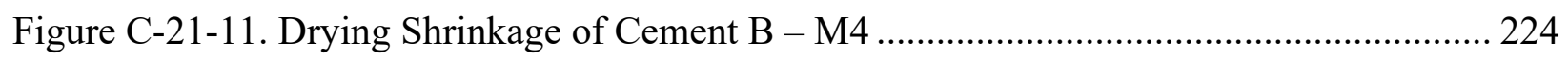

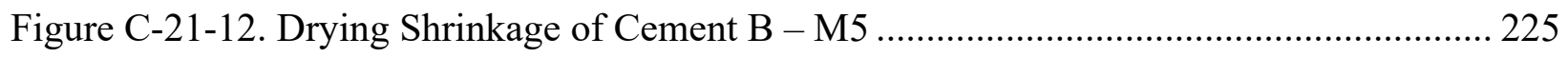

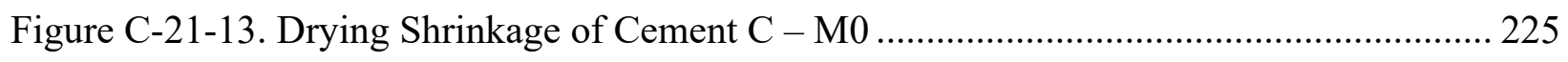

Figure C-21-14. Drying Shrinkage of Cement C - M1 ........................................................... 226

Figure C-21-15. Drying Shrinkage of Cement C - M2 ……................................................. 226

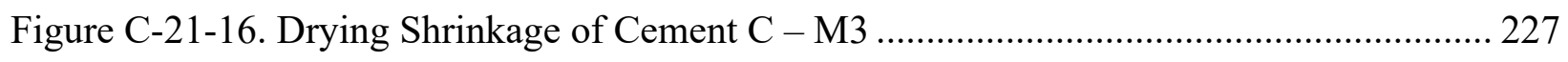

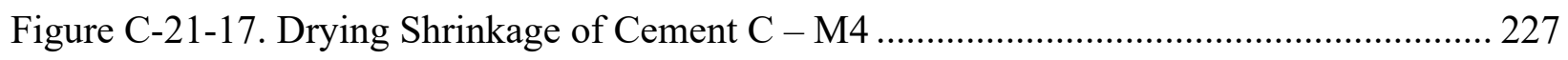

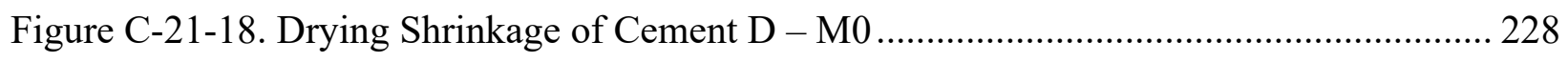

Figure C-21-19. Drying Shrinkage of Cement D - M1 ........................................................ 228

Figure C-21-20. Drying Shrinkage of Cement D - M2 ......................................................... 229

Figure C-21-21. Drying Shrinkage of Cement D - M3 ……………………………............... 229 
Figure C-21-22. Drying Shrinkage of Cement D - M4 ....................................................... 230

Figure C-21-23. Drying Shrinkage of Cement D - M5 ....................................................... 230

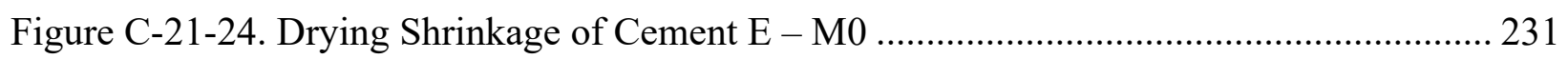

Figure C-21-25. Drying Shrinkage of Cement E - M1 ...................................................... 231

Figure C-21-26. Drying Shrinkage of Cement E - M2 ………............................................ 232

Figure C-21-27. Drying Shrinkage of Cement E - M3 …………………………................ 232

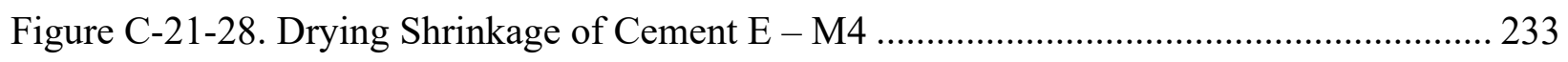

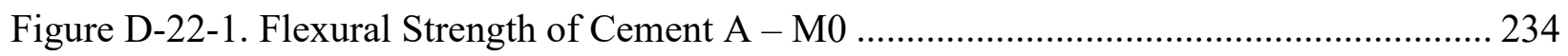

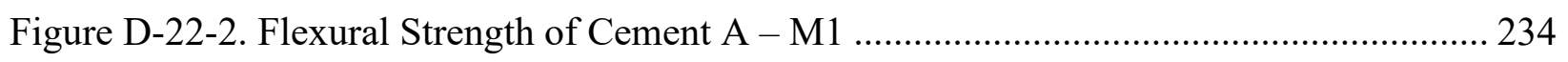

Figure D-22-3. Flexural Strength of Cement A - M2 …………......................................... 235

Figure D-22-4. Flexural Strength of Cement A - M3 …………………………………….... 235

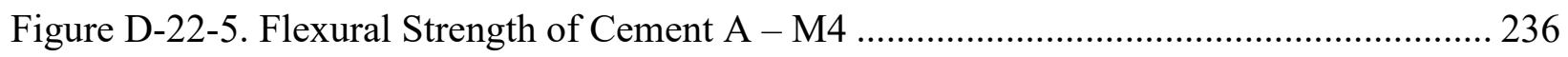

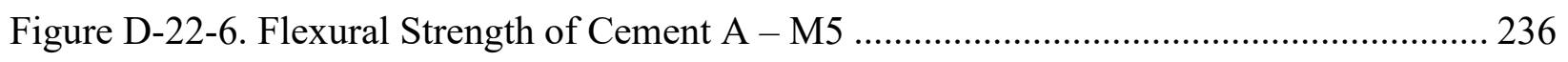

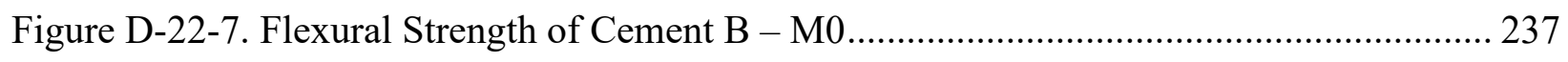

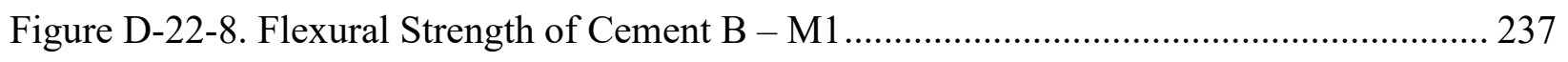

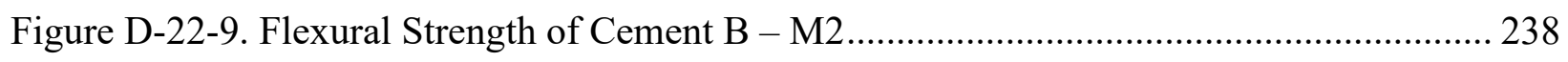

Figure D-22-10. Flexural Strength of Cement B - M3 …….................................................... 238

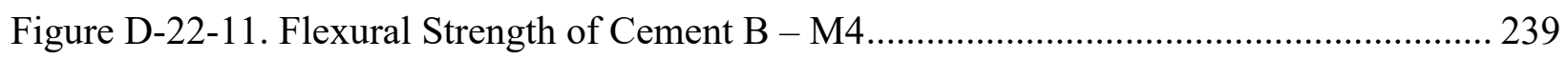

Figure D-22-12. Flexural Strength of Cement B - M5 ........................................................... 239

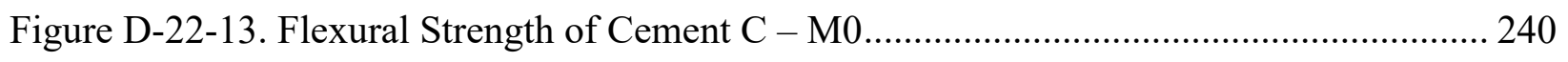

Figure D-22-14. Flexural Strength of Cement C - M1 ……………………........................... 240

Figure D-22-15. Flexural Strength of Cement C - M2 …………………………………........ 241

Figure D-22-16. Flexural Strength of Cement C - M3 …………………………………....... 241 
Figure D-22-17. Flexural Strength of Cement C - M4 ........................................................... 242

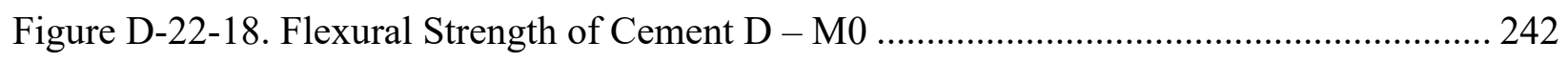

Figure D-22-19. Flexural Strength of Cement D - M1 _......................................................... 243

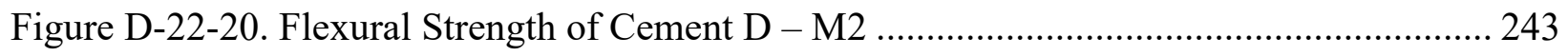

Figure D-22-21. Flexural Strength of Cement D - M3 ……………………………….......... 244

Figure D-22-22. Flexural Strength of Cement D - M4 ....................................................... 244

Figure D-22-23. Flexural Strength of Cement D - M5 ………………................................. 245

Figure D-22-24. Flexural Strength of Cement E - M0 ........................................................... 245

Figure D-22-25. Flexural Strength of Cement E - M1 ……………....................................... 246

Figure D-22-26. Flexural Strength of Cement E - M2 …...................................................... 246

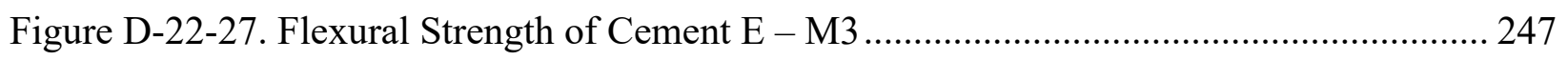

Figure D-22-28. Flexural Strength of Cement E - Mixture....................................................... 247

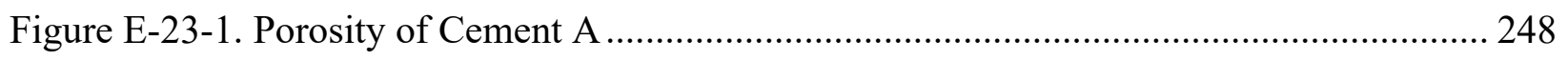

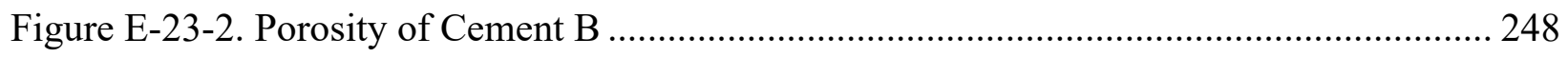

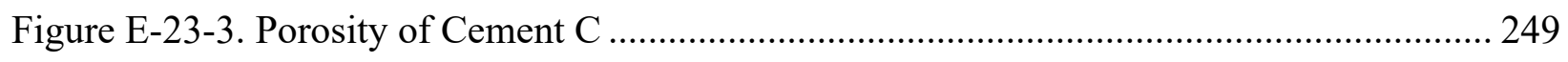

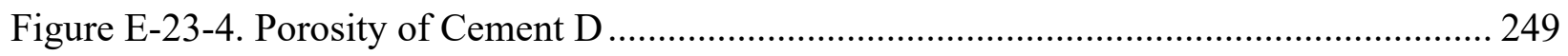

Figure E-23-5. Electrical resistivity of Cement A ……….................................................... 250

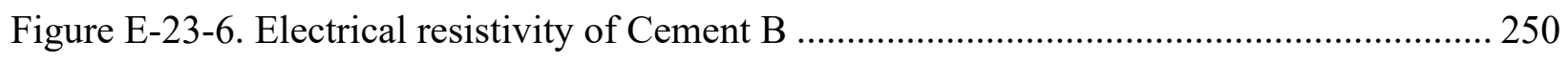

Figure E-23-7. Electrical resistivity of Cement C ................................................................... 251

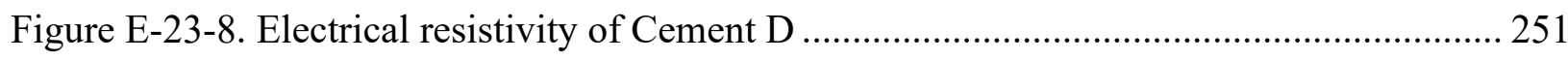

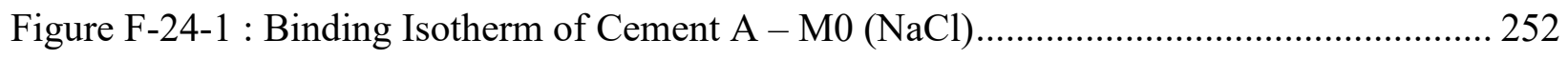

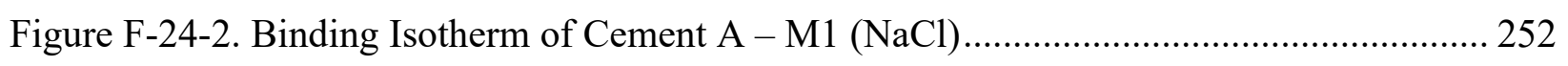

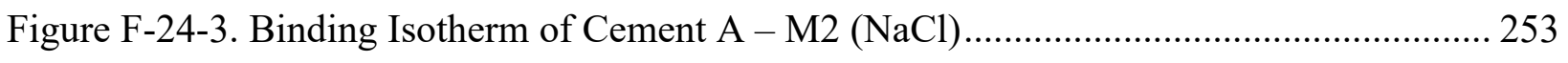




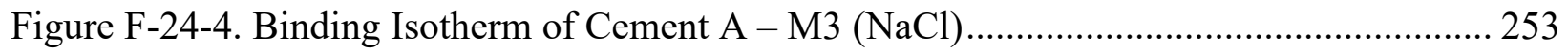

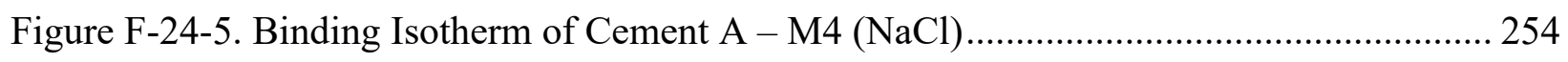

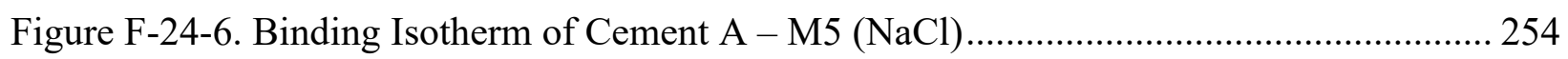

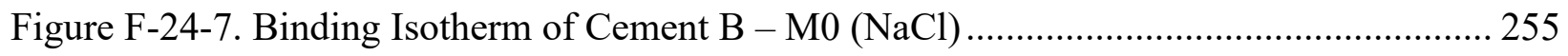

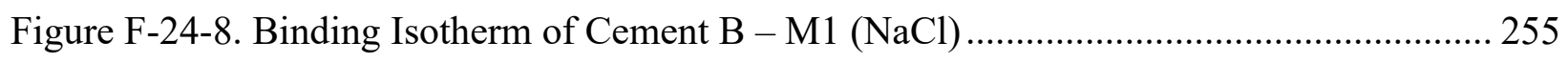

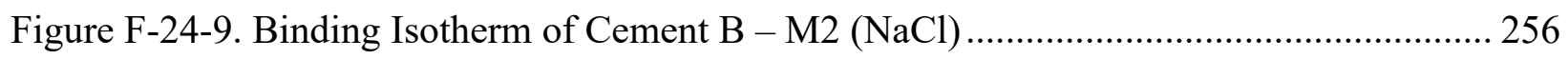

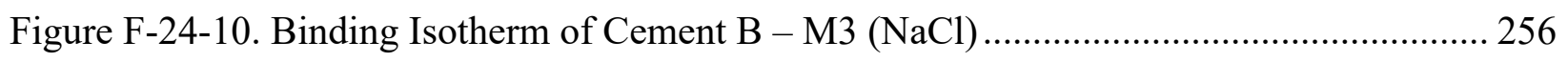

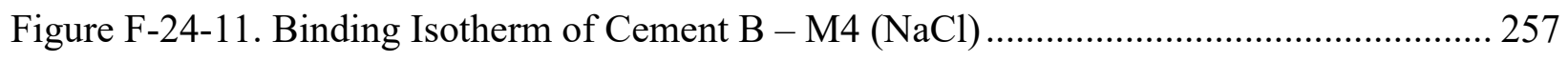

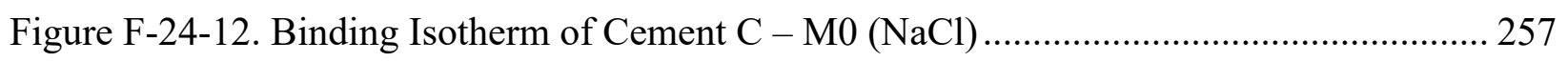

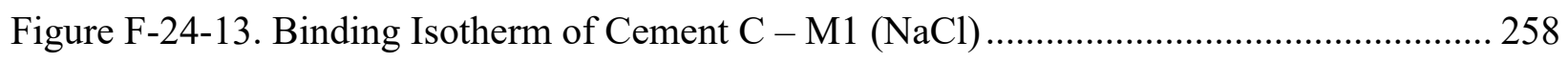

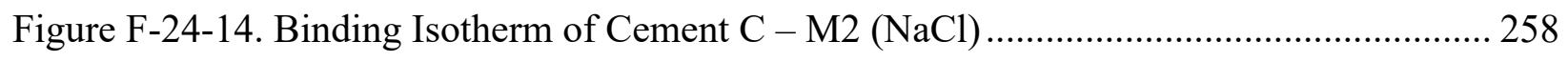

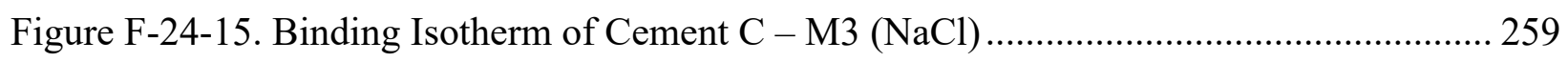

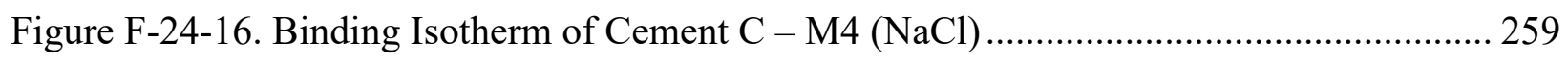

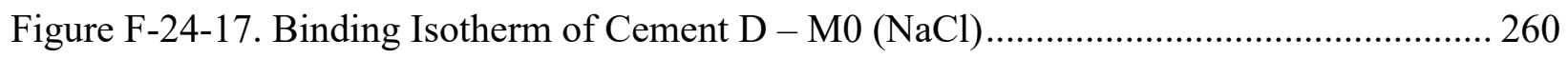

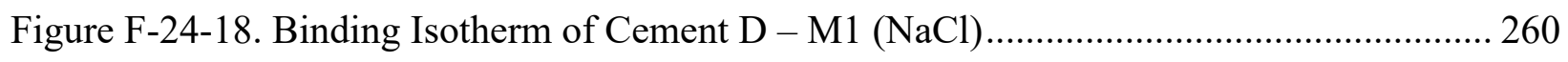

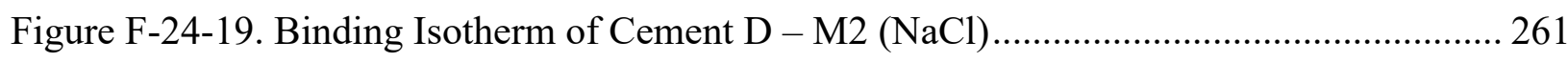

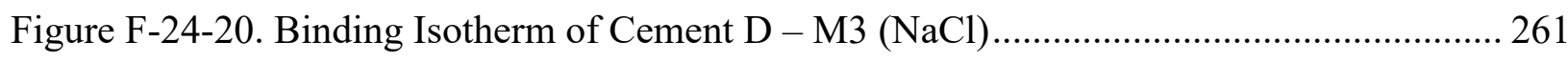

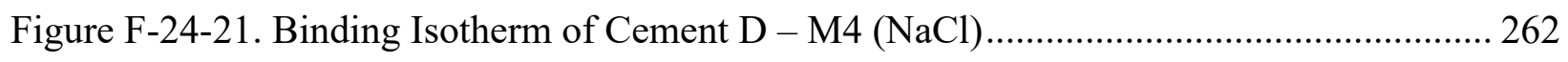

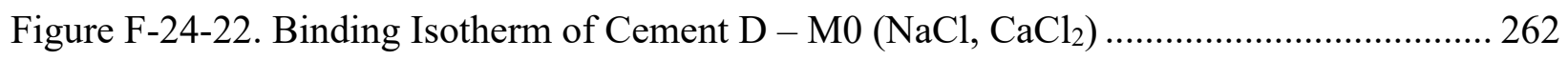

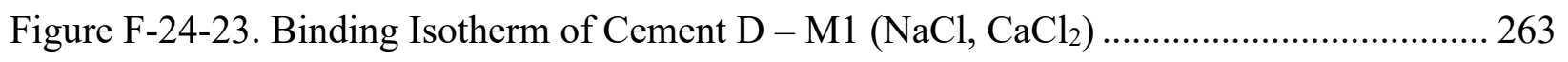

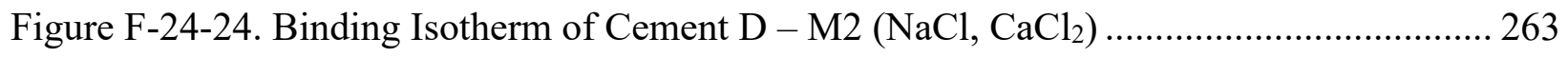

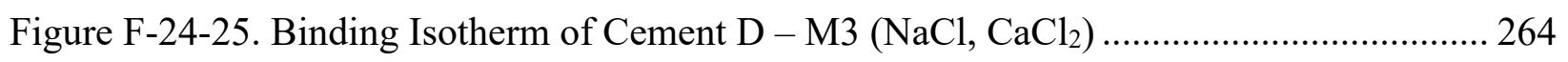

Figure F-24-26. Binding Isotherm of Cement D - $\mathrm{M} 4\left(\mathrm{NaCl}, \mathrm{CaCl}_{2}\right)$...................................... 264 


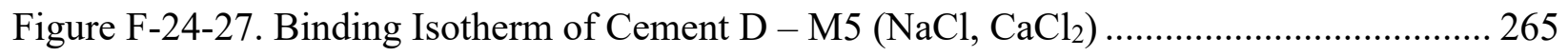

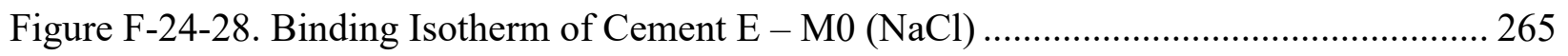

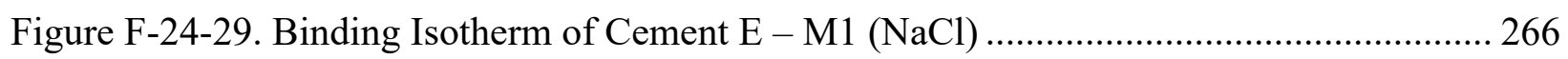

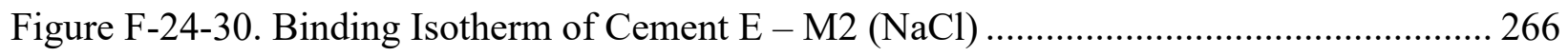

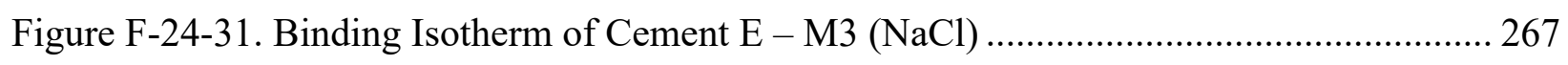

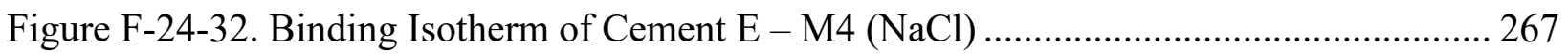


While the assembling of this report was a group effort, the significant contributing authors of each 2 section is noted below.

3 1. Executive summary - Prof. Jason Weiss

2. Background - Prof. Jason Weiss, Prof. Burkan Isgor, Prof. Jason Ideker, Prof. David Trejo

3. Constituent materials and mixtures considered in the experimental plan - Prof. Jason Weiss, Prof. Burkan Isgor, Prof. Jason Ideker, Prof. David Trejo

4. Constituent material characterization - Antara Choudhary, Prof. Jason Weiss, Prof. Burkan Isgor

5. Alkali-silica reactivity - Siva Teja Chopperla, Prof. Jason Ideker

6. Shrinkage and restrained shrinkage cracking - Antara Choudhary, Prof. Jason Weiss, Prof. Burkan Isgor

7. Mechanical properties - Antara Choudhary, Prof. Jason Weiss, Prof. Burkan Isgor

8. Transport properties - Antara Choudhary, Dr. Rita Ghantous, Prof. Jason Weiss, Prof. Burkan Isgor

9. Chloride binding - Nathalene Then, Dr. Rita Ghantous, Prof. Jason Weiss, Prof. Burkan Isgor

10. Resistance to Chloride Ingress in Concrete - Dr. Rita Ghantous, Nathalene Then, Gokul Vasudevan, Prof. Jason Weiss, Prof. Burkan Isgor

11. Corrosion of reinforcing steel - Gokul Dev Vasudevan, Prof. David Trejo

12. Air entrainment - Siva Teja Chopperla, Prof. Jason Ideker

13. External sulfate attack - Siva Teja Chopperla, Prof. Jason Ideker

14. Construction schedule - Gokul Vasudevan, Antara Choudary, Prof. David Trejo

15. Environmental impact - Dr. Sabbie Miller, Siva Teja Chopperla, Prof. Jason Ideker

16. Thermodynamic simulations - Keshav Bharadwaj, Prof. Jason Weiss, Prof. Burkan Isgor 17. Conclusions - Prof. Jason Weiss, Professor Burkan Isgor

The list of study advisory members is also noted below:

- Dr. Paul Tennis (PCA)

- Jacquelyn Wong S (CALTRANS)

- Zihui Li

- Joe R Harline

- Kirk McDonald

- S Lim

- Tom Tietz

- Joe Holland

- Patrick Lo

- Craig Knapp 


\section{Executive Summary}

38 CALTRANS does not currently allow Portland-Limestone Cements (PLC) to replace Ordinary Portland Cement (OPC) in concrete. PLC has been proposed for consideration in CALTRANS specifications due to potential benefits in reducing greenhouse gas (GHG) emissions. This report outlines a comprehensive plan to provide both experimental and computational analysis results to address whether PLC may replace OPC without loss of mechanical and durability performance of concrete materials and mixtures specific to California. The objective of this study was to provide data for CALTRANS to make informed decisions on whether specification changes to permit use of PLC would be appropriate. Additionally, the research team was asked to assess the impact of added limestone (LS) powder as an alternative to using ASTM C 595/AASHTO M 240 cement.

In general, the following observations were made (as compared to OPC):

The results indicate that PLCs can be used as a direct 1:1 substitution for OPC in concrete mixtures. It should also be noted that PLC can replace OPC in systems containing supplementary cementitious materials (SCM). Further, when PLCs are used with SCM, there can be a synergistic

71 behavior between the limestone and alumina that improves overall performance. As such, we 72 recommend that specifications that permit the use of OPC (ASTM C 150, AASHTO M 85) could 
73 also permit the use of PLC (ASTM C 595, AASHTO M 240). Specifications could also be 74 developed to permit the use of up to $10 \%$ limestone with OPC; however, some details will be 75 needed on the chemical and physical properties of the limestone to ensure its size (packing and 76 reaction) and chemical purity. 


\section{Background}

78 Portland-limestone cements (PLC) are not currently specified for use by CALTRANS. A plan was developed to determine whether the performance of concrete made with PLC using clinker used

80 for producing Type II/V cement would be adversely impacted as compared to a similar OPC-based 81 concrete in California [1]. These materials were evaluated both experimentally and 82 computationally. The ultimate goal of this work is to provide CALTRANS with data to make 83 informed decisions on the potential specification of PLC and indicate whether any limitations on 84 PLC should be considered.

85 ASTM C150 (AASHTO M 85) currently allows up to 5\% limestone in hydraulic cement. ASTM 86 C595 (AASHTO M 240) allows up to 15\% limestone in blended cements. There have been several 87 recent reviews on the use of PLC as a replacement for OPC [2]. This report will not repeat the 88 review of the literature performed in those studies. However, while several studies have been 89 conducted on the use of Type I OPC and PLC [3-6], there are limited studies on the use of PLC 90 made with the clinkers that are typical of those used in California for the production of Type II/V 91 cements.

92 An anonymous survey was submitted to ask state highway materials engineers whether they permit 93 the use of PLC. Currently, approximately $83 \%$ of the State Highway Agencies (SHAs) that 94 responded reported allowing PLC in a wide range of applications (one state did note that it did not 95 allow this in cement bases, and another did not allow this where Type II cement is used). Of the 9630 state representatives that responded, only $10 \%$ do not permit the use of PLC. Sixty-three 97 percent of the SHA have approximated that PLC is used less than $10 \%$ of the time; however, $7 \%$ 98 of the states suggesting it is used more than $65 \%$ of the time. Over $80 \%$ of the response reported 99 an interest (moderate to great) in learning more about the results of the CALTRANS study when 100 it is completed.

101 Furthermore, there are limited studies using the Type II/V clinkers used to make PLC with SCM. 102 This research addresses the need to provide CALTRANS with data to make informed decisions on 103 the potential specification of PLC or OPC + LS as an alternative to OPC. 
106 Mixture proportions investigated in this project have been developed based on the CALTRANS 107 specifications and after consultations with the study advisory committee (SAC), consisting of 108 CALTRANS members and industry. The constituent materials were also selected based on a 109 discussion with CALTRANS and industry. Samples were made using OPC and PLC cements 110 made from the same clinkers. Specifically, this study considered five clinkers (listed as A to E) 111 that result in five OPC cements and six PLC cements (one of the clinkers was used with two 112 limestone addition levels). Additional mixtures were made by adding a ground limestone powder 113 to mixtures as a replacement for cement (these mixtures are listed as OPC + LS). A water-to114 cementitious materials ratio $(\mathrm{w} / \mathrm{cm})$ of $0.40^{1}$ was selected for all mixtures. While it was initially 115 proposed in the contract that approximately 28 mixtures would be studied with less testing for 116 some test series, 86 different mixture compositions were studied in this project. The samples were 117 produced in the form of pastes (no aggregate), mortar (with sand), and concrete (with sand and 118 coarse aggregate).

119 Five OPCs and six corresponding PLCs were provided in large quantities by five different cement 120 producers, which have their manufacturing operations established to deliver cements to the 121 California region. These cements have been designated with letters A, B, C, D, and E throughout 122 the report in reference to the sources of the cements. The naming convention for the cementitious 123 materials refers to the parent plant (A through E); whether the mixture is OPC, PLC, or OPC+LS 124 (designated with $\mathrm{O}, \mathrm{P}$, or O+LS, respectively); the type of cement (II, $\mathrm{V}$ or IIV); and the 125 approximate percentage of LS used (e.g., L10 or 10LS). The details on the constituent materials 126 are provided later in this document (Section 4); however, Table 3.1 provides an example of the 127 cementitious materials nomenclature used in this report.

${ }^{1}$ ACI 211.7 and other guides note that limestone is not an SCM, although it is a cement ingredient when used in a PLC. Therefore, when added limestone is used, this ratio includes limestone as part of the denominator; i.e., w/(cm $+1 \mathrm{~s})$ $=0.40$. For simplicity, this ratio will be designated as $\mathrm{w} / \mathrm{cm}$ throughout the report. 
Table 3.1. Nomenclature of cements used in this study

\begin{tabular}{|c|c|c|c|c|}
\hline Parent clinker & Type & OPC & PLC & OPC + Limestone \\
\hline $\mathrm{A}$ & II & A_OII & A_L15 & A_OII+10LS \\
\hline $\mathrm{B}$ & $\mathrm{II} / \mathrm{V}$ & B_OIIV & B_L15 & B_OIIV+10LS \\
\hline \multirow[t]{2}{*}{$\mathrm{C}$} & \multirow[t]{2}{*}{ V } & \multirow[t]{2}{*}{ C_OV } & C_L10 & \multirow[t]{2}{*}{ C_OV+10LS } \\
\hline & & & C_L15 & \\
\hline $\mathrm{D}$ & $\mathrm{V}$ & D_OV & D_L15 & D_OV+10LS \\
\hline $\mathrm{E}$ & $\mathrm{II} / \mathrm{V}$ & E_OIIV & E_11 & E_OIIV+10LS \\
\hline
\end{tabular}

130 The study predominantly uses low CaO fly ash (FA1), slag cement (SL), silica fume (SF), and natural pozzolan (NP) as SCMs. FA1 has a $\mathrm{CaO}$ content $<10 \%$. Fly ash from another source (FA2) with $\mathrm{CaO}$ content between $10 \%-15 \%$ was procured only for ASR testing. Details on these materials are provided in Section 4.

134 In 2018, members of the OSU team met with CALTRANS and industry members and determined 135 that the following mixtures would be studied in in this project. Mixtures 0 to 5 (denoted as M0 to 136 M5) were identified as general binders, with a sixth mixture specifically added for the ASR study. 137 The specific details of these mixtures are provided in Section 6.

138 General binders:

139 M0. No SCM

$140 \quad$ M1. 25\% Fly Ash replacement - $(\mathrm{CaO} \sim 8.5 \%)$, consistent throughout except mixture 6

141 M2. 20\% Fly Ash + 5\% Silica Fume

142 M3. 50\% Slag

$143 \quad$ M4. 25\% Fly Ash + 25\% Slag

144 M5. 25\% Natural Pozzolan (NP)

146 Binder for the ASR study:

147 M6. 25\% Fly Ash $2(\mathrm{CaO} \sim 12.5 \%)$

148 The mixture proportions were sent to the committee for review, and approval for these mixture 149 proportions was received. These mixture proportions are presented later in the Section 6. The 150 aggregate used in the concrete mixture was also characterized, and trial mixtures were evaluated 151 for workability and consistency. 


\section{Constituent Materials Characterization}

\section{$154 \quad 4.1 \quad$ Research Objective}

155 This section aims to provide materials characterization for the materials used throughout this study. 156 This includes the chemical compositions and particle size distributions of cementitious materials, 157 and information on the heat of hydration of the clinkers, and the degree of reactivity of the SCM.

\subsection{Background/Literature Review}

159 Material characterization was performed to classify the reference binder materials used in the 160 remainder of the study. Commercially available OPCs, PLCs, limestone powders, SCMs were 161 analyzed [7]. Specifically, this section of the report provides background on chemical 162 composition, particle size, hydration heat, and pozzolanic reactivity.

163 This project examines the use of SCM such as fly ash, slag, silica fume. CALTRANS widely use 164 these to improve long term durability while reducing the carbon footprint of the concrete. The 165 replacement of clinker by SCM contributes to reducing greenhouse gas emissions and energy 166 consumption during cement manufacturing $[8,9]$.

167 There is a general agreement in the scientific community that when limestone is used with 168 hydraulic cement at contents up to $15 \%$, it generally has a similar mechanical performance as OPC. 169 Shaker et al. [10] demonstrated that concrete made with CEM II (A-L) has similar mechanical resistance to the one made with OPC CEM I 42.5N. A minor difference in the mechanical performance between CEM I 42.5R and 15\% PLC concrete has been observed in the study of Dhir et al. [11]. Chen et al. [12] found that the addition of limestone in a quantities less than $8 \%$ increases concrete strength. Meddah et al. [13] reported that the addition of $15 \%$ limestone as a

174 partial replacement of OPC had an insignificant effect on the modulus of elasticity (5\% reduction compared with OPC). De Weerdt et al. [14] observed that up to $15 \%$ of OPC could be replaced by limestone powder without impairing the compressive strength development. When more than 15\% of the OPC is replaced by a limestone, a reduction in the mechanical strength has been observed at later ages along with a decrease in the modulus of elasticity [11,15-18] due to the dilution effect. The similarity between OPC and PLC is particularly true in North America, where limestone is interground (typically) to result in a finer mixture (of the OPC and limestone) that is explicitly designed to have similar performance. Huang et al.[19] proved that the replacement of part of the

182 OPC with limestone in ultra-high performance concrete mixtures (using silica fume) improves the 183 mechanical properties of concrete as well as its hydration.

184 It should be noted that there can be some benefits of adding limestone to cement such as an increase 185 in the compressive strength and a decrease in porosity [20]. Limestone can participate to some 186 extent in chemical reaction with aluminum-rich phases in OPC and SCM by forming 
187 carboaluminates $[6,21,22]$, which can reduce the induction period, setting time and stabilize

188 ettringite which can lead to the reduction in the volume of hydrates and porosity [16, 23-27]. These

189 potential aluminate reactions that cause a decrease in porosity and an increase in compressive

190 strength were the basis for the limitation of limestone addition to $15 \%$. Early studies note that the

191 porosity and strength in PLCs with a $12 \%-15 \%$ limestone content is nearly equal to the porosity

192 and strength for a cement of the same chemistry containing no limestone [20].

193 Menéndez et al.[28] reported benefits from combining limestone with blast furnace slag and OPC 194 to improve the early and later age compressive strength of mortar or concrete. At early ages (e.g., 195 during the first 48h), the hydration is improved by limestone because the limestone particles can 196 act as nucleation sites for cement hydrates [29-31]. Sun et al. [32] showed that the limestone is 197 preferentially ground to be among the finest particles because it is softer than conventional clinker. 198 This resulted in high performing blends of OPC, limestone, and slag [28].

199 Bentz et al. [33] demonstrated that high volume fly ash benefited explicitly from the addition of 200 finely ground limestone, which helped it offset the retardation of hydration and delayed setting 201 time. As a result, the use of fine limestone powder in high volume fly ash mixtures may be very 202 promising for ready-mix producer, however, the fineness of the limestone is crucial.

203 These studies show that there is a general agreement in the literature that the replacement of cement 204 with limestone (when finely ground) can be beneficial for the mechanical properties, hydration, 205 and microstructural properties of cementitious materials when SCM are used. Nevertheless, to the 206 best of the authors' knowledge, most of the studies in the literature focused on type I or II cement. 207 This study aims to determine the impact of the replacement of OPC with limestone in a wider 208 range of cement types on both the mechanical and chemical characteristics of cementitious 209 materials.

\subsection{An Overview of the Experimental Characterization Performed for the Cementitious Powders}

212 This section describes testing for chemical composition using $\mathrm{x}$-ray fluorescence, loss on ignition, 213 particle size distribution of the cement, and SCM. Five OPCs, 6 PLCs, the limestone powder, and 214 SCM were evaluated. The heat of hydration was assessed for the mixtures shown in section 4.6 215 (mixtures 0 to 5) using a fixed w/cm. Finally, the reactivities of the SCM were assessed using the 216 Pozzolanic Reactivity Test described in [34-37]. 


\subsection{Chemical Composition and Loss on Ignition}

\section{4.4.1 Experimental Methods}

219 A PANalytical Epsilon 3XLE bench-top energy dispersive X-Ray fluorescence (XRF) 220 spectrometer (Figure 4-1a) was used to analyze the chemical composition of the cement and SCMs 221 used in this study. The XRF samples were prepared in the form of fused beads. For each sample 222 type (cement or SCM), a calibration was performed with known standards to quantify the elements 223 studied [7] accurately. The calibration involved measuring high purity samples with known 224 concentrations and establishing a relationship between the measured intensities and 225 concentrations. The XRF was demonstrated to comply with ASTM C114-18 [38] standards for 226 chemical analysis of hydraulic cement.

227 Before the XRF analysis, the loss on ignition (LOI) was obtained for each sample by heating $3 \mathrm{~g}$ 228 of the samples in a furnace up to $970{ }^{\circ} \mathrm{C}$ for 3 hours in accordance with ASTM C114-18 [38]. It 229 should be noted that the LOI at $750^{\circ} \mathrm{C} \pm 50^{\circ} \mathrm{C}$ was the same as the LOI at $970^{\circ} \mathrm{C}$ as there was no 230 mass loss between $750^{\circ} \mathrm{C}$ and $970^{\circ} \mathrm{C}$. The mass loss observed in the sample after being heated to $231970{ }^{\circ} \mathrm{C}$ gives the LOI of the cement or fly ash. The cement beads were prepared by combining $1 \mathrm{~g}$ 232 of cement and $5 \mathrm{~g}$ of flux in LeNeo Fluxer for 20 minutes. While the cement was fused in its original 233 state, a slightly different procedure was used for the SCM. To prepare the SCM beads, $0.55 \mathrm{~g}$ of 234 the SCM (obtained after the LOI test) was combined with $5.5 \mathrm{~g}$ of flux (consisting of $49.75 \%$ 235 lithium metaborate, $49.75 \%$ lithium tetraborate, and $0.50 \%$ lithium iodide) in a platinum flat 236 bottomed crucible. The reduced SCM and flux were mixed gently but thoroughly using a spatula 237 and then transferred to LeNeo Fluxer for fusion at a temperature of $1450{ }^{\circ} \mathrm{C}$.

238 The device uses a predetermined fusing program for general oxides for 25 minutes. The clear 239 cement/SCM beads after fusion were further analyzed in the XRF spectrometer, shown in Figure $240 \quad 4-1$. 


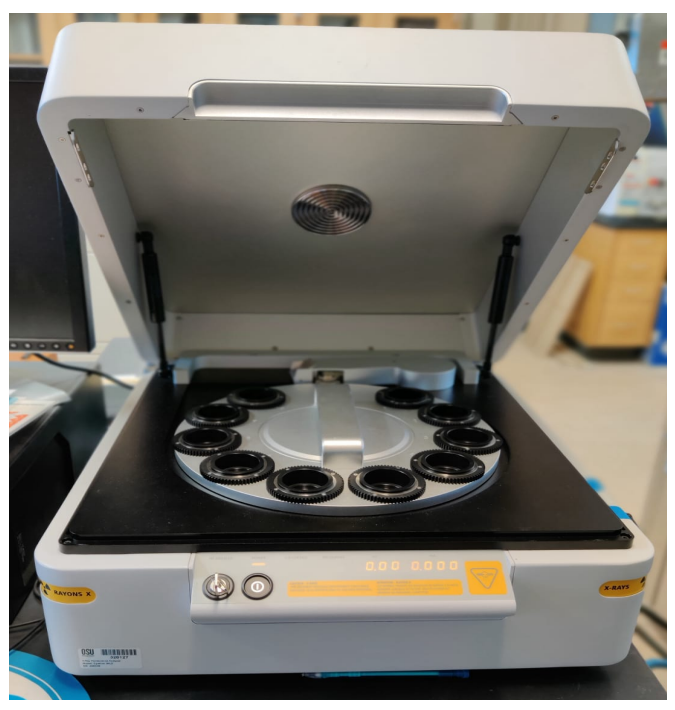

(a)

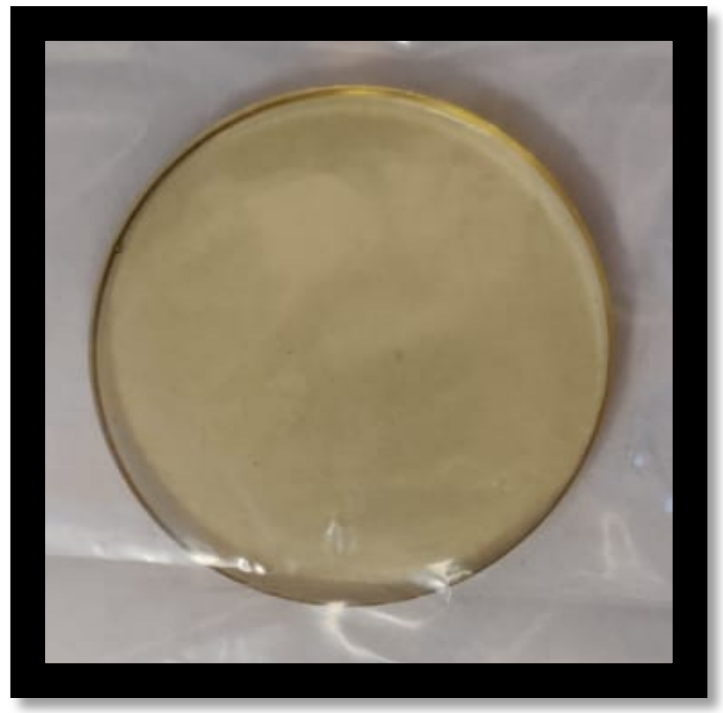

(b)

Figure 4-1. (a) PANalytical Epsilon 3XLE XRF spectrometer (b) a cement bead

\subsubsection{Typical Experimental Measurement and Interpretation}

The chemical composition obtained from the XRF spectrometer provides the mass of each elemental oxide (in g) per 100g of cement/SCM used to make the bead. For the PLC, the limestone content was also measured. The cement's chemical oxide composition was used to classify the parent clinker type based on ASTM C150.

The companies producing the cements A, B, D, and E also sent out samples of the limestone rocks, which are added to the clinker during the grinding phase to produce the OPC and PLCs. The $\mathrm{CaCO}_{3}$ contents of these limestones was determined by grinding the limestone rocks into fine powder and determining the mass of $\mathrm{CO}_{2}$ released when the powder is heated from $600^{\circ}$ $800^{\circ} \mathrm{C}$ using Thermo-Gravimetric Analysis (TGA) [38-41] $]^{2}$. Stoichiometry dictates that $100 \mathrm{~g}$ of calcite - $\left(\mathrm{CaCO}_{3}\right)$ releases $40 \mathrm{~g}$ of $\mathrm{CO}_{2}$ upon decomposition. The received cements were also analyzed for mass loss between $600^{\circ}-800^{\circ} \mathrm{C}$ using TGA, and their limestone content was determined.

\footnotetext{
${ }^{2} \mathrm{The} \mathrm{CaCO}_{3}$ content of the rocks was also measured using a furnace (mass loss between $550^{\circ} \mathrm{C}$ to $950^{\circ} \mathrm{C}$; see ASTM C114) and the $\mathrm{CaCO}_{3}$ contents obtained from the TGA approach, and the furnace approach were within $1 \%$ of each other.
} 


\subsubsection{Experimental Results and Discussion}

257 As determined from XRF, the chemical oxide composition and limestone content of all the cements

258 received from Companies A, B, C, D, and E are provided in Table 4.1.

259

260

Table 4.1a. Chemical composition of OPCs and PLCs from Company A and B

\begin{tabular}{|c|c|c|c|c|}
\hline & \multicolumn{2}{|c|}{ Cement Source A } & \multicolumn{2}{|c|}{ Cement Source B } \\
\hline$\%$ & A_OII & A_L15 & B_OIIV & B_L15 \\
\hline $\mathrm{SiO}_{2}$ & 19.95 & 18.38 & 20.54 & 18.46 \\
\hline $\mathbf{A l}_{2} \mathbf{O}_{3}$ & 3.95 & 3.62 & 4.05 & 3.71 \\
\hline $\mathrm{Fe}_{2} \mathrm{O}_{3}$ & 2.28 & 2.07 & 3.62 & 3.46 \\
\hline $\mathrm{CaO}$ & 63.32 & 61.69 & 61.72 & 60.45 \\
\hline MgO & 1.43 & 1.33 & 2.52 & 2.28 \\
\hline $\mathrm{SO}_{3}$ & 2.55 & 2.48 & 1.80 & 1.71 \\
\hline LOI & 2.71 & 6.42 & 1.96 & 6.75 \\
\hline $\mathrm{Na}_{2} \mathrm{O}$ & 0.21 & 0.22 & 0.17 & 0.13 \\
\hline $\mathrm{K}_{2} \mathrm{O}$ & 0.48 & 0.44 & 0.69 & 0.63 \\
\hline $\mathrm{TiO}_{2}$ & 0.19 & 0.18 & 0.22 & 0.20 \\
\hline $\mathbf{P}_{2} \mathbf{O}_{5}$ & 0.10 & 0.10 & 0.11 & 0.10 \\
\hline $\mathrm{ZnO}$ & 0.01 & 0.01 & 0.03 & 0.03 \\
\hline $\mathrm{Mn}_{2} \mathrm{O}_{3}$ & 0.07 & 0.07 & 0.07 & 0.06 \\
\hline Cl & 0.003 & 0.003 & 0.007 & 0.004 \\
\hline Limestone & 4.31 & 13.32 & 1.79 & 13.11 \\
\hline Clinker Type & II & II & II $/ \mathrm{V}$ & $\mathrm{II} / \mathrm{V}$ \\
\hline
\end{tabular}


Table 4.1b. Chemical composition of OPCs and PLCs from Company C, D, and E

\begin{tabular}{|c|c|c|c|c|c|c|c|}
\hline & \multicolumn{3}{|c|}{ Cement Source C } & \multicolumn{2}{|c|}{ Cement Source D } & \multicolumn{2}{|c|}{ Cement Source E } \\
\hline$\%$ & C_OV & C_L10 & C_L15 & D_OV & D_L15 & E_OIIV & E_L11 \\
\hline $\mathrm{SiO}_{2}$ & 19.45 & 18.77 & 18.36 & 20.54 & 18.46 & 19.98 & 19.38 \\
\hline $\mathbf{A l}_{2} \mathbf{O}_{3}$ & 3.68 & 3.65 & 3.50 & 4.05 & 3.71 & 3.72 & 3.61 \\
\hline $\mathrm{Fe}_{2} \mathrm{O}_{3}$ & 3.35 & 3.18 & 3.01 & 3.62 & 3.46 & 3.49 & 3.30 \\
\hline $\mathrm{CaO}$ & 60.32 & 59.43 & 58.37 & 61.72 & 60.45 & 61.97 & 61.65 \\
\hline MgO & 4.45 & 4.33 & 4.24 & 2.52 & 2.28 & 1.71 & 1.73 \\
\hline $\mathrm{SO}_{3}$ & 2.73 & 3.03 & 2.91 & 1.80 & 1.71 & 2.66 & 2.70 \\
\hline LOI & 2.53 & 4.37 & 6.12 & 1.96 & 6.75 & 2.35 & 4.71 \\
\hline $\mathrm{Na}_{2} \mathrm{O}$ & 0.22 & 0.22 & 0.24 & 0.17 & 0.13 & 0.21 & 0.23 \\
\hline $\mathrm{K}_{2} \mathrm{O}$ & 0.36 & 0.27 & 0.30 & 0.69 & 0.63 & 0.51 & 0.46 \\
\hline $\mathrm{TiO}_{2}$ & 0.23 & 0.22 & 0.20 & 0.22 & 0.20 & 0.22 & 0.21 \\
\hline $\mathbf{P}_{2} \mathrm{O}_{5}$ & 0.06 & 0.05 & 0.05 & 0.11 & 0.10 & 0.13 & 0.13 \\
\hline $\mathrm{ZnO}$ & 0.02 & 0.02 & 0.02 & 0.03 & 0.03 & 0.01 & 0.01 \\
\hline $\mathrm{Mn}_{2} \mathrm{O}_{3}$ & 0.06 & 0.05 & 0.05 & 0.07 & 0.06 & 0.05 & 0.05 \\
\hline Cl & 0.006 & 0.005 & 0.005 & 0.007 & 0.004 & 0.003 & 0.006 \\
\hline Limestone & 4.2 & 10 & 14 & 3.48 & 13.71 & 5 & 11.11 \\
\hline $\begin{array}{c}\text { Clinker } \\
\text { Type }\end{array}$ & V & V & V & V & V & $\mathrm{II} / \mathrm{V}$ & $\mathrm{II} / \mathrm{V}$ \\
\hline
\end{tabular}

264 The SCMs used to produce cement paste/mortar or concrete based on CALTRANS specifications 265 were also characterized, and the results are shown in Table 4.2. 
Table 4.2. Chemical composition of SCMs and limestone

\begin{tabular}{|c|c|c|c|c|c|c|c|c|c|}
\hline$\%$ & $\begin{array}{c}\text { Fly } \\
\text { Ash } 1\end{array}$ & Slag & $\begin{array}{l}\text { Natural } \\
\text { Pozzolan }\end{array}$ & $\begin{array}{l}\text { Silica } \\
\text { Fume }\end{array}$ & $\begin{array}{c}\text { Fly } \\
\text { Ash } 2\end{array}$ & $\begin{array}{l}\text { Lime- } \\
\text { stone }\end{array}$ & $\begin{array}{c}\text { Lassen } \\
\text {-ite* }\end{array}$ & $\begin{array}{c}\text { Bassal } \\
\text {-ite* }\end{array}$ & $\begin{array}{c}\text { Blended } \\
\text { Natural } \\
\text { Pozzolan } \\
*\end{array}$ \\
\hline $\mathrm{SiO}_{2}$ & 51.86 & 31.58 & 66.42 & 95.88 & 47.15 & 2.93 & 67.76 & 74.13 & 30.05 \\
\hline $\mathbf{A l}_{2} \mathbf{O}_{3}$ & 21.70 & 12.13 & 11.98 & 0.69 & 16.57 & 0.79 & 14.70 & 13.39 & 5.89 \\
\hline $\mathrm{Fe}_{2} \mathrm{O}_{3}$ & 5.04 & 0.55 & 0.86 & 0.12 & 5.88 & 0.41 & 6.34 & 0.84 & 1.46 \\
\hline $\mathrm{CaO}$ & 8.61 & 41.34 & 4.06 & 0.70 & 12.54 & 86.50 & 2.11 & 0.91 & 3.02 \\
\hline MgO & 2.58 & 6.97 & 0.18 & 0.26 & 4.80 & 5.74 & 0.84 & 0.09 & 0.37 \\
\hline $\mathrm{SO}_{3}$ & 0.78 & 3.75 & 0.19 & 0.15 & 0.60 & 0.13 & 1.03 & 0.09 & 0.18 \\
\hline LOI & 1.42 & 0.00 & 4.09 & 4.30 & 2.43 & 42.27 & 8.71 & 3.33 & 8.73 \\
\hline $\mathrm{Na}_{2} \mathrm{O}$ & 2.58 & 0.24 & 3.57 & 0.16 & 3.65 & 0.14 & 1.85 & 3.68 & 1.23 \\
\hline $\mathbf{K}_{2} \mathbf{O}$ & 1.45 & 0.28 & 4.35 & 0.49 & 1.72 & 0.12 & 1.70 & 4.69 & 1.36 \\
\hline $\mathrm{TiO}_{2}$ & 1.19 & 0.47 & 0.09 & 0.01 & 1.17 & 0.04 & 0.58 & 0.09 & 0.14 \\
\hline $\mathbf{P}_{2} \mathbf{O}_{5}$ & 0.23 & 0.00 & 0.00 & 0.05 & 0.24 & 0.09 & 0.19 & 0.01 & 0.07 \\
\hline $\mathrm{ZnO}$ & 0.02 & 0.00 & 0.00 & 0.06 & 0.01 & 0.00 & 0.01 & 0.00 & 0.00 \\
\hline $\mathrm{Mn}_{2} \mathrm{O}_{3}$ & 0.03 & 0.19 & 0.07 & 0.04 & 0.09 & 0.02 & 0.15 & 0.07 & 0.04 \\
\hline Cl & 0.01 & 0.00 & 0.02 & 0.01 & 0.00 & 0.01 & 0.01 & 0.02 & 0.01 \\
\hline
\end{tabular}

267

268

269

270

271

272

273

274

275

276

277

278

279

280

281

282

283

284

285

*Note that the chemical compositions of Lassenite, Bassalite, and Blended Natural Pozzolans are mentioned in the table as CALTRANS had provided these materials for testing; however, they are not used anywhere else in the report.

Table 4.1 illustrates that the project has only Type II, Type II/V, and Type V cements, which provide moderate to high sulfate resistance to comply with CALTRANS specifications. Determination of the materials' chemical composition shows that the OPC and PLCs from the same parent company have similar chemical composition when the limestone content is accounted for as expected. The OPCs had limestone contents between 3\%-6\% (ASTM C 150, AASHTO M 85 permit up to $5 \%$ limestone). The PLCs had limestone contents ranging from 10\%-15\% (which was consistent with the values noted by the producer). Table 4.1 provides the chemical composition of the cement used in this study, while Table 4.2 provides the chemical composition of the SCMs used in this study. This information is valuable to interpret the remainder of the experiments performed in this project and computational modeling.

\subsection{Particle Size Analysis}

\subsubsection{Experimental Methods}

Particle size analysis was performed on both the cement and SCMs using a Horiba LA-920 particle size analyzer shown in Figure 4-2. Isopropyl alcohol (IPA) was used as the solvent to disperse the particles in the front of a laser-lamp assembly, which measures the particle size distribution using laser light scattering. 
Before each test, the device's solution pathway was rinsed several times with IPA to remove any contaminants following the standard operating procedure. Rinsing and draining cycles were repeated until there was no observed scatter (solid particles scatter the light) on the real-time scatter sensor (frequency-diameter plot) on the software (i.e., the solid impurities had been flushed out and only the solvent remained). An initial alignment function was then performed on the device to realign the laser-lamp assembly axis with the measuring station. After initial alignment, the lamp and laser power raised to a full $100 \%$ [42]. The system was reset before every reading to the

293 baseline solution signal.

294 Approximately 3 to $5 \mathrm{~g}$ of powder was added in small increments of $0.5 \mathrm{~g}$ through the inlet hole into the solvent below. The solution was then circulated several times to prevent the clumping of powder particles together. The powder was added to the solvent and circulated until the laser's power and the lamp was reduced to be in the range of 80 to $90 \%$, and a visible scatter was observed on the real-time scatter monitor. After this, the actual measurement was performed for 30 seconds, and the particle size distribution was measured.

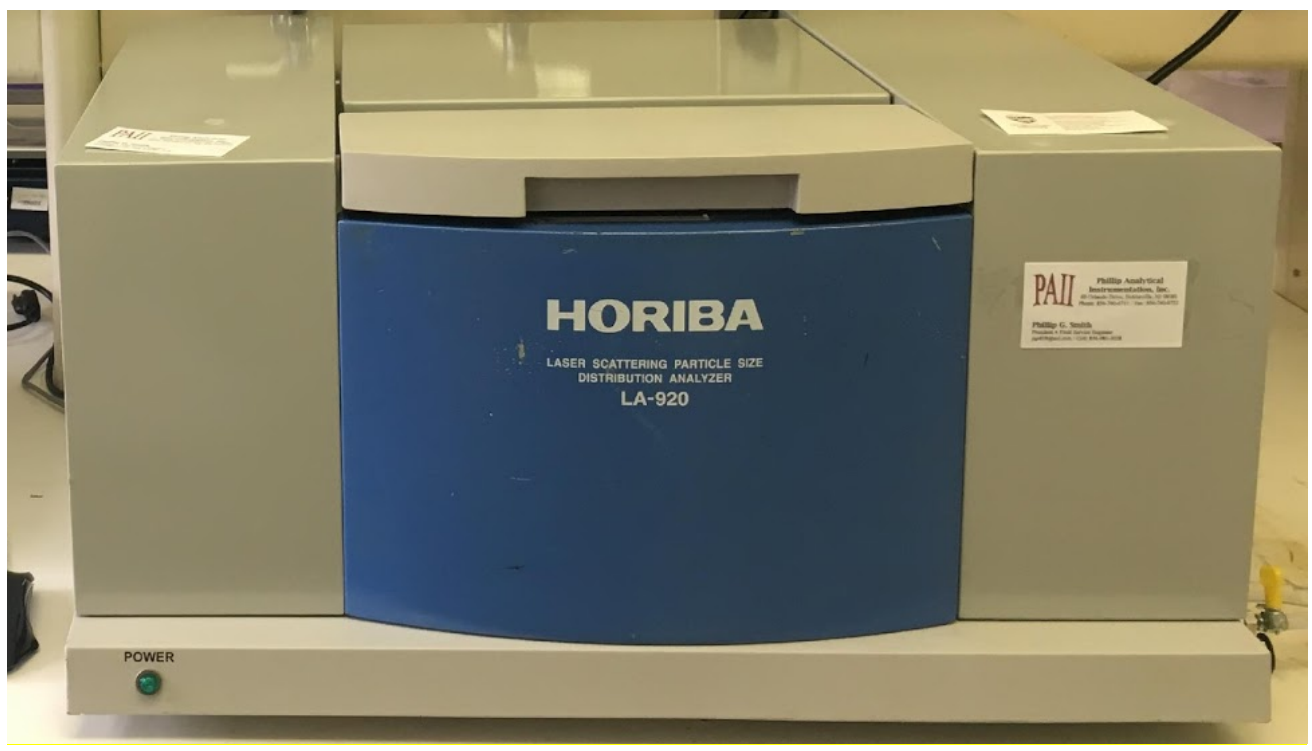

\section{2}

Figure 4-2. Horiba LA-920 Particle Size Analyzer

\subsubsection{Typical Experimental Measurement and Interpretation}

A typical particle size distribution is shown in Figure 4-3. The y-axis describes the relative frequency of the volume fraction (\%) of particles in a unit volume of measured material detected at a given size (diameter) in Figure 4-3a. In contrast, the y-axis describes the cumulative volume fraction of particles with a size (diameter) lower than a particular size in a unit volume of measured material in Figure 4-3b. The x-axis of these plots is the average size (diameter) of the powder 
308 particles in microns. The particle sizes of evenly ground materials like cements usually follow a 309 normal distribution; however, bimodal distributions are sometimes observed in SCMs. Figure 4$3103 \mathrm{~b}$ also shows the $\mathrm{d} 50$ and the $\mathrm{d} 90$ values of the particle size distribution, which is defined as the $31150^{\text {th }}$ and $90^{\text {th }}$ percentile particle size respectively.

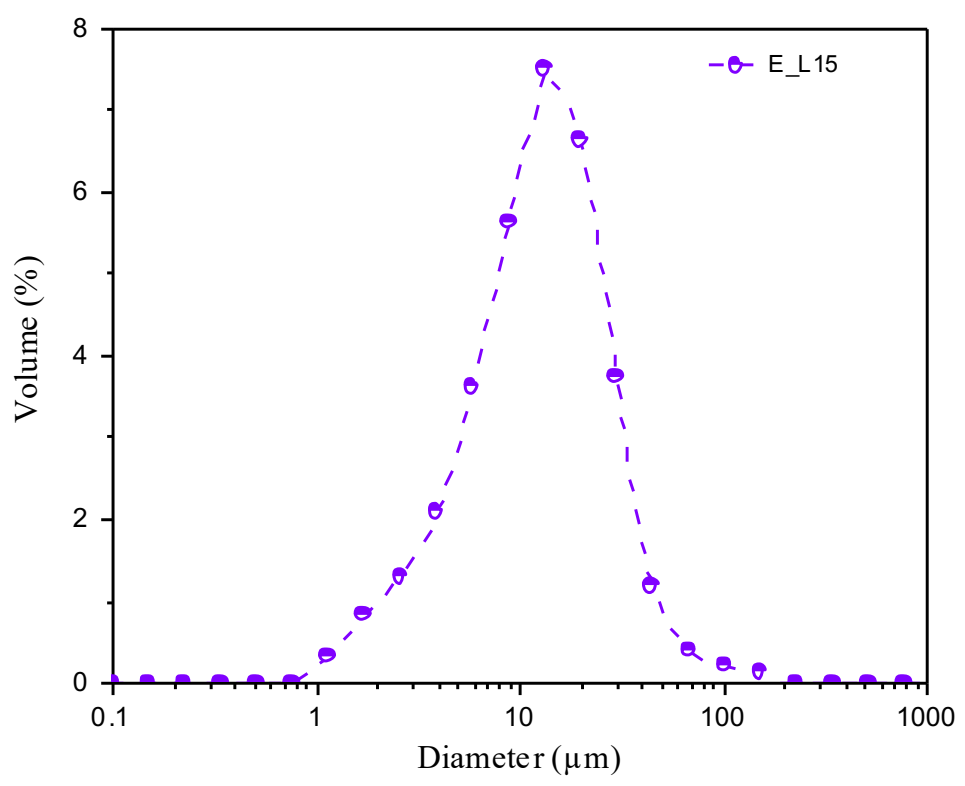

(a)

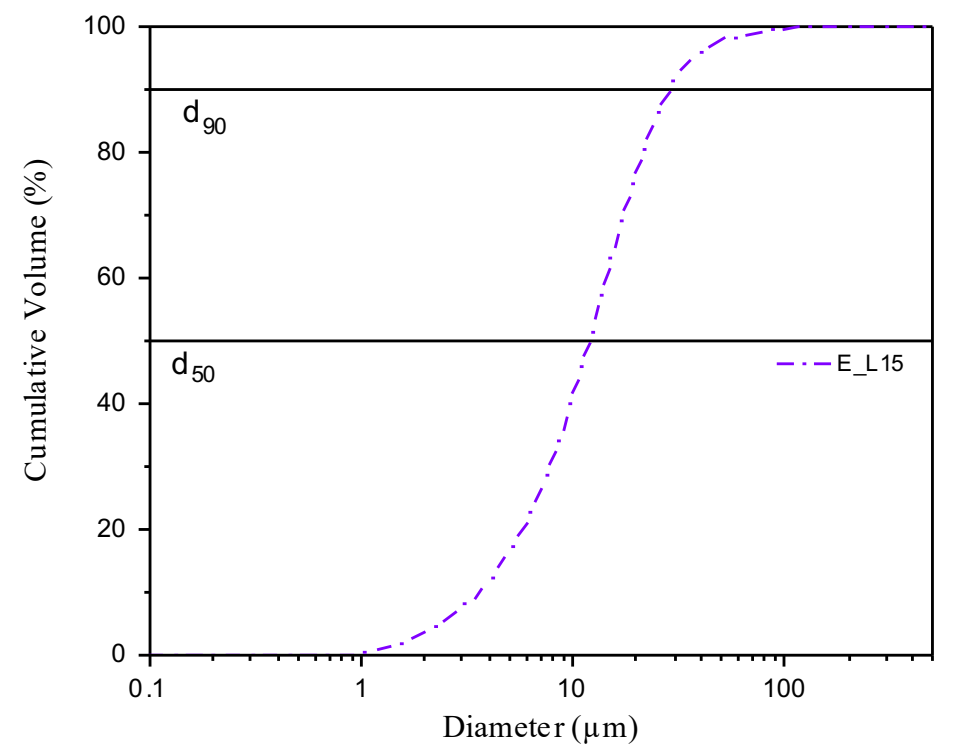

(b)

312 Figure 4-3. Typical particle size distributions of cements showing (a) volume vs. diameter 


\section{$314 \quad$ 4.5.3 Experimental Results and Discussion}

315 The results of particle size analysis of all the cements and SCMs are presented in Figure 4-4 (a)316 (d). It should be noted that in general, the OPC and PLC all have a similar particle size distribution 317 with the exception of A_L15, which appears to have a proportion of larger clinker particles, and 318 E_OIIV has a higher concentration of more finely ground particles. As expected, silica fume was 319 the finest and the slag was the coarsest cementitious material,

320 Table 4.3 summarizes the $\mathrm{d} 50$ and $\mathrm{d} 90$ values of these materials. The comparison of particle size 321 distributions for the cements shows that when the $\mathrm{d}_{50}$ is used as a measure of particle size are 322 similar (+/-5\%). Cement A has a coarser particle size distribution than PLC; however, in general, 323 the average particle size of PLC is smaller than the OPC.

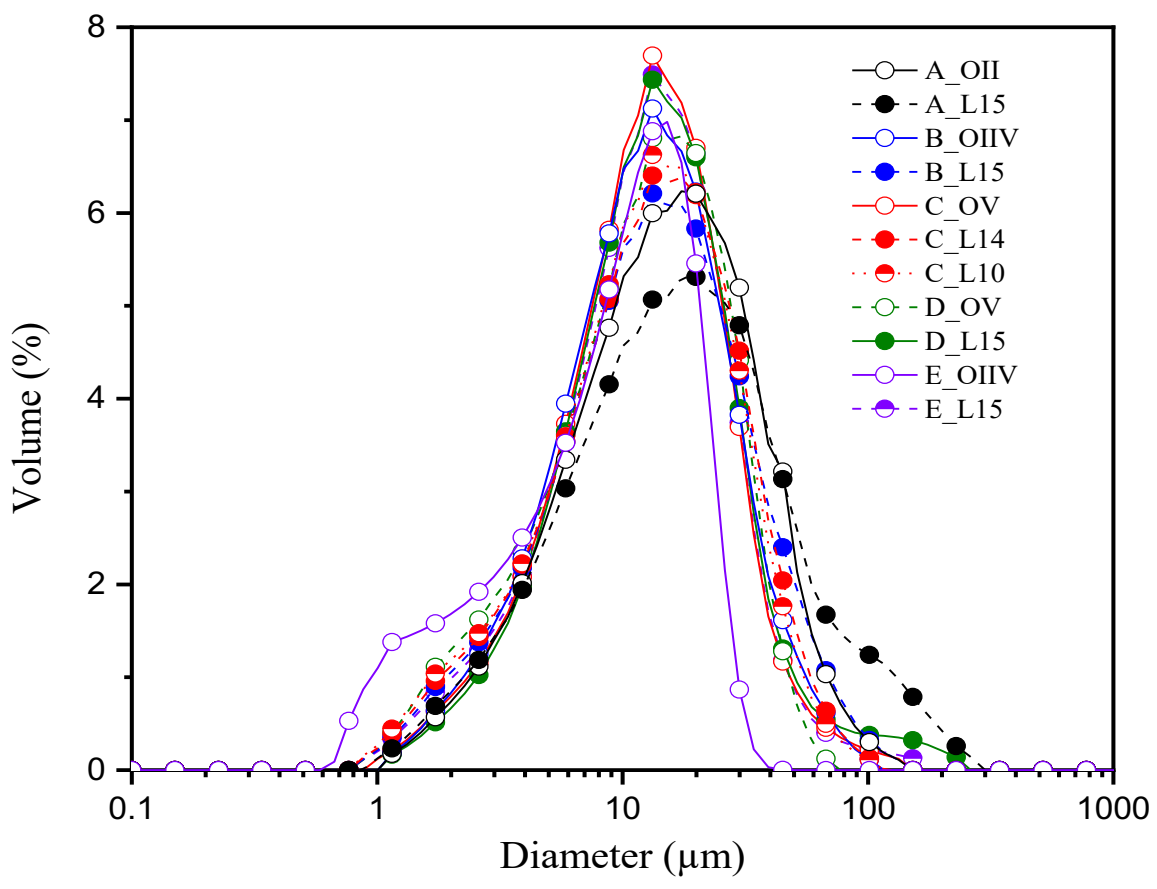

(a) 


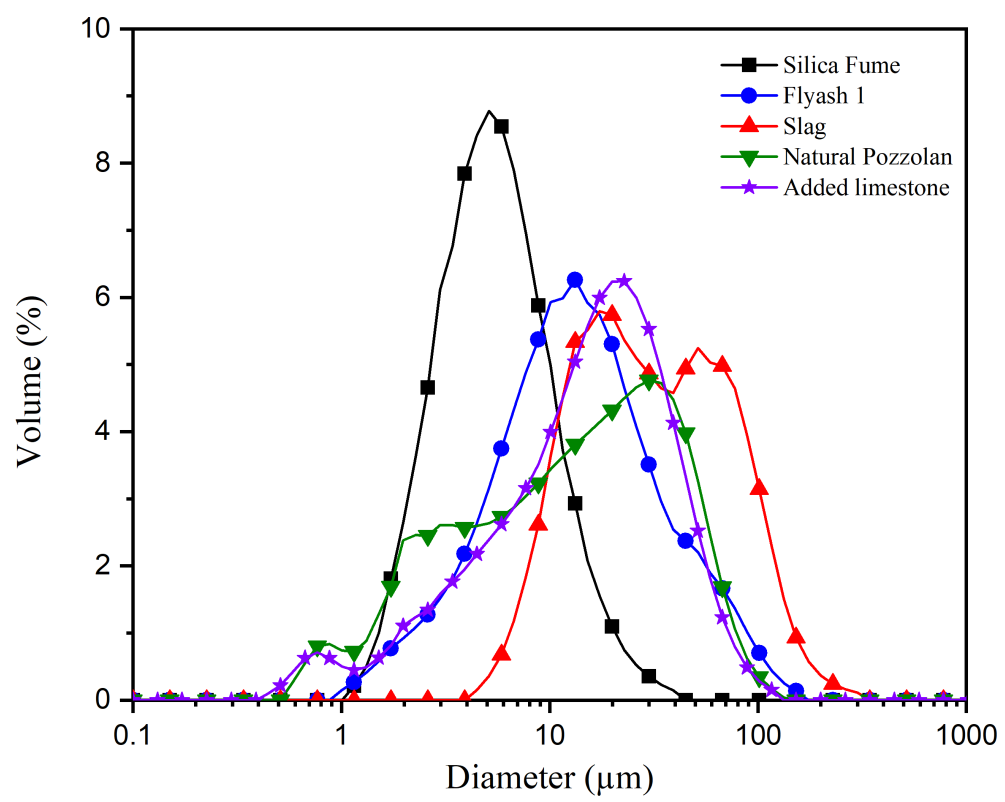

(b)

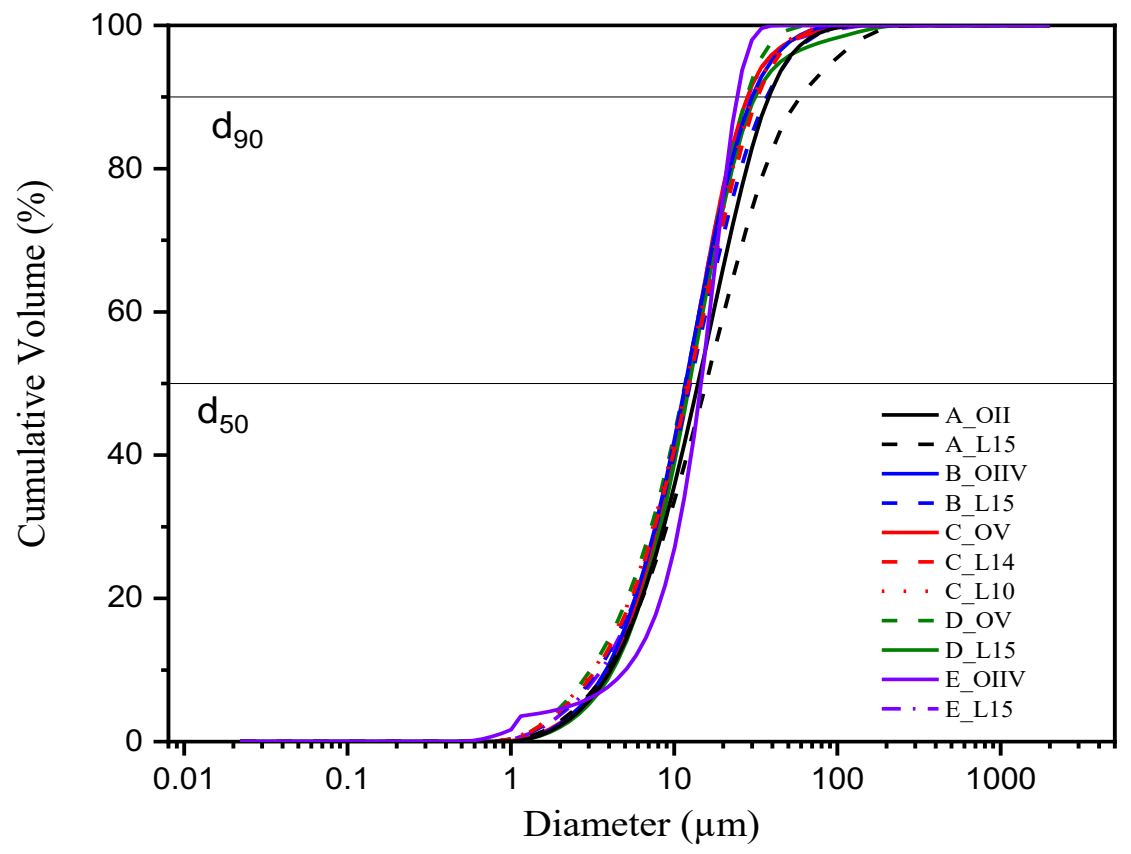

(c) 


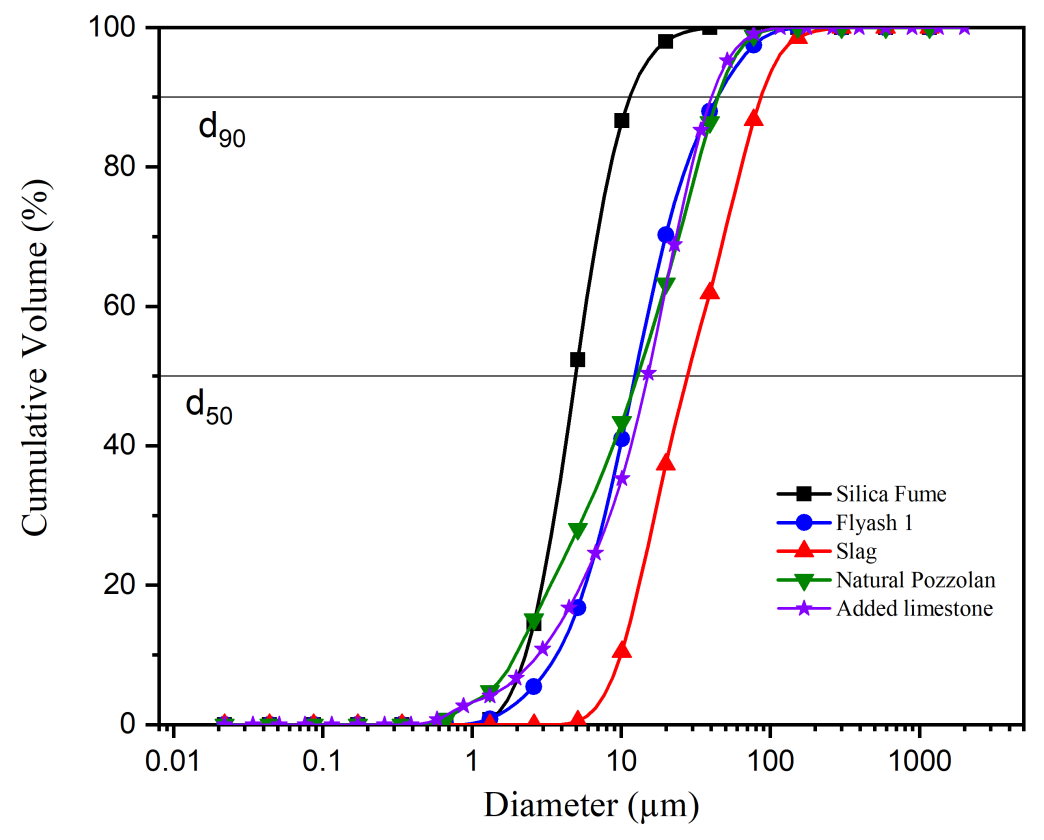

(d)

325 Figure 4-4. Particle size distributions depicting volume fraction vs particle diameter of (a)

SCMs.

Table 4.3. $\mathrm{d}_{50}$ and $\mathrm{d}_{90}$ values of tested materials

\begin{tabular}{|l|c|c|}
\hline & $\mathbf{d s}_{\mathbf{0}}(\boldsymbol{\mu m})$ & $\mathbf{d} \mathbf{0}(\boldsymbol{\mu m})$ \\
\hline A_OII & 10.52 & 27.89 \\
\hline A_L15 & 15.70 & 59.18 \\
\hline B_OIIV & 11.77 & 29.94 \\
\hline B_L15 & 12.57 & 37.06 \\
\hline C_OV & 12.53 & 27.90 \\
\hline C_L10 & 12.33 & 32.53 \\
\hline C_L14 & 11.95 & 30.75 \\
\hline D_OV & 12.45 & 31.46 \\
\hline D_L15 & 11.72 & 27.88 \\
\hline E_OIIV & 11.15 & 30.98 \\
\hline E_L15 & 11.87 & 28.40 \\
\hline Fly Ash 1 & 13.01 & 44.62 \\
\hline Silica Fume & 4.96 & 11.28 \\
\hline Slag & 29.08 & 87.81 \\
\hline Natural Pozzolan & 13.11 & 44.28 \\
\hline Addded Limestone & 15.03 & 40.27 \\
\hline
\end{tabular}




\subsection{Heat of Hydration}

\subsubsection{Experimental Methods}

332 The influence of limestone replacement on the reaction rate was measured using heat release values 333 of the OPC, PLC, OPC+SCM, and PLC+SCM systems. This involved performing cement paste 334 mixtures tests in isothermal calorimeter (TAM Air, TA Instruments shown in Figure 4-5) at $23^{\circ} \mathrm{C}$ $335 \pm 0.1^{\circ} \mathrm{C}$ for seven days.

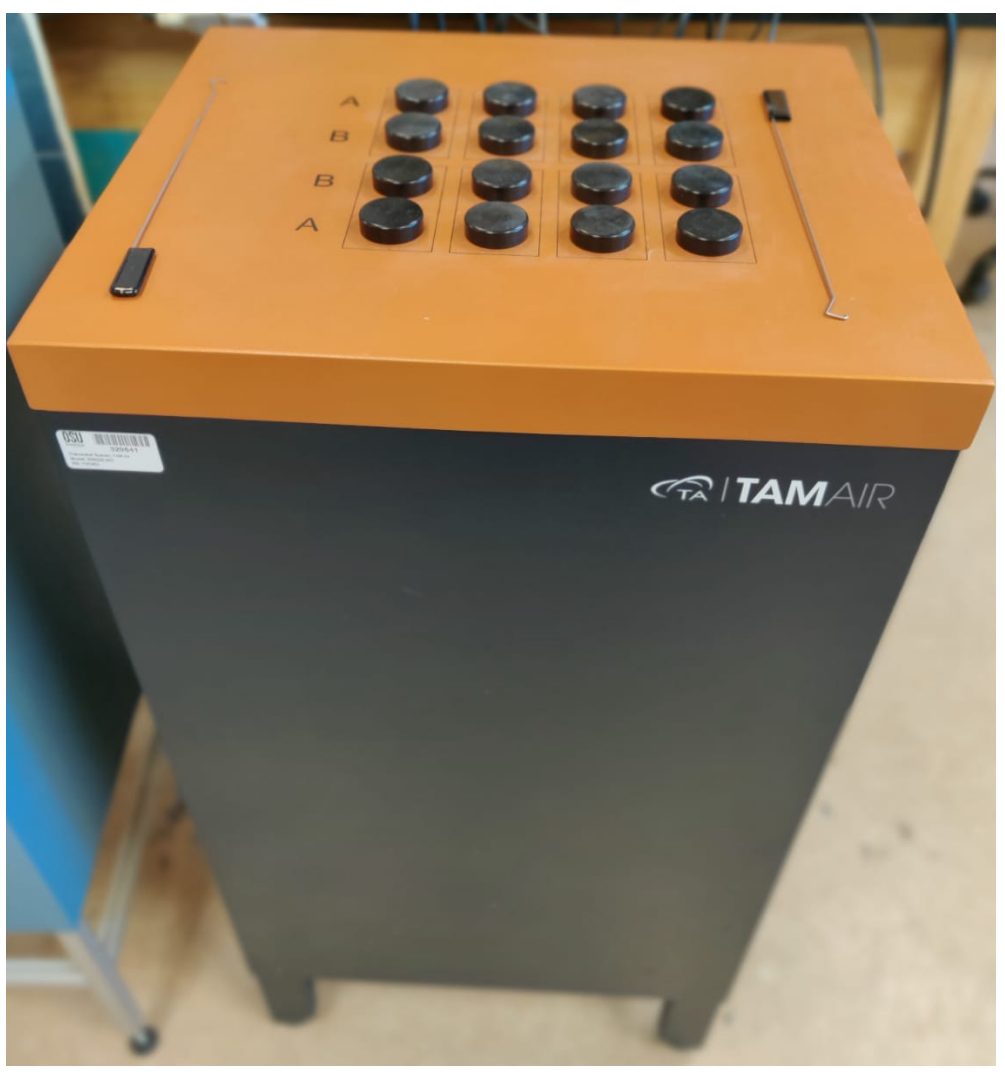
samples

339 The cement paste samples were mixed in a vacuum mixer at 250 revolutions per minute for four 340 minutes and immediately transferred to a glass ampoule. These ampoules were then sealed and 341 lowered into the isothermal calorimeter stabilized at $23^{\circ} \mathrm{C} \pm 0.1{ }^{\circ} \mathrm{C}$. After a short delay (twenty 342 minutes) for baseline correction, the heat values were recorded continuously for seven days. The 
343 paste mixture proportions are described in Table 4.4. The $\mathrm{w} / \mathrm{cm}$ for all mixtures was kept constant 344 at 0.40 .

Table 4.4. Mixture proportions of cement paste mixtures

\begin{tabular}{|l|l|l|l|l|l|l|l|}
\hline & $\begin{array}{l}\text { Mixture short name } \\
\text { example using } \\
\text { B_OIIV }\end{array}$ & Cement $^{\#}$ & Fly Ash & Slag & $\begin{array}{l}\text { Silica } \\
\text { fume }\end{array}$ & $\begin{array}{l}\text { Natural } \\
\text { Pozzolan }\end{array}$ & water \\
\hline M0 & B_OIIV & 200 & ---- & ---- & ---- & ---- & 80 \\
\hline M1 & B_OIIV-25FA1 & 150 & 50 & ---- & ---- & ---- & 80 \\
\hline M2 & B_OIIV-20FA1-5SF & 150 & 40 & ---- & 10 & ---- & 80 \\
\hline M3 & B_OIIV-50SL & 100 & ---- & 100 & ---- & ---- & 80 \\
\hline M4 & B_OIIV-25FA1-25SL & 100 & 50 & 50 & ---- & ---- & 80 \\
\hline M5 & B_OIIV-25NP & 150 & ---- & ---- & ---- & 50 & 80 \\
\hline
\end{tabular}

$347 *$ All the mixture proportion values are in $\mathrm{g}$

348 \# Cement: OPC, PLC, OPC $+10 \%$ limestone

349 All the mixtures described in Table 4.4 were performed using all five cements (A, B, C, D, and E) 350 with their respective OPC, PLC, and OPC $+10 \%$ LS systems. The experimental matrix describing 351 the progress is shown in Table 4.5. Each mixture in Table 4.5 was tested twice, and an average is 352 reported. 
Table 4.5. Experimental matrix of heat of reaction tests

\begin{tabular}{|l|l|l|l|l|l|l|}
\hline & M0 & M1 & M2 & M3 & M4 & M5 \\
\hline A_OII & $\sqrt{ }$ & $\sqrt{ }$ & $\sqrt{ }$ & $\sqrt{ }$ & $\sqrt{ }$ & $\sqrt{ }$ \\
\hline A_L15 & $\sqrt{ }$ & $\sqrt{ }$ & $\sqrt{ }$ & $\sqrt{ }$ & $\sqrt{ }$ & $\sqrt{ }$ \\
\hline A_OII+10LS & $\sqrt{ }$ & $\sqrt{ }$ & $\sqrt{ }$ & $\sqrt{ }$ & $\sqrt{ }$ & $\sqrt{ }$ \\
\hline B_OIIV & $\sqrt{ }$ & $\sqrt{ }$ & $\sqrt{ }$ & $\sqrt{ }$ & $\sqrt{ }$ & $\sim$ \\
\hline B_L15 & $\sqrt{ }$ & $\sqrt{ }$ & $\sqrt{ }$ & $\sqrt{ }$ & $\sqrt{ }$ & $\sim$ \\
\hline B_OIIV+10LS & $\sqrt{ }$ & $\sqrt{ }$ & $\sqrt{ }$ & $\sqrt{ }$ & $\sqrt{ }$ & $\sim$ \\
\hline C_OV & $\sqrt{ }$ & $\sqrt{ }$ & $\sqrt{ }$ & $\sqrt{ }$ & $\sqrt{ }$ & $\sim$ \\
\hline C_L10 & $\sqrt{ }$ & $\sqrt{ }$ & $\sqrt{ }$ & $\sqrt{ }$ & $\sqrt{ }$ & $\sim$ \\
\hline C_L14 & $\sqrt{ }$ & $\sqrt{ }$ & $\sqrt{ }$ & $\sqrt{ }$ & $\sqrt{ }$ & $\sim$ \\
\hline C_OV+10LS & $\sqrt{ }$ & $\sqrt{ }$ & $\sqrt{ }$ & $\sqrt{ }$ & $\sqrt{ }$ & $\sim$ \\
\hline D_OV & $\sqrt{ }$ & $\sqrt{ }$ & $\sqrt{ }$ & $\sqrt{ }$ & $\sqrt{ }$ & $\sqrt{ }$ \\
\hline D_L15 & $\sqrt{ }$ & $\sqrt{ }$ & $\sqrt{ }$ & $\sqrt{ }$ & $\sqrt{ }$ & $\sqrt{ }$ \\
\hline D_OV+10LS & $\sqrt{ }$ & $\sqrt{ }$ & $\sqrt{ }$ & $\sqrt{ }$ & $\sqrt{ }$ & $\sqrt{ }$ \\
\hline E_OIIV & $\sqrt{ }$ & $\sqrt{ }$ & $\sqrt{ }$ & $\sqrt{ }$ & $\sqrt{ }$ & $\sim$ \\
\hline E_11 & $\sqrt{ }$ & $\sqrt{ }$ & $\sqrt{ }$ & $\sqrt{ }$ & $\sqrt{ }$ & $\sim$ \\
\hline E_OIIV+10LS & $\sqrt{ }$ & $\sqrt{ }$ & $\sqrt{ }$ & $\sqrt{ }$ & $\sqrt{ }$ & $\sim$ \\
\hline
\end{tabular}

$\sqrt{ }$ completed

$355 \sim$ not tested

\subsubsection{Typical Experimental Measurement and Interpretation}

358 Figure 4-6 shows a typical heat release (cumulative heat of hydration) determined using an 359 isothermal calorimeter. The vertical axis represents the total heat released values from each sample 360 normalized by the per unit mass of binder (powder), while the horizontal axis represents the age 361 of the cement paste sample in days. The tests were run for approximately seven days. The systems 362 with higher overall heat release have a greater extent of reaction than the systems with a lower 363 heat release. For the systems shown in Figure 4-6, Binder 1 has undergone exhibits a greater extent 364 of reaction than Binder 2 at the end of seven days. Several factors can influence this mixture to 365 exhibit a greater extent of reaction, such as the proportion of active clinker in the system, the 366 fineness, or the materials' reactivity. 


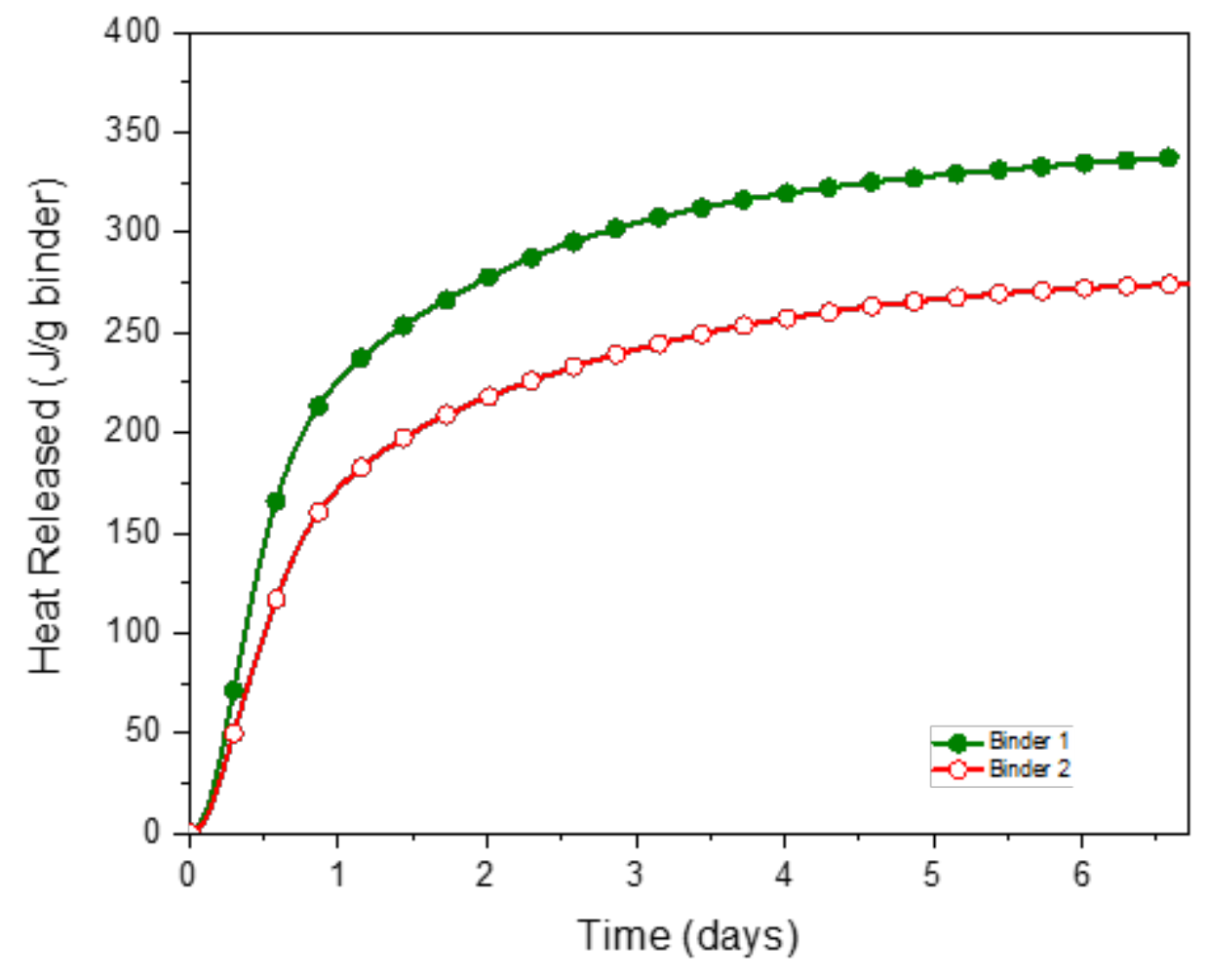

369 Figure 4-7 (a)-(f) shows the heat of reaction results comparing OPC, PLC, and OPC $+10 \% \mathrm{LS}$ 370 systems for cement $\mathrm{A}$ for the different mixture proportions. The heat of reaction results of the 371 remaining cements is provided in Appendix B. 


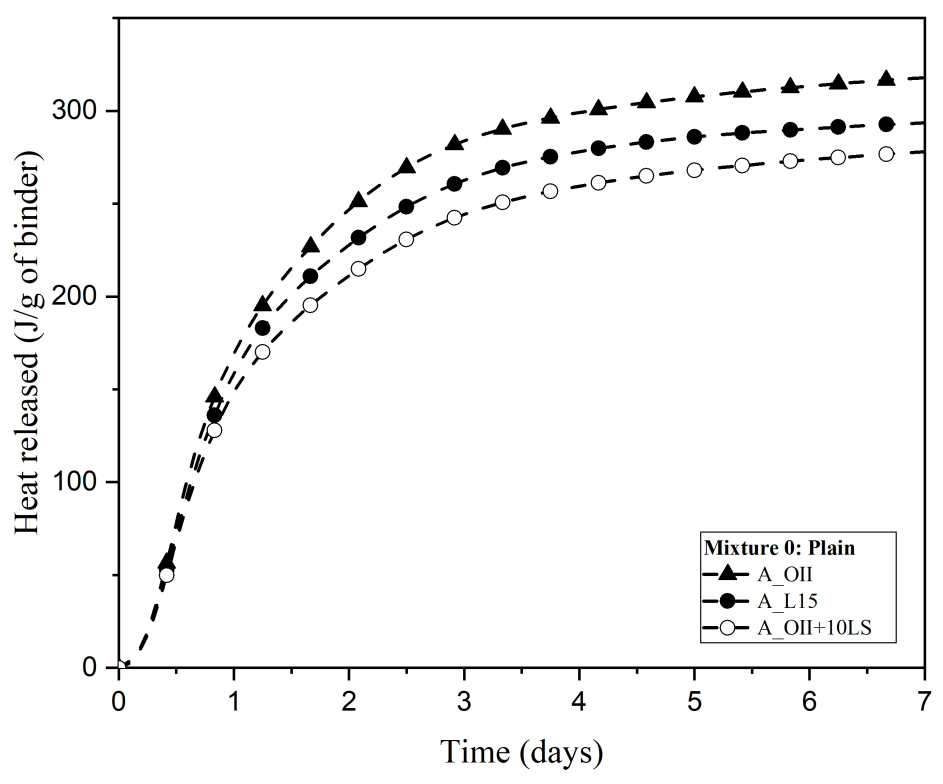

(a)

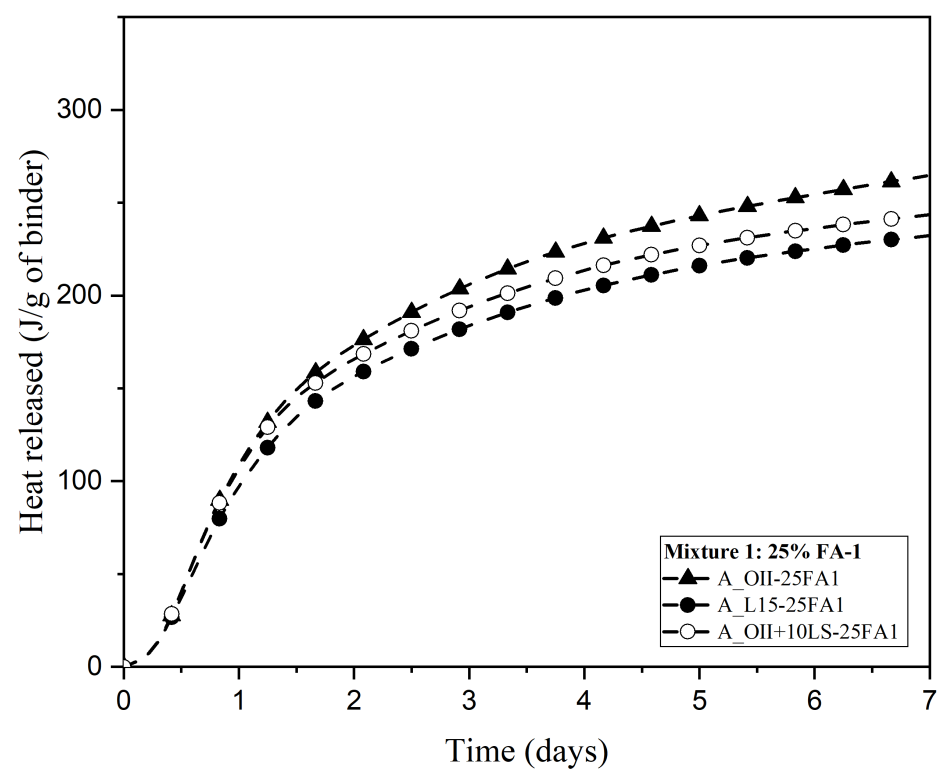

(b) 


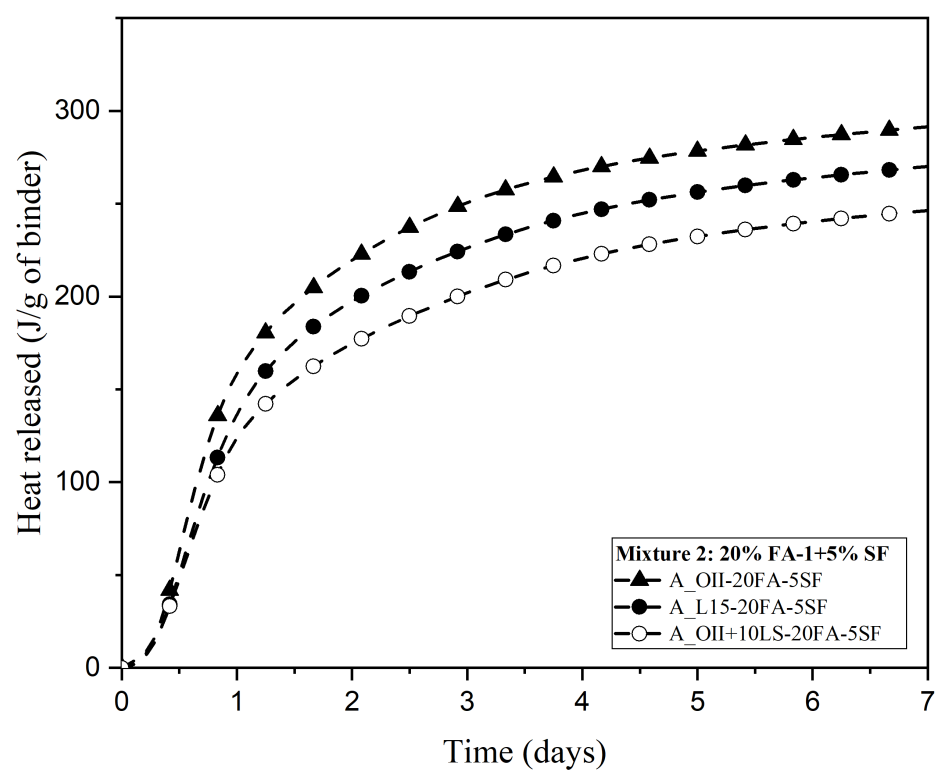

(c)

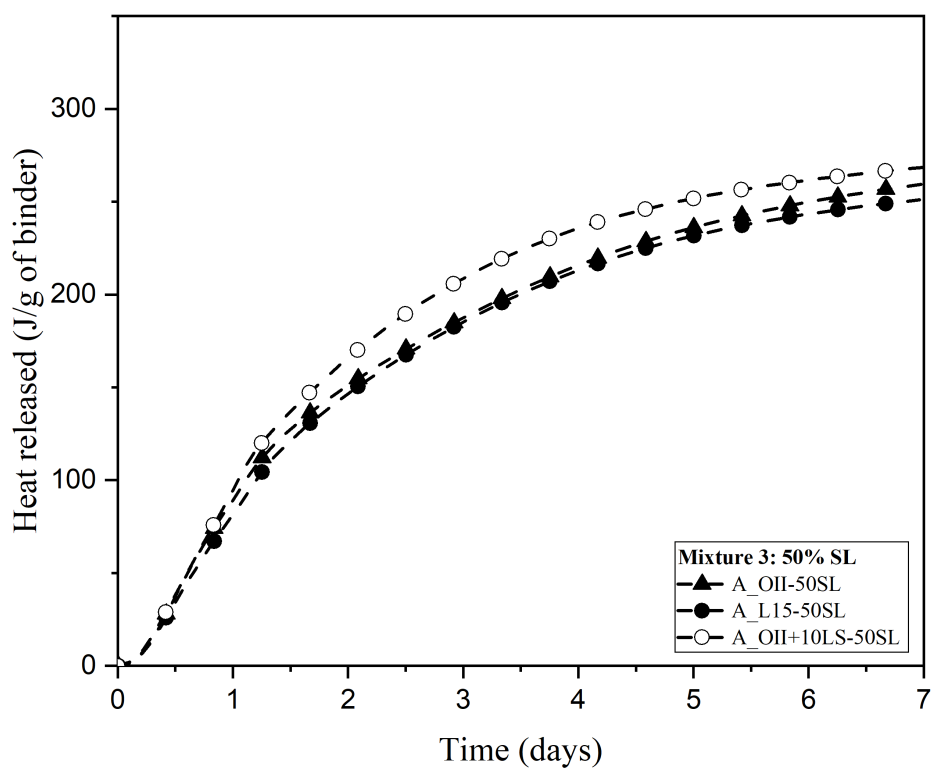

(d) 


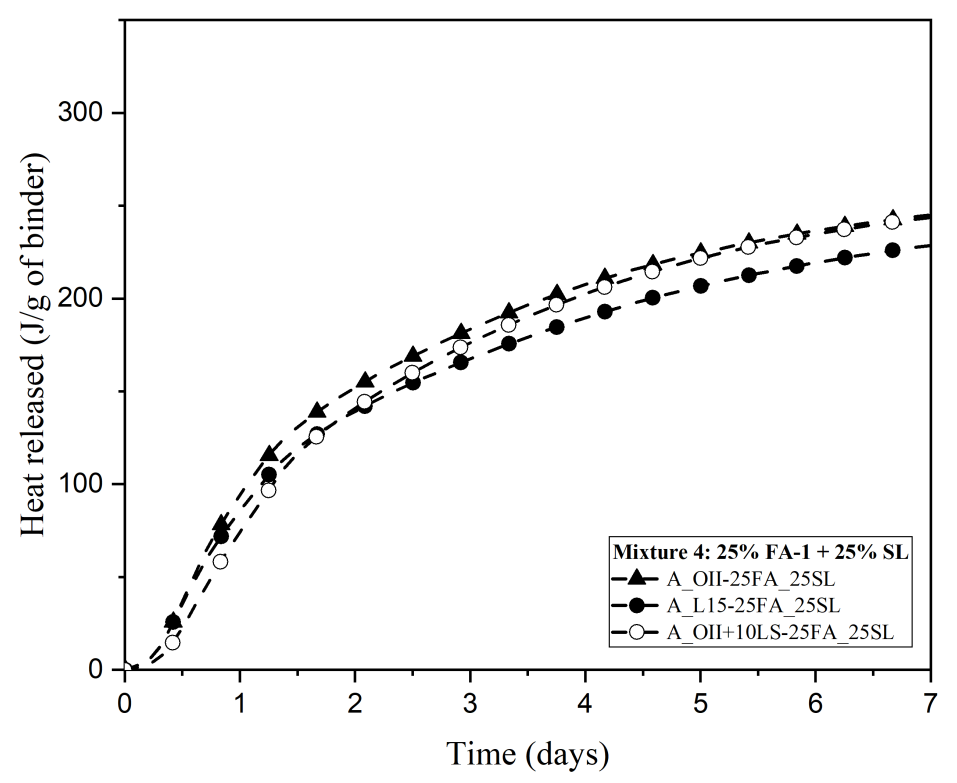

(e)

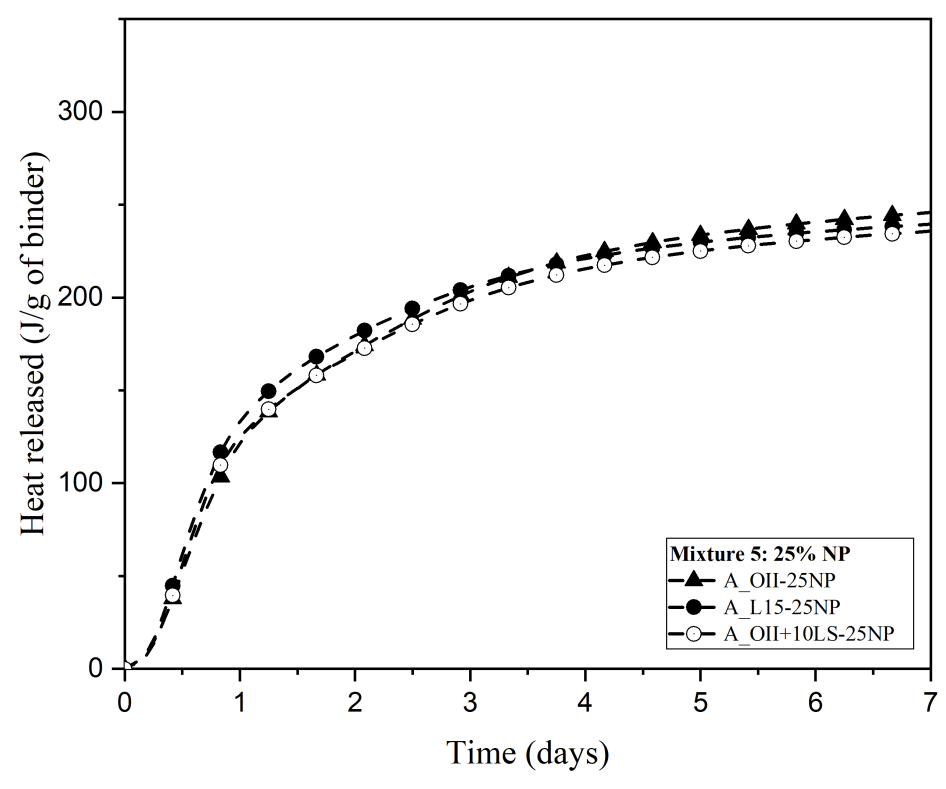

(f)

373 Figure 4-7. (a-f) Total heat released per unit binder in cement paste measured for seven 374 days using Isothermal Calorimeter for Cement A for M0, 1, 2, 3, 4 and 5 respectively 
While Figure 4-7 was performed for Cement A, similar results were obtained for the other cements. The total heat released (when normalized per unit of binder) has approximately $\pm 10 \%$ variation when the OPC, PLC, and OPC+LS systems are compared. In general, a slightly lower heat release occurs with the PLC (1 to 8\%) and OPC+LS (3 to 15\%) systems, indicating less overall reactivity consistent with dilution. This decrease is consistent with a reduction in clinker content in the PLC and OPC+10LS systems. This was not found to be statistically significant for the PLC mixtures; however, it was statistically significant for the OPC + LS mixtures.

When the verticals axis's normalization was done by clinker content, the PLC and OPC+10LS have a higher heat of reaction, illustrating that more of the cement clicker reacts in those systems. The reaction of fly ash, being primarily pozzolanic, can be seen at later ages as this requires more time for the reaction to occur. The reaction between the hydraulic slag and the limestone present in the system is responsible for additional heat release in PLC and OPC+10LS systems in M3 and 4.

\subsection{The Reactivity of SCMs}

\subsubsection{Experimental Methods}

A reactivity test is used to quantify the maximum degree of reactivity (DOR*) of the SCMs [3537, 43]. The DOR* of an SCM is the maximum \% amount of the SCM that reacts with calcium hydroxide $(\mathrm{CH})$ in a pozzolanic reaction. The test method uses a combination of experimentally determined $\mathrm{CH}$ consumption, as quantified by TGA, and heat release obtained from isothermal calorimetry (IC). These values are superimposed on a plot with thermodynamically calculated $\mathrm{CH}$ consumption and heat release lines for reference $\mathrm{SiO}_{2}$ and $\mathrm{Al}_{2} \mathrm{O}_{3}$ systems at equilibrium at different theoretical maximum reactivities. To obtain reference lines, thermodynamic modeling was performed using GEMS3K software [44-47] and the CemData v.18.01 database [48]. Depending on the relative position of the point represented by $\mathrm{CH}$ consumption and heat release with respect to the theoretical lines, the DOR* can be quantified. This test provides a methodology for measuring the amount of reactive versus non-reactive components of a pozzolan..

The blends for measuring reactivity were made by dry mixing reagent-grade $\mathrm{CH}$ and the SCM in a 3:1 mass ratio [34, 37]. The powder was then mixed with $0.5 \mathrm{M}$ potassium hydroxide $(\mathrm{KOH})$ solution while keeping the liquid-to-powder mass ratio constant at 0.9 . For each test, $38 \mathrm{~g}$ of materials were mixed for 4 minutes in a plastic container using a spatula. After mixing, approximately $7 \mathrm{~g}$ of the paste was immediately sealed in a glass ampoule. The ampoules were transferred to an isothermal calorimeter (TAM Air, TA Instruments) that had been preconditioned at $50^{\circ} \mathrm{C} \pm 2^{\circ} \mathrm{C}$ for 24 hours. Following signal stabilization, about 45 minutes after the ampoule was 
410 After 240 hours of IC testing, the ampoules were removed from the calorimeter, and $20 \mathrm{mg}$ of the 411 removed sample was analyzed using TGA (Q50, TA Instruments). The TGA involved heating the 412 sample from $23^{\circ} \mathrm{C}$ to $1000^{\circ} \mathrm{C}$ in a nitrogen-purged atmosphere at a rate of $10^{\circ} \mathrm{C} / \mathrm{min}$. TGA was 413 performed within 12 hours of removing the samples from the isothermal calorimeter. The mass 414 loss between $350^{\circ} \mathrm{C}$ to $450^{\circ} \mathrm{C}$ corresponds to the decomposition of $\mathrm{CH}$. The decomposition of $\mathrm{CH}$ 415 was used to calculate $\mathrm{CH}$ remaining in the system after 240 hours (21). $\mathrm{CH}$ decomposition occurs 416 between $350-450^{\circ} \mathrm{C}[38,39,43]$. Testing and analysis followed the method described in Appendix

417 A. $\mathrm{CH}$ consumption versus heat release was plotted for each SCM. Each SCM's reactivity was 418 determined using thermodynamically calculated reference lines for the $\mathrm{SiO}_{2}$ and $\mathrm{Al}_{2} \mathrm{O}_{3}$ reactions 419 with $0.5 \mathrm{M} \mathrm{KOH}$ at $50^{\circ} \mathrm{C}$. This method provides the maximum degree of reactivity (DOR*) at 420 equilibrium.

\section{$421 \quad$ 4.7.2 Analysis Results and Discussion}

422 The SCMs used in the project Fly Ash 1 (FA-1), Slag, Silica Fume, Natural Pozzolan, and Fly Ash 4232 (FA-2) were all tested for their maximum degree of reactivity (DOR*) and results were plotted 424 against the reference reaction lines depicting the reaction of pure silica and alumina with $0.5 \mathrm{M}$ $425 \mathrm{KOH}$ in Figure 4-8. The DOR* are summarized in Table 4.6.

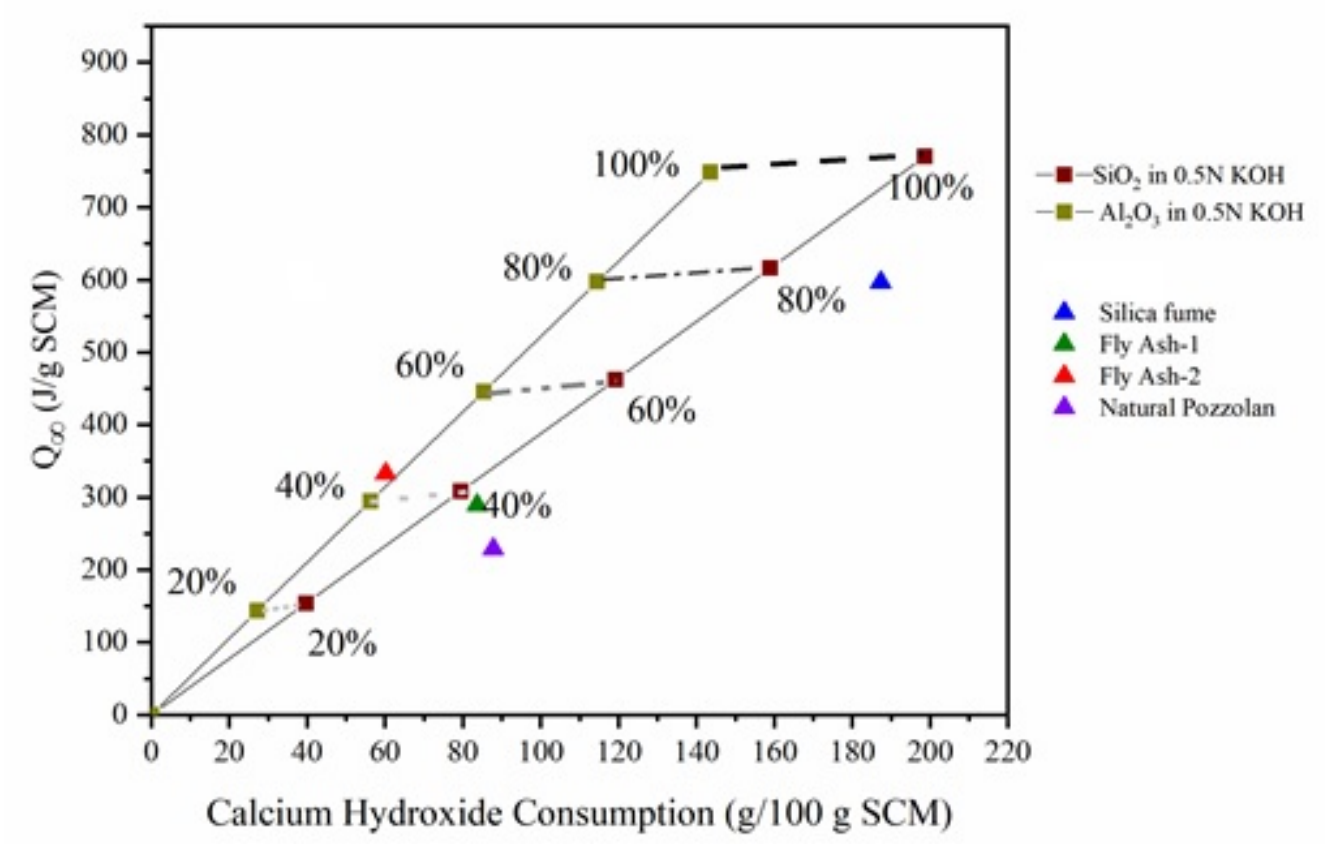


Table 4.6. SCM Heat $\left(Q_{\infty}\right), C H$, and DOR values

\begin{tabular}{|l|c|c|c|}
\hline & $\begin{array}{c}\text { CH consumed } \\
\text { (g/g 100 SCM) }\end{array}$ & $\mathbf{Q}_{\infty}(\mathbf{J} / \mathbf{g}$ SCM) & DOR* \\
\hline Silica fume & 189.2 & 599.8 & $76.15 \%$ \\
\hline Fly Ash-1 & 83.7 & 289.4 & $37.15 \%$ \\
\hline Fly Ash-2 & 60.2 & 332.9 & $44.67 \%$ \\
\hline Natural Pozzolan & 87.8 & 228.7 & $28.19 \%$ \\
\hline
\end{tabular}

431 The DOR* of SCM corresponds to the maximum degree of pozzolanic reactivity shown by the 432 SCM under relatively idealistic conditions.

433 The silica fume, a fine powder composed of almost pure reactive silica dioxide, has a reactivity of $43476 \%$. The natural pozzolan contains reactive silica-based SCM shows a DOR* of 28\%. Fly Ash 435 1, and Fly Ash 2 were observed to have a similar DOR* of 37 and 45\%; however, their position 436 with respect to the reference $\mathrm{SiO}_{2}$ and $\mathrm{Al}_{2} \mathrm{O}_{3}$ reaction is impacted by the fact that Fly Ash 2 has 437 higher $\mathrm{CaO}$ content than Fly Ash 1. Note that the PRT was developed to determine the reactivity 438 of pozzolanic materials (materials which primarily contains $\mathrm{SiO} 2$ and $\mathrm{Al} 2 \mathrm{O} 3$ ) and since slag is a 439 mostly a hydraulic material containing significant $\mathrm{CaO}$ the PRT in its current form cannot be 440 directly used for measuring the reactivity of slag. Although, the PRT has been extended to quantify 441 the reactivity of slags in recent work, but this is beyond the scope of this report.

\section{$442 \quad 4.8$ Significant Findings}

443 This section of the report has provided information on the loss of ignition, chemical composition, 444 oxide composition, the particle size of the raw materials, and the SCMs reactivity. The results are 445 useful for providing reference values for use in the remainder of the study. In general, a similar 446 particle size analysis exists for the OPC and PLC. The heat of hydration was found to be with +/$44710 \%$ when the OPC, PLC, and OPC+LS systems were compared based on total cumulative heat; 448 however, the PLC and OPC+LS systems were found to have a greater degree of reaction. The 449 degree of reactivity (DOR*) was determined for each of the SCM used, with the silica fume 450 generally having a value of over $75 \%$, with the other SCMs having values in the range of 28 to $45145 \%$. 


\section{$5 \quad$ Alkali-Silica Reactivity}

454 Alkali-silica reaction (ASR) in concrete was first studied by Thomas E. Stanton of the California 455 State Division of Highways during the 1930s and published in a study in 1940 [49]. ASR is a 456 chemical reaction between amorphous silica found in some aggregates and alkali hydroxides in 457 the concrete pore solution. Cement is the primary source of alkalis. The other sources of alkali can 458 be SCMs, deicers, aggregates, chemical admixtures, and seawater. Given sufficient moisture, the 459 reaction product, ASR gel, swells, and exerts pressure on the surrounding concrete leading to 460 cracking.

461 Since the discovery of ASR, significant research has been done on understanding the mechanism 462 of ASR and the expansion caused by it, developing test methods to evaluate ASR, and developing 463 strategies to mitigate the distress caused by the reaction in concrete. Rajabipour et al. [50] 464 summarized the current understanding of reaction mechanisms and the current knowledge of ASR 465 mitigation.

466 The use of SCMs in sufficient quantities and lithium compounds have been found to be the most effective ways to mitigate ASR so far. Thomas summarized the effect of the use of SCMs on ASR expansion [51]. The quantity of SCM required to mitigate ASR depends on the SCM composition mainly calcium oxide, silica, and alkali content [51]. It also depends on the reactivity level of aggregate and the amount of alkalis available from the cement and other sources. SCMs mitigate ASR mainly by lowering the pore solution alkalinity through binding the alkalis by C-S-H hydrates $[52,53]$. The replacement level of cement with SCM needs to be increased to control ASR expansion with an increase in its calcium content, increase in alkalis available, increase in aggregate reactivity, and decrease in SCM's silica content. Also, SCMs rich in alumina are considered to be effective in controlling ASR expansion as aluminum in pore solution was found to lower the reactive silica dissolution rate from aggregates $[54,55]$.

477 Using a sufficient amount of certain lithium compounds, especially $\mathrm{LiNO}_{3}$, as an admixture in new concrete has also been found to be a viable way to mitigate ASR [56]. Folliard et al. [56] published a report summarizing the proposed mechanisms on how lithium compounds work and guidelines for using lithium compounds to mitigate or prevent ASR. The main conclusions from the report were: (i) the effectiveness of lithium admixtures is a function of its dosage (in terms of lithium-toalkali molar ratio) in the mixture and of the petrographic nature of the reactive aggregate to the control, (ii) required lithium-to-alkali molar ratio to mitigate ASR vary significantly for different aggregates (as low as 0.56 to $>1.11$ ), (iii) it was recommended to use SCMs along with lithium admixture to reduce the cost using lithium compounds and to produce low-permeability concrete to provide better durability, and, (iv) the most recommended method to assess the effectiveness of lithium admixture was ASTM C1293, with a 2-year duration. Due to lithium's cost, however, it is becoming less and less frequently used in concrete construction. 
490 As California has siliceous aggregate sources that show the potential for ASR and damage, SCMs

491 are included as a prescriptive specification to address ASR concerns. In two recent studies, PLC 492 generally showed a synergistic benefit when used with SCM [5, 57]; however, the influence of 493 PLC on mitigation of ASR has not been extensively evaluated.

\subsection{Research Objective}

495 The main objective in this part of the project is to extensively study the influence of PLC usage in 496 conjunction with SCMs on the expansion due to ASR. Ultimately, the results of this task will 497 inform CALTRANS if their current mitigation options for a range of alkali-silica reactivity levels 498 can be utilized as-is, increased, or decreased when combined with a PLC.

\subsection{Background/Literature Review}

500 There are several test methods to detect the alkali-silica reactivity of aggregates. These range from 501 tests on aggregates, mortar, and concrete, to petrographic examination of aggregates and concrete. 502 There are also modifications to these tests that allow for the use of SCMs in the mixture to test 503 their efficacy in mitigating the reactivity of the aggregates.

504 The most commonly used laboratory test methods to evaluate the effectiveness of the usage of 505 SCMs to mitigate or control ASR are accelerated mortar bar test - AMBT (ASTM C1567 [58]) 506 and concrete prism test - CPT (ASTM C1293 [59]). AMBT involves immersion of mortar bars of 507 dimensions $25 \times 25 \times 285 \mathrm{~mm}$ cast with the reactive fine aggregate (crushed, if the reactive 508 aggregate is a coarse aggregate) in $1 \mathrm{~N} \mathrm{NaOH}$ solution at $80^{\circ} \mathrm{C}$ for 14 days. CPT involves storing 509 concrete bars of dimensions $75 \times 75 \times 285 \mathrm{~mm}$ over water at $38^{\circ} \mathrm{C}$ for a year (with no mitigation 510 measures) or two years (mixtures with mitigation measures). Thomas et al. discussed these tests 511 in detail, and the authors also discussed how the AMBT and CPT methods are different in testing 512 the effectiveness of SCMs controlling ASR expansion [60]. It was concluded that none of the 513 current ASTM methods to evaluate the mitigation measures for ASR are ideal methods due to their 514 limitations. The AMBT is mostly criticized for its severe test conditions, which results in a loss in 515 reliability. The CPT is mainly criticized for its long test duration and its inability to test job 516 mixtures (e.g., capture low-alkali loading mixtures). The authors also concluded that the CPT 517 method is the most reliable ASTM method available currently, and the use of AMBT is possible 518 to determine the required minimum amount of SCM to control ASR expansion as there is a low 519 risk of damaging expansion in the field when the combination of materials pass the AMBT 520 performance criteria [60]. The miniature concrete prism test - MCPT (AASHTO T 380 [61]) is a 521 newer test method, which is also of interest as the preliminary results regarding the prediction of 522 the field performance show promising results in shorter duration (up to 84 days) when compared 523 to the CPT method $[62,63]$. 
524 Laker and Smartz used PLC with 10-12\% interground limestone to evaluate alkali-silica 525 reactivity. ASTM C 1567 was used in the study. They observed that PLC performed similarly to 526 PC in terms of expansions [64].

527 Thomas et al. [65] tested PLC with 12\% interground limestone for its resistance to ASR and 528 compared the results to PC. AMBT (ASTM C1260), CPT, and accelerated concrete prism test 529 (ACPT) were done using alkali-silica reactive Spratt aggregate. ACPT is similar to ASTM C1293, 530 with the exception of storage temperature is $60^{\circ} \mathrm{C}$. The authors observed no consistent difference 531 in expansion results produced with PLC compared with OPC [65]. Figure 5-1 shows the reported 532 expansion results of AMBT at 14 days, CPT at one year, and ACPT at three months.

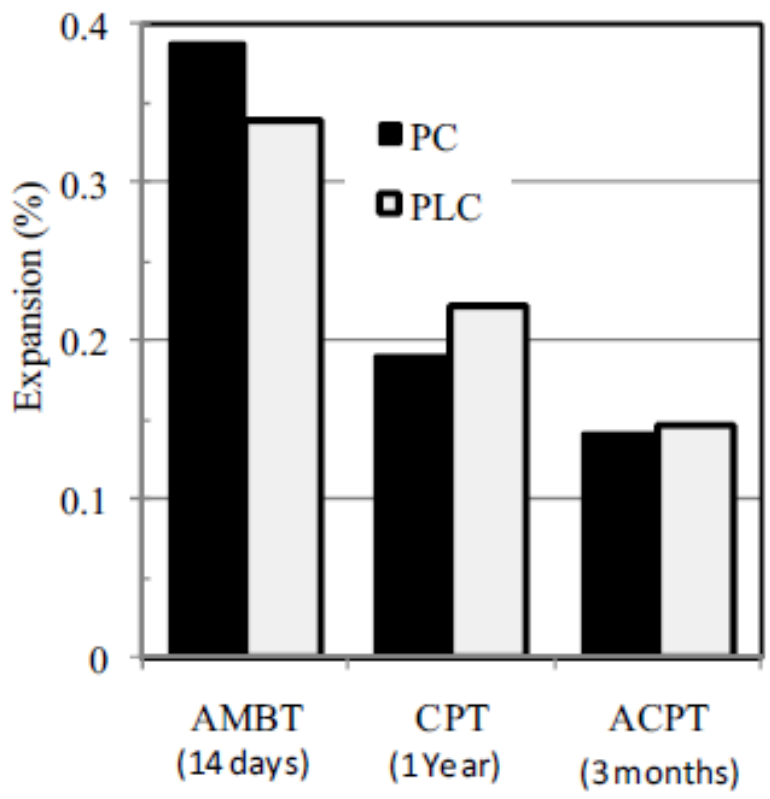

Figure 5-1. Expansion results of mortar and concrete prisms [65]

535 Hooton et al. [66] compared the performance of three cements with different levels of limestone $536-3.5 \%, 10 \%$, and 15\% and labeled as GU (OPC), PLC10, and PLC15, respectively. Both the 537 AMBT and CPT methods were done for various mixes. Figure 5-2 shows the reported AMBT 538 expansions. 


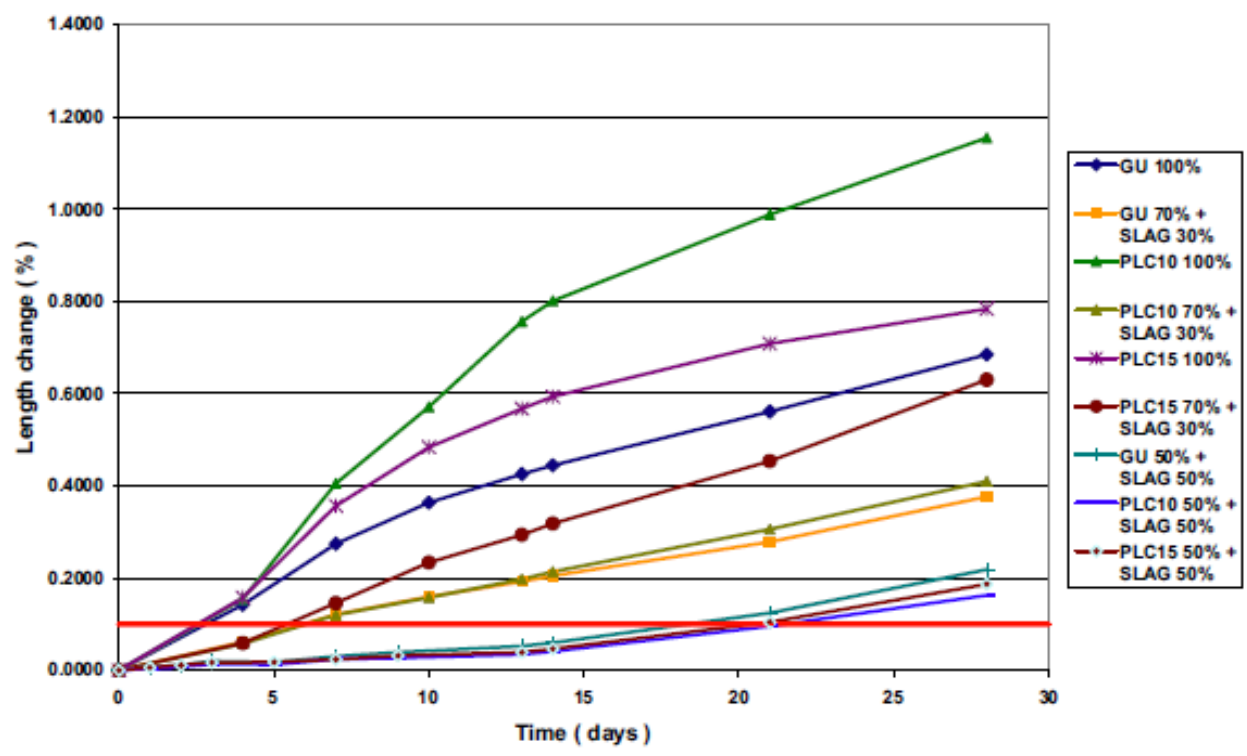

541 When no slag was used, none of the mixtures passed the AMBT test, and PLC10 and PLC15 had 542 higher expansions than GU. Even replacing the cements with 30\% slag was not sufficient. When $54350 \%$ of the cements were replaced with slag, all the cements passed the AMBT test. It was 544 observed that both the PLC10 and PLC15 cements had less expansion than the GU cement [66]. 545 Figure 5-3 shows the reported CPT expansions.

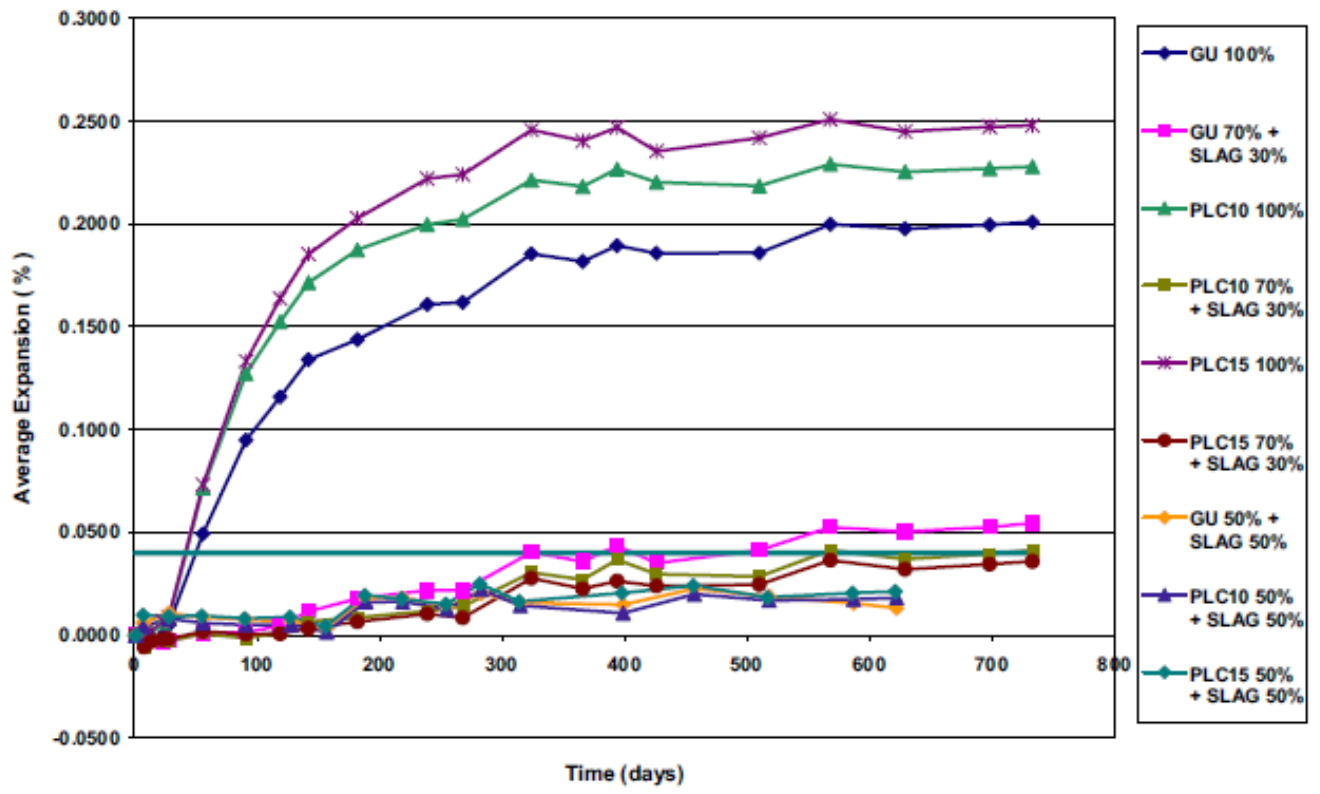


548 From the CPT results - GU, PLC10, PLC15, GU Slag 30\%, and PLC10 Slag, 30\% failed the test.

549 Whereas, PLC15 Slag 30\%, GU Slag 50\%, PLC10 Slag 50\%, and PLC15 Slag 50\% passed the 550 test with less than $0.04 \%$ expansion even after two years. It was observed that expansions were in 551 an increasing order with the limestone content when no SCM was used or in the case of the cements 552 replaced with 50\% slag. This was not observed in the case of cements replaced with $30 \%$ slag [66].

553 From this literature review, concrete with PLCs and no SCMs expanded more than their respective $554100 \%$ OPC. Whereas in the case of mixtures containing SCMs, no consistent difference in 555 expansion results produced with PLC compared with OPC. Therefore, there is a need for study to 556 evaluate the performance of PLCs in the presence of various types of SCMs to verify appropriate 557 SCM contents for ASR prevention.

\section{$558 \quad 5.3 \quad$ Experimental Test Matrix}

559 To evaluate the cements' relative ASR performance in this study, the ASTM C441 [67] test that 560 uses borosilicate glass as fine aggregate was conducted. The testing was divided into two phases. 561 As part of ASTM C441 - phase I, all the received cements were tested. As part of phase II, cement $562+$ SCMs were tested. The cements or mixtures tested as part of ASTM C441 - phase II and ASTM $563 \mathrm{C} 1567$ with Bishop fine aggregate are summarized in Table 5.1. The concrete mixtures with SCMs 564 that were tested according to AASHTO T 380 are shown in Table 5.2.

Table 5.1. Experimental matrix for ASTM C441 phase-II and ASTM C1567 testing

\begin{tabular}{|c|c|c|c|c|c|}
\hline \multirow[b]{2}{*}{ Cement } & \multirow[b]{2}{*}{ Mixture \# } & \multirow[b]{2}{*}{$\begin{array}{l}\text { SCM } \\
\text { proportions }\end{array}$} & \multirow{2}{*}{$\begin{array}{l}\text { ASTM } \\
\text { C441 phase- } \\
\text { II }\end{array}$} & \multicolumn{2}{|c|}{ ASTM C1567 } \\
\hline & & & & $\begin{array}{l}\text { F1 fine } \\
\text { aggregate }\end{array}$ & $\begin{array}{l}\text { F2 fine } \\
\text { aggregate }\end{array}$ \\
\hline \multirow{6}{*}{ A_OII } & 1 & 25FA1 & $\mathrm{x}$ & $\mathrm{x}$ & - \\
\hline & 2 & 20FA1-5SF & $\mathrm{x}$ & - & - \\
\hline & 3 & 50SL & $\mathrm{x}$ & - & - \\
\hline & 4 & $\begin{array}{c}\text { 25FA1- } \\
\text { 25SL }\end{array}$ & $\mathrm{x}$ & - & - \\
\hline & 5 & 25NP & - & $\mathrm{x}$ & - \\
\hline & 6 & 25FA2 & $\mathrm{x}$ & - & - \\
\hline \multirow{6}{*}{ A_L15 } & 1 & 25FA1 & $\mathrm{x}$ & $\mathrm{x}$ & - \\
\hline & 2 & 20FA1-5SF & $\mathrm{x}$ & - & - \\
\hline & 3 & 50SL & $\mathrm{x}$ & - & - \\
\hline & 4 & $\begin{array}{c}\text { 25FA1- } \\
\text { 25SL }\end{array}$ & $\mathrm{x}$ & - & - \\
\hline & 5 & 25NP & - & $\mathrm{x}$ & - \\
\hline & 6 & 25FA2 & $\mathrm{x}$ & - & - \\
\hline \multirow{2}{*}{ B_OIIV } & 1 & 25FA1 & $\mathrm{x}$ & $\mathrm{x}$ & $\mathrm{x}$ \\
\hline & 2 & 20FA1-5SF & $\mathrm{x}$ & $\mathrm{x}$ & $\mathrm{x}$ \\
\hline
\end{tabular}




\begin{tabular}{|c|c|c|c|c|c|}
\hline & 3 & 50SL & $\mathrm{x}$ & $\mathrm{x}$ & $\mathrm{x}$ \\
\hline & 4 & $\begin{array}{l}\text { 25FA1- } \\
\text { 25SL }\end{array}$ & $\mathrm{x}$ & $\mathrm{x}$ & $\mathrm{X}$ \\
\hline & 5 & 25NP & - & $\mathrm{x}$ & - \\
\hline & 6 & 25FA2 & $\mathrm{x}$ & $\mathrm{x}$ & $\mathrm{x}$ \\
\hline \multirow{6}{*}{ B_L15 } & 1 & 25FA1 & $\mathrm{x}$ & $\mathrm{x}$ & $\mathrm{x}$ \\
\hline & 2 & 20FA1-5SF & $\mathrm{x}$ & $\mathrm{x}$ & $\mathrm{x}$ \\
\hline & 3 & 50SL & $\mathrm{x}$ & $\mathrm{x}$ & $\mathrm{x}$ \\
\hline & 4 & $\begin{array}{l}\text { 25FA1- } \\
\text { 25SL }\end{array}$ & $\mathrm{X}$ & $\mathrm{x}$ & $\mathrm{x}$ \\
\hline & 5 & 25NP & - & $\mathrm{x}$ & - \\
\hline & 6 & 25FA2 & $\mathrm{x}$ & $\mathrm{x}$ & $\mathrm{X}$ \\
\hline \multirow{6}{*}{$\underset{\text { LS }}{\text { B_OIIV }+10}$} & 1 & 25FA1 & - & $\mathrm{x}$ & $\mathrm{X}$ \\
\hline & 2 & 20FA1-5SF & - & $\mathrm{x}$ & $\mathrm{x}$ \\
\hline & 3 & 50SL & - & $\mathrm{x}$ & $\mathrm{X}$ \\
\hline & 4 & $\begin{array}{c}\text { 25FA1- } \\
\text { 25SL }\end{array}$ & - & $\mathrm{x}$ & $\mathrm{X}$ \\
\hline & 5 & 25NP & - & $\mathrm{x}$ & - \\
\hline & 6 & 25FA2 & - & $\mathrm{x}$ & $\mathrm{X}$ \\
\hline \multirow{4}{*}{ C_OV } & 1 & 25FA1 & $\mathrm{x}$ & - & - \\
\hline & 2 & 20FA1-5SF & $\mathrm{x}$ & - & - \\
\hline & 3 & 50SL & - & $\mathrm{x}$ & - \\
\hline & 6 & 25FA2 & - & - & $\mathrm{x}$ \\
\hline \multirow{4}{*}{ C_L10 } & 1 & 25FA1 & $\mathrm{x}$ & - & - \\
\hline & 2 & 20FA1-5SF & $\mathrm{x}$ & - & - \\
\hline & 3 & 50SL & - & $\mathrm{x}$ & - \\
\hline & 6 & 25FA2 & - & - & $\mathrm{x}$ \\
\hline D_OV & 5 & 25NP & - & - & $\mathrm{x}$ \\
\hline D_L15 & 5 & 25NP & - & - & $\mathrm{x}$ \\
\hline E_OIIV & 1 & 25FA1 & - & $\mathrm{x}$ & - \\
\hline E_L11 & 1 & 25FA1 & - & $\mathrm{x}$ & - \\
\hline
\end{tabular}


Table 5.2. Experimental matrix for AASHTO T 380 testing

\begin{tabular}{|c|c|c|c|c|}
\hline \multirow[b]{2}{*}{ Cement } & \multirow[b]{2}{*}{ Mixture \# } & \multirow[b]{2}{*}{$\begin{array}{c}\text { SCM } \\
\text { proportions }\end{array}$} & \multicolumn{2}{|c|}{ AASHTO T 380} \\
\hline & & & $\begin{array}{c}\text { F1 fine } \\
\text { aggregate }\end{array}$ & $\begin{array}{c}\text { F2 fine } \\
\text { aggregate }\end{array}$ \\
\hline \multirow{6}{*}{ B_OIIV } & 1 & 25FA1 & $\mathrm{x}$ & - \\
\hline & 2 & 20FA1-5SF & $\mathrm{x}$ & - \\
\hline & 3 & 50SL & $\mathrm{x}$ & - \\
\hline & 4 & 25FA1-25SL & $\mathrm{x}$ & - \\
\hline & 5 & $25 \mathrm{NP}$ & - & $\mathrm{x}$ \\
\hline & 6 & 30FA2 & $\mathrm{x}$ & $\mathrm{x}$ \\
\hline \multirow{6}{*}{ B_L15 } & 1 & 25FA1 & $\mathrm{x}$ & $\mathrm{x}$ \\
\hline & 2 & 20FA1-5SF & $\mathrm{x}$ & - \\
\hline & 3 & 50SL & $\mathrm{x}$ & - \\
\hline & 4 & 25FA1-25SL & $\mathrm{x}$ & - \\
\hline & 5 & $25 \mathrm{NP}$ & - & $\mathrm{x}$ \\
\hline & 6 & 30FA2 & $\mathrm{x}$ & $\mathrm{x}$ \\
\hline \multirow{6}{*}{ B_OIIV+10LS } & 1 & 25FA1 & $\mathrm{x}$ & - \\
\hline & 2 & 20FA1-5SF & - & - \\
\hline & 3 & 50SL & $\mathrm{x}$ & - \\
\hline & 4 & 25FA1-25SL & - & - \\
\hline & 5 & $25 \mathrm{NP}$ & - & - \\
\hline & 6 & 30FA2 & $\mathrm{x}$ & - \\
\hline
\end{tabular}

\subsection{ASTM C441 - Phase I}

\subsubsection{Experimental Methods}

ASTM C441 [67] is a test method that involves casting mortar bars ( $25 \times 25 \times 285 \mathrm{~mm})$ using borosilicate glass with a standard gradation as fine aggregate. This test method is used to assess the relative effectiveness of a potential SCM source to reduce expansion caused by ASR. For this part of the project, ASTM C441 was used to compare PLCs' performance to their respective OPCs as well as PLCs plus SCMs. As the fine aggregate used in this method is borosilicate glass, which is not used in the field mixtures, the test method only indicates the relative performance of the cement with respect to alkali-silica reaction. It does not predict a combination of cementitious material to prevent ASR for a potentially reactive aggregate.

The test method involved casting three mortar bars per mixture. The mortar bars are stored in a vertical position in an air-tight container over water at $38^{\circ} \mathrm{C}$. Each container accommodated two mixtures (six bars) per ASTM C441, as shown in Figure 5-4. The total testing period for the method was 14 days. The water to cementitious ratios used for the mixtures were based on the flow test (ASTM C1437) to produce a flow between 100 and 115 as per ASTM C441. 


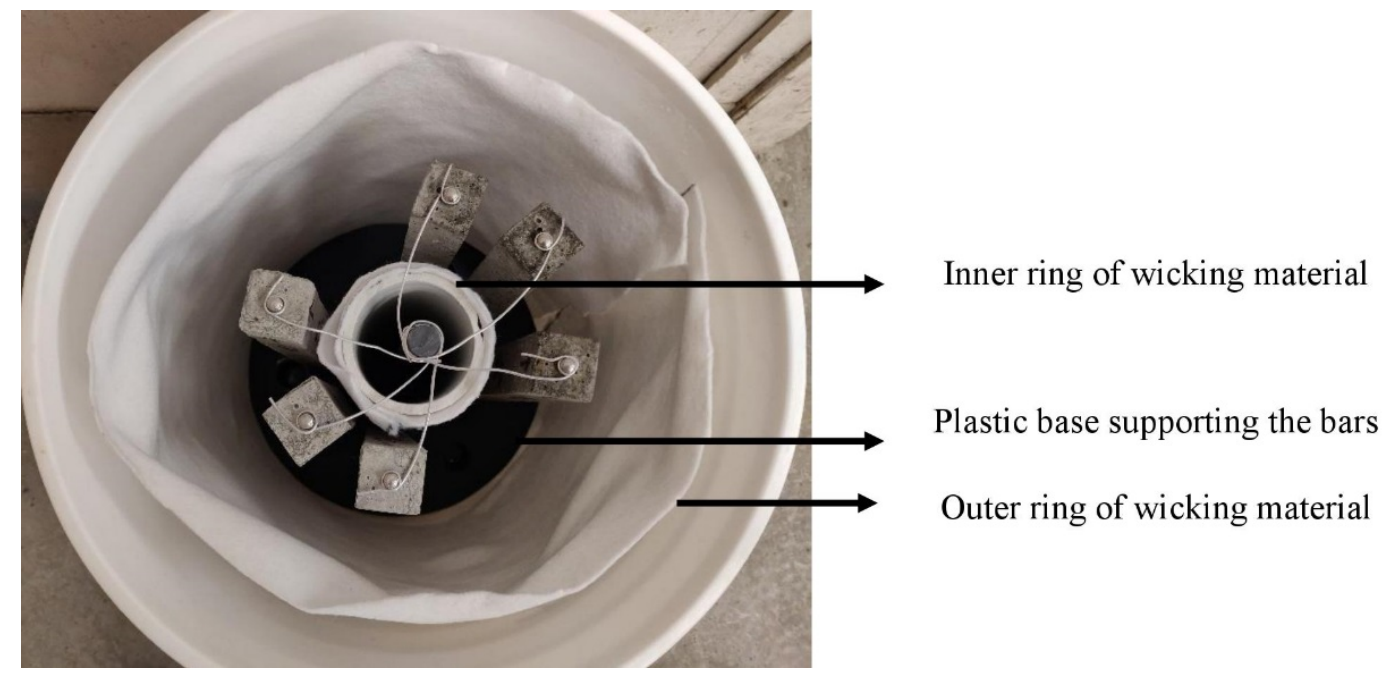

Figure 5-4. Top view of the ASTM C441 set up used

586 All the received cements were tested using ASTM C441. The test set-up was initially validated 587 using a control set of mortar bars cast with high-alkali cement $\left(0.96 \% \mathrm{Na}_{2} \mathrm{O}_{\mathrm{e}}\right)$. The water to cement 588 ratio for all the mixtures were either 0.54 or 0.55 to produce a flow between 100 and 115 according 589 to ASTM C441.

\subsubsection{Typical Experimental Measurement and Interpretation}

591 The length and mass measurements were made after demolding (initial measurements) and on the 592 fourteenth day (final measurements). The length measurements were made using a length 593 comparator with an accuracy of 0.0001 in. The measured lengths were used to calculate the 594 expansion of the bars. Then the average expansions of the mixture were calculated and reported.

\subsubsection{Experimental Results}

596 Figure 5-5 shows the average 14-day expansion results of the bars with all the cements received 597 from CALTRANS and were tested according to ASTM C441. The expansions of the PLCs were 598 compared to their respective parent OPCs. The percentage reduction in average expansion for each 599 PLC compared to the parent OPC was also reported in the figure. 


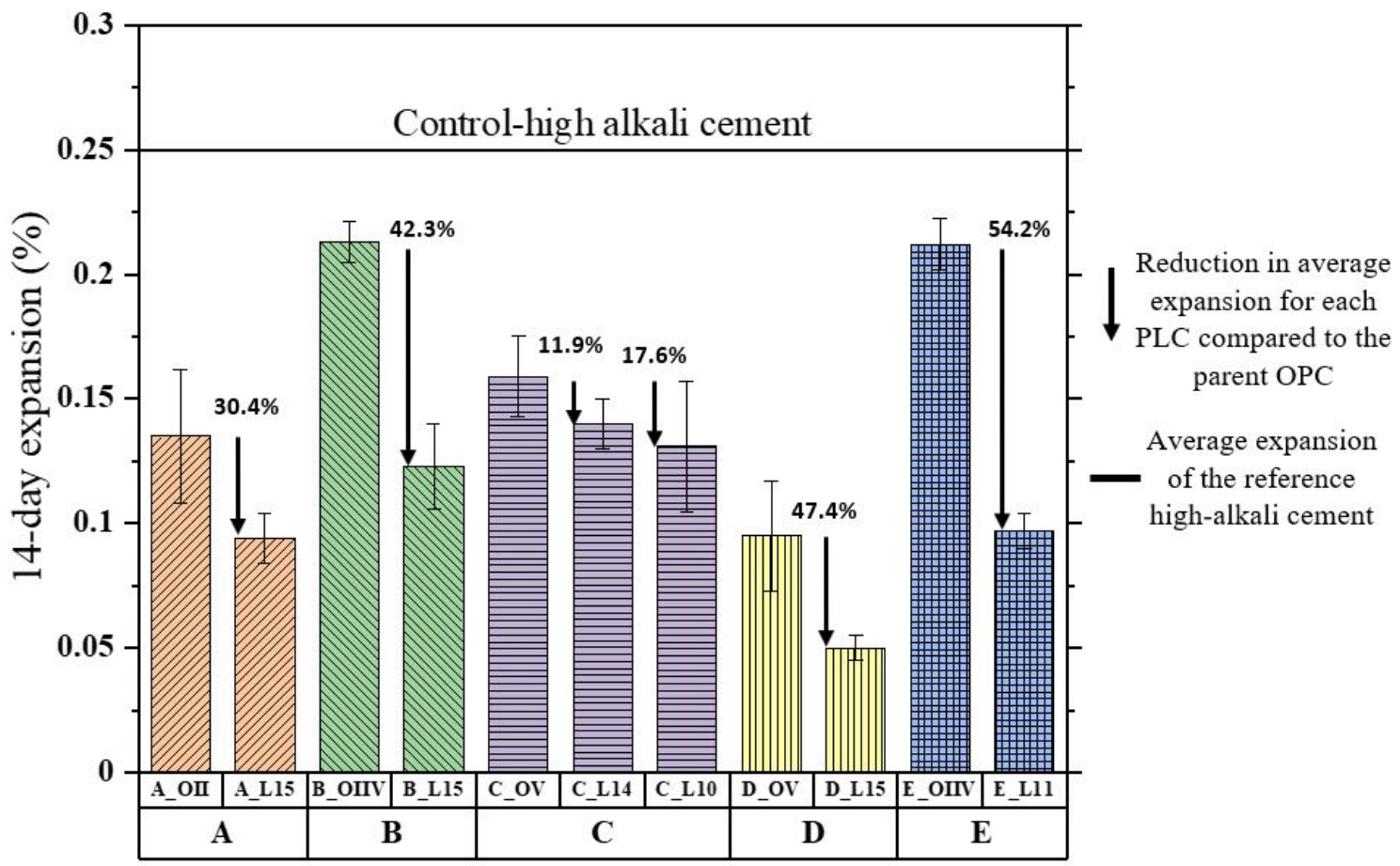

601 Figure 5-5. ASTM C441 - phase I 14-d expansion results of received PLCs and OPCs

602 Table 5.3 shows the results of ASTM C441 average 14-day expansion, standard deviation of the 603 expansions, maximum allowed according to the standard, and percentage reduction in expansions 604 of the PLCs compared to the parent OPCs. 
Table 5.3. ASTM C441 - phase I results

\begin{tabular}{|l|l|l|l|l|}
\hline $\begin{array}{l}\text { Mixtures } \\
\text {-C441 } \\
\text { phase I }\end{array}$ & $\begin{array}{l}\text { Average } \\
\text { 14-d } \\
\text { expansion } \\
\text { (\%) }\end{array}$ & $\begin{array}{l}\text { Standard } \\
\text { deviation } \\
\text { of the } \\
\text { expansion } \\
(\%)\end{array}$ & $\begin{array}{l}\text { \% SD } \\
\text { allowance } \\
\text { max. } \\
\text { (according } \\
\text { to ASTM } \\
\text { C441) }\end{array}$ & $\begin{array}{l}\text { \% } \\
\text { reduction } \\
\text { in \% } \\
\text { expansion } \\
\text { compared } \\
\text { to parent } \\
\text { OPC }\end{array}$ \\
\hline Control & 0.250 & 0.0359 & 0.0375 & $\sim$ \\
\hline A_OII & 0.135 & 0.0270 & 0.0216 & $\sim$ \\
\hline A_L15 & 0.094 & 0.0100 & 0.0141 & 30.4 \\
\hline B_OIIV & 0.213 & 0.0080 & 0.0319 & $\sim$ \\
\hline B_L15 & 0.123 & 0.0170 & 0.0184 & 42.3 \\
\hline C_OV & 0.159 & 0.0160 & 0.0238 & $\sim$ \\
\hline C_L14 & 0.140 & 0.0101 & 0.0210 & 11.9 \\
\hline C_L10 & 0.131 & 0.0261 & 0.0196 & 17.6 \\
\hline D_OV & 0.095 & 0.0220 & 0.0142 & $\sim$ \\
\hline D_L15 & 0.050 & 0.0050 & 0.0075 & 47.4 \\
\hline E_OIIV & 0.212 & 0.0103 & 0.0318 & $\sim$ \\
\hline E_L11 & 0.097 & 0.0070 & 0.0145 & 54.2 \\
\hline
\end{tabular}

\subsubsection{Discussion of the Results}

608 From Figure 5-5, it is evident that the PLCs performed better than their respective OPCs. All the 609 cements expanded less than the control-high alkali cement, likely due to their lower alkali content 610 than the control high alkali cement. Cement B_IIOV expanded the highest among the CALTRANS 611 cements, likely due to its high alkali content among the received cements. It was observed that all 612 the mixtures except A_OII, C_L10, and D_OV had met the maximum allowed standard deviation 613 limit in ASTM C441. It should be noted that the precision and bias statement for ASTM C441 614 was done with high alkali cement $\left(0.95\right.$ to $\left.1.05 \% \mathrm{Na}_{2} \mathrm{O}_{\mathrm{e}}\right)$ and not for cements with finely ground 615 limestone.

\subsubsection{Significant Findings}

617 The main take away from the Phase I ASTM C441 testing is that the PLCs performed better than 618 their respective OPCs in reducing ASR associated expansion. PLCs with similar amounts of finely 619 ground limestone also performed comparably to each other (i.e., the OPC and OPC+LS had similar 620 performance). As a result, this allowed us to move forward with a smaller number of PLCs in 621 Phase II to focus on the evaluation of SCMs in conjunction with PLCs. 


\subsection{ASTM C441 - Phase 2}

\section{$623 \quad$ 5.5.1 Experimental Methods}

624 The experimental method is the same as described for the ASTM C441 - Phase I. ASTM C441 625 Phase II involves testing the mixtures with SCMs. This test aims to determine if the PLCs perform 626 equal or better than their respective OPCs when a portion of the PLC is replaced with SCMs.

\section{$627 \quad 5.5 .2 \quad$ Typical Experimental Measurement and Interpretation}

628 The length and mass measurements were made after demolding (initial measurements) and on the 629 fourteenth day (final measurements). The length measurements were made using a length 630 comparator. The measured lengths were used to calculate the expansion of the bars. Then the 631 average expansions of the mixture were calculated and reported.

\section{$632 \quad$ 5.5.3 Experimental Results}

633 Figure 5-6 to Figure 5-10 show the results of ASTM C441 - phase II. For an even comparison, 634 efforts were made to maintain the same water to binder ratio for a PLC and its parent OPC. Please 635 note that HA stands for high-alkali cement $\left(0.96 \% \mathrm{Na}_{2} \mathrm{O}_{e}\right)$ and was used as a control per ASTM 636 C441.

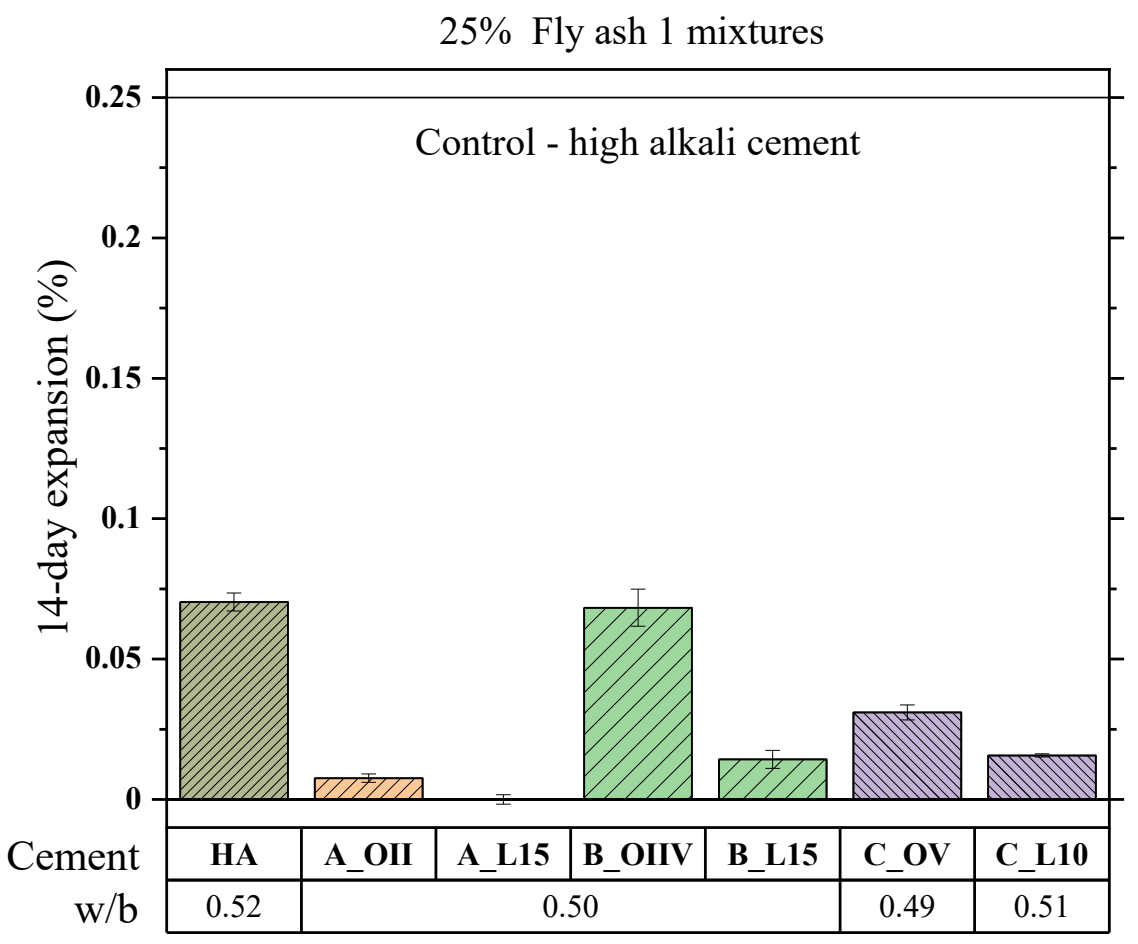




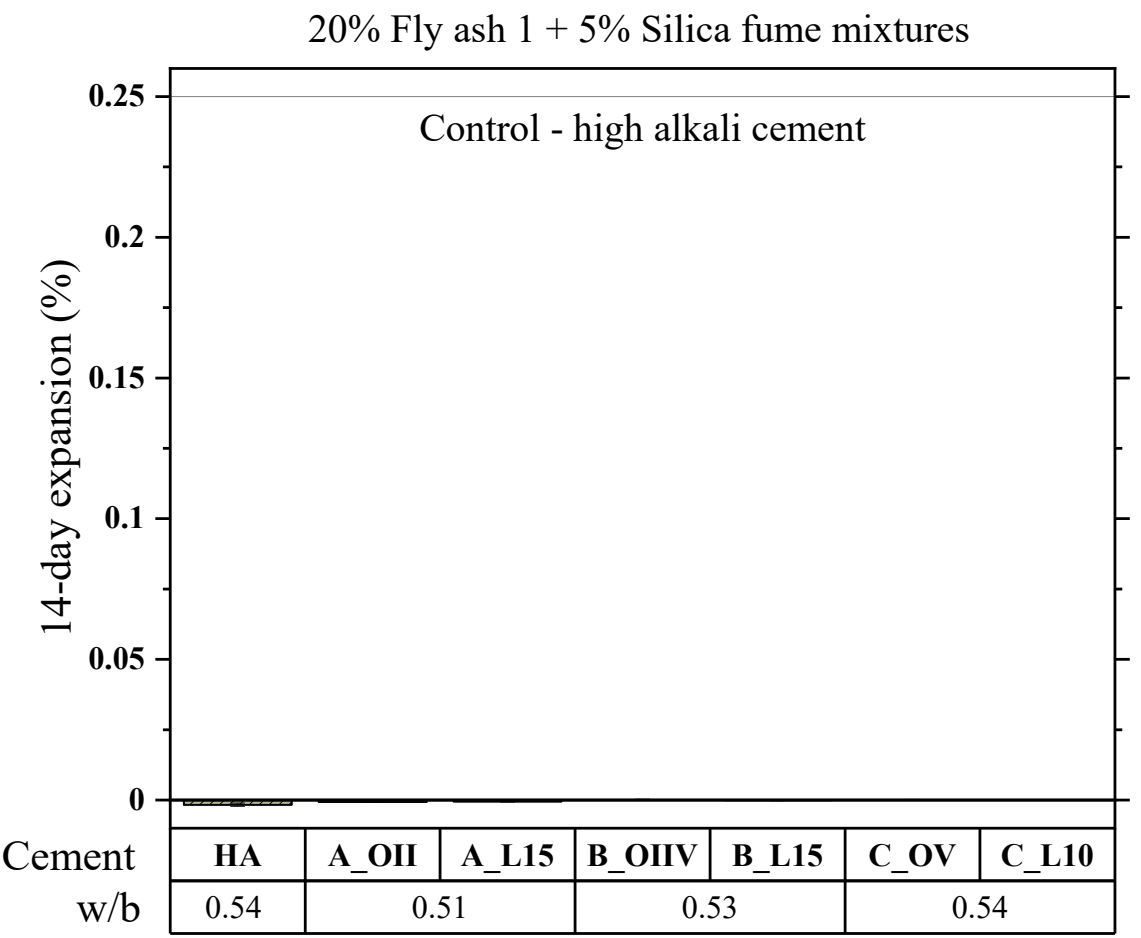

$50 \%$ Slag mixtures

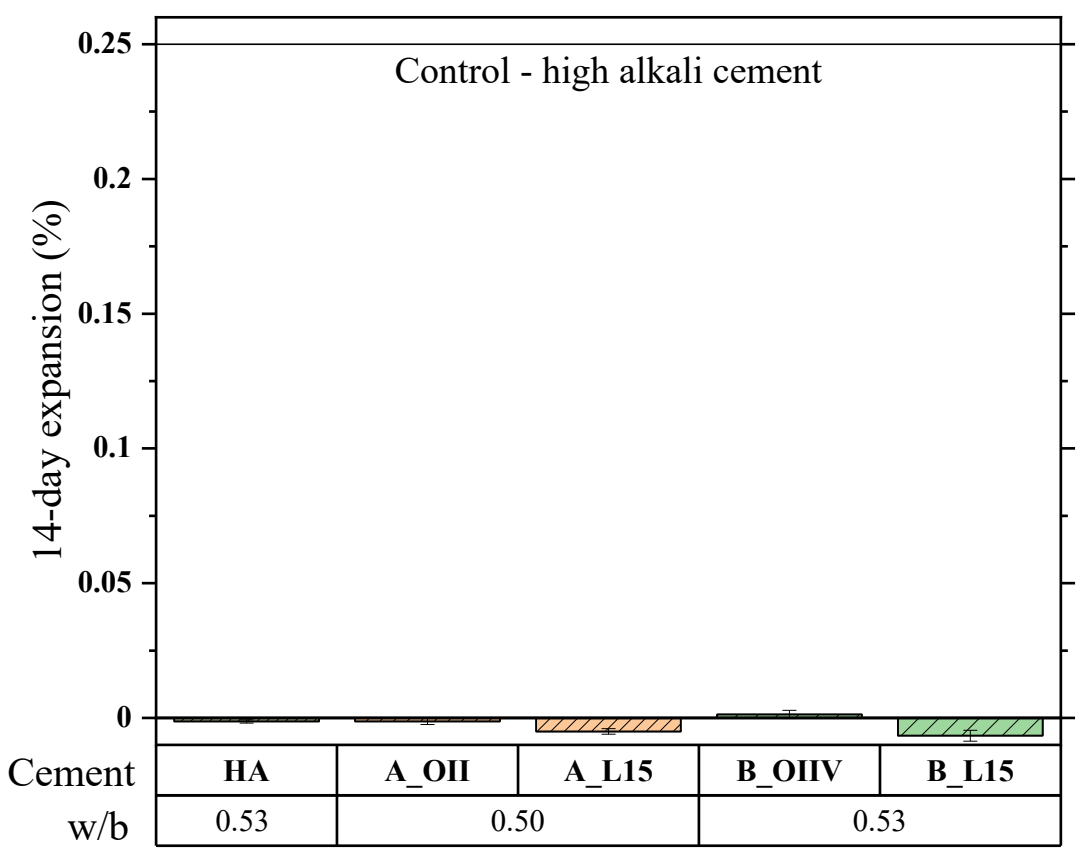

Figure 5-8. ASTM C441 results for 50\% slag mixtures 


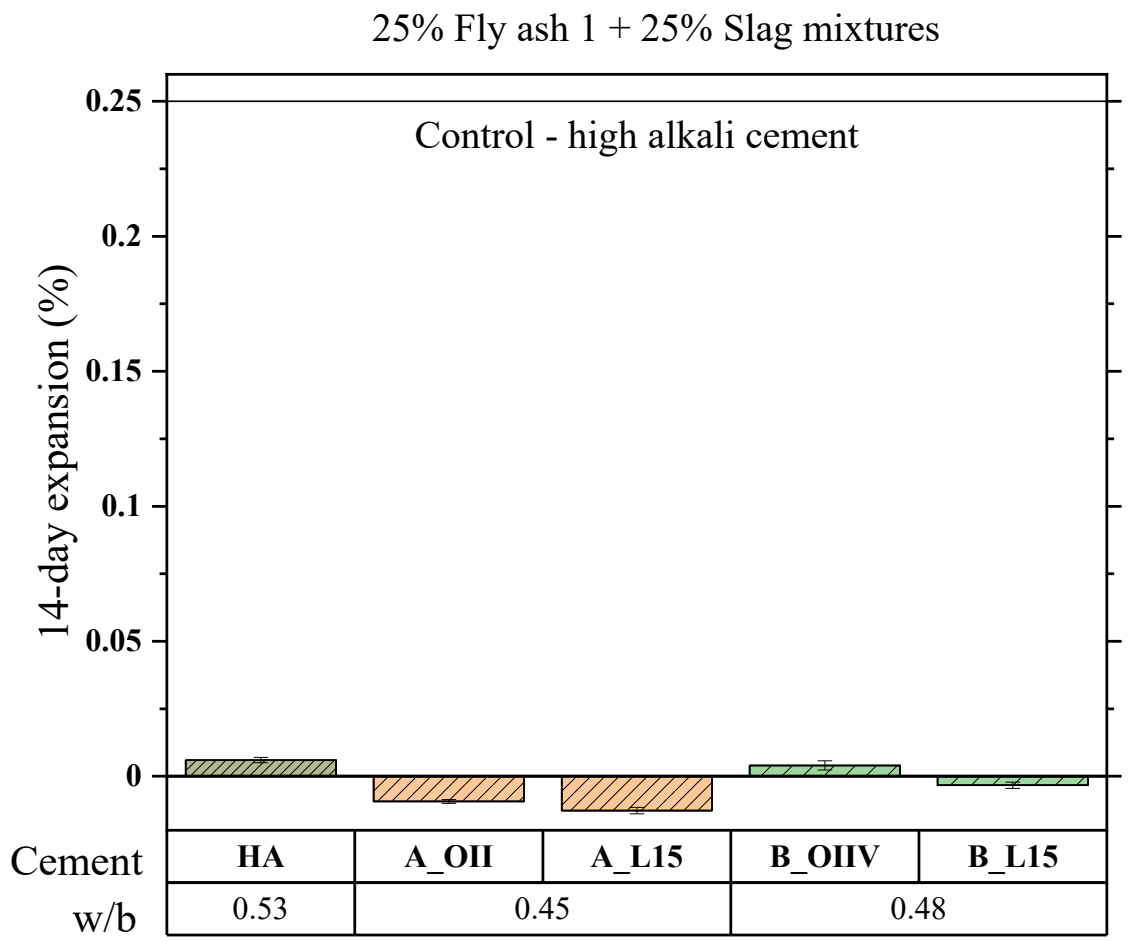

Figure 5-9. ASTM C441 results for 25\% FA1 + 25\% slag mixtures

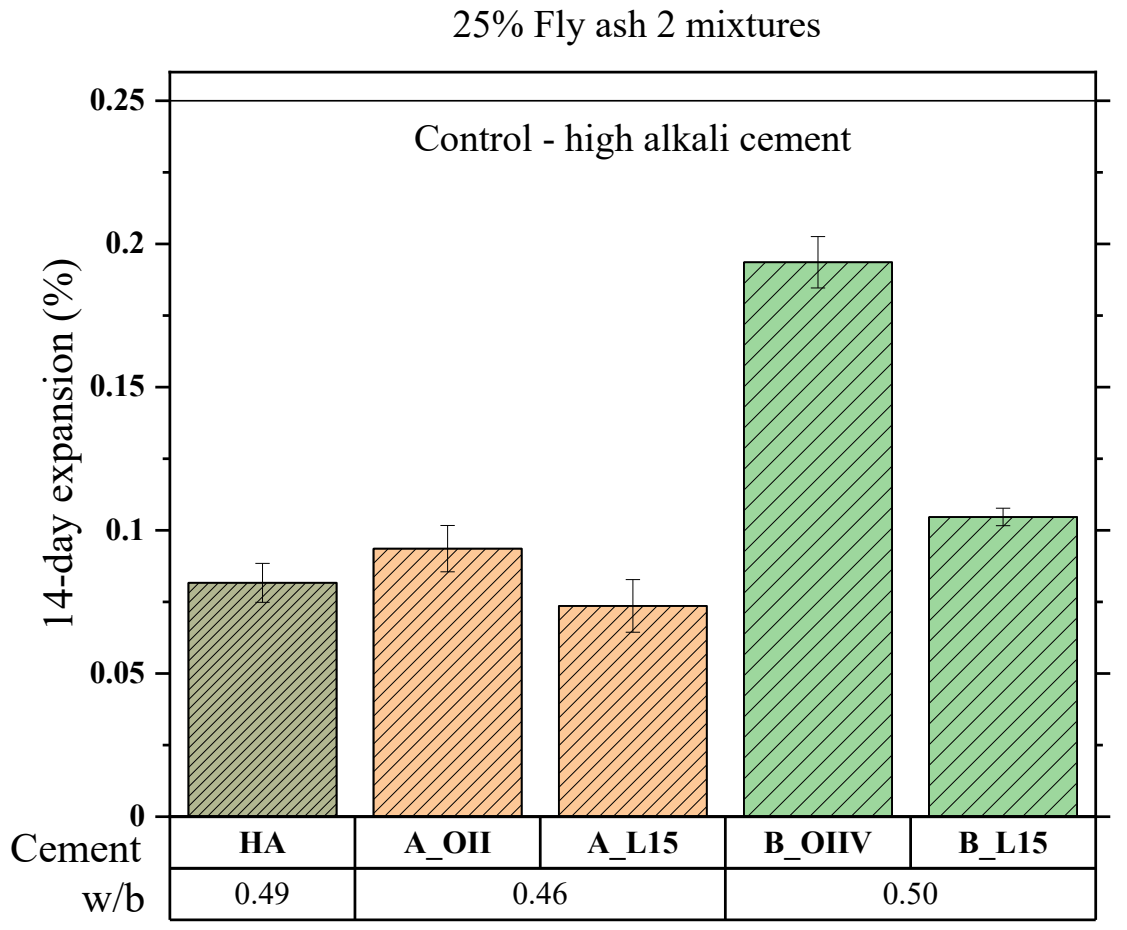




\subsubsection{Discussion of the Results}

648 In all the cases, the expansions of the mixtures (with SCMs) were less than the control - high alkali 649 cement (with no SCM). It was observed that all the PLCs performed better than their respective 650 OPCs in the presence of SCMs.

\section{$651 \quad$ 5.5.5 Significant Findings}

652 The main takeaway from the ASTM C441 testing is that all the PLCs performed better than their 653 respective OPCs in the presence of SCMs. Further, there was generally good agreement among 654 the PLCs. This allowed for the selection of a smaller number of PLCs to test in Phase 2 of the 655 project, as decided at the November 2019 project meeting.

\subsection{ASTM C1260 - Phase 2}

\subsubsection{Experimental Methods}

658 ASTM 1260 [58] is a standard test method for detecting potential alkali reactivity of aggregates in 659 mortar bars. Mortar bars of dimensions $25 \times 25 \times 285 \mathrm{~mm}$ are prepared according to ASTM C1260.

660 It involves immersion of mortar bars in $1 \mathrm{~N} \mathrm{NaOH}$ solution at $80^{\circ} \mathrm{C}$ for 14 days. Each test container 661 contains four bars soaked in $1 \mathrm{~N} \mathrm{NaOH}$ solution, as shown in Figure 5-11. To test coarse 662 aggregates, they are crushed to meet the required gradation stated in the standard.

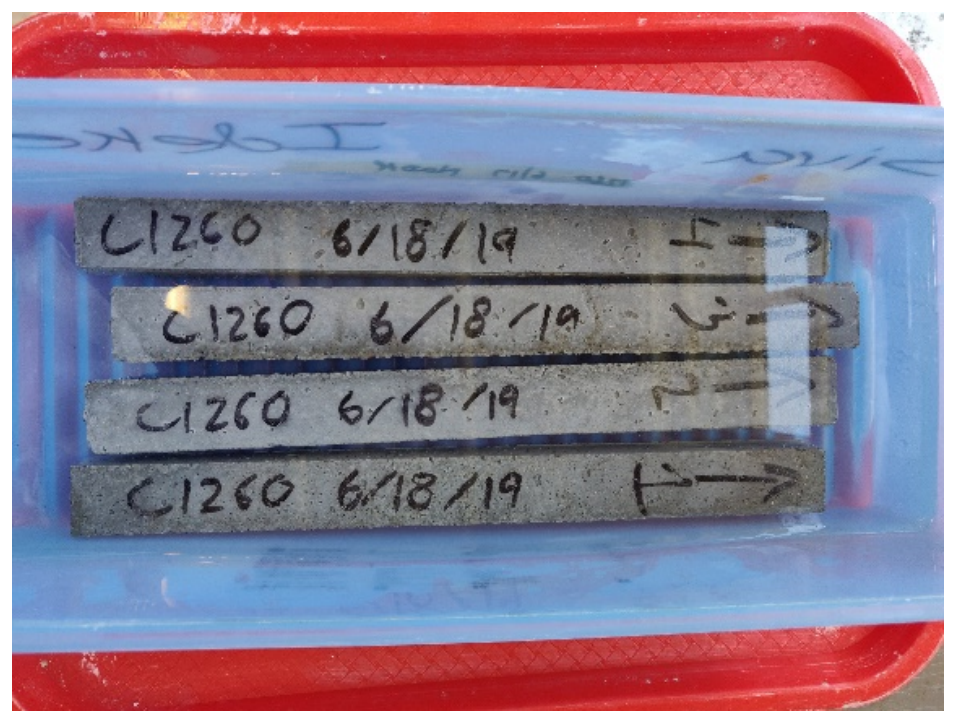




\subsubsection{Typical Experimental Measurement and Interpretation}

667 The length and mass measurements were made before immersing the bars in sodium hydroxide 668 solution (initial measurements) and various days after immersion until fourteen days. The length 669 measurements were made using a length comparator. The measured lengths were used to calculate 670 the expansion of the bars. Then the average expansions of the mixture were calculated and 671 reported. Based on the average 14-day expansion of the mixture, the aggregate reactivity was 672 determined according to ASTM C1778 [68].

\section{$673 \quad$ 5.6.3 Experimental Results}

674 A selection of reactive aggregates from four potential sources was collected. Table 5.4 shows the 675 results of the ASTM C1260 [58] testing. Table 5.5 shows the estimated major constituents in the 676 reactive fine aggregates determined using petrographic analysis according to ASTM C295 done 677 by an independent testing firm.

Table 5.4. ASTM C1260 results

\begin{tabular}{|l|l|l|l|l|}
\hline No. & $\begin{array}{l}\text { Type of } \\
\text { aggregate }\end{array}$ & Source & $\begin{array}{l}\text { ASTM C1260 14-day } \\
\text { average expansion (\%) }\end{array}$ & ASTM C1778 classification \\
\hline $\mathbf{1}$ & Fine (F1) & S1 & 0.54 & R3 Very highly-reactive \\
\hline $\mathbf{2}$ & Fine (F2) & S2 & 0.47 & R3 Very highly-reactive \\
\hline $\mathbf{3}$ & Coarse (C1) & S2 & 0.33 & R2 Highly-reactive \\
\hline $\mathbf{4}$ & Coarse (C2) & S3 & 0.24 & R1 Moderately-reactive \\
\hline
\end{tabular}

679 
Table 5.5. Estimated major constituents in the reactive fine aggregates by petrographic examination

\begin{tabular}{|c|c|}
\hline Constituent & Approximate \% by weight \\
\hline \multicolumn{1}{|c|}{ Fine aggregate - 1 (F1) } \\
\hline Granitic Rock & 44.7 \\
\hline Quartz & 17.6 \\
\hline Feldspar & 16.3 \\
\hline Diorite & 8.8 \\
\hline Volcanic Rock & 6.4 \\
\hline \multicolumn{2}{|c|}{ Fine aggregate - 2 (F2) } \\
\hline Intermediate Volcanic Rock & 59.2 \\
\hline Intermediate Volcanic Rock (Oxidized) & 12.1 \\
\hline Felsic Volcanic Rock & 12.6 \\
\hline Feldspar & 4.4 \\
\hline Quartz & 2.3 \\
\hline Granitic Rock & 1.7 \\
\hline
\end{tabular}

683 F1 aggregate was primarily comprised of granitic rock, diorite, and volcanic rock as well as quartz and feldspar mineral grains. The strained quartz and volcanic glass contained within the aggregate were identified as being potentially susceptible to ASR. F2 aggregate was primarily comprised of volcanic rock fragments of intermediate to felsic composition, with minor to trace amounts of granitic rock fragments and individual mineral grains including quartz, feldspar, pyroxene, amphibole, biotite, and opaques. The volcanic rock fragments predominate throughout the various

689 size fractions. The microcrystalline quartz and/or volcanic glass were identified as the greatest 690 potential contributors to ASR.

\subsubsection{Discussion of the Results}

692 Fine aggregates - F1 and F2, which both are very-highly reactive fine aggregate, were selected to 693 use and study for this project.

\section{$694 \quad$ 5.6.5 Significant Findings}

695 It was decided between OSU and CALTRANS Project Leaders to use the two fine aggregates 696 investigated for the remainder of the reactive aggregate assessment.

\section{$697 \quad 5.7 \quad$ ASTM C1567 - Phase 2}

\subsubsection{Experimental Methods}

699 ASTM C1567 [58] is used to evaluate the effectiveness of SCMs to mitigate or control ASR 700 expansion. The sample preparation procedure and the testing procedure for ASTM C1567 is the 
same as for ASTM C1260. The only difference is that a portion of the OPC is replaced with the desired amount, by mass, of SCM or a combination of SCM (e.g., fly ash + silica fume) to be evaluated.

\subsubsection{Typical Experimental Measurement and Interpretation}

705 The experimental measurement and interpretation are the same as for ASTM C1260. If the average 14-day expansion of a mixture is less than $0.10 \%$, it is considered that the SCM dosage effectively mitigates deleterious ASR expansion.

\subsubsection{Experimental Results}

709 Figure 5-12 and Figure 5-13 show the results of ASTM C1567 for all the mixtures tested with F1 710 aggregate. Figure 5-14 shows the results of ASTM C1567 for all the mixtures tested with F2 711 aggregate.

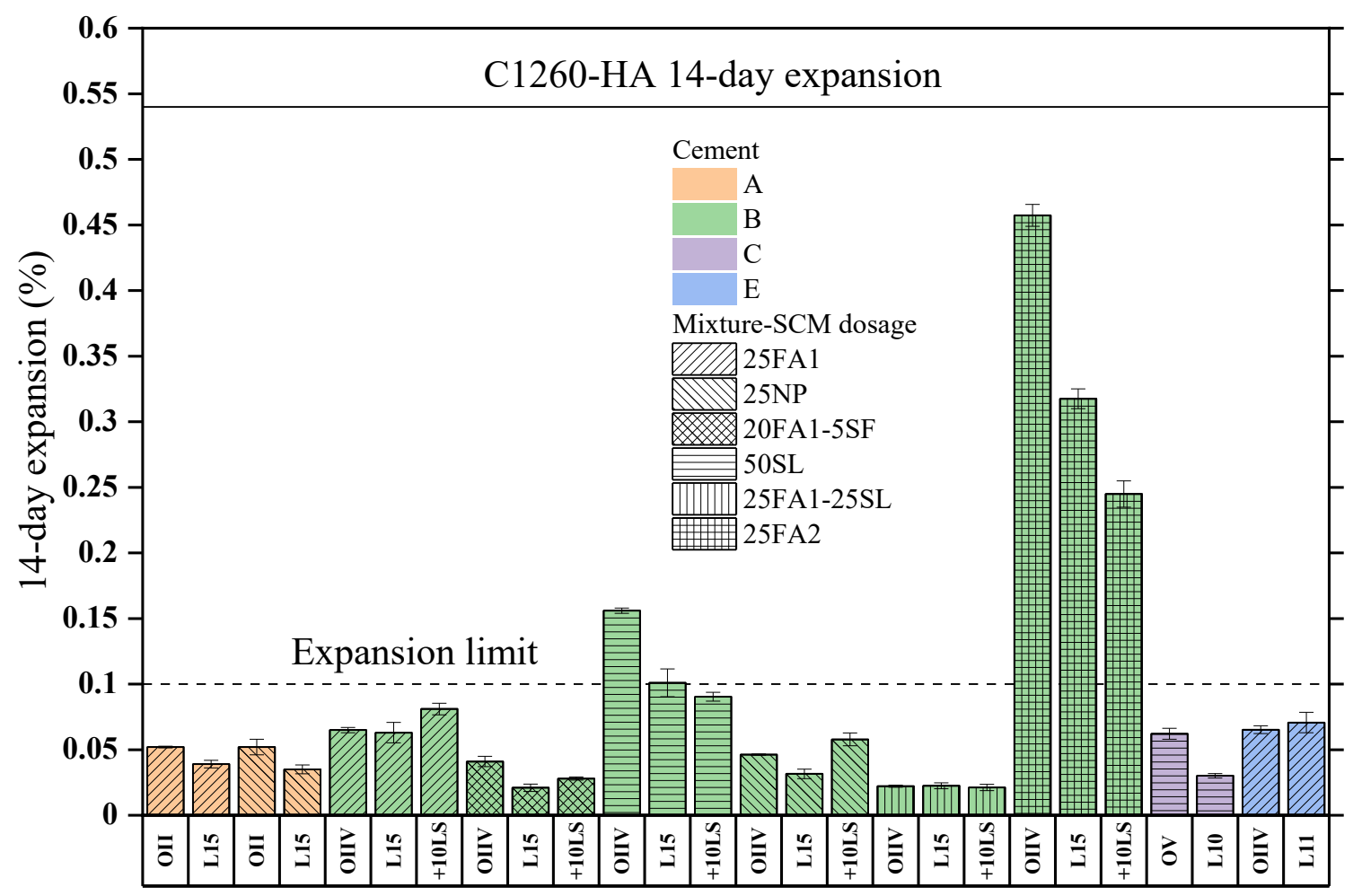
$10 \%$ LS systems with F1 aggregate (very-highly reactive as per ASTM C1778) 


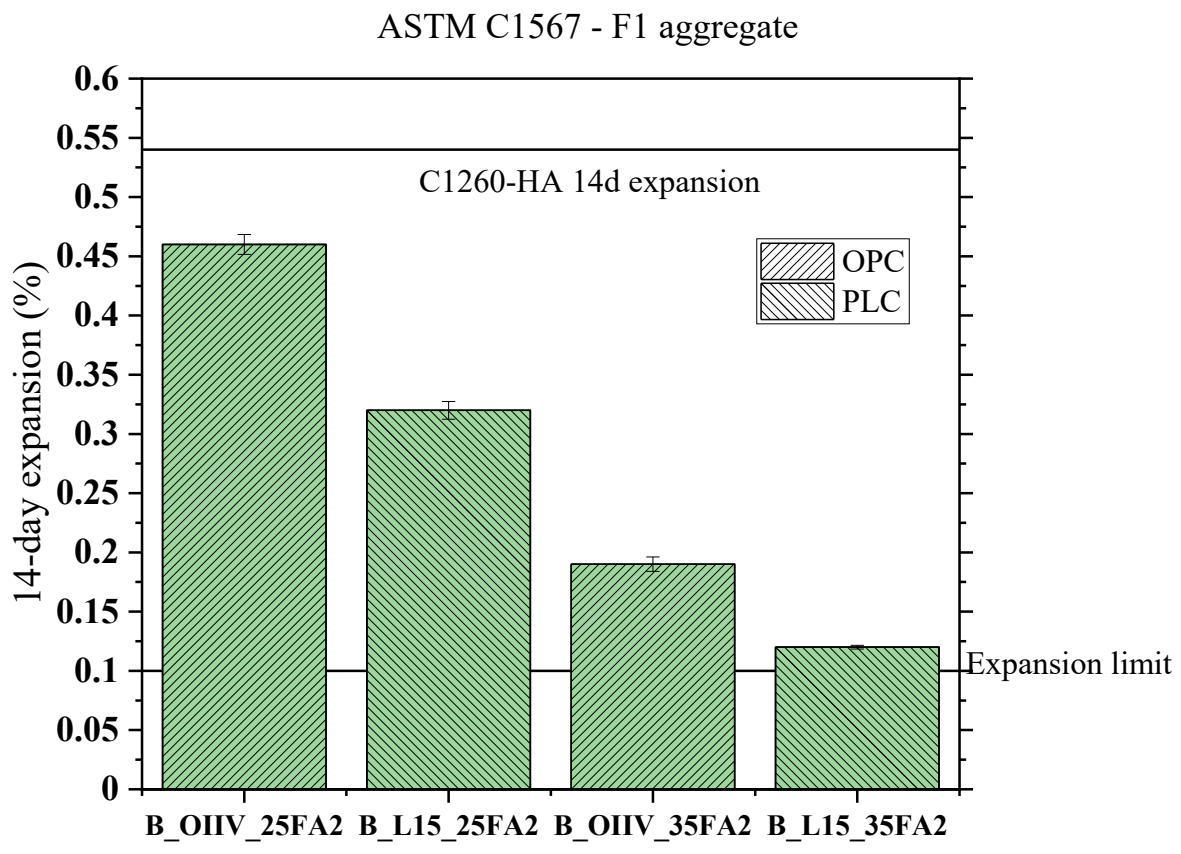

717 Figure 5-13. Comparison of ASTM C1567 expansions of mixtures with F1 aggregate and 718 cements B_OIIV and B_L15 with $25 \%$ and $35 \%$ FA2.

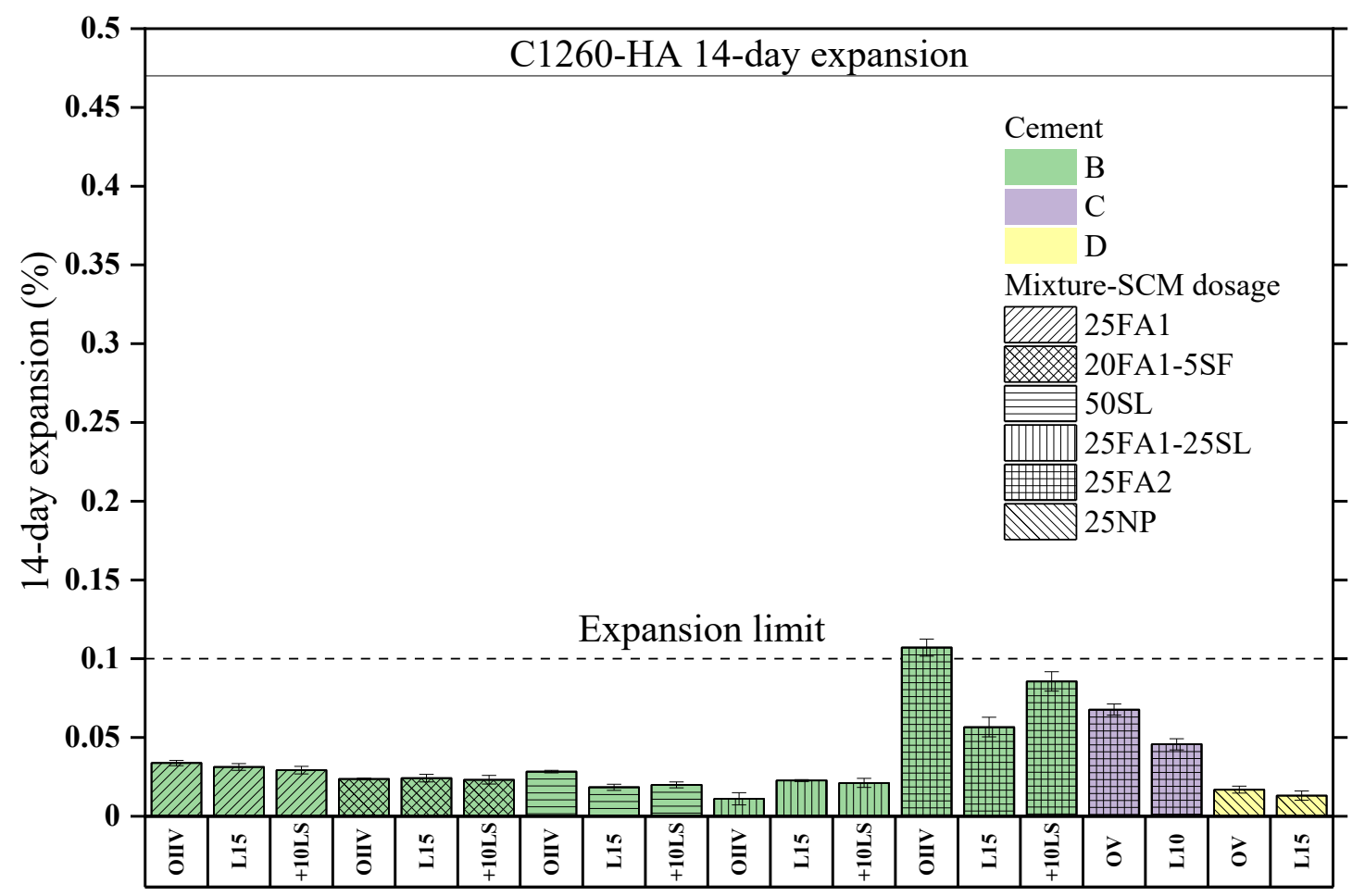




\subsubsection{Discussion of the Results}

723 Figure 5-12 shows that the use of SCMs significantly reduced the expansion of the mortar bars.

724 PLCs performed equal or better than the parent OPCs. All the mixtures expanded less than 725 expansion limit of $0.10 \%$ except the $50 \%$ slag (B_OIIV_50SL, B_L15_50SL) and 25\% FA2 726 (B_OIIV_25FA2, B_L15_25FA2, B_OIIV_25FA2 + 10LS) mixtures that included cement B. The 727 blended OPC $+10 \%$ limestone systems expanded equal or more than the PLCs except in the $50 \%$

728 slag mixture and 25\% FA2 mixtures of cement B. In most cases, OPC $+10 \%$ LS mixtures 729 performed equal or better than their parent OPCs, and their expansions were lower than the 730 expansion limit in the cases where they had more expansion than the parent OPCs.

731 Figure 5-13 shows that increasing the replacement level of FA2 from 25\% to $35 \%$ decreased the 732 expansions considerably. However, even at 35\% FA2, the mortar bars failed the ASTM C1567 733 test. Nevertheless, it should be noted that B_L15 performed better than B_OIIV with both $25 \%$ 734 and $35 \% \mathrm{FA} 2$.

Figure 5-14 observed that the use of SCMs significantly reduced the expansion of the mortar bars. All the mixtures expanded less than the expansion limit of 0.10\% except B_OIIV_25FA2. Similar to the case for F1 aggregate, 25FA2 mixtures expanded the most among the SCM mixtures. The PLCs and the blended OPC $+10 \%$ limestone systems expanded equal or less than the parent OPCs with an exception of B_OIIV_25FA1-25SL mixture. It was observed that B_OIIV_25FA1-25SL expanded slightly less than its limestone cements. However, the expansions of all the three mixtures of B_25FA1-25SL were very low, and the difference in the expansions was negligible compared to the expansion limit.

Among all the mixtures with SCMs, the mixtures with FA2 expanded the most. This could be due to its higher alkali content $\left(4.0 \% \mathrm{Na}_{2} \mathrm{O}_{e}\right)$ and $\mathrm{CaO}(12.5 \%)$ content compared to FA1 $\left(3.5 \% \mathrm{Na}_{2} \mathrm{O}_{\mathrm{e}}\right.$ and $8.6 \% \mathrm{CaO}$ ). In addition, it should be noted that one of the other important factors for controlling ASR related expansion is using SCMs that are high in alumina. FA2 (16.6\% $\left.\mathrm{Al}_{2} \mathrm{O}_{3}\right)$ has a considerably lower $\mathrm{Al}_{2} \mathrm{O}_{3}$ content compared to FA1 $\left(21.7 \% \mathrm{Al}_{2} \mathrm{O}_{3}\right)$, and this likely accounts for the higher expansion observed at the same replacement level of $25 \%$. If this ash were to be used with this very highly reactive fine aggregate, a higher replacement level would be needed and could be verified through ASTM C1567 testing. It may also be effective in a ternary blend with $5 \%$ silica fume or metakaolin while retaining the $25 \%$ replacement level. However, further performance-based testing would be needed to determine that amount.

754 and either of the following shall be used:

755

756
- Available alkali as $\mathrm{Na}_{2} \mathrm{O}+0.658 \mathrm{~K}_{2} \mathrm{O}$ must not exceed 1.5 percent when tested under ASTM C311. 
- Total alkali as $\mathrm{Na}_{2} \mathrm{O}+0.658 \mathrm{~K}_{2} \mathrm{O}$ must not exceed 5.0 percent when tested under AASHTO T 105.

759 Both FA1 and FA2 have total alkali content less than $5.0 \% \mathrm{Na}_{2} \mathrm{O}_{\mathrm{e}}$, thus complying with the second 760 requirement. The available alkali (ASTM C311) of the ashes are not determined as part of this 761 project. There exists a linear correlation between total equivalent alkali and available alkali 762 (ASTM C311), as shown in Figure 5-15. The data in Figure 5-15 were collected from literature $763[69,70]$ to determine the correlation between available alkali and total alkali for Class $\mathrm{F}$ fly ashes. 764 The calculated available alkali (ASTM C311) for FA1 and FA2 according to the equation in Figure $7655-15$ were $1.4 \% \mathrm{Na}_{2} \mathrm{O}_{\mathrm{e}}(<1.5 \%$ limit $)$ and $1.7 \% \mathrm{Na}_{2} \mathrm{O}_{\mathrm{e}}(>1.5 \%$ limit $)$, respectively. This indicates 766 that FA2 contributed higher alkali compared to FA1.

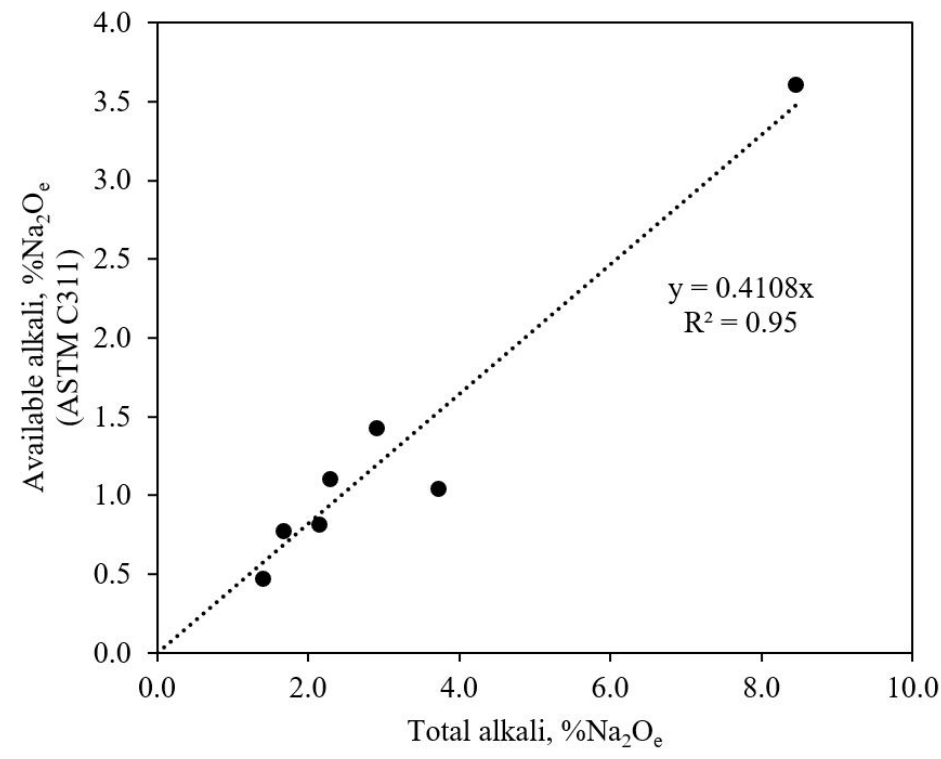

Figure 5-15. Correlation between available alkali (ASTM C311) and total alkali of Class F fly ashes (Data from [69] and [70]).

770 To further confirm the contribution of alkalis from FA2 to pore solution, cement pastes were cast 771 in $50 \mathrm{~mm}$ (diameter) x $100 \mathrm{~mm}$ (length) cylinder molds and were sealed cured for 28 days at $23^{\circ} \mathrm{C}$.

772 After the curing period, the cylinders were demolded, and the specimens were squeezed in a pore 773 press to extract the pore solutions. The extracted pore solutions were filtered and analyzed using 774 ICP-OES to determine their ion compositions. Figure 5-16 shows the sum of sodium and potassium 775 ion concentrations in the pore solutions of hardened cement pastes (for the materials in this project) 776 that were sealed cured at $23^{\circ} \mathrm{C}$ for 28 days. 


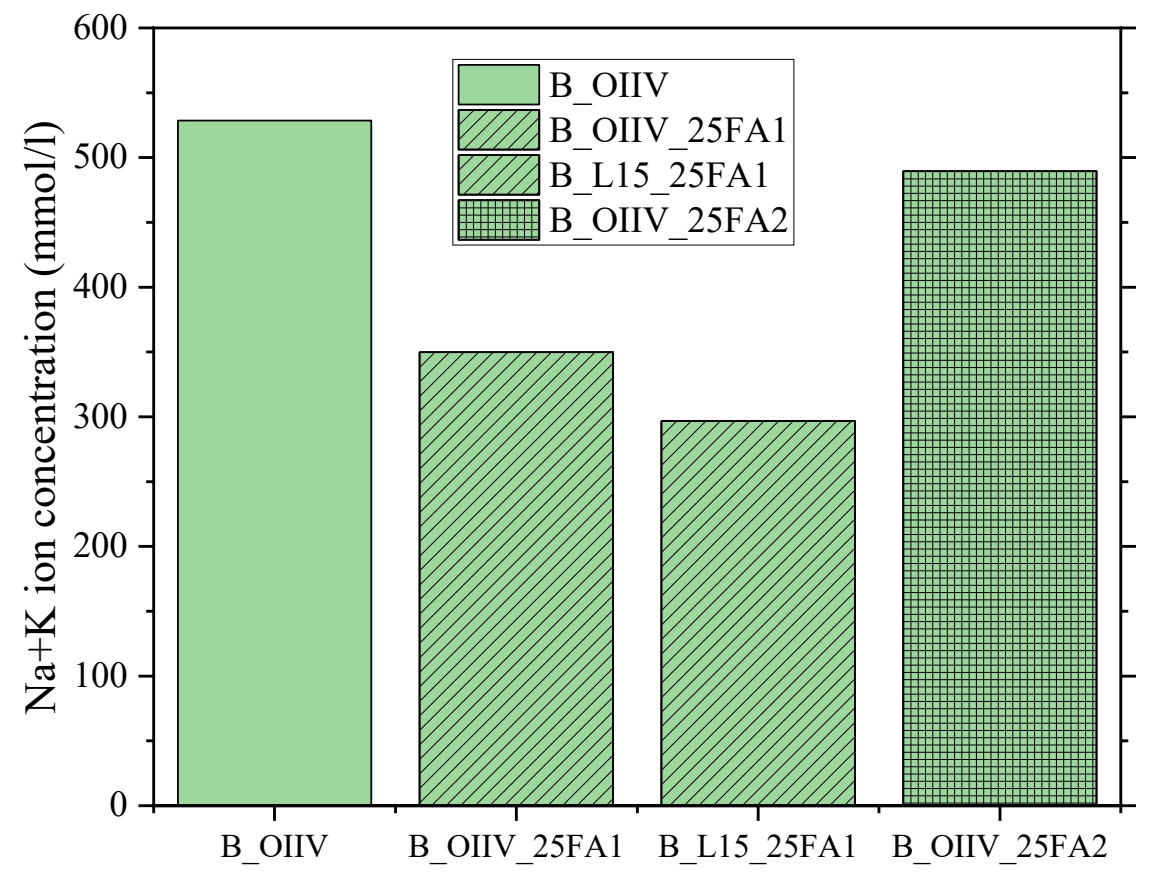

Figure 5-16. Sum of sodium and potassium ion concentrations in the pore solutions of hardened cement pastes that were sealed cured at $23^{\circ} \mathrm{C}$ for 28 days for the materials in this

It was observed that pore solution alkalinity of B_OIIV_25FA2 is higher than B_OIIV_25FA1 giving further evidence that FA2 provides more alkalis to pore solution than it binds, and it has higher available alkali than FA1.

\subsubsection{Significant Findings}

The main take away from the C1567 testing is that the PLCs perform similar to or better than their parent OPCs. In addition, it was observed that the presence of all SCMs reduced expansions compared to the control for all the mixtures. This resulted in the majority of the mixtures ( 39 of 45) being below the $0.10 \%$ expansion limit with the six exceptions, as noted in section 5.7.4.

\subsection{AASHTO T 380 (Miniature Concrete Prism Test)}

\subsubsection{Experimental Methods}

791 The miniature concrete prism test (MCPT) is a recently developed test method by Latifee and 792 Rangaraju [71] to overcome the limitations of the AMBT and the CPT. The method was recently 793 standardized by AASTHO, and it is available as AASHTO T 380. The MCPT involved casting 794 concrete bars of dimensions $50 \mathrm{~mm} \times 50 \mathrm{~mm} \times 285 \mathrm{~mm}$ with $\mathrm{w} / \mathrm{cm}$ of 0.45 according to AASHTO 795 T 380. F1 and F2 reactive aggregates were used as fine aggregate, and a non-reactive limestone 796 aggregate was used as a coarse aggregate in the study. The coarse aggregate consisted of $9.5 \mathrm{~mm}$ 
797 sieve retained rock ( $57.5 \%$ by mass) and $4.8 \mathrm{~mm}$ sieve retained rock ( $42.5 \%$ by mass). The alkali 798 content of the mixture was boosted to $1.25 \%$ of the cement mass according to the standard. The 799 concrete bars were immersed in $1 \mathrm{~N}$ sodium hydroxide solution (as shown in Figure 5-17) and 800 stored at $60^{\circ} \mathrm{C}$ for a period of 56 days, and the expansions were monitored periodically.

\subsubsection{Typical Experimental Measurement and Interpretation}

804 The length and mass measurements were made before immersing the bars in sodium hydroxide 805 solution (initial measurements) and various days after immersion until 84 days. The length 806 measurements were made using a length comparator. The measured lengths were used to calculate 807 the expansion of the bars. Then the average expansions of the mixture were calculated and 808 reported. The criteria for determining the efficacy of SCMs in preventing ASR expansion 809 according to AASHTO T 380 are:

810 - ineffective if the 56-day average expansion greater than $0.025 \%$

811 - effective if the 56-day average expansion is less than $0.020 \%$

812 - uncertain if the 56-day average expansion is in between $0.020 \%$ and $0.025 \%$

\section{$813 \quad$ 5.8.3 Experimental Results}

814 Figure 5-18 and Figure 5-19 show the AASHTO T 380 expansion results at 56 days for the 815 mixtures with F1 and F2 aggregates, respectively. 


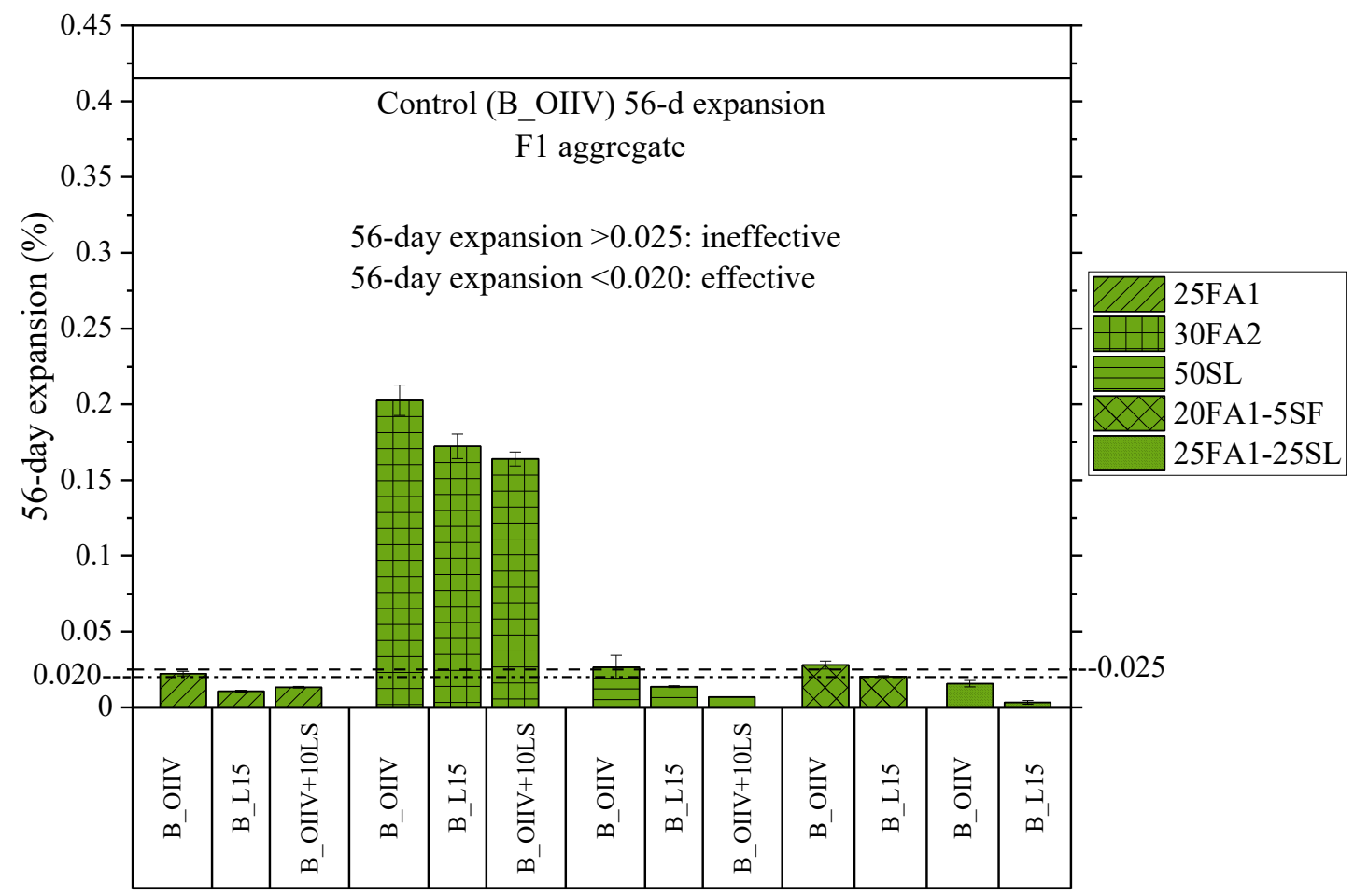

817 Figure 5-18. 56-day AASHTO T 380 expansion results of the mixtures with F1 aggregate 818 and portland cement $B$

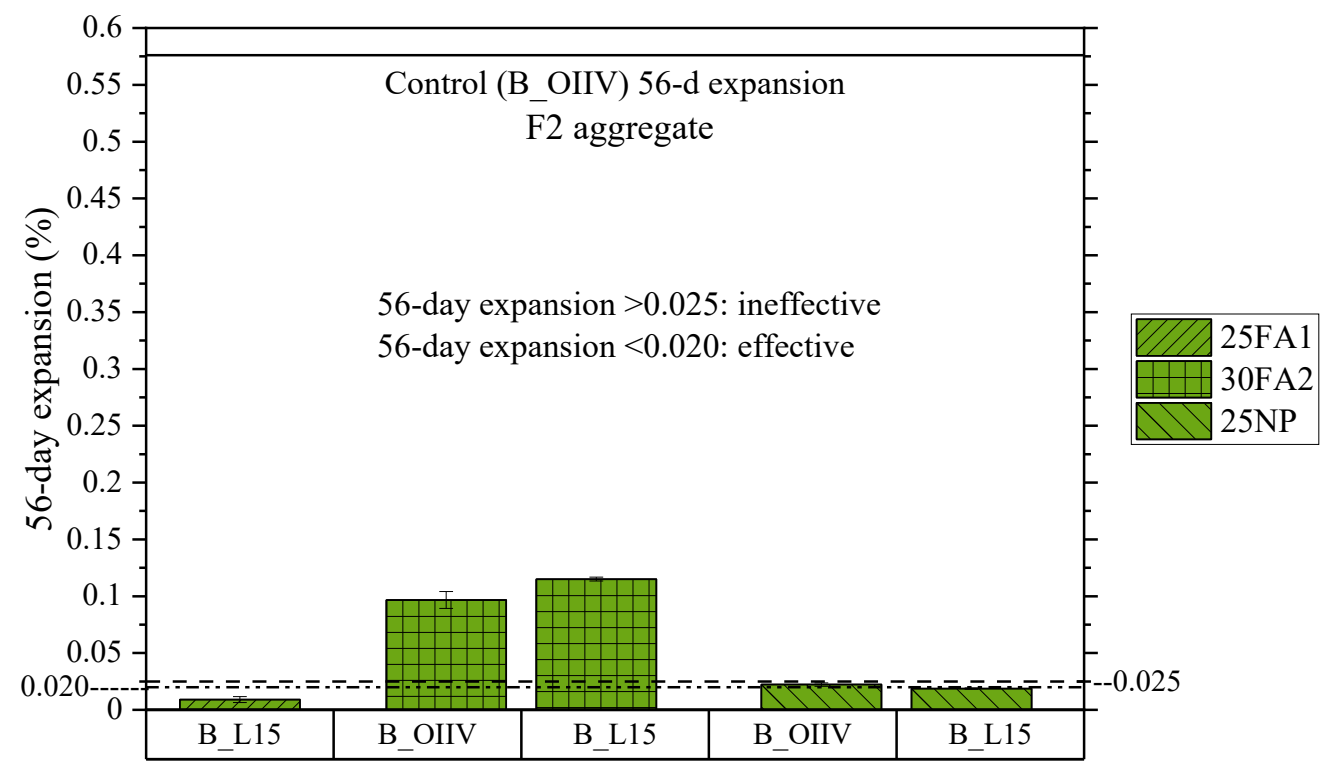

820 Figure 5-19. 56-day AASHTO T 380 expansion results of the mixtures with F2 aggregate 


\subsubsection{Discussion of the Results}

823 Figure 5-18 shows that the use of SCMs significantly reduced the expansions compared to the

824 control mixture with F1 aggregate. All the concrete mixtures with PLCs (interground and added 825 limestone) expanded lower than the mixtures with the parent OPCs. The percentage reduction in 826 the expansions of the mixtures with PLCs ranged from $15 \%$ to $79 \%$ with respect to their parent 827 OPC mixture in combinations with SCMs. In the case of the mixtures with $50 \%$ slag, the outcome 828 of the test method (effective or ineffective) varied based on the presence of OPC or PLC in the 829 mixture. Similar to the observation made from the ASTM C1567 results, the mixtures with 25FA1830 25SL expanded the least, and the mixtures with FA2 expanded the most among the SCM 831 combinations tested and failed in AASHTO T 380.

832 From Figure 5-19, it was observed that the concrete mixtures with F2 aggregate and SCMs 833 performed better than the control mixture as expected. It was observed that the B_L15-30FA2 834 mixture expanded slightly more than the B_OIIV-30FA2 mixture. However, both the mixtures 835 with 30FA2 failed the test due its higher alkali content and lower alumina content. Either a higher 836 replacement of FA2 or a ternary blend of FA2+(SCM) would be needed to reduce expansions 837 below the expansion limit. It was observed that B_L15-25NP mixture expanded 14\% less than 838 B_OIIV-25NP mixture.

\section{$839 \quad 5.8 .5 \quad$ Significant Findings}

840 The main take away from AASHTO T 380 testing is that the PLCs perform similar to or better 841 than their parent OPCs. The only exception observed was the mixture with 30FA2 and F2 842 aggregate, where the PLC mixture expanded slightly higher than the OPC mixtures; however, both 843 the mixtures failed the test.

\subsection{ASTM C1293 (Concrete Prism Test)}

\subsubsection{Experimental Methods}

846 ASTM C1293 is a standard test method for detecting potential alkali reactivity of aggregates in 847 concrete bars and determining the efficiency of SCMs to prevent ASR. In this study, ASTM C1293 848 test was used to determine the aggregate reactivity level of F1 and F2 aggregates. Concrete bars 849 of dimensions $75 \times 75 \times 285 \mathrm{~mm}$ are prepared according to ASTM C1293. A non-reactive 850 limestone aggregate was used as a coarse aggregate in the study. The coarse aggregate consisted 851 of $12.5 \mathrm{~mm}$ sieve retained rock ( $33.3 \%$ by mass), $9.5 \mathrm{~mm}$ sieve retained rock ( $33.3 \%$ by mass) and $8524.8 \mathrm{~mm}$ sieve retained rock (33.3\% by mass). The alkali content of the mixture was boosted to $8531.25 \%$ of the cement mass according to the standard. It involved storage of concrete bars in sealed 854 buckets over water at $38^{\circ} \mathrm{C}$ for a period of one year. Each test container contained three bars. 


\subsubsection{Typical Experimental Measurement and Interpretation}

856 The length and mass measurements were made before storing the bars in sealed buckets (initial 857 measurements) and periodically after that as mentioned in ASTM C1293. The length 858 measurements were made using a length comparator. The measured lengths were used to calculate 859 the expansion of the bars. Then the average expansions of the mixture were calculated and 860 reported. The criteria for determining the alkali reactivity of aggregates according to ASTM C1778 861 is

- Non-reactive if the 1-year average expansion is less than $0.04 \%$

- Moderately reactive if the 1-year average expansion is less than $0.12 \%$ and greater than or equal to $0.04 \%$

- Highly reactive if the 1-year average expansion is less than $0.24 \%$ and greater than or equal to $0.12 \%$

- Very highly reactive if the 1-year average expansion is greater than or equal to $0.24 \%$

869 Figure 5-20 shows the expansion results of all the four mixtures tested according to ASTM C1293.

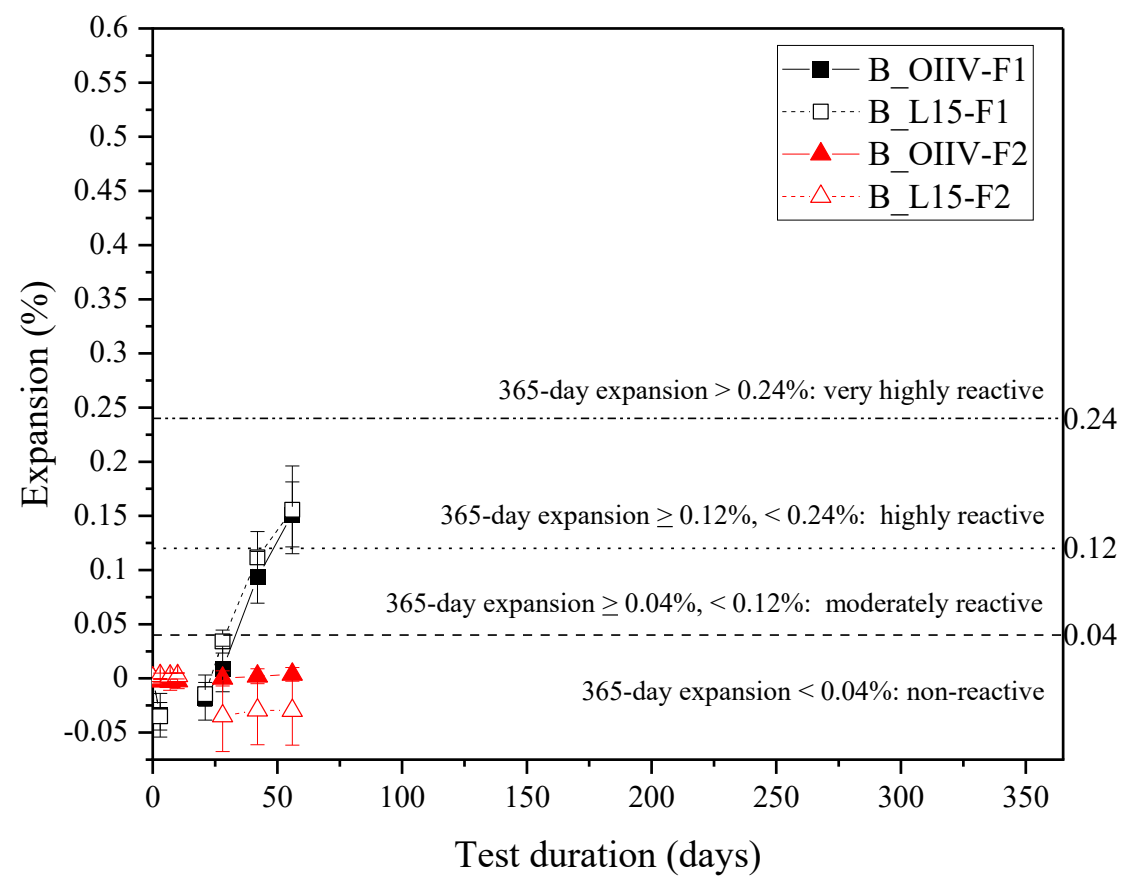

\subsubsection{Experimental Results}

871 Figure 5-20. ASTM C1293 expansion results (Note: the total test duration is 365 days) 


\section{$872 \quad$ 5.9.4 Discussion of the Results}

873 The total test duration of ASTM C1293 method to classify the reactivity of aggregates is 365 days.

874 The mixtures have been monitored for only 56-days at the time of writing the report. The reactivity 875 of F1 and F2 aggregates will be determined according to ASTM C1778 after 365 days of 876 monitoring the specimens.

$877 \quad 5.9 .5 \quad$ Significant Findings

878 While testing for ASTM C1293 is still in progress and a final conclusion cannot be drawn, at the 879 time report has been issued, it can be noticed that the PLC behaves similarly to the OPC system 880 for both the reactive aggregates. As such, the preliminary indication suggests that the aggregate 881 reactivity level is expected to be similar for both the OPC and PLC systems. 
884 Drying shrinkage occurs primarily due to the capillary pressure that develops as fluid leaves the 885 cement paste pores. The Kelvin-Laplace-Young equation [72] states that as the size of a pore is 886 reduced, the capillary pressure that would develop increases, which would result in increased 887 shrinkage. Many state highway agencies have been concerned that the increased fineness of PLC 888 may make mixtures made using PLC more susceptible to shrinkage and shrinkage cracking than 889 those made with OPC [73, 74]. This portion of the research investigated the shrinkage for paste 890 made using OPC, PLC, and OPC+ LS for both plain mixtures (M0) and systems containing SCM 891 (M1 to M5).

\subsection{Research Objective}

893 This portion of the research's primary objective was to determine whether the replacement of OPC 894 with PLC or OPC with OPC + LS in conjunction with SCMs impacts cement paste shrinkage. This 895 will be evaluated using ASTM C157. Ultimately, this research will inform CALTRANS whether 896 PLC can be used as an equivalent replacement of OPC without impacting the shrinkage and 897 cracking performance. This would enable language to be added to the specifications, if deemed 898 necessary, stating what aspects would need to be measured through trial batches using the actual 899 mixture design and raw materials if PLC is used.

\subsection{Background/Literature Review}

901 Some state highway agencies are concerned that the increased fineness associated with the PLC's 902 manufacture may mixtures made using PLC more susceptible to shrinkage and shrinkage cracking $903[5,57,75,76]$. Previous studies on PLC shrinkage have also provided mixed results related to the 904 PLC shrinkage compared to an OPC made from the same clinker. Adams and Race [77] reported 905 that limestone addition increased the drying shrinkage of type I and type II cements. However, 906 this study is nearly 20 years old, and as a result, the PLC that was used had been made using a

907 fundamentally different approach than the current approach. Some studies have reported that the 908 addition of limestone does not affect or reduces overall shrinkage in concrete. Alunno-Rossetti 909 and Curcio [15] produced comparable concrete mixtures using two sets of OPC and PLC 910 (produced at two different plants) and observed their creep and shrinkage behavior for one year. 911 They noted that OPCs and their respective PLCs exhibited essentially the same shrinkage rate and 912 total drying shrinkage for one year. Stubstad et al. [75] measured shrinkage and shrinkage cracking 913 of mixtures with up to 5\% limestone and observed less shrinkage in the OPC cements. Dhir et al. 914 [11] compared OPC concrete with comparable PLC concretes $\left(310 \mathrm{~kg} / \mathrm{m}^{3}\right.$ and $\left.\mathrm{w} / \mathrm{cm}=0.60\right)$ and 915 reported marginal reduction in shrinkage for concretes produced with ground limestone $(15 \%$ to 916 45\%). Cost [78] and Smartz and Lankar [3] reported similar shrinkage in OPC and PLC systems 917 (however, detailed data was not provided). As a result, it appears that, in general, the OPC and 918 PLC have similar shrinkage; however, this can be dependent on the fineness of the cement and 
919 limestone. Bucher et al. [73, 74] measured autogenous shrinkage, unrestrained shrinkage, and 920 restrained drying shrinkage of mortars produced using three cements (with an OPC with $0 \%$, a 921 PLC with 5\%, and a PLC with 10\% LS) as shown in Figure 6-1. The tests concluded that both 922 autogenous and unrestrained shrinkage in mortar samples were slightly less with increasing 923 limestone content. Bucher et al. [73, 74] examined the addition of limestone of different fineness 924 to OPC as a replacement to evaluate the shrinkage cracking. Bucher et al. [73, 74]observed that 925 the coarser limestone had a slower rate of stress development and a longer time to develop the first 926 restrained shrinkage crack. However, it should be noted that the study by Bucher et al. [73, 74] 927 was not on PLC that was designed to be 'equivalent' OPC in terms of strength development. They 928 also noted that the coarser limestone had a slower rate of stress development, and thus addition of 929 limestone also increased the time to cracking slightly compared to OPC mortar (Figure 6-2). Piasta 930 and Sikora [79] examined the shrinkage of concrete with limestone cements combined with SCMs 931 and concluded that SCMS were useful in reducing shrinkage. Similar observations have been 932 reported by Barrett et al. [2, 76] (Figure 6-3). Bentz et al. [80] attributed the reduced shrinkage in 933 PLC mortars to the differences in particle size distributions of constituents.

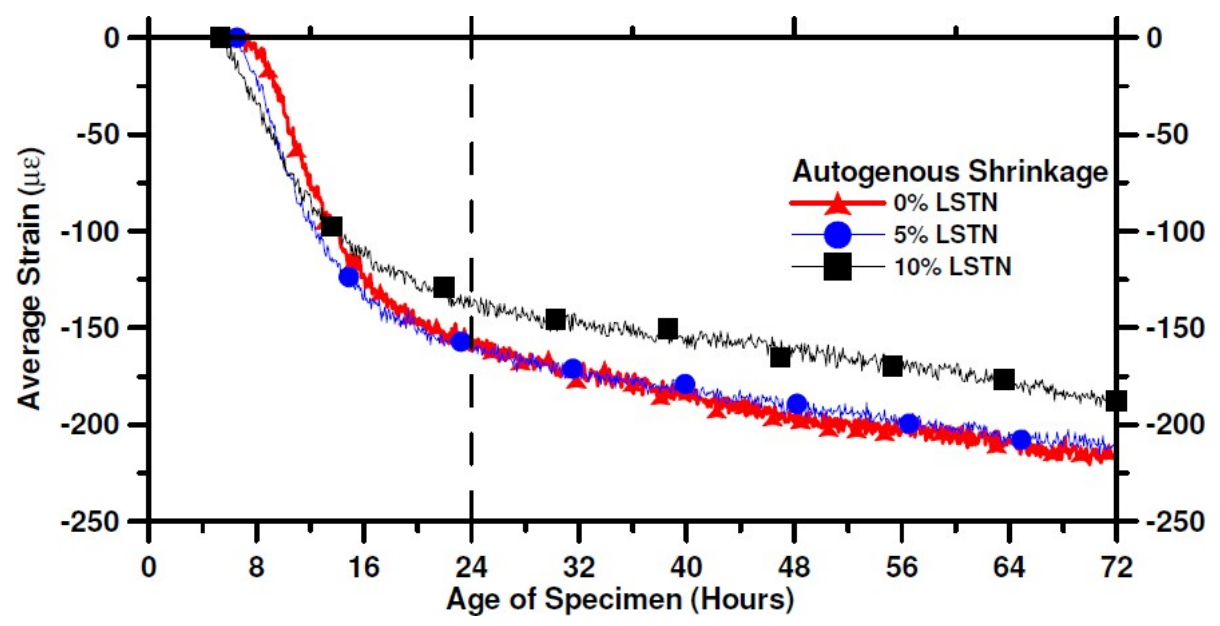

(a) 


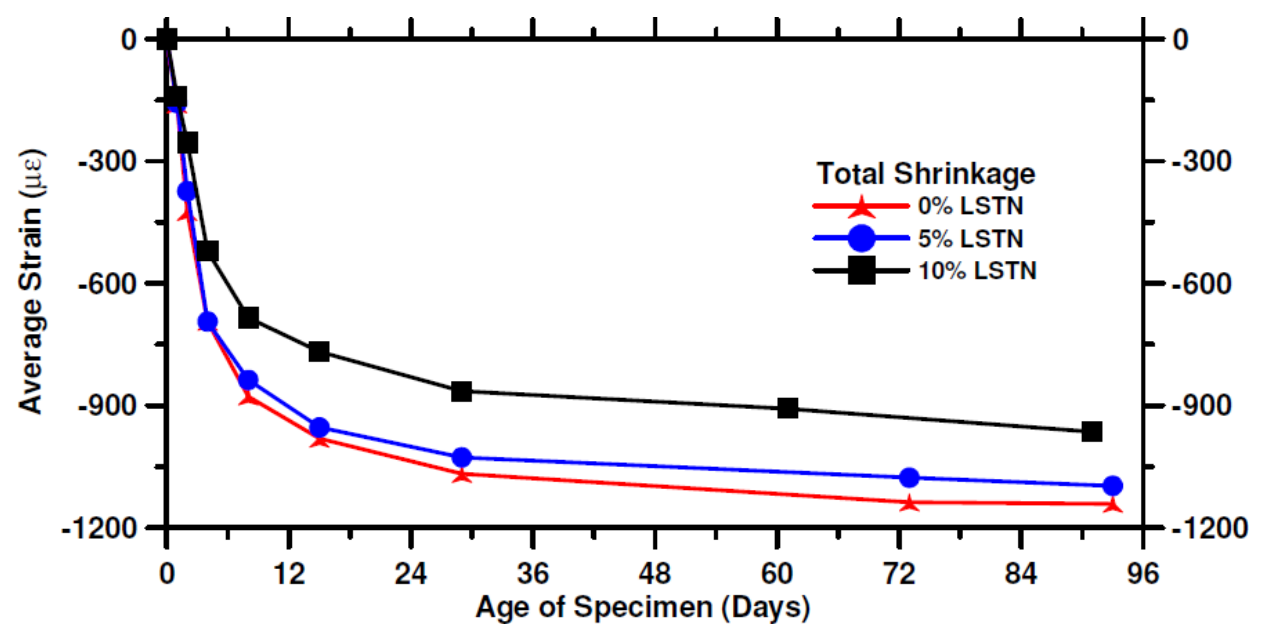

(b)

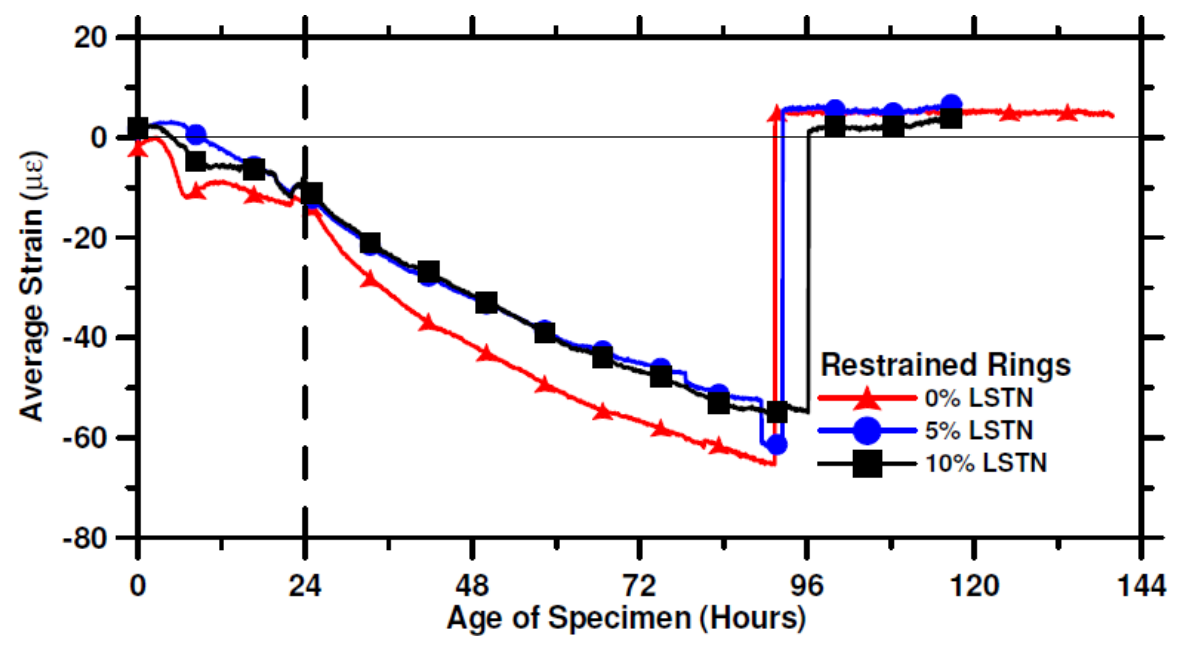

(c)

935 Figure 6-1. (a) Autogenous, (b) unrestrained, and (c) restrained shrinkage (ASTM C1581) 


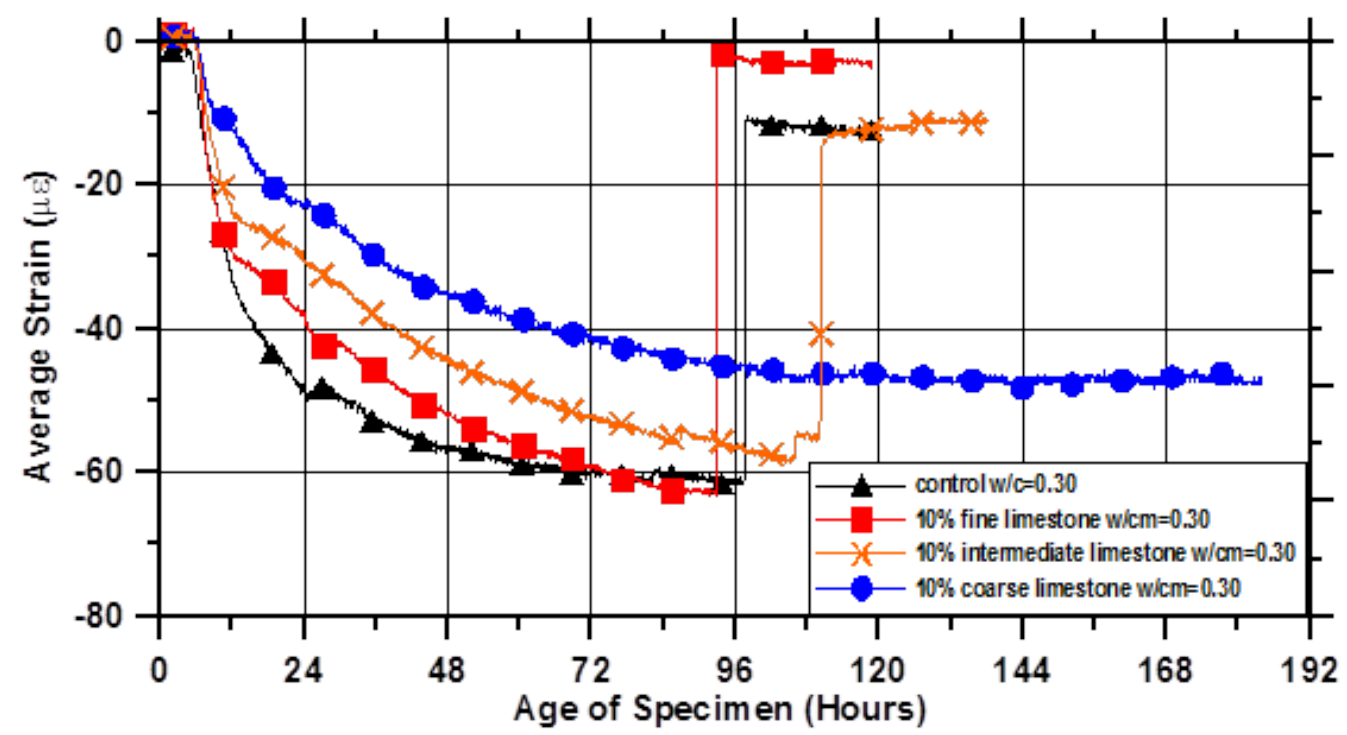

939

940

Figure 6-2. The influence of the fineness of limestone on the restrained shrinkage stress development in OPC $+10 \%$ LS systems [74]

941

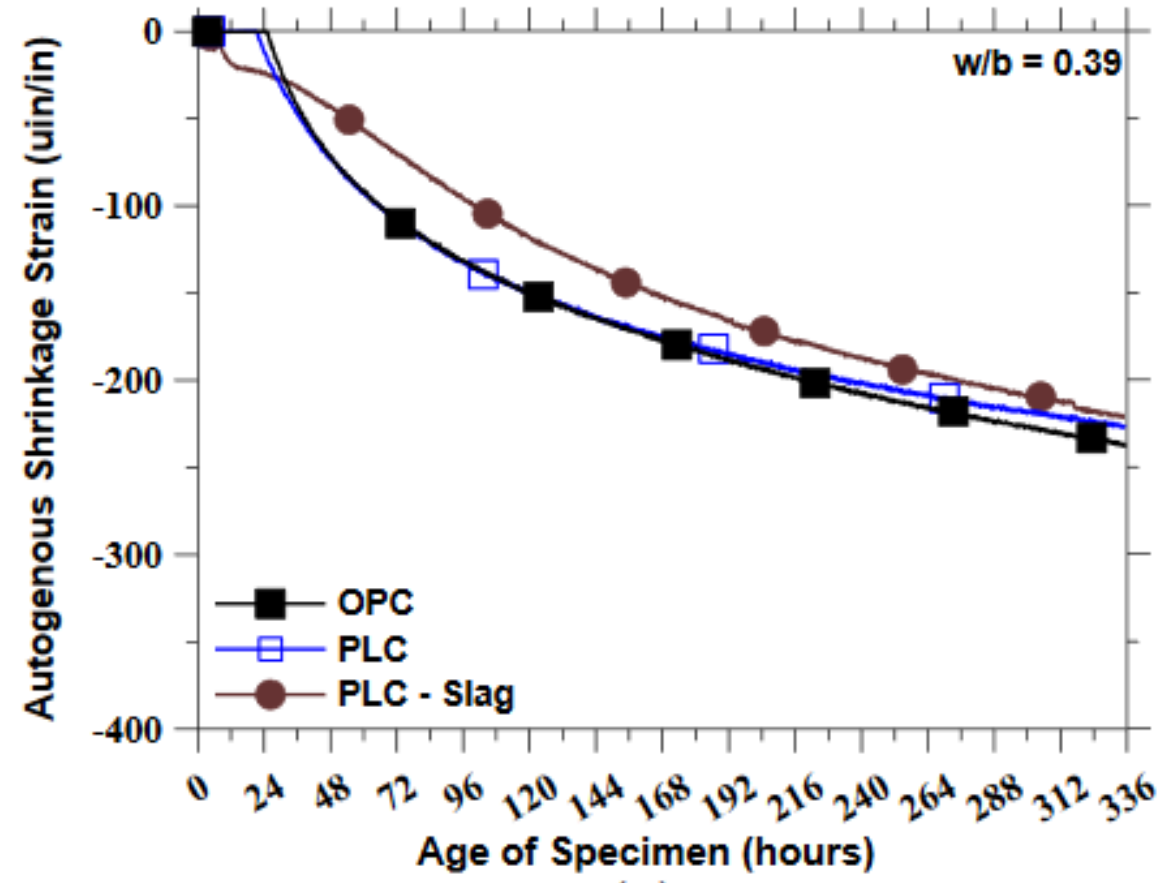

(a) 


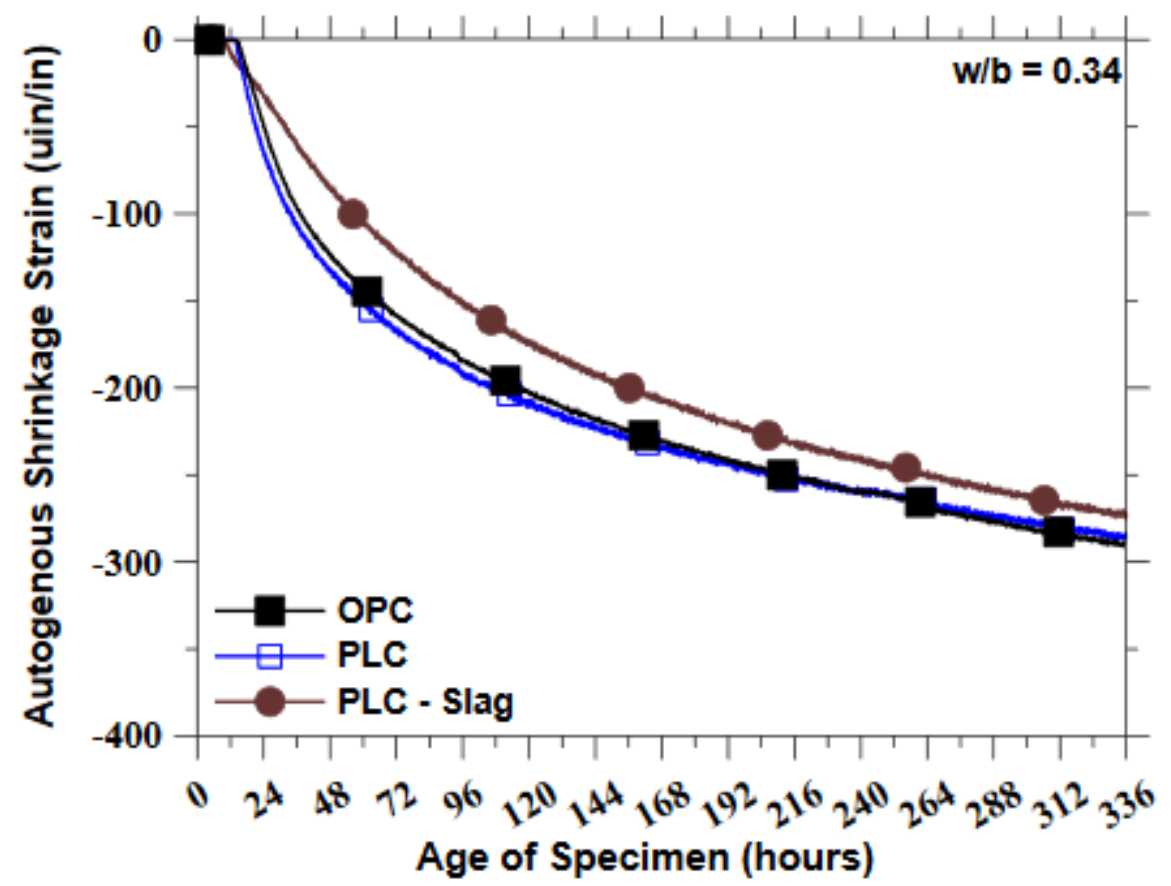

(b)

Figure 6-3. Autogenous shrinkage measurements for OPC, PLC, and PLC-slag systems with: (a) w/b 0.39; and (b) w/b 0.34 [76]

945 Barrett et al. [76] studied commercial OPC and PLC systems that were 'equivalent' (manufactured 946 to meet ASTM C595 based on the principle of similar 28-day strengths) and reported no significant 947 change in drying shrinkage or restrained shrinkage cracking (Figure 6-4) with the one exception 948 of a PLC cement that was ground to a fineness level that was much finer $(30 \%$ more than that 949 typically observed).

950

951 


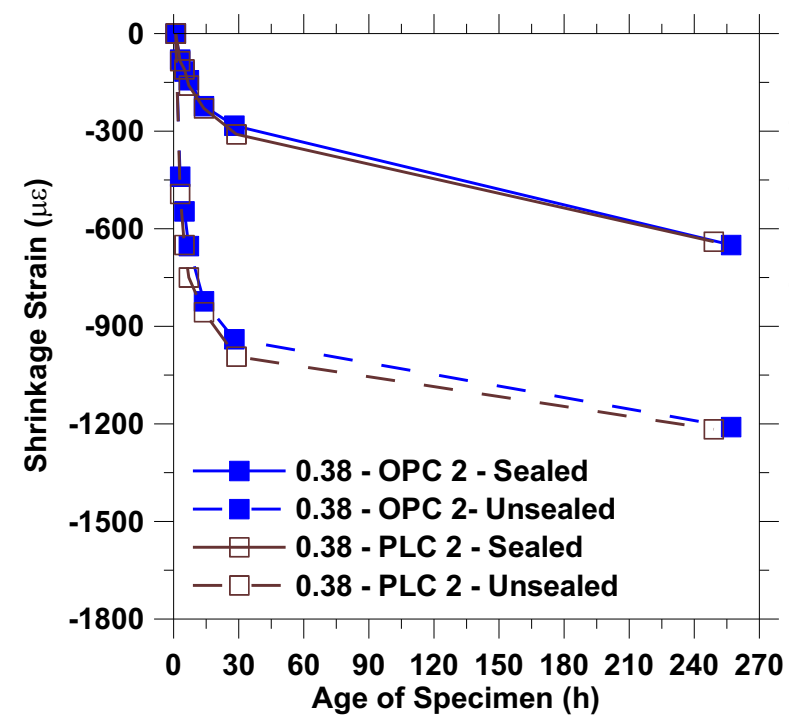

(a)

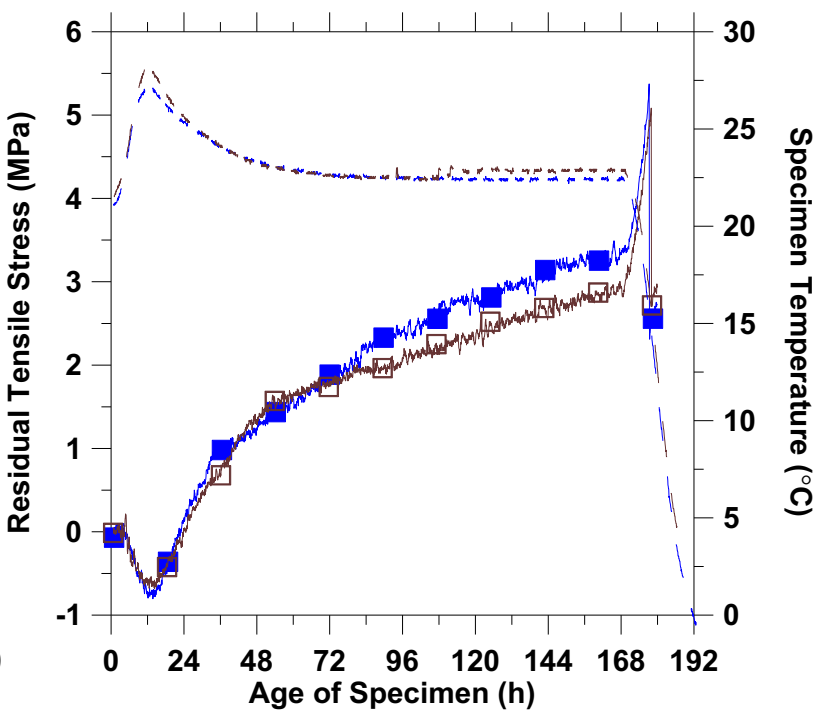

(b)

954 Figure 6-4. (a) Free shrinkage of sealed and unsealed OPC and PLC concrete and (b) Stress 955 Developed for OPC and PLC when restrained from shrinking freely. [76]

\section{$956 \quad 6.3$ Experimental Test Matrix}

957 To evaluate the relative performance of all cements in this study, drying shrinkage testing was 958 performed following the ASTM C 157 [81] (as used by CALTRANS). The shrinkage tests were 959 performed on mortar prisms $(25 \times 25 \times 285 \mathrm{~mm})$. Two samples were tested for each mixture. The 960 mixtures for the test using the proportions provided in Table 6.1 based on the SCM replacement 961 levels (by mass) provided in Section 3.

962 The test matrix comprising 80 mortar mixtures, which were cast and tested for drying shrinkage 963 tests, is shown in Table 6.2. 
Table 6.1. Mixture proportions for mixtures evaluated for shrinkage

\begin{tabular}{|c|c|c|c|c|c|c|c|}
\hline & & \multicolumn{7}{|c|}{ Mass given in $\mathbf{( k g}^{\left.-\mathbf{m}^{3}\right)}$} \\
\hline Material & S.G. & M0 & M1 & M2 & M3 & M4 & M5 \\
\hline $\begin{array}{c}\text { Cement or } \\
\text { cement+powder* }\end{array}$ & 3.15 & 696.9 & 498.8 & 492.8 & 339.0 & 328.2 & 503.9 \\
\hline Fly Ash & 2.34 & 0 & 166 & 131 & 0 & 164 & 0 \\
\hline Silica Fume & 2.20 & 0 & 0 & 33 & 0 & 0 & 0 \\
\hline Slag & 2.83 & 0 & 0 & 0 & 339 & 164 & 0 \\
\hline $\begin{array}{c}\text { Natural } \\
\text { Pozzolan }\end{array}$ & 2.36 & 0 & 0 & 0 & 0 & 0 & 168 \\
\hline \#23 Sand (SSD) & 2.67 & 1335 & 1335 & 1335 & 1335 & 1335 & 1335 \\
\hline Water & 1 & 279 & 266 & 263 & 271 & 263 & 269 \\
\hline
\end{tabular}

* OPC, PLC or OPC+10LS

Table 6.2. Test matrix for drying shrinkage tests on mortar specimens

\begin{tabular}{|c|c|c|c|c|c|c|}
\hline & M0 & M1 & M2 & M3 & M4 & M5 \\
\hline A_OII & $\sqrt{ }$ & $\sqrt{ }$ & $\sqrt{ }$ & $\sqrt{ }$ & $\sqrt{ }$ & $\sqrt{ }$ \\
\hline A_L15 & $\sqrt{ }$ & $\sqrt{ }$ & $\sqrt{ }$ & $\sqrt{ }$ & $\sqrt{ }$ & $\sqrt{ }$ \\
\hline A_O $\overline{\mathrm{II}}+10 \mathrm{LS}$ & $\sqrt{ }$ & $\sqrt{ }$ & $\sqrt{ }$ & $\sqrt{ }$ & $\sqrt{ }$ & $\sqrt{ }$ \\
\hline B_OIIV & $\sqrt{ }$ & $\sqrt{ }$ & $\sqrt{ }$ & $\sqrt{ }$ & $\sqrt{ }$ & $\sim$ \\
\hline B_L15 & $\sqrt{ }$ & $\sqrt{ }$ & $\sqrt{ }$ & $\sqrt{ }$ & $\sqrt{ }$ & $\sim$ \\
\hline B_OIIV+10LS & $\sqrt{ }$ & $\sqrt{ }$ & $\sqrt{ }$ & $\sqrt{ }$ & $\sqrt{ }$ & $\sim$ \\
\hline C_OV & $\sqrt{ }$ & $\sqrt{ }$ & $\sqrt{ }$ & $\sqrt{ }$ & $\sqrt{ }$ & $\sim$ \\
\hline C_L10 & $\sqrt{ }$ & $\sqrt{ }$ & $\sqrt{ }$ & $\sqrt{ }$ & $\sqrt{ }$ & $\sim$ \\
\hline C_L14 & $\sqrt{ }$ & $\sqrt{ }$ & $\sqrt{ }$ & $\sqrt{ }$ & $\sqrt{ }$ & $\sim$ \\
\hline $\mathrm{C} \_\mathrm{OV}+10 \mathrm{LS}$ & $\sqrt{ }$ & $\sqrt{ }$ & $\sqrt{ }$ & $\sqrt{ }$ & $\sqrt{ }$ & $\sim$ \\
\hline D_OV & $\sqrt{ }$ & $\sqrt{ }$ & $\sqrt{ }$ & $\sqrt{ }$ & $\sqrt{ }$ & $\sqrt{ }$ \\
\hline D_L15 & $\sqrt{ }$ & $\sqrt{ }$ & $\sqrt{ }$ & $\sqrt{ }$ & $\sqrt{ }$ & $\sqrt{ }$ \\
\hline D_OV $+10 \mathrm{LS}$ & $\sqrt{ }$ & $\sqrt{ }$ & $\sqrt{ }$ & $\sqrt{ }$ & $\sqrt{ }$ & $\sqrt{ }$ \\
\hline E_OIIV & $\sqrt{ }$ & $\sqrt{ }$ & $\sqrt{ }$ & $\sqrt{ }$ & $\sqrt{ }$ & $\sim$ \\
\hline$\overline{\mathrm{E}} \_11$ & $\sqrt{ }$ & $\sqrt{ }$ & $\sqrt{ }$ & $\sqrt{ }$ & $\sqrt{ }$ & $\sim$ \\
\hline E_OIIV+10LS & $\sqrt{ }$ & $\sqrt{ }$ & $\sqrt{ }$ & $\sqrt{ }$ & $\sqrt{ }$ & $\sim$ \\
\hline
\end{tabular}

971

\section{$972 \quad 6.4 \quad$ Experimental Methods}

973 Drying shrinkage tests were performed on mortar specimens using the mixture proportions shown

974 in Table 6.1. The mortar mixtures were prepared using concrete sand. The mixtures were prepared 975 with a batch size of $0.002 \mathrm{~m}^{3}$. The samples were mixed in a Hobart (N50 5-Quart) mixer. The 976 cementitious powders were dry mixed for 15 seconds at low agitation (60 rotation per minute), 977 and then water was added to the mixing bowl, and the paste was mixed for 30 seconds. The sand 
978 was added in the next 30 seconds of mixing. After sand addition, the agitation speed was increased 979 to intermediate (120 rotations per minute), and the mortar was mixed at this speed for 30 seconds 980 and then stopped for a quick scraping of the bowl and the attachments. Following this, the mixture 981 was mixed for another 1 minute at intermediate agitation before it was poured into molds. Three 982 mixtures with the same mixture design but different cements (OPC, PLC, and OPC+10\%LS 983 respectively) of the same parent clinker were cast on the same day to enable direct comparison of 984 the samples.

985 The mortar mixture was placed in two prism-shaped molds $(25 \times 25 \times 285 \mathrm{~mm}$.). Metal pins (gauge 986 studs) were cast in the ends of the beams to facilitate length change measurements using a 987 comparator. Figure 6-5 illustrates the comparator that was used for shrinkage measurements. In 988 addition to the shrinkage prisms, six cylinders ( $50 \mathrm{~mm}$ diameter $\mathrm{x} 40 \mathrm{~mm}$ length) were also cast 989 for testing the mixture's mechanical properties as described in section 7.

990
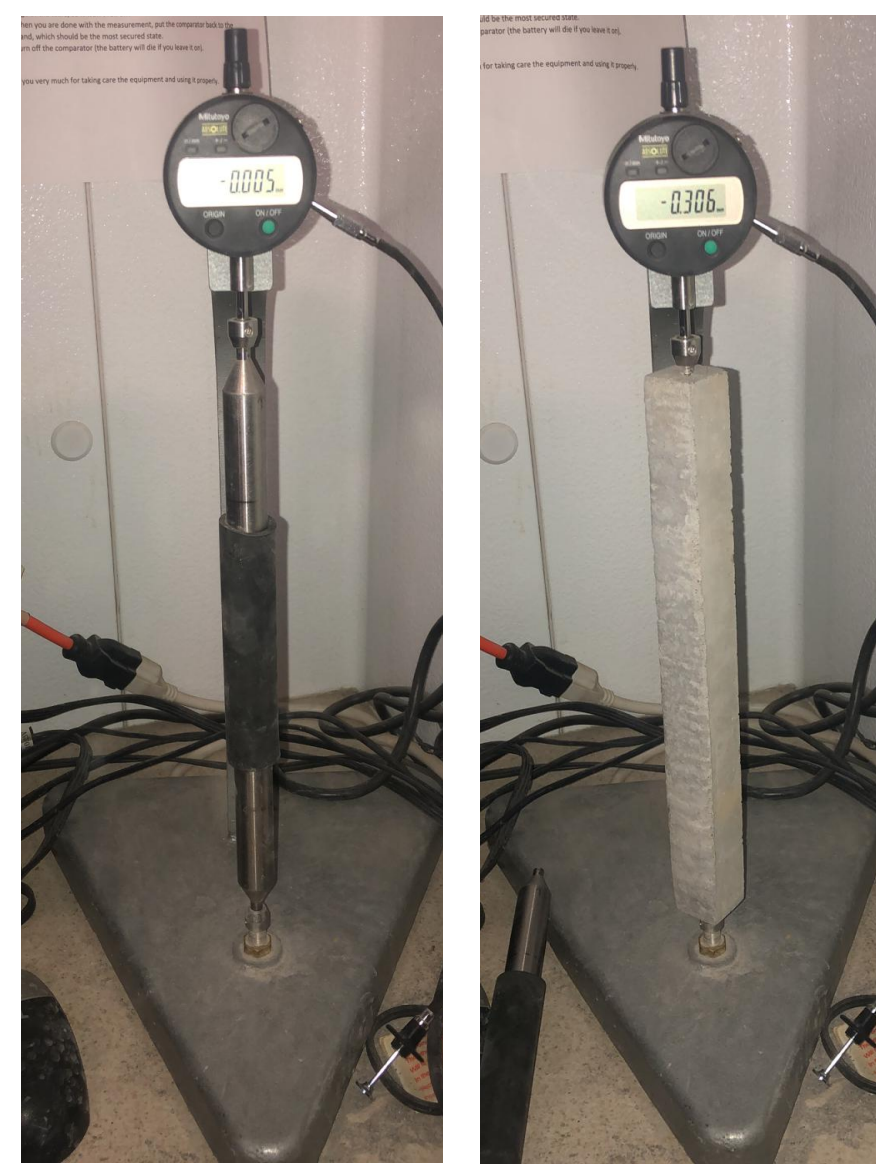
measurement and b) shows the sample's measurement. 
993 The drying shrinkage measurements were performed using ASTM C157 (AASHTO T 160) on the

994 mortar prisms. Fresh mortar samples were stored under wet burlap at $23 \pm 2^{\circ} \mathrm{C}$ for 24 hours. The 995 samples were then demolded and placed in water at $23 \pm 2^{\circ} \mathrm{C}$ for 30 minutes, after which initial 996 length measurements were recorded. After the initial length measurements, the samples were again 997 stored in water up to 3 days from casting time. The specimens were then removed from the water, 998 and their length was measured. They were stored in the drying chamber up to 28 days from casting. 999 The length change measurements were performed at every $1,3,5,7,14$, and 28 days.

\subsection{Typical Experimental Measurement and Interpretation}

1001 Figure 6-6 depicts a typical drying shrinkage plot. The y-axis depicts the drying shrinkage strain 1002 in micro-strain $(\mu \varepsilon)$. The $\mathrm{x}$-axis represents the duration of exposure to drying (in days) at which 1003 the shrinkage measurements were made. The typical values of drying shrinkage strains observed 1004 in mortar beams is between 1000-1500 $\mu \varepsilon$. Each plot compares the shrinkage measurements of (i) 1005 OPC (ii) PLC and (iii) OPC+ limestone bars with the same parent clinker corresponding to one 1006 particular mixture described in the graph legend.

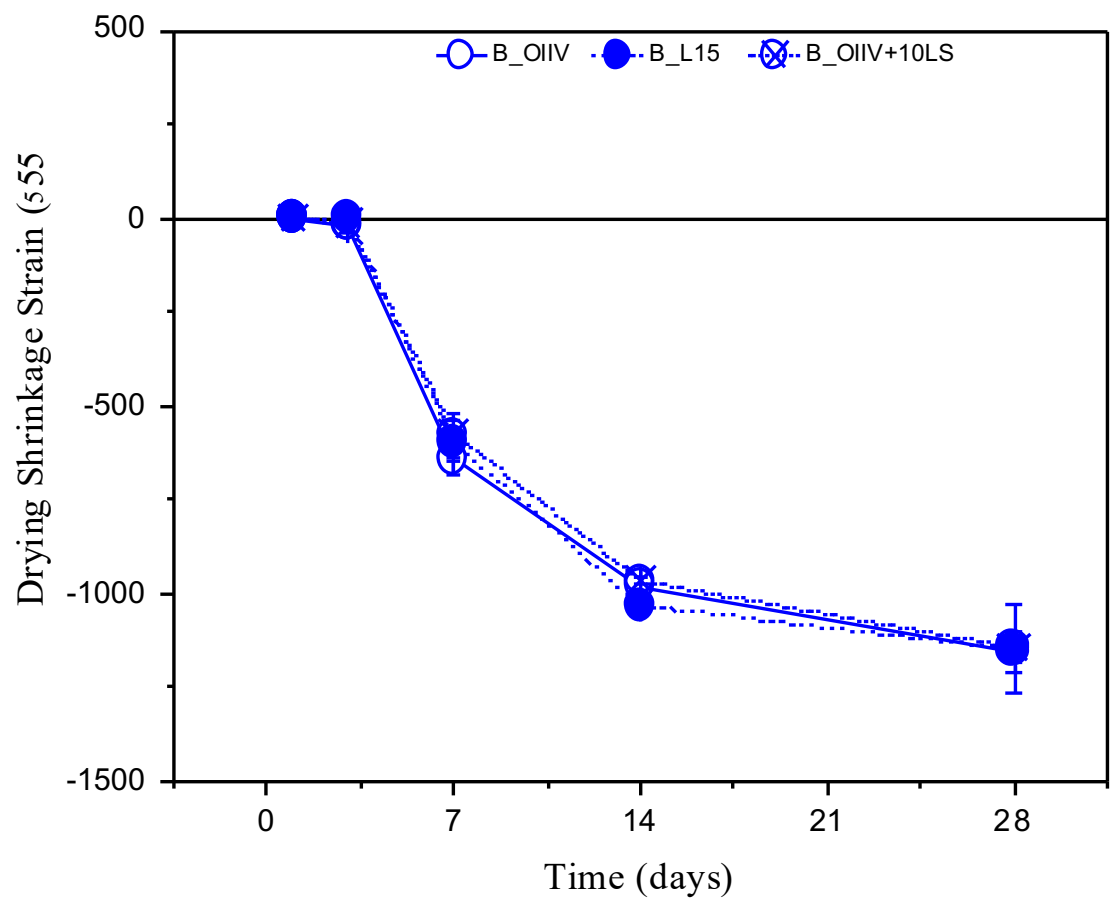




\section{$1009 \quad 6.6 \quad$ Experimental Results}

1010 Drying shrinkage results are shown for cement $\mathrm{D}$ for the different mixture proportions are provided 1011 in Figure 6-7(a-f). The drying shrinkage results of the remaining cements are provided in Appendix 1012 C.

1013

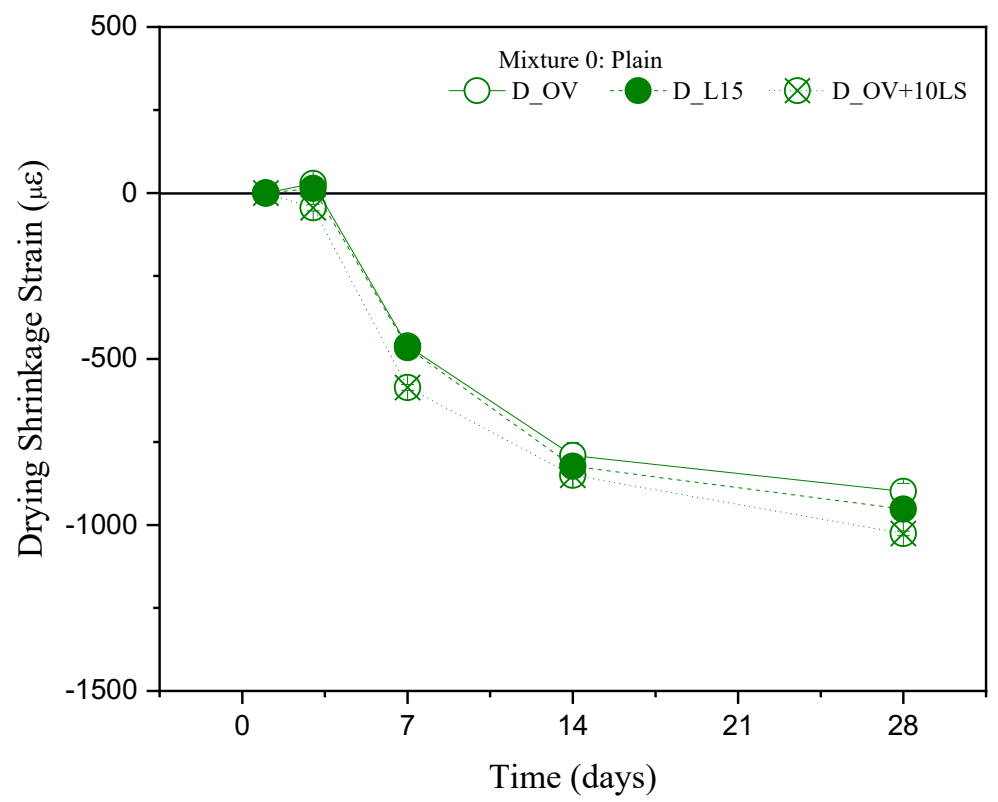

(a)

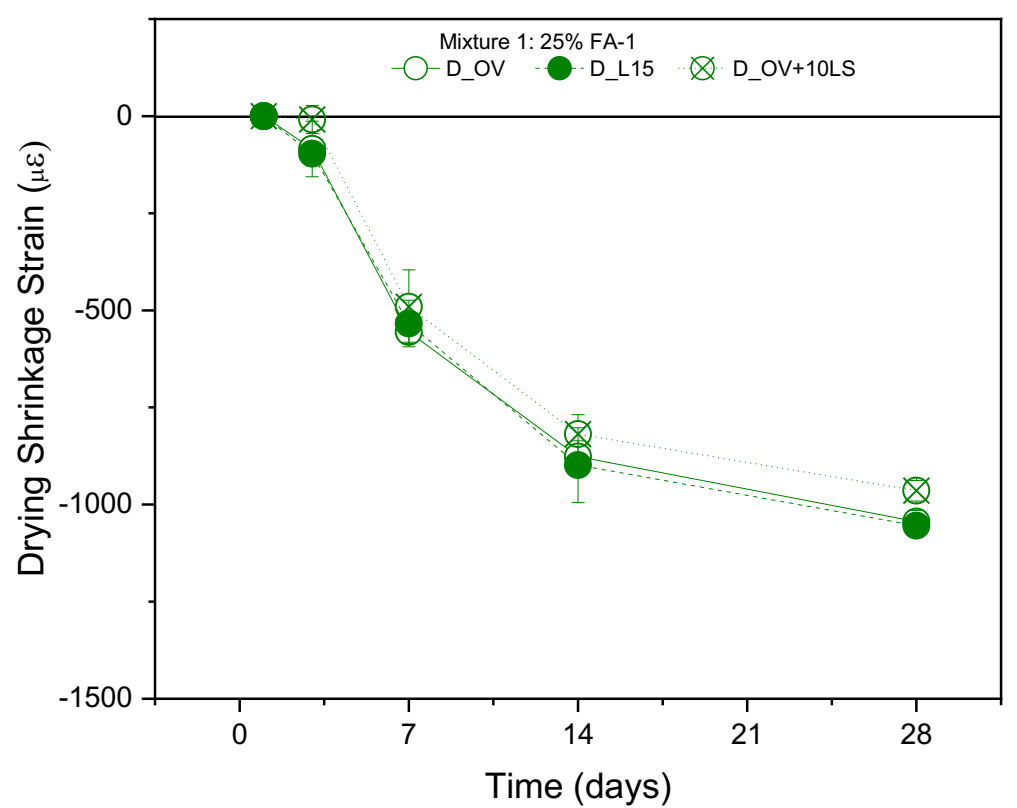

(b) 


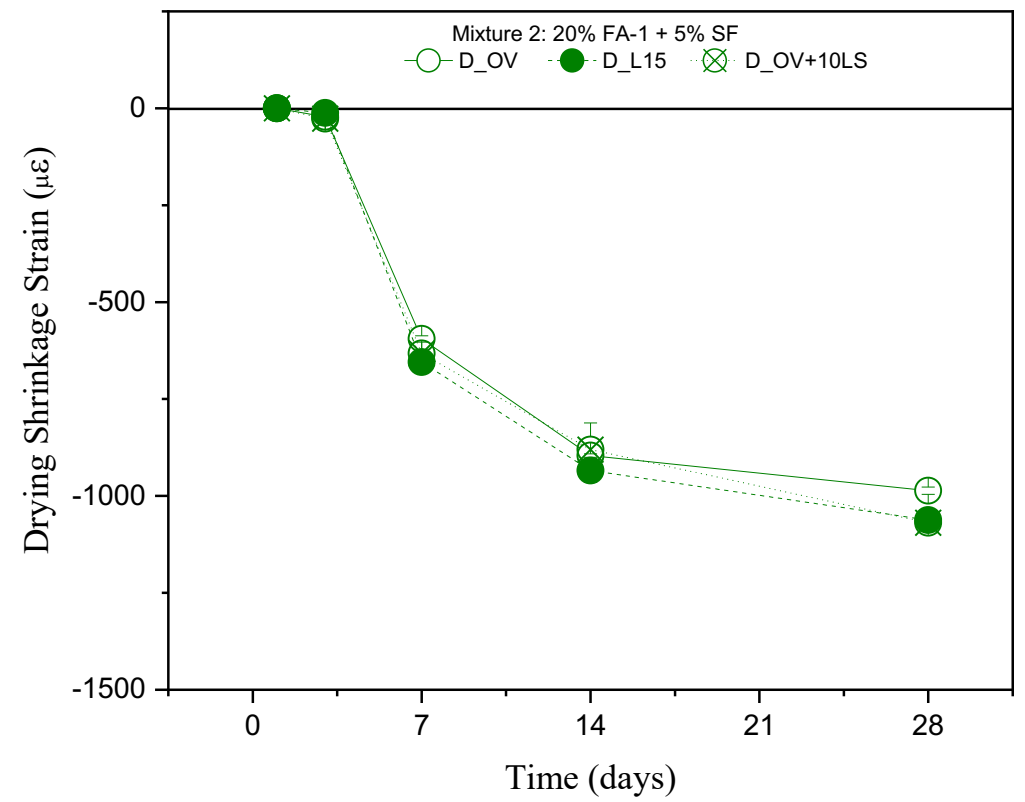

(c)

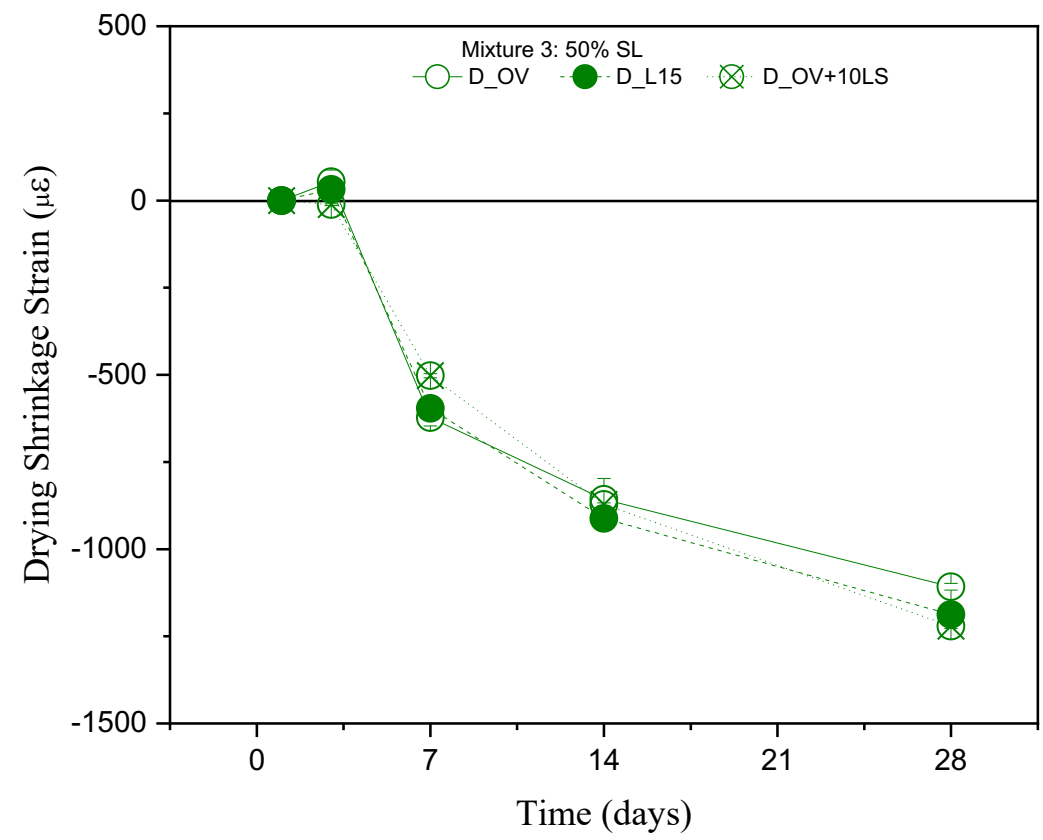

(d) 


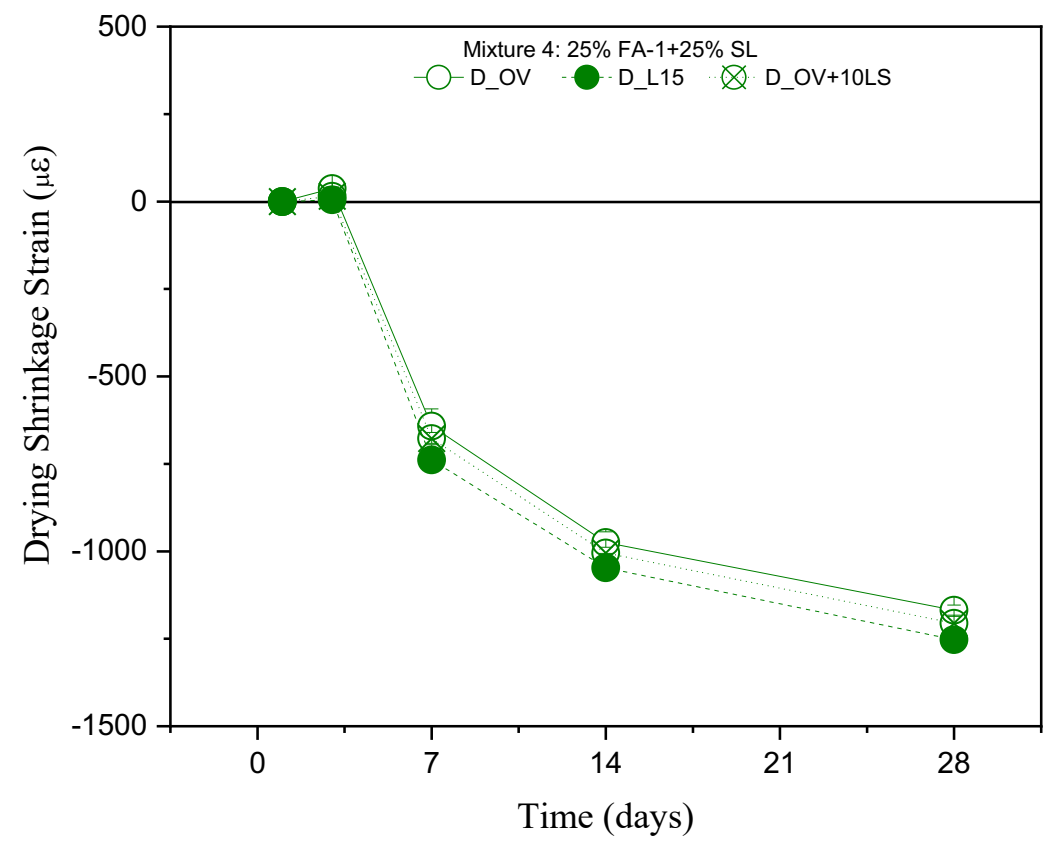

(e)

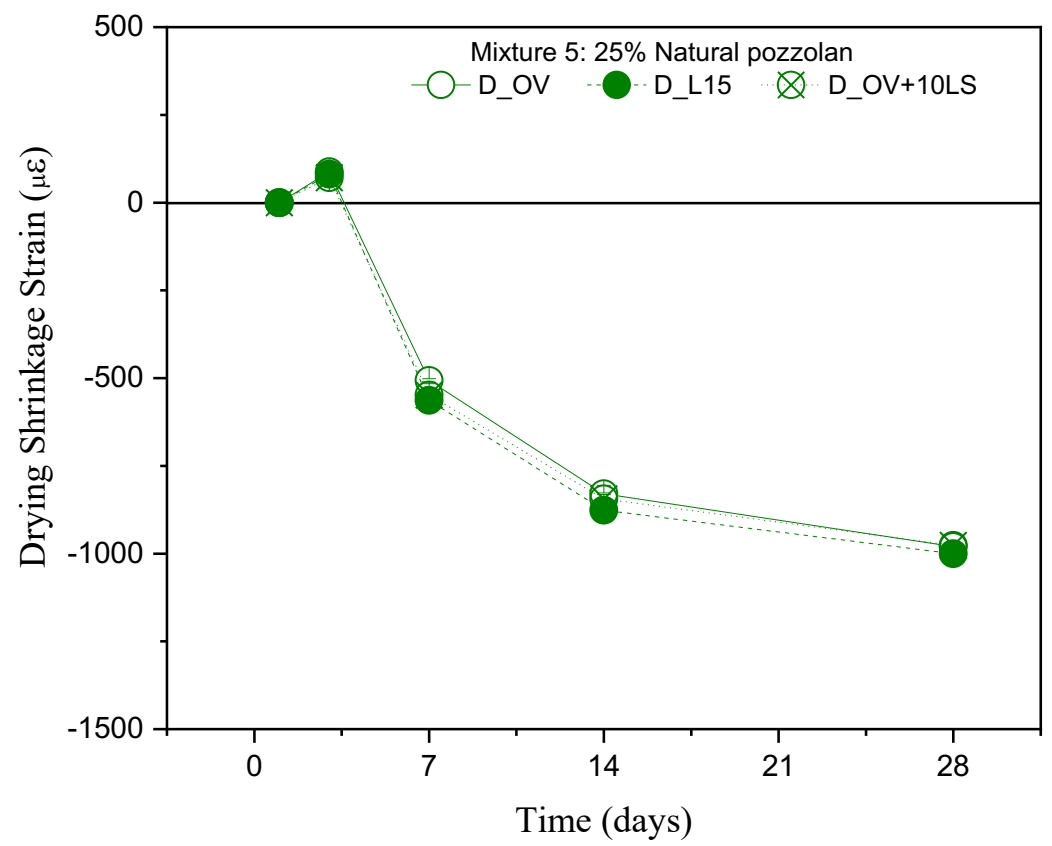

(f)

1014 1015

Figure 6-7. (a)-(f) Drying shrinkage results of Cement D for M0, 1, 2, 3, 4, and 5, respectively

\section{$1016 \quad 6.7$ Discussion of the Results}

1017 As clearly seen in Figure 6-7(a) and Figure 6-7(b), for M0 and M1 no discernable difference 1018 observed between drying shrinkage strains in OPC and corresponding PLC samples at all ages. 
1019 For OPC+10LS samples, the drying shrinkage at early ages were slightly higher than OPC and 1020 PLC systems. However, by the age of 28 days, the difference in shrinkage strains was almost non1021 discernable. Figure 6-7 (c)-(f) shows that OPC, PLC, and OPC + LS systems have no marked 1022 difference in drying shrinkage strains. The shrinkage of mixtures 2, 3, and 4 displayed a higher 1023 drying shrinkage strain than M1 and M2. The addition of natural pozzolan did not affect the drying 1024 shrinkage of mortar samples.

1025 Figure 6-8 illustrates the shrinkage of all 80 mixtures. The $\mathrm{Y}$-axis represents the shrinkage of the 1026 PLC or OPC + LS mixtures, while the X-axis represents the OPC systems' shrinkage. A plot that 1027 follows the 1:1 solid diagonal line would imply no difference between the OPC and PLC or OPC 1028 and OPC + LS. Dashed lines represent a variation of $20 \%$ from equivalence. It can be noted that, 1029 as expected, the shrinkage increases over time (i.e., moving from Figure 6-7(a) to Figure 6-7(d)).

1030 To determine if the results were statistically significant, $t$-tests of the shrinkage tests were 1031 performed. It was determined that the vast majority of the samples were found to be statistically 1032 similar. The only exception for the OPC versus PLC system occurred for M3 and M4 at 14 and 103328 days, with the PLC systems' shrinkage being 7-8\% higher. Similarly, the OPC's exceptions 1034 versus OPC + LS systems for M0 occurred at 3 and 7 days, M2 at 28 days (6\% higher), and M4 at 103514 and 28 days ( 3 and $4 \%$ higher on average). At this point, it should be remembered that a $1 \%$ 1036 variation in paste content can result in shrinkage variations of 6 to $10 \%[73,74,82]$. Further, M3 1037 and M4 mixtures made using OPC have approximately 10\% more shrinkage than M0.

\section{$1038 \quad 6.8 \quad$ Significant Findings}

1039 Preliminary testing on shrinkage cracking showed no statistically significant difference in cracking 1040 performance, consistent with the literature. Due to similar drying shrinkage and the literature, the 1041 Study Advisory Committee determined that additional restrained shrinkage cracking testing was 1042 not needed.

1043 As a result, variations in shrinkage with replacing OPC with PLC or OPC with OPC + LS do not 1044 appear to be a sufficient concern as it would relate to shrinkage cracking. 


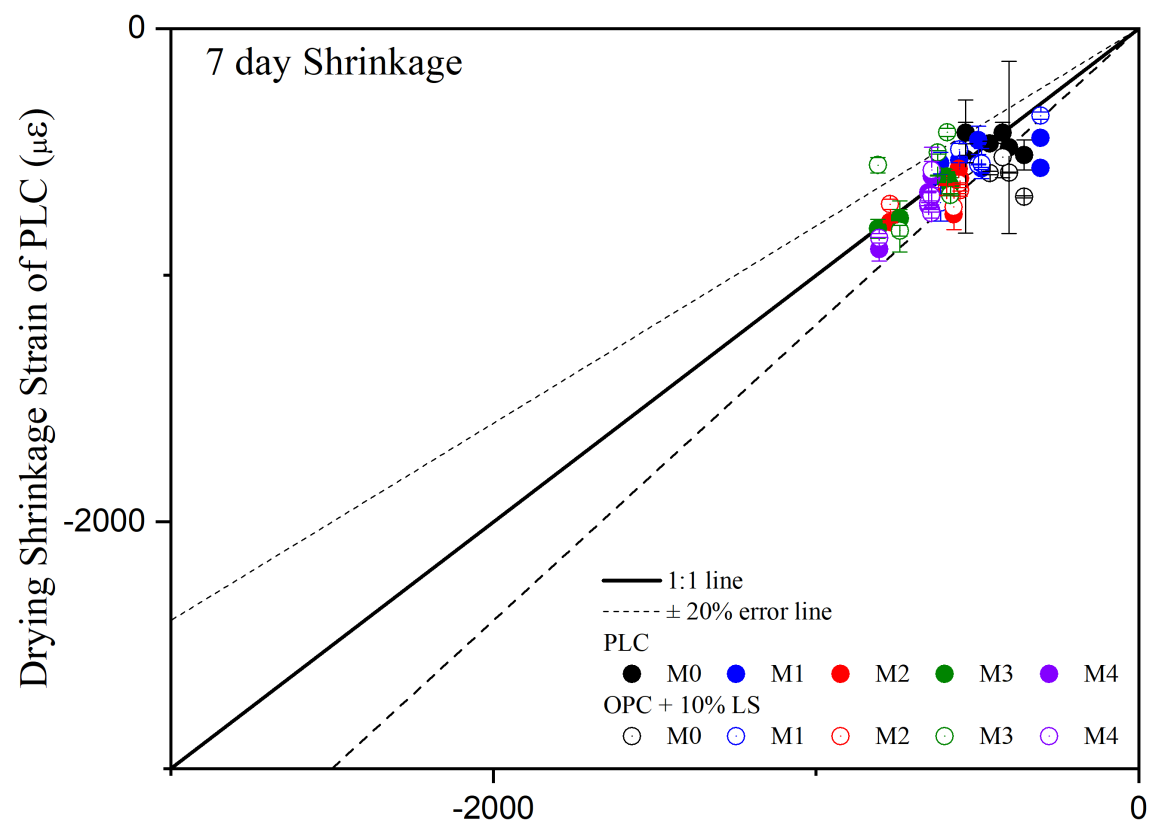

Drying Shrinkage Strain of OPC $(\mu \varepsilon)$

(a)

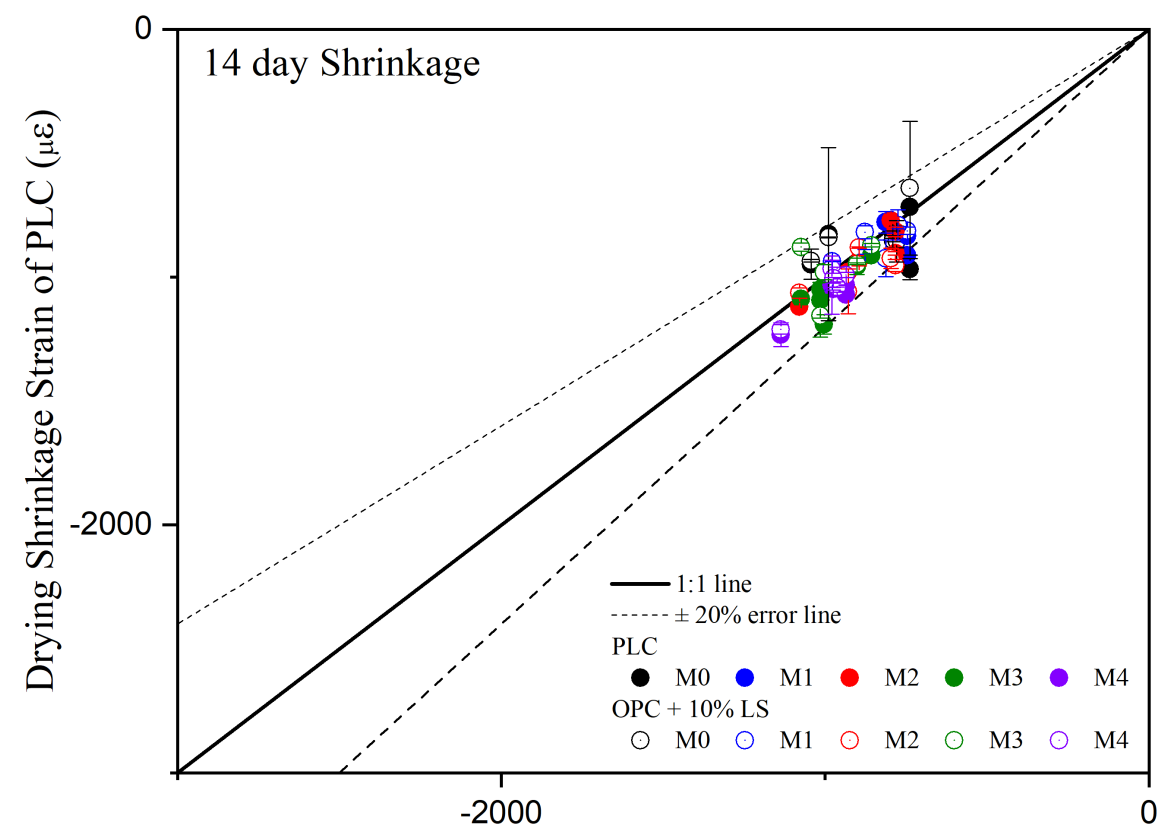

Drying Shrinkage Strain of OPC $(\mu \varepsilon)$

(b) 


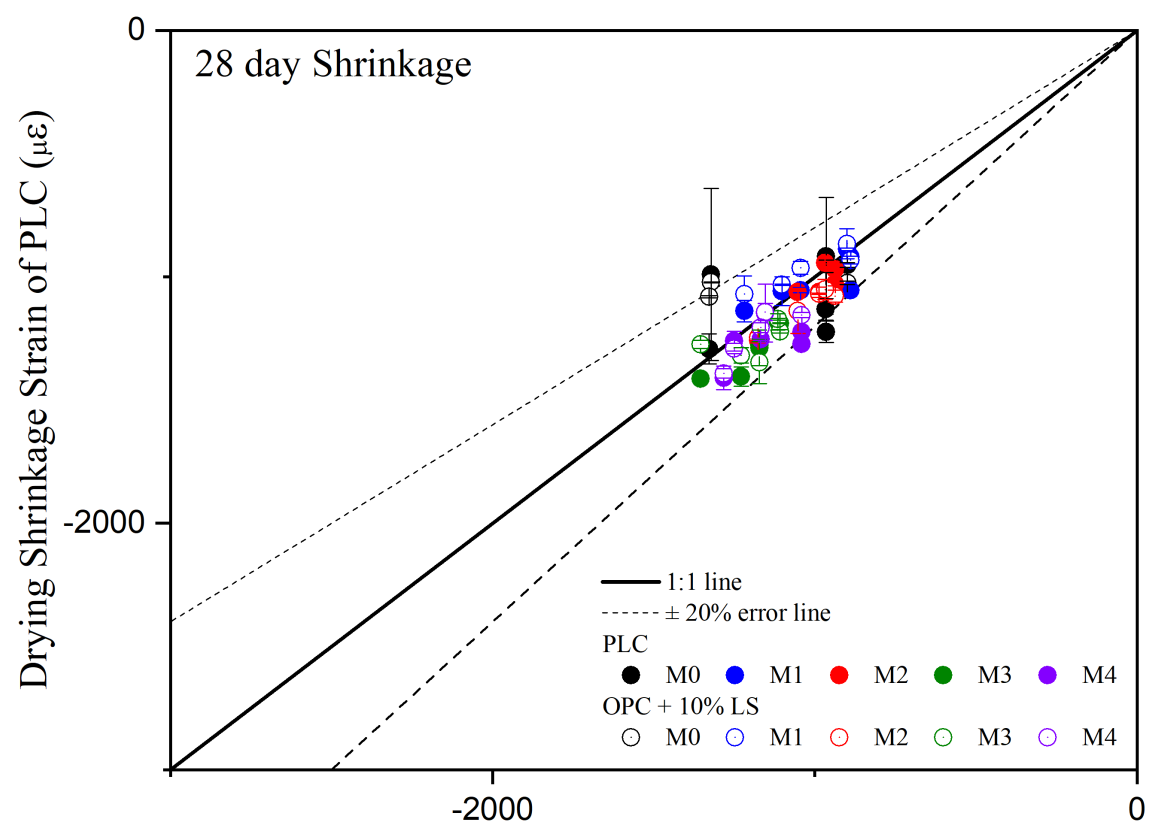

Drying Shrinkage Strain of OPC $(\mu \varepsilon)$

(c)

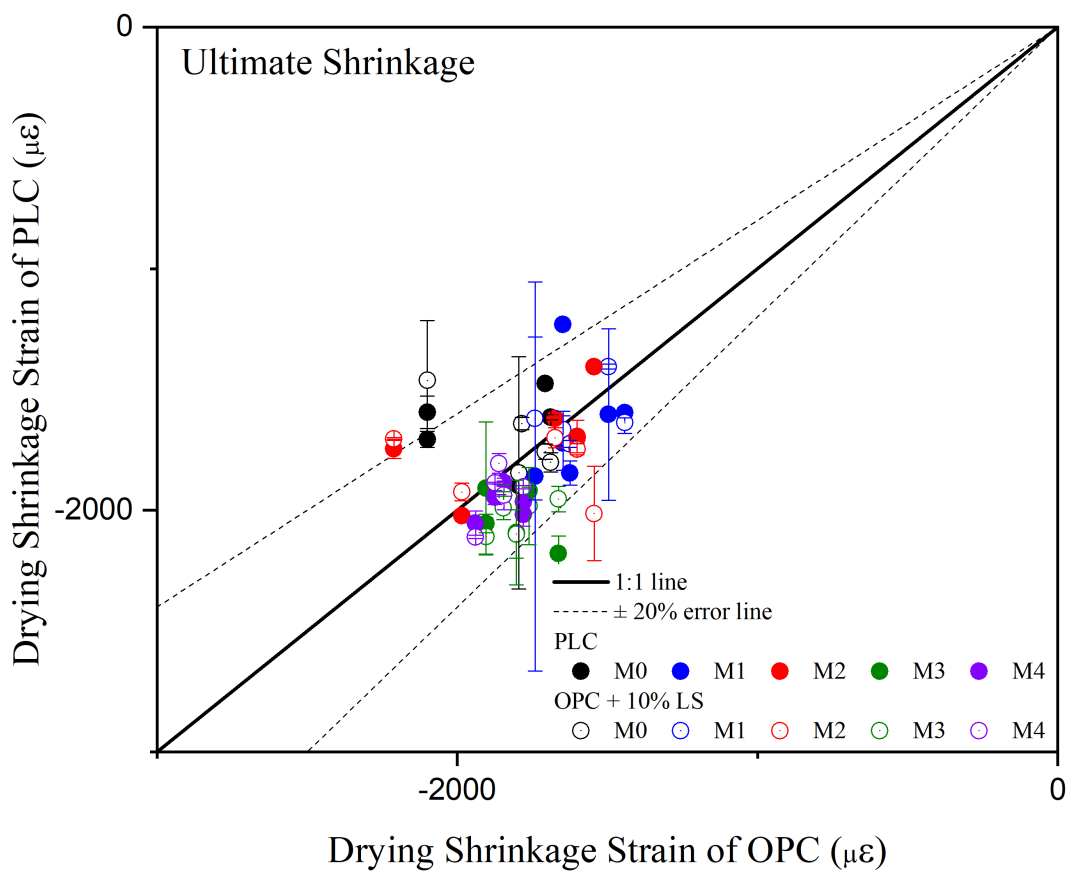

(d) drying, b) 14 days of drying, c) 28 days of drying, and d) ultimate drying 


\section{Mechanical Properties}

1050

1051

1052

1053

1054

1055

1056

1057

1058

1059

1060

1061

1062

1063

1064

1065

1066

1067

1068

1069

1070

1071

1072

1073

1074

1075

1076

1077

1078

1079

1080

1081

1082

1083

\subsection{Research Objective}

Flexural strength development is an essential factor in assessing the performance of PLC. Some have been concerned that replacing $15 \%$ of the cement clinker with limestone would result in substantial strength reduction, especially at early ages. However, it should be noted that in North America, ASTM C595/AASTHO M240 cements (PLC) are designed to target a similar 28-day performance. As a result, the fineness of the PLC is generally increased to offset early age strength reduction. This portion of the research investigated the flexural strength for paste made using OPC, PLC, and OPC + LS for both plain systems (M0) and systems containing SCM (M1 to M5).

\subsection{Background/Literature review}

Dhir et al. [11] showed a slight reduction in mechanical performance between CEM I 42.5R and a similar PLC containing 15\% limestone. Chen et al. [12] found that the replacement of clinker with limestone increased in compressive strength of up to 8\%. De Weerdt et al. [83] observed that up to $15 \%$ of portland cement could be replaced by limestone powder without impairing the compressive strength development. This is pointed out as a reminder that care must be taken when reviewing international literature since many regions do not target similar 28-day performance as has been done in North America for PLC (ASTM C595/AASTHO M240).

North American PLC with a limestone content up to $15 \%$ has a similar mechanical performance as ordinary portland cement (OPC) at 28 days [6]. Barrett et al. [2, 76] examined North American PLCs (ASTM C 595/ASTM M240) relating the performance of systems made using OPC with systems made from PLC from the same clinker using primarily Type I and Type III cements. Figure 7-1 shows the study results for the compressive strength, elastic modulus, and flexural strength. In general, it was found that PLCs show an increase in compressive strength at early ages that diminishes with time, resulting in similar compressive strengths at 28 days of age. Systems that had OPC-blended limestone cements had an average reduction in strength of $8.5 \%$ at 28 days. This has been attributed to the fact that the cements were not ground finer to account for clinker dilution.

Menéndez et al. [28] showed that when limestone additions occurred in cements containing blast furnace slag, early and later age compressive strength improved. They concluded that during the first $48 \mathrm{~h}$, the limestone acts as a nucleation site increasing hydration reactions [16, 23]. They also suggested that after seven days, a synergy occurs with limestone and slag. Barrett et al. $[2,76]$ demonstrated the benefits of limestone addition in mixtures containing fly ash. Bentz et al. [84] showed substantial improvements in high volume fly ash when limestone was added. Limestone was suggested as a potential method to compensate for high volume fly ash's impact on the retardation of hydration and delayed setting time. It has been suggested that limestone participates 
1084 in the hydration reaction by forming carboaluminates. The carboaluminates may reduce the 1085 induction period, set time, and stabilize ettringite, leading to the reduction in the volume of 1086 hydrates and porosity [24, 25].
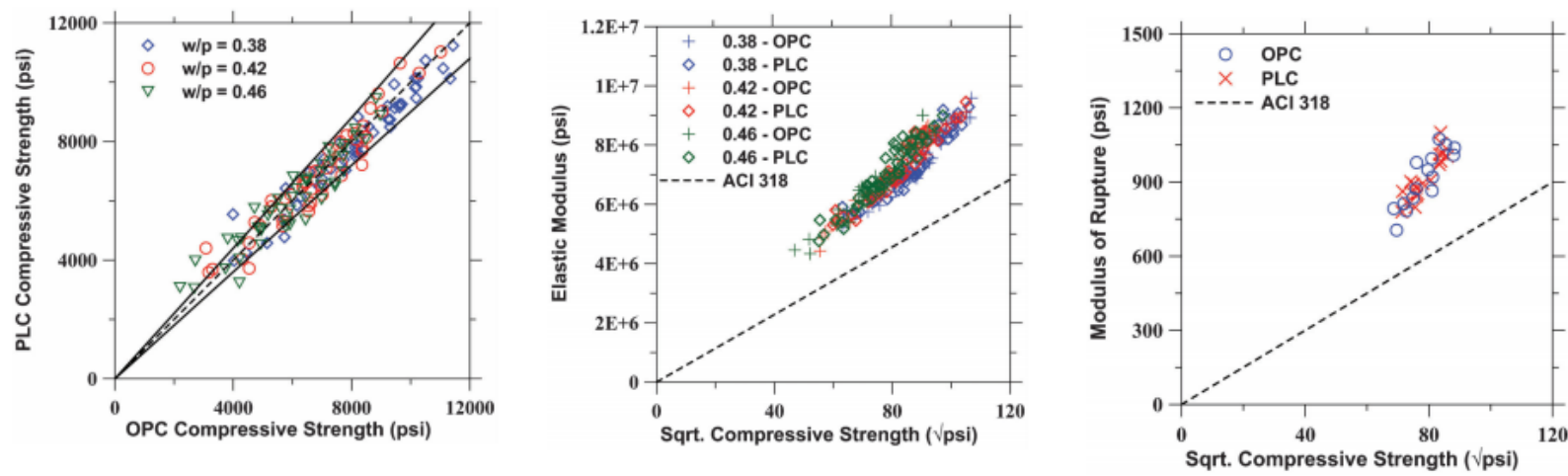

1087

1088

1089

1090

1091

1092

1093

1094

1095

1096

1097

1098

1099

1100

1101

1102

1103

1104
Figure 7-1. A Comparison of the mechanical properties of OPC and PLC: (a) compressive strength, (b) Elastic modulus vs. square root of compressive Strength, and, (c) Flexural Strength vs. Square Root of Compressive Strength [76]

Meddah et al. [13] also showed that 15\% replacement of OPC with limestone had a 5\% reduction in the modulus of elasticity for concrete produced with PLC when compared with OPC-based concrete. Barrett et al. [76] concluded that the modulus of elasticity and flexural strength of concrete produced with OPC and PLC are similar. Figure 7-1b shows their similarity and compares them to the equations used to estimate these properties by ACI. The modulus estimate is substantially larger (COV of $40 \%$ ) than any minor variation caused by PLC addition.

When OPC is replaced by a limestone content higher than $15 \%$, a reduction in the mechanical strength at later ages has been observed as well as a decrease in the modulus of elasticity $[11,15-$ 18]. However, it should be noted that these cements are not consistent with the ASTM C 595/AASHTO M 240 specification.

There is a consensus in the literature that the replacement of cement clinker with interground limestone levels up to $15 \%$ can benefit the mechanical properties. Most literature focuses on cement type I or III cements with little, if any, work done on type II/V cements. This study aims to determine the impact of PLC on paste's mechanical properties, specifically here focusing on flexural strength.

\subsection{Experimental Test Matrix}

The Ball on Three Ball (B3B) test was performed to flexural strength testing described in the following section [85-89]. The flexural strength was measured on mortar cylinders $(50 \mathrm{~mm}$ diameter $\times 100 \mathrm{~mm}$ length) that were sectioned to a nominal thickness of $2.65 \mathrm{~mm}$. Samples were cast along with the shrinkage samples, as discussed in section 6 . The mixtures for the test using the proportions provided in Table 6-1 based on the SCM replacement levels (by mass) provided in 
1111 Section 3 The test matrix comprises 80 mortar mixtures, which were cast and tested for flexural 1112 strength in accordance with Table 6.2.

\section{$1113 \quad 7.4 \quad$ Experimental Methods}

1114 Fresh mortar samples were mixed using the procedure described in section 6.4. The fresh mortar 1115 was cast in plastic cylinders (52 $\mathrm{mm}$ diameter and $100 \mathrm{~mm}$ length) in two layers. Each layer was 1116 consolidated using a tamping rod. The samples were then sealed with duct tape to prevent the loss 1117 of any moisture. The sealed samples were stored at $23 \pm 1^{\circ} \mathrm{C}$ for curing. The samples were 1118 demolded and prepared for flexural strength using the ball-on-three-ball (B3B) test at 7, 14, 28 and 111990 days [89].

1120 At the age of testing, the sealed cylinder samples were demolded and cut with a diamond-tipped 1121 saw blade that was water-cooled to produce disc specimens with a nominal thickness of $2.65 \mathrm{~mm}$

1122 (the samples were measured after they were broken, and the average of 3 thickness measurements 1123 was used as the thickness). The ends of the samples (approximately the last $10 \mathrm{~mm}$ ) were discarded 1124 to avoid end defects. The sliced discs were gently wiped with a towel to remove excess moisture 1125 and then tested using the B3B machine (Mark-10 Tester with a Series 5 force gauge). 1126 Approximately 6-7 slices were sampled from each cylinder to obtain a representative data set. The 1127 B3B test set-up is shown in Figure 7-1. The rate of loading is $0.22 \mathrm{~N} / \mathrm{sec}$. The peak load $(\mathrm{F})$ and 1128 the thickness of the broken pieces $(\mathrm{t})$ were noted down for each tested disc. The flexural strength $1129(\sigma)$ was determined for each disc of radius $(\mathrm{R})$ using the equation provided in Börger's work [851130 88] which was modified and adapted for mortar specimens by Fu and Weiss [89]

$$
\sigma=\left[c_{0}+\frac{\left(c_{1}+c_{2} \alpha+c_{3} \alpha^{2}+c_{4} \cdot \alpha^{3}\right)\left(1+c_{6} \beta\right)}{1+c_{5} \alpha}\right] \frac{F}{t^{2}}
$$

1131 where, $\alpha=\mathrm{t} / \mathrm{R}, \quad \beta=\mathrm{R}_{\mathrm{a}} / \mathrm{R}, \mathrm{c}_{0}=-14.671, \mathrm{c}_{1}=17.988, \mathrm{c}_{2}=567.22, \mathrm{c}_{3}=-80.945, \mathrm{c}_{4}=$ $1132-53.486, c_{5}=36.01$, and $c_{6}=0.0709$ (for Poisson's ratio $=0.2$ for cement mortar). 

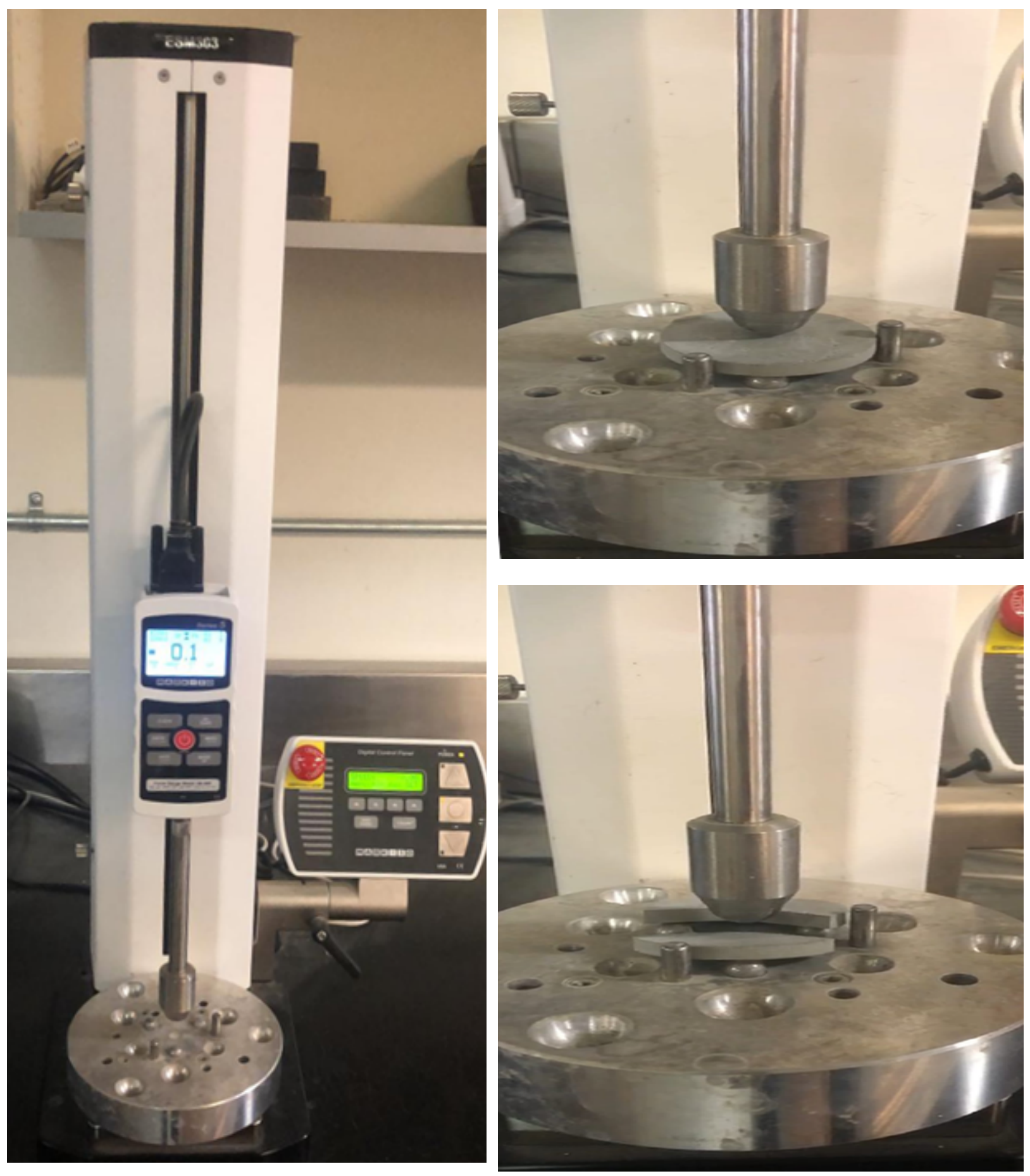

Figure 7-1. The Experimental Setup for the B3B test: a) without the sample b) showing a mortar disk during testing and c) showing a mortar disk after testing (note the three vertical pins provide no restraint and are only used to locate the sample) 


\subsection{Typical Experimental Measurement and Interpretation}

1137 Figure 7-2 provides a typical plot of flexural strength for the mortars made using PLC and OPC. 1138 The strength data was collected at the ages of 7, 14, and 28 days is shown on the $\mathrm{X}$-axis. The $\mathrm{Y}$ 1139 axis denotes the flexural strength of PLC and OPC+ limestone samples at different ages 1140 normalized by the average flexural strength on OPC samples of the same parent clinker at that age. 1141 The reference line drawn at $100 \%$ corresponds to the strength of the OPC average sample strength 1142 at that age. The points lying above this line signify that the PLC has a higher strength than OPC 1143 samples, and the points below correspond to lower strength. Similar to what has been observed by 1144 others, at early ages, the PLC system may have slightly lower or similar strength; however, at the 1145 age of 28 days, the PLC and OPC mixtures have similar performance.

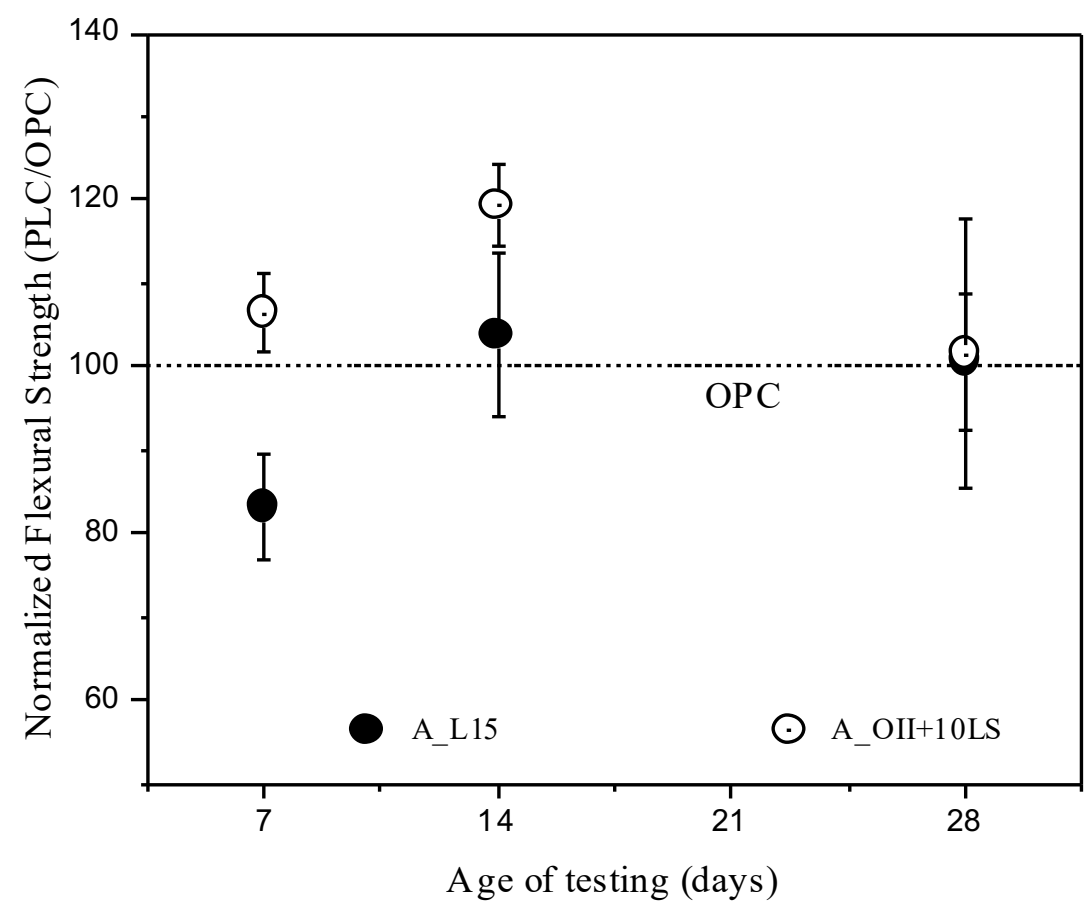

\section{$1147 \quad 7.6 \quad$ Experimental Results}

1148 The flexural strength for the series of mixture made using Cement B is provided in Figure 7-3 (a)1149 (e). Again, the samples made using PLC and OPC + LS are normalized to the flexural strength of 1150 the OPC samples. The flexural strength results of the other cements are provided in Appendix D. 
1152 The strength at early ages for both the PLC and the OPC+LS were lower than the OPC at early 1153 ages for M0, 1, 2 (no SCM, 25\% fly ash and 20\% fly ash $/ 5 \%$ silica fume respectively). A $10 \%$ 1154 reduction in flexural strength was noted at later ages as well. Mixtures 3-5 did not show this delay 1155 (50\% slag, and $25 \%$ slag $/ 25 \%$ fly ash, $25 \%$ natural pozzolan, respectively).

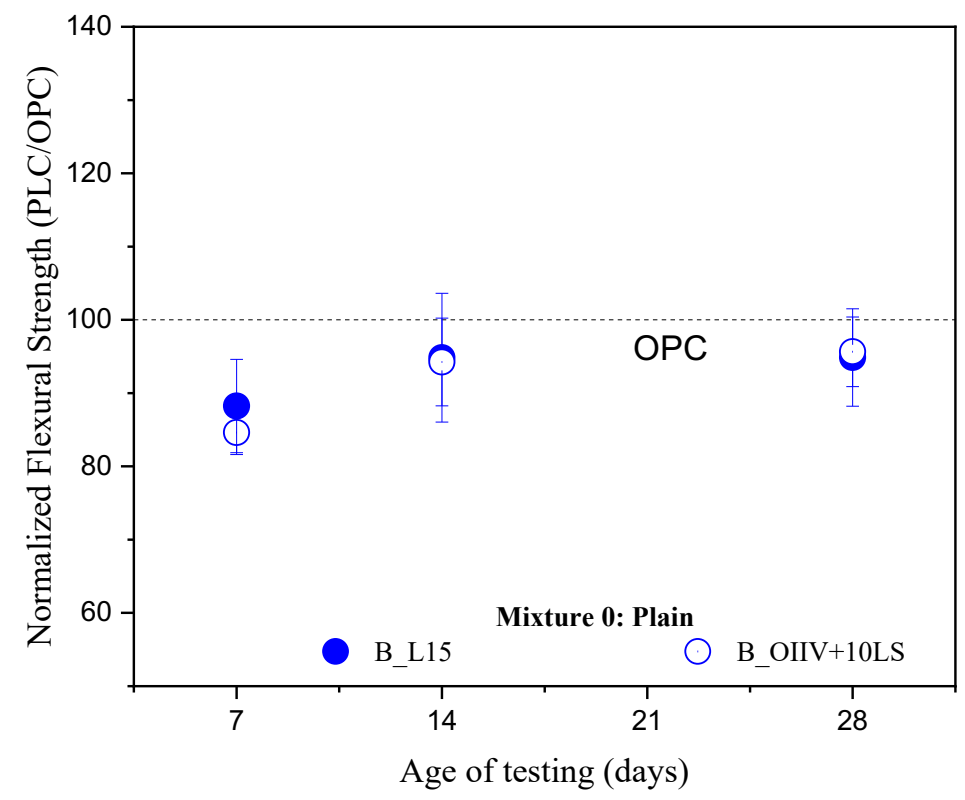

(a)

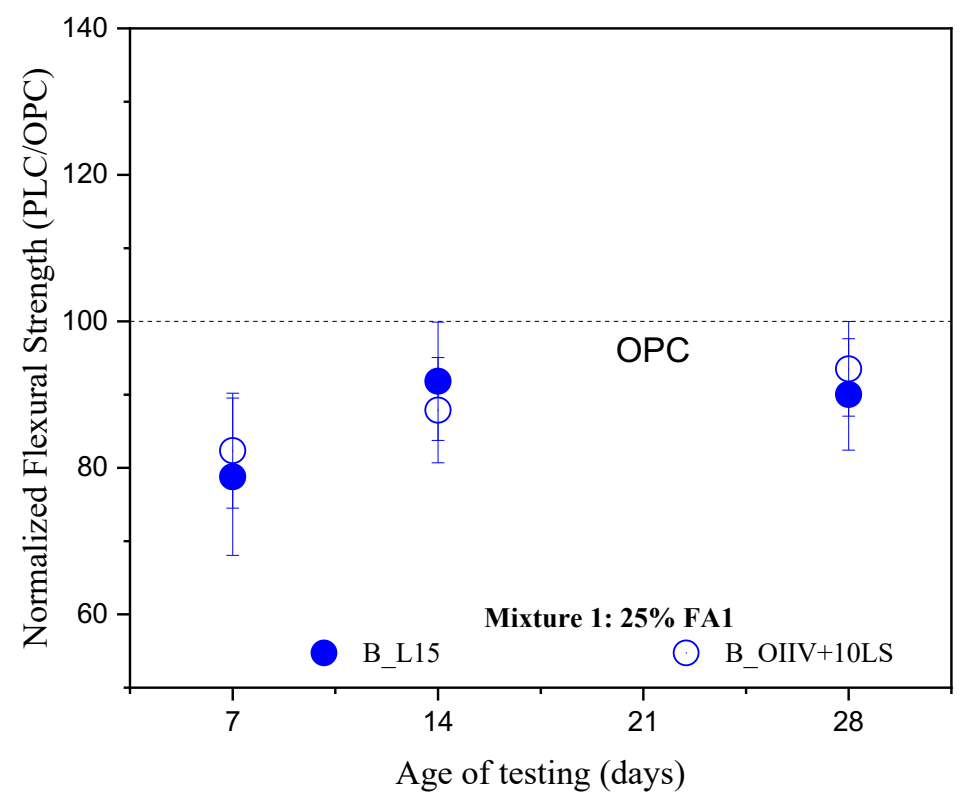

(b) 


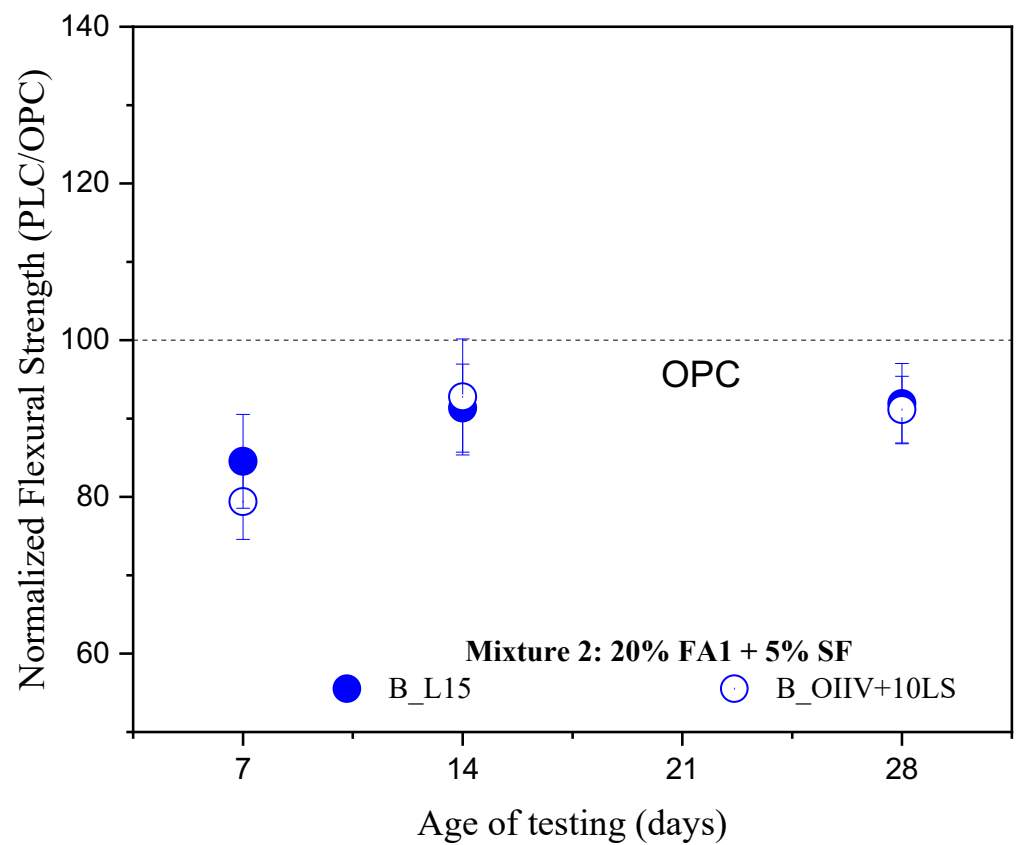

(c)

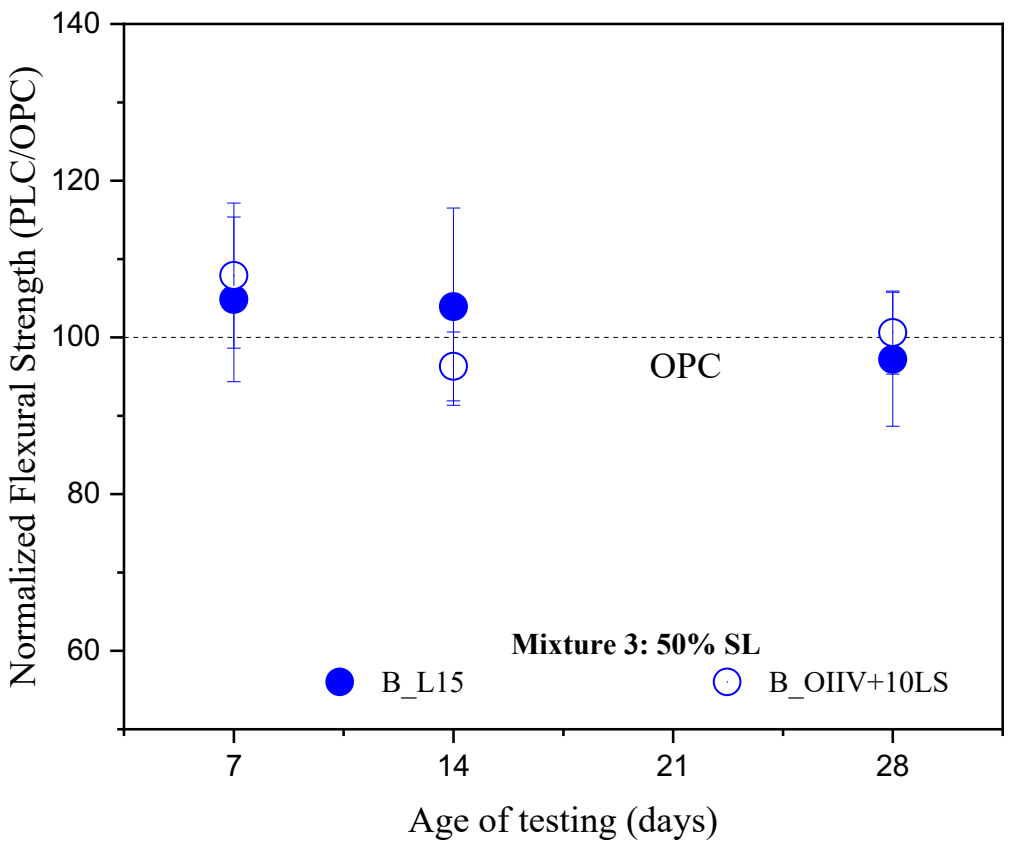

(d) 


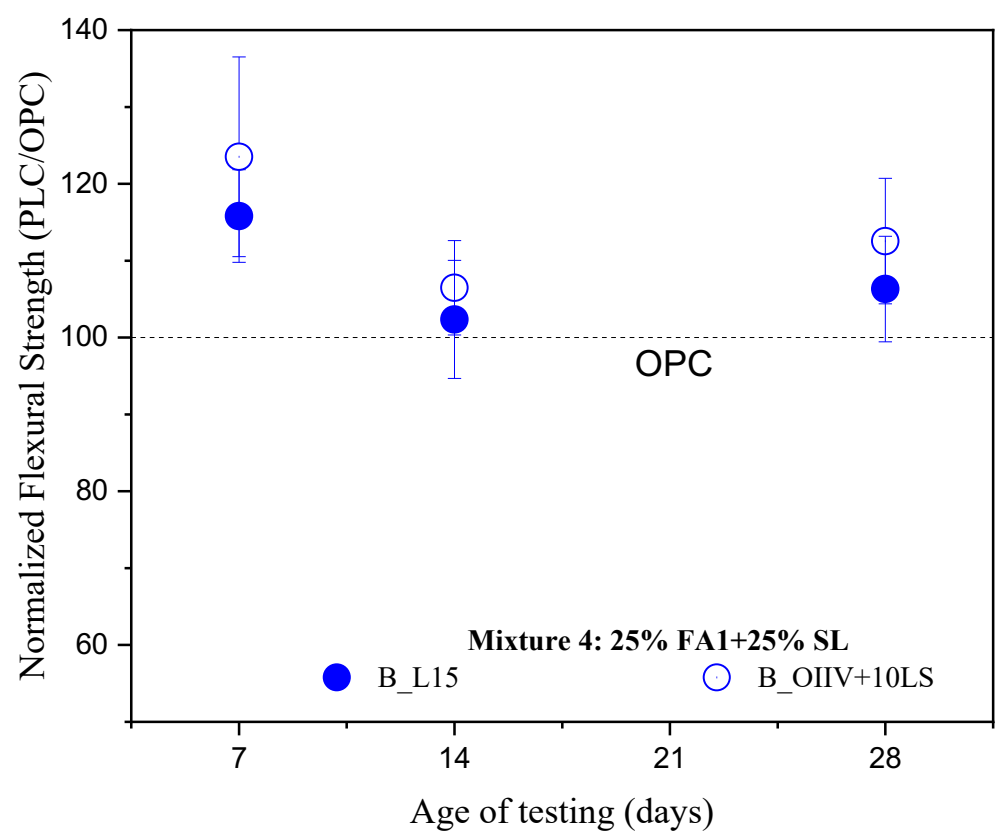

(e)

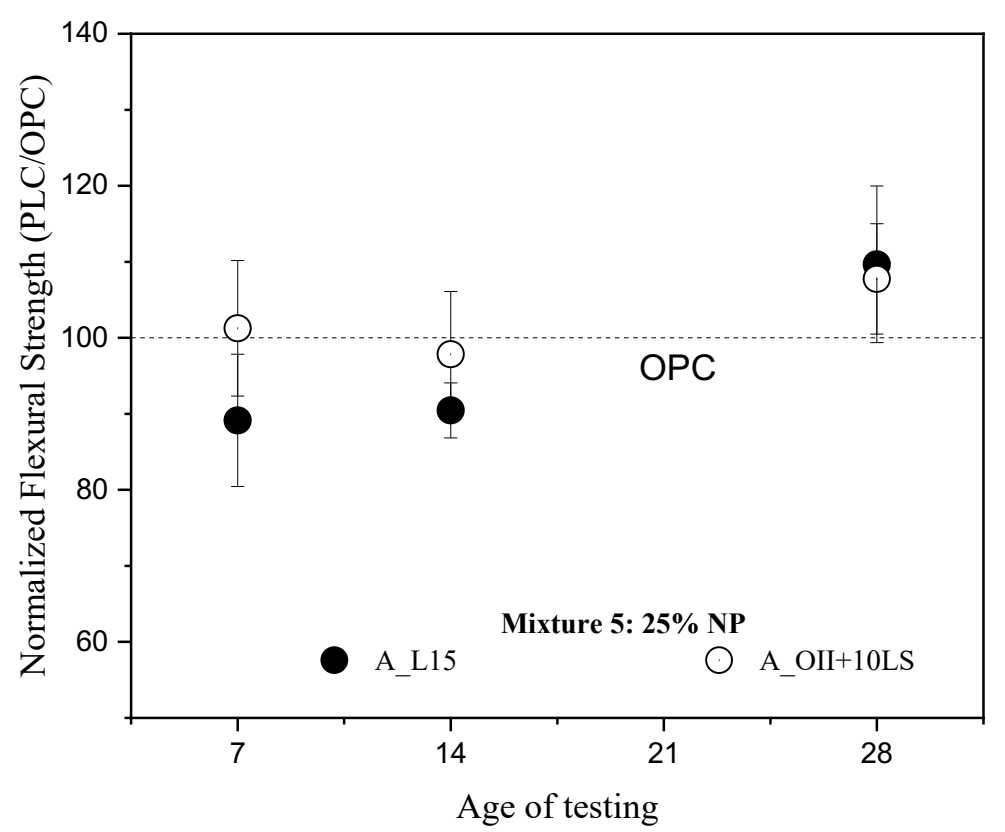

(f) and 5 respectively 


\subsection{Discussion of the Results}

1159 In general, for all the cement clinkers studied, the PLC and OPC + LS mortar samples had a flexural 1160 strength that was less than 5\% lower than the OPC mixtures. The following section describes the 1161 trends for cements A through E.

1162 M0 (plain) demonstrated PLC strength that was $10 \%$ lower at seven days and $5 \%$ lower at the other 1163 ages, while the OPC + LS was $18.5 \%$ lower at seven days, $10 \%$ lower at 14 days, and $5 \%$ at other 1164 ages. M1 (25\%FA) had a PLC and OPC +LS strength was approximately $15 \%$ lower at early ages, 1165 and this difference reduced over time. M2 (25\% FA +SF) was at most $8 \%$ lower. M3 (slag) and 1166 M4 (silica fume and slag) were found to have statistically similar strengths in comparing OPC, 1167 PLC, and OPC + LS. M5 (natural pozzolan) was found to have statistically similar strengths in 1168 comparing OPC, PLC, and OPC + LS with one exception in that the PLC was on average 14\% 1169 less at 90 days. Figures 7-4 show that the addition of limestone reduces flexural strength at an 1170 early age for plain systems (M0) and fly ash systems (M1). Still, the effect of limestone 1171 replacement and clinker reduction is almost inconsequential when considering sample to sample

1172 variability by 14, 28, and 90 days (Figure 7-4 b, c, d). Thermodynamic models (Part II of the 1173 report) and physical testing will correlate the strength with porosity. Initial results indicate that 1174 M0, M1, and M2 have a slight increase in porosity for the PLC and OPC + LS systems than the 1175 OPC systems.

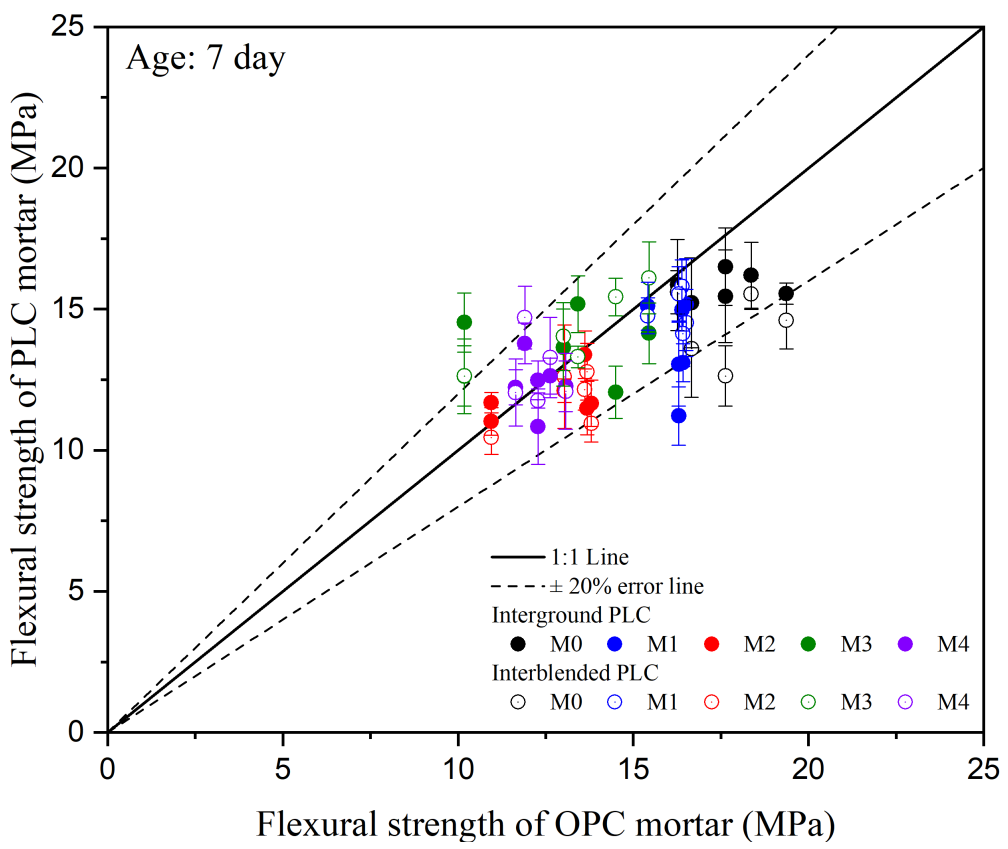

(a) 


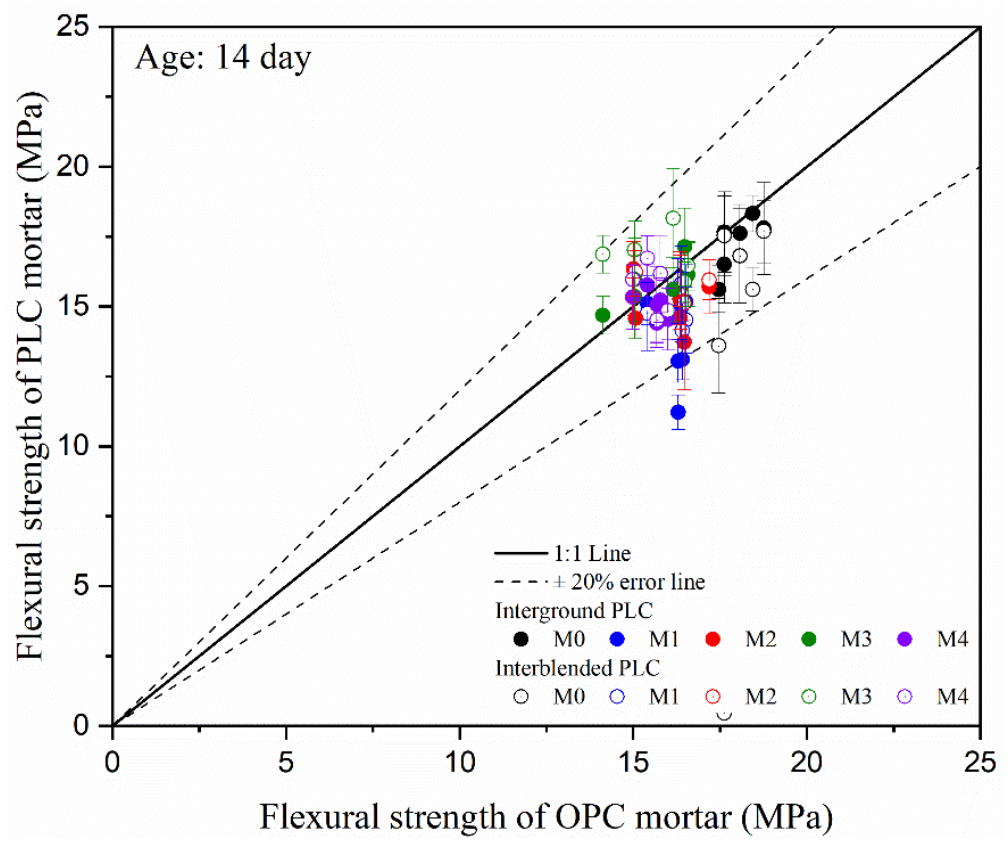

(b)

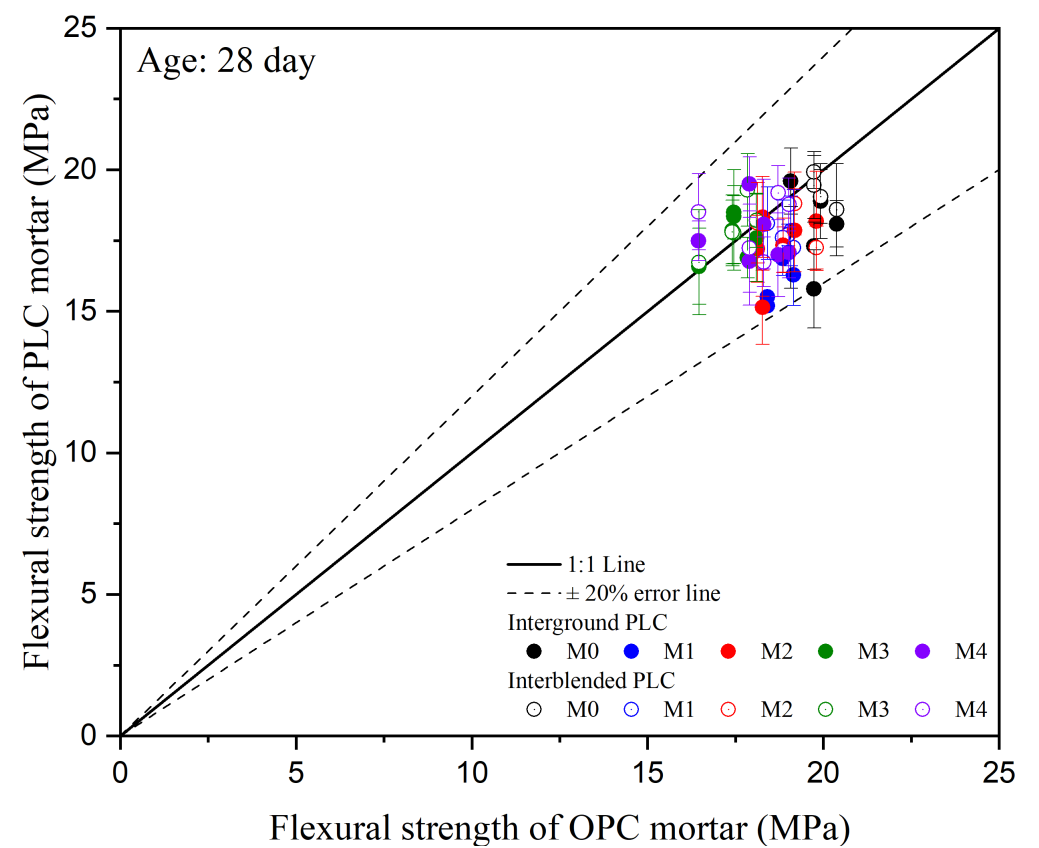

(c) 


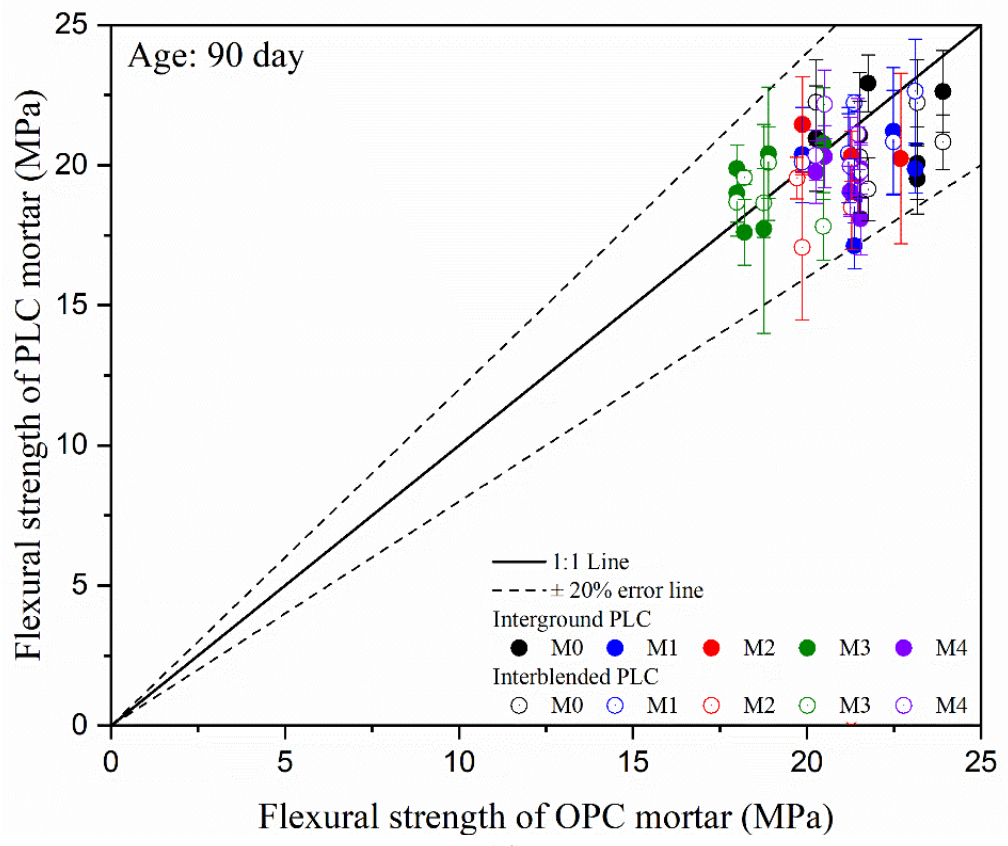

(d)

\section{$1178 \quad 7.8$ Significant Findings}

1179 This study examined the performance of PLC made with clinkers that are typically used for Type 1180 II/V OPC that contain SCM. Eighty different mixtures were prepared to evaluate the influence of 1181 the PLC flexural strength. The flexural strength in the PLC and OPC + LS mixtures was on average 1182 less than 5\% lower than the OPC mixtures. However, the flexural strength was up to $13 \%$ greater 1183 for PLC when combined with slag. Overall, the flexural strength was consistently within the +/$118420 \%$ range compared with the parent system. In conclusion, PLC can be used as an alternative to 1185 OPC for systems made with type II/V clinker with and without SCM. 


\section{Transport Properties}

\section{$1188 \quad 8.1 \quad$ Research Objective}

1189 The durability of concrete is impacted by its ability to resist ingress of fluids and ions. This chapter examines the transport properties of mortars made using OPC, PLC, and OPC+LS systems. The objective is to determine the influence of PLC and OPC+LS on the pore structure of concrete and provide data to inform CALTRANS on the potential replacement OPC related to fluid transport.

1193 Transport in cementitious systems is generally categorized as 1) absorption of fluids, 2) hydraulic 1194 permeation, 3) diffusion of ions. Several test methods exist to determine these factors; however, 1195 recent work has shown that these critical factors can be related to a measurable property called the 1196 formation factor (F, AASHTO PP-84). The formation factor is generally measured using electrical 1197 testing (e.g., resistivity) and is inversely related to the product of the total porosity and the 1198 connectivity of the pores. This chapter will measure the formation factor of OPC, PLC, and 1199 OPC + LS mortars and related properties. Similar properties for concrete mixtures are presented 1200 separately in Chapter 10 as part of the discussion on chloride ingress in concrete.

\section{$1201 \quad 8.2$ Background/Literature review}

1202 As previously mentioned, the transport properties of concrete are often evaluated using electrical 1203 measurements (e.g., RCPT (ASTM C1202), electrical conductivity, or electrical resistivity (ASTM 1204 C1876, AASHTO TP-119)). In an earlier study, Stubstad et al. [1] compared OPC and PLC and noted that PLC performed better than OPC in the ASTM C1202 test (for rapid chloride penetrability). Barrett et al. [31] measured the bulk resistivity of PLCs and found that while the results were generally similar to the OPC, some PLC mixtures performed better than the OPC, and some did not perform as well. Barrett et al. did conclude that the results were within $\pm 25 \%$ of the reference OPC mixtures and stated that more information was needed on the role of pore solution resistivity to understand the variations [37]. It is worth noting that the bulk resistivity of PLC systems containing fly ash exhibited an improvement of up to one order of magnitude compared to OPC systems. Hooton et al. [5] and Lakar and Smart [64] also reported similar performance

1214 Elgahud et al. [41] reported results from a wide variety of tests over the last 30 years and found 1215 that, in general, limestone addition increased the rate of chloride diffusion and ingress. However, 1216 it should be noted that care needs to be taken in these systems as the design of PLC around the 1217 world can vary. It should be noted that North American PLC has been designed to have a similar 1218 28-day compressive strength to that of the OPC and tends to perform differently from around the 1219 world. Barrett et al. found that while North American PLC systems have similar volumes of 1220 permeable voids as OPC, the chloride diffusion coefficients in these systems could be up to $30 \%$ 1221 higher than the OPC systems. This trend, however, was substantially reversed when SCMs were 
1222 used. The PLC-SCM systems outperformed the OPC-SCM systems. Further, the PLC systems

1223 containing fly ash have chloride diffusion coefficients that are up to $90 \%$ lower than systems 1224 without fly ash.

1225 Others have investigated fluid sorption directly. Barrett et al. [31] measured a series of PLC 1226 systems and found that the initial and secondary rates of absorption of the PLCs were within \pm $122730 \%$ of the reference OPCs. Ramazeianipour et al. [22] showed that there was no impact on 1228 absorption at low levels of limestone addition. Still, at higher levels of limestone addition, the 1229 absorption increased slightly at early ages but decreased at later ages. Tsivilis et al. [32,33,34] 1230 reported that for high w/cm (0.7), the PLC had a similar water absorption response as the OPC.

\section{$1231 \quad 8.3$ Experimental Test Matrix}

1232 The formation factor is measured using electrical resistivity (AASTHO TP-119) and is inversely 1233 related to the product of the total porosity (AASTHO TP-135) and the connectivity of the pores. 1234 This chapter describes experiments to determine the porosity and electrical resistivity of mortar 1235 samples. These samples were cast along with the shrinkage bars (section 6), and flexural strength 1236 (section 7) using the proportions provided in Table 6.1 are based on the SCM replacement levels 1237 (by mass) provided in Table 6.2. The test matrix comprises 80 mortar mixtures.

\section{$1238 \quad 8.4 \quad$ Experimental Methods}

1239 Fresh mortar samples were mixed and cast using the procedure described in Section 6. The sealed 1240 samples were stored at $23 \pm 1^{\circ} \mathrm{C}$ for curing. The samples were demolded and prepared for porosity 1241 (AASHTO TP 135-20 [90]) and resistivity (AASHTO TP-119-20 [91]) testing at the age of 90 1242 days.

\section{$1243 \quad$ 8.4.1 Porosity}

1244 Porosity was measured following AASHTO T 135-20 [90] using two samples per mixture. The 1245 ends were cut using a saw. The samples were surface dried before vacuum saturation at a pressure 1246 of 6 Torr for four hours. During the third hour of vacuum saturation, standard pore solution (13250 $1247 \mathrm{~g}$ water, $102.6 \mathrm{~g} \mathrm{NaOH}, 143.9 \mathrm{~g} \mathrm{KOH}$, and $\left.27 \mathrm{~g} \mathrm{Ca}(\mathrm{OH})_{2}\right)$ was introduced into the bucket via an 1248 external tube to saturate the pores with the standard pore solution. After the samples had been 1249 exposed to the solution under vacuum for an hour, they were removed from the vacuum and stored 1250 in the solution for three days. After this storage, each specimen was removed from the solution, 1251 and its saturated mass was recorded in the SSD condition. The apparent mass of the specimen 1252 underwater was also measured. The sample was then dried in oven conditioned at $105^{\circ} \mathrm{C}$, and the 1253 mass of sample after five days of drying (i.e., a stabilized mass) was recorded. The specimen 1254 porosity was determined using: 


$$
\phi_{s}=\frac{m_{s}-m_{a}}{m_{s}-m_{d}}
$$

1255 where, $\phi_{s}$ is the porosity of the specimen, $m_{s}$ is the saturated surface-dry mass of the specimen in 1256 air, $m_{a}$ is the apparent mass of the saturated specimen in water, and $m_{d}$ is the oven-dry mass of 1257 the specimen in air

\section{$1258 \quad$ 8.4.2 Electrical Resistivity Test}

1259 Electrical resistivity was measured following AASHTO TP 119-15. the resistivity was measured 1260 after the samples were saturated in the standard pore solution for three days. The resistance was 1261 measured along with temperature, and sample geometry. The resistivity of the specimen was 1262 calculated using:

$$
\rho_{s}=R \cdot \frac{A}{L}
$$

1263 Where, $\rho_{s}$ is the resistivity of specimen, $R$ is the resistance of the specimen $(\Omega), A$ is specimen 1264 cross-sectional area $\left(\mathrm{m}^{2}\right), L=$ average specimen length $(\mathrm{m})$. Temperature corrections were made 1265 using the Arrhenius approach following the guidance of Coyle et al. [92] with an activation energy 1266 of $15 \mathrm{~kJ} / \mathrm{mol}$.

\section{$1267 \quad$ 8.4.3 Formation Factor and Pore Connectivity}

1268 The formation factor was calculated using

$$
F=\frac{\rho_{s}}{\rho_{p s}}
$$

1269 where, $F$ is the formation factor and $\rho_{p s}$ is the resistivity of standard pore solution $(\Omega-m)$, assumed 1270 to be $0.127 \Omega-\mathrm{m}$.

1271 The connectivity of each sample was calculated using.

$$
\beta=\frac{1}{\phi_{s} \cdot F}
$$

1272 where, $\beta$ is the measure of connectivity of pores. 


\subsection{Typical Experimental Measurement and Interpretation}

1274 Figure 8-1 provides a typical plot of the measured porosity for the mortars made using PLC and 1275 OPC+10LS normalized by the porosity of OPC mortars. The Y-axis denotes the porosity of PLC and $\mathrm{OPC}+$ limestone samples at 90 days normalized by the average porosity of the OPC samples of the same parent clinker at that age. The reference line drawn at 100\% corresponds to the porosity of the OPC average sample at that age. The points lying above this line signify that the PLC mortar has a higher porosity than OPC mortar, and the points below correspond to lower porosity. Similar

1280 plots were developed for the measured electrical resistivity.

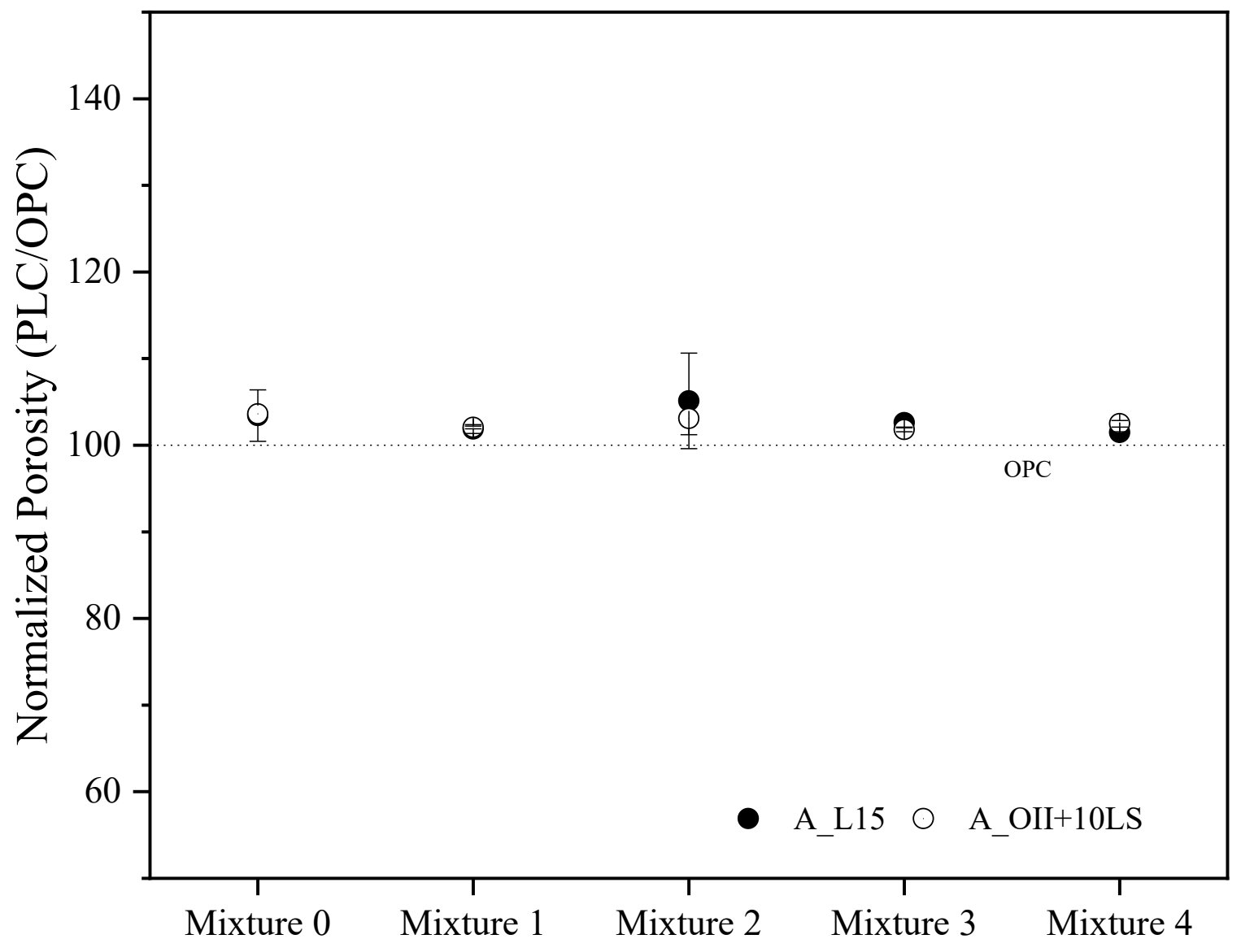

Figure 8-1. Typical plot of normalized porosity results of Cement A for all mixtures

1283 Figure 8-2 is a typical 1:1 consolidated plot of porosity of PLC and OPC+10LS mortars as 1284 compared to the porosity of OPC mortars. The x-axis denotes the OPC porosity and the y-axis 1285 denotes the PLC and OPC+LS samples at 90 days for M0-M4. The solid 1:1 reference line corresponds to an equivalent porosity of the OPC and PLC samples. Points lying above this line signify that the PLC mortar has a higher porosity than OPC mortar, and the points below correspond to lower porosity. $\pm 20 \%$ and $\pm 40 \%$ error lines are also provided. Similar plots were made to represent other properties. 


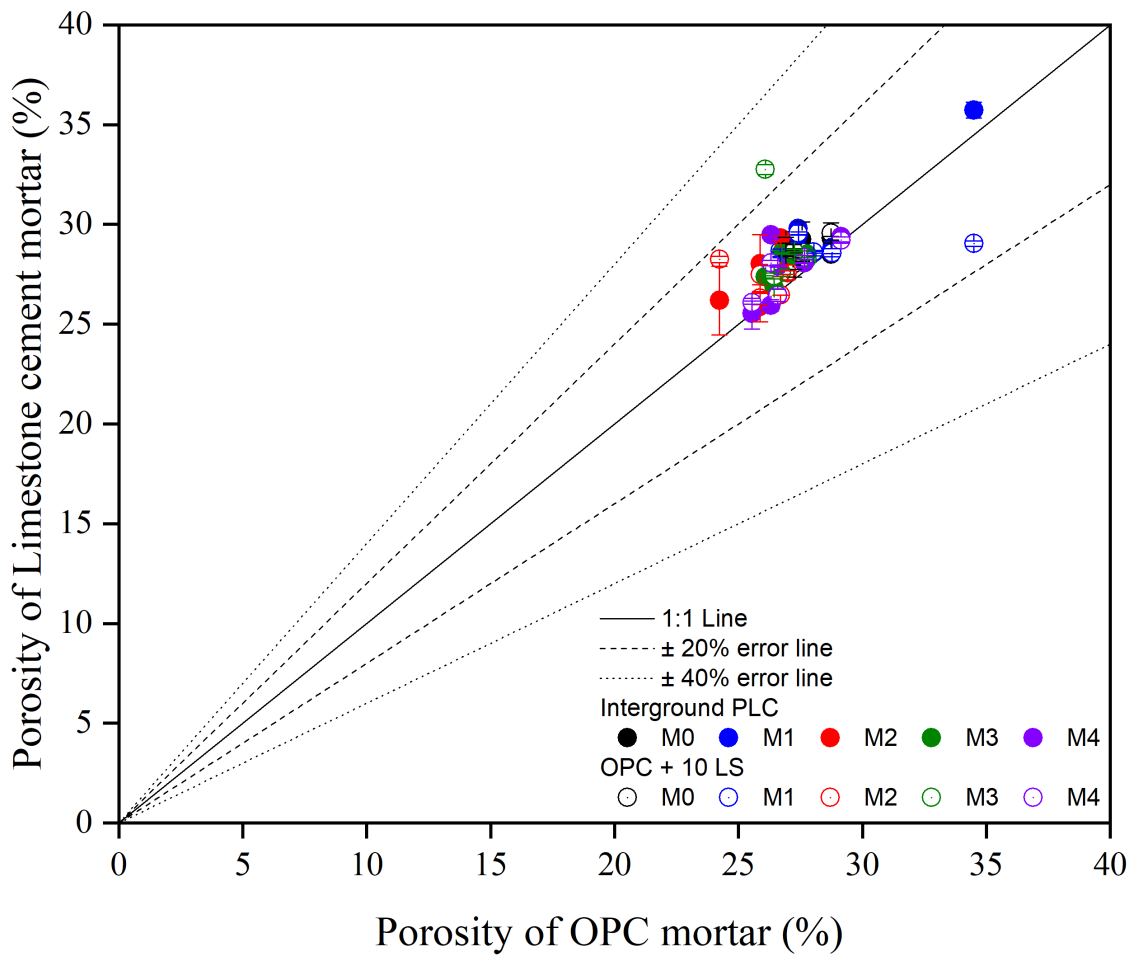

1292

Figure 8-2. Typical plot of porosity results comparing porosities of PLC mixtures with OPC mixtures

\section{$1294 \quad 8.6 \quad$ Experimental Results}

1295 The porosity and resistivity are provided in Figure 8-3 for mortars made using Cement E while the 1296 porosity and resistivity results of the other cements are provided in Appendix E. The 90-day 1297 porosity of the PLC and OPC+10LS mortars for all the mixtures are shown in Figure 8-4. The 901298 day resistivity of the PLC and OPC+10LS mortars for all the mixtures are shown in Figure 8-4 


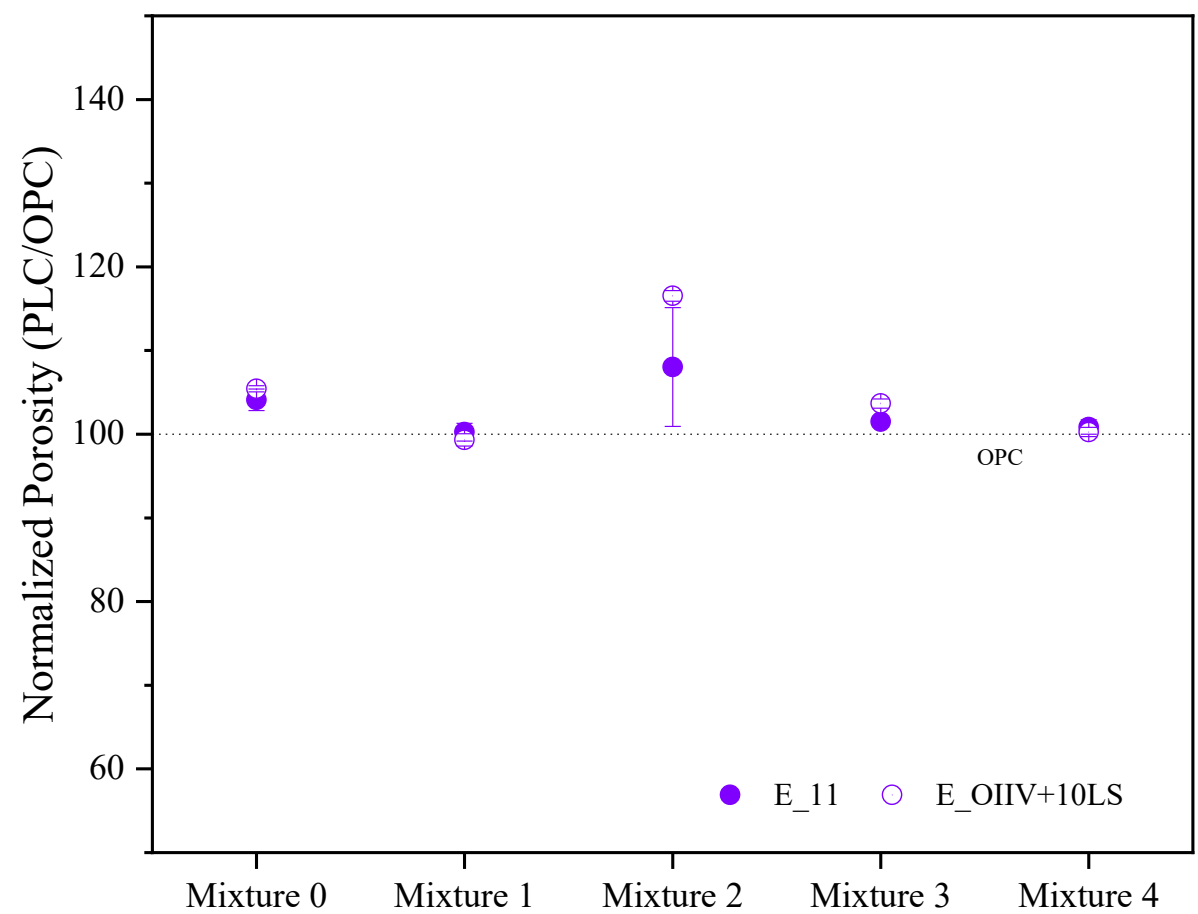

(a)

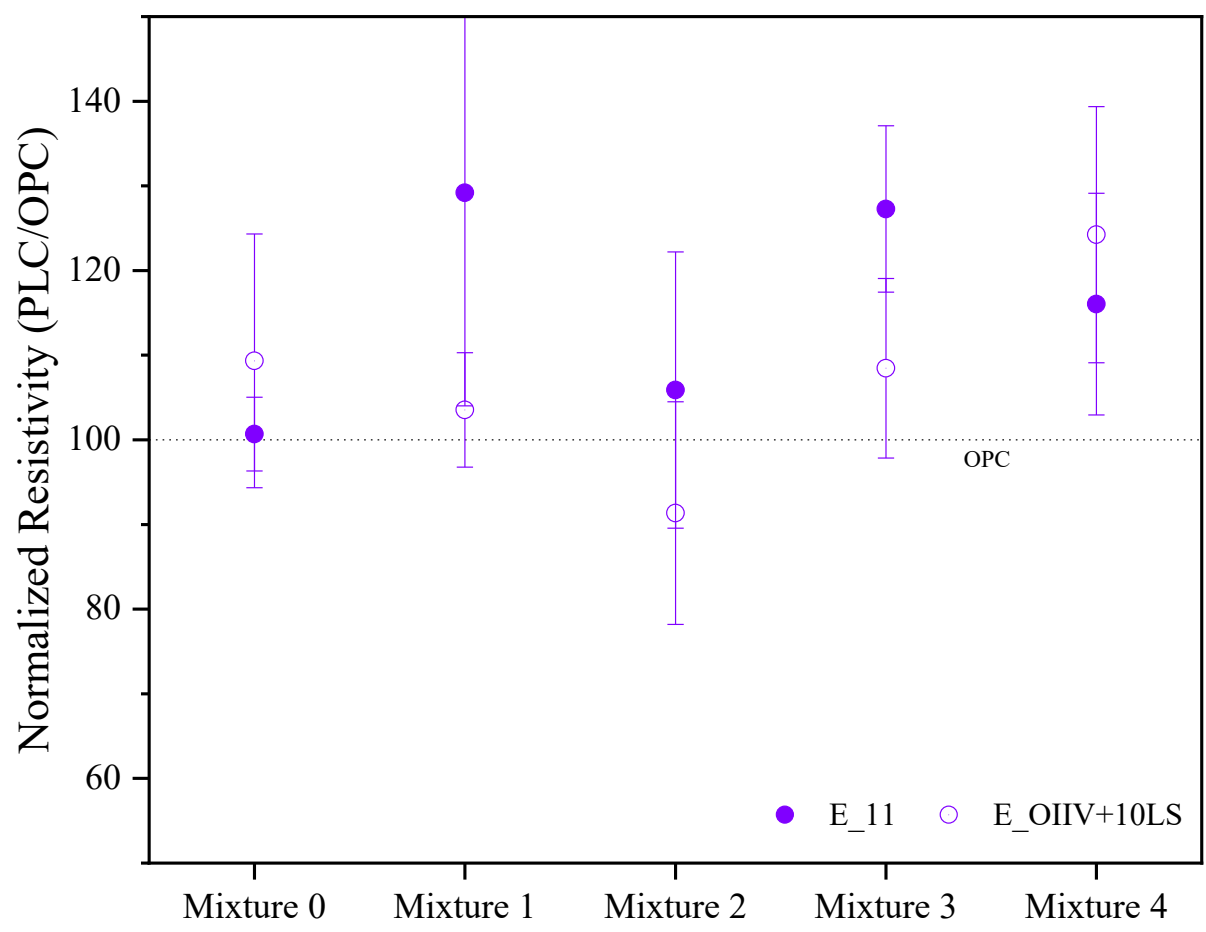

(b) the mixtures made using Cement $E$ 


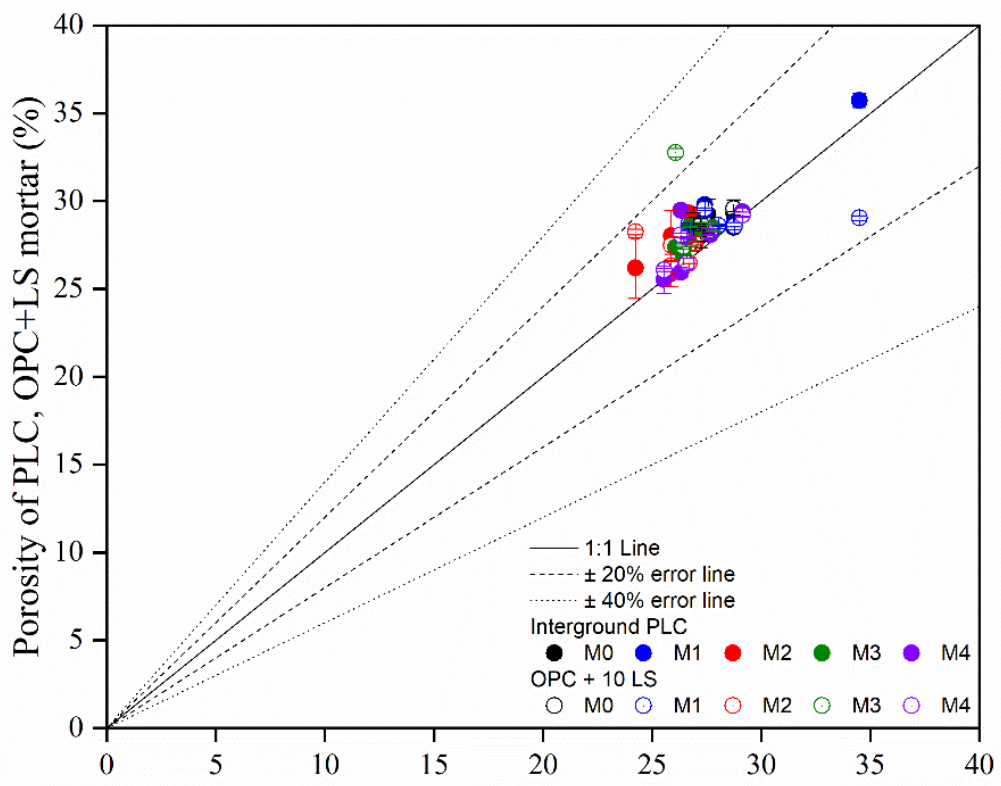

Porosity of OPC mortar (\%)

Figure 8-4. Porosity of PLC and OPC + 10 LS mortar compared to porosity of OPC mortars after 90 days

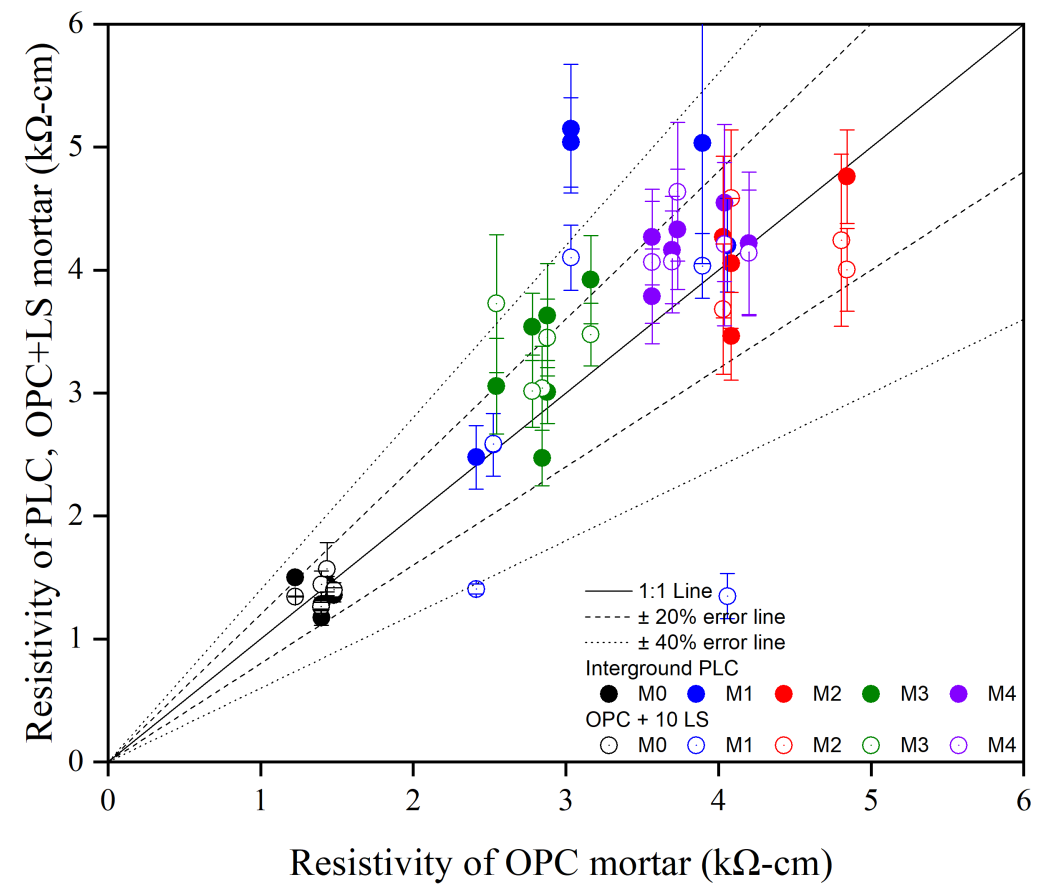




\section{$1308 \quad 8.7$ Discussion of the Results}

1309 The porosity of the PLC and OPC + 10LS mortar samples are observed to be consistently 5\% 1310 higher than the porosity observed in OPC mortar samples (Figure 8-4). This occurred in both the 1311 plain systems and the systems containing SCM. The resistivity of the PLC and OPC + 10LS mortar 1312 samples is observed to be greater than the OPC samples (Figure 8-5). This occurred in both the 1313 plain systems and the systems containing SCM. Mixtures with SCMs have a greater resistivity 1314 than plain systems. The electrical resistivity of PLC mortar samples was 10\%-30\% higher than the 1315 OPC. The OPC+10LS mortars also had a higher resistivity than the OPC samples, barring a few 1316 outliers (suspected of having undergone inadequate blending during mixing). In general, it can be 1317 concluded that PLC and OPC+LS systems had an improved electrical resistivity.

1318 The formation factor and pore connectivity were calculated (using equation (8-3) and (8-4) 1319 respectively) for the mixtures tested and are shown in Figure 8-6. Mixtures containing SCMs had 1320 a higher formation factor and lower pore connectivity than plain samples. The PLC + SCM mortar 1321 samples generally performed better than the OPC + SCM systems with a lower pore connectivity 1322 (approximately 10\%) due to pore refinement. The OPC+10LS + SCM mortar samples also display 1323 a greater spread in results due potentially to the cement not being ground as finely and the LS also 1324 being coarser. 


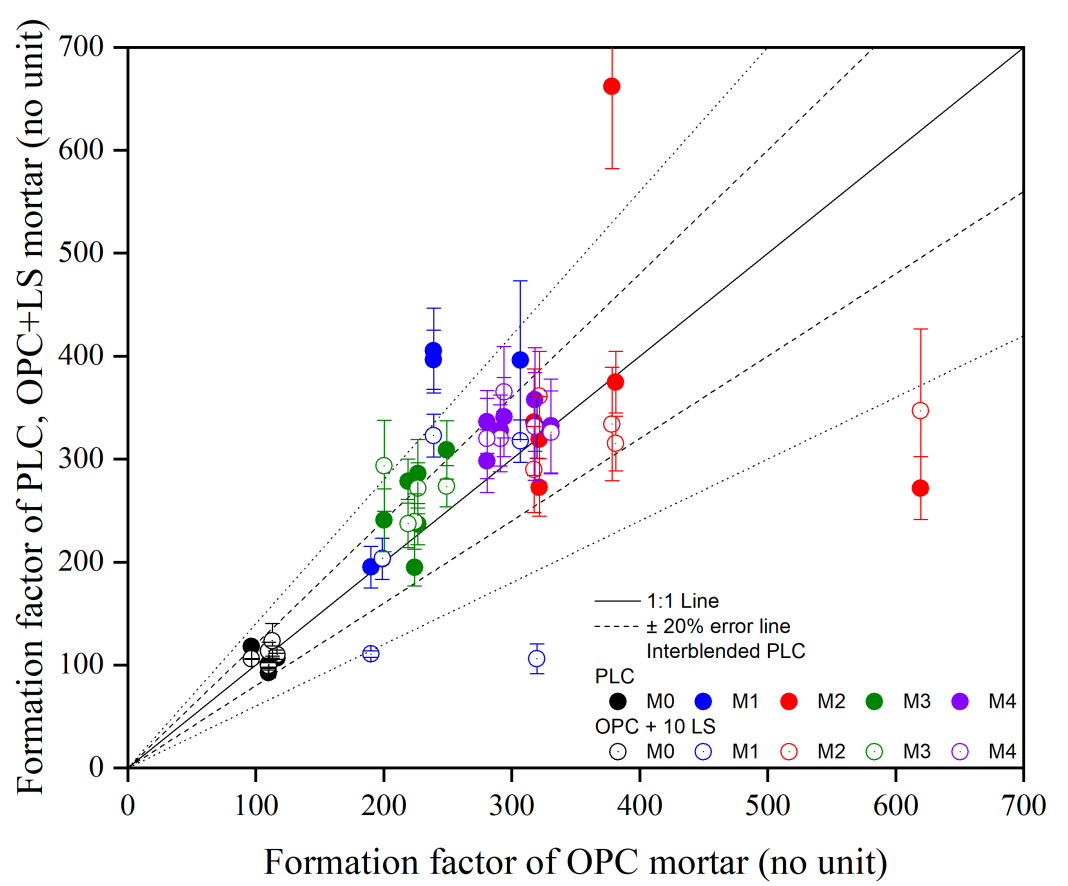

(a)

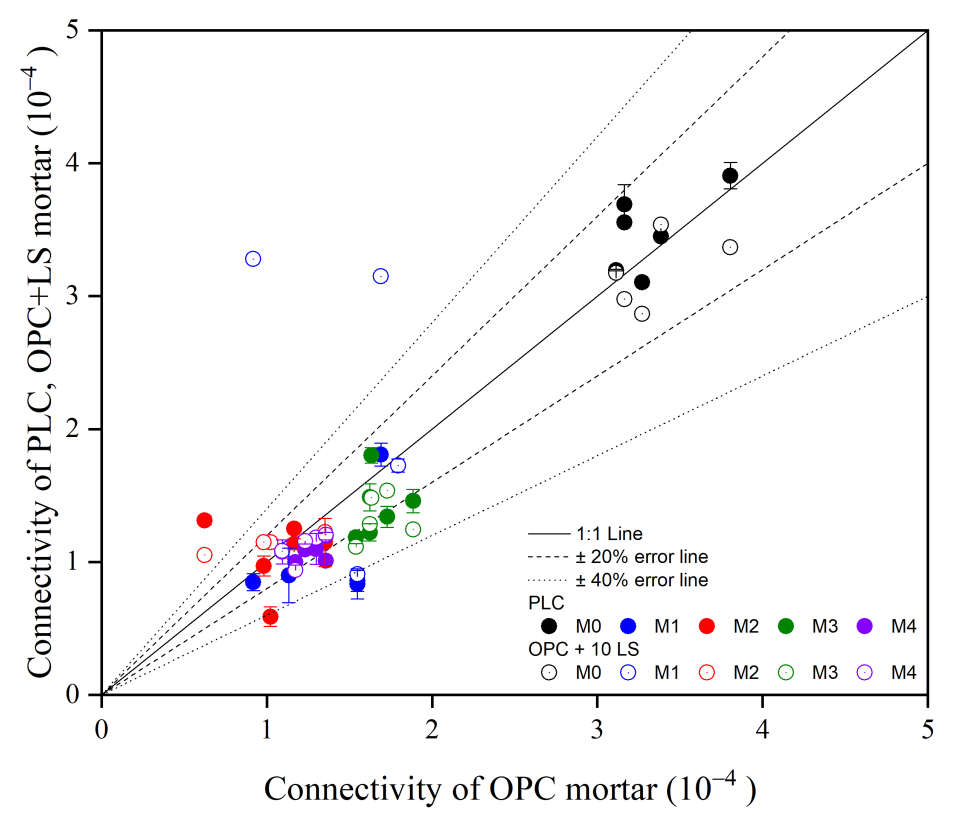

(b) mortar compared to pore connectivity of OPC mortars after 90 days 


\subsection{Significant Findings}

1329 This section examined the transport properties of OPC, PLC, and OPC+LS mortars. Porosity and 1330 electrical resistivity were measured. The formation factor and pore connectivity were computed. 1331 In general, the PLC and OPC +LS mortar samples displayed 5\% greater porosity than samples 1332 made with OPC. However, the resistivity of PLC and OPC+LS mortar samples were $10 \%$ and 5\% 1333 greater than OPC samples on average, respectively due to a reduction in pore connectivity. 


\section{Chloride Binding}

1335 Chlorides from seawater or deicing salts [93-97] can enter the concrete's pore structure and cause steel reinforcement corrosion. While a portion of these ions are transported through the pores, a portion of the chlorides are bound chemically or physically adsorbed on the pore walls. Changes have been made to ACI 318-19 to quantify the allowable chloride content in concrete mixtures in terms of total cementitious material content, not simply the cement content as was the case in prior code additions. Despite this advancement, questions remain regarding chlorides and concrete containing limestone. Specifically, there is uncertainty regarding whether the limestone portion of a PLC would contribute to the binding chlorides, and therefore, whether PLC and OPC+LS be treated differently than other cementitious systems. This portion of the research is intended to answer these questions by investigating chloride binding for mortar made using OPC, PLC, and OPC+ LS for both plain mixtures (M0) and SCM systems (M1 to M5).

\section{$1346 \quad 9.1 \quad$ Research Objectives}

1347 The primary objective is to determine whether the replacement of OPC with PLC or OPC with 1348 OPC + LS in conjunction with SCMs impacts chloride binding in concrete. Ultimately, this 1349 research will inform CALTRANS whether PLC can be used as an equivalent replacement of OPC

1350 without impacting the chloride binding performance. The work will also determine the variation 1351 in the chloride binding of pastes made using five sources of ASTM Type II/V OPCs (A, B, C, D, 1352 E). The work will also determine SCM's impact (fly ash, silica fume, slag) on the binding capacity 1353 of mixtures with limestone.

\subsection{Background/Literature Review}

1355 Reports published in 2010 and 2011 discussed the durability of concrete made with PLC [98]. It 1356 was concluded that PLC containing up to $15 \%$ limestone does not negatively impact the chloride 1357 ingress in PLC-based systems. PLC can refine pore size distribution due to particle size, increase 1358 strength, and develop chemical reactions that produce carbo-aluminate phases, reducing porosity. 1359 Unfortunately, however, none of the work specifically examined the chloride binding in mixtures 1360 made using PLC.

1362 Chlorides are typically introduced to hardened concrete from seawater or deicing salts [93-97]. A 1363 portion of the chloride remains in the pore solution and can move inside the concrete's pore 1364 structure, while a portion is chemically and physically bound. Chloride binding isotherms are 1365 typically developed for a concrete mixture as a plot of the free chloride content against the bound 1366 chloride content. These binding isotherms can be used in service life predictions and select 1367 appropriate cementitious materials to increase the durability of concrete structures [99]. Therefore, 1368 it is critical to characterize the effect of limestone on the chloride binding of concrete. 


\subsubsection{Binding Mechanisms}

1370 The two mechanisms involved in chloride binding are chemical binding (through Friedel's salt,

1371 Kuzel's salt, and calcium oxychloride) and physical adsorption (e.g., on C-S-H). Chemical binding 1372 can mainly be attributed to AFm and alumina-containing unhydrated phases such as $\mathrm{C}_{3} \mathrm{~A}$ and $\mathrm{C}_{4} \mathrm{AF}$ $1373[96,100,101]$.

1374 In an OPC-based mixture, AFm chemically reacts with chlorides to transform into Kuzel's 1375 salt $\left(3 \mathrm{CaO} \cdot \mathrm{Al}_{2} \mathrm{O}_{3} \cdot 0.5 \mathrm{CaSO}_{4} \cdot 0.5 \mathrm{CaCl}_{2} \cdot 10 \mathrm{H}_{2} \mathrm{O}\right)$ at low chloride concentrations $(<0.1 \mathrm{M})$ and 1376 Friedel's salt $\left(3 \mathrm{CaO} \cdot \mathrm{Al}_{2} \mathrm{O}_{3} \cdot \mathrm{CaCl}_{2} \cdot 10 \mathrm{H}_{2} \mathrm{O}\right)$ at higher concentrations $(>0.1 \mathrm{M})[96,100,102] . \mathrm{AFt}$ 1377 becomes unstable at higher chloride concentrations $(>2 \mathrm{M})$ and partially decomposes to form AFm 1378 and Friedel's salt. The aluminate phases in $\mathrm{C}_{3} \mathrm{~A}$ and $\mathrm{C}_{4} \mathrm{AF}$ may also chemically bind chlorides to 1379 form Friedel's salt [97, 100, 103]. Balonis et al. [101] suggest that a typical portland cement 1380 contains sulfates and/or carbonates, and as a result, initially formed AFm is a monosulfoaluminate 1381 type (Ms) and converts to $\mathrm{OH}-\mathrm{AFm}$ (hydroxy $\mathrm{AFm}$ ) at $25^{\circ} \mathrm{C}$ or monocarboaluminate (Mc) [53]. 1382 Depending on the phases' stability, the sulfate, hydroxyl, and carbonate anions can be displaced 1383 by chloride ions to form chloride AFm, which is essentially Friedel's salt [104, 105]. Physical 1384 binding can be attributed to the physical adsorption of chlorides on the surface of the C-S-H, C-A$1385 \mathrm{H}$, and C-A-S-H phases [96]. The phenomenon occurs due to the electrostatic Van der Waals forces 1386 between charged particles, in this case, between chloride ions and the surface of these phases.

\section{$1387 \quad 9.2 .2 \quad$ Limestone in Chloride Binding}

1388 Limestone may react with calcium aluminate hydrates (C-A-H) in SCM blends to form 1389 carboaluminate hydrates, (monocarboaluminate (Mc, $\left.\mathrm{Ca}_{4} \mathrm{Al}_{2}\left(\mathrm{CO}_{3}\right)(\mathrm{OH})_{12} \cdot 5 \mathrm{H}_{2} \mathrm{O}\right)$ and 1390 hemicarboaluminate $\left(\mathrm{Hc}, \mathrm{Ca}_{4} \mathrm{Al}_{2}\left(\mathrm{CO}_{3}\right)_{0.5}(\mathrm{OH})_{13} \cdot 5.5 \mathrm{H}_{2} \mathrm{O}\right)$ [106]). Ms, Mc, and $\mathrm{Hc}$ are all part of 1391 the monosulfate (AFm) family and can chemically bind chloride ions to form Friedel's salt (Fs, $\left.1392 \mathrm{Ca}_{4} \mathrm{Al}_{2} \mathrm{Cl}_{2}(\mathrm{OH})_{12} \cdot 4 \mathrm{H}_{2} \mathrm{O}\right)$ [106-108]. Ipavec et al. [109] found that the chloride binding capacity of 1393 limestone cements is strongly dependent on the external solution's $\mathrm{pH}$. The chloride binding 1394 capacity decreases at $\mathrm{pH} \sim 13.5$ but remains unchanged at $\mathrm{pH} \sim 12.8$.

\section{$1395 \quad$ 9.2.3 Binding Isotherms}

1396 Chloride binding isotherms relate the free $\left(\mathrm{C}_{\mathrm{f}}\right)$ and bound $\left(\mathrm{C}_{\mathrm{b}}\right)$ chlorides in concrete at a constant 1397 temperature [96]. The binding isotherm is dependent on the chemical composition of the system, 1398 including the total aluminate content, limestone content, the type and amount of SCMs used in the 1399 mixture, and the $\mathrm{pH}$ of the pore solution. The isotherms are typically represented quantitatively 1400 using either the Langmuir equation (equation (9-1)) or the Freundlich equation (equation (9-2)).

$$
C_{b}=\frac{\alpha C_{f}}{\left(1+\beta C_{f}\right)}
$$




$$
C_{b}=\alpha C_{f}^{\beta}
$$

1401 where $\alpha$ and $\beta$ coefficients need to be determined experimentally.

1402 Tang and Nilsson [102] found that the Langmuir isotherm is a good fit at low chloride 1403 concentrations $(<0.05 \mathrm{M})$ and the Freundlich isotherm is a good fit at high chloride concentrations $1404(>0.01 \mathrm{M})$. The Freundlich isotherm will be used in this work to fit the chloride binding data, as 1405 the chosen chloride concentrations start from 0.1 M.

\section{$1406 \quad$ 9.2.4 Factors Affecting Chloride Binding}

1407 Several factors affect chloride binding capacity. Increased chloride binding is observed when 1408 cement paste is exposed to $\mathrm{CaCl}_{2}$ solution compared to $\mathrm{NaCl}$. This can be attributed to $\mathrm{NaCl}$ having to react first with $\mathrm{Ca}(\mathrm{OH})_{2}$ to form $\mathrm{CaCl}_{2}$ before reacting with the hydrated aluminate phases [110, 111]. If available, $\mathrm{Ca}(\mathrm{OH})_{2}$ will then react with the $\mathrm{CaCl}_{2}$ solution to form calcium oxychloride

1412 The $\mathrm{pH}$ of the pore solution is also an important factor. Delagrave et al. conducted chloride binding 1413 tests for cement powder immersed in saturated lime solutions $\left(\mathrm{Ca}(\mathrm{OH})_{2}+\mathrm{NaCl}\right)(\mathrm{pH} 12.5)$ and in 1414 alkaline solutions $(\mathrm{NaOH}+\mathrm{KOH}+\mathrm{NaCl})(\mathrm{pH}$ 13.5) [119]. They observed a higher amount of 1415 bound chlorides when immersed in the lime saturated solution, indicating that a lower $\mathrm{pH}$ of the 1416 external solution will cause an increase in bound chlorides [113, 119]. Zibara also observed an 1417 increase in the $\mathrm{pH}(13-14)$ of the storage solution reduced chloride binding [96]. Song et al. studied 1418 the influence of $\mathrm{pH}$ of hydration products on chloride binding and found that the $\mathrm{pH}$ of the external 1419 solution increased with time, likely because of the leaching out of alkalis such as $\mathrm{K}+$ and $\mathrm{Na}+$ from 1420 the cement matrix [37].

$1421 \mathrm{Bu}$ and Weiss investigated the influence of alkali content on the microstructure, chloride binding, 1422 and electrical resistivity of concrete by fully saturating concrete specimens in $\mathrm{NaOH}$ solution 1423 [120]. They found that a higher alkali content in the external solution can decrease the resistivity 1424 of the pore solution, causing a reduction of bound chlorides. The higher alkali content also resulted 1425 in a denser microstructure, a higher formation factor, an increase in bulk resistivity, and a lower 1426 diffusion rate. They concluded that high alkali content in a cementitious system could improve its 1427 resistance to chloride ingress [38]; however, the alkali can impact other aspects of the concrete 1428 negatively (e.g., ASR). Ipavec et al. found that the presence of limestone decreased chloride 1429 binding at a $\mathrm{pH} 13.5$ and did not significantly affect binding at $\mathrm{pH} \sim 12.8$ [109].

1430 In simulated solution tests, saturated lime solution $\left(\mathrm{Ca}(\mathrm{OH})_{2}\right)$, sodium hydroxide $(\mathrm{NaOH})$, 1431 potassium hydroxide $(\mathrm{KOH})$, and sodium sulfate $\left(\mathrm{NaSO}_{4}\right)$ can be added to chloride solutions to 1432 increase the $\mathrm{pH}$. A saturated lime solution has a $\mathrm{pH} \sim 12.4$, and in the presence of $\mathrm{CaCl}_{2}$, it will 
1433 form calcium oxychlorides, complicating the quantification of chloride binding. Sodium, 1434 hydroxide, and potassium ions in $\mathrm{NaOH}$ and $\mathrm{KOH}$ are alkali and contribute to the solution's 1435 alkalinity. However, sulfates in $\mathrm{NaSO}_{4}$ are bound first to $\mathrm{C}_{3} \mathrm{~A}$ before chlorides, and this interrupts 1436 chloride binding. Hence, it is advisable to use $\mathrm{NaOH}$ and/or $\mathrm{KOH}$ to increase the $\mathrm{pH}$ of a chloride 1437 solution to maintain a $\mathrm{pH}$ like that in a concrete matrix.

1438 The aluminate content in the cementitious matrix is another important factor in chloride binding. 1439 Increasing the amounts of $\mathrm{C}_{3} \mathrm{~A}$ was initially found to increase chloride binding [1,5]. Later it was 1440 discovered that it is not only the $\mathrm{C}_{3} \mathrm{~A}$ but the total aluminate content $\left(\mathrm{C}_{3} \mathrm{~A}+\mathrm{C}_{4} \mathrm{AF}\right)$ that increases 1441 chloride binding $[14,25]$. Aluminates can come from SCMs as well. The binding capacity is 1442 dependent on the alumina content of the binder. This is due to the increase of C-A-H and C-A-S$1443 \mathrm{H}$, which provide a larger surface area for the physical adsorption of chlorides when compared to 1444 OPC. For example, silica fume, which contains less alumina than OPC, decreases chloride binding. 1445 Slag, which contains more alumina than OPC, increases chloride binding. Fly ash, which typically 1446 has the largest alumina content than slag and silica fume, strongly increases binding capacity [96, $1447109,121]$.

\section{$1448 \quad 9.3 \quad$ Experimental Test Matrix}

1449 Chloride binding testing was performed to evaluate the relative performance of all cements in this 1450 study. The binding test will be described in the following section 9.5. The mortars described in 1451 section 9.4.1 were tested after flexural strength testing. These samples included mortars made from

1452 Type II/V OPCs from five different clinkers, namely A, B, C, D, and E. The samples exposed to 1453 sodium chloride $(\mathrm{NaCl})$ as concrete in marine environments are constantly exposed to seawater. 1454 The chloride binding isotherms for each of the clinkers were compared to determine if there is 1455 variation among the chlorides bound by OPC. The same was done for PLC and OPC+10LS 1456 systems. The binding isotherm was measured for OPC, PLC, and OPC+10LS systems made using 1457 supplementary cementitious materials (SCMs), namely fly ash, silica fume, and slag. Finally, 1458 exposure to calcium chloride $\left(\mathrm{CaCl}_{2}\right)$ on the chloride binding capacity of PLCs was investigated. 1459 Only cement $\mathrm{D}$ was exposed to $\mathrm{CaCl}_{2}$. The effect of $\mathrm{CaCl}_{2}$ exposure was studied because it is 1460 commonly used as a deicing salt, and this work would provide useful insight into the chloride 1461 binding capacity in limestone cements.

1462 The test matrix, comprising 80 mortar mixtures, is provided in Table 6.2.

\section{$1463 \quad 9.4 \quad$ Experimental Methods}

\section{$1464 \quad 9.4 .1 \quad$ Mortar samples}

1465 The $\mathrm{w} / \mathrm{cm}$ of mortar pastes was 0.40 . The mixing procedure is described in Section 6.4. The mortar 1466 samples were cast into cylindrical molds measuring 4 inches $(101.6 \mathrm{~mm})$ in diameter and 8 inches 
$1467(203.2 \mathrm{~mm})$ in height. The cylinders were sealed, cured at $23^{\circ} \mathrm{C}$ for 91 days, demolded, then sliced 1468 into discs of $2.65 \mathrm{~mm}$ width. The discs were tested for tensile strength using the ball-on-three-ball 1469 (B3B) test [89], then double-bagged to prevent carbonation. The discs were crushed into smaller pieces using a mortar and pestle and passed through a 3/8-inch $(9.5 \mathrm{~mm})$ sieve. The crushed samples were stored in double plastic bags to avoid carbonation before chloride immersion.

\subsubsection{Simulated pore solution}

1473 A simulated pore solution was prepared to maintain the $\mathrm{pH}$ of concrete $(\sim 13.5)$ in the chloride 1474 solutions. $102.6 \mathrm{~g} \mathrm{NaOH}$ and $143.9 \mathrm{~g} \mathrm{KOH}$ were mixed with 13,250 g DI water according to 1475 ASTM C1876-08 and stored in a 5-gallon bucket. The simulated pore solution was mixed with varying concentrations of $\mathrm{NaCl}$ and $\mathrm{CaCl}_{2}$ salts to prepare chloride solutions.

\section{$1477 \quad$ 9.4.3 Chloride solutions}

1478 Sodium chloride was dissolved in simulated pore solution to prepare $\mathrm{NaCl}$ concentrations of 0.1 $1479 \mathrm{M}, 0.5 \mathrm{M}, 1 \mathrm{M}, 2 \mathrm{M}, 3 \mathrm{M}$, and $5 \mathrm{M}$, respectively. Granular calcium chloride dihydrate $1480\left(\mathrm{CaCl}_{2} \cdot 2 \mathrm{H}_{2} \mathrm{O}\right.$, EMD Millipore Inc., reagent grade) was dissolved in simulated pore solution to 1481 prepare $\mathrm{CaCl}_{2}$ solutions with concentrations of $0.1 \mathrm{M}, 0.5 \mathrm{M}, 1 \mathrm{M}, 2 \mathrm{M}$, and $3 \mathrm{M}$, respectively (Note 1482 the $5 \mathrm{M}$ concentration was not prepared due to the formation of Calcium Oxychloride). All cement 1483 mixtures were immersed in $\mathrm{NaCl}$ solutions, while only cement $\mathrm{D}$ mixtures were immersed in $\mathrm{CaCl}_{2}$ 1484 solutions.

\subsection{Typical Experimental Measurement and Interpretation}

\section{$1486 \quad 9.5 .1$ Determination of bound chlorides}

1487 Approximately $5 \mathrm{~g}$ of crushed mortar sample was weighed and stored in a capped centrifuge tube 1488 (15 ml, VWR Inc.) with $5 \mathrm{ml}$ chloride solutions $\left(\mathrm{NaCl}\right.$ and $\left.\mathrm{CaCl}_{2}\right)$ of varying concentrations sealed 1489 at $23 \pm 1^{\circ} \mathrm{C}$ for 30 days to reach equilibrium. $50 \mu 1$ of the equilibrated solutions was extracted using 1490 a pipette, and the chloride concentrations were determined by an automatic titration device shown 1491 in Figure 9-1. The bound chloride content was calculated using (Equation (9-3).

$$
C_{b}=\frac{\left(c_{0}-c_{1}\right) \cdot V_{\text {sol }} \cdot M_{C l}}{m_{\text {powder }}}
$$

1492 where $C_{b}$ is the bound chloride content ( $\mathrm{mg} / \mathrm{g}$ powder), and $c_{0}$ and $c_{1}$ are the initial and final $\mathrm{Cl}^{-}$ 1493 concentrations of the exposure solution (M), respectively, $V_{s o l}$ is the volume of the solution $(\mathrm{ml})$, $1494 M_{C l}$ is the molar mass of chloride, and $m_{\text {powder }}$ is the mass of the powder $(\mathrm{g})$. The curves were 1495 fitted using the Freundlich isotherm. 


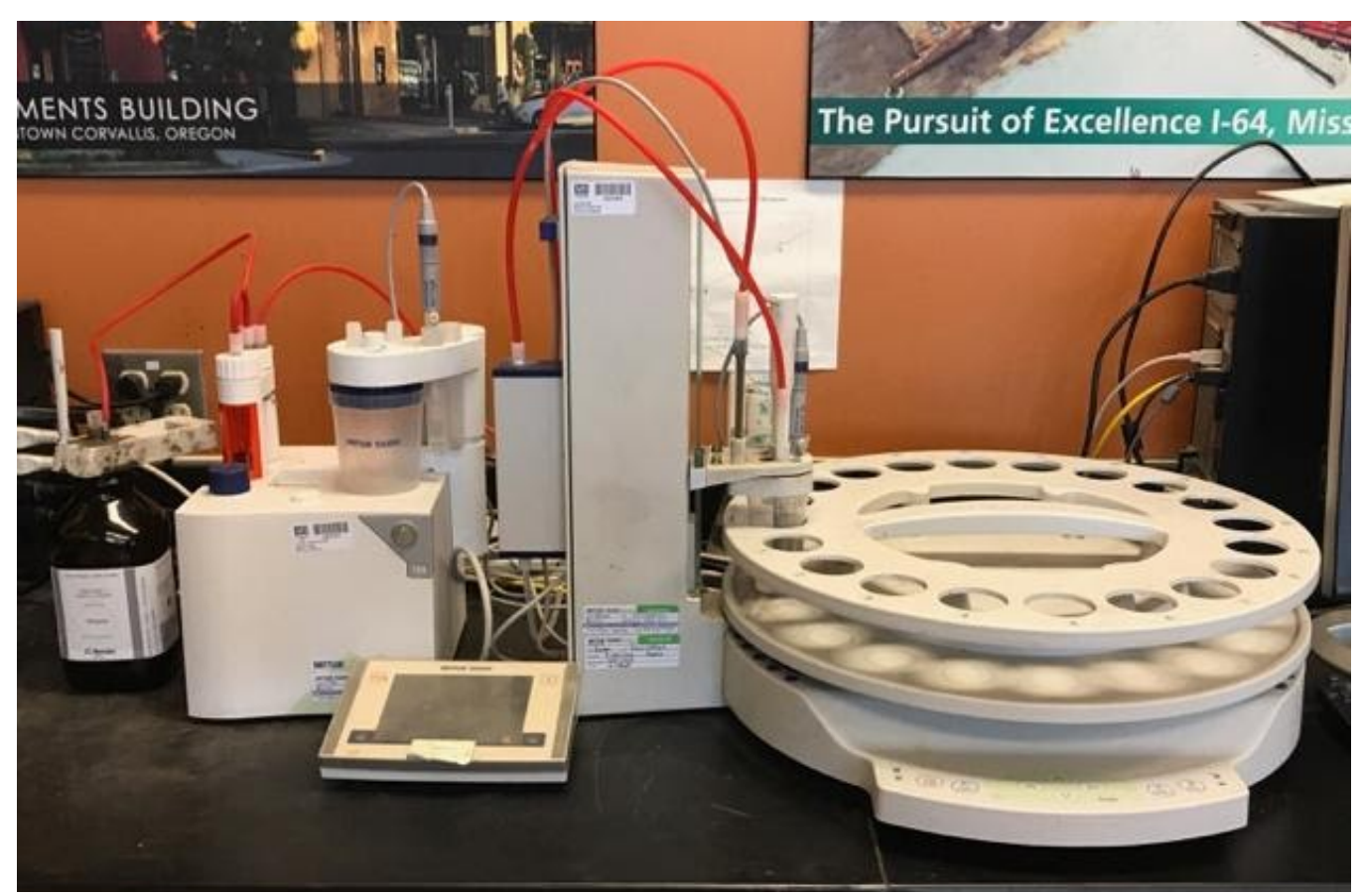

Figure 9-1. Automatic titration device used for determination of bound chlorides

1500 Statistical analyses were performed to determine if there is a statistical difference between the 1501 chlorides bound by OPC, and PLC, OPC and OPC+10LS, and between mixtures with no SCMs 1502 (M0) and mixtures with SCMs (M1-M5). Because the data set contained several groups of data, 1503 the analysis of variance (ANOVA) was performed to determine the statistical significance between 1504 the means of the different data groups [122]. When one comparison test is conducted, the error 1505 rate of 0.05 is used. There will be many comparison tests in such a large set of data, which means 1506 the error rate must be adjusted accordingly (familywise error rate, FWER). The means were 1507 calculated from the bound chloride data among the different cements A-E. Tukey's honestly 1508 significant test (HSD) was performed for the ANOVA tests to correct for FWER.

1509 For the comparison between M0 and M5, t-tests were performed instead, and a Bonferroni 1510 correction was used to correct for the FWER. The t-test is used here because the comparisons were 1511 for each pair only, so multiple comparisons were unnecessary.

\section{$1512 \quad 9.6 \quad$ Experimental Results}

1513 This chapter provides information on the binding of chloride ions in hydrated cement pastes made 1514 using OPC, PLC, and OPC+LS. In Appendix F, Tables E-OPC, E-PLC, E-OPC+10LS, and E$1515 \mathrm{CaCl}_{2}$ provide results of the experimentally measured values for the bound chloride, comparing 1516 systems with varying limestone content (OPC, PLC, OPC+10LS) as determined using the 1517 procedure described in section 9.5.1. Specifically, Table E-OPC illustrates the bound chlorides by 
1518 OPC for the five different cement clinkers tested (A to E) with six sodium chloride $(\mathrm{NaCl})$ 1519 concentrations (from 0 to $5 \mathrm{M}$ ) mixed with simulated pore solution to maintain concrete $\mathrm{pH} \sim 13$. 1520 Mixtures were prepared without supplementary cementitious materials (SCMs) and with SCMs as indicated through the mixture designations M0 to M5. M0 was prepared without SCM replacement, M1 was prepared with 25\% fly ash, M2 was prepared with $20 \%$ fly ash and $5 \%$ silica fume, M3 was prepared with 50\% slag, M4 was prepared with 25\% slag, and 25\% fly ash and M5 was prepared with $25 \%$ natural pozzolan. Similarly, experimental data is provided in Table E-PLC for the PLC clinker systems, Table E-OPC+10LS for the OPC plus LS systems, and in Table E$\mathrm{CaCl}_{2}$ for the cement clinker D systems that utilized calcium chloride $\left(\mathrm{CaCl}_{2}\right)$ as the salt solution as opposed to sodium chloride $(\mathrm{NaCl})$.

1528 As the chloride concentration increases, so does the binding, consistent with the trends commonly observed in the literature $[96,113]$. A rise in molarity from $0.1 \mathrm{M}$ to $5 \mathrm{M}$ corresponds with an increase in free chloride concentration. This increase in free chloride concentration resulted in an increase in binding from $\sim 0.9$ to $13 \mathrm{mg} \mathrm{Cl} / \mathrm{g}$ powder. This increase in binding with concentration can be seen in Figure 9-2, which shows the binding isotherm of M0 with clinker A. In general, the binding does not change significantly based on the parent cement clinker. The full set of binding isotherms of M0-M5 for cements A-E comparing isotherms with varying limestone content (OPC, 1535 PLC, OPC+10LS) can be found in Appendix F.

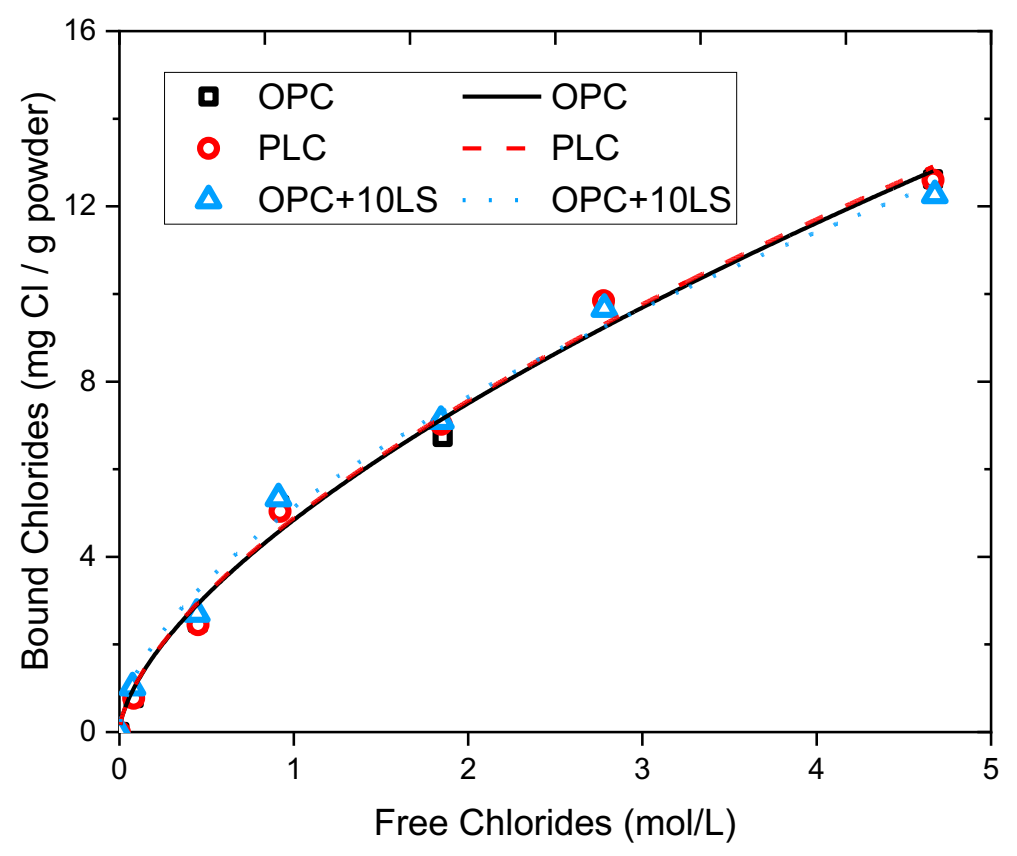


1538 It can also be seen that when SCMs are added, as shown in Figure 9-3 for cement A, binding curves 1539 are slightly different for mixtures 0 to 4 in OPC, PLC, and OPC+10LS systems. Fitted parameters 1540 for the Freundlich isotherm are also provided in Appendix F.

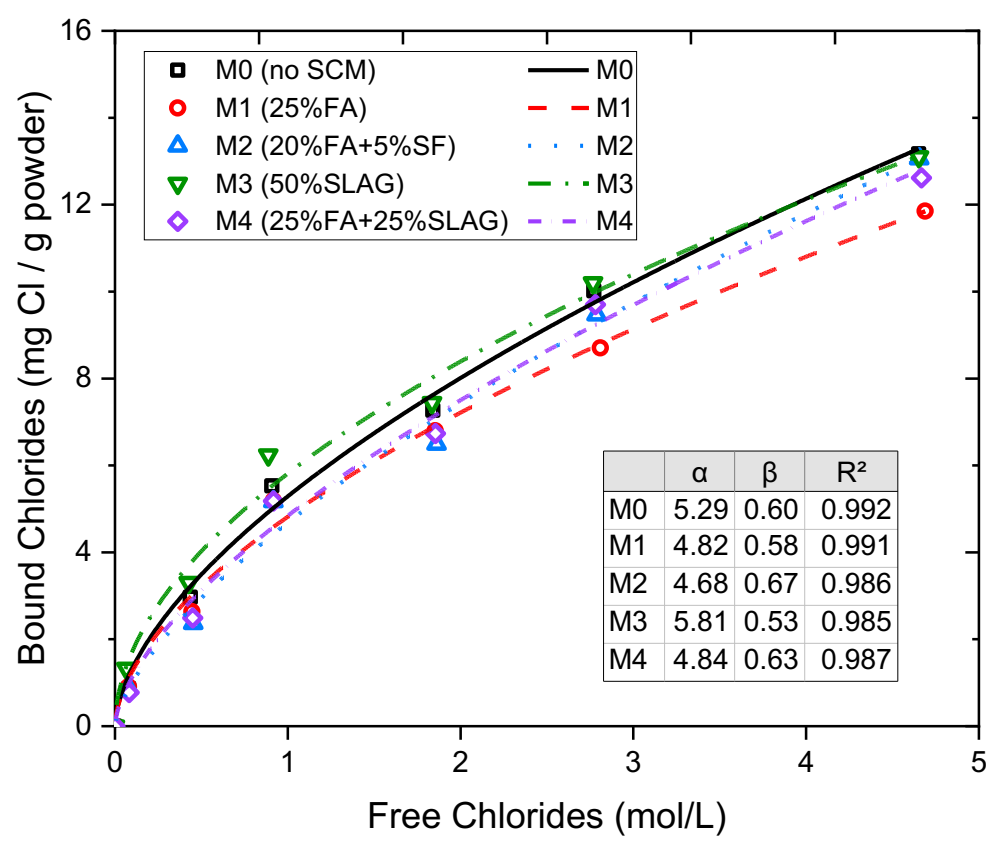

(a)

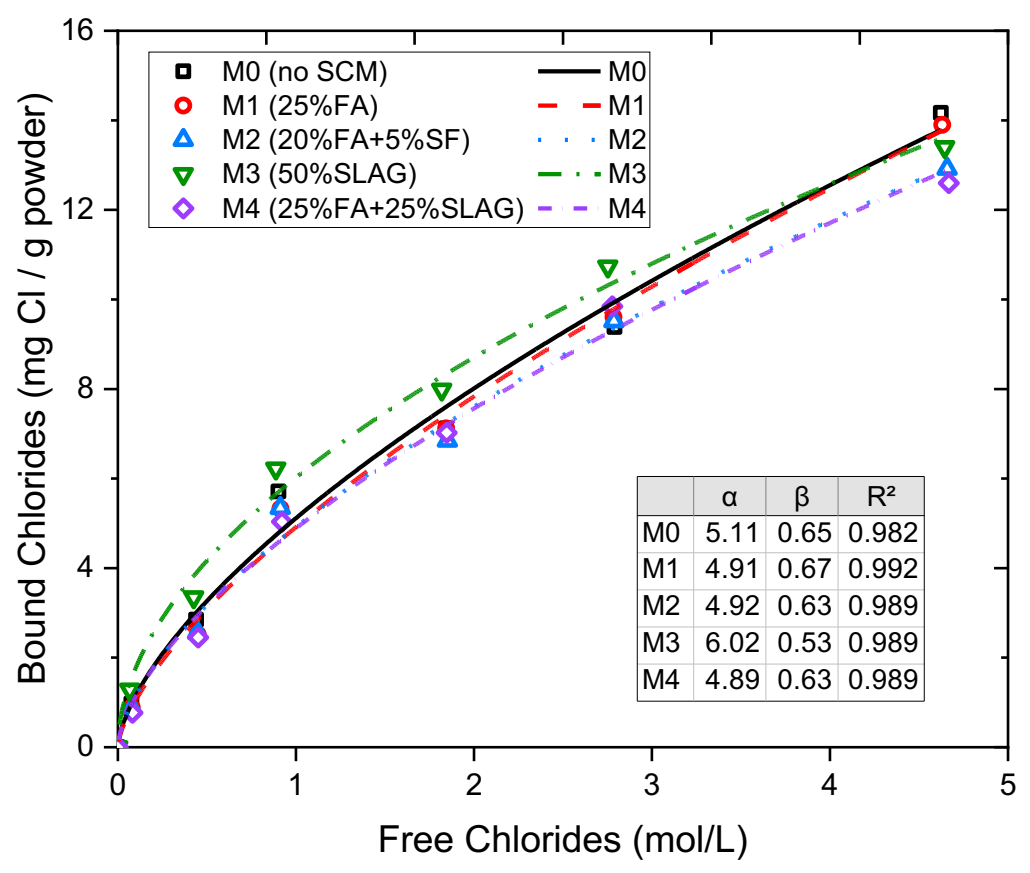

(b) 


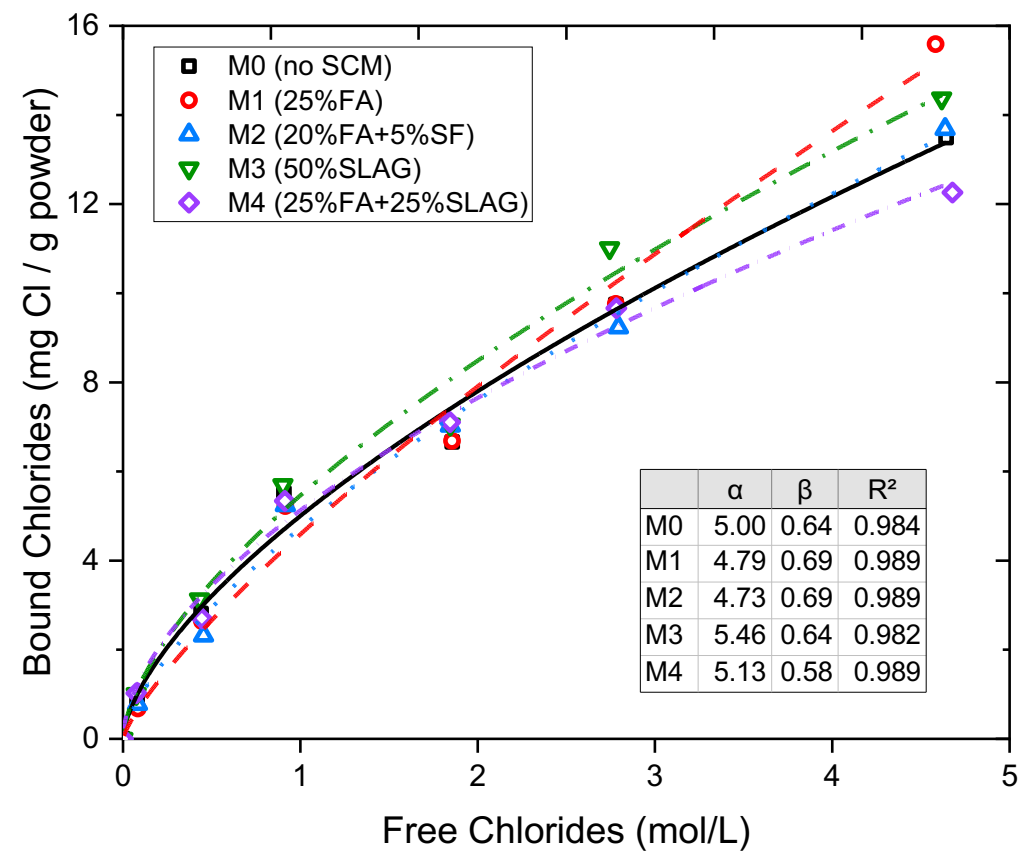

(c)

\subsection{Discussion of results}

\section{$1544 \quad$ 9.7.1 Difference in binding of OPC, PLC, and OPC $+10 L S$}

1545 The statistically significant difference between the binding isotherms of varying limestone content 1546 (OPC, PLC, OPC+10LS) and varying SCM content (M0-M5) was determined through statistical 1547 analysis (t-tests and Bonferroni; ANOVA and Tukey's HSD).

1548 No statistical difference was found between the chloride binding of cement pastes made using 1549 clinkers A, B, C, D, or E. A statistical difference between bound chlorides of OPC vs. PLC at only 1550 one chloride concentration was investigated. A decision was made to require multiple indicators 1551 to conclude that there is a statistical difference between the entire system's bound chlorides. As 1552 such, the single indicators will not be considered statistically meaningful. No statistical difference 1553 was found between the chlorides bound by OPC, PLC, and OPC+10LS. It can be recommended 1554 that, based on chloride binding, these binders can be used as a direct substitute for one another.

\subsubsection{Difference in binding of M0 and M1-M5}

1556 The majority of the SCM showed no statistical difference, except the mixture that contains 50\% 1557 slag, or M4, which contains 25\% slag and 25\% fly ash. The slag mixtures tend to increase binding. 
1558 There was no statistical difference (as determined through Tukey's HSD procedure) between bound chloride in all the mixtures except M3. As such, all data (except M3) was used to obtain an overall Freundlich fit ( $\alpha=4.79$ and $\beta=0.62$ ), as shown in Figure 9-4. The error ranges shown represent one standard deviation and two standard deviations.

1562 The implications of potentially using one single binding isotherm can be quite large. Considering 1563 the papers by Azad [123], the binding isotherm is combined with the formation factor to predict 1564 the apparent diffusion coefficient, using the approach described in Section 10.3.3. The use of a 1565 single series of alpha and beta terms could enable service life based on the apparent diffusion to 1566 be directly related to the formation factor.

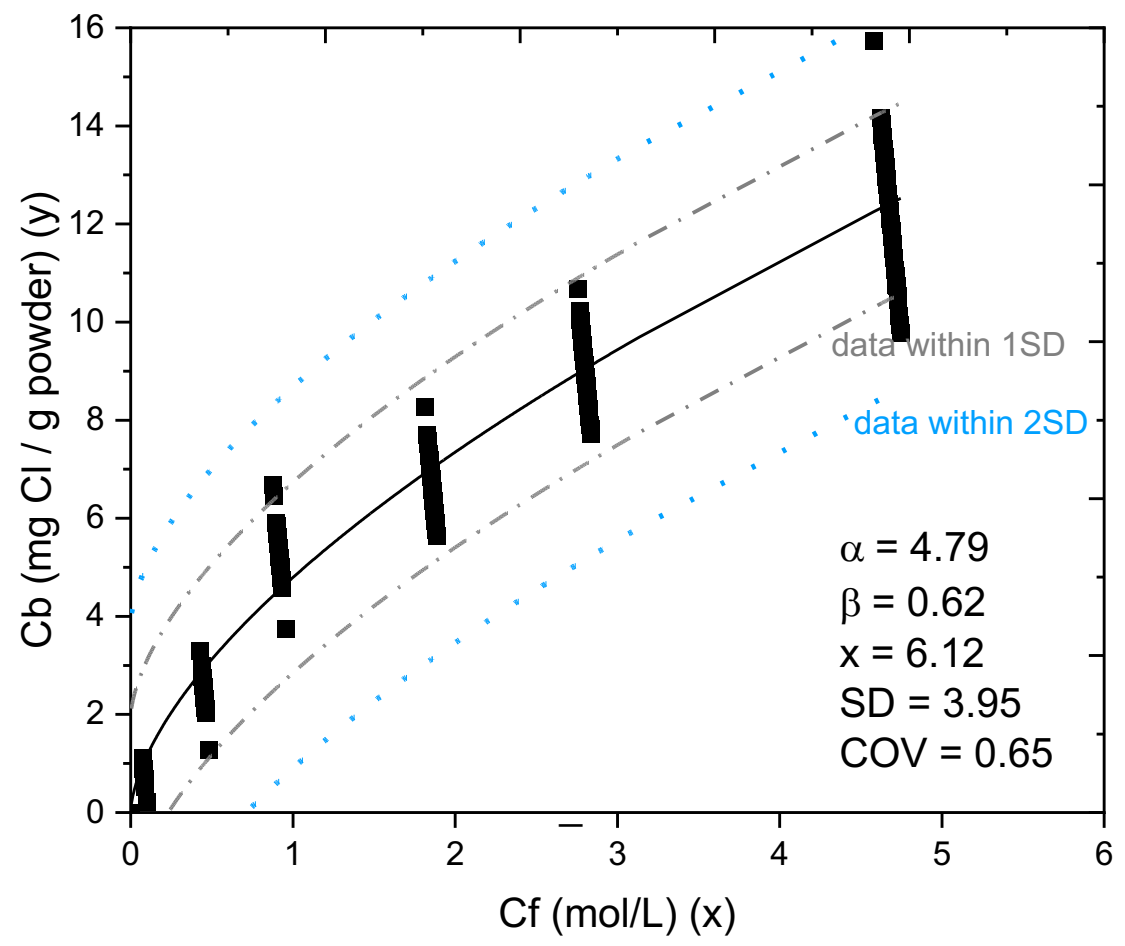

Figure 9-4. Freundlich fit for all data except for slag mixtures

\subsubsection{Difference in binding of $\mathrm{NaCl}$ and $\mathrm{CaCl}_{2}$}

1570 The difference in chloride binding between $\mathrm{NaCl}$ and $\mathrm{CaCl}_{2}$ salts at $23^{\circ} \mathrm{C}$ is shown in Figure 9-5

1571 for the plain (M0) and fly ash system (M1). An increase in binding capacity was observed when 1572 chlorides are exposed to $\mathrm{CaCl}_{2}$ salt compared to $\mathrm{NaCl}$. This has also been observed in earlier 1573 literature $[113,124,125]$. Similar observations can be made for the other mixtures, as shown in 1574 Appendix F.

1575 The increase in chloride binding is not likely to be caused by calcium oxychloride formation due 1576 to the temperatures involved. Qiao et al. [126] compared systems with $0 \%$ and $40 \%$ fly ash, 
1577 respectively, and observed that the amount of Friedel's salt formed reaches a plateau at $>2.0 \mathrm{M}$ for $15780 \%$ fly ash; however, the plateau starts later at $>2.5 \mathrm{M}$ for $40 \%$ fly ash. This was attributed to the 1579 reaction of all available aluminate phases with $\mathrm{CaCl}_{2}$ solutions to form Friedel's salt at high 1580 chloride concentrations [113]. The result is that cementitious systems with higher alumina content 1581 produce more Friedel's salt at higher chloride concentrations, which changes the slope and 1582 inflection point of the binding isotherm. This is a possible explanation for the increase in bound 1583 chloride content for limestone and $\mathrm{SCM}$ blends exposed to $\mathrm{CaCl}_{2}$ solutions.

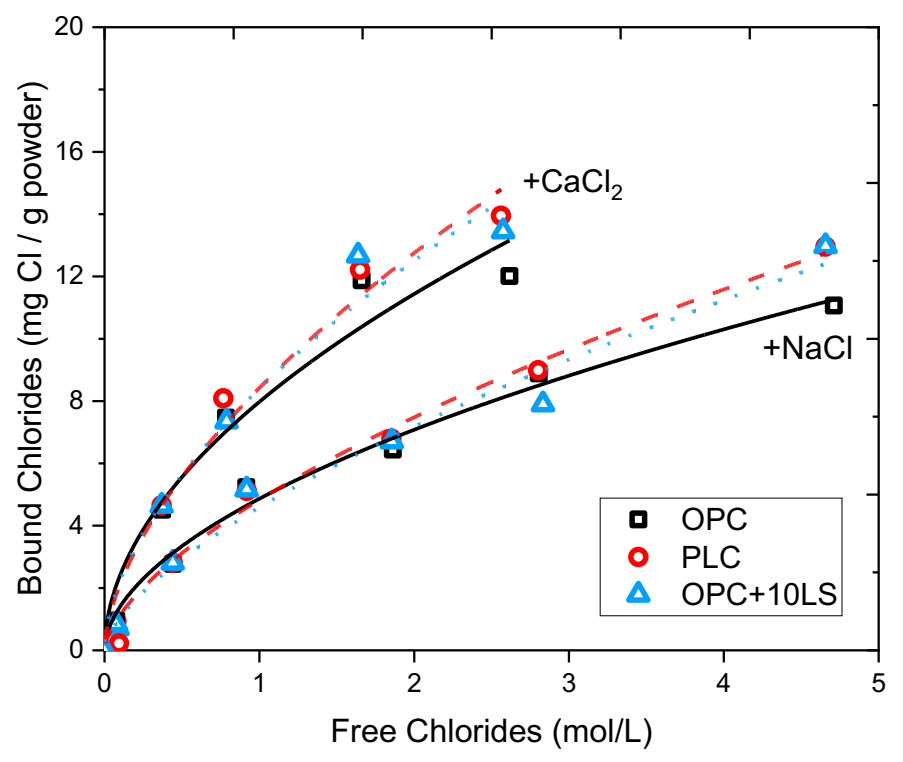

(a)

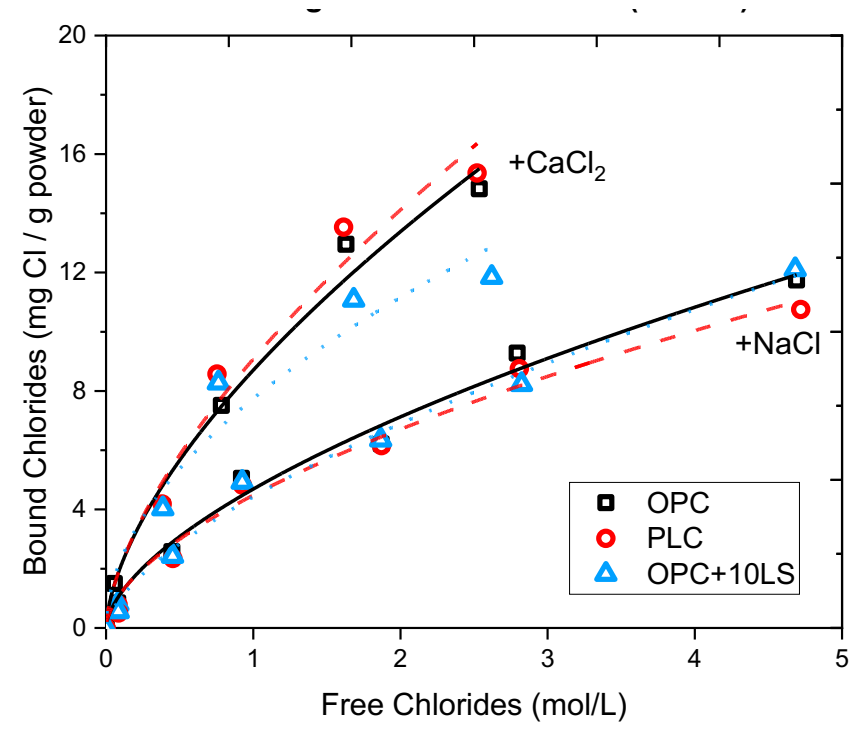

(b) 


\subsection{Significant Findings}

1588 The following conclusions can be drawn. No statistically significant difference was observed in the bound chloride contents of mixtures comparing OPC (i.e., ASTM C 150/AASHTO M 85), PLC (i.e., ASTM C 595/AASHTO M 240), and OPC+LS (provided that a specific size and quality of limestone is used). No statistically significant difference was observed in the bound chloride contents for mixtures comparing OPC, PLC, and OPC+LS with SCM (i.e., fly ash, silica fume, natural pozzolan) in more than one salt concentration. Mixtures containing PLC and OPC + LS mixtures made with ground granulated blast furnace slag outperformed the OPC+Slag systems.

1595 The variation in chloride binding observed between all the commercial OPC mixtures made using 1596 different commercially available Type II and V cements is $9 \%$ at a chloride concentration of $0.1 \mathrm{M}$ 1597 and $3 \%$ at a 5M. As such, it appears feasible that a single binding isotherm can be used to satisfy 1598 the behavior of all Type II and V mixtures. Fly ash, silica fume, and natural pozzolans do not affect 1599 the chloride binding capacity in cementitious systems containing up to $15 \%$ interground limestone. 1600 Slag increased the bound chloride content by $21 \%$ at a chloride concentration of $0.1 \mathrm{M}$ and $6 \%$ at $16015 \mathrm{M}$. The chloride binding was $30 \%$ higher for $\mathrm{CaCl}_{2}$ than $\mathrm{NaCl}$ at $0.1 \mathrm{M}$ chloride concentration 1602 and $40 \%$ higher from $0.5 \mathrm{M}$ to $3 \mathrm{M}$.

1603 It should also be noted that for the calculation of the allowable chloride content for new mixtures 1604 and comparison with set limits by ACI 318, limestone should be included in the total binder 1605 content. In other words, the entire OPC+SCM, PLC+SCM content or OPC+SCM+LS content be 1606 considered as binder. Note that as per ASTM C 595 and AASHTO M 240, the chloride content 1607 for a PLC is reported as part of the finished cement, which is in line with this suggestion. 
$1610 \quad \mathbf{1 0 . 1}$ Research Objectives

1611 This work compares the resistance of concrete made using OPC, PLC and OPC+LS to chloride

1612 ingress. This chapter builds on concepts from chapters 8 and 9 to predict performance.

\section{$1613 \quad 10.2$ Experimental Test Matrix}

1614 Concrete samples were prepared using the mixture proportions in Table 10.1. Cylindrical concrete 1615 samples were prepared (101.6 $\mathrm{mm}$ diameter and $203.2 \mathrm{~mm}$ height), demolded 24 hours after the 1616 casting, and were kept in a moist room for 90 days. The samples were stored at relative humidity 1617 of $50 \pm 5 \%$ and temperature of $23 \pm 5{ }^{\circ} \mathrm{C}$ for 90 days, then tested according to the experimental 1618 plan.

Table 10.1. Mixture proportions of concrete samples

\begin{tabular}{|l|c|c|c|c|c|c|c|}
\hline & & \multicolumn{7}{|c|}{ Mass $\mathbf{( k g / \mathbf { m } ^ { 3 } )}$} \\
\hline Material & S.G. & M0 & M1 & M2 & M3 & M4 & M5 \\
\hline Cement* & 3.15 & 504.3 & 378.2 & 378.2 & 252.1 & 252.1 & 378.2 \\
\hline Fly Ash & 2.34 & 0 & 126.1 & 100.9 & 0 & 126.1 & 0 \\
\hline Silica Fume & 2.20 & 0 & 0 & 25.2 & 0 & 0 & 0 \\
\hline Slag & 2.83 & 0 & 0 & 0 & 252.1 & 126.1 & 0 \\
\hline $\begin{array}{l}\text { Natural } \\
\text { Pozzolan }\end{array}$ & 2.36 & 0 & 0 & 0 & 0 & 0 & 126.1 \\
\hline \#23 Sand & 2.67 & 732.1 & 732.1 & 732.1 & 732.1 & 732.1 & 732.1 \\
\hline $\begin{array}{l}\text { \#8 Coarse } \\
\text { aggregate }\end{array}$ & 2.48 & 813.4 & 813.4 & 813.4 & 813.4 & 813.4 & 813.4 \\
\hline Water & 1.00 & 201.7 & 201.7 & 201.7 & 201.7 & 201.7 & 201.7 \\
\hline
\end{tabular}

$1620 *$ Cement: OPC, PLC, OPC + LS

\section{$1621 \quad \mathbf{1 0 . 3}$ Experimental Methods}

1622 Table 10.2 summarizes the experimental characterizations performed on the concrete samples. One 1623 concrete sample was used for porosity measurements and formation factor. A second concrete 1624 sample was measured for acid soluble chloride profile.

Table 10.2. Experimental characterizations performed on concrete samples

\begin{tabular}{|l|l|c|c|c|c|}
\hline \multirow{2}{*}{ Cement } & \multirow{2}{*}{ Mixture } & Porosity & \multirow{2}{*}{$\begin{array}{c}\text { Formation } \\
\text { factor }\end{array}$} & \multicolumn{2}{|c|}{$\begin{array}{c}\text { Acid-Soluble Chloride } \\
\text { Profile }\end{array}$} \\
\cline { 5 - 6 } & & & & $\begin{array}{c}\text { ASTM C1152- } \\
\text { 04 }\end{array}$ & $\begin{array}{c}\text { Calculated } \\
*\end{array}$ \\
\hline & M1 & $\checkmark$ & $\checkmark$ & -- & $\checkmark$ \\
\hline
\end{tabular}




\begin{tabular}{|c|c|c|c|c|c|}
\hline \multirow{3}{*}{$\begin{array}{l}\text { A (OPC, } \\
\text { PLC) }\end{array}$} & M2 & $\checkmark$ & $\checkmark$ & -- & $\checkmark$ \\
\hline & M3 & $\checkmark$ & $\checkmark$ & -- & $\checkmark$ \\
\hline & M4 & $\checkmark$ & $\checkmark$ & -- & $\checkmark$ \\
\hline \multirow{4}{*}{$\begin{array}{l}\text { C (OPC, } \\
\text { PLC) }\end{array}$} & M1 & $\checkmark$ & $\checkmark$ & -- & $\checkmark$ \\
\hline & M2 & $\checkmark$ & $\checkmark$ & -- & $\checkmark$ \\
\hline & M3 & $\checkmark$ & $\checkmark$ & -- & $\checkmark$ \\
\hline & M4 & $\checkmark$ & $\checkmark$ & -- & $\checkmark$ \\
\hline \multirow{6}{*}{$\begin{array}{l}\text { E (OPC, } \\
\text { PLC, } \\
\text { OPC+10\% } \\
\text { limestone) }\end{array}$} & M0 & OPC, PLC & OPC, PLC & OPC, PLC & OPC, PLC \\
\hline & M1 & $\checkmark$ & $\checkmark$ & OPC, PLC & $\checkmark$ \\
\hline & M2 & $\checkmark$ & $\checkmark$ & OPC, PLC & $\checkmark$ \\
\hline & M3 & $\checkmark$ & $\checkmark$ & OPC, PLC & $\checkmark$ \\
\hline & M4 & $\checkmark$ & $\checkmark$ & -- & $\checkmark$ \\
\hline & M5 & $\checkmark$ & $\checkmark$ & -- & -- \\
\hline
\end{tabular}

$\checkmark \quad$ means all cement types were tested,

-- means experiments were not performed,

Calculated using the formation factor values and porosity

\subsubsection{Porosity, Resistivity, and Apparent Formation Factor}

Porosity was measured using AASHTO TP135-20 [90] (section 8.4.1); however, these concrete samples were saturated using a saturated lime solution $\left(2 \mathrm{~g} / \mathrm{L} \mathrm{Ca}(\mathrm{OH})_{2}\right)$. The uniaxial resistance was measured using AASHTO TP 119 [91] after 7 and 14 days of immersion in the simulated pore solution (Option A). The resistivity of the concrete sample $\left(\rho_{s}\right)$ was calculated as described in chapter 8, section 8.4.2. The resistivity of the simulated pore solution $\left(\rho_{p s}\right)$ was measured using the VWR B40PCID meter after 7- and 14-days duration of samples immersion (Table 10.3). The formation factor after 7 days and 14 days of immersion in solution was then calculated according 1638 to equation (8-3).

Table 10.3. Resistivity of pore solution after 7 and 14 days of immersion

\begin{tabular}{|c|c|c|}
\hline Bucket number & $\begin{array}{c}\text { Resistivity of pore solution } \\
\text { after } 7 \text { days of samples' } \\
\text { immersion }(\Omega \cdot \mathbf{m})\end{array}$ & $\begin{array}{c}\text { Resistivity of pore solution } \\
\text { after 14 days of samples' } \\
\text { immersion }(\Omega \cdot \mathbf{m})\end{array}$ \\
\hline Average & 0.115 & 0.116 \\
\hline Standard Deviation & 0.001 & 0.001 \\
\hline
\end{tabular}




\subsubsection{Experimental determination of acid soluble chloride profile and chloride apparent diffusion coefficient}

The apparent diffusion coefficient $\left(D_{a}\right)$ (using acid soluble chlorides) was determined experimentally using ASTM C1556-11a [127]. At an age of 6 months (section 10.2), the concrete samples were cut, sealed, and immersed in an aqueous sodium chloride solution $(\mathrm{NaCl})$ with a concentration of $165 \pm 1 \mathrm{~g} \mathrm{NaCl}$ per 1 liter of solution for a duration of at least 35 days. At the end of the immersion period in $\mathrm{NaCl}$ solution, powder was collected from 8 different layers by grinding off materials in layers parallel to the surface that was in contact with $\mathrm{NaCl}$ solution. In this study, the powder was collected at $0.5,2,4,6,8.5,11.5,14.5$, and $18 \mathrm{~mm}$ from the exposed surface. Following ASTM C1152-04 [128], the ground powders were exposed to nitric acid before titration in order to determine the acid-soluble chloride contents at different depths in the concrete sample.

The total apparent surface chloride content $\left(\mathrm{C}_{\mathrm{s}-\mathrm{a}}\right)$ and apparent diffusion coefficient $\left(\mathrm{D}_{\mathrm{a}}\right)$ are obtained by fitting the data to Eq.10-1 using the approach described in ASTM C1556 as illustrated in Figure 10-1.

$$
\frac{C_{t}(x, t)-C_{0}}{C_{s-a}-C_{0}}=\operatorname{erfc}\left[\frac{x}{2 \sqrt{D_{a} \cdot t}}\right]
$$

where, $C_{0}$ is the initial chloride concentration of the concrete before exposure to the $\mathrm{NaCl}$ solution, $C_{t}(x, t)$ is the chloride concentration at a given depth in the concrete specimen and a given exposure duration to $\mathrm{NaCl}$ solution, $x(\mathrm{~m})$ is the depth of the powder in the concrete sample, $t$ is the time (s) at which the samples were extracted for chloride profiles testing [127].

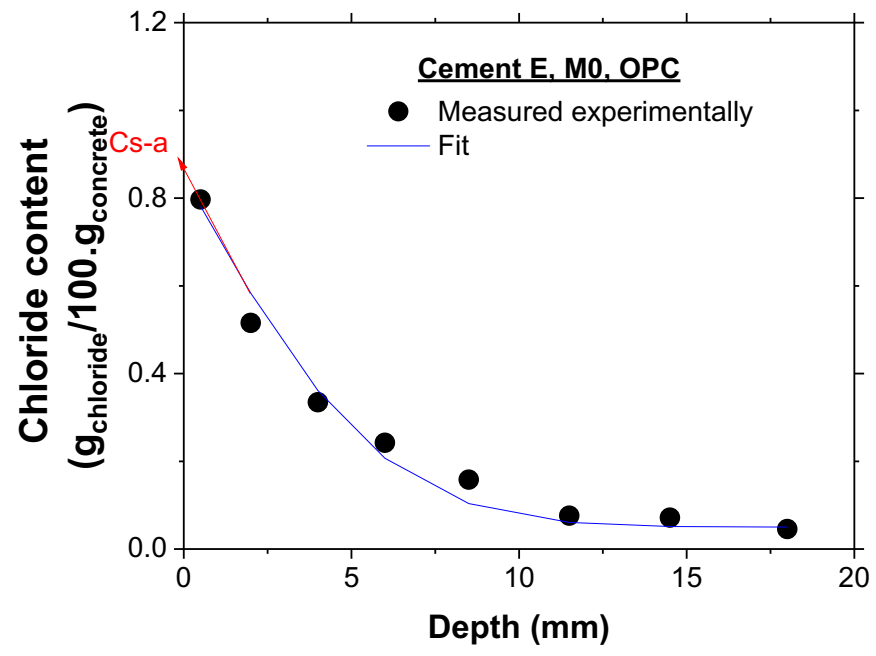

1662 


\subsubsection{Theoretical determination of acid soluble chloride profile and $D_{a}$}

1664 As an alternative to the fitting approach described in 10.3.2, Azad et al. [99] defined equation 1665 (10-2) to relate the formation factor, porosity and chloride binding parameters to the $\mathrm{D}_{\mathrm{a}}$ of concrete 1666 and $\mathrm{C}_{\text {s-a. }}$.

$$
D_{a}=\frac{D_{0}}{\phi F\left(1+\frac{1.25}{\phi} \alpha \beta C_{\text {exp }}^{\beta-1}\right)}
$$

1667 where, $D_{0}$ is the self-diffusion coefficient of chloride ion in water $\left(1.38 \times 10^{-9} \mathrm{~m}^{2} / \mathrm{s}\right), \phi$ is the 1668 porosity of the concrete sample, $F$ is the measured formation factor of concrete, $\alpha$ and $\beta$ are the 1669 Freundlich binding parameters for concrete samples, $C_{\text {exp }}$ is the free chloride concentration on the 1670 surface that is in equilibrium with the chloride concentration of the exposure solution $(100 \mathrm{~kg}$ 1671 chloride per $1 \mathrm{~m}^{3} \mathrm{NaCl}$ solution).

1672 The Freundlich binding parameters $(\alpha, \beta)$ were experimentally determined on all the mortar 1673 samples as described in chapter 9, section9.5. Equation (10-3) is used to scale these to concrete 1674 based on the assumption that the paste is the only portion of the system that binds chloride. The 1675 free chloride remains the same for both mortar and concrete.

$$
C_{b-c}=C_{b} \cdot\left(W_{f-m}\right)^{-1} \cdot W_{f-c}
$$

1676 where, $\mathrm{C}_{\mathrm{b}-\mathrm{c}}$ is the bound chlorides in concrete $\left(\mathrm{mg} / \mathrm{g}_{\text {concrete }}\right), \mathrm{C}_{\mathrm{b}}$ is the bound chlorides in mortar $1677\left(\mathrm{mg} / \mathrm{g}_{\text {mortar }}\right), \mathrm{W}_{\mathrm{f}-\mathrm{m}}$ is the mass fraction of cement paste in mortar ( $\left.\mathrm{g}_{\text {paste }} / \mathrm{g}_{\text {mortar }}\right), \mathrm{W}_{\mathrm{f}-\mathrm{c}}$ is the paste 1678 content by mass in concrete ( $\left.\mathrm{g}_{\text {paste }} / \mathrm{g}_{\text {concrete }}\right)$. The results from this scaling are shown in Figure 10-2.

1679 The Freundlich model was then used to fit the data points calculated in Figure 10-2 (b) to determine 1680 the Freundlich binding parameter for the concrete samples. 


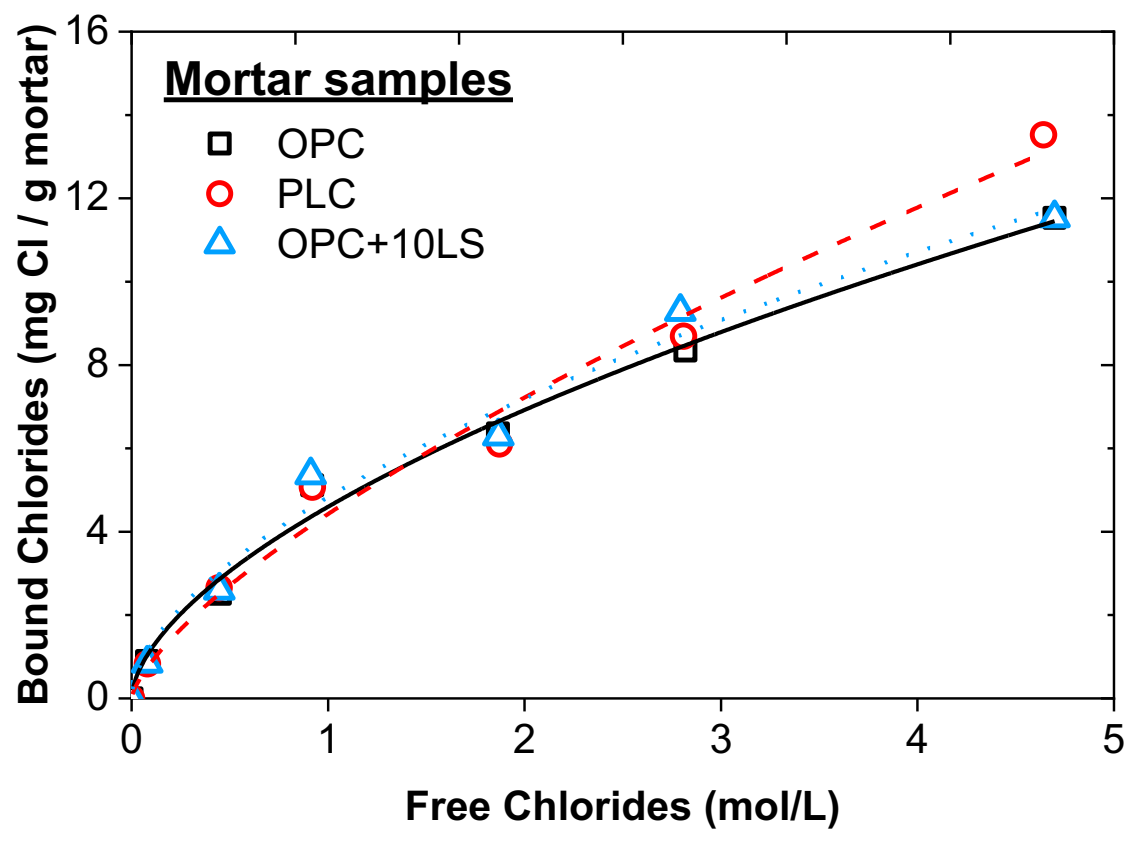

(a)

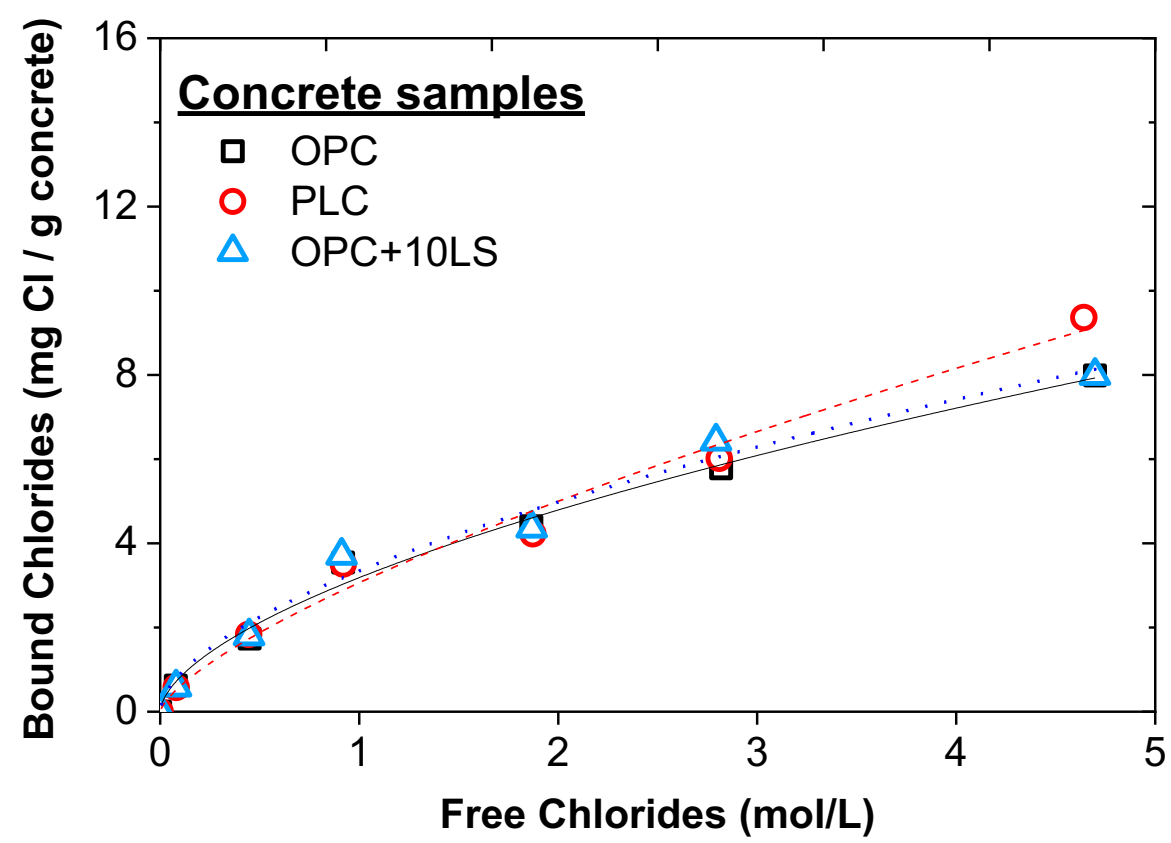

(b)

1683 In order to determine the acid soluble chloride profile theoretically using equation (10-1), $C_{\text {s-a }}$ 1684 needs to be calculated as shown in equation (10-4). 


$$
C_{s-a}=\phi C_{e x p}+C_{s-b}
$$

1685 where, $\phi$ is the porosity, $\mathrm{C}_{\mathrm{exp}}$ is the free chloride content on the surface, $\mathrm{C}_{\mathrm{s}-\mathrm{b}}$ is the bound chloride 1686 content on the surface. In $165 \mathrm{~g} \mathrm{NaCl} /$ liter of solution there is 2.8 moles of free chloride per liter 1687 of solution. Consequently, $\mathrm{C}_{\mathrm{s}-\mathrm{b}}$ is the bound chloride that corresponds to $2.8 \mathrm{~mol} / \mathrm{liter}$ of free 1688 chloride in the binding isotherm of concrete.

\subsection{Experimental results}

\subsubsection{Porosity}

1691 Figure 10-3 shows the porosity of the OPC vs the PLC or OPC + LS concrete samples. In general, 1692 the OPC and PLC and OPC + LS system were similar with the exception of a mixture made using 1693 OLC + LS which showed a higher porosity than the corresponding OPC sample. This likely reflects 1694 insufficient consolidation. For this reason, results from this sample will not be presented in the 1695 next sections of this report. Further, this suggests that when LS is added separately special attention is needed to ensure that the sample can be properly consolidated.

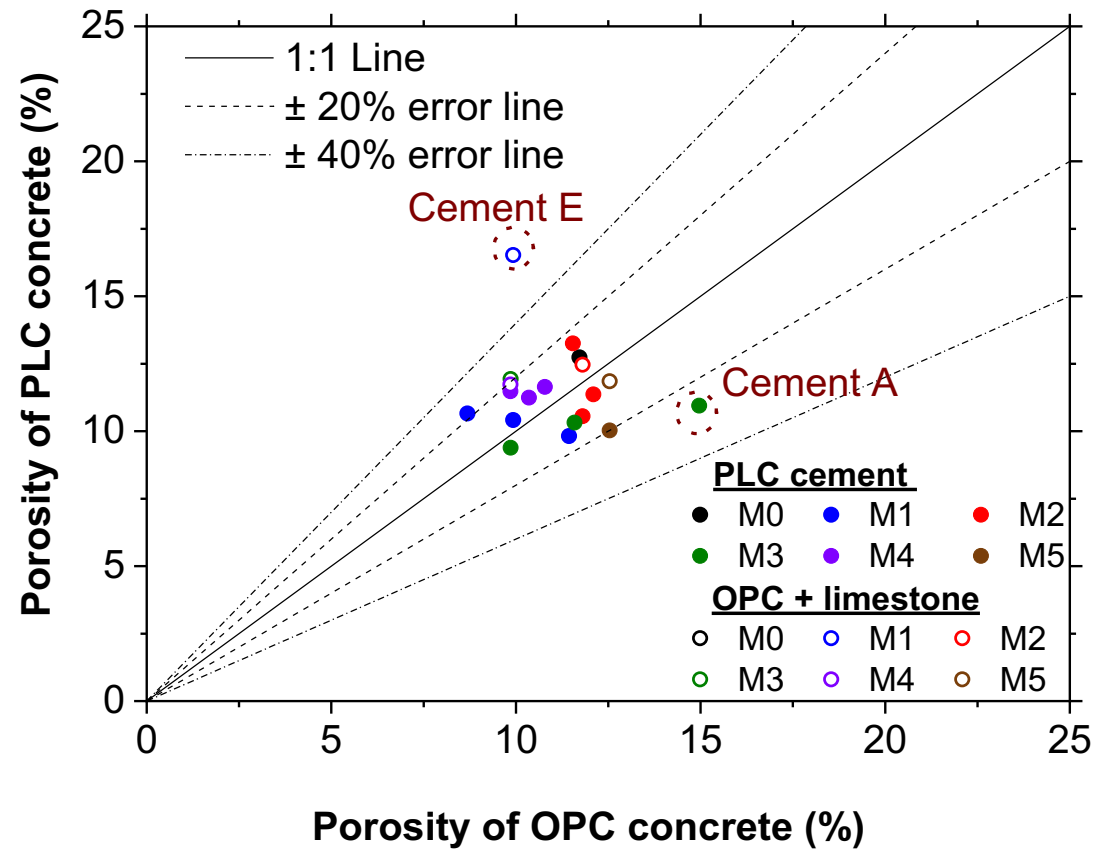

1699 The formation factor calculated after 7 days of samples' immersion were similar to those calculated 1700 after 14 days of samples' immersion in the simulated pore solution. Therefore, in this study, the 1701 results obtained after 14 days of samples' immersion are presented and used for the calculations 1702 of the chloride profiles. 
1703 Figure 10-4 is a plot of formation factor of all concrete made using OPC, PLC and OPC + LS. The 1704 points lying above this line signify that the PLC has a higher formation factor than OPC, and the 1705 points below correspond to lower formation factor. The plots also show $\pm 20 \%$ and $\pm 40 \%$ variation 1706 lines.

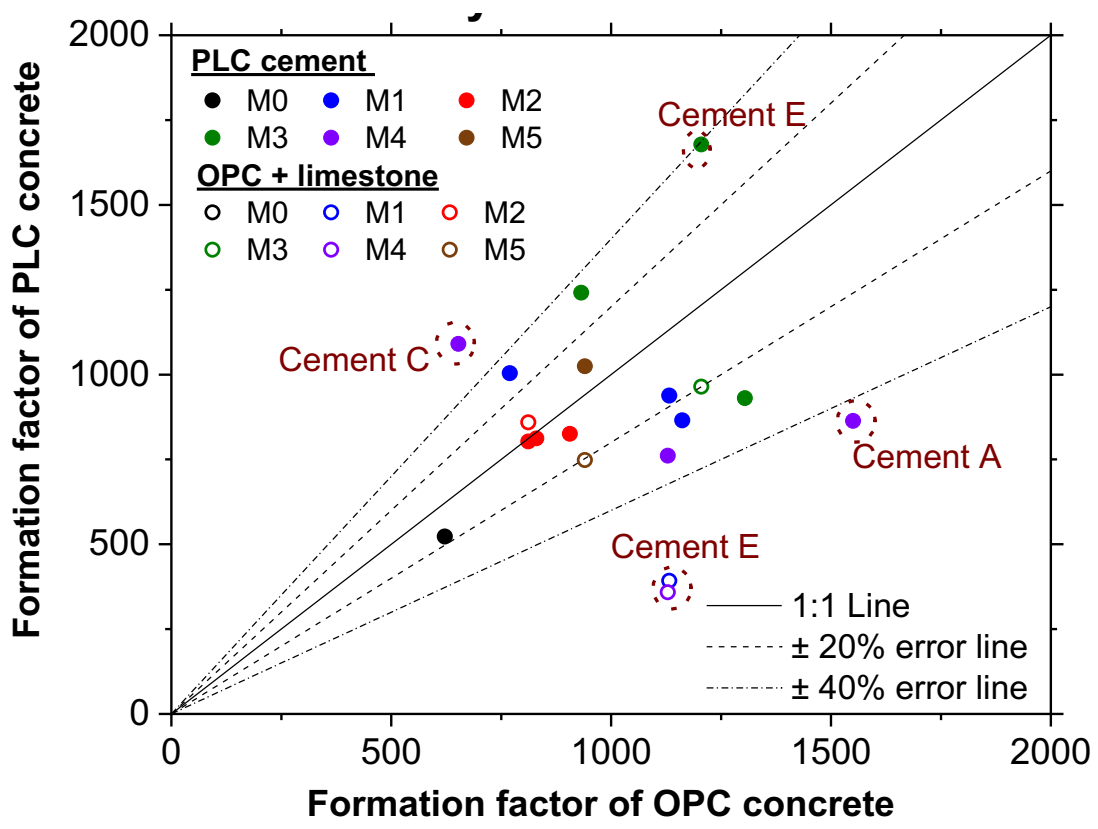

\section{Figure 10-4. Formation factor of OPC, PLC and OPC $+L S$ concrete samples. Circles show} data outside the $40 \%$ error lines.

\subsubsection{Experimental determination of acid soluble chloride profile and chloride apparent diffusion coefficient (ASTM C 1556)}

1712 The experimentally determined chloride profiles are shown in Figure 10-5. It can be noticed that 1713 for M1-M3 the profiles are nearly identical when comparing OPC and PLC. The only mixture that

1714 appears to indicate an increase in ingress is M0. Since there appeared to be an issue with concrete 1715 sample preparation, Figure 10-6 was developed to show the theoretical acid-soluble chloride 1716 profile obtained from the porosity, formation factor and binding parameters of both M0-OPC and 1717 M0-PLC concrete samples. Based on Figure 10-5 and Figure 10-6, it can be noted that both OPC 1718 and PLC samples have nearly identical chloride profiles. 

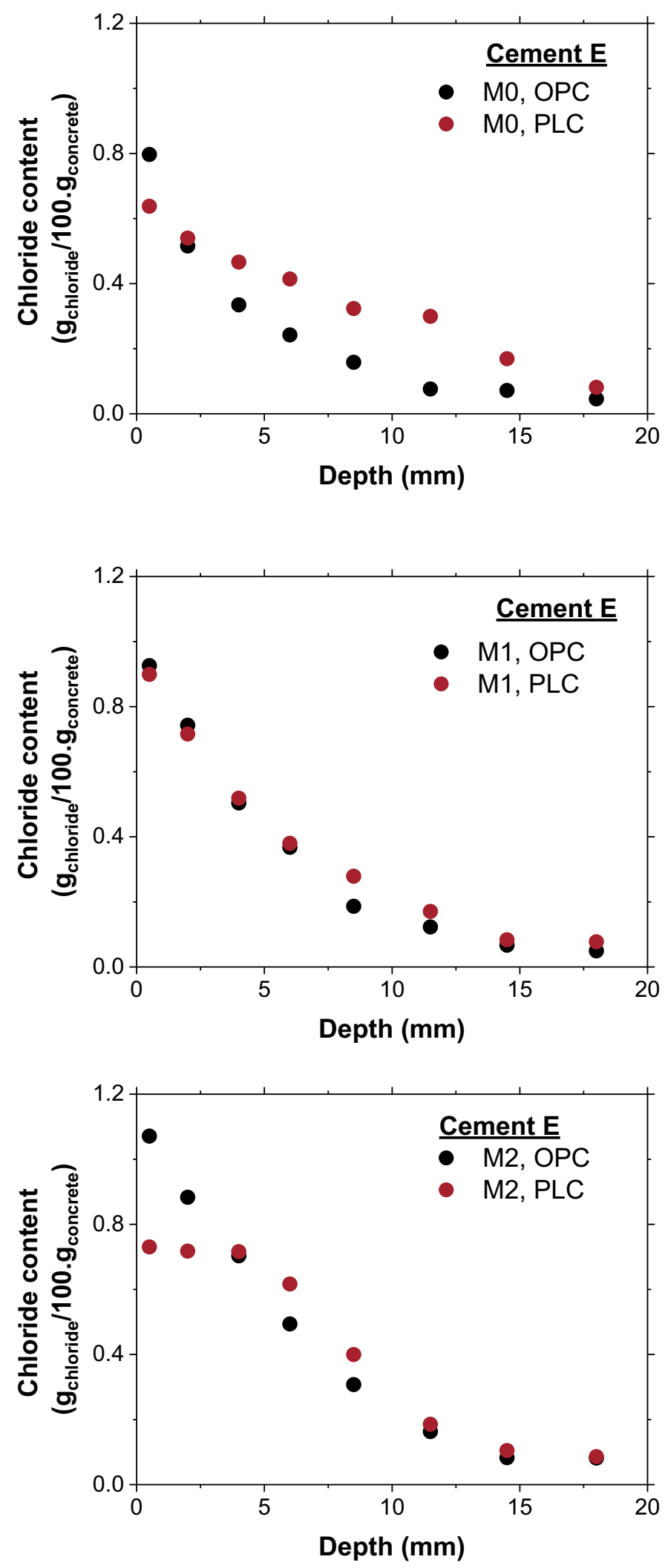


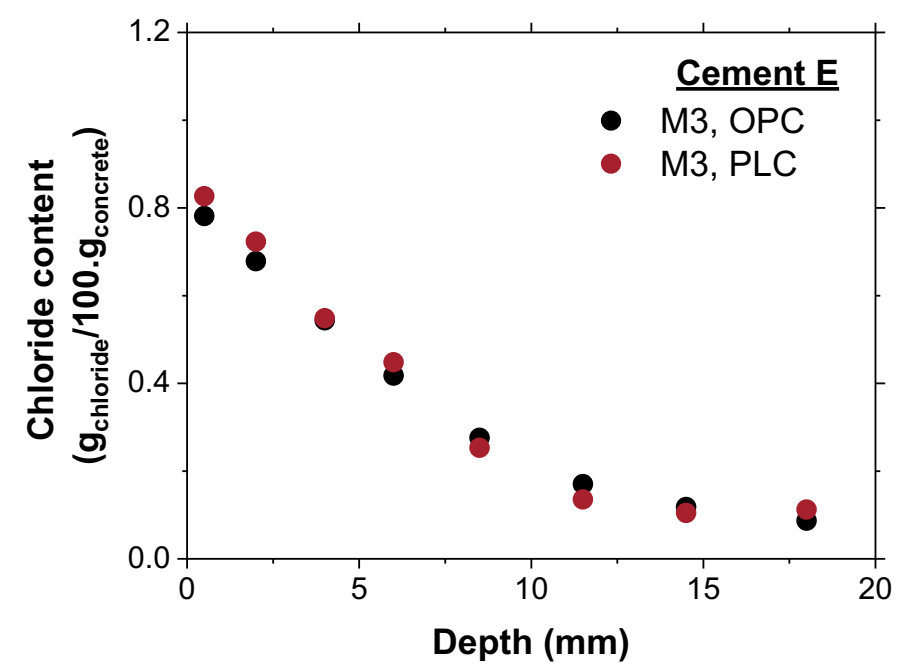

Figure 10-5. Acid-soluble chloride profiles obtained from experimental data for both OPC and PLC concrete samples

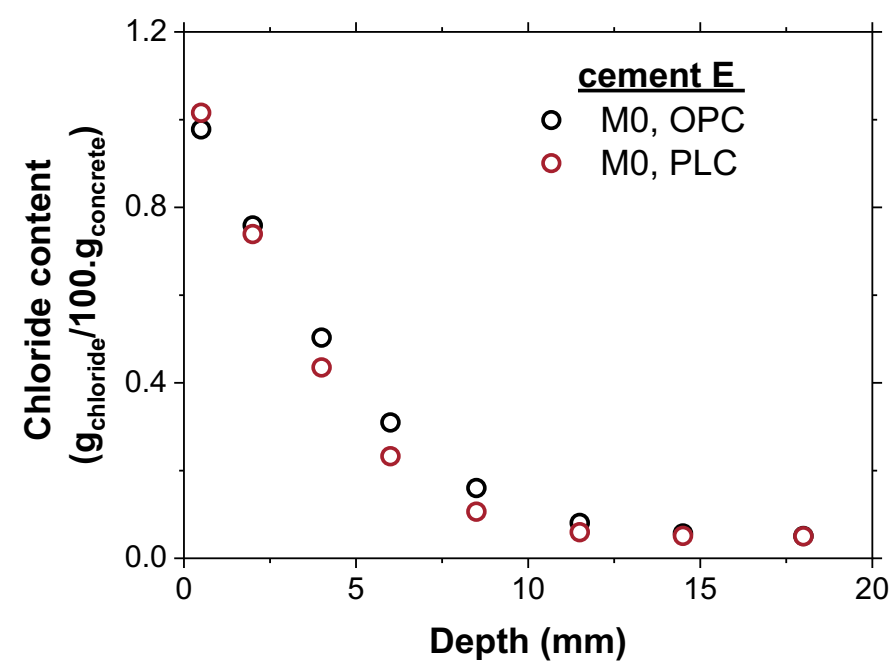
M0, PLC concrete samples

1725 The experimentally determined chloride apparent diffusion coefficient values of PLC concrete with respect to OPC concrete are illustrated in Figure 10-7. Figure 10-8 shows the experimental values of the total apparent surface chloride content in PLC samples with respect to OPC. It can be seen that for these mixtures, PLC and OPC are comparable with the exception of mixture 0 which, as we have understood from the SAC, is not used in environments where corrosion is a primary concern. 


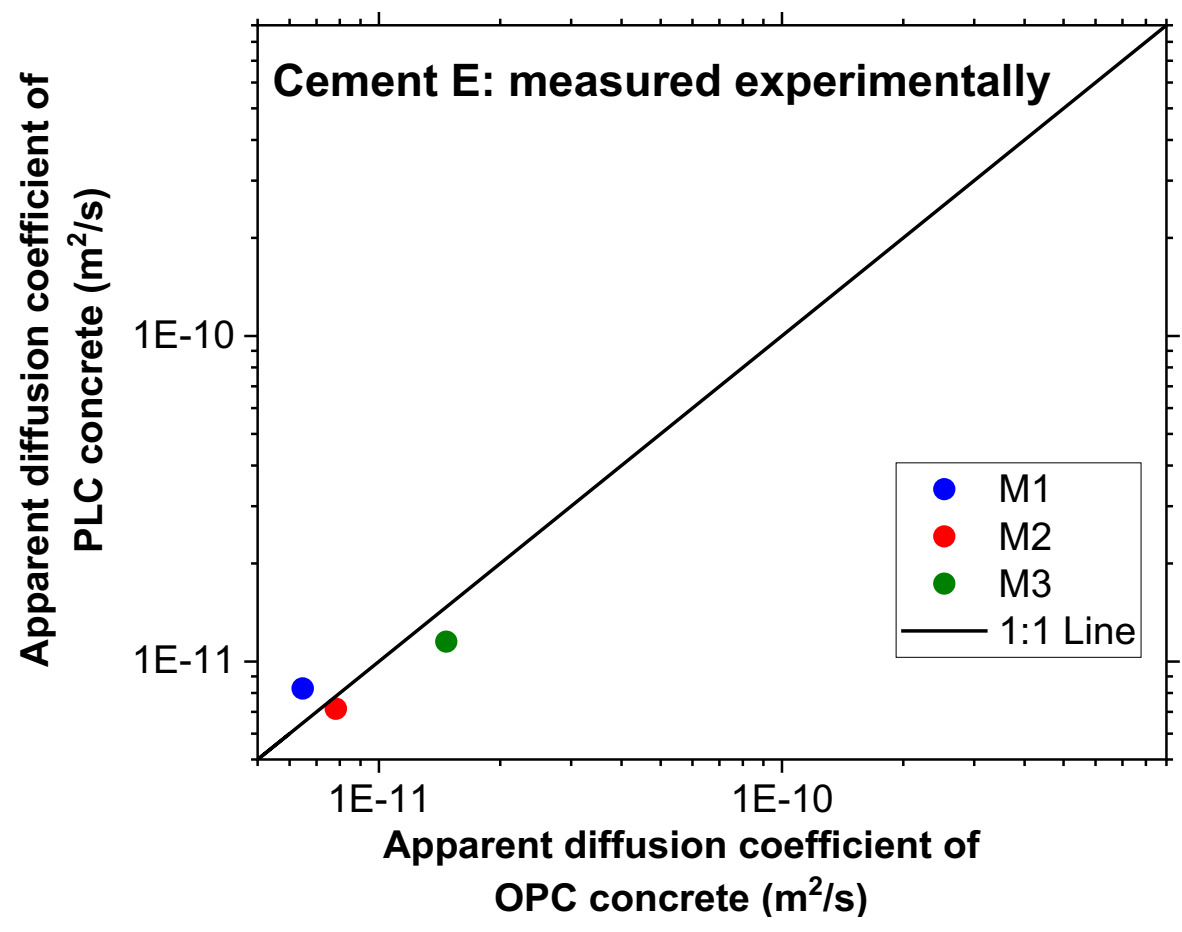

1733 Figure 10-7. Experimental values of $D_{\mathbf{a}}$ for OPC and PLC concrete samples

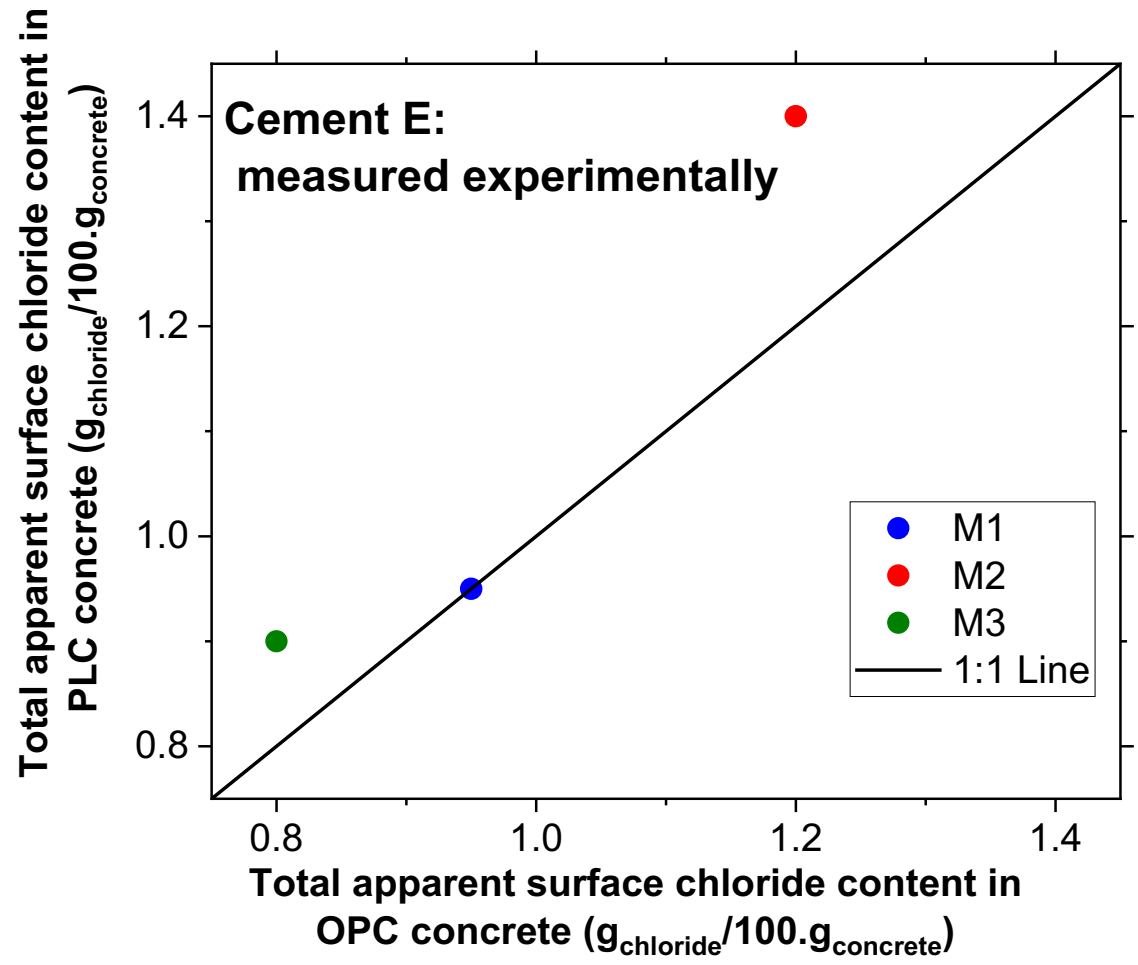
OPC concrete $\left(g_{\text {chloride }} / 100 . g_{\text {concrete }}\right)$

Figure 10-8. Experimental values of $\mathrm{C}_{\mathrm{s}-\mathrm{a}}$ for $\mathrm{OPC}$ and PLC concrete samples 
Table 10.4. Experimental values of $C_{s-a}$ and $D_{a}$ for OPC and PLC concrete samples

\begin{tabular}{|c|c|c|c|c|}
\hline Cement E & \multicolumn{2}{|c|}{ Da $\left(\mathbf{m}^{\mathbf{2}} / \mathbf{s}\right)$} & \multicolumn{2}{c|}{$\mathbf{C}_{\text {s-a }}\left(\mathbf{g}_{\text {chloride }} / \mathbf{1 0 0} g_{\text {concrete }}\right)$} \\
\hline & OPC & PLC & OPC & PLC \\
\hline M1 & $6.47 \mathrm{E}-12$ & $8.26 \mathrm{E}-12$ & 0.95 & 0.95 \\
\hline M2 & $7.82 \mathrm{E}-12$ & $7.16 \mathrm{E}-12$ & 1.2 & 1.4 \\
\hline M3 & $1.47 \mathrm{E}-11$ & $1.15 \mathrm{E}-11$ & 0.8 & 0.9 \\
\hline
\end{tabular}

10.4.3 Theoretical determination of acid soluble chloride profile and chloride apparent diffusion coefficient parameters

1741 Table 10.5 summarizes the Freundlich binding parameters of concrete samples (obtained from 1742 equation 10-3 using the data in chapter 9).

Table 10.5. Freundlich binding parameters of concrete samples

\begin{tabular}{|c|c|c|c|c|c|c|c|c|c|c|}
\hline \multirow{2}{*}{$\begin{array}{l}\text { Cement } \\
\text { Sample }\end{array}$} & & \multicolumn{3}{|l|}{$\mathbf{A}$} & \multicolumn{3}{|l|}{ C } & \multicolumn{3}{|l|}{$\mathbf{E}$} \\
\hline & & OPC & PLC & $\begin{array}{l}\text { OPC } \\
+ \text { LS }\end{array}$ & OPC & PLC & $\begin{array}{l}\text { OPC } \\
+\mathrm{LS}\end{array}$ & $\overline{\text { OPC }}$ & PLC & $\begin{array}{l}\text { OPC } \\
+ \text { LS }\end{array}$ \\
\hline \multirow[t]{3}{*}{ M0 } & $\alpha_{c}$ & 3.66 & 3.54 & 3.46 & 3.23 & 3.3 & 3.2 & 3.18 & 3.06 & 3.34 \\
\hline & $\boldsymbol{\beta}_{\mathbf{c}}$ & 0.6 & 0.65 & 0.64 & 0.61 & 0.6 & 0.59 & 0.59 & 0.71 & 0.57 \\
\hline & $\mathbf{R}^{2}$ & 0.994 & 0.986 & 0.987 & 0.988 & 0.989 & 0.986 & 0.989 & 0.982 & 0.979 \\
\hline \multirow[t]{3}{*}{ M1 } & $\alpha_{c}$ & 3.42 & 3.49 & 3.39 & 3.26 & 3.19 & 3.13 & 3.14 & 3.29 & 2.94 \\
\hline & $\boldsymbol{\beta}_{\mathbf{c}}$ & 0.58 & 0.67 & 0.69 & 0.53 & 0.61 & 0.58 & 0.66 & 0.56 & 0.59 \\
\hline & $\mathbf{R}^{2}$ & 0.993 & 0.993 & 0.991 & 0.968 & 0.987 & 0.991 & 0.985 & 0.978 & 0.991 \\
\hline \multirow[t]{3}{*}{ M2 } & $\boldsymbol{\alpha}_{\mathfrak{c}}$ & 3.34 & 3.51 & 3.37 & 3.15 & 3.01 & 3.33 & 3.01 & 3.16 & 3.42 \\
\hline & $\boldsymbol{\beta}_{\mathbf{c}}$ & 0.67 & 0.63 & 0.69 & 0.56 & 0.65 & 0.58 & 0.58 & 0.55 & 0.66 \\
\hline & $\mathbf{R}^{2}$ & 0.989 & 0.991 & 0.991 & 0.97 & 0.988 & 0.971 & 0.98 & 0.96 & 0.987 \\
\hline \multirow[t]{3}{*}{ M3 } & $\boldsymbol{\alpha}_{\mathfrak{c}}$ & 4.08 & 4.23 & 3.83 & 4.23 & 3.77 & 3.71 & 4.28 & 3.73 & 3.97 \\
\hline & $\beta_{c}$ & 0.53 & 0.53 & 0.64 & 0.5 & 0.59 & 0.57 & 0.44 & 0.63 & 0.6 \\
\hline & $\mathbf{R}^{2}$ & 0.988 & 0.991 & 0.986 & 0.985 & 0.988 & 0.979 & 0.968 & 0.982 & 0.989 \\
\hline \multirow[t]{3}{*}{ M4 } & $\alpha_{c}$ & 3.46 & 3.49 & 3.66 & 3.85 & 3.77 & 4.01 & 3.87 & 3.83 & 3.64 \\
\hline & $\boldsymbol{\beta}_{\mathbf{c}}$ & 0.63 & 0.63 & 0.58 & 0.54 & 0.63 & 0.58 & 0.59 & 0.57 & 0.63 \\
\hline & $\mathbf{R}^{2}$ & 0.99 & 0.991 & 0.992 & 0.994 & 0.99 & 0.97 & 0.991 & 0.966 & 0.993 \\
\hline
\end{tabular}

1745 Figure 10-9 (a) illustrates the apparent diffusion coefficient based on the formation factor and 1746 porosity calculated using equation (10-2). The total apparent surface chloride content $\left(\mathrm{C}_{\mathrm{s}-\mathrm{a}}\right)$ 1747 calculated using equation (10-4) is shown in Figure 10-9 (b). It can be noted that both $\mathrm{C}_{\mathrm{s}-\mathrm{a}}$ and $\mathrm{D}_{\mathrm{a}}$ 1748 values determined on OPC samples are comparable to those determined on the corresponding PLC 1749 samples. 


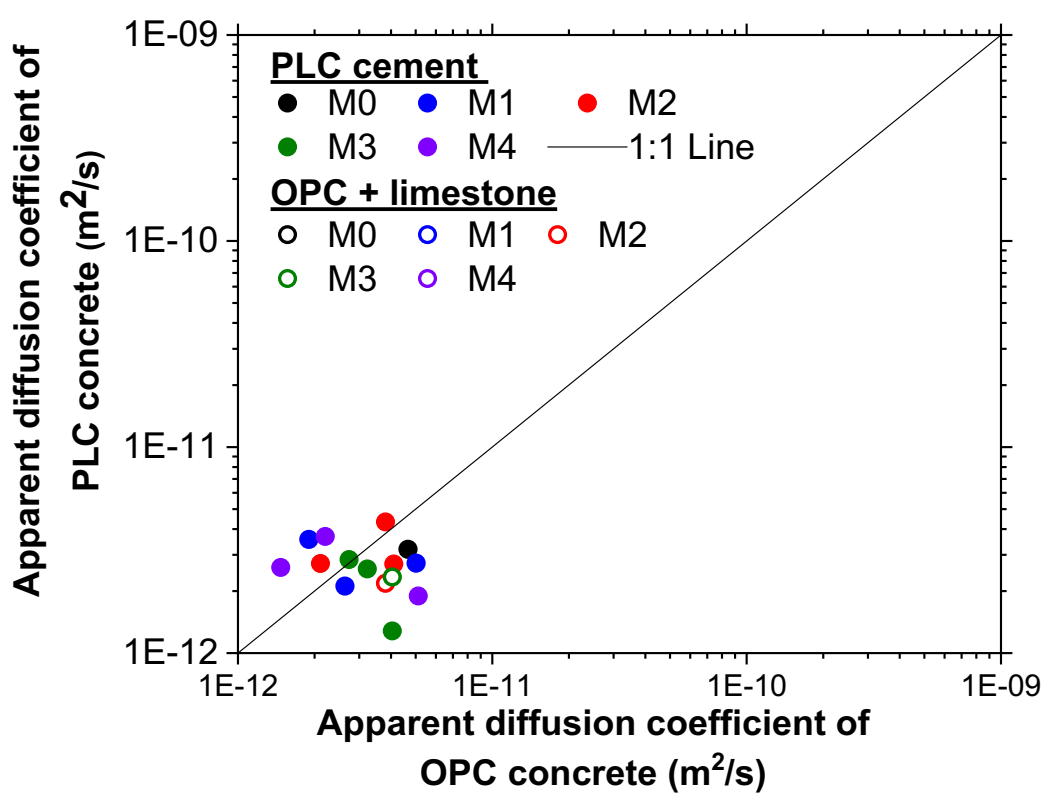

(a)

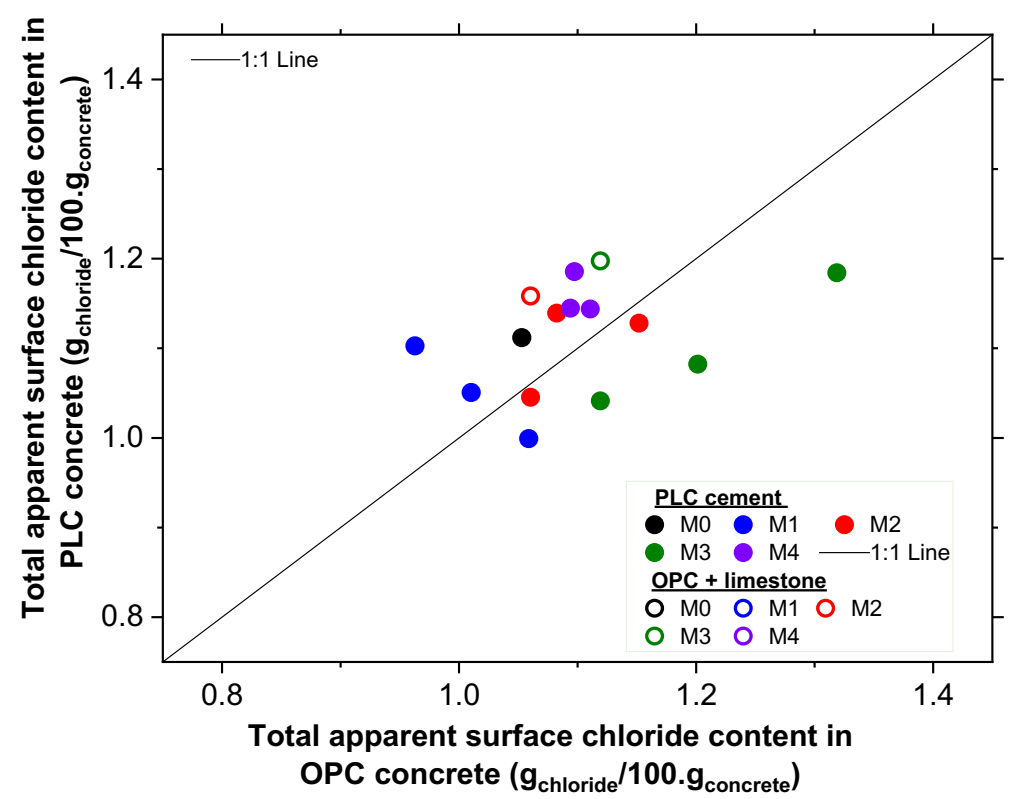

(b) measurements for OPC and PLC concrete samples, (b) Calculated values of $\mathrm{C}_{\mathrm{s}-\mathrm{a}}$ based on porosity and formation factor measurements for OPC and PLC concrete samples

\section{$1753 \quad 10.5$ Discussion of Results}

1754 The porosity, chloride binding, and formation factor of PLC concrete samples are similar to their 1755 OPC counterparts. Samples containing SCM in the mixture design show a higher formation factor. 1756 This is due to the refinement of the microstructure induced by SCMs addition. 
1757 The acid soluble chloride profiles were calculated for all the mixtures listed in Table 10.1 using 1758 equation (10-1) with the parameters from section 10.4.3, the porosities and formation factor for an 1759 exposure duration to chloride solution of 20 years $\left(\mathrm{Co}=0.05\right.$ ( $\left.\left.\mathrm{g}_{\text {chloride }} / 100 \mathrm{~g}_{\text {concrete }}\right)\right)$. These acid 1760 soluble profiles for all the mixtures are shown in Appendix G. No significant difference can be 1761 noted between the acid soluble profiles of both PLC and OPC samples. The chloride content at 20 1762 years and $50 \mathrm{~mm}$ depth (a typical rebar level) are shown in Figure 10-10. The chloride content 1763 for all these mixtures at $50 \mathrm{~mm}$ depth after 20 years of exposure to salt solution range between 2.7 $1764 \mathrm{~g} / 100 \mathrm{~g}$ cement and $5.4 \mathrm{~g} / 100 \mathrm{~g}$ cement. Based on these results, it can be seen that the chloride 1765 content in PLC samples is either comparable to or slightly lower than that of the OPC samples.

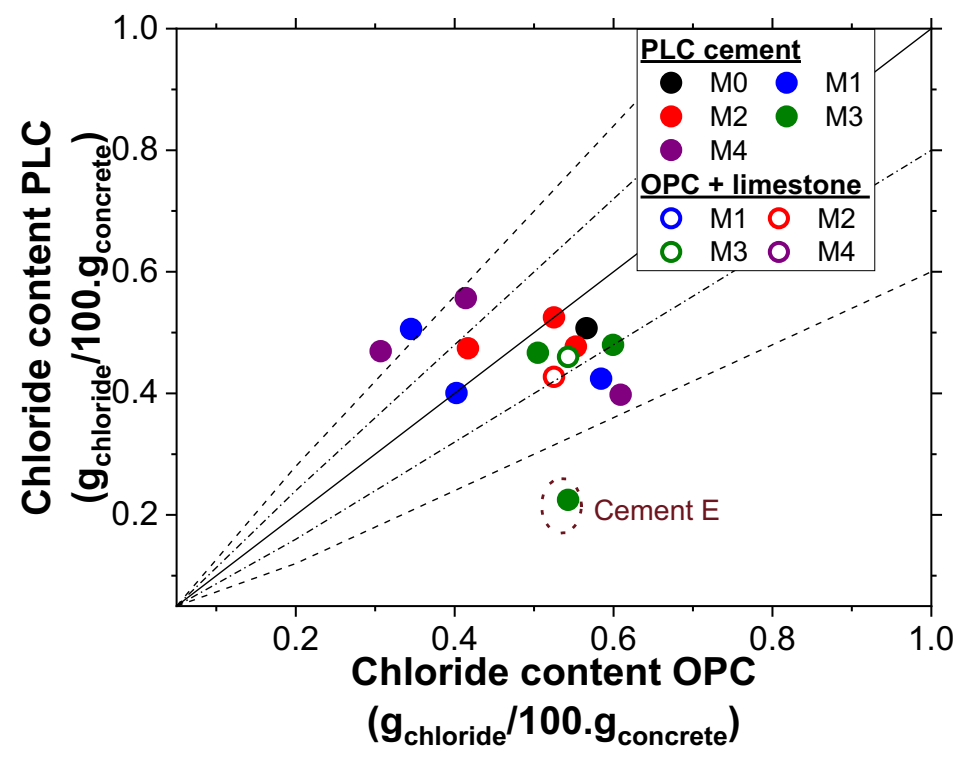

Figure 10-10. Comparison between the chloride content of OPC and PLC concrete at 50 mm depth determined at an exposure period 20 years

1770 Based on the collected measurements, it can be concluded that the porosity, formation factor, and 1771 chloride apparent diffusion coefficient of PLC concrete are comparable to those obtained on OPC 1772 concrete. 


\section{Corrosion of Reinforcing Steel}

1774 The critical chloride threshold $\left(\mathrm{Cl}_{\text {crit }}\right)$ is defined as the concentration of chlorides at the steel1775 concrete interface that is required to initiate corrosion. $\mathrm{Cl}_{\text {crit }}$ draws its prominence from the fact 1776 that it is a critical parameter to estimate the service life of a reinforced concrete structure. Steel1777 cementitious system with a high $\mathrm{Cl}_{\text {crit }}$ value is expected to have a longer service life when 1778 compared to the systems with a low $\mathrm{Cl}_{\text {crit }}$ values. As prominent as this parameter may be, it is 1779 surprising that there is very little agreement on $\mathrm{Cl}_{\text {crit }}$ values. Even though several test setups have 1780 been used in the past to assess $\mathrm{Cl}_{\text {crit }}$ [129], these tests each have limitations and drawbacks and all 1781 result in different $\mathrm{Cl}_{\text {crit }}$ values; this has led to little consensus on realistic $\mathrm{Cl}_{\text {crit }}$ values. A new test 1782 method, the $\mathrm{OC}_{\text {crit }}$ method, has been developed to quantify the $\mathrm{Cl}_{\text {crit }}$ of steel-cementitious systems $1783[130,131]$. This method was developed at Oregon State University with an objective to establish 1784 a standardized, reliable, and timely test to quantify the amount of chlorides necessary to initiate 1785 corrosion. Past studies have shown that the $\mathrm{OC}_{\text {crit }}$ could yield results faster and more reliably than 1786 other accelerated corrosion tests $[130,131]$.

\section{$1787 \quad 11.1$ Research Objectives}

1788 In this chapter, the corrosion performance of reinforcing steel embedded in OPC, PLC, and $1789 \mathrm{OPC}+\mathrm{LS}$ is evaluated using the $\mathrm{OC}_{c r i t}$ method and the modified ASTM G109 method. The primary 1790 objective is to determine whether the replacement of OPC with PLC or OPC + LS in combination 1791 with SCMs impacts the corrosion performance of reinforcing steel embedded in these systems. A 1792 standardized, fast, and reliable test method can help the SHAs assess these products, in a timely 1793 manner, and will provide data to ensure the use of these materials results in acceptable long-term 1794 performance [129]. In this study, the $\mathrm{OC}_{\text {crit }}$ method is used to assess the $\mathrm{Cl}_{\text {crit }}$ of OPC and PLC 1795 cementitious systems with and without SCM replacements. The experimental program in this study 1796 assesses the corrosion performance of PLC as opposed to OPC cementitious systems. The 1797 specimens were also evaluated using the modified ASTM G109 test method to compare the $\mathrm{Cl}_{\text {crit }}$ 1798 values and time to activation with that of the $\mathrm{OC}_{\text {crit }}$ specimens. It should be noted that both these 1799 test methods have different criteria for corrosion activation and hence may not indicate the same 1800 corrosion activity.

\subsection{Background/Literature Review}

1802 Corrosion is an electrochemical process that involves an anode, a cathode, an electrolyte, and an 1803 electrical connection between the anode and cathode for the transfer of electrons. Reactions take 1804 place on the surface of both the anodic and cathodic sites whereas mass loss takes place exclusively 1805 at the anodic site. The pore water in mortar or concrete acts as the electrolyte making the 1806 cementitious system behave as a conducting medium. Cathodic and anodic reaction sites may be 1807 located on the same reinforcement (microcell) or on different reinforcing bars (macrocell). 
1809 The oxidation and reduction reactions that take place at the anodic and cathodic sites respectively 1810 are referred to as half-cell reactions. At the anode, iron is oxidized and goes into solution as ferrous 1811 ions, as follows:

$$
F e \rightarrow F e^{2+}+2 e^{-}
$$

1812 Depending on the availability of oxygen and the $\mathrm{pH}$ of the environment, the following reduction 1813 reaction takes place at the cathode:

$$
2 \mathrm{H}_{2} \mathrm{O}+\mathrm{O}_{2}+4 e^{-} \rightarrow 4 \mathrm{OH}^{-}
$$

1814 The ferrous ions that are produced and in solution at the anodic site react with hydroxyl ions to 1815 form ferrous hydroxide.

$$
\mathrm{Fe}^{2+}+2 \mathrm{OH}^{-} \rightarrow \mathrm{Fe}(\mathrm{OH})_{2}
$$

1816 Ferrous hydroxide can be further oxidized to form hydrated ferric oxide (red brown rust) and 1817 hydrated magnetite (green rust). Hydrated ferric oxide and hydrated magnetite can further 1818 dehydrate to produce red rust, ferric oxide, $\mathrm{Fe}_{2} \mathrm{O}_{3}$, and black magnetite, $\mathrm{Fe}_{3} \mathrm{O}_{4}$. In highly alkaline 1819 concrete pore solution environments, ferrous hydroxide can also oxidize to gamma ferric 1820 oxyhydroxide $(2 \gamma-\mathrm{FeOOH})$. The reactions follow:

$$
\begin{aligned}
& 4 \mathrm{Fe}(\mathrm{OH})_{2}+\mathrm{O}_{2} \rightarrow 2 \mathrm{Fe}_{2} \mathrm{O}_{3} \cdot \mathrm{H}_{2} \mathrm{O}+2 \mathrm{H}_{2} \mathrm{O} \\
& 6 \mathrm{Fe}(\mathrm{OH})_{2}+\mathrm{O}_{2} \rightarrow 2 \mathrm{Fe}_{3} \mathrm{O}_{4} \cdot \mathrm{H}_{2} \mathrm{O}+4 \mathrm{H}_{2} \mathrm{O} \\
& 2 \mathrm{Fe}(\mathrm{OH})_{2}+\frac{1}{2} \mathrm{O}_{2} \rightarrow 2 \gamma-\mathrm{FeOOH}+\mathrm{H}_{2} \mathrm{O}
\end{aligned}
$$

1822 The products from the above reactions can form a passive layer around the steel reinforcing bar 1823 and the extent to which each product can protect the steel is still a topic of interest. This stability 1824 of this protective passive film can be affected by two environmental exposure conditions: 1) 1825 chlorides and 2) carbonation. Both phenomena can break down the passive layer and initiate 1826 corrosion of the reinforcement. The corrosion products generally have a larger volume than the 1827 base steel reinforcement which can result in internal pressure on the concrete cover, leading to 1828 development of tensile stresses. These tensile stresses can lead to cracking, spalling, and 1829 deterioration of structural capacity. Loss of steel ductility, reduction in bond strength between the 1830 steel reinforcement and concrete, and loss of cross-sectional area of the steel reinforcement are 1831 also other consequences of corrosion [132]. In the presence of chlorides, oxygen and moisture, the 
1832 passive layer between the steel reinforcement and the concrete is disrupted and localize corrosion 1833 initiates. Calcium chloroaluminate (Freidel's Salt) is formed when the chloride ions form a 1834 complex with hydration products [133]. If the total concentration of chlorides exceeds the binding 1835 capacity of cementitious system, the remaining (e.g., free) chlorides can induce corrosion. The 1836 study presented in the current chapter focuses on the chloride-induced corrosion of reinforcing 1837 steel.

1838 Corrosion studies on PLCs are crucial because the performance of embedded steel reinforcing bar 1839 in a PLC cementitious system when exposed to these environments will ultimately determine the 1840 service life of these structures. The type and characteristics of the cementitious cover surrounding 1841 the reinforcing bar directly influences the time to corrosion initiation and the propagation of this 1842 corrosion. As such, there is a need to assess the corrosion behavior of steel-embedded in PLC 1843 cementitious systems. Limited research has been performed on chloride-induced steel corrosion in 1844 concrete containing PLC [134-136]. These past studies demonstrate that, up to a certain 1845 replacement level of interground limestone filler, PLCs can reduce the corrosion rate of embedded 1846 steel. They also report that cement content and fineness have a significant impact on the corrosion 1847 behavior. One study reports that mortars with OPC exhibit better durability than mortars 1848 containing limestone cement [136]. Because of this, corrosion studies are needed to determine the 1849 potential influence of PLCs on the corrosion of steel reinforcement corrosion when exposed to 1850 chlorides.

1851 PLC systems, in combination with SCMs such as fly ash, have shown promising results, gaining 1852 more credibility as an eco-friendly material. Concrete specimens with PLC have been reported to 1853 produce higher compressive strengths than OPC when fly ash is incorporated into the mixture 1854 [137]. However, limited research has been performed on the corrosion performance of these 1855 systems. This research investigates that gap in knowledge.

\subsection{Experimental Test Matrix}

1857 The performance of OPC and PLC with select SCM combinations, as shown in Table 11-1, were 1858 evaluated. The results include the average chloride concentration in the mortar cover, $\mathrm{Cl}_{\text {test }}$, and 1859 the times to corrosion initiation. For each SCM combination, specimens were cast with portland

1860 cement E_OIIV and PLC E_L11. Comparisons of the corrosion behavior of the steel reinforcement 1861 embedded in these systems is then made. Note that the $\mathrm{Cl}_{\text {test }}$ is the average chloride concentration 1862 of the mortar cover and this is not the $\mathrm{Cl}_{\text {crit }}$. Trejo and Vaddey (2020) developed a correlation 1863 between the $\mathrm{Cl}_{\text {test }}$ and $\mathrm{Cl}_{\text {crit }}$ as follows [131]:

$$
\frac{C l_{\text {crit }}}{C l_{\text {test }}}=0.147+0.844 \cdot \mathrm{w} / \mathrm{cm}+0.083 C l_{\text {exp }}-0.014 p H_{\text {exp }}+0.004 t_{\text {exp }}
$$


1864 where $\mathrm{w} / \mathrm{cm}$ is the water to cementitious materials ratio, $\mathrm{Cl}_{\exp }$ is the concentration of the chloride

1865 exposure solution, $\mathrm{pH}_{\exp }$ is the $\mathrm{pH}$ of the exposure solution, and $\mathrm{t}_{\exp }$ is the time for the specimen to 1866 initiate corrosion. Note that $\mathrm{Cl}_{\text {test }}$ and $\mathrm{Cl}_{\text {crit }}$ are expressed as percent by cement mass, $\mathrm{Cl}_{\text {exp }}$ is 1867 expressed as percent, and $t_{\text {exp }}$ is expressed in days. For this testing, the $\mathrm{Cl}_{\exp }$ was $2 \%$ for all testing, 1868 the $\mathrm{pH}_{\exp }$ was 12.5 for all testing, and the $\mathrm{w} / \mathrm{cm}$ was 0.4 for all specimens. Using this information, 1869 equation (11-7) can be reduced as follows:

$$
\frac{C l_{\text {crit }}}{C l_{\text {test }}}=0.457+0.004 t_{\text {exp }}
$$

1871 In addition to the cases identified in Table 11.1, OPCs and PLCs from two other clinker sources, 1872 namely C_OV, C_L10, A_O11, and A_L15, were also evaluated using the modified ASTM G109 1873 method. For the modified ASTM G109 testing, all cements were evaluated with a $25 \%$ fly ash 1874 replacement.

\section{$1875 \quad 11.4$ Experimental Methods}

\section{$1876 \quad 11.4 .1$ OC $_{\text {crit }}$ method}

1877 A water to cementitious material ratio $(\mathrm{w} / \mathrm{cm})$ of 0.4 was used for all the cases. Once the specimens 1878 were cast, they were cured in a moist room with $100 \% \mathrm{RH}$ for 56 days. At the end of the curing 1879 period, the anodic specimens were transferred in to a $2 \% \mathrm{Cl}^{-}$solution saturated with calcium 1880 hydroxide $\left(\mathrm{Ca}(\mathrm{OH})_{2}\right)$. The cathodic specimens were stored in a container with saturated $\mathrm{Ca}(\mathrm{OH})_{2}$ 1881 solution. The open circuit potential (OCP) of the anodic specimens were measured using a $\mathrm{Cu}-$ $1882 \mathrm{CuSO}_{4}$ on all weekdays since the start of exposure. Previous work indicated that microcell current 1883 ( $\left.\mathrm{I}_{\text {macro }}\right)$ and linear polarization resistance $\left(\mathrm{R}_{p}\right)$ testing exhibited large scatter [130] when compared 1884 to OCP measurements. Because of this, OCP was selected to monitor the corrosion initiation in 1885 this program. OCP was recorded on a daily basis and a period of 2 consecutive days during which 1886 the OCP was more negative than $-350 \mathrm{mV}$ was considered to represent initiation of corrosion. Note 1887 that the activation criterion is defined based on the OCP measured using a $\mathrm{Cu}-\mathrm{CuSO}_{4}$ electrode 1888 and will differ for other standard electrodes. The setup for this test method is illustrated in Figure 1889 11-1. 
Table 11.1. Experimental program for OCcrit study

\begin{tabular}{|c|c|c|}
\hline No. & Cement type & SCM addition \\
\hline 1 & \multirow{5}{*}{ E_OIIV } & Control \\
\hline 2 & & 25FA1 \\
\hline 3 & & 20FA1-5SF \\
\hline 4 & & $50 \mathrm{SL}$ \\
\hline 5 & & 25FA1-25SL \\
\hline 6 & \multirow{4}{*}{ E_L11 } & 25FA1 \\
\hline 7 & & 20FA1-5SF \\
\hline 8 & & $50 \mathrm{SL}$ \\
\hline 9 & & 25FA1-25SL \\
\hline
\end{tabular}

1892 Once the specimens have activated, the cover was broken from the test specimen and tested for 1893 both water and acid soluble chlorides using the AASHTO TP 260 method. In addition to 1894 determining the average chloride concentration of the cover, this testing also provides information 1895 on the ratio of free to bound chlorides. Chlorides are present in concrete in two forms: 1) free chlorides that are dissolved in the pore solution and 2) bound chlorides that are physically and chemically bound to the cement hydrates. The free chlorides are responsible for initiating the corrosion process. There are two types of mechanisms by which chloride binding occurs. In the first mechanism, chloride ions are physically adsorbed onto the surface of cement hydrates, especially C-S-H. In the second mechanism, which chemically occurs, chlorides react with monosulfate (AFm) compounds resulting in chloride containing AFm compounds such as 1902 Freidel's salt $\left(\mathrm{C}_{3} \mathrm{~A} \cdot \mathrm{CaCl}_{2} \cdot 10 \mathrm{H}_{2} \mathrm{O}\right)$. The ratio of water to acid soluble chlorides (WSC/ASC) 1903 indicates the relative quantity of bound chlorides in the specimens. If the WSC/ASC ratio is close 1904 to unity, there are large concentrations of free chlorides in the pore solution and this indicates a 1905 lower binding capacity.

1906 Studies have suggested that the chloride binding capacity of specimens depend on the aluminum 1907 content of the binder. Specimens blended with fly ash, slag, or metakaolin have been reported to 1908 exhibit increased chloride binding capacities when compared to specimens that were blended with 1909 silica fume [138]. This was reported to be attributed to the higher aluminum content of the 1910 supplementary cementitious materials. A higher CSH content generally leads to an increased 1911 chloride binding [95]. 


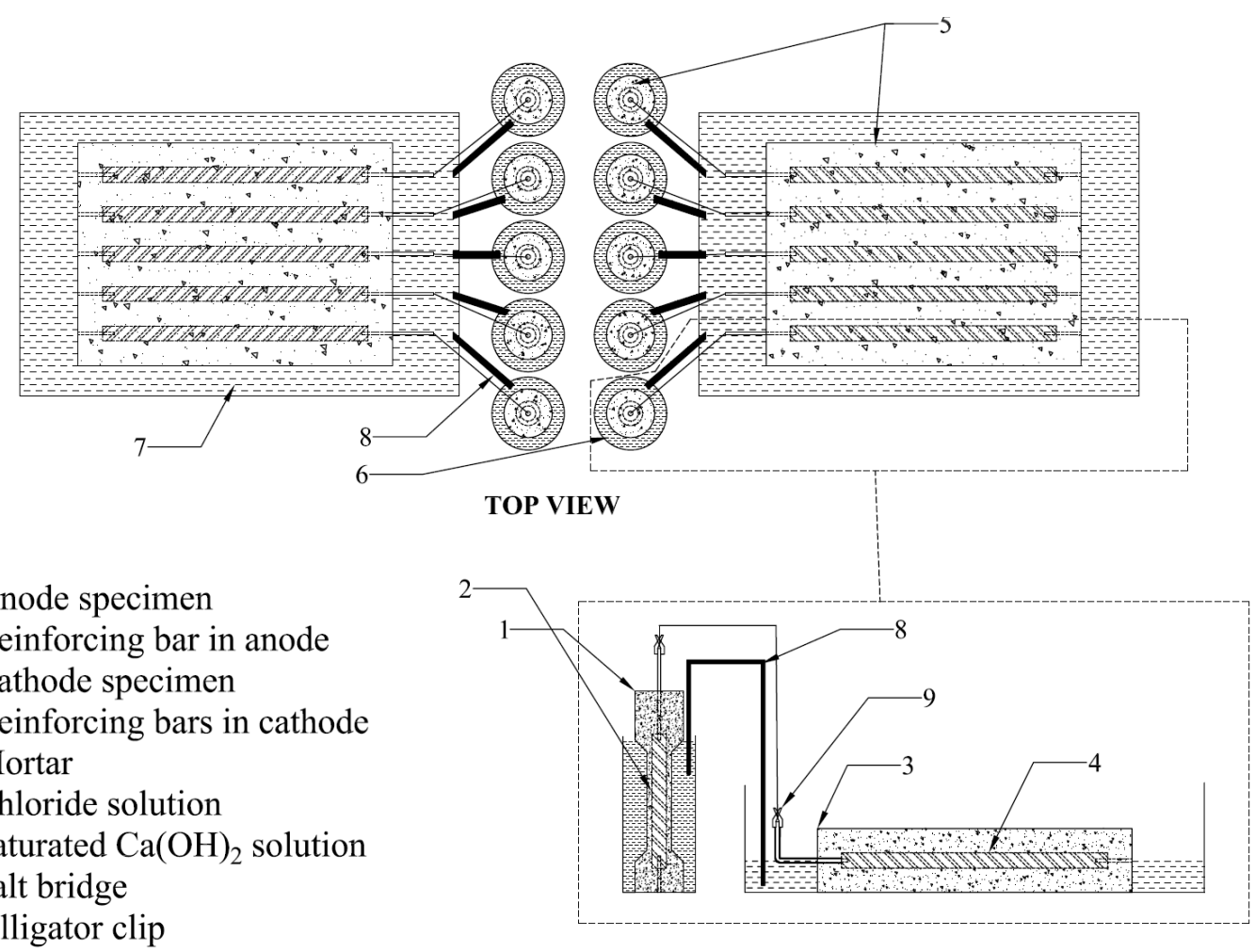

1. Anode specimen

2. Reinforcing bar in anode

3. Cathode specimen

4. Reinforcing bars in cathode

5. Mortar

6. Chloride solution

7. Saturated $\mathrm{Ca}(\mathrm{OH})_{2}$ solution

8. Salt bridge

9. Alligator clip

FRONT CROSS SECTIONAL VIEW

Figure 11-1. Setup for the $\mathrm{OC}_{\text {crit }}$ test method.

\subsubsection{Modified ASTM G109 method}

1916 ASTM G109 method is based on the macrocell corrosion process and is commonly used to study 1917 corrosion of steel reinforcing bars embedded in concrete [139, 140]. In this method, concrete 1918 prisms are cast with one anodic reinforcement bar and two cathodic reinforcement bars (cathode 1919 to anode ratio of 2). The corrosion is monitored by: 1) monitoring the OCP of the anodic 1920 reinforcing bar and 2) measuring the macrocell current between the anodic and cathodic 1921 reinforcing bars. The original method was devised to check the effectiveness of corrosion 1922 inhibitors. This method, however, has been used extensively to assess and compare corrosion of 1923 reinforcing bars embedded in different cementitious systems. In this study, the ASTM G109 1924 method is used to compare the performance of OPC concrete with PLC or OPC+LS concrete and 1925 no corrosion inhibitors were used in this study. Additionally, instead of constructing a dam to store 1926 the ponding solution as suggested in ASTM G109, the ponding solution was stored above the cover 1927 surrounding the anodic rebar as shown in Figure 11-2.

1928 In this program, concrete specimens were subjected to 56 days of curing in a moist room with 1929 $100 \%$ RH. Post curing, the specimens were then dried for two weeks in an environment with a $193050 \%$ RH. The cured specimens were then epoxy-coated on all sides except the top and bottom 1931 surfaces. The ponding salt solution was prepared by dissolving 3 parts of sodium chloride to 97 
1932 parts of water. The ponding solution, atop the specimen, was applied for two weeks and then

1933 vacuumed off where the specimen was dry for the following two weeks (i.e., biweekly wet-dry 1934 cycles). The macrocell current and the OCP were measured once every four weeks, starting from 1935 the second week of ponding. Imacro, the macro-current flowing from the anode to the cathode, was 1936 measured using a $100 \Omega$ resistor. One end of the resistor was connected to the insulating wire 1937 protruding from the copper wire of the cathodic rebars and the other end of the resistor was 1938 connected to the anodic rebar. The voltage $(\mathrm{V})$ across the $100 \Omega$ resistor was measured by attaching 1939 both terminals of the voltmeter to the opposite ends of the resistor. The macro-cell corrosion 1940 current density, $\mathrm{I}_{\text {macro, }}$, was then estimated as $\mathrm{V} /\left(\mathrm{R}^{*} \mathrm{~A}\right)$, where $\mathrm{V}$ represents the voltage across the

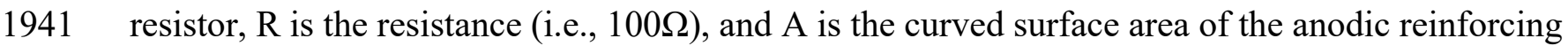
1942 bar exposed to the chlorides. The steel reinforcement located between the epoxied ends of the 1943 anodic reinforcing bar is considered as the exposed reinforcing steel. The value of 'A' is $0.01 \mathrm{~m}^{2}$ $1944\left(15.5 \mathrm{in}^{2}\right)$. The setup for this test method is illustrated in Figure 11-2.

1945 The test was carried out until the average integrated charge, based on the macrocell current, 1946 reached 150 coulombs, C. At the conclusion of testing, the bars were visually inspected for 1947 corrosion and the acid soluble chloride content was measured at the depth corresponding to the top 1948 of the reinforcement bar.

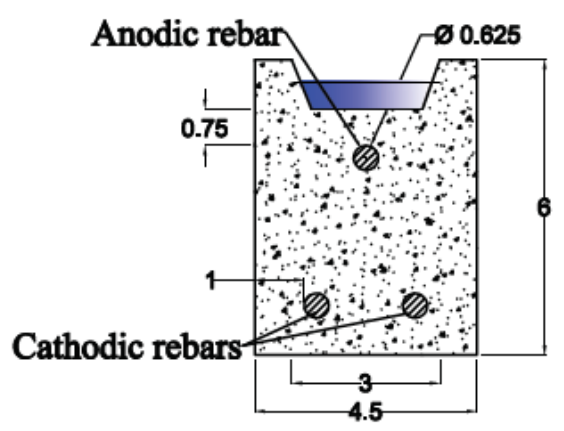

Front View

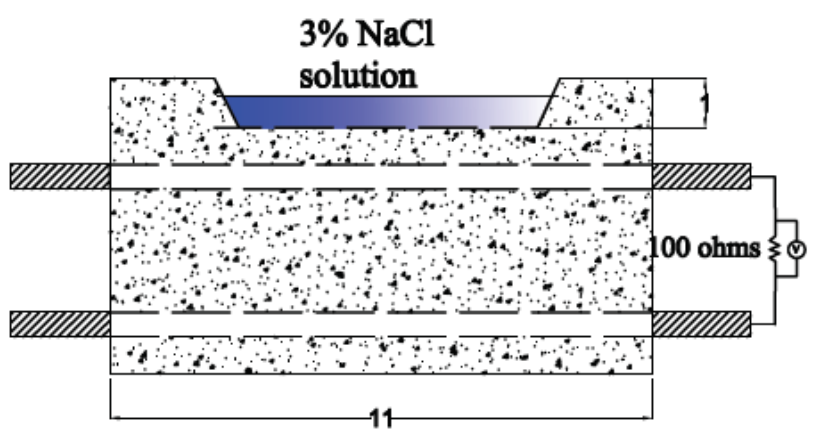

Side View

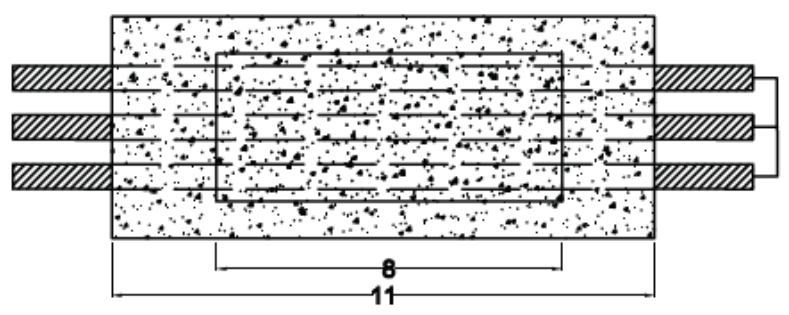

Top View 


\subsection{Experimental Results}

1952

1953

1954

1955

1956

1957

1958

1959

1960

1961

1962

1963

1964

1965

1966

1967

1968

1969

1970

1971

1972

1973

1974

1975

1976

1977

1978

1979

1980

1981

1982

1983

1984

1985

1986

1987

\subsubsection{OC crit $_{\text {method }}$}

The $\mathrm{OC}_{\text {crit }}$ method provides a reliable and fast way to estimate the critical chloride threshold of steel reinforcement embedded in cementitious systems. In this test, the specimens are exposed to $2 \%$ chloride solutions and the OCP is assessed every day with a digital multimeter. The specimens are considered "activated," that is corrosion has initiated, when the OCP is less than $-350 \mathrm{mV}$ for 2 consecutive days. Once the specimens have activated, they are removed from the chloride solution and the thin cover surrounding the reinforcing bar is removed and tested for average chloride concentration. Figure 11-3 shows the OCP for anodic specimens cast with E_OIIV. In this research these are considered as the control group and are henceforth used as a benchmark to compare and contrast the corrosion performance of the cements and the SCMs in the remaining experimental program. It can be seen in the figure that the activation time range from 22 to 63 days with a mean activation time of 39.8 days. Figure 11-4 shows the OCP for all mortar specimens cast with $25 \%$ FA1 for both E_OIIV and E_L11. It can be seen that both cases exhibit similar mean activation times of 55.3 and 49.4 days, respectively. In Figure 11-5, the OCP values of specimens made with $20 \% \mathrm{FA} 1$ and $5 \%$ silica fume are shown. It can be seen that specimens with E_OIIV exhibit a much higher mean activation time when compared to those of E_L11. Figure 11-6 shows the OCP of specimens made with $25 \%$ FA1 and $25 \%$ slag. It can be seen that both E_OIIV and E_L11 specimens have similar mean activation times of 28.5 and 25.6 days, respectively. Figure 11-7 shows a similar trend for specimens cast with $50 \%$ slag for both and E_OIIV and E_L11 cements. Note that the specimens with 50\% slag activated much earlier when compared to the other specimens. Figure 11-8 summarizes the time to activation for $\mathrm{OC}_{\text {crit }}$ specimens. It can be observed that both E_OIIV and E_L11 specimens with 25\% FA1 have a higher mean time to activation when compared to the control specimens. Time to corrosion initiation and critical chloride threshold are critical factors in determining overall service life. The OCcrit test method was developed to quantify critical chloride threshold values. The test was not developed to determine the transport rates of chlorides into a cementitious system. The reporting of times to corrosion was presented to provide the reader with a general idea of test times. The time to corrosion has not been correlated with critical chloride threshold; specimens with shorter test times could exhibit higher critical chloride values and alternatively, longer test times could result in lower critical chloride test values. Chloride transport rate is independent of critical chloride threshold value.

The $\mathrm{C}_{\text {test }}$ of the specimens was measured by extracting the thin cover surrounding the reinforcing bar. It is subsequently pulverized, sieved, and then tested based on AASHTO T 260 for both water and acid soluble chlorides. Figure 11-9 shows the results for average water-soluble chlorides. It can be seen that, except for the $25 \%$ fly ash and 50\% slag mixtures, both E_OIIV and E_L11 specimens had approximately similar quantities of $\mathrm{C}_{\text {test. }}$. Figure 11-10 shows the results for average 
1988 acid-soluble chlorides for the same set of specimens. This figure shows the same trend observed 1989 in Figure 11-9. The ratios between the water-soluble and acid-soluble chloride values are shown 1990 in Figure 11-11. For the control specimens, this ratio is 0.72. It can be seen that E_OIIV and E_L11 1991 specimens with 25\% FA1 has the lowest WSC/ASC values, indicating higher binding capacities. 1992 This could be attributed to the high concentration of alumina in FA1 (21.7\%). A higher 1993 concentration of alumina leads to the formation of Friedel's salt that helps bind the free chlorides 1994 and slows down the transport of chlorides to the surface of the rebar. This also helps explain the 1995 higher mean activation times of $\mathrm{OC}_{\text {crit }}$ specimens made with $25 \% \mathrm{FA} 1$.

1996 Figure 11-12 shows the $\mathrm{Cl}_{\text {crit }}$ for "partner" specimens. These "partner" specimens were used to 1997 calculate the $\mathrm{Cl}_{\text {crit }}$ of specimens because testing the cover mortar of the $\mathrm{OC}_{\text {crit }}$ specimens only 1998 provides information on the average chloride concentration of the mortar cover. It can be seen that 1999 most of the specimens have a $\mathrm{Cl}_{\text {crit }}$ of about $0.4 \%$ by weight of binder. Specimens E_OIIV-25FA1 2000 and E_L11-50SL have higher $\mathrm{Cl}_{\text {crit }}$ values of $0.59 \%$ and $0.63 \%$, respectively.

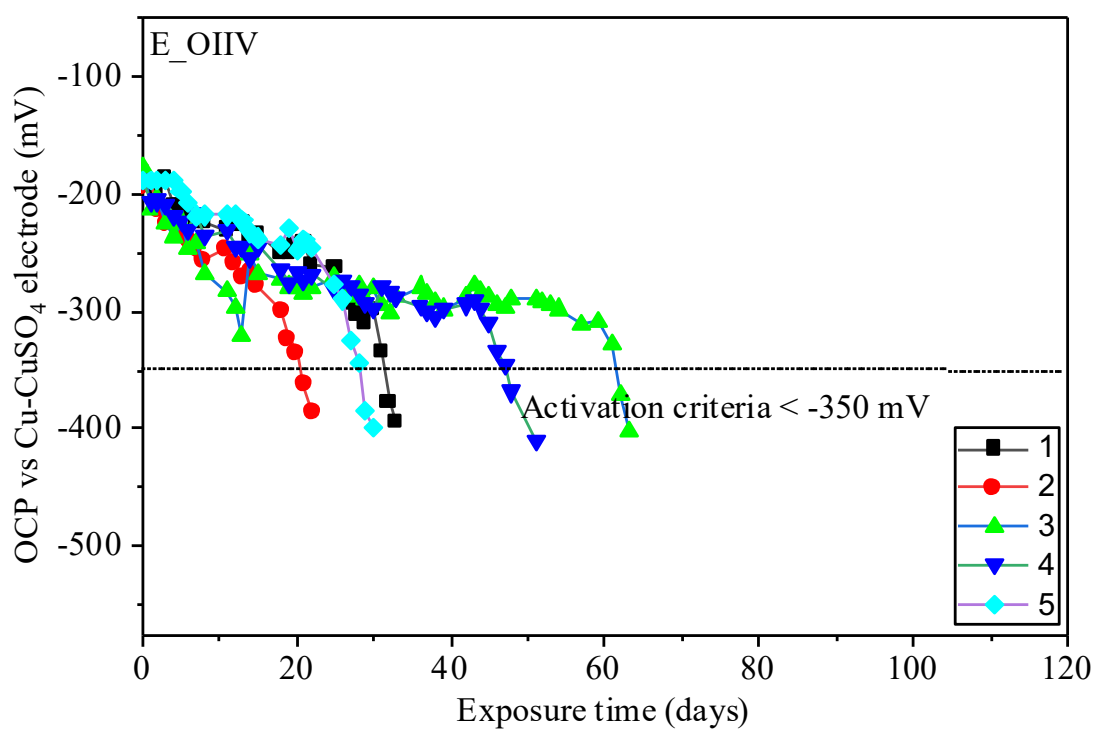




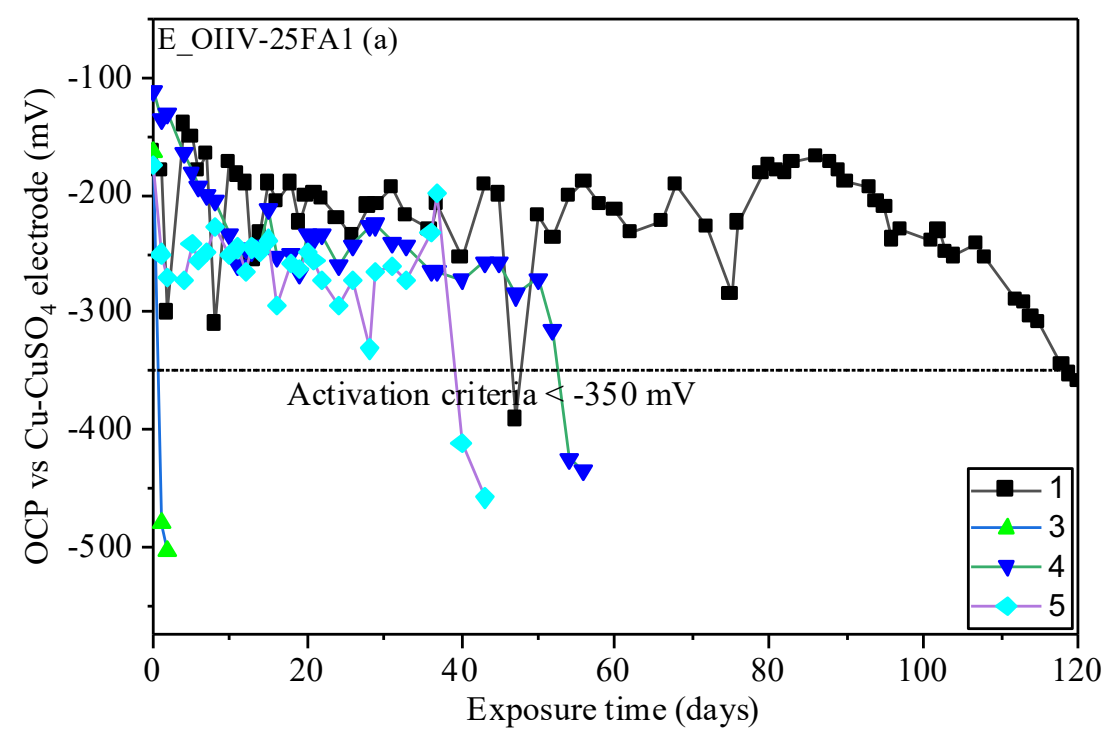

(a)

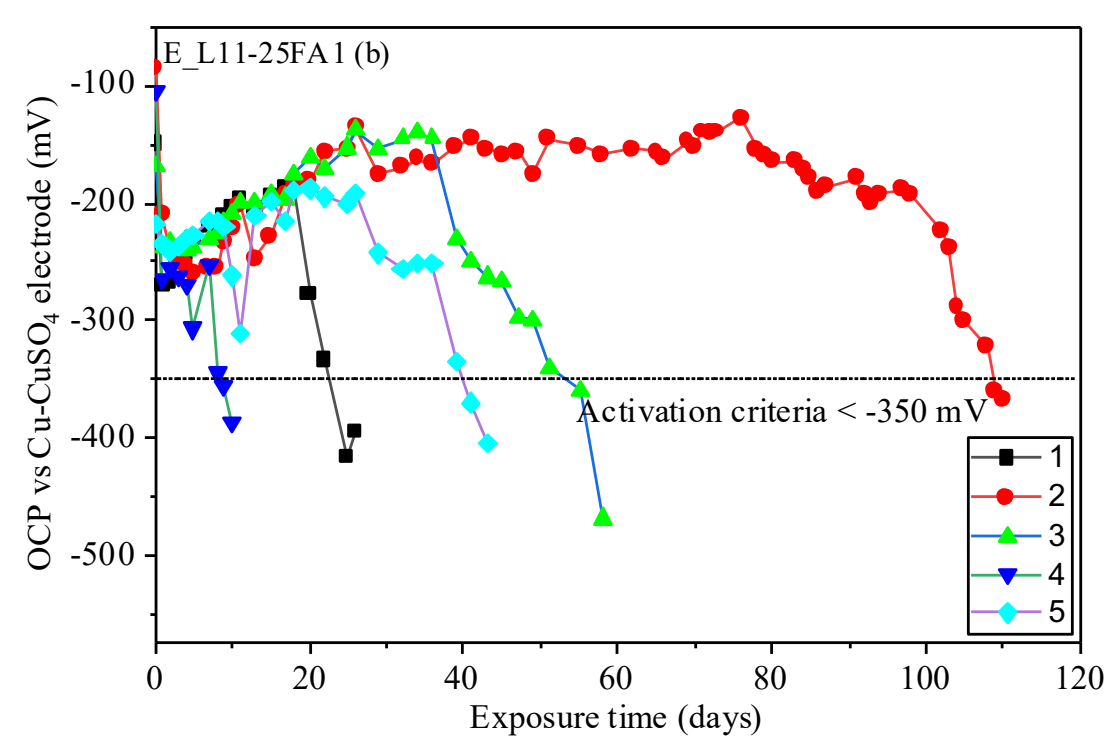

(b) 


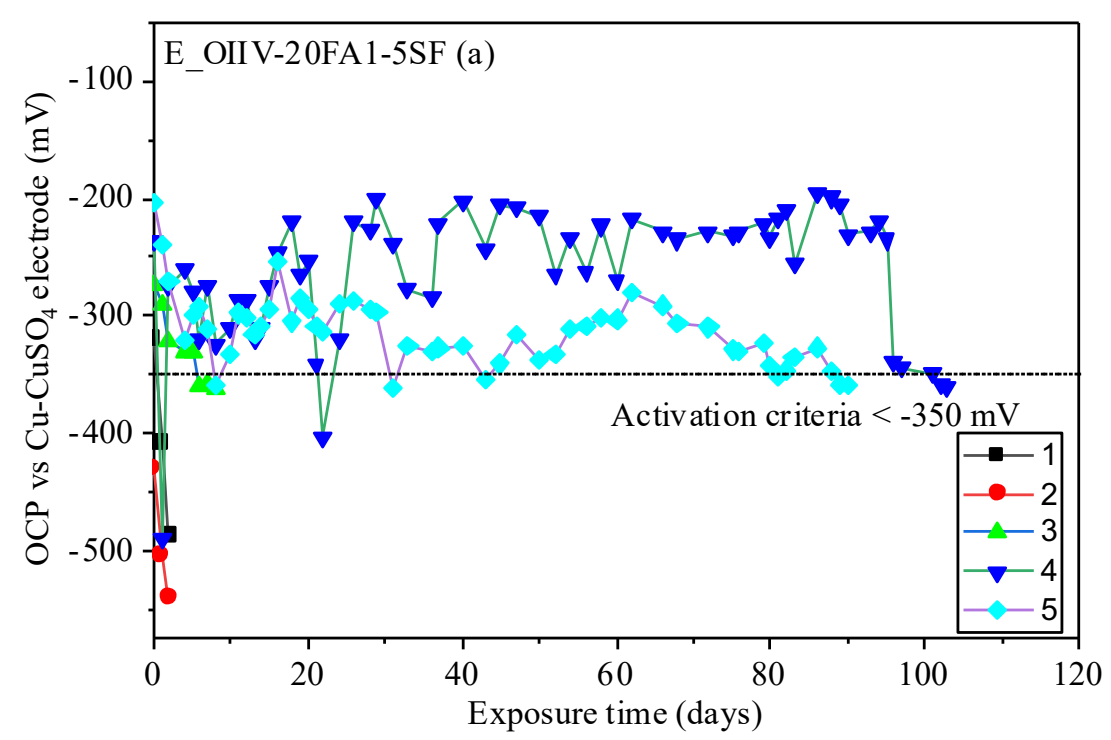

(a)

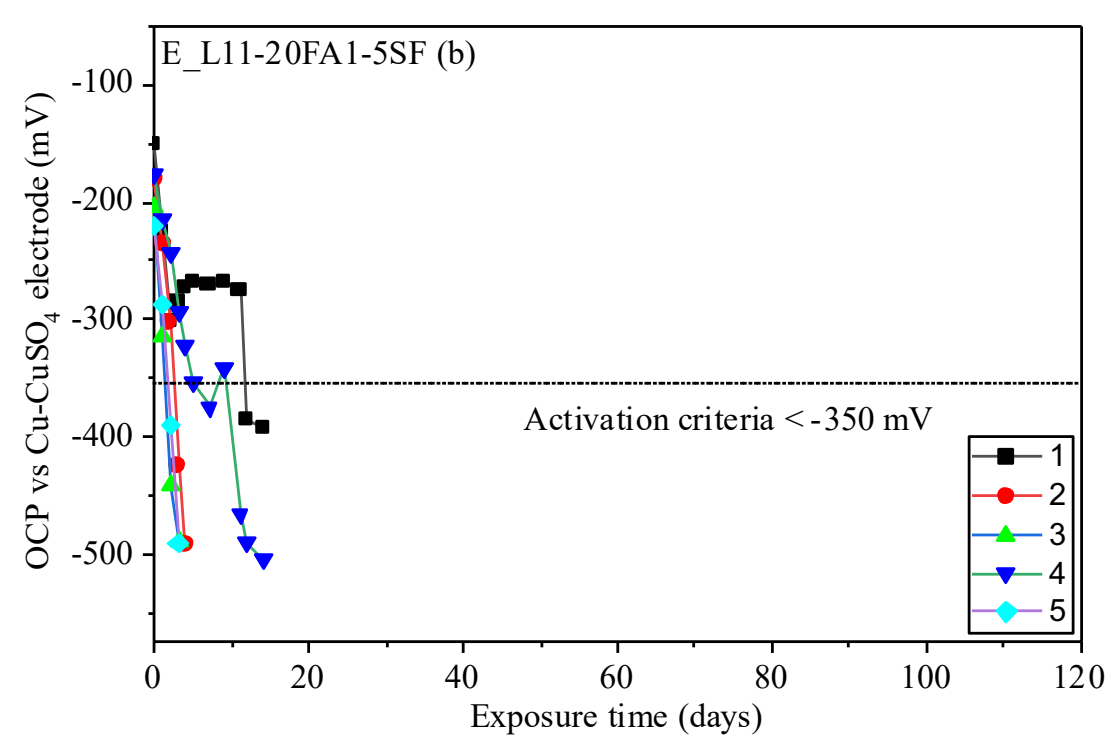

(b)

Figure 11-5. (a) Open circuit potential of anode specimens made with E_OIIV-20FA1-5SF and (b) Open circuit potential of anode specimens made with $E_{-} L \overline{11}-20 F A 1-5 S F$ 


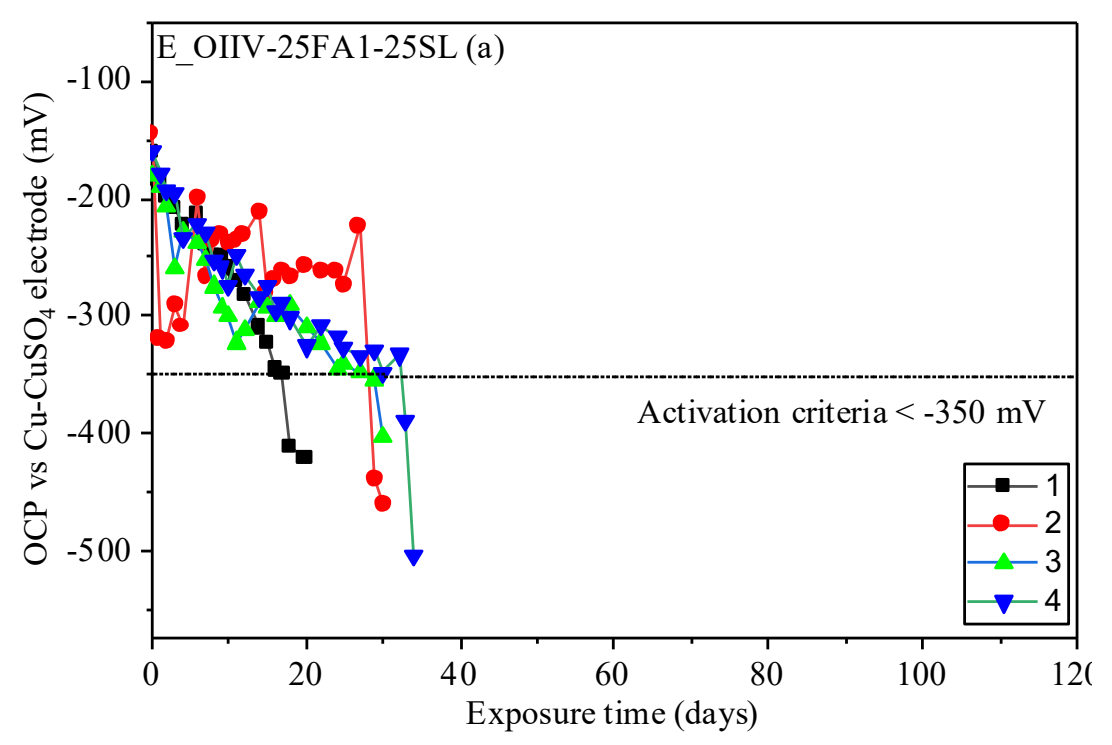

(a)

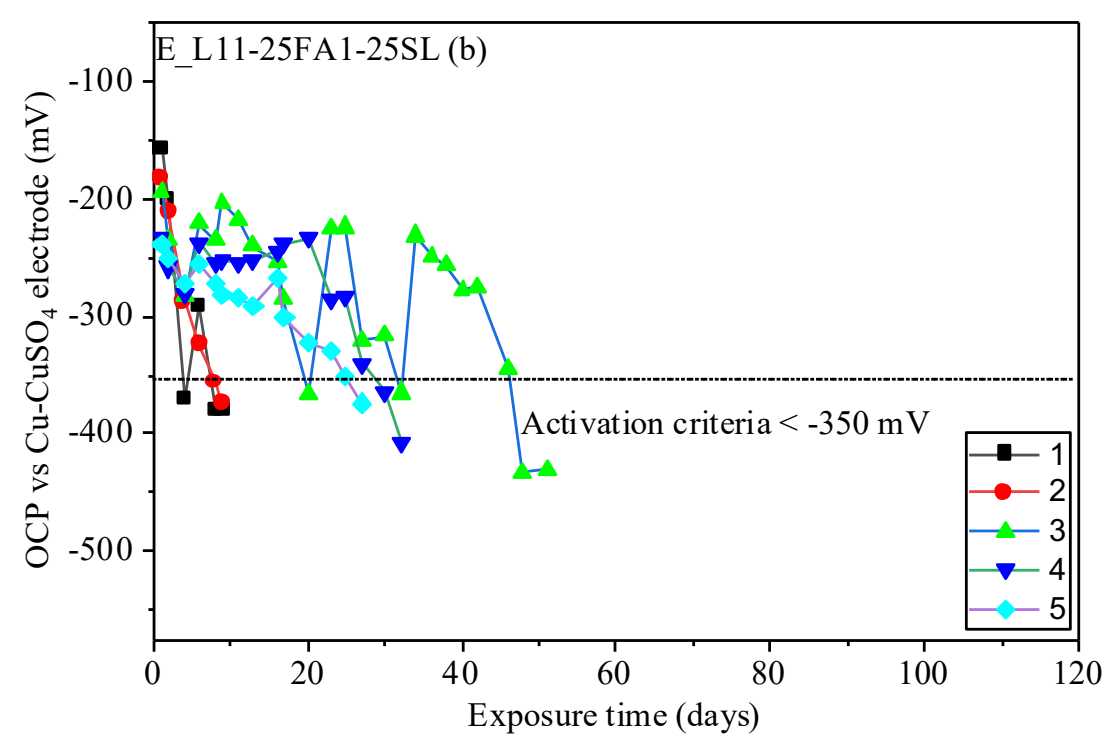

(b)

Figure 11-6. (a) Open circuit potential of anode specimens made with E_OIIV-25FA1-25SL and (b) Open circuit potential of anode specimens made with $E \_$L11-25FA1-25SL 


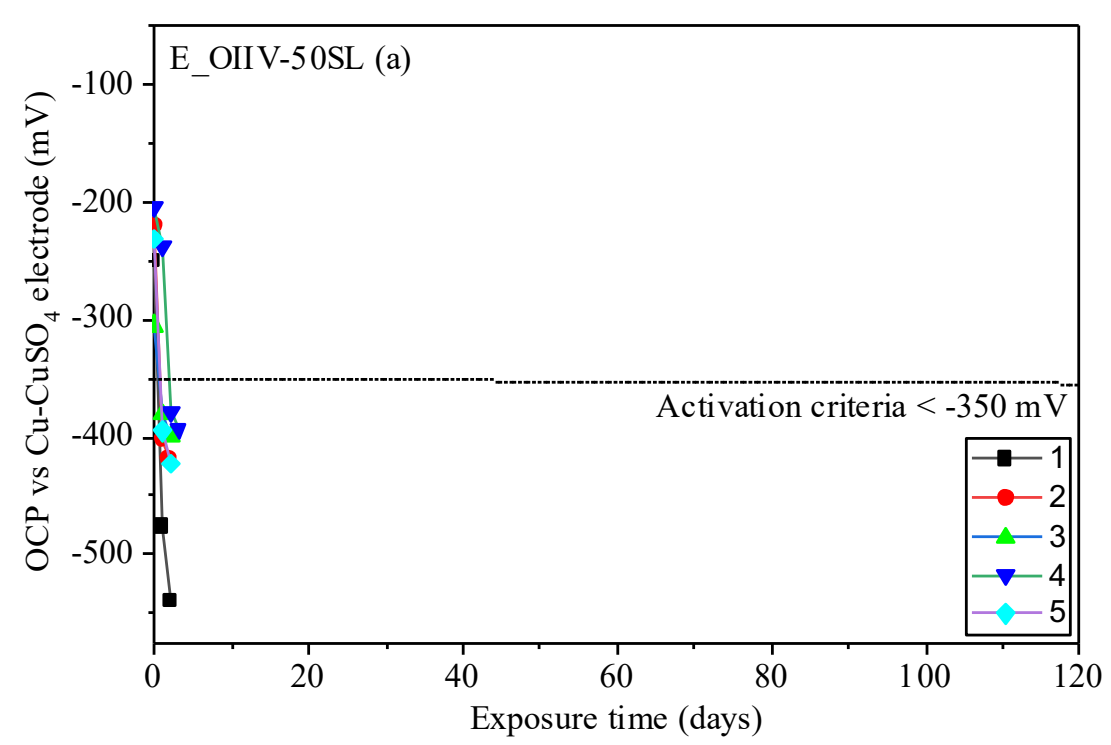

(a)

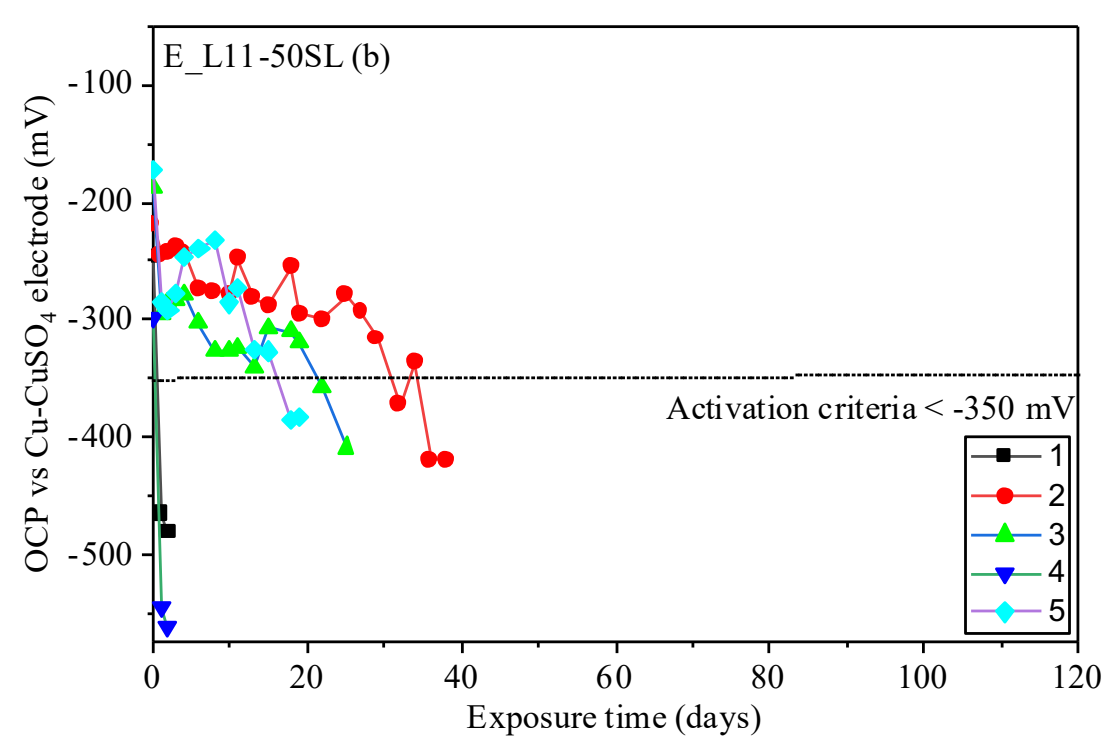

(b)

2013 Figure 11-7. (a) Open circuit potential of anode specimens made with E_OIIV-50SL and (b) 


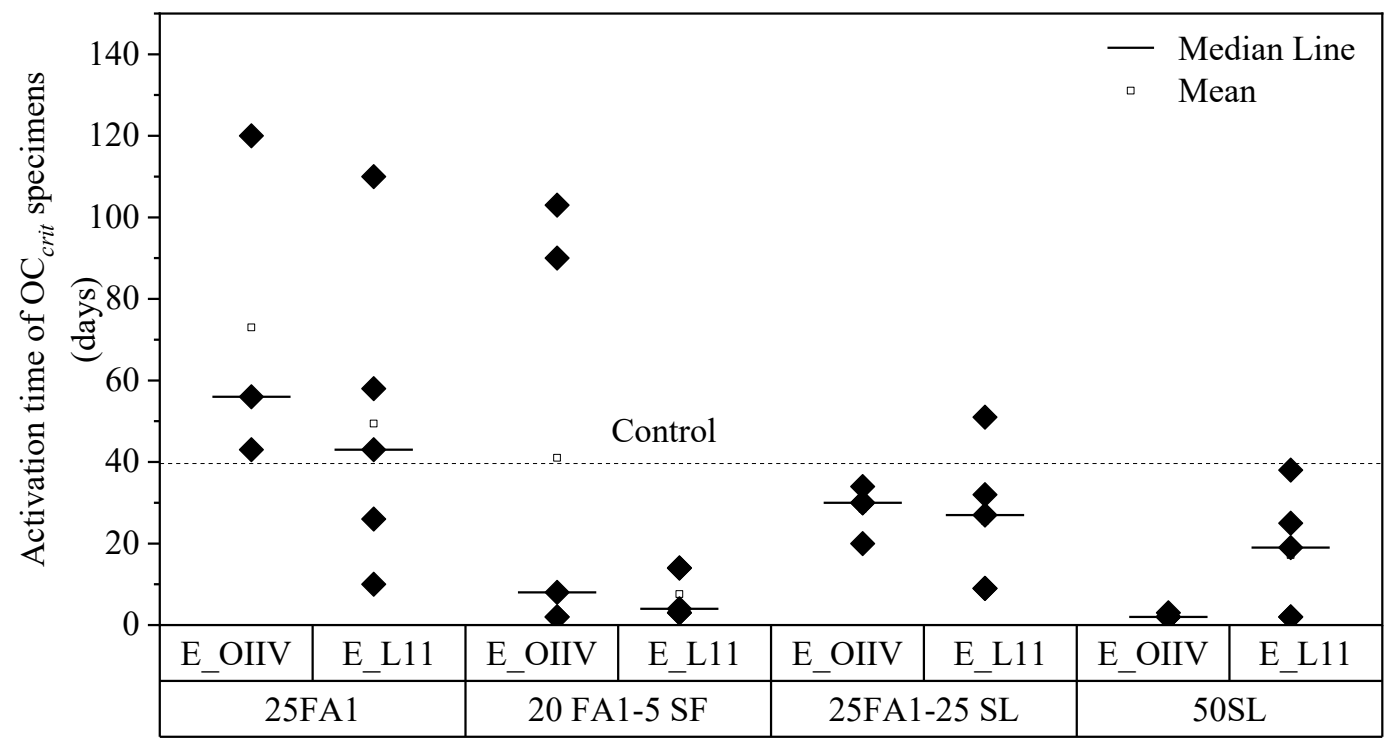

Figure 11-8. Time to activation of $\mathrm{OC}_{\text {crit }}$ specimens

2018

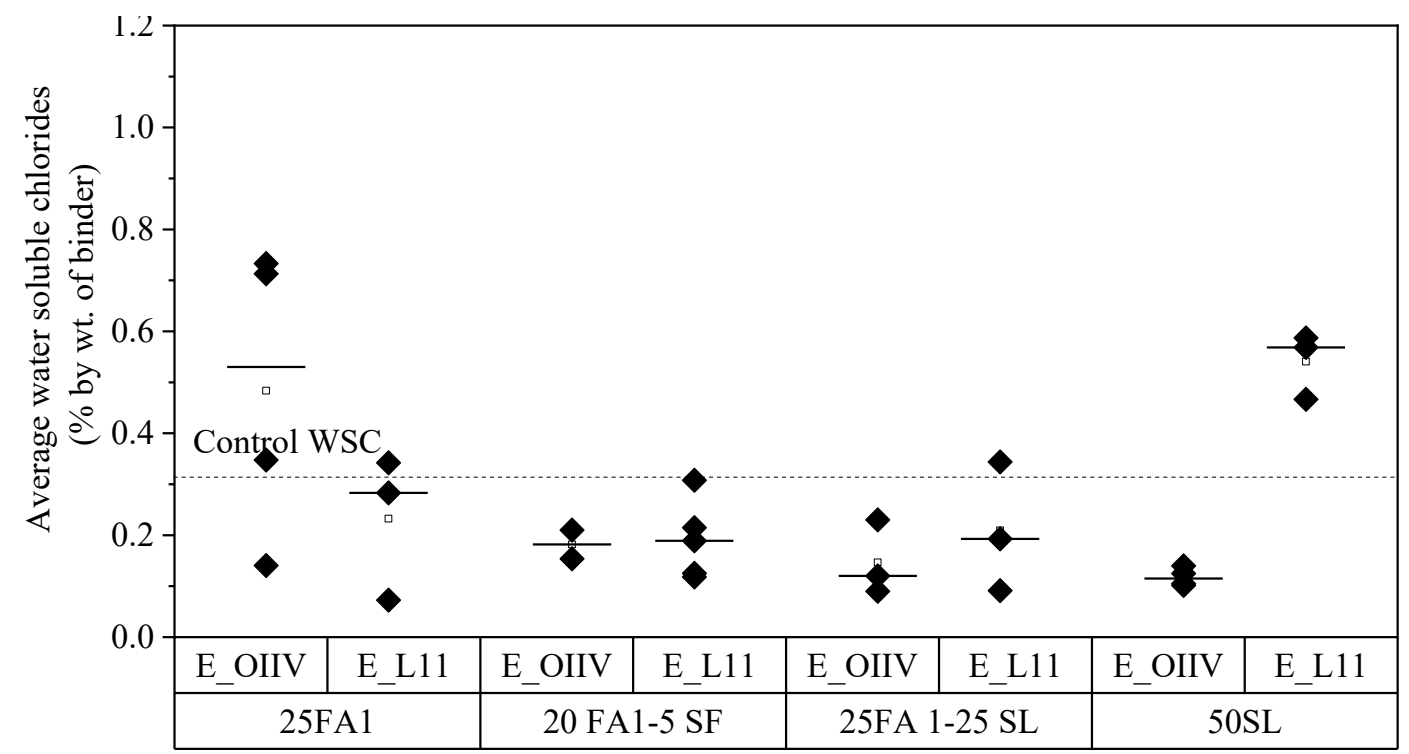
260 water-soluble method. 


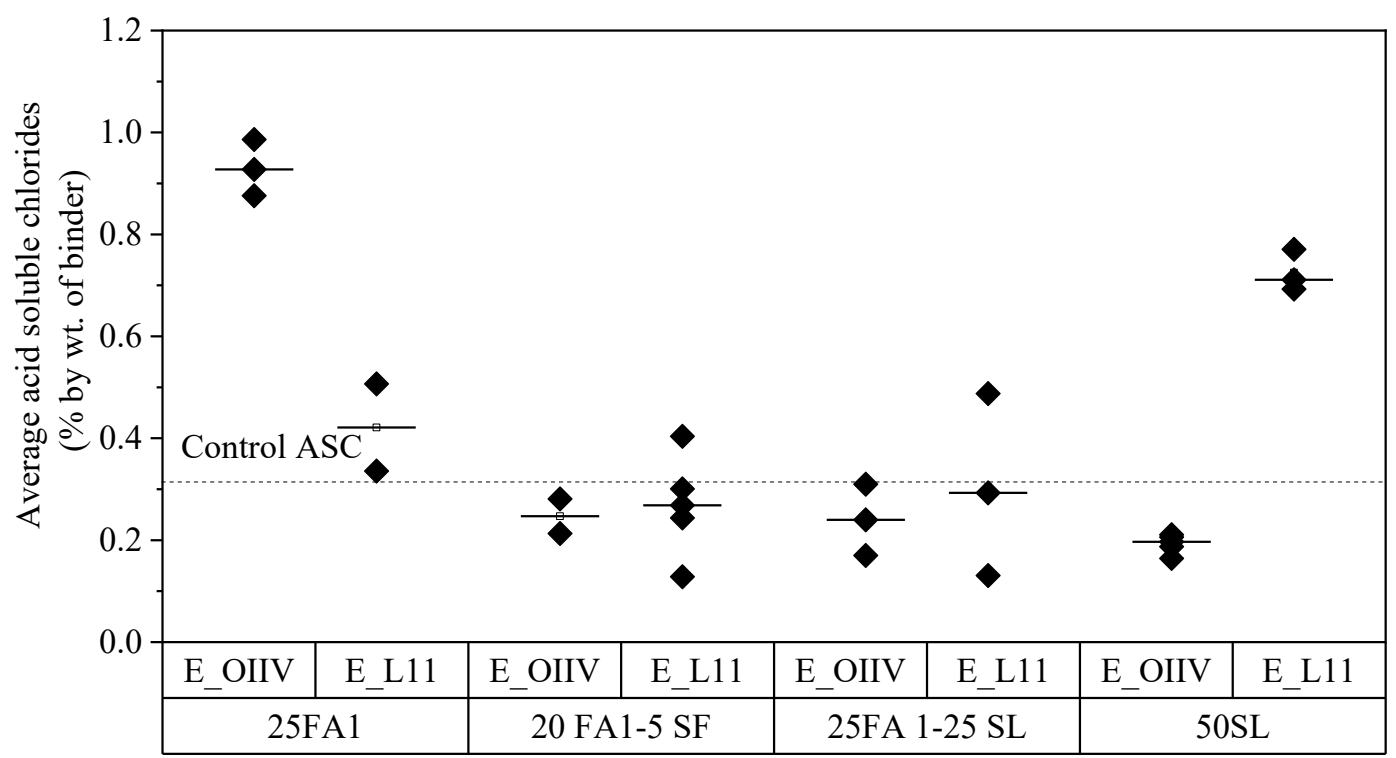

2023 2024 2025 2026
Figure 11-10. Average chloride concentration of activated specimens based on AASHTO T 260 acid-soluble method

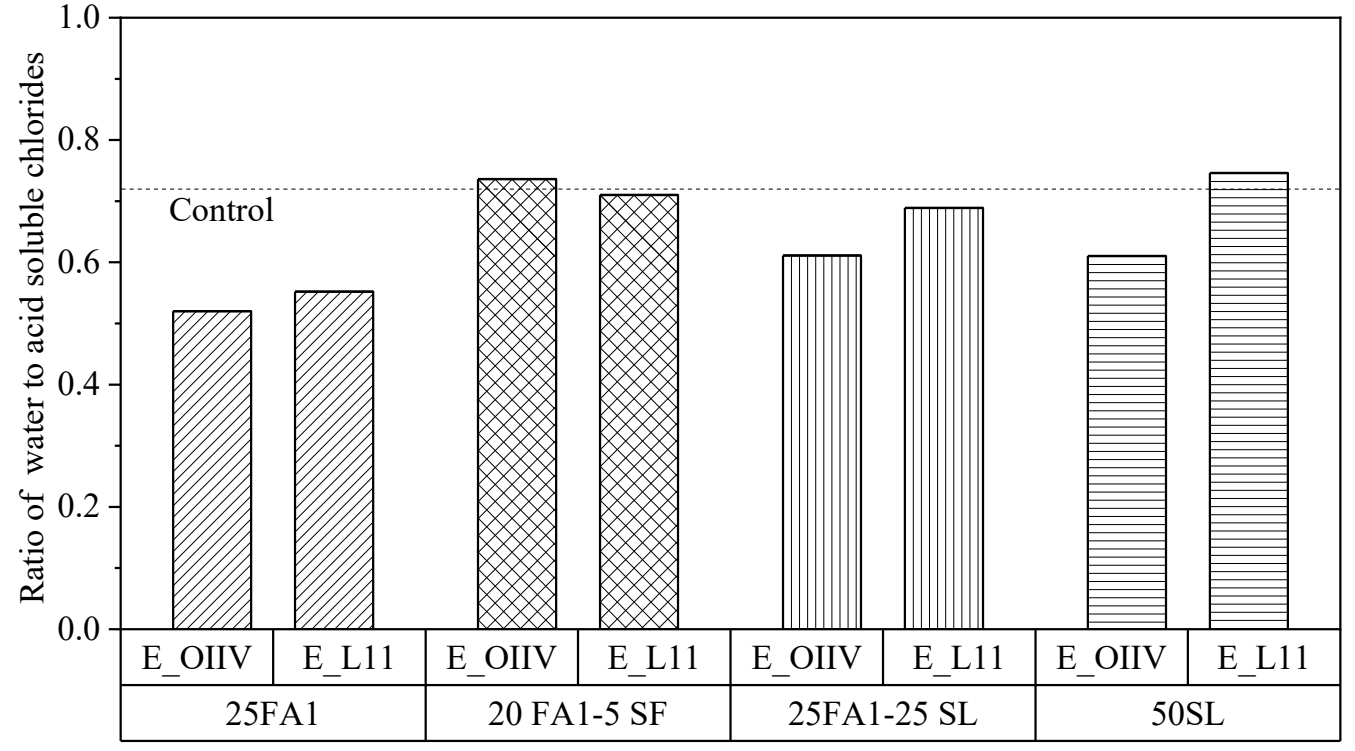

Figure 11-11. Ratio of water to acid soluble chlorides of activated specimens 


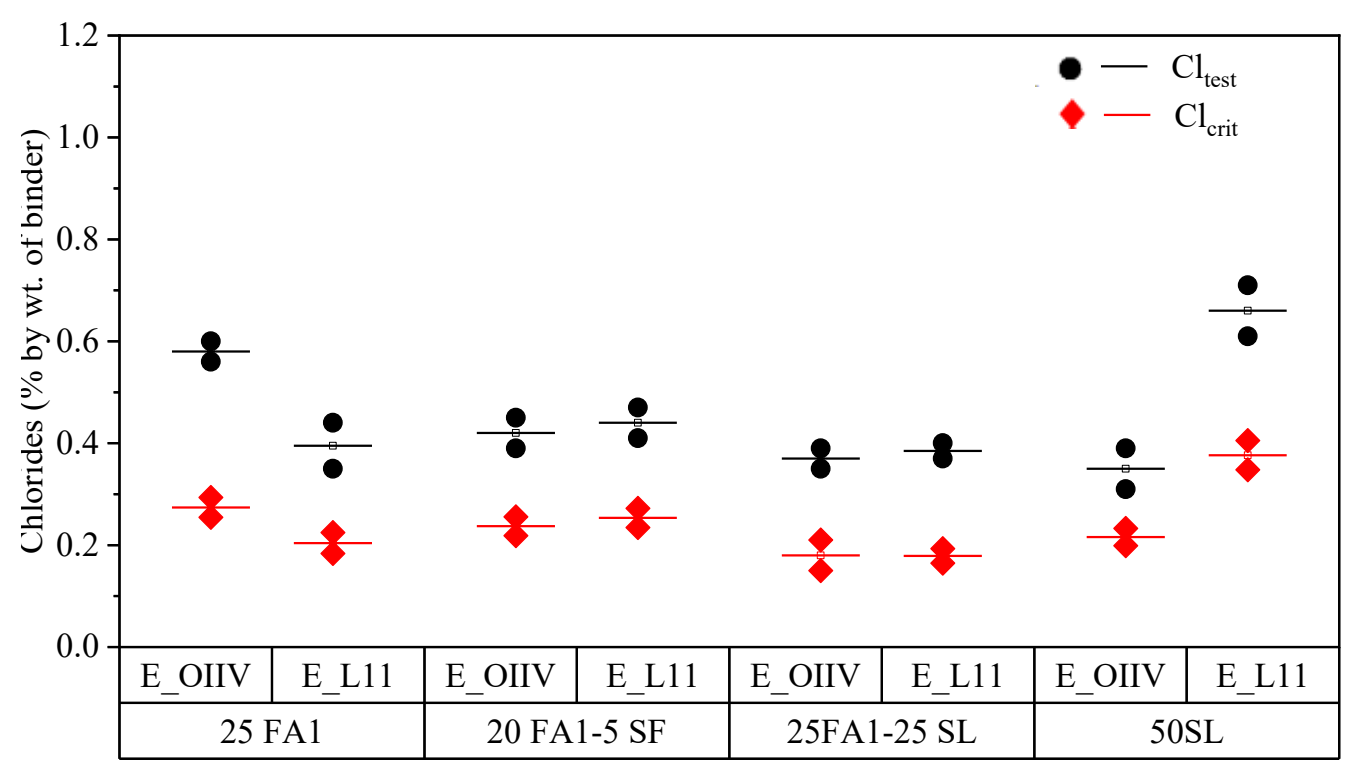

Figure 11-12. Critical and average chloride concentration

\subsubsection{Modified ASTM G109 method}

2035 Figure 11-13 shows the total charge passed for the modified G109 specimens. It can be seen that 2036 specimens with interground limestone exhibit higher charge passed for all three cement sources, 2037 throughout the exposure cycles. However, it also should be noted at earlier exposure ages there is 2038 significant overlap between the error bars. This indicates that the difference in total charge passed may not be statistically significant. Note that each cycle represents two weeks of exposure to the ponding solution followed by two weeks of drying. Figure 11-14 shows the critical chloride concentration for the same set of specimens. Once the specimens reach the activation criteria, samples are extracted from the top surface of the anodic reinforcement bar and were tested for acid-soluble chlorides following the AASHTO TP-260 standard. It can be seen that $25 \%$ FA1 specimens with OPC+10LS have a higher critical chloride concentration when compared to the rest of the specimens. The E_L11 mixtures evaluated with the $O C_{c r i t}$ test (initiation in 40 days) and modified G109 test (initiation in 7 months) exhibited similar $\mathrm{Cl}_{\text {crit }}$ values. Comparison of OPC and $\mathrm{PLC} \mathrm{Cl}_{\text {crit }}$ for each clinker source reveals that there is no statistically significant difference. 


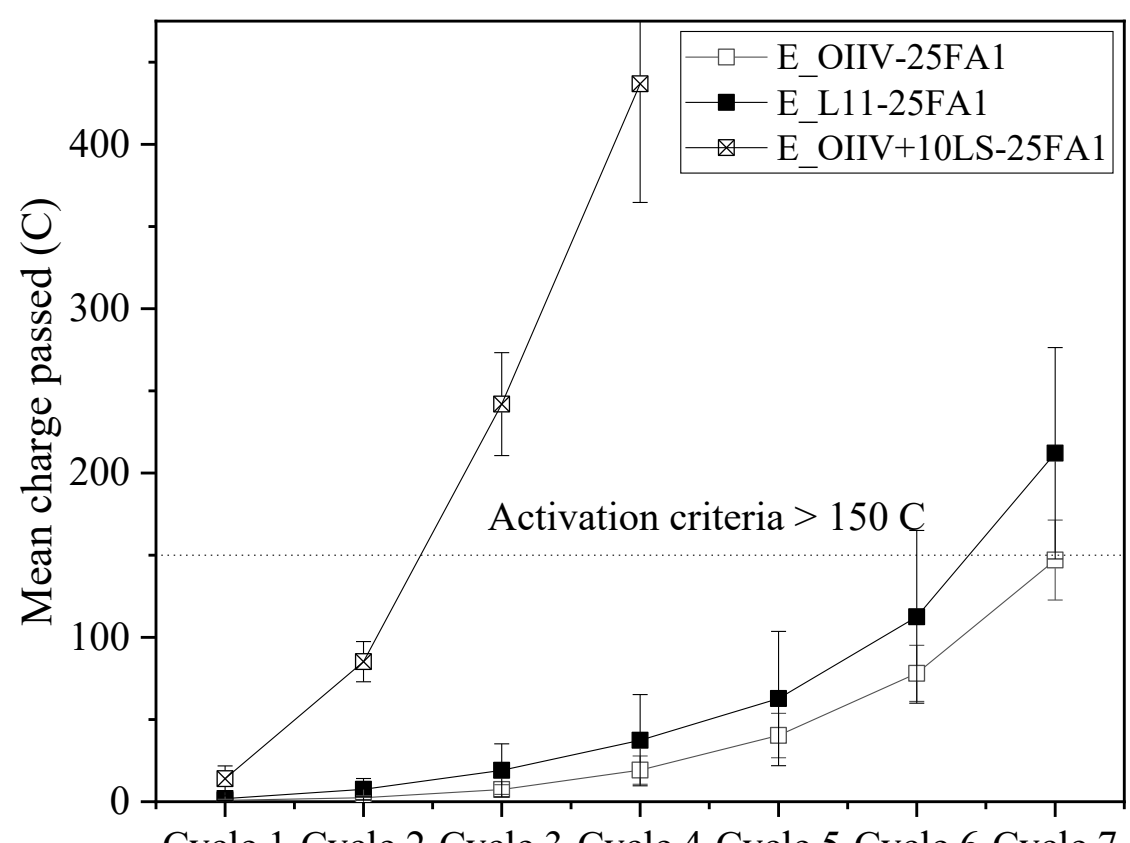

Cycle 1 Cycle 2 Cycle 3 Cycle 4 Cycle 5 Cycle 6 Cycle 7

Exposure cycles

(a)

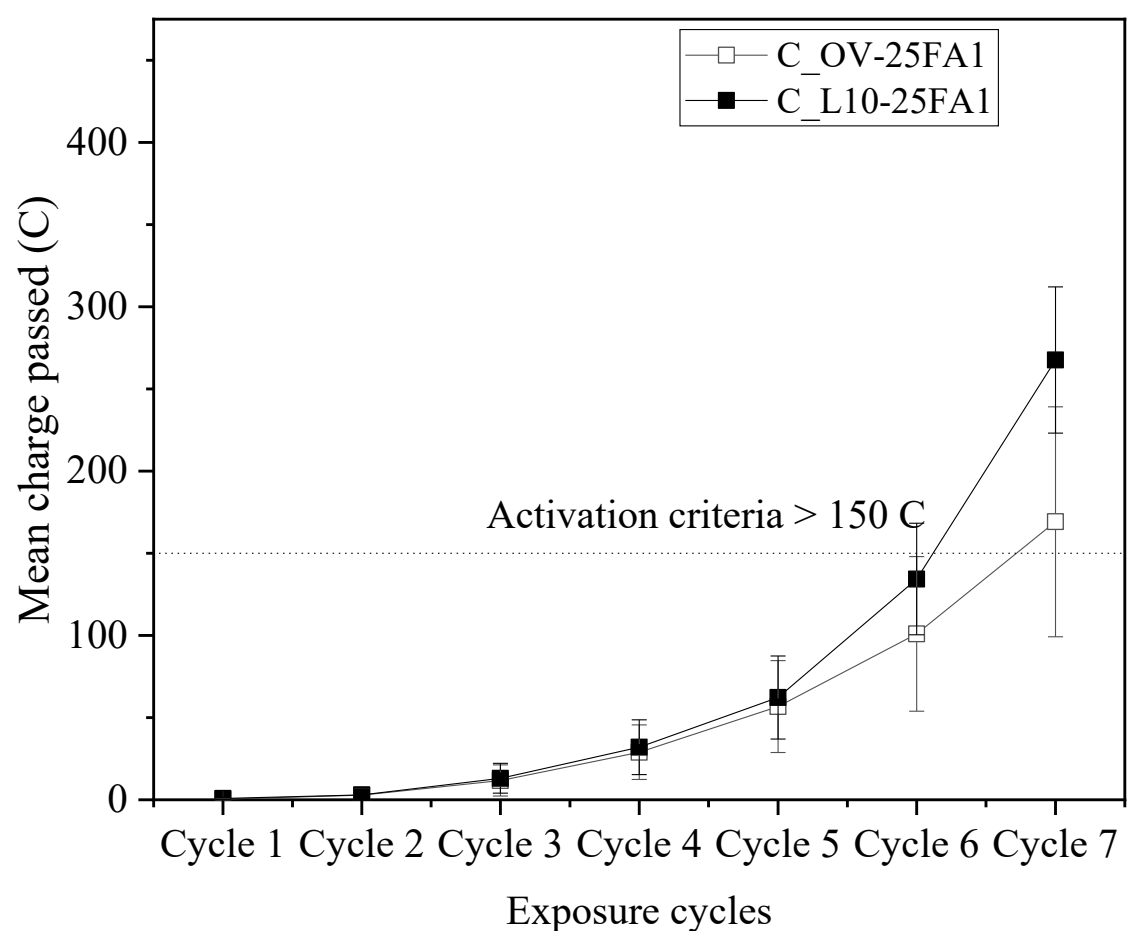

(b) 


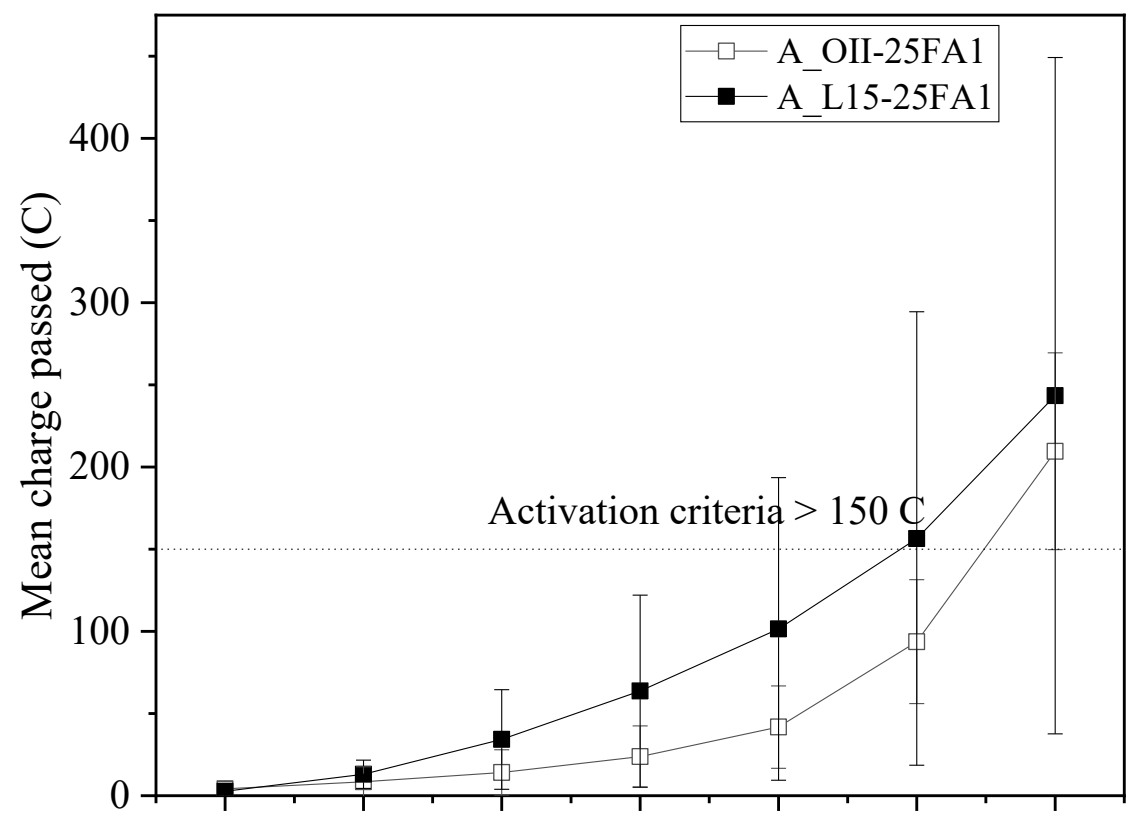

Cycle 1 Cycle 2 Cycle 3 Cycle 4 Cycle 5 Cycle 6 Cycle 7

Exposure cycles

(c)

Figure 11-13. Total integrated charge passed for modified ASTM G109 specimens

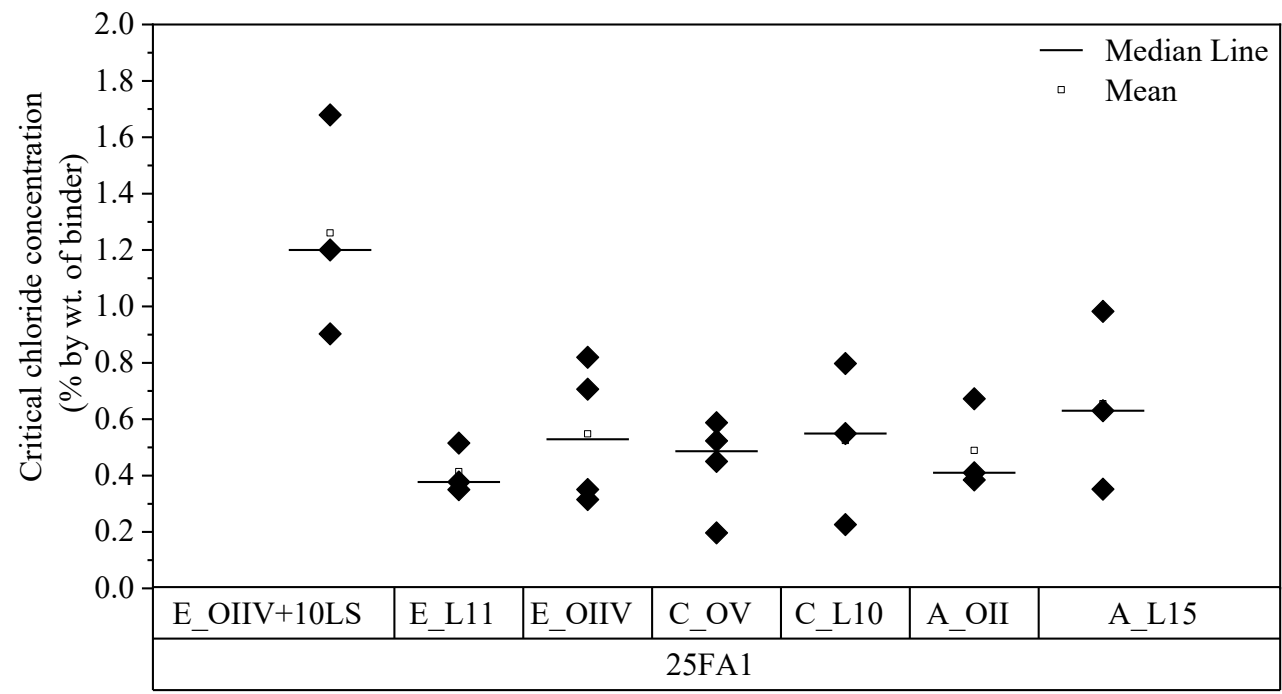




\section{$2053 \quad 11.6$ Significant Findings}

2054 In this research, OPC and PLC specimens, in combination with SCMs, were evaluated for 2055 corrosion performance using the $\mathrm{OC}_{\text {crit }}$ and modified ASTM G109 methods. The following 2056 conclusions can be drawn from the results that were shown in the earlier section:

2057 The findings from $\mathrm{OC}_{\text {crit }}$ testing indicates that OPC and PLC systems have comparable times to 2058 corrosion initiation. Both OPC and PLC systems with 25\% FA1 exhibit longer times to corrosion 2059 initiation than the control systems without fly ash. Both systems also exhibit higher $\mathrm{Cl}_{\text {crit }}$ values 2060 when compared to the control specimens. For specimens with $50 \%$ slag, the research indicates that 2061 the OPC specimens exhibited a mean time to corrosion initiation of 2.2 days.

2062 The $\mathrm{Cl}_{\text {crit }}$ of most of the specimens observed in the study is around $0.4 \%$ by weight of binder, with 2063 the exception of E_OIIV-25FA1 and E_L11-50SL. Both E_OIIV-25FA1 and E_L11-50SL 2064 exhibited higher mean $\mathrm{Cl}_{\text {crit }}$ values. This indicates that concrete containing just PLC will likely 2065 exhibit similar times to corrosion as systems containing only OPC. This also indicates that PLC 2066 systems with fly ash will likely exhibit longer times to corrosion than systems containing only 2067 OPC. Although the time to corrosion initiation of the specimens containing $50 \%$ slag was short, 2068 the mean $\mathrm{Cl}_{\text {crit }}$ values for the PLC is higher than the control, indicating longer times to corrosion 2069 initiation (assuming all other factors are the same).

2070 The findings from the modified ASTM G109 test indicate that OPC and PLC specimens exhibit 2071 similar total charge passed until the time of activation and most of the specimens were observed 2072 to activate after 7 cycles. Even though PLC specimens have a higher mean charge passed, it is 2073 deemed insignificant due to the large error bars. 


\section{Air Entrainment}

2076 Concrete is air-entrained to improve its freezing and thawing (FT) resistance. Air-entraining concrete is done by adding an air-entraining admixture (AEA) during concrete mixing. In readymixed concrete and precast this is typically done with a liquid admixture. Powdered air entrainers are also available, therefore, there is the possibility to intergrind or interblend this with the cement. The air-entraining admixture stabilizes air bubbles formed during the mixing process. The surface charge on the bubbles prevents the coalescence of bubbles and causes adherence to cement and aggregate particles. The interior of the entrained air bubbles is inherently hydrophobic so that under normal temperature and pressure conditions, the bubbles are vapor filled. However, during a freezing event the increased pressure from the expansion of freezing pore solution forces the liquid into the air bubbles. The air bubbles in hardened concrete provide extra space for the freezing pore solution to expand, thus relieving the pressure and preventing damage. Therefore, air-entrainment is recommended for all concretes exposed to freezing and thawing and as well as deicing chemicals to improve concrete durability. Air-entrainment also has other benefits such as reducing bleeding and increasing plasticity in the fresh state. In general, both minimum and maximum limits of air-entrainment are provided to offer freezing and thawing resistance and prevent compressive strength reduction [141].

\subsection{Research Objective}

2093 The objective of this part of the project is to investigate if the same amount of air-entrainment (by $\%$ volume) can be achieved with PLCs as with OPCs by using liquid air-entraining admixtures.

\subsection{Background and Literature Review}

2096 The cyclic freezing and thawing test - ASTM C666 is commonly used to evaluate the FT resistance 2097 by determining the durability factor. Thomas et al. [98] evaluated and compared the FT resistance 2098 of concretes made with OPCs and PLCs (with 12\% interground LS) using ASTM C666. The 2099 concrete specimens were made with and without SCMs. The target air content was 5-7\%. The 2100 measured air content in fresh concrete is shown in Table 12.1.

Table 12.1. Comparison of measured air contents for OPC and PLC mixtures; note that the target air content was 5-7\% [98]

\begin{tabular}{|c|c|c|c|c|c|c|c|c|}
\hline Cement & OPC & \multicolumn{1}{c|}{ PLC } & OPC & PLC & OPC & PLC & OPC & PLC \\
\hline w/cm & \multicolumn{2}{|c|}{$\mathbf{0 . 4 0}$} & \multicolumn{2}{|c|}{$\mathbf{0 . 4 5}$} & \multicolumn{2}{c|}{$\mathbf{0 . 4 5}$} & \multicolumn{2}{c|}{$\mathbf{0 . 4 5}$} \\
\hline SCM & \multicolumn{2}{|c|}{ No SCM } & \multicolumn{2}{c|}{ No SCM } & \multicolumn{2}{c|}{$35 \%$ slag } & \multicolumn{2}{c|}{$20 \%$ fly ash } \\
\hline $\begin{array}{c}\text { Measured } \\
\text { air (\%) }\end{array}$ & 6.2 & 5.4 & 6.2 & 5.3 & 6.0 & 5.6 & 5.2 & 5.0 \\
\hline
\end{tabular}


2104 As observed from Table 12.1, the measured amount of air content for the PLC mixtures was 2105 slightly lower than the OPC mixtures. The authors mentioned that the PLC mixtures required 2106 slightly higher AEA than the OPC mixtures (this is consistent with mixtures that have a higher 2107 surface area of the cementitious materials). However, it is important to note that all the mixtures 2108 had achieved the target air content. The mixtures were tested using ASTM C666 (Procedure A) to 2109 compare the FT resistance of OPC and PLC mixtures. The ASTM C666 results are presented in 2110 Figure 12-1. Personal communications have noted that about 3 million cubic yards of concrete 2111 have been made and placed using PLC with air entraining admixtures without any issues related 2112 to the PLC cement.

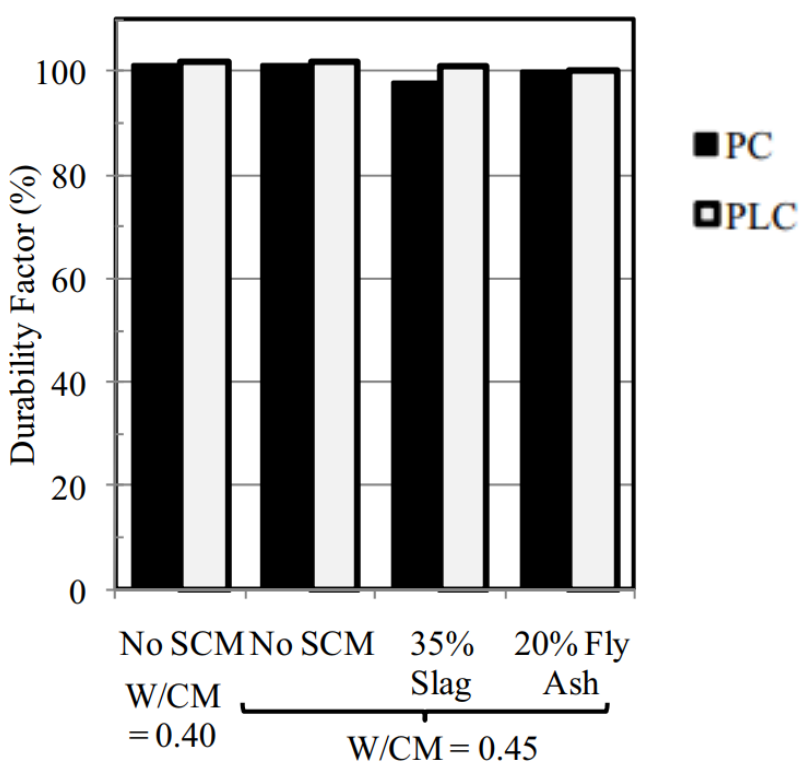

2114 Figure 12-1. Durability factor of the mixtures tested using ASTM C666 (Procedure A) [98]

2115 All of the concrete mixtures performed well in cyclic FT environment per ASTM C666 with 2116 durability factors ranging from 98 to $102 \%$. No significant difference in FT performance of OPC 2117 and PLC mixtures was observed.

\section{$2118 \quad 12.3$ Experimental Test Matrix}

2119 Two sets of concrete mixtures were cast to compare the air-entrainment of OPC and PLC mixtures.

2120 Two different fine aggregates (F1 and F2) were used for each set of mixtures. High purity 2121 limestone was used as coarse aggregate. The coarse aggregate used has proportions following 2122 ASTM C1293 as the same mixtures were used for making concrete prism test specimens for alkali2123 silica reactivity (ASR) testing. Air entrainment was done after the ASR prisms were cast. More 2124 information on the concrete prism test can be found in section 5.9. Equal proportions of coarse 2125 aggregate particles retained on $12.5 \mathrm{~mm}, 9.5 \mathrm{~mm}$, and $4.75 \mathrm{~mm}$ sieves were used for all the mixtures. 
2126 Cements B_OIIV and B_L15 were used for this task. The AEA used for this task was BASF Master 2127 AE90.

\section{$2128 \quad 12.4$ Experimental Methods}

2129 The pressure method following ASTM C231/C231M was used to measure the air content in fresh 2130 concrete. For each mixture, the batch amounts were calculated to determine air contents at three 2131 different AEA dosages starting with $0 \mathrm{ml} / 100 \mathrm{~kg}$ cement (i.e., no added air). After measuring the 2132 air content of fresh concrete when no AEA was added, the first dose of AEA was added to the 2133 concrete to obtain an air content of $3.5 \pm 1 \%$ and mixed the concrete for two minutes. After 2134 measuring the air content of concrete for the second AEA dosage, more AEA was added to obtain $21356.5 \pm 1 \%$ air content. During this mixing and testing, the concrete that was used to measure air 2136 content was not re-used.

\section{12.5 Typical Experimental Measurement and Interpretation}

2138 The measured air content of fresh concrete is reported in terms of the percentage of total concrete

2139 volume. At each dosage of AEA, only one measurement of air content was made.

\section{$2140 \quad$ 12.6 Experimental Results}

2141 The measured air contents at different dosages of the AEA for both sets of concrete mixtures are 2142 listed in Table 12.2 and Table 12.3. The manufacturer's recommended AEA dosage range was 16 $2143-260 \mathrm{ml} / 100 \mathrm{~kg}$ cement.

Table 12.2. The measured air contents of the mixtures with $F 1$ fine aggregate

\begin{tabular}{|c|c|c|c|}
\hline \multicolumn{2}{|c|}{ B_OIIV - F1 } & \multicolumn{2}{c|}{ B_L15 - F1 } \\
\hline $\begin{array}{c}\text { AEA dose (ml/100kg } \\
\text { cement) }\end{array}$ & $\begin{array}{c}\text { Measured air } \\
\text { content (\%) }\end{array}$ & $\begin{array}{c}\text { AEA dose (ml/100kg } \\
\text { cement) }\end{array}$ & $\begin{array}{c}\text { Measured air } \\
\text { content (\%) }\end{array}$ \\
\hline 0 & 1.0 & 0 & 1.0 \\
\hline 48 & 4.0 & 48 & 3.0 \\
\hline 79 & 7.5 & 79 & 7.0 \\
\hline
\end{tabular}


Table 12.3. The measured air contents of the mixtures with $F 2$ fine aggregate

\begin{tabular}{|c|c|c|c|}
\hline \multicolumn{2}{|c|}{ B_OIIV - F2 } & \multicolumn{2}{c|}{ B_L15 - F2 } \\
\hline $\begin{array}{c}\text { AEA dose (ml/100kg } \\
\text { cement) }\end{array}$ & $\begin{array}{c}\text { Measured air } \\
\text { content (\%) }\end{array}$ & $\begin{array}{c}\text { AEA dose (ml/100kg } \\
\text { cement) }\end{array}$ & $\begin{array}{c}\text { Measured air } \\
\text { content (\%) }\end{array}$ \\
\hline 0 & 1.8 & 0 & 1.8 \\
\hline 56 & 2.8 & 64 & 3.1 \\
\hline 181 & 3.1 & 189 & 4.1 \\
\hline
\end{tabular}

\section{$2149 \quad 12.7$ Discussion of the Results}

2150 From Table 12.2 and Table 12.3, it was observed that with the increase in AEA dosage, the amount 2151 of measured air increased, as expected. For the mixtures with F1 fine aggregate, it was observed 2152 that the PLC concrete had air content equal to or slightly less than the OPC concrete. It should be 2153 noted that both the OPC and PLC concretes reached the target air contents and were within a 2154 desired range of $\pm 1 \%$. For the mixtures with F2 fine aggregate, it was observed that the target air 2155 content was not achieved for the highest AEA dosage for both cements. The reason for this could 2156 be a high moisture content (12\%) and absorption capacity $(4.2 \%)$ of the F2 fine aggregate. 2157 However, the amount of measured air contents for both the OPC and PLC concrete mixtures were 2158 similar at all AEA dosages. From the study by Thomas et al. [98], it was observed that even though 2159 PLC concrete had slightly lower (by $\sim 1 \%$ ) air content than the OPC concrete, the measured 2160 durability factor from ASTM C666 testing was very similar for both OPC and PLC concretes. 2161 Therefore, the OPC and PLC concrete mixtures made in this project are expected to perform 2162 similarly in the cyclic FT environment.

\section{$2163 \quad 12.8$ Significant Findings}

2164 There was no significant difference in measured air contents between OPC and PLC concrete 2165 mixtures. The OPC and PLC concrete mixtures with similar air contents are expected to perform 2166 similarly in the FT environment. 
Table 8. Concrete Mix Proportions and Test Results - Study 3

\begin{tabular}{l|cc|cc|cc|cc}
\multirow{2}{*}{} & \multicolumn{2}{|c|}{ No SCM } & \multicolumn{2}{c|}{$25 \%$ SCM } & \multicolumn{2}{c|}{$40 \%$ SCM } & \multicolumn{2}{c}{$50 \%$ SCM } \\
\cline { 2 - 9 } & PC & PLC & PC & PLC & PC & PLC & PC & PLC \\
\hline W/CM & 0.45 & 0.44 & 0.44 & 0.45 & 0.44 & 0.44 & 0.44 & 0.44 \\
\hline Plastic air, \% & 6.8 & 6.0 & 6.2 & 6.6 & 6.8 & 6.0 & 6.8 & 6.5 \\
Slump, mm & 100 & 80 & 75 & 100 & 95 & 80 & 95 & 95 \\
Slump, in. & 3.9 & 3.1 & 3.0 & 3.9 & 3.7 & 3.1 & 3.7 & 3.7 \\
\hline Hardened air, \% & 5.3 & 5.6 & 4.9 & 5.4 & 5.6 & 5.3 & 5.6 & 6.6 \\
Spacing factor, $\mu \mathrm{m}$ & 173 & 187 & 148 & 149 & 164 & 165 & 150 & 147 \\
Spacing factor, in. & 0.068 & 0.074 & 0.058 & 0.059 & 0.065 & 0.065 & 0.059 & 0.058 \\
\hline
\end{tabular}

2168 This study from Thomas and Hooton 2010 (reference \#98 in v05) shows that the spacing factor is 2169 nearly identical for all mixtures with SCMs whether the binder was portland cement or portland2170 limestone cement. The spacing factor was slightly higher in the case of $100 \%$ portland or portland2171 limestone cement. 


\section{External Sulfate Attack}

2174 California is known to have sulfate-rich soils. Sulfates from external sources can ingress into 2175 concrete and cause damage. The damage due to external sulfate attack (ESA) in concrete is 2176 primarily caused by secondary ettringite formation. Calcium sulfate in the form of gypsum in 2177 cement reacts with hydrated calcium aluminate to form ettringite $2178\left(\mathrm{Ca}_{6}(\mathrm{Al}, \mathrm{Fe})_{2}\left(\mathrm{SO}_{4}\right)_{3}(\mathrm{OH})_{12} \cdot 26 \mathrm{H}_{2} \mathrm{O} ; \mathrm{AFt}\right)$ during early cement hydration. The ettringite forms early 2179 during hydration reacts with remaining anhydrous aluminate to form monosulfoaluminate $2180 \quad\left(3 \mathrm{CaO} .(\mathrm{Al}, \mathrm{Fe})_{2} \mathrm{O}_{3} \cdot \mathrm{CaSO}_{4} \cdot 12 \mathrm{H}_{2} \mathrm{O} ; \mathrm{AFm}\right)$. The sulfates present in hydrated cement are mainly these 2181 ettringite and monosulfoaluminate. These two phases are finely intermixed with the C-S-H. When 2182 external sulfates are present, sulfates ingress and react with AFm and form AFt. This secondary 2183 formation of $\mathrm{AFt}$ from AFm can develop stresses in hardened concrete as AFt occupies more than 2184 twice the volume of AFm [20]. Sulfate attack can also be from internal sources such as concrete 2185 having sulfide-bearing aggregates or delayed ettringite formation due to thermal activation 2186 occurring during initial hydration.

2187 The reaction products of sulfates and cement hydrates that induce damage are mainly of three 2188 types: $\mathrm{AFt}$, gypsum $\left(\mathrm{CaSO}_{4} \cdot 2 \mathrm{H}_{2} \mathrm{O}\right)$, and thaumasite $\left(3 \mathrm{CaO} \cdot \mathrm{SiO}_{2} \cdot \mathrm{SO}_{4} \cdot \mathrm{CO}_{3} \cdot 15 \mathrm{H}_{2} \mathrm{O}\right)$. AFt formed in 2189 pores smaller than $100 \mathrm{~nm}$ generates crystallization pressure enough to develop cracks in the 2190 cementitious matrix, and the supersaturation of the pore solution is needed in the pores to generate 2191 crystallization pressure [142, 143]. Mullauer et al. [144] observed that AFt formed in small pores $2192(10-50 \mathrm{~nm})$ generated stresses that overcame the tensile strength of the cementitious matrix. This 2193 results in expansion and eventually damage of the matrix.

2194 Gypsum can form when calcium hydroxide reacts with ingressed sulfates, as shown in equation $2195 \quad(13-1)$

$$
\mathrm{CH} \text { (from cement hydration) }+\mathrm{N} \underline{\mathrm{S}} / \mathrm{M} \underline{\mathrm{S}}+2 \mathrm{H} \rightarrow \mathrm{CS}_{2}+\mathrm{NH} / \mathrm{MH}
$$

2197 Gypsum formation can also contribute to the expansion of cement mortars under exposure to high 2198 sulfate concentrations [145]. The formed gypsum can further react with unhydrated calcium 2199 aluminate, hydrated calcium aluminate or AFm to form AFt, as shown in equations (13-2), (13-3), 2200 and (13-4) [146].

$$
\begin{aligned}
& \mathrm{C}_{4} \mathrm{AH}_{13}+3 \mathrm{C} \underline{\mathrm{S}} \mathrm{H}_{2}+14 \mathrm{H} \rightarrow \mathrm{C}_{6} \mathrm{~A}_{3} \mathrm{H}_{32}+\mathrm{CH} \\
& \mathrm{C}_{4} \mathrm{~A} \underline{\mathrm{S}} \mathrm{H}_{12-18}+2 \mathrm{C} \underline{\mathrm{S}}_{2}+(10-16) \mathrm{H} \rightarrow \mathrm{C}_{6} \mathrm{~A}_{3} \mathrm{H}_{32} \\
& \mathrm{C}_{3} \mathrm{~A}+3 \mathrm{C} \underline{\mathrm{S}}_{2}+26 \mathrm{H} \rightarrow \mathrm{C}_{6} \mathrm{~A}_{3} \underline{\mathrm{S}}_{32}
\end{aligned}
$$


Thaumasite forms in the presence of limestone (as a filler or aggregate) and when exposed to sulfate solution. Structurally, thaumasite is similar to ettringite. Thaumasite forms as a non-binding calcium carbonate silicate sulfate hydrate and needs specific conditions, such as low temperatures $\left(<15^{\circ} \mathrm{C}\right)$, presence of carbonates or bi-carbonates, and moisture. According to Hobbs who studied thaumasite attack in laboratory and field concretes, the combination of conditions needed for thaumasite attack are a low temperature, wet conditions, exposure to a sulfate source, the prior formation of AFt from classical sulfate attack, presence of limestone in the system (at least 15\% to $35 \%$ calcium carbonate by mass of cement from laboratory observations) [147]. Thaumasite was also found to be formed at relatively higher temperatures; however, high limestone contents $(>15 \%)$ and prior damage by secondary AFt formation are needed $[148,149]$.

The cation associated with sulfate affects the extent of damage caused by sulfate attack. The most common cations that are in compound with sulfate and thus attack concrete are calcium, sodium, and magnesium sulfates. Calcium sulfate is the least aggressive, and magnesium sulfate is the most aggressive in terms of sulfate attack. The higher solubility of sodium and magnesium sulfates the more aggressive the attack and resulting damage. AFt and gypsum are formed from AFm, hydrated aluminates, $\mathrm{C}_{3} \mathrm{~A}$, and in severe cases, $\mathrm{C}-\mathrm{S}-\mathrm{H}$ under sodium sulfate attack. Sodium sulfate can also cause physical salt attack. AFt, gypsum, brucite, and silica gel are formed under magnesium sulfate attack. The formation of brucite lowers the $\mathrm{pH}$ of the pore solution, and this encourages gypsum formation. These reactions continue until $\mathrm{CH}$ and $\mathrm{C}-\mathrm{S}-\mathrm{H}$ are exhausted, resulting in loss of cohesion and softening of the cementitious matrix [150].

2221 To limit the formation of AFt from sulfate attack, low $\mathrm{C}_{3} \mathrm{~A}$ portland cements or sulfate resistant cements such as ASTM C150 Type II and Type V cements are generally recommended. However, it was observed that usage of low $\mathrm{C}_{3} \mathrm{~A}$ cements alone might not prevent damage due to sulfate attack if the permeability of the concrete is high or the degradation of concrete is due to physical salt attack [151]. Therefore, SCMs and lower water to binder ratio are also used to improve the sulfate resistance of concrete.

\section{$2227 \quad 13.1$ Research Objective}

2228 The main objective in this part of the project is to extensively study the influence of usage of PLC 2229 in conjunction with SCMs on the expansion due to ESA. The results of this Task will inform 2230 CALTRANS if their current mixtures with SCMs can be utilized as-is when combined with a PLC 2231 with up to $15 \%$ limestone.

\section{$2232 \quad 13.2$ Background and Literature Review}

2233 Using limestone in a cementitious system can impart changes in the capillary porosity due to 2234 physical effects such as filler effect, dilution effect, and nucleation effect [152]. These physical 2235 effects depend on the fineness, amount, and purity of limestone filler used. The effective water to 
cement ratio increases due to the dilution effect when a certain portion of cement is replaced with limestone filler. Therefore, an increase in cement replacement with limestone increases the water to cement ratio of the paste due to the dilution effect, and it is only slightly compensated by the other two effects. These physical effects, in turn, affect the water transport of the cementitious system [153]. Transport properties of concrete can govern the ESA resistance. Some studies [154, 155] reported that high limestone contents $(>15 \%)$ increased the porosity and pore interconnectivity, thus reducing the ESA resistance. However, the mixtures with limestone contents less than $15 \%$ when used in conjunction with appropriate levels of SCMs were observed to perform similar to OPCs [150].

Hooton and Thomas [150] evaluated the sulfate resistance of mortars (in laboratory conditions) and concrete (in simulated field conditions) produced with PLCs with up to $15 \%$ limestone. The PLCs were produced with both higher- $\mathrm{C}_{3} \mathrm{~A}(11-12 \%)$ and moderate $\mathrm{C}_{3} \mathrm{~A}(8-9 \%)$ clinkers. The authors observed that when the expansions were mitigated with appropriate levels of SCMs, there was no influence of limestone on sulfate attack resistance. The concrete samples produced with PLCs and appropriate amounts of SCMs under simulated field conditions (very severe sulfate exposure) performed similar or better than ASTM Type V cements for up to 5 years [150].

Hooton and Thomas [150] also studied sulfate resistance of OPCs and PLCs (with up to 15\% limestone) at a low temperature of $5^{\circ} \mathrm{C}$. The mortar specimens evaluated with modified ASTM $\mathrm{C} 1012\left(5^{\circ} \mathrm{C}\right)$ showed that the non-sulfate resistant mixtures are initially damaged by ettringitebased sulfate attack and thaumasite is only observed after significant deterioration. Therefore, by using appropriate amounts of SCMs and low $\mathrm{C}_{3} \mathrm{~A}$ cements to prevent classical sulfate attack, thaumasite sulfate attack can also be prevented. Also, the authors recommended not to use modified ASTM C1012 $\left(5^{\circ} \mathrm{C}\right)$ to evaluate low-temperature sulfate attack as it is overly aggressive and did not provide reliable results relating to the performance of cementitious materials at cold temperature in field exposure. It was observed that the amount of limestone up to $15 \%$ in the PLCSCM blends had little impact on the performance of mortars or concretes in sulfate environment at $5^{\circ} \mathrm{C}$ when tested in either laboratory or field simulated conditions. Moreover, the authors observed that the concrete containing PLC and SCMs generally perform similar, if not better, than concrete containing ASTM C150 Type II or Type V cement at the same $\mathrm{w} / \mathrm{cm}$ and in the same sulfate-exposure condition [150].

Figure 13-1 presents the expansion ratio (PLC/OPC) of mortars with plain cements subjected to sodium sulfate solution according to ASTM C1012 at different amounts of limestone in the mixture [156-159]. Similarly, Figure 13-2 presents the expansion ratio (PLC/OPC) of mortars with SCMs subjected to sodium sulfate solution according to ASTM C1012 at different amounts of limestone in the mixture [160-163]. The data in Figure 13-1 and Figure 13-2 include expansion measurements obtained at $6,12,18$ months of exposure to $50 \mathrm{~g} / \mathrm{L} \mathrm{Na}_{2} \mathrm{SO}_{4}$ solution at $23^{\circ} \mathrm{C}$. 


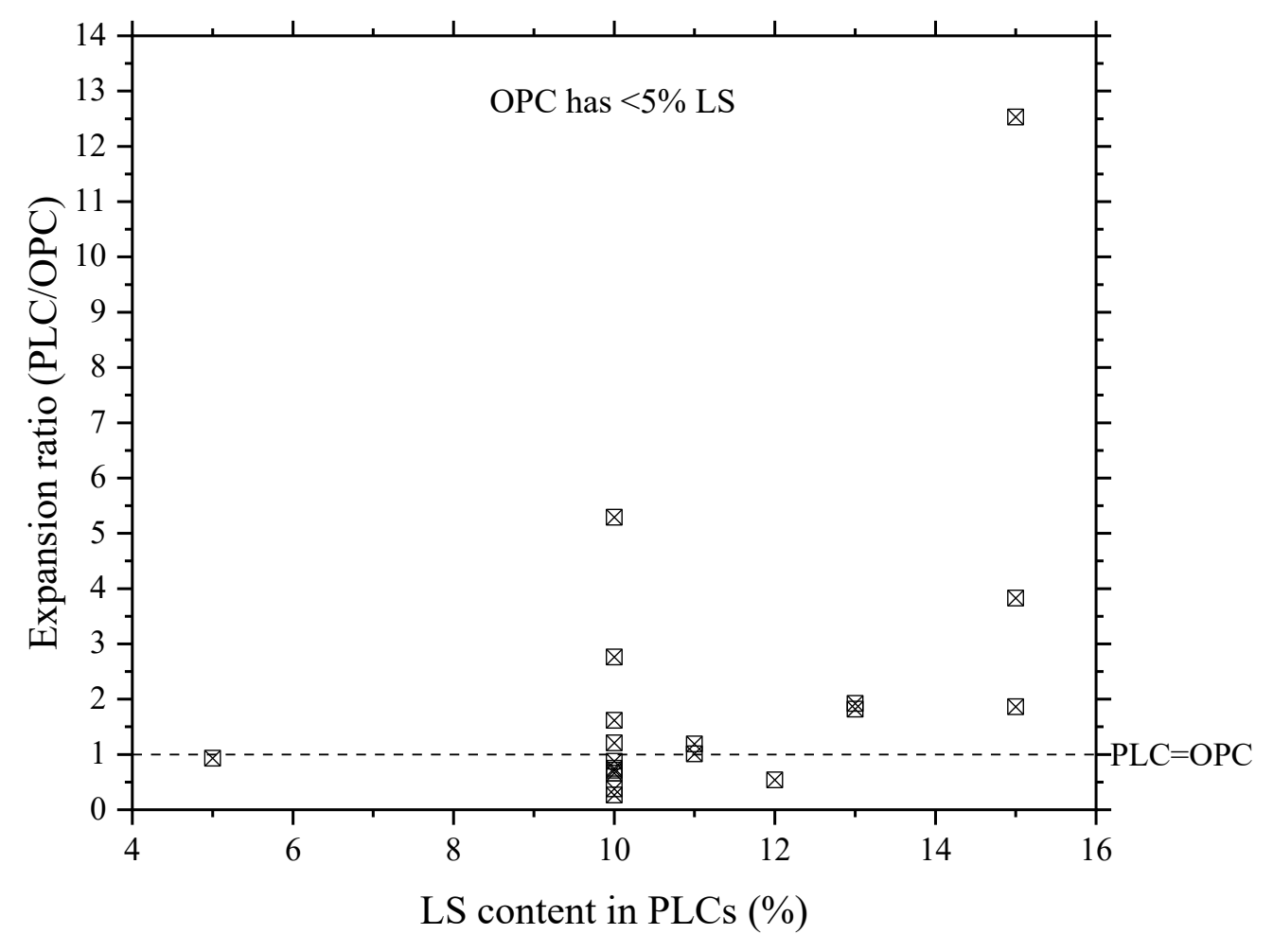

Figure 13-1. External sulfate attack expansion ratio (PLC/OPC) of mortars with plain cements tested according to ASTM C1012 [156, 158, 159, 162]

2275 In the absence of SCMs, the mixtures with PLCs (up to 15\% limestone) expanded more than the 2276 mixtures with OPC (with $<5 \%$ limestone) with an expansion ratio as high as 12.5 , as shown in 2277 Figure 13-1. As discussed earlier, the expansion mainly depends on $\mathrm{C}_{3} \mathrm{~A}$ content of cement, the 2278 fineness and amount of limestone, and how that affects porosity and pore connectivity of the 2279 mixtures. 


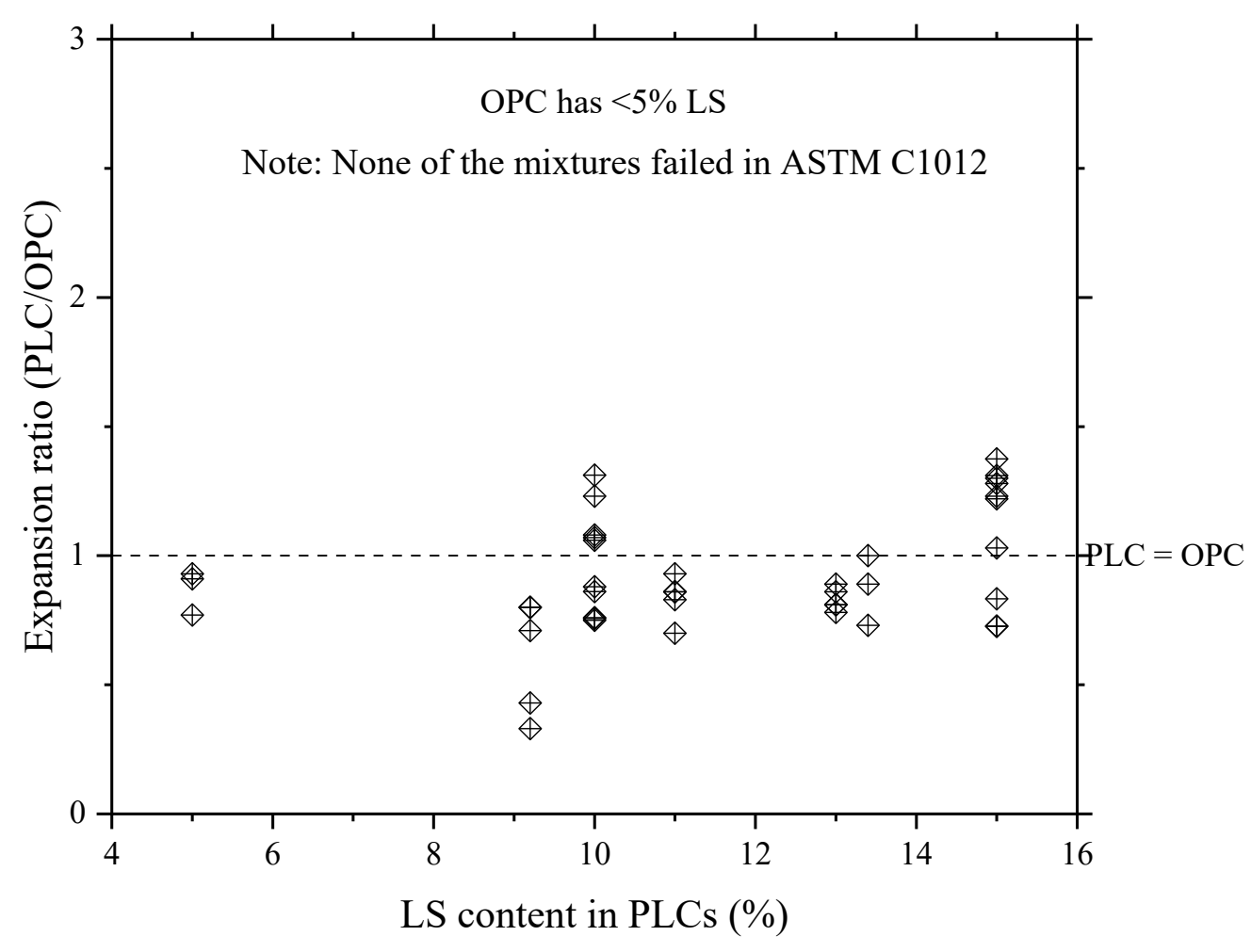

\section{Figure 13-2. External sulfate attack expansion ratio (PLC/OPC) of mortars with SCMs tested according to ASTM C1012 [160-163]}

2283 In the presence of SCMs, the expansion ratio (PLC/OPC) did not exceed 1.4, and in most cases, 2284 the expansion ratio is less than 1.0, as shown in Figure 13-2. In addition, for the data shown in 2285 Figure 13-2, none of the PLC mixtures failed ASTM C1012 even though some of the mixtures 2286 with PLCs expanded slightly more than their respective OPCs.

2287 In this project, moderate and high sulfate resistant cements were used to evaluate PLCs (with up 2288 to $15 \%$ limestone) performance in the presence of SCMs using ASTM C1012. Damage due to 2289 thaumasite formation is not expected in the mortars tested in this project as the limestone content 2290 is not greater than $15 \%$, low $\mathrm{C}_{3} \mathrm{~A}$ cements were used, high amounts of SCMs were used, the 2291 specimens were stored at room temperature, and completely submerged in sodium sulfate solution.

2293 Two OPCs with different $\mathrm{C}_{3} \mathrm{~A}$ contents - A_OII and D_OV were selected for this Task to evaluate 2294 and compare them to their respective PLC mixtures in conjunction with SCMs according to ASTM 2295 C1012. A total of 32 mixtures were cast including the controls (A_OII and D_OV, without SCMs) 2296 as shown in Table 13.1. 


\begin{tabular}{|c|c|}
\hline Cements & $\begin{array}{c}\text { SCM } \\
\text { proportions }\end{array}$ \\
\hline A_OII & 25FA1 \\
A_L15 & 20FA1-5SF \\
A_OII+10LS & 50SL \\
D_OV & 25FA1-25SL \\
D_L15 & 25NP \\
D_OII+10LS & \\
\hline Controls & A_OII, D_OV \\
\hline
\end{tabular}

\subsection{Experimental Methods}

2300 The sulfate resistance of the cements was evaluated in accordance with ASTM C1012. Standard 2301 Ottawa sand was used to cast mortar bars and cubes. Six mortar bars of dimensions $25 \times 25 \times 285$ $2302 \mathrm{~mm}$ were cast for each mixture to monitor their expansions. Mortar cubes of dimensions $50 \times 50$ $2303 \mathrm{~mm}$ were cast to determine their compressive strength. The bars and cubes were cured at $35 \pm 3^{\circ} \mathrm{C}$ 2304 and $100 \% \mathrm{RH}$ for the first 24 hours after casting. Later, the mortar bars and cubes were cured in a 2305 lime saturated solution at $23^{\circ} \mathrm{C}$ until they reached a compressive strength of $20 \mathrm{MPa}$. Then, the 2306 bars were immersed in a $50 \mathrm{~g} / \mathrm{L}$ of sodium sulfate $\left(\mathrm{Na}_{2} \mathrm{SO}_{4}\right)$ solution as shown in Figure 13-3, and 2307 their length change was measured periodically for one year according to ASTM C1012. The 2308 sodium sulfate solution was renewed after every measurement.

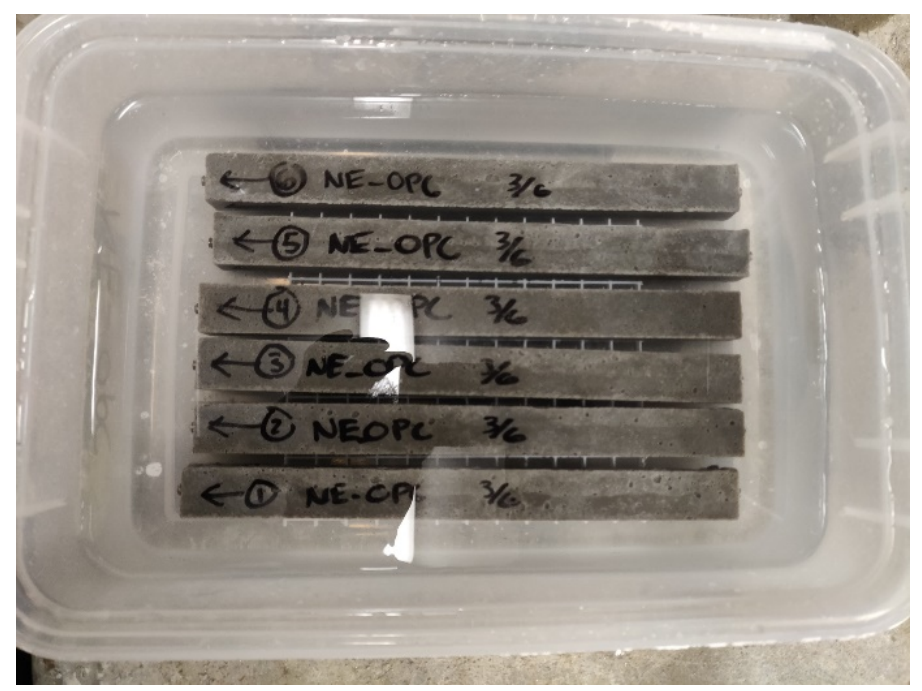




\subsection{Typical Experimental Measurement and Interpretation}

2312 Length and mass measurements were taken before immersing the bars in sodium sulfate solution 2313 (as initial measurements) and at various days after immersion until one year. After every 2314 measurement, the sodium sulfate solution was renewed. The length measurements were made 2315 using a length comparator. The measured lengths were then used to calculate the expansion of the 2316 bars. Then the average expansions of six bars per mixture were calculated and reported. According 2317 to ACI 318-19, the 6-month ASTM C1012 expansion limit is $0.05 \%$, and the 1-year ASTM C1012 2318 expansion limit is $0.10 \%$. If the average expansion of a particular mixture is more than the 2319 expansion limit, the mixture has failed the test. And, it can be interpreted as the cementitious 2320 material used in the mixture cannot mitigate external sulfate attack.

\section{$2321 \quad$ 13.6 Experimental Results}

2322 The expansion results of the mortar bars at six months (26 weeks) and one year (52 weeks) for 2323 cement A are provided in Figure 13-4 and Figure 13-5, respectively. Similarly, the expansion 2324 results for the mortar bars at six months (26 weeks) and one year (52 weeks) for cement D are 2325 provided in Figure 13-6 and Figure 13-7, respectively.

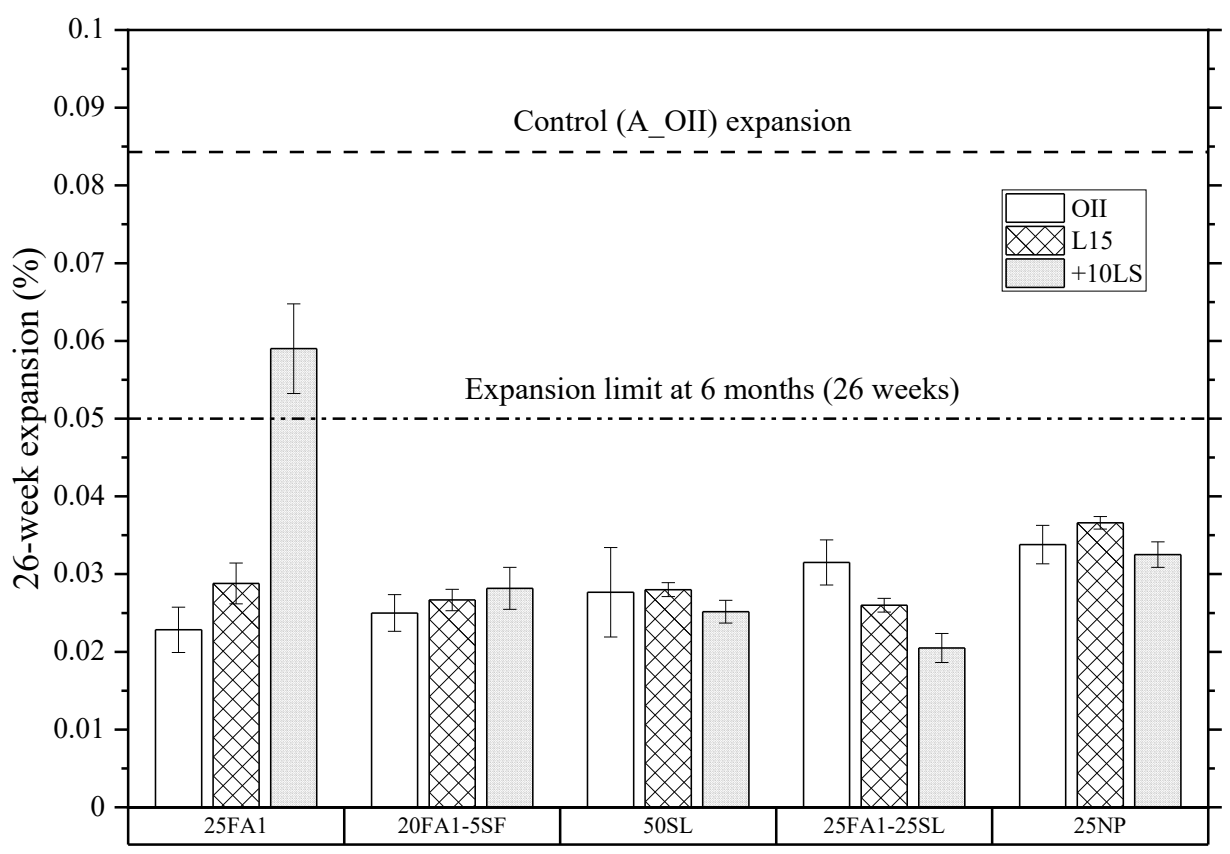




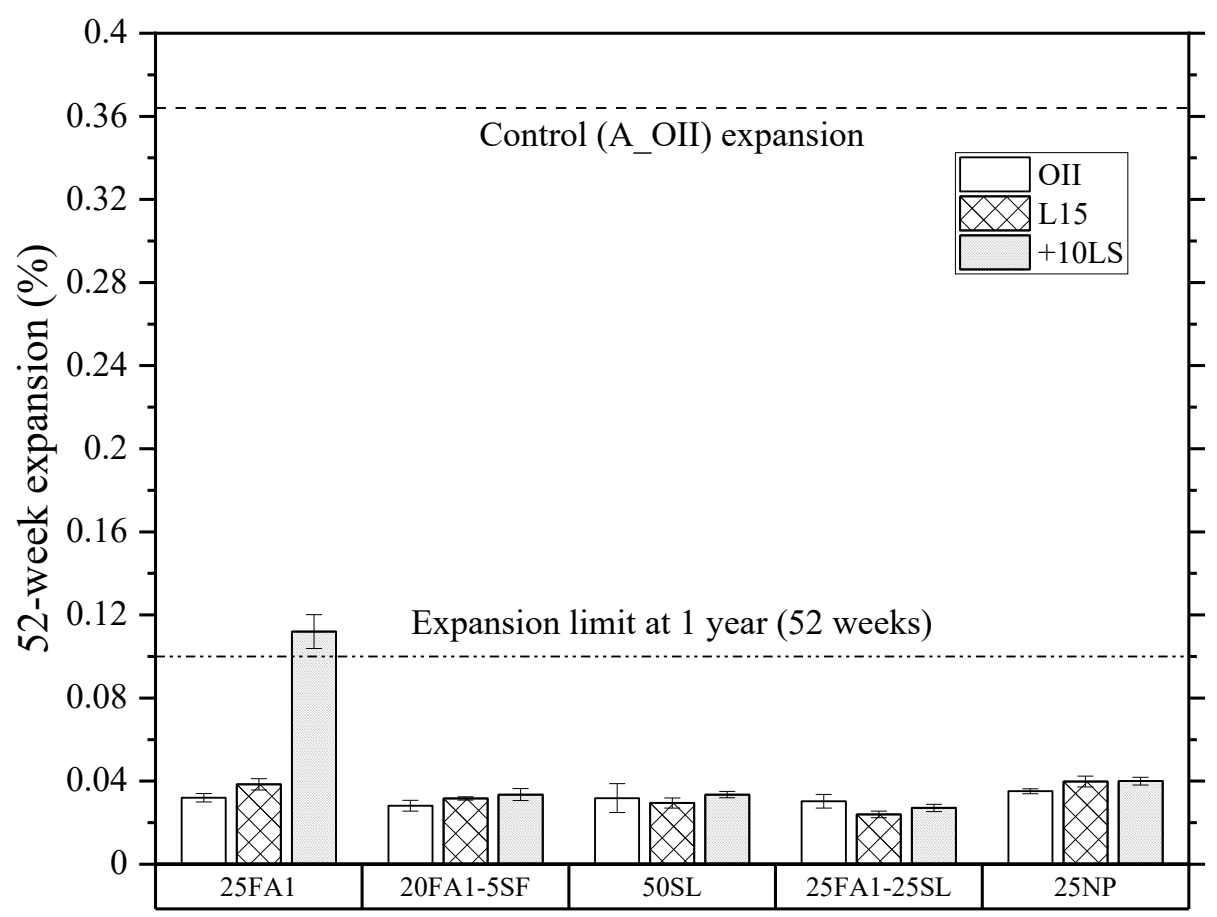

Figure 13-5. 1-year (52 weeks) expansion data of the mortar bars with clinker $\mathrm{A}$

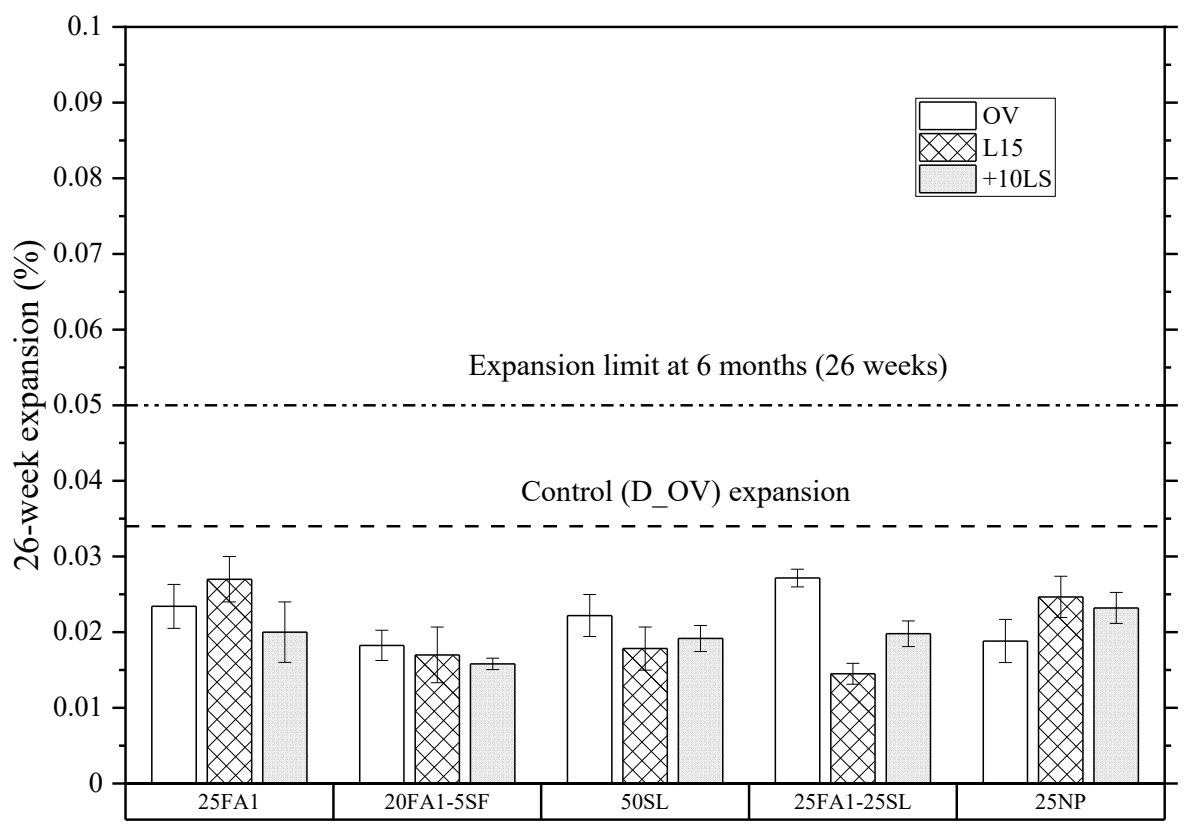




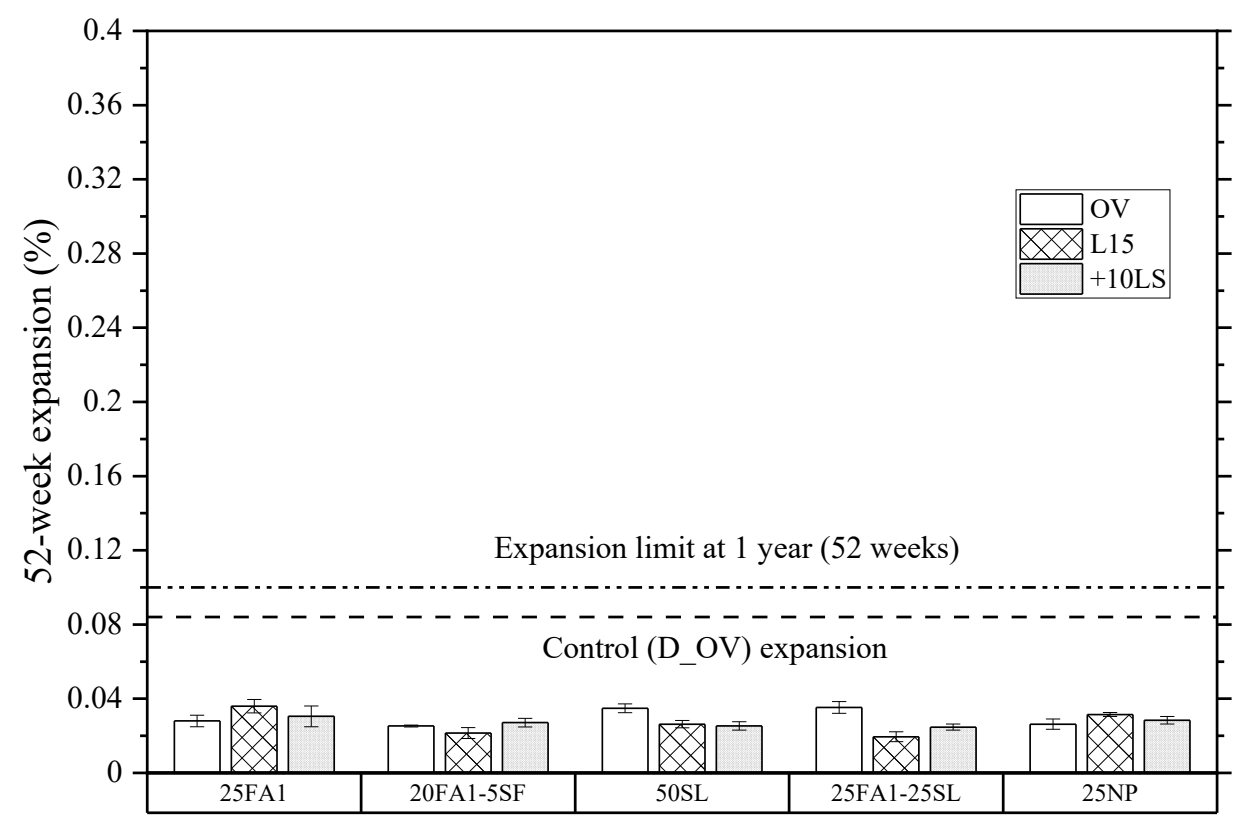

Figure 13-7. 1-year (52 weeks) expansion data of the mortar bars with clinker D

\subsection{Discussion of the Results}

As seen in Figure 13-4 and Figure 13-5, the mixtures with SCMs reduced the expansion significantly when compared to the control mixture with no SCMs. Except in the case of cement A and 25\% FA1, there was no significant difference in both 6-month (26-week) and 1-year (52week) expansions observed between the A_OII, A_L15, and A_OII +10LS mixtures in the presence of SCMs. In the case of mixtures with 25FA1-25SL, PLC mortars were observed to perform slightly better than the OPC mortar. The mixture - A_OII+10LS _25FA1 was observed to be expanding higher than A_OII_25FA1 and A_L15_25FA1 mixtures. The reason for this is unknown. Potential reasons include incorrect initial length measurements or incorrect material proportions used for mixing. Therefore, the expansion result of A_OII+10LS_25FA1 is considered to be an outlier.

From Figure 13-6 and Figure 13-7, the SCMs' ability to reduce the expansion significantly when compared to the control mixture (D_OV) is evident. There was no significant difference observed in both 6-month (26-week) and 1-year (52-week) expansions between the D_OV, D_L15, and D_OV+10LS mixtures in the presence of SCMs. In the case of mixtures with 25FA1-25SL, PLC mortars were observed to perform slightly better than the OPC mortar, probably due to better pore refinement. Figure 13-8 illustrates the comparison of 6-month expansions of OPC and PLC mortars of both cements $\mathrm{A}$ and $\mathrm{D}$ (30 mixtures). The Y-axis represents the 6-month expansion of the PLC (interground) and OPC+LS mortars. The X-axis represents the 6-month expansion of the 
2354 OPC mortars. The data points along the 1:1 solid line imply that there is no difference in the 2355 expansion of the mortars with OPC and PLC. The dashed lines represent a variation of $20 \%$ from 2356 equivalence.

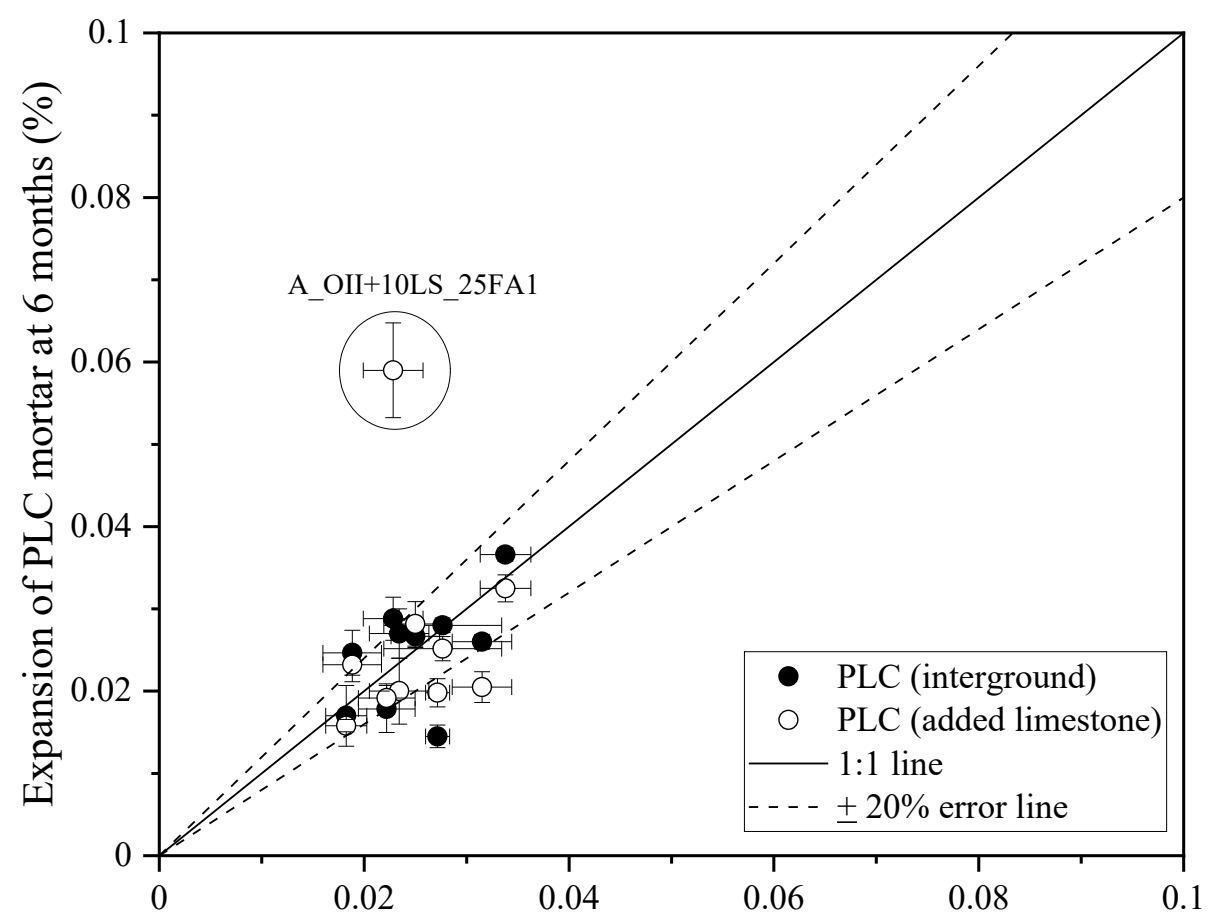

Expansion of OPC mortar at 6 months (\%)

Figure 13-8. ASTM C1012 6-month expansion results: comparison of OPC and PLC mixtures

2360 From Figure 13-8, it was observed that most of the mixtures fall very close to the 1:1 line and 2361 within the $20 \%$ error lines, implying that there is no significant difference in the expansions 2362 observed between the OPC and PLC mortars. The mixture- A_OII+10LS _25FA1 can be clearly 2363 identified as an outliner in the plot.

\section{$2364 \quad 13.8$ Significant Findings}

2365 The presence of all $\mathrm{SCM}(\mathrm{s})$ reduced expansions significantly compared to the control (with no 2366 SCM). The main takeaway from this Task is that, overall, the PLCs performed similar to, if not 2367 better than, their corresponding OPC mixtures in the presence of SCMs. Therefore, CALTRANS 2368 can use their current SCM mixtures as is with ASTM C 150 Type II or V clinker that is then 2369 interground or interblended with PLC up to 15\% limestone and expect similar external sulfate 2370 resistance to ASTM C150 Type II or V cements with no or very low amounts of 2371 interground/interblended limestone. 


\section{Construction Schedule}

2373 Certain concrete characteristics can influence the effort required and rate at which the concrete is placed in the field. Two commonly measured characteristics that can influence the constructability and scheduling of concrete construction projects is the workability and setting of the concrete mixtures. Workability, or more specifically slump of the concrete mixtures, is shown in Figure 141. This figure shows the slump of concrete mixtures made from the three clinker sources with their respective SCM combinations. The target slump was 4 inches in most cases. It can be seen that the majority of the slump values fall within \pm 2.5 inches. It should also be noted that slump was measured on the fresh concrete and the heat of hydration data were obtained from a different set of paste samples. This section will focus on the setting times of the concrete mixtures evaluated.

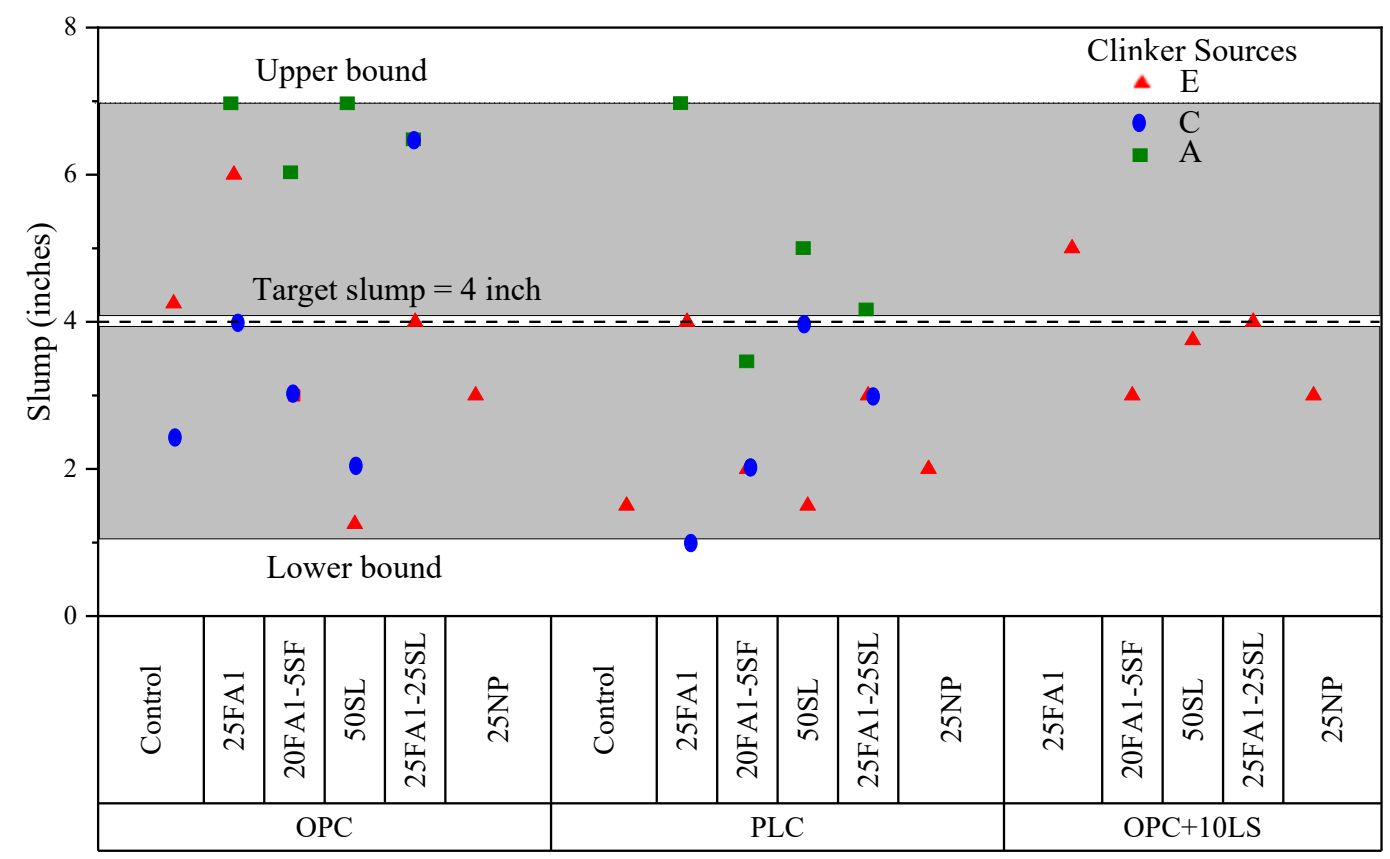

Figure 14-1. Slump values of fresh concrete mixtures.

\subsection{Research Objectives}

2386 The objective of this section is to quantify the relative time of setting of the different mixtures. 2387 This is accomplished using the heat of hydration data as described in Section 4.6 of this report. 2388 Testing was performed following ASTM C1753-15. Statistical analyses are used to compare these 2389 relative times of setting. 


\subsection{Background/Literature Review} Mixtures Using Thermal Measurements, was used to evaluate the mixtures in this research [164]. The standard indicates that the thermal profiles can be used to evaluate the hydration behavior of hydraulic cementitious mixtures after the addition of water and this can provide indications concerning setting. Sandberg and Liberman (2007) reported that $21 \%$ of the measured main peak temperature correlates with the initial setting time and $42 \%$ of the measured main peak temperature correlates with the final set [165]. This research will evaluate the relative initial and final set and will use the $30 \%$ and $50 \%$ values for comparing the relative initial and final setting times.

\subsection{Experimental Test Matrix}

All five clinker sources (A, B, C, D, and E) with their respective OPC, PLC, and OPC+10\%LS systems were evaluated. In addition, the systems with $25 \%$ fly ash, $20 \%$ fly ash $+5 \%$ silica fume, $50 \%$ slag, and $25 \%$ fly ash $+25 \%$ slag were evaluated for relative setting times. Each mixture included two tests and the average values are reported. The experimental test matrix is shown in Table 4.5.

\subsection{Experimental and Analytical Methods}

2406 The process stipulated in ASTM C1753-15, Standard Practice for Evaluating Early Hydration of 2407 Hydraulic Cementitious Mixtures Using Thermal Measurements, was used to evaluate the 2408 mixtures in this research. To assess the significance of the potential difference in setting times, $t$ 2409 tests were performed. These tests provide a comparison of the setting times at $t_{30 \%}$ and $t_{50} \%$ for the 2410 various paste samples. Initially, specimens from all cement sources (A, B, C, D, and E) made with 2411 OPC, PLC, and OPC+10LS containing no SCMs were grouped are compared. Following this, 2412 comparisons are made between the different mixtures (e.g., PLC from all cements with no SCM 2413 and PLC with all cements containing $25 \%$ fly ash). A p-value of less than 0.05 indicates that there 2414 is a significant difference between the two datasets. Table 14.1 shows the comparisons assessed in 2415 this research. Note that comparisons were made for both $t_{30 \%}$ and $t_{50 \%}$ data. 
Table 14.1. Statistical comparisons

\begin{tabular}{|c|c|c|c|c|c|c|}
\hline \multirow{3}{*}{$\begin{array}{l}\text { Cement Type } \\
\text { (Manufacturers) }\end{array}$} & \multicolumn{6}{|c|}{ SCM Addition } \\
\hline & \multicolumn{3}{|c|}{ None (MO) } & \multicolumn{3}{|c|}{$\begin{array}{c}25 \% \text { FA, } 20 \% \text { FA+5\% SF } \\
50 \% \text { Slag, } 25 \% \text { FA + } 25 \% \\
\text { Slag (M1-M4) } \\
\end{array}$} \\
\hline & $\begin{array}{l}\text { OPC } \\
\text { (A, B, } \\
\text { C, D, } \\
\& \text { E) } \\
\end{array}$ & $\begin{array}{l}\text { PLC } \\
\text { (A, B, } \\
\text { C, D, } \\
\text { \& E) } \\
\end{array}$ & $\begin{array}{l}\text { PLC+LS } \\
(\mathbf{A}, \mathbf{B}, \mathrm{C}, \\
\text { D, \& E) }\end{array}$ & $\begin{array}{l}\text { OPC } \\
(\mathrm{A}, \mathrm{B}, \\
\mathrm{C}, \mathrm{D}, \\
\& \mathbf{E}) \\
\end{array}$ & $\begin{array}{l}\text { PLC } \\
\text { (A, B, } \\
\text { C, D, } \\
\& \text { E) } \\
\end{array}$ & $\begin{array}{l}\text { PLC+LS } \\
(\mathrm{A}, \mathrm{B}, \mathrm{C}, \\
\text { D, \& E) }\end{array}$ \\
\hline $\begin{array}{c}\text { OPC } \\
(\mathrm{A}, \mathrm{B}, \mathrm{C}, \mathrm{D}, \boldsymbol{\&} \\
\mathrm{E})\end{array}$ & & $\mathrm{X}$ & $\mathrm{X}$ & $X$ & & \\
\hline $\begin{array}{c}\text { PLC } \\
(\mathrm{A}, \mathrm{B}, \mathrm{C}, \mathrm{D}, \boldsymbol{\&} \\
\mathrm{E}) \\
\end{array}$ & $X$ & & $X$ & & $X$ & \\
\hline $\begin{array}{c}\text { PLC+LS } \\
(A, B, C, D, \& \\
\text { E) }\end{array}$ & $X$ & $X$ & & & & $\mathrm{X}$ \\
\hline
\end{tabular}

2419 In this chapter, the apparent setting times were measured and compared. Statistical t-tests were performed to compare and contrast these setting times. Both initial $\left(t_{30}\right)$ and final $\left(t_{50 \%}\right)$ setting times were compared. A p-value of less than 0.05 indicates that there is a significant difference between the two datasets. In the first set of t-tests, OPCs, PLCs, and OPC+10LS' were compared within the same mixture (i.e., no SCM, 25\% FA, 20\% FA+5\% SF, 50\% slag, and 25\% FA and $25 \%$ slag). This was done for all mixtures (M0 to M4). For example, for mixture M0, the following three comparisons were made:

- $\quad$ OPC and PLC

- PLC and OPC +10LS

- $\quad$ OPC and OPC +10LS

The findings from the two-sample t-tests reveal that, for mixtures M0 to M4, there is no statistically significant difference between the comparison for mixtures without SCMs for both initial and final setting times. The $\mathrm{p}$-values from the $\mathrm{t}$-tests for initial set were: OPC/PLC -0.95 , OPC/OPC+LS 0.63 , and PLC/OPC+LS -0.51 . The p-values from the t-tests for final set were: OPC/PLC -0.93 , $\mathrm{OPC} / \mathrm{OPC}+\mathrm{LS}-0.54$, and PLC/OPC+LS -0.37 . This indicates that there is likely no significant difference in setting time between the OPC systems with the inter-grinding or addition of the 
2435 limestone. The p-values for all the comparisons were significantly higher than 0.05 threshold by a 2436 comfortable margin.

2437 In the second set of t-tests, the OPC, PLC, and OPC+10LS samples of M0 were compared with 2438 the OPC, PLC, and OPC+10LS samples of the M1, M2, M3, and M4. shows the p-values for these 2439 comparisons (for initial set/for final set). Shaded cells indicate statistically significant values.

2440 Comparisons between M0 and M1 reveal that there is no statistically difference between most OPC and PLC mixtures. However, a comparison of the OPC+LS mixtures between mixtures M0 and M1 (25\% fly ash addition) indicates a statistically significant difference. The data indicates that when limestone (not interground) and fly ash are included in a mixture, the initial and final setting times can increase. However, it should be noted that the p-values for the other comparisons in this group are relatively close to the p-value threshold of 0.05 , indicating the difference between cement types is likely not significant.

2447 For the M0-M2 and M0-M3 comparisons, it can be seen that there are no statistically significant differences for all cases. Comparison of mixtures M0 and M4 indicate the initial and final setting times are statistically significant for all cement types. This indicates that the addition of $25 \%$ FA and $25 \%$ slag to mixtures leads to longer initial and final setting times. However, note that the initial and final setting times for the mixtures without limestone also exhibited increased setting times and the increase is likely not a result of the limestone additions.

Table 14.2 shows the p-values for these comparisons (for initial set/for final set). Shaded cells indicate statistically significant values. Comparisons between M0 and M1 reveal that there is no statistically difference between most OPC and PLC mixtures. However, a comparison of the OPC + LS mixtures between mixtures M0 and M1 (25\% fly ash addition) indicates a statistically significant difference. The data indicates that when limestone (not interground) and fly ash are included in a mixture, the initial and final setting times can increase. However, it should be noted that the $p$-values for the other comparisons in this group are relatively close to the $p$-value threshold of 0.05 , indicating the difference between cement types is likely not significant.

2461 For the M0-M2 and M0-M3 comparisons, it can be seen that there are no statistically significant 2462 differences for all cases. Comparison of mixtures M0 and M4 indicate the initial and final setting 2463 times are statistically significant for all cement types. This indicates that the addition of $25 \% \mathrm{FA}$ 2464 and $25 \%$ slag to mixtures leads to longer initial and final setting times. However, note that the 2465 initial and final setting times for the mixtures without limestone also exhibited increased setting 2466 times and the increase is likely not a result of the limestone additions.

Table 14.2. p-values for different mixtures.

\begin{tabular}{|c|c|c|c|c|}
\hline \multirow{2}{*}{ Mixture } & \multirow{2}{*}{ Cement Type } & \multicolumn{3}{|c|}{ Mixture M0 } \\
\cline { 3 - 5 } & & OPC & PLC & OPC+LS \\
\hline
\end{tabular}




\begin{tabular}{|c|c|c|c|c|}
\hline \multirow{3}{*}{ M1 } & OPC & $0.22 / 0.07$ & & \\
\hline & PLC & & $0.10 / 0.07$ & \\
\hline & OPC $+\mathbf{L S}$ & & & $0.04 / 0.03$ \\
\hline \multirow{3}{*}{ M2 } & OPC & $0.12 / 0.19$ & & \\
\hline & PLC & & $0.08 / 0.15$ & \\
\hline & OPC+LS & & & $0.20 / 0.36$ \\
\hline \multirow{3}{*}{ M3 } & OPC & $0.26 / 0.16$ & & \\
\hline & PLC & & $0.14 / 0.06$ & \\
\hline & OPC+LS & & & $0.40 / 0.41$ \\
\hline \multirow{3}{*}{ M4 } & OPC & $0.03 / 0.02$ & & \\
\hline & PLC & & $0.08 / 0.02$ & \\
\hline & OPC+LS & & & $0.01 / 0.02$ \\
\hline
\end{tabular}

$p$-value on the left corresponds to the initial set, $p$-value on the right corresponds to the final set

\section{$2469 \quad 14.6$ Significant Findings}

2470 The fresh characteristics of concrete can influence the constructability of concrete projects. This

2471 research evaluated the setting time of concretes containing various cements from different 2472 producers with the additions of limestone and various combinations of SCMs. In general, the 2473 addition of limestone, whether interground or added to the cement, had no statistically significant 2474 influence on the initial or final setting times. Of course, in some cases the addition of SCMs did 2475 influence the initial and final setting times. 


\section{Impact on Greenhouse Gas Emissions}

$2478 \quad 15.1$ Background of Sustainability Efforts in the Cement Industry

2479 The production of portland cement clinker produces approximately $6 \%$ of the world's 2480 anthropogenic $\mathrm{CO}_{2 \text { eq }}$ emissions $[166,167]$. The main contribution $(\sim 60 \%)$ comes from the 2481 calcination (heating) process in which limestone $\left(\mathrm{CaCO}_{3}\right)$ is decomposed into $\mathrm{CaO}$ (for cement) 2482 and $\mathrm{CO}_{2}$ (released). Note that while the global values of the contribution from calcinations are 2483 typically $50 \%$, the typical values in the state of California are closer to $60 \%$ [168]. The other main 2484 component $(\sim 40 \%)$ of the $\mathrm{CO}_{2 \text { eq }}$ emissions comes from the fuel source used to heat the raw 2485 materials (limestone and clay) to approximately $1450^{\circ} \mathrm{C}$ (note that the emissions at this stage are primarily associated with energy resources). Minor emissions come from the grinding of the clinker material and the transportation of materials to and from the cement production facility [166]. The production of OPC is highly efficient ( approximately $85 \%$ efficient $[6,168,169]$ ) and the emission of $\mathrm{CO}_{2 \text { eq }}$ from the process is lower than other building materials such as steel, wood and aluminum [170]. The challenge is that we use more cementitious materials than all other building materials combined. Therefore, the overall percent contribution is higher than other building materials.

The portland cement industry and the research community have been focused on reducing $\mathrm{CO}_{2 \mathrm{eq}}$ emissions for many years $[166,169,171,172]$. Some of the early efforts were to simply optimize the production process, move toward a dry rather than wet production process, increase waste heat recovery and to use alternative fuel sources. There is still work to do in the area of alternative fuel sources and energy where more $\mathrm{CO}_{2 \text { eq }}$ savings can be realized. The use of supplementary cementitious materials (SCMs) such as ground granulated blast furnace slag, fly ash and silica fume - which are all by-products of other industrial processes which would most likely be landfilled - provided a second wave of lowering $\mathrm{CO}_{2 \text { eq }}$ emissions. The third wave of reducing $\mathrm{CO}_{2 \mathrm{eq}}$ emissions the past $\sim 20$ years have been on increasing the amount of finely ground limestone as a replacement for a portion of the portland cement. This can be done either as an interground product with the clinker phase of portland cement, or blended product once the portland cement is ground. Most ASTM C 150 Type I, II, III and V cements produced in the United States contain up to $5 \%$ finely ground limestone today. The amount of finely ground limestone is increasing beyond $5 \%$ to upwards of $15 \%$ in the United States. Europe has been using higher amounts of finely ground limestone for many years and certain cements may contain up to $35 \%$ FGL $[4,173]$. The most recent work ( last 10 years) to reduce $\mathrm{CO}_{2 \mathrm{eq}}$ emissions has centered around $\mathrm{LC} 3$ systems where both finely ground limestone and calcined clay are used to replace as much as $50 \%$ of the portland cement. Calcined clay does require energy to heat and thus activate the material, though the calcining temperatures $\left(\sim 650^{\circ} \mathrm{C}-800^{\circ} \mathrm{C}\right)$ are about $1 / 2$ that needed to calcine portland cement. Further there is little $\mathrm{CO}_{2}$ emissions from the calcining process itself as clays typically do not contain $\mathrm{CaCO}_{3}$. The "tool box" for $\mathrm{CO}_{2 \text { eq }}$ reduction in cements has widened and this will provide 
2514 even more options in the future as these technologies are further refined and verified for long-term 2515 durability (LC3 investigations underway) [174]. While there is much "buzz" about alternative 2516 cementitious materials having lower $\mathrm{CO}_{2 \text { eq }}$ footprints most, if not all, of these technologies cannot 2517 be produced on the same scale that ordinary portland cement is used. There may be a potential for 2518 these materials to be part of a suite of solutions to reduce $\mathrm{CO}_{2 \text { eq }}$ emissions [175]. The impact from 2519 this will be minor compared to the impact for calcined clays and/or limestone to reduce $\mathrm{CO}_{2 \mathrm{eq}}$ at a 2520 global scale.

\subsection{Life Cycle Inventory (LCI) of PLC Concrete}

2522 In this project CALTRANS is interested in increasing the amount of limestone in their portland cements to as much as $15 \%$. The research team partnered with the University of California at Davis to implement a life cycle inventory tool recently developed for CALTRANS [176, 177]. The process flow diagram for this GHG emissions assessment tool is shown below in Figure 15-1.

This GHG emissions assessment tool was used to evaluate four example CALTRANS concrete mixtures representing bridge decks, pavements and pre-cast concrete replacement panels for transportation infrastructure. The baseline mixture data was input into the software tool and then the portland cement portion was adjusted to reflect a 10 or $15 \%$ interground limestone replacement. The overall reduction in GHG, as compared to the baseline mixtures (assuming no limestone replacement) was then calculated. A second set of calculations was done to capture the influence of supplementary cementitious material replacement in addition to the 10 or $15 \%$ interground limestone replacement, in terms of GHG savings. The mixture designs provided to the team from Caltrans for the pavement and precast panel contained 100\% OPC. A third set of calculations was done to demonstrate the impact of also including SCMs in the mixture.

In this GHG emissions assessment tool, several key assumptions are of note:

- Fly ash is considered to be a waste material that is ready for use without processing. Transportation is considered in the calculation of GHG emissions. Thus, for fly ash, GHG emissions reduction are an upper bound.

- Ground granulated blast furnace slag and silica fume require additional processing before incorporation into portland cement concrete. This is captured in the EPDs and LCA documentation that supports this LCI tool. This, as well as transportation, are included in GHG emission calculations.

- Transportation distances (Table 15.1) are assumed based on market analysis [176, 177] and generally expected transportation distances for the State of California's concrete market.

The calculations shown are examples to illustrate the GHG emissions reduction potential for more sustainably designed concrete mixtures. For specific projects the referenced GHG emissions assessment tool can be used to produce data that incorporates project specific information. 
2549 In Figure 15-1, the process flow as well as boundary conditions used in the GHG emissions 2550 assessment tool are given. The raw materials acquisition, manufacturing, batching for the specific 2551 concrete mixture are included in the LCI GHG tool. The "in use" phase of the concrete is not 2552 included in this tool.

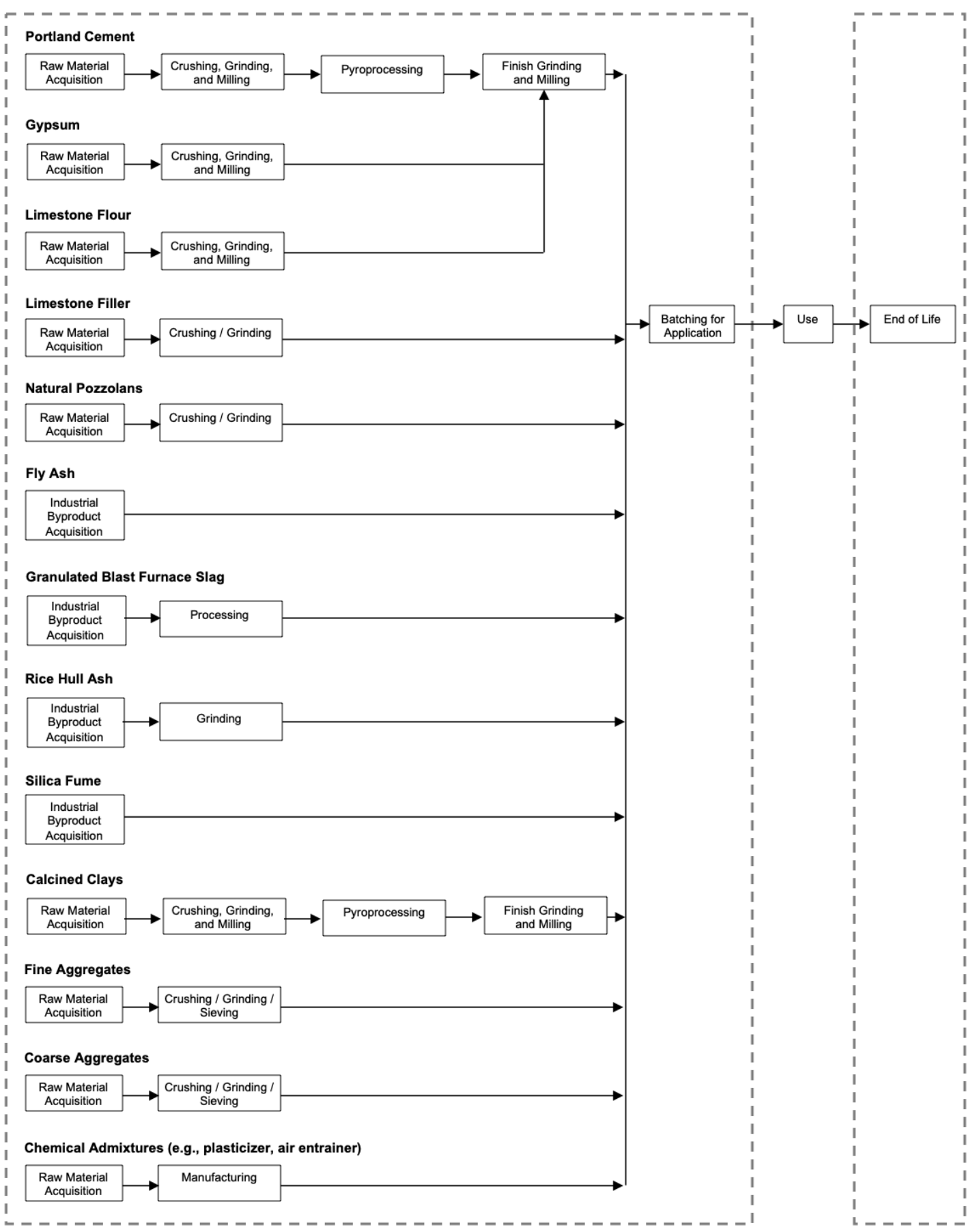


Table 15.1. Transportation distances used to calculate greenhouse gas reduction in the LCI GHG tool (from [176, 177]).

\begin{tabular}{|c|c|}
\hline \multicolumn{2}{|c|}{ Transportation Distances } \\
\hline Constituent & Distance (km) \\
\hline Portland Cement & 20 \\
\hline Limestone, interground & 20 \\
\hline Limestone Filler & 150 \\
\hline Natural Pozzolans & 150 \\
\hline Shale Ash & 150 \\
\hline Calcined Clay & 150 \\
\hline Silica Fume & 150 \\
\hline Fly Ash & 2000 \\
\hline Blast Furnace Slag & 2000 \\
\hline Fine Aggregates & 100 \\
\hline Coarse Aggregates & 100 \\
\hline Superplasticizer & 1000 \\
\hline Water & 0 \\
\hline
\end{tabular}

2559 The example CALTRANS concrete mixture designs that were investigated are shown below in 2560 Table 15.2.

Table 15.2. Example CALTRANS concrete mixture designs evaluated using the GHG reduction in the LCI GHG tool [176]

\begin{tabular}{|l|c|c|c|c|c|c|c|}
\hline & $\begin{array}{c}\text { Cement } \\
\left(\mathbf{k g} / \mathbf{m}^{3}\right)\end{array}$ & $\mathbf{w} / \mathbf{c m}$ & $\begin{array}{c}\text { Water } \\
\left(\mathbf{k g} / \mathbf{m}^{\mathbf{3}}\right)\end{array}$ & $\begin{array}{c}\text { Slag } \\
\left(\mathbf{k g} / \mathbf{m}^{3}\right)\end{array}$ & $\begin{array}{c}\text { Fly ash } \\
\left(\mathbf{k g} / \mathbf{m}^{\mathbf{3}}\right)\end{array}$ & $\begin{array}{c}\text { Coarse } \\
\text { aggregate } \\
\left(\mathbf{k g} / \mathbf{m}^{3}\right)\end{array}$ & $\begin{array}{c}\text { Fine } \\
\text { aggregate } \\
\left(\mathbf{k g} / \mathbf{m}^{\mathbf{3}}\right)\end{array}$ \\
\hline $\begin{array}{l}\text { Bridge } \\
\text { Deck 1 }\end{array}$ & 334 & 0.38 & 171 & - & 112 & 979 & 682 \\
\hline $\begin{array}{l}\text { Bridge } \\
\text { Deck 2 }\end{array}$ & 218 & 0.38 & 168 & 218 & - & 1085 & 753 \\
\hline $\begin{array}{l}\text { Precast } \\
\text { Panel }\end{array}$ & 409 & 0.38 & 154 & - & - & 1066 & 675 \\
\hline $\begin{array}{l}\text { Jointed } \\
\text { Plain } \\
\text { Concrete } \\
\text { Pavement }\end{array}$ & 400 & 0.43 & 172 & - & - & 1075 & 662 \\
\hline
\end{tabular}


2564 In Table 15.2, four example CALTRANS concrete mixture designs are shown. It should be noted 2565 that the two bridge deck mixture designs used either a $50 \%$ replacement by slag cement or a $25 \%$ 2566 replacement by fly ash. The precast panel and jointed plain concrete pavement were $100 \%$ OPC 2567 mixtures. Those two mixtures had very similar cement contents. The cementitious materials 2568 content in bridge deck 2 was also similar but slightly higher than the previously mentioned 2569 mixtures. Bridge deck 1 had the highest cementitious materials content at $446 \mathrm{~kg} / \mathrm{m}^{3}$. Overall, 2570 three of the mixtures had the same $\mathrm{w} / \mathrm{cm}$ at 0.38 while the joined plain concrete pavement was a 2571 bit higher at 0.43 .

2572 Figure 15-2 shows the results of the analysis on the four example CALTRANS Concrete mixture 2573 designs for potential GHG reduction.

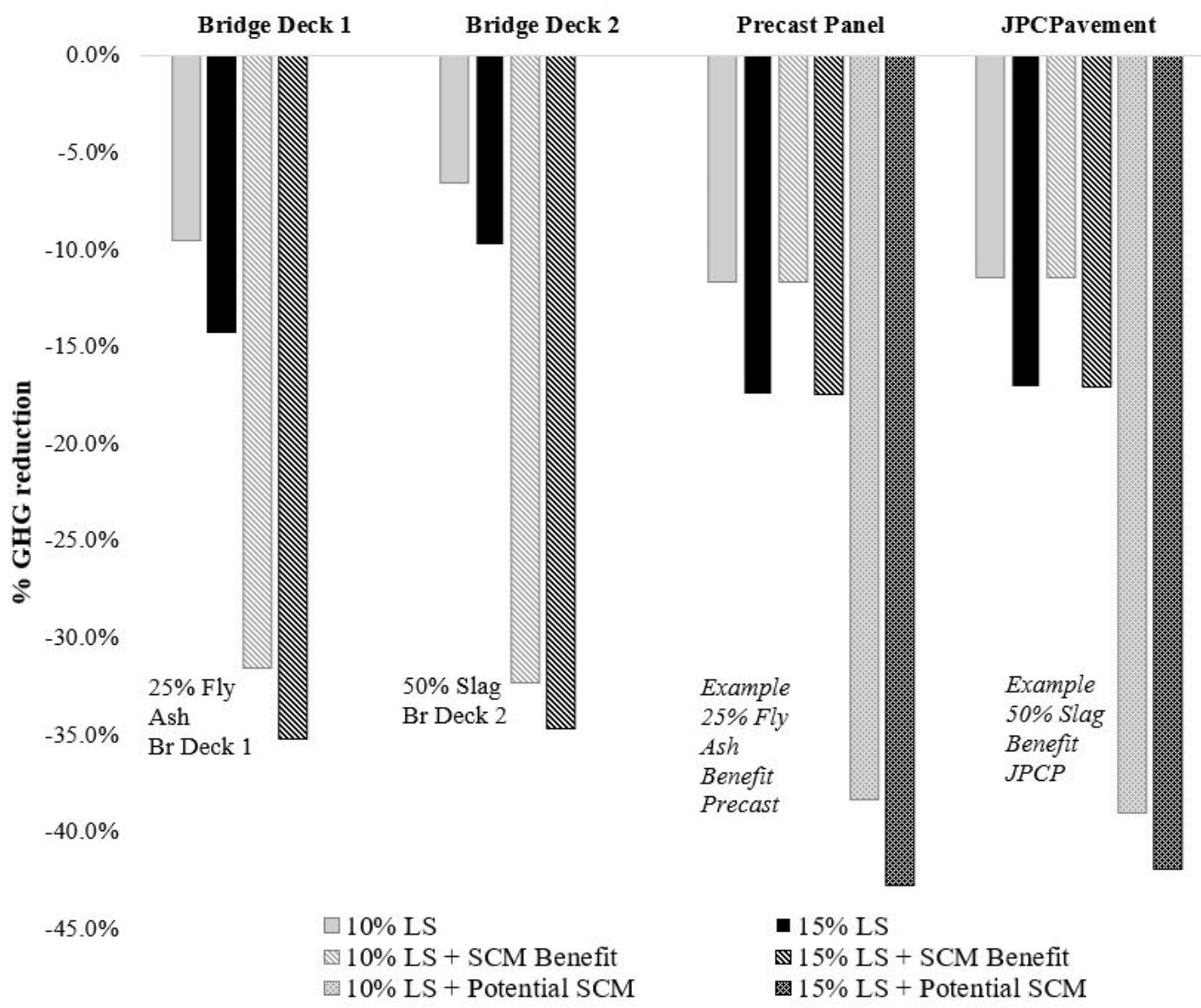

\section{Figure 15-2. Greenhouse gas reductions compared for 10 and $15 \%$ interground limestone} replacements for example CALTRANS concrete mixtures.

Figure 15-2 shows groups of bar charts where the lighter color in each pair represents $10 \% \mathrm{LS}$ and the darker, $15 \%$. This figure shows that there is an approximate $6.5-17 \%$ potential for greenhouse gas reduction in all the example mixtures evaluated when SCMs are not included in the analysis. 
2580 For the 10\% LS replacement levels the savings ranged from $6.5-12.1 \%$. For the $15 \%$ LS 2581 replacement levels the savings ranged from 9.8-17.5\%. These variations are due to the mixture 2582 design specifics as well as associated transportation distances with the various materials in the 2583 mixtures. When SCMs are included in the analysis (e.g., compared against the same mixture 2584 design except with $100 \%$ OPC) there is a substantially higher reduction in greenhouse gas 2585 emissions, roughly around $30-35 \%$.

2586 It is important to note that the precast panel or jointed plain concrete pavement contained $100 \%$ 2587 OPC. To show further potential for greenhouse gas reduction in these mixtures a third set of 2588 calculations was done, and those results are shown in the last set of bars (light and dark gray dotted 2589 bars). This shows that there is a potential greenhouse gas reduction of up to $41.9 \%$ and $42.7 \%$ in 2590 the case of the jointed plain concrete pavement or the precast panel mixture, respectively, if PLC 2591 is combined with SCM in these mixtures.

\section{$2593 \quad 15.3$ Significant Findings}

2594 Overall, there is significant potential for GHG when using Portland-limestone cement to replace 2595 portland cement from $6.5 \%$ to $17.1 \%$ with an average of approximately $10 \%-12 \%$. Further 2596 reductions can be realized with the incorporation of SCMs. Further, there appear to be benefits of 2597 SCM with portland-limestone cements to realize the most significant GHG reductions. 


\section{Thermodynamic Simulations}

$2600 \quad 16.1$ Research Objective

2601 This section reports the influence of cement clinker chemistry on PLC performance. Specifically, 2602 thermodynamic simulations were performed for the clinkers used in the experimental portion of 2603 this work. The research compares the results of the model with experiments. Furthermore, the 2604 synergy between PLCs and SCMs is explored parametrically using thermodynamic modeling.

\subsection{Background and Literature Review}

2606 The use of PLCs as a replacement for OPCs in concrete has been gaining momentum due to 2607 environmental benefits associated with the reduction of $\mathrm{CO}_{2}$ emissions during production [6]. 2608 Although some consider limestone an inert material, others have shown that it can affect the 2609 reaction products of hydrated OPC systems [22, 48, 98, 150, 157, 178]. In typical OPC systems, 2610 limestone content can stabilize ettringite and result in the formation of monocarbonate instead of 2611 monosulfate $[14,22,48,98,179]$. This change in the phase assemblage of reaction products due 2612 to the presence of limestone can sometimes directly impact the porosity and pore volume 2613 distribution in concrete as ettringite is a more space-filling phase $[6,20,180]$.

2614 Matschei et al. [20] showed that the porosity of OPC-Limestone systems decreased (and the 2615 compressive strength increased) when the limestone content increased up to clinker replacement 2616 levels of $2 \%$. Any further increase in limestone content led to a porosity increase (and a decrease 2617 in compressive strength). However, many have used Matschei's work as a guide for 'equivalent 2618 porosity' or 'equivalent strength' in PLC which has driven the design and proportioning of PLC2619 based mixtures in North America.

2620 Several authors have experimentally studied the impact of partial replacement of clinker in 2621 OPC+SCM systems with limestone. The synergistic effect of using SCMs containing alumina (like 2622 fly ash, slag or metakaolin) with limestone has been documented on the compressive strength [14, 2623 181] or transport properties [2]. While the synergy of limestone and alumina is noted in the 2624 literature $[180,181]$, there is lack of a robust recommendation on switching between OPC to PLC 2625 to exploit this synergy. For example, some have questioned how well PLC will work with SCM. 2626 Thermodynamic modeling is one such tool which can be used in a predictive capacity to study the 2627 impact of replacement of OPC with PLC in systems made with cement and SCMs.

2628 Thermodynamic modeling is gaining popularity as a predictive tool to evaluate the solid and liquid 2629 reaction products of OPC and OPC+SCM systems [8, 22, 44, 48, 182-185]. Thermodynamic 2630 modeling has also been used in conjunction with the concepts of Power's and Brownyard's model $2631[186,187]$ to determine the pore structure of OPC [123] and OPC+SCM pastes [188, 189]. The 
2632 work on pastes has also been extended to concrete to predict the porosity, pore volumes, and 2633 service life of concrete [190]. In this work, thermodynamic modeling is used to study the impact 2634 of using PLCs as a direct replacement for OPCs.

\subsection{Modeling Framework}

\subsubsection{Thermodynamic Modeling}

2637 The GEMS3K [191] software is used to perform thermodynamic modeling, and it is coupled with 2638 the CEMDATA thermodynamic database [48]. Thermodynamic modeling is performed by 2639 calculating the phase assemblage at equilibrium, which minimizes the system's Gibbs Free Energy. 2640 The GEMS-CEMDATA framework has been used to calculate the volumes and compositions of solids, liquid, and gaseous products at thermodynamic equilibrium. The framework has been used previously to obtain the reaction product volumes and pore solution composition of OPC [44, 182] and OPC+SCM systems [192]. While all phases are available to form in the GEMS-CEMDATA framework, siliceous hydrogarnet [123, 192, 193], hydrotalcite [123], and carbonate-ettringite phases $[83,178,185]$ were blocked from forming based on empirical evidence from the literature that these phases do not form in significant quantities in cementitious systems at typical temperatures (less than $\left.60^{\circ} \mathrm{C}\right)$ in the time frames studied $(<20$ years).

\subsubsection{Kinetic Models}

2649 While powerful, thermodynamic models calculate only the phase assemblage of the systems studied at equilibrium (i.e., the final phases). In practice, most cementitious systems have not yet reached thermodynamic equilibrium. Kinetic models (such as the Parrot-Killoh model for OPCclinker [194] or the Modified Parrot-Killoh Model for clinker + SCM [195]) are often used to predict the mass fraction of the clinker that reacts at a given age. Thermodynamic models are often coupled with kinetic models to predict cementitious systems' reaction products at a given age. The literature has shown that the phase assemblage of cementitious systems depends on the amount of clinker, SCM, and limestone available to react [48] and the kinetics of dissolution of the three components of the systems studied (i.e.,, clinker, SCM, limestone) are essential to understand.

\subsubsection{Modified Parrot Killoh Model for Clinker and SCM}

2659 The Modified Parrot Killoh (MPK) model $[195,196]$ was used to predict the mass fraction of the 2660 clinker phases $\left(\mathrm{C}_{3} \mathrm{~S}, \mathrm{C}_{2} \mathrm{~S}, \mathrm{C}_{3} \mathrm{~A}, \mathrm{C}_{4} \mathrm{AF}\right)$ and oxide phases in $\mathrm{SCMs}\left(\mathrm{SiO}_{2}, \mathrm{Al}_{2} \mathrm{O}_{3}, \mathrm{CaO}\right)$ that react at 2661 a given age. The inputs to the MPK model are: (i) the chemical composition of the OPC-clinker 2662 and SCM used, (ii) the reactivity of the SCM (fraction of SCM that can react at equilibrium, usually 2663 the amorphous fraction of the SCM [196]), (iii) w/b, and, (iv) the temperature of curing.

2664 The MPK model outputs are the degree of reaction of the clinker phases $\left(\mathrm{C}_{3} \mathrm{~S}, \mathrm{C}_{2} \mathrm{~S}, \mathrm{C}_{3} \mathrm{~A}, \mathrm{C}_{4} \mathrm{AF}\right)$ 2665 and pozzolanic oxide phases $\left(\mathrm{SiO}_{2}, \mathrm{Al}_{2} \mathrm{O}_{3}, \mathrm{CaO}\right)$ as a function of time. The degree of reaction of 
each phase at a given time $\left(D O R_{p h}(t)\right)$ is the fraction of the component that is available to react

2667 at that time. The dissolution of the alkali oxide phases in the clinker $\left(\mathrm{Na}_{2} \mathrm{O}, \mathrm{K} 2 \mathrm{O}, \mathrm{MgO}, \mathrm{SO}_{3}\right)$ was 2668 scaled based on their distribution in the clinker phases obtained from the literature [197]. The 2669 dissolution of alkali oxide phases from the SCM were scaled with the reactivity $\left(D O R^{*}\right)$ of the $2670 \mathrm{SCM}$ and the degree of reaction of the SCM. The degree of reaction of the system $\left(D O R_{s y s}\right)$ is the mass averaged degree of reaction of clinker and $\mathrm{SCM}$ oxide phases $\left(\mathrm{C}_{3} \mathrm{~S}, \mathrm{C}_{2} \mathrm{~S}, \mathrm{C}_{3} \mathrm{~A}, \mathrm{C}_{4} \mathrm{AF}, \mathrm{SiO}_{2}\right.$, $\mathrm{Al}_{2} \mathrm{O}_{3}, \mathrm{CaO}$ ). A sample output of the MPK Model for one of the systems studied (M1, $\mathrm{PLC}+25 \% \mathrm{FA}$ ) is shown in Figure 16-1 below. The figure shows the degree of reaction of each phase as well as the degree of reaction of the whole system as a function of time from 1 day up to 365 days of hydration. Early age predictions of the model ( $<1$ day) are not shown as they are not very accurate, which is a known issue with the Parrot and Kiloh model [194].

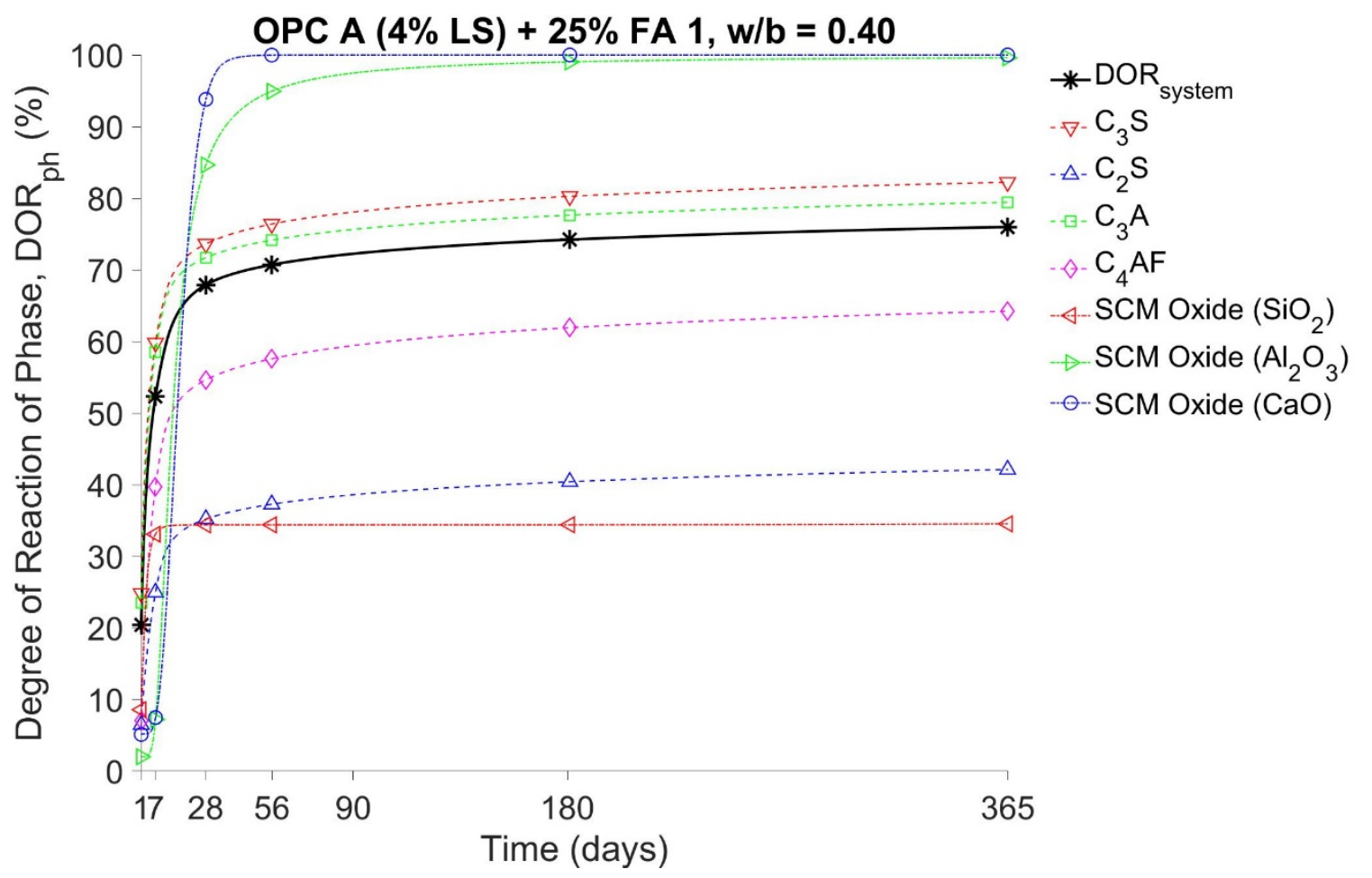

Figure 16-1. Output of the MPK model for clinker and SCM oxides

\subsubsection{Modeling the dissolution of limestone}

2681 The mass of limestone available to react is an essential input parameter to thermodynamic 2682 calculations, which impacts the phase assemblage [48] and porosity $[20,22,198]$ of these systems. 2683 The amount of $\mathrm{CaCO}_{3}$ available to react at any given time is considered the total amount of $\mathrm{CaCO}_{3}$ 2684 in the system. This is justified below. 
Calcium carbonate dissolves to some extent at ambient temperature to saturate typical cementitious pore solutions with carbonate within the first few hours of mixing [14, 83, 199]. The total volume and fineness also plays only a minor role in the amount of calcium carbonate dissolved at equilibrium [200]. It has also been observed that the solubility of limestone in the pore solution of typical $\mathrm{OPC}+\mathrm{SCM}$ systems is high enough to saturate the solution with carbonates within a few hours [201, 202], and often the effects of limestone dissolution kinetics disappear after the first hour of mixing [203]. Therefore, the kinetics of limestone dissolution is governed by the kinetics of product formation and not the rate at which carbonate dissolves from the limestone. In this work, since the thermodynamic models are run at ages greater than one day (typically $D O R_{\text {sys }}>30 \%$ ), the entire mass of calcium carbonate is considered to be available to react at all times. The material that does not react simply reforms as calcite and is considered unreacted. Preliminary studies investigated these details

While it is possible for some of the calcium carbonate to be encapsulated by reaction products rendering the rest of the calcite unable to react, it is assumed in this work that this does not occur to a significant degree in the systems studied due to the limestone being ground finely enough.

2700 It should also be noted that the input to the thermodynamic model is the total mass of calcium carbonate, and as such, systems with inter-ground limestone (OPC+Ls) and blended limestone (PLC) are assumed to be thermodynamically equivalent.

\subsection{Typical Results}

2704 Typical outputs of thermodynamic modeling at 56-days of hydration $\left(\mathrm{DOR}_{\text {system }} \sim=72 \%\right)$ and the 2705 Pore Partitioning Model (PPM) are shown in Figure 16-2. Figure 16-2(a) shows the phase assemblage of systems containing Type II/V clinker with varying levels of limestone addition, while Figure 16-2(b) shows the results for Type I/III clinker. It can be seen that as the limestone content increases, the volume of monosulfate decreases and is replaced by ettringite and carboaluminate phases (hemi-/monocarbonate). Above a 2\% limestone content, no further limestone reacts, and unreacted calcite (limestone) remains in the system. It should be noted that the mass of alumina in Type II/V systems (which contain $4.29 \mathrm{~g}$ alumina/ $100 \mathrm{~g}$ clinker) is typically lower than the alumina in Type I/III systems (which contain 4.92g alumina/100g clinker) [204], it should be noted that the phase assemblage in these two systems are similar. It can also be noted that the relative proportions of the different types of C-S-H that form (and therefore the $\mathrm{C} / \mathrm{S}$ ratio of the C-S-H) stay constant, indicating that the presence of limestone does not significantly affect the C-S-H gel chemistry.

2718

Figure 16-2(c, d) shows a typical output of the PPM in terms of the volumes of gel solids, gel water, capillary water, and chemical shrinkage that forms in the same system. From Figure 16-2(c, d), it can be seen that the volume of gel solids increases and the volumes of capillary water 
2720 decreases when limestone content is increased from $0-2 \%$, and at $2 \%$ the system is at the lowest 2721 porosity. Above a $2 \%$ limestone content, any further addition of limestone causes the volumes of 2722 gel solids to decrease and the volume of capillary pores to increase. This is due to the dilution of 2723 clinker with unreacted limestone. 


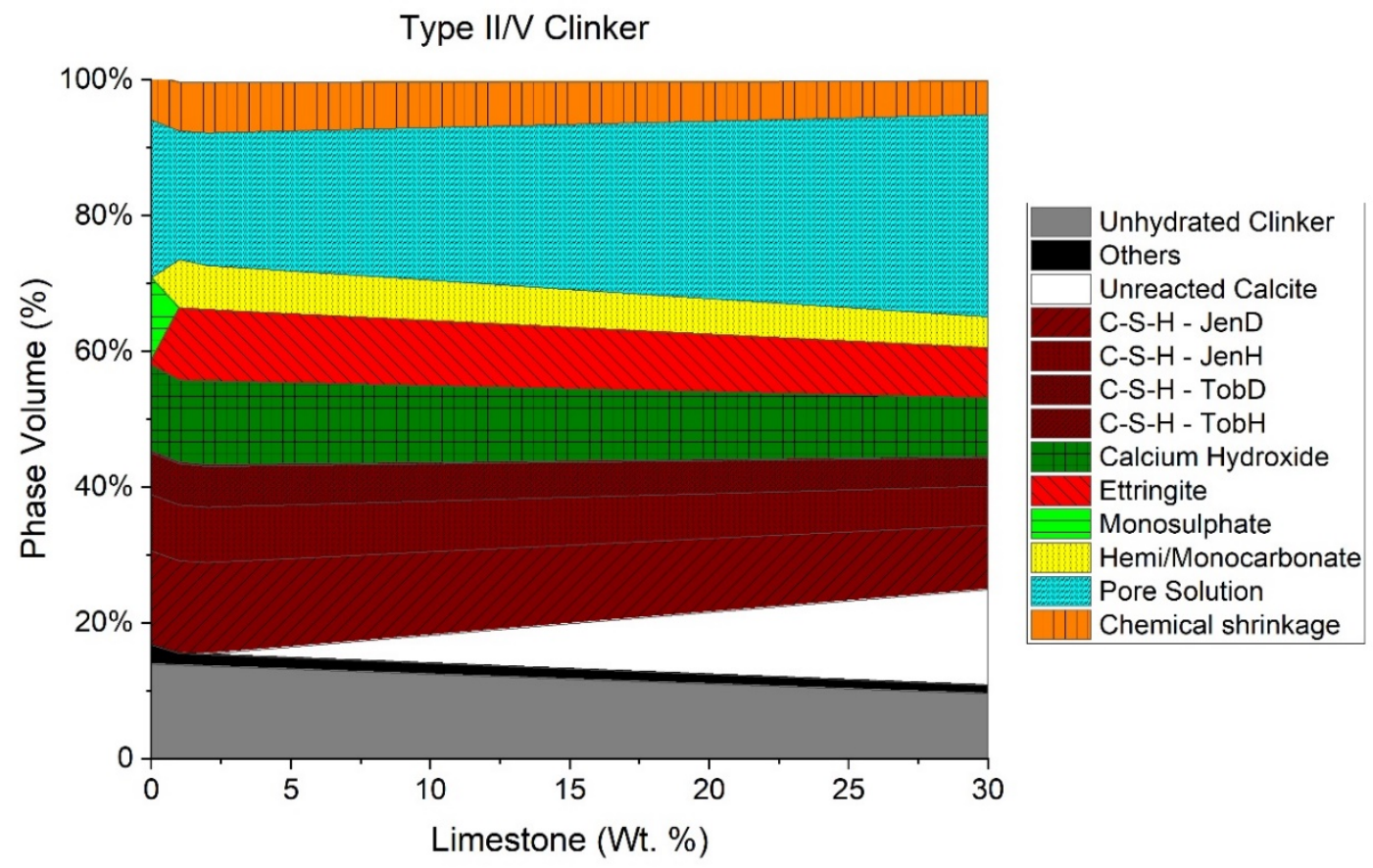

(a) The phase assemblage of Type II/V Clinker + Limestone systems

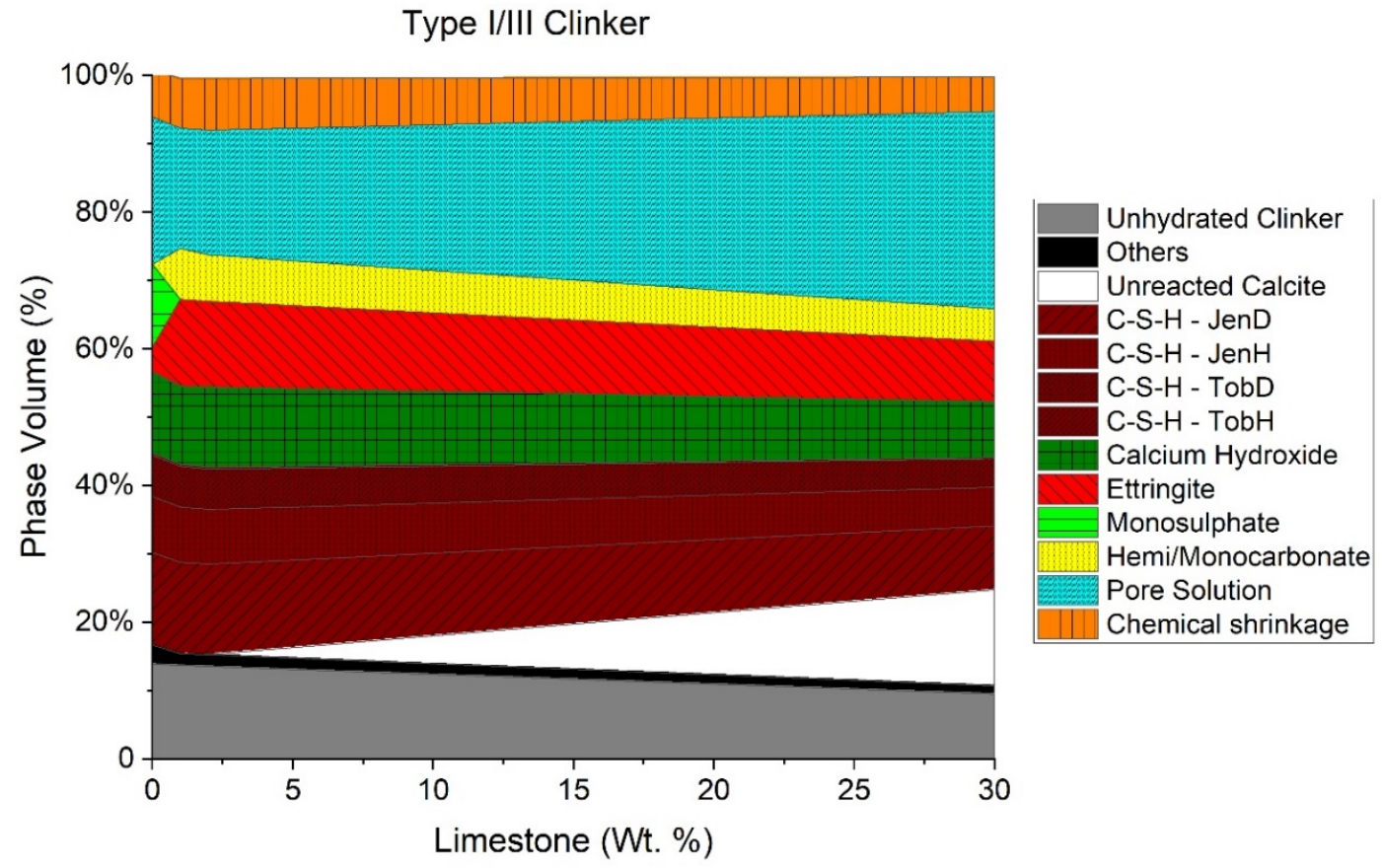

(b) The phase assemblage of Type I/III Clinker + Limestone systems 


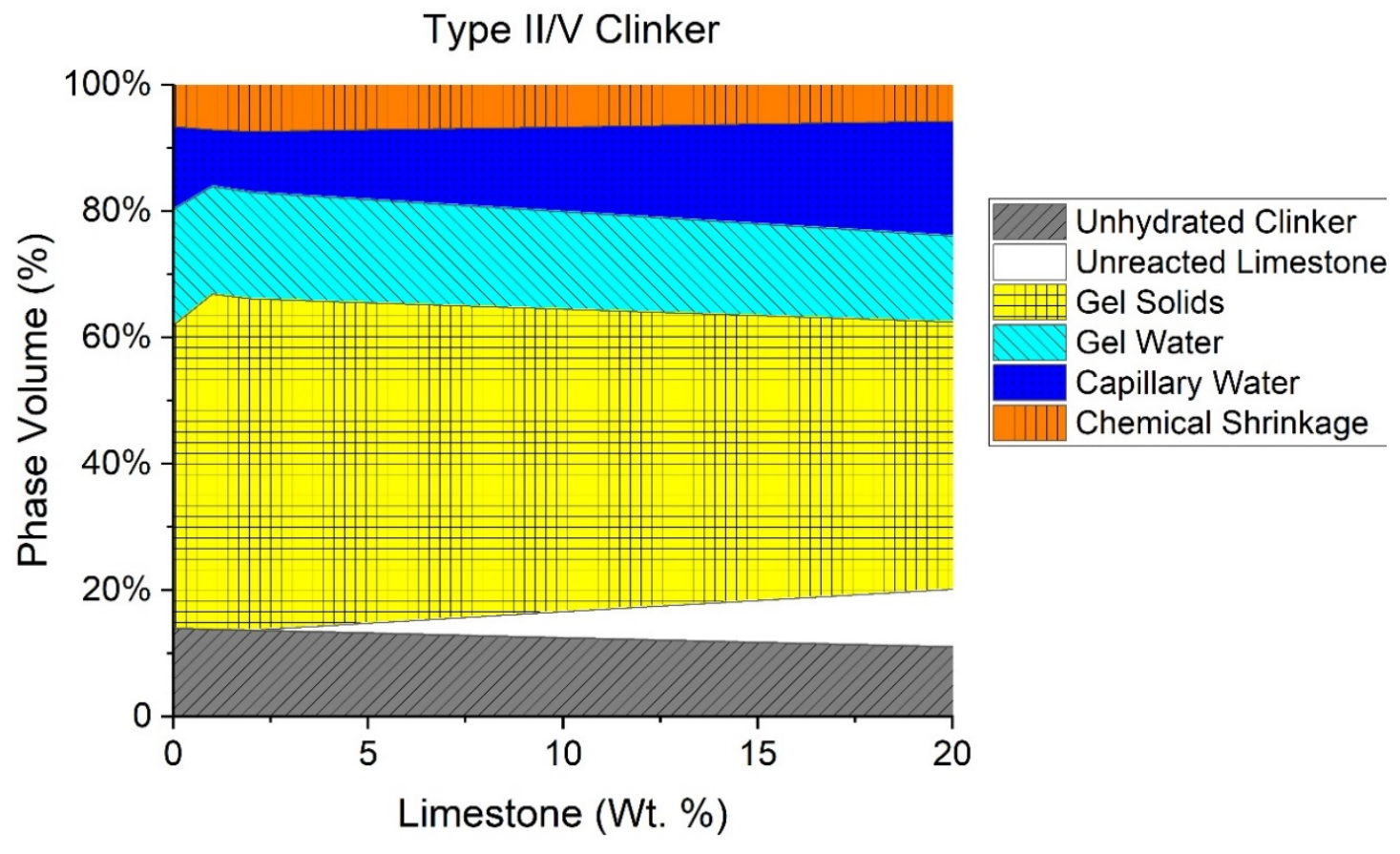

(c) Output of PPM for Type II/V clinker

Type I/III Clinker

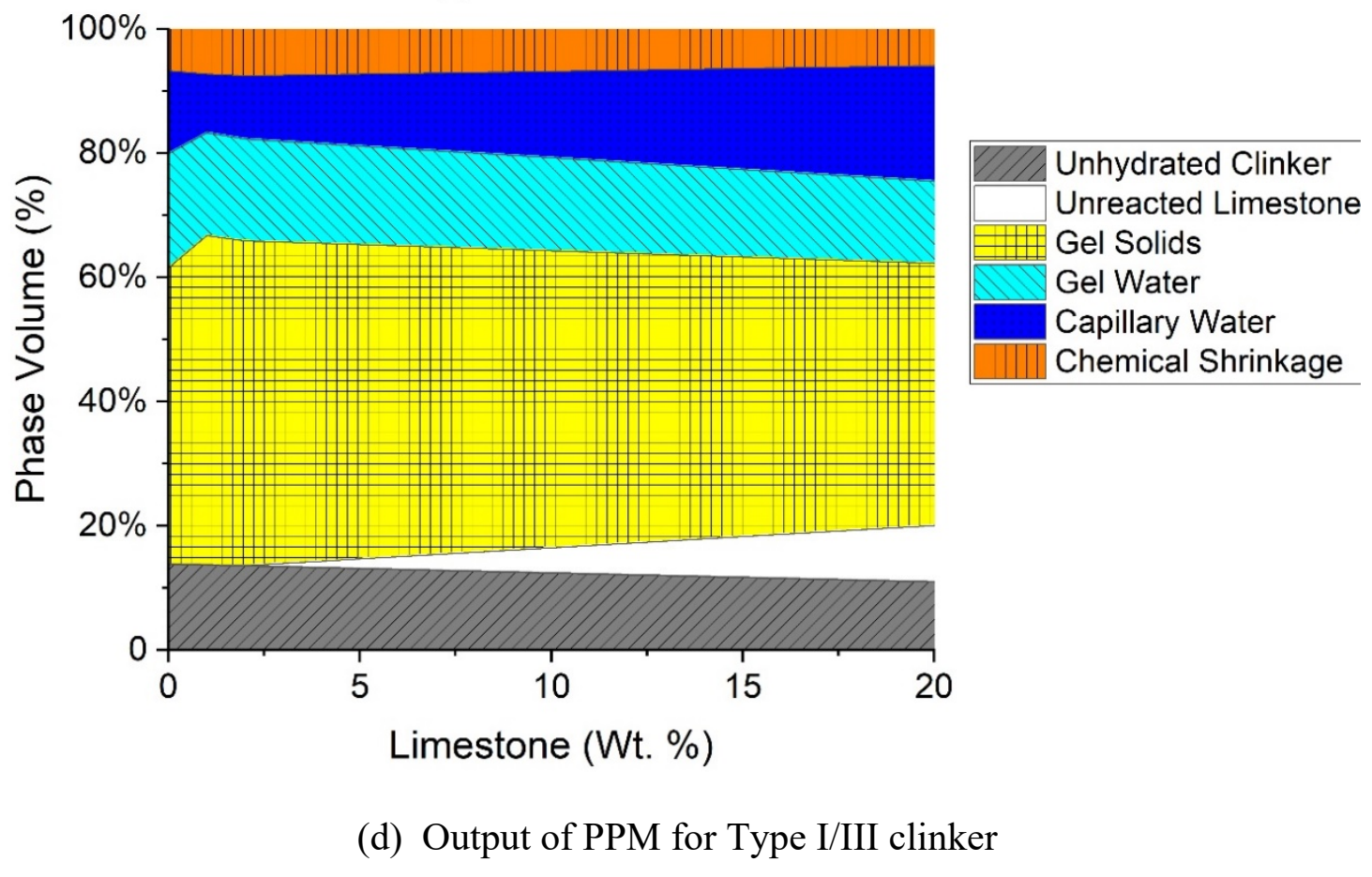

2724 Figure 16-2. Typical outputs of thermodynamic modeling showing the phase assemblage and the output of the PPM showing the different volumes of phases in the Clinker + Limestone systems 


\section{$2728 \quad 16.5$ Modeling Predictions of Experimental Results}

2729 Simulations were run to predict the porosity for the experimentally tested mixtures. The results of 2730 the simulation are plotted against the results of the experiments to compare the experimentally 2731 obtained results to the theoretical values.

2732 16.5.1 Porosity of Mortar Mixtures

2733 The predicted porosity of the mortar mixtures (M0-M5, mixture proportions in section 3) versus 2734 the experimentally measured porosity of these mixtures is shown in Figure 16-3. The mortars were 2735 considered to have 5\% entrapped air. The root mean square error between the model and the 2736 experiment is 4\% for OPC systems and 4.4\% for PLC systems. Most mixtures fall within $\pm 5 \%$ 2737 porosity of the predicted value. The outlier mixtures are the mixtures made with clinker D 2738 containing 25\% FA (D_OV:M1 and D_L15:M1). It is possible that this mixture was not compacted 2739 properly and had entrapped air. It can be seen that the model underpredicts the porosity of the 2740 mixtures, which is documented in the literature [123, 189]. The output data of the PPM for OPC 2741 and PLC systems is provided in the Appendix H, as Table H.2. 


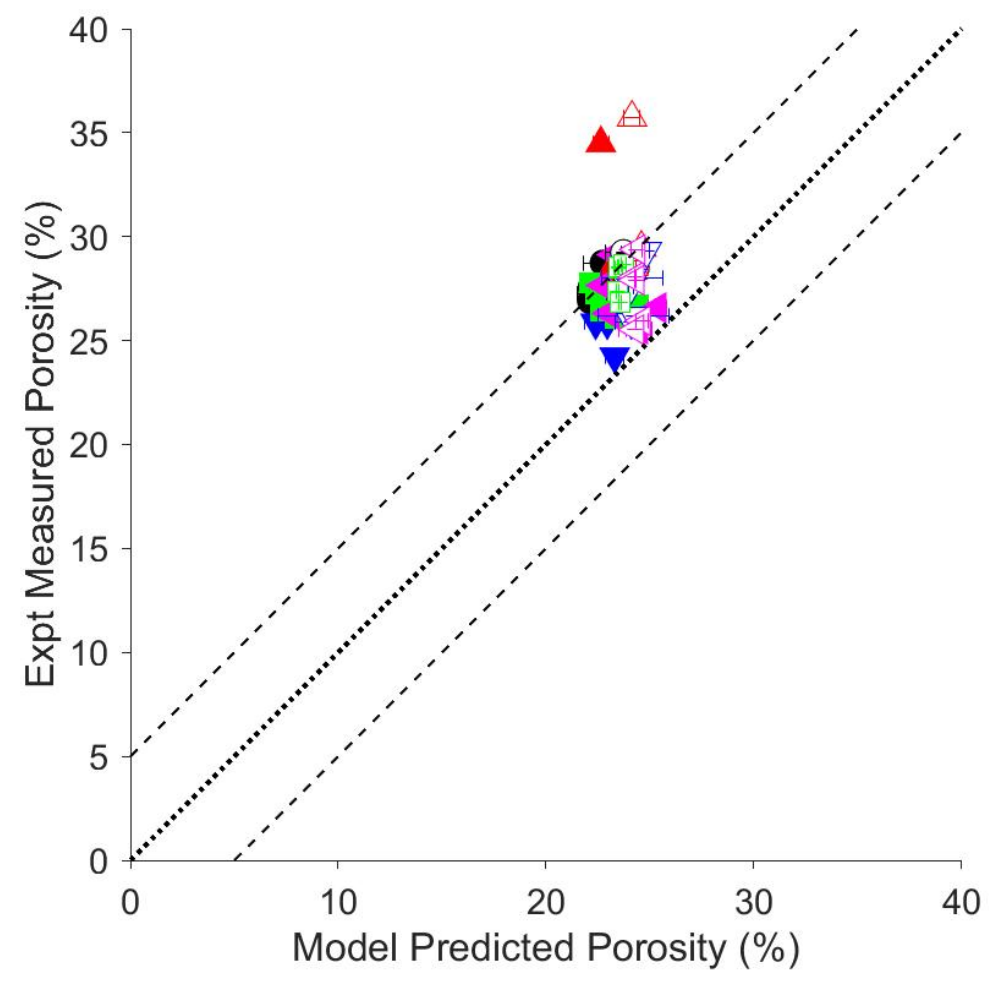
M0: OPC Control
$M 1: O P C+25 \% F A$
$\mathrm{M} 2: \mathrm{OPC}+20 \% \mathrm{FA}+5 \% \mathrm{SF}$
$\mathrm{M} 3: \mathrm{OPC}+50 \% \mathrm{SL}$
M4: OPC + 25\%FA + $25 \% S L$
MO: PLC Control
M1: PLC + 25\%FA
I- $\mathrm{M} 2: \mathrm{PLC}+20 \% \mathrm{FA}+5 \% \mathrm{SF}$
M3: PLC $+50 \% S L$
퍽 M4: PLC $+25 \% \mathrm{FA}+25 \% \mathrm{SL}$
........ 1:1 line
- - - +/-5\% Porosity

Figure 16-3. Porosity of the mortar mixtures of OPC and PLC (Model vs. Experiment)

\subsection{Comparison of PLC and OPC Using Modeling}

In this section, the properties of OPC and PLC systems will be directly compared against one another to compare their performance. The properties that will be studied are the paste porosity, unreacted calcite (limestone), and $\mathrm{CH}$ consumed. The raw data for all simulations run is available in tabular form in Appendix F.

\subsubsection{Porosity}

2751 Figure 16-4 is a plot of the predicted porosity of PLC systems plotted against their corresponding 2752 OPC systems for the mixtures M0-M5 and clinkers A-E. From Figure 16-4, it can be seen that 2753 most of the results lie above the 1:1 line, indicating that the porosity of PLC pastes is slightly 2754 higher (about 2\%) than the porosity of the corresponding OPC paste. This is because of the dilution 2755 of reactive clinker with unreacted limestone. The OPCs contain 3-6\% limestone, which is near the 2756 optimal point of minimum porosity (2-3\% Limestone), and any further limestone addition causes 2757 dilution in the system. The only exception to this observation is three mixtures (all three mixtures 2758 are M3 \& M4), which contain enough reactive alumina to react with almost all the limestone in 
2759 the PLC systems, thus causing enough carboaluminate reactions to lower the porosity. The porosity 2760 difference between OPC and PLC systems is tabulated in Table 16.1. Practically, a 3\% higher 2761 volume of pores in an OPC/PLC system is approximately equivalent to a 0.05 increase in the w/c 2762 or a $15 \%$ decrease in the degree of hydration.

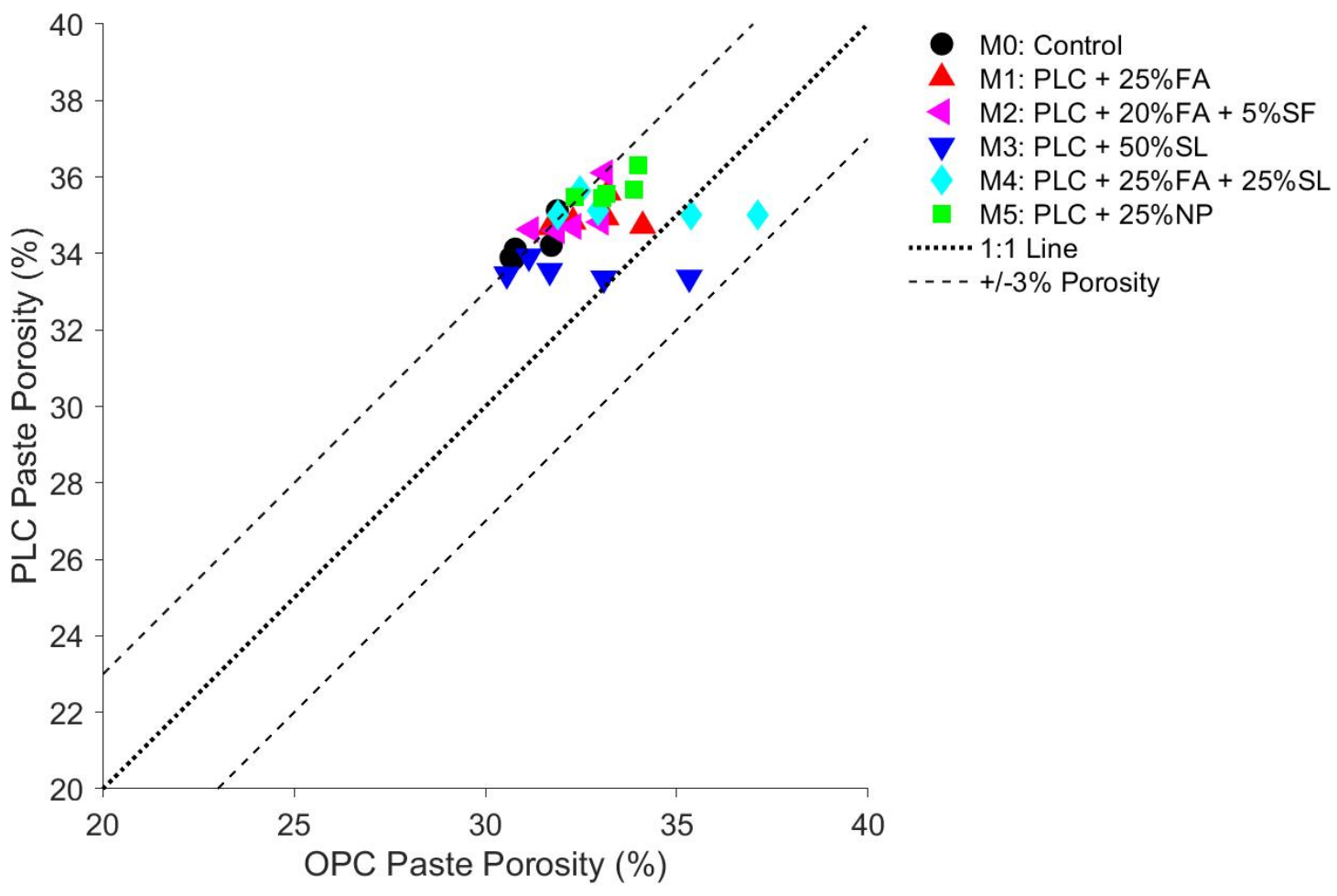

Figure 16-4. Plot of predicted porosity of PLC systems vs OPC systems.

2765

Table 16.1. Difference in porosity between OPC and PLC systems

\begin{tabular}{|l|c|c|c|c|c|c|}
\hline $\begin{array}{l}\boldsymbol{\phi}_{\text {PLC }} \\
-\boldsymbol{\phi}_{\text {OPC }}\end{array}$ & $\mathbf{M 0}$ & $\mathbf{M 1}$ & $\mathbf{M 2}$ & $\mathbf{M 3}$ & $\mathbf{M 4}$ & $\mathbf{M 5}$ \\
\cline { 2 - 7 } & PC+FA & $\begin{array}{c}\text { PC+FA+S } \\
\mathbf{F}\end{array}$ & $\mathbf{P C}+\mathbf{S L}$ & $\begin{array}{c}\text { PC+SL+F } \\
\mathbf{A}\end{array}$ & PC+NP \\
\hline Clinker A & $3 \%$ & $3 \%$ & $2 \%$ & $3 \%$ & $3 \%$ & $2 \%$ \\
\hline Clinker B & $3 \%$ & $1 \%$ & $3 \%$ & $-2 \%$ & $-2 \%$ & $3 \%$ \\
\hline Clinker C & $3 \%$ & $2 \%$ & $3 \%$ & $3 \%$ & $3 \%$ & $2 \%$ \\
\hline Clinker D & $3 \%$ & $3 \%$ & $3 \%$ & $0 \%$ & $0 \%$ & $2 \%$ \\
\hline Clinker E & $2 \%$ & $2 \%$ & $2 \%$ & $2 \%$ & $2 \%$ & $2 \%$ \\
\hline Average & $3 \%$ & $2 \%$ & $3 \%$ & $1 \%$ & $1 \%$ & $2 \%$ \\
\hline St. Dev. & $0 \%$ & $1 \%$ & $1 \%$ & $2 \%$ & $2 \%$ & $0 \%$ \\
\hline
\end{tabular}


The synergistic effects of alumina $\left(\mathrm{Al}_{2} \mathrm{O}_{3}\right)$ with limestone in $\mathrm{PLC}+\mathrm{Al}_{2} \mathrm{O}_{3}$ systems [180] are shown in Figure 16-5 below. It can be seen in a system with no alumina that the point of minimum porosity occurs at a limestone content of $1.5 \%$. As alumina is added to the system, it reacts with the limestone to form carboaluminate phases that reduce the porosity. Therefore, the point of minimum porosity shifts to a higher limestone content (the point of minimum porosity is $2 \%$ limestone for an $\mathrm{Al}_{2} \mathrm{O}_{3}$ content of $2.5 \%, 3.5 \%$ for an $\mathrm{Al}_{2} \mathrm{O}_{3}$ content of $5 \%$, and $4 \%$ for an $\mathrm{Al}_{2} \mathrm{O}_{3}$ content of $7.5 \%$ ). The value of porosity at this minimum is also lower for higher alumina contents (porosity for $0 \%$ $\mathrm{Al}_{2} \mathrm{O}_{3}$ is $34 \%$, for $2.5 \% \mathrm{Al}_{2} \mathrm{O}_{3}$ is $32 \%$, for $5 \% \mathrm{Al}_{2} \mathrm{O}_{3}$ is $29 \%$, and for $7.5 \% \mathrm{Al}_{2} \mathrm{O}_{3}$ is $27 \%$ ). Beyond the point of minimum porosity, any additional limestone addition dilutes the system and increases the porosity. This however shows the synergy that can be obtained with aluminous SCMs. However, even systems with $15 \%$ limestone have a lower porosities than systems containing no limestones, demonstrating the performance improvement (in terms of porosity reduction) when

2780 limestone is used.

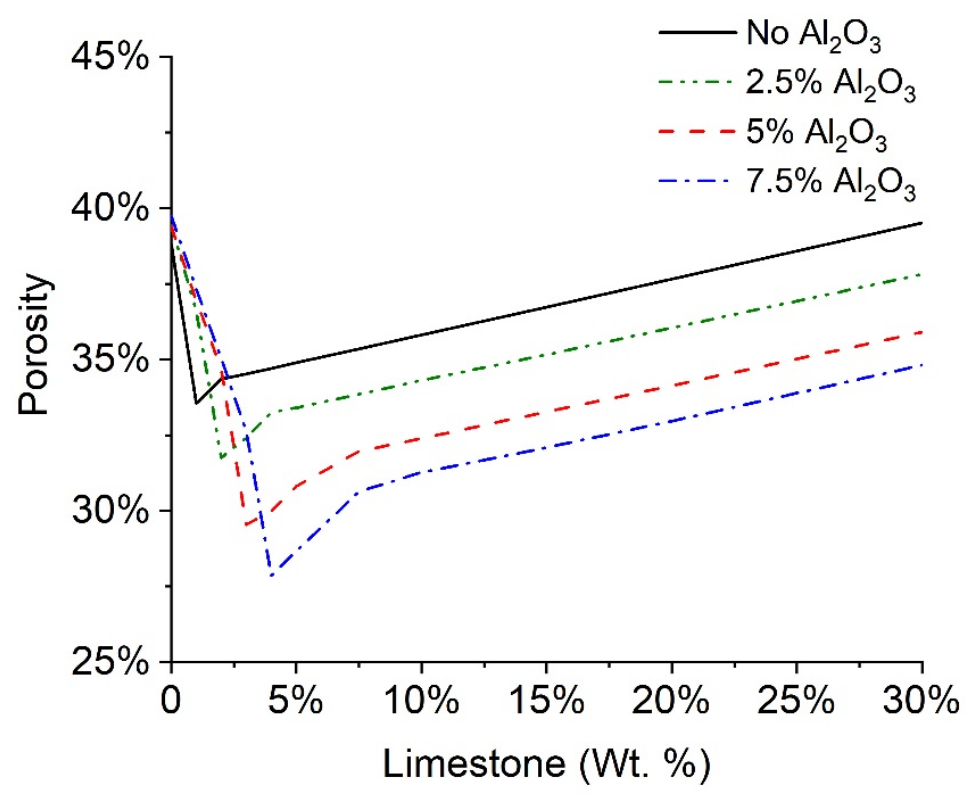

Figure 16-5. Plot of porosity of $\mathrm{PLC}+\mathrm{Al}_{2} \mathrm{O}_{3}$ systems showing the synergy between limestone and alumina.

\subsubsection{Reacted and Unreacted Calcite (From Limestone)}

2786 Figure 16-6 shows the reactive alumina in each of the mixtures and Figure 16-7 shows the amount of limestone that reacts and the unreacted limestone for each mixture. The reactive alumina is the total amount of reactive alumina from both the clinker and the SCM (calculated as the total sum

2789 of the products of degree of reactions of alumina-containing phases in the cement and SCM and 
2790 the total mass of alumina in the alumina-containing compound). It must be remembered that in 2791 this section of the report the limestone is considered to be pure calcite. From Figure 16-7 it can be 2792 seen that in neat systems (M0) only up to $2 \mathrm{~g}$ limestone reacts per $100 \mathrm{~g}$ binder. In systems 2793 containing little reactive alumina (M1, M2, and M5), between $4 \mathrm{~g}$ and $7 \mathrm{~g}$ of limestone reacts per 2794 100g binder. In systems containing more reactive alumina (M3 and M4), 9g-12g of limestone 2795 reacts. A clear synergy is seen between limestone and alumina in these systems. This can also be 2796 seen in Figure 16-4, where PLC systems of M4 and M5 have a lower porosity than their OPC 2797 counterparts due to the high reaction potential of these materials with limestone.

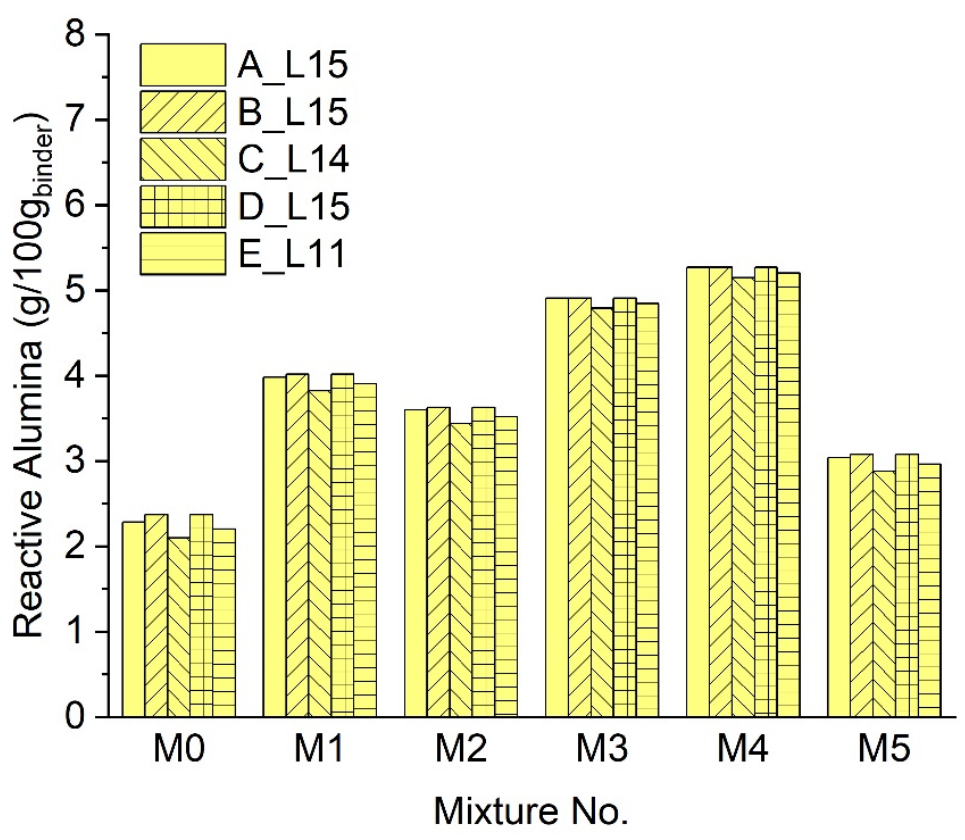

2798

2799 Figure 16-6. Reactive alumina in all mixtures. 


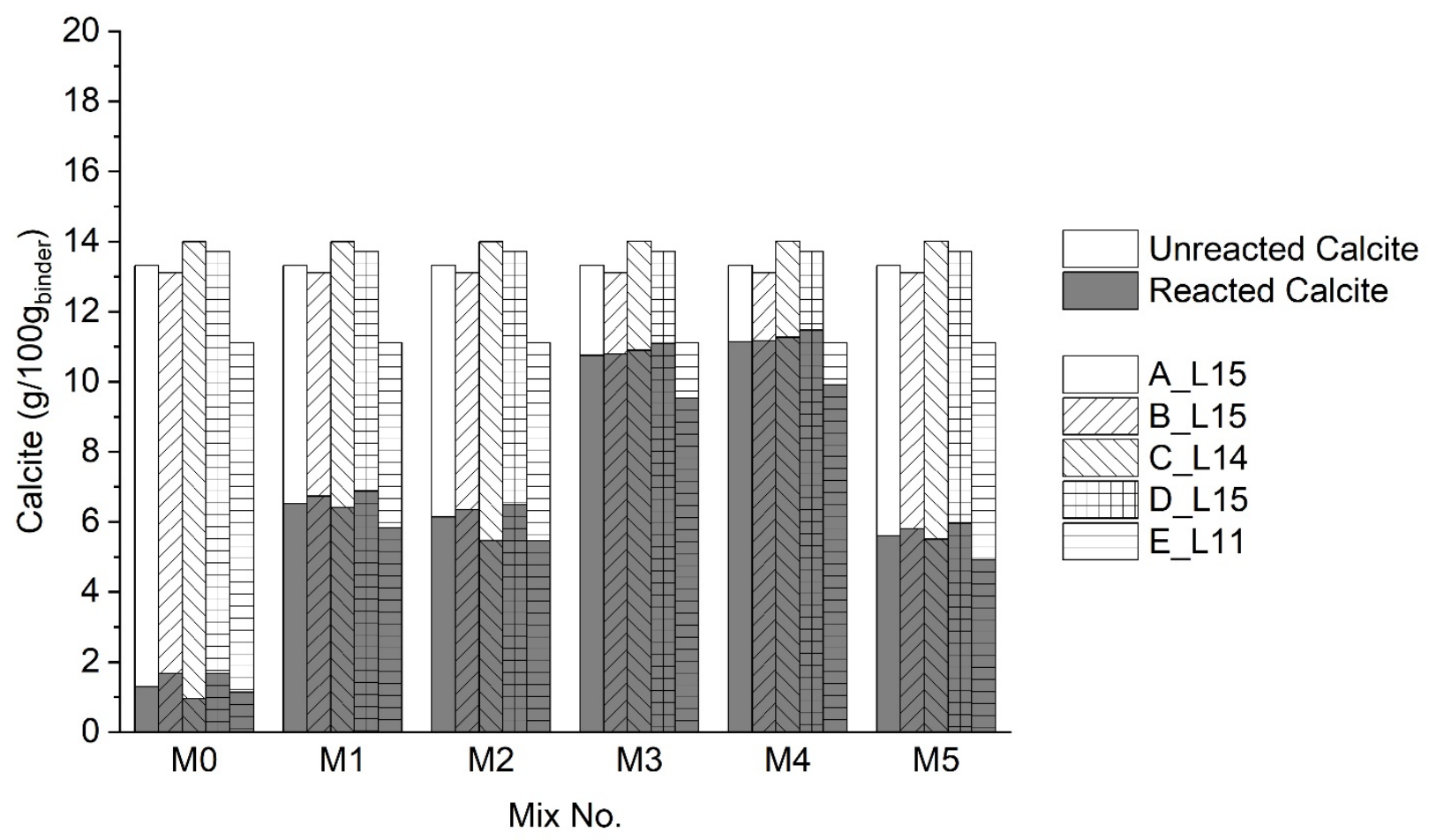

Figure 16-7. (a) Reacted calcite, and, (b) Unreacted calcite in PLC systems.

2803 The reactive alumina in each mixture is shown in Table 16.2 and the amount of reacted limestone 2804 is shown in Table 16.3 below.

2805

Table 16.2. Reactive alumina in each PLC mixture

\begin{tabular}{|c|c|c|c|c|c|c|}
\hline \multirow[b]{2}{*}{$\begin{array}{l}\text { Reactive } \mathrm{Al}_{2} \mathrm{O}_{3} \\
\text { (g /100g ginder) }\end{array}$} & M0 & M1 & M2 & M3 & M4 & M5 \\
\hline & PC & $\mathbf{P C}+\mathbf{F A}$ & $\begin{array}{c}\mathbf{P C}+\mathbf{F A}+\mathbf{S} \\
\mathbf{F}\end{array}$ & $\mathbf{P C}+\mathrm{SL}$ & $\underset{\mathbf{A}}{\mathrm{PC}+\mathrm{SL}+\mathrm{F}}$ & $\mathbf{P C}+\mathbf{N P}$ \\
\hline Clinker A & 2.29 & 3.98 & 3.60 & 4.91 & 5.27 & 3.04 \\
\hline Clinker B & 2.37 & 4.02 & 3.63 & 4.91 & 5.27 & 3.07 \\
\hline Clinker C & 2.10 & 3.82 & 3.44 & 4.79 & 5.15 & 2.88 \\
\hline Clinker D & 2.37 & 4.02 & 3.63 & 4.91 & 5.27 & 3.07 \\
\hline Clinker E & 2.20 & 3.90 & 3.52 & 4.84 & 5.20 & 2.96 \\
\hline Average & 2.27 & 3.95 & 3.56 & 4.87 & 5.23 & 3.01 \\
\hline St. Dev. & 0.12 & 0.08 & 0.08 & 0.05 & 0.05 & 0.08 \\
\hline
\end{tabular}


Table 16.3. Reacted limestone in each PLC mixture

\begin{tabular}{|l|c|c|c|c|c|c|}
\hline $\begin{array}{l}\text { Reacted CaCO } \\
\text { (g / } 100 g_{\text {binder }}\end{array}$ & M0 & M1 & M2 & M3 & M4 & M5 \\
\cline { 2 - 7 } & PC & PC+FA & $\begin{array}{c}\text { PC+FA+S } \\
\text { F }\end{array}$ & PC+SL & $\begin{array}{c}\text { PC+SL+F } \\
\text { A }\end{array}$ & PC+NP \\
\hline Clinker A & 1.29 & 6.52 & 6.14 & 10.75 & 11.14 & 5.60 \\
\hline Clinker B & 1.68 & 6.73 & 6.34 & 10.79 & 11.17 & 5.81 \\
\hline Clinker C & 0.95 & 6.41 & 5.46 & 10.89 & 11.27 & 5.50 \\
\hline Clinker D & 1.68 & 6.88 & 6.49 & 11.09 & 11.47 & 5.96 \\
\hline Clinker E & 1.13 & 5.83 & 5.45 & 9.53 & 9.92 & 4.92 \\
\hline Average & 1.35 & 6.47 & 5.97 & 10.61 & 10.99 & 5.56 \\
\hline St. Dev. & 0.32 & 0.40 & 0.49 & 0.62 & 0.62 & 0.40 \\
\hline
\end{tabular}

\section{16.6.3 Calcium Hydroxide (CH) Consumed}

2811 Figure 16-8 is a plot of the predicted $\mathrm{CH}$ consumed of PLC systems plotted against their 2812 corresponding OPC systems for the mixtures M0-M5 and clinkers A-E. From Figure 16-8, it can 2813 be seen that most of the results lie above the 1:1 line, indicating that the $\mathrm{CH}$ consumed in PLC 2814 pastes is slightly higher (about $1-2 \mathrm{~g} \mathrm{CH} / 100 \mathrm{~g}$ binder higher) than the $\mathrm{CH}$ consumed in the 2815 corresponding OPC paste. This is because of the carboaluminate reactions and the formation of 2816 different carboaluminate phases (monocarbonate forms at higher calcite contents and 2817 hemicarbonate forms at lower calcite contents $[22,48]$ ). It can also be seen that the $\mathrm{CH}$ consumed 2818 in PLC+SCM systems (M1 to M5) is higher than the CH consumed in PLC systems (M0). This is 2819 due to pozzolanic reactions that take place. Recent work [189] has also shown that the $\mathrm{CH}$ 2820 consumed in a system is an indicator of the secondary reactions that occur in cementitious systems 2821 and can be related to the extent of pore refinement. This would indicate that PLC systems have 2822 slightly more refined pore networks than the corresponding OPC systems. This is seen in Figure 2823 8-6. Whether this is due to the effect of finer grinding or changes to the phase assemblage needs 2824 to be investigated. 


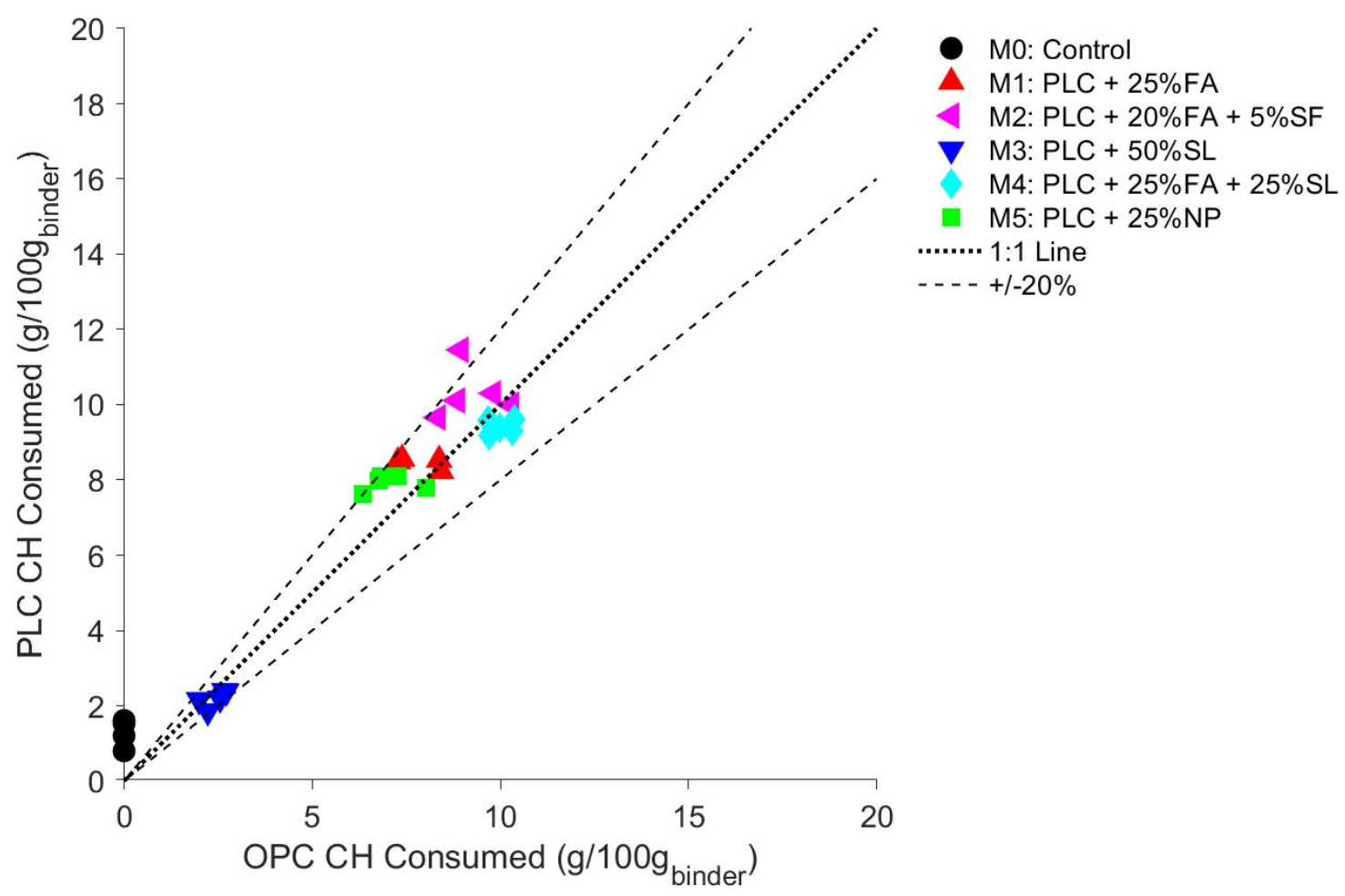

Figure 16-8. Plot of predicted CH consumed in PLC systems vs OPC systems.

\subsubsection{C-S-H}

2829 Figure 16-9 is a plot of the predicted C-S-H volume fraction PLC systems plotted against their 2830 corresponding OPC systems for the mixtures M0-M5 and clinkers A-E. From Figure 16-9, it can 2831 be seen that most of the results lie below the 1:1 line, indicating that the C-S-H volume in PLC 2832 pastes is slightly lower (approximately 3\% lower volume of C-S-H forms in PLC systems than 2833 OPC systems). This is because dilution of the clinker with limestone in PLC systems. It can also 2834 be seen that the C-S-H in neat systems (M0) is higher than the PLC+SCM systems (M1 to M5). 2835 This is because of the low reactivity of the SCMs in this study. This is underscored by the 2836 observation that system with a highly reactive SCM like silica fume (M2) has the closest volume 2837 of C-S-H to the neat system (M0). 


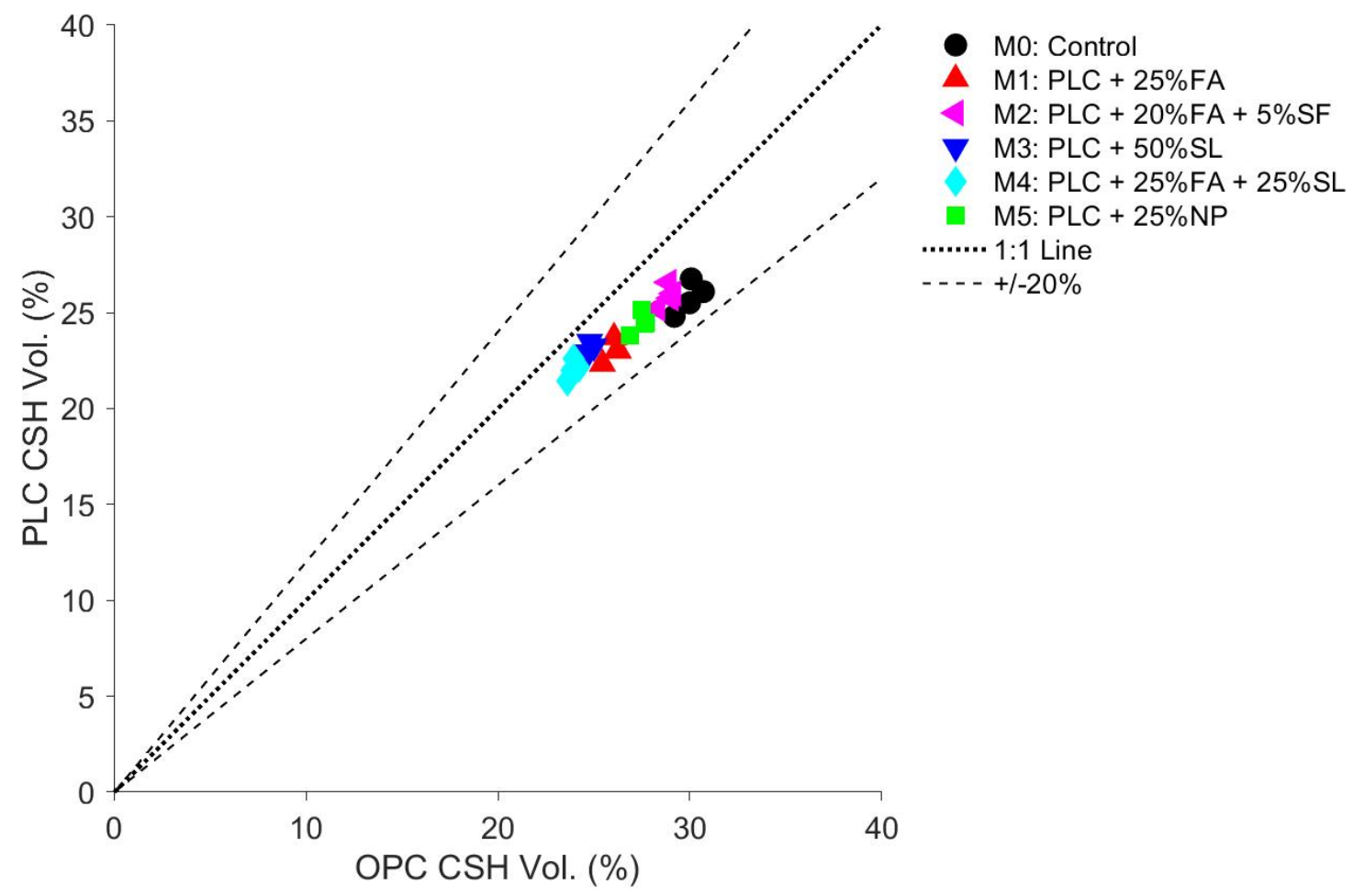

Figure 16-9. Plot of predicted C-S-H volume in PLC systems vs OPC systems.

2840 The volume difference of C-S-H between OPC and PLC systems is tabulated in Table 16.4 below.

Table 16.4. Decrease in C-S-H volume when OPC is replaced with PLC

\begin{tabular}{|l|l|l|l|l|l|l|}
\hline $\begin{array}{l}\text { C-S-H } \\
\text { Volume } \\
\text { Difference }\end{array}$ & M0 & M1 & M2 & M3 & M4 & M5 \\
\cline { 2 - 7 } Clinker A & $5 \%$ & $3 \%$ & $3 \%$ & $2 \%$ & $2 \%$ & $3 \%$ \\
\hline Clinker B & $4 \%$ & $3 \%$ & $3 \%$ & $2 \%$ & $2 \%$ & $3 \%$ \\
\hline Clinker C & $4 \%$ & $3 \%$ & $3 \%$ & $2 \%$ & $2 \%$ & $3 \%$ \\
\hline Clinker D & $4 \%$ & $3 \%$ & $3 \%$ & $2 \%$ & $2 \%$ & $3 \%$ \\
\hline Clinker E & $3 \%$ & $2 \%$ & $2 \%$ & $1 \%$ & $1 \%$ & $2 \%$ \\
\hline Average & $4 \%$ & $3 \%$ & $3 \%$ & $2 \%$ & $2 \%$ & $3 \%$ \\
\hline St. Dev. & $1 \%$ & $0 \%$ & $0 \%$ & $0 \%$ & $0 \%$ & $0 \%$ \\
\hline
\end{tabular}

2842 


\subsubsection{Carboaluminate Phases}

2845 Figure 16-10 is a plot of the predicted carboaluminate (hemi-/monocarbonate) phase volume 2846 fraction PLC systems plotted against their corresponding OPC systems for the mixtures M0-M5 2847 and clinkers A-E. From Figure 16-10, it can be seen that most of the results except for five data 2848 points lie below the 1:1 line, indicating that the carboaluminate phase volume in PLC pastes is 2849 slightly lower (approximately $0.4 \%$ lower volume of C-S-H forms in PLC systems than OPC 2850 systems). This is because dilution of the clinker with limestone in PLC systems without significant contribution of carboaluminate reaction from the SCMs. It can also be seen that the carboaluminate in neat systems is (M0) is much lower than in the PLC+SCM systems (M1 to M5). This is because of the reactive alumina in the SCMs used, which reacts with the limestone to form carboaluminate reactions. The mixtures M3 and M4 have the highest volumes of carboaluminate as they have the highest amounts of reactive alumina. This is also noted in Figure 16-7 where it can be seen that in mixtures M3 and M4, more limestone reacts.

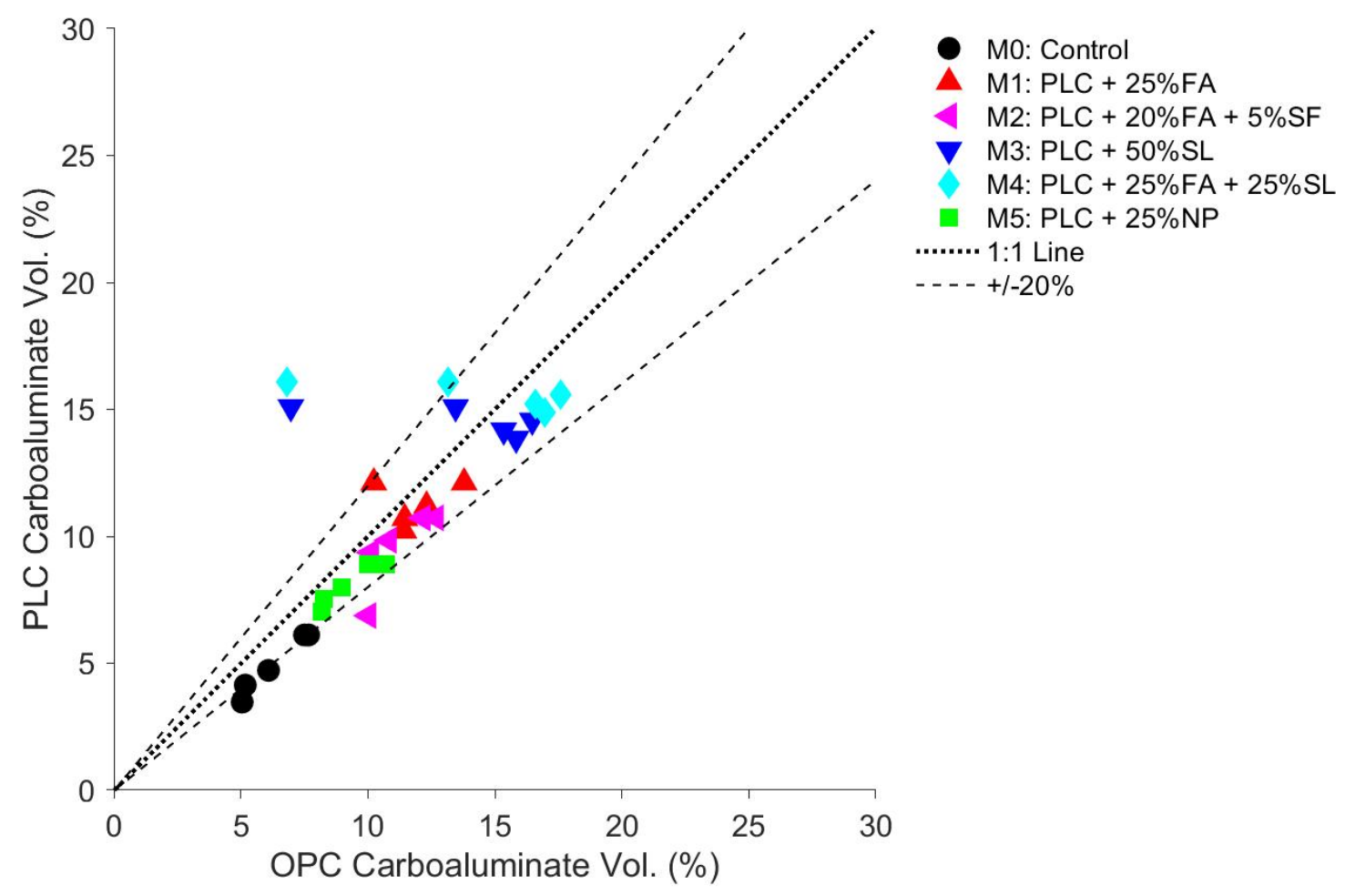

Figure 16-10. Plot of predicted volume of hemi-/monocarbonates in PLC systems vs OPC systems.

2860 The volumes of the carboaluminate phases formed in PLC systems is shown in Table 16.5 below. 
Table 16.5. Volume fraction of carboaluminate phases in the PLC systems

\begin{tabular}{|l|c|c|c|c|c|c|}
\hline $\begin{array}{l}\text { Carboaluminat } \\
\text { es (\% Vol.) }\end{array}$ & M0 & M1 & M2 & M3 & M4 & M5 \\
\cline { 2 - 6 } & PC & PC+FA & $\begin{array}{c}\text { PC+FA+S } \\
\text { F }\end{array}$ & PC+SL & $\begin{array}{c}\text { PC+SL+F } \\
\text { A }\end{array}$ & PC+NP \\
\hline Clinker A & $5 \%$ & $11 \%$ & $10 \%$ & $15 \%$ & $16 \%$ & $8 \%$ \\
\hline Clinker B & $6 \%$ & $12 \%$ & $11 \%$ & $15 \%$ & $16 \%$ & $9 \%$ \\
\hline Clinker C & $3 \%$ & $10 \%$ & $7 \%$ & $14 \%$ & $15 \%$ & $7 \%$ \\
\hline Clinker D & $6 \%$ & $12 \%$ & $11 \%$ & $15 \%$ & $16 \%$ & $9 \%$ \\
\hline Clinker E & $4 \%$ & $11 \%$ & $9 \%$ & $14 \%$ & $15 \%$ & $8 \%$ \\
\hline Average & $5 \%$ & $11 \%$ & $10 \%$ & $15 \%$ & $16 \%$ & $8 \%$ \\
\hline St. Dev. & $1 \%$ & $1 \%$ & $2 \%$ & $1 \%$ & $1 \%$ & $1 \%$ \\
\hline
\end{tabular}

\subsubsection{The pH of Pore Solution}

2865 Figure 16-11 is a plot of the predicted $\mathrm{pH}$ of pore solution in pastes made of OPC and PLC for the mixtures M0-M5 and clinkers A-E. From Figure 16-11, it can be seen that most of the results lie 2867 on the 1:1 line, indicating that the $\mathrm{pH}$ of PLC systems is not significantly different from the $\mathrm{pH}$ in 2868 OPC systems (the results all lie within $0.02 \mathrm{pH}$ of each other). This is likely because the dilution of the clinker with limestone in PLC systems counteracts the increased hydration due to finer grinding. These competing effects combined with the alkali binding due to the $\mathrm{C}-(\mathrm{A})-\mathrm{S}-\mathrm{H}$ phases present cause the $\mathrm{pH}$ to remain nearly similar. 


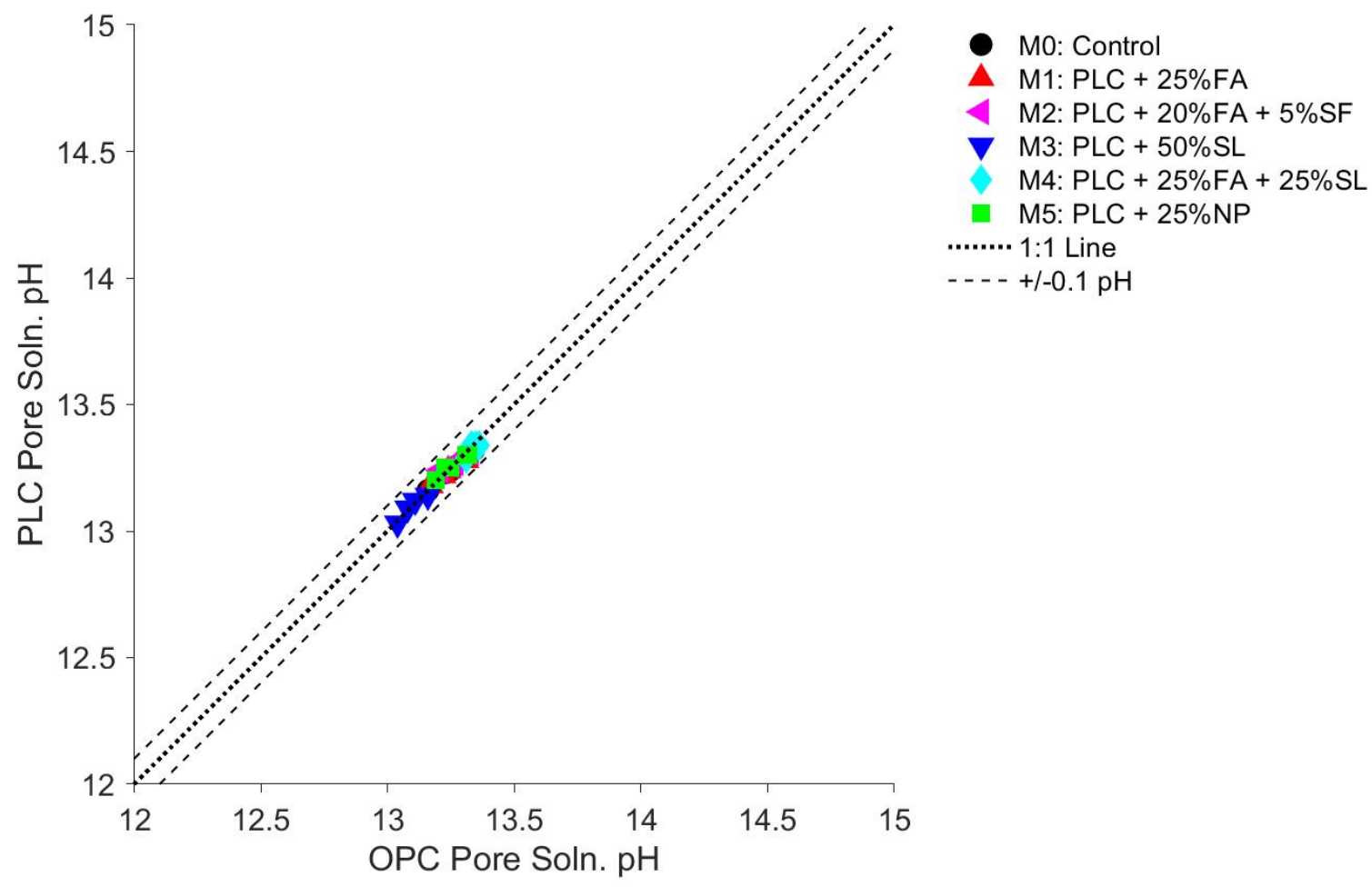

Figure 16-11. Plot of predicted pH of pore solution of PLC systems vs OPC systems.

2874 The difference in $\mathrm{pH}$ of pore solution between OPC and PLC systems is tabulated in Table 16.6 2875 below.

2876

Table 16.6. Difference in pore solution $\mathrm{pH}$ when OPC is replaced with PLC

\begin{tabular}{|l|c|c|c|c|c|c|}
\hline $\begin{array}{l}\text { pH } \\
\text { difference }\end{array}$ & M0 & M1 & M2 & M3 & M4 & M5 \\
\cline { 2 - 7 } & PC+FA & $\begin{array}{c}\text { PC+FA+S } \\
\text { F }\end{array}$ & PC+SL & $\begin{array}{c}\text { PC+SL+F } \\
\text { A }\end{array}$ & PC+NP \\
\hline Clinker A & 0.00 & 0.00 & -0.01 & -0.01 & -0.01 & -0.02 \\
\hline Clinker B & 0.02 & 0.01 & 0.02 & 0.02 & 0.01 & 0.02 \\
\hline Clinker C & 0.00 & -0.01 & -0.03 & 0.01 & 0.02 & -0.01 \\
\hline Clinker D & 0.02 & 0.03 & 0.02 & 0.02 & 0.02 & 0.01 \\
\hline Clinker E & 0.01 & 0.00 & 0.00 & -0.01 & -0.01 & 0.00 \\
\hline Average & 0.01 & 0.01 & 0.00 & 0.01 & 0.01 & 0.00 \\
\hline St. Dev. & 0.01 & 0.02 & 0.02 & 0.02 & 0.02 & 0.02 \\
\hline
\end{tabular}


2880 It is well known that the presence of limestone causes a change in the phase assemblage of 2881 cementitious systems. In the presence of limestone, ettringite is stabilized and hemi2882 /monocarbonate forms instead of monosulfate [21, 22, 48]. It is also known from previous 2883 experiments that SCMs containing alumina can react with the limestone in PLCs to improve 2884 performance $[14,83,181]$. In this section thermodynamic modeling was used to model 2885 experimental mixtures and it was found that the results of the model and the results of the 2886 experiment correlated well. The performance of PLC systems with respect to the heat released, 2887 porosity, quantity of limestone that reacts, Calcium Hydroxide consumed, the key phases that form 2888 (C-S-H, carboaluminates), and $\mathrm{pH}$ of pore solution is compared to the OPC counterparts. It is 2889 noteworthy that similar trends were obtained in both experiments and models. In general, the 2890 porosity of PLC systems is $1-3 \%$ higher than OPC systems, and the pore solution $\mathrm{pH}$ is not 2891 significantly different in PLC and OPC systems. When SCMs rich in alumina are used, they show 2892 synergistic behavior with the limestone in PLCs and the mass of limestone that reacts is directly 2893 proportional to the mass of reactive alumina in the SCMs. Therefore, we conclude that in typical 2894 OPC systems, PLCs can be used as a direct replacement for OPC, and in OPC+SCM systems (for 2895 typical SCM replacement levels), PLCs can be used instead of OPCs due to the synergistic 2896 behavior of limestone and alumina. 


\section{Conclusions}

2899 A comprehensive plan was undertaken to provide both experimental and computationally modeled 2900 results to address whether PLC may significantly impact the mechanical performance and 2901 durability of concrete materials specific to California, as compared to OPC. This work aims to 2902 provide CALTRANS with data to make informed decisions on the potential specification of PLC.

2903 The loss on ignition, chemical composition, oxide composition, the particle size of the raw 2904 materials, and the SCMs reactivity were determined. The heat of hydration was found to be within $2905+/-10 \%$ when the OPC, PLC, and OPC+LS systems were compared based on total cumulative 2906 heat; however, the PLC and OPC+LS systems were found to have a greater degree of reaction.

2907 ASR testing showed that the PLCs perform similar to or better than their parent OPCs. In addition, 2908 all the SCMs reduced expansions compared to the control for most of the mixtures. This resulted 2909 in the majority of the mixtures being below the $0.10 \%$ expansion limit recommended in ASTM 2910 C1778 (AASHTO R 80) for ASTM C1567 testing with the six exceptions, as noted in Section 5.7.4.

2912 Shrinkage measurements were performed for eighty mixtures. The majority of the samples were 2913 found to be statistically similar when comparing OPC, PLC and OPC + LS. The only exception 2914 for the OPC versus PLC system occurred for M3 and M4 at 14 and 28 days, with the PLC systems' 2915 shrinkage being 7-8\% higher. It should be noted that M3 and M4 mixtures made using OPC have 2916 approximately 10\% more shrinkage than M0. It is anticipated that this increase in shrinkage is due 2917 to increased reaction product. As a result, variations in shrinkage with replacing OPC with PLC 2918 or OPC with OPC + LS do not appear to be a concern as related to a potential increase in the 2919 shrinkage cracking.

2920 The flexural strength in the PLC and OPC + LS mixtures was on average less than 5\% lower than the OPC mixtures. However, the flexural strength was up to $13 \%$ greater for PLC when combined with slag. Overall, the flexural strength was consistently within the $+/-15 \%$ range compared with the parent system. In conclusion, PLC can be used as an alternative to OPC for systems made with type II/V clinker with and without SCM.

2925 The initial or final setting times of the PLC and OPC + LS mixtures was statistically similar. In 2926 some cases, the addition of SCMs did influence the initial and final setting times.

2927 No statistically significant difference was observed in the bound chloride contents of mixtures 2928 comparing OPC (i.e., ASTM C 150/AASHTO M 85), PLC (i.e., ASTM C 595/AASHTO M 240), and $\mathrm{OPC}+\mathrm{LS}$ (provided that a specific size and quality of limestone is used). No statistically 2930 significant difference was observed in the bound chloride contents for mixtures comparing OPC, 2931 PLC, and OPC+LS with SCM (i.e., fly ash, silica fume, natural pozzolan). Mixtures containing 2932 PLC and OPC + LS mixtures made with ground granulated blast-furnace slag, which is generally 
2933 referred to as slag cement if it meets ASTM C 989, outperformed the OPC+Slag system. It should

2934 also be noted that for the calculation of the allowable chloride content for new mixtures and 2935 comparison with set limits by ACI 318, limestone should be included in the total binder content.

2936 In other words, the PLC+SCM content, or OPC+SCM+LS content be considered as binder.

2937 The measured porosity, formation factor, and chloride apparent diffusion coefficient of PLC 2938 concrete are comparable to those obtained on OPC concrete. The OPC +LS mixture may result in 2939 excessive porosity due to insufficient consolidation and as such care should be taken to examine 2940 the PSDs when these materials are combined.

2941 The OPC and PLC systems have comparable times to corrosion initiation. The critical chloride 2942 concentration was approximately $0.4 \%$ by weight of binder. The findings from the modified 2943 ASTM G109 test indicate that OPC and PLC specimens exhibit similar total charge passed until 2944 the time of activation and most of the specimens were observed to activate after 7 cycles. The OPC 2945 and PLC will likely exhibit similar times to corrosion.

2946 There was no significant difference in measured air contents between OPC and PLC concrete 2947 mixtures that are not consistent with variations in the grind of the cement. As such, OPC and PLC 2948 can be used in concrete mixtures to obtain air contents that perform well in a FT environment.

2949 The PLCs performed similar to, if not better than, their corresponding OPC mixtures when exposed 2950 to sulfate when SCMs are used. Therefore, CALTRANS can use their current SCM mixtures as is 2951 with ASTM C 150 Type II or V clinker that is then interground or interblended with PLC and 2952 expect similar external sulfate resistance to ASTM C150 Type II or V cements.

2953 Replacement of OPC with PLC (or OPC +LS) offers the potential for a reduction of GHG of 10$295412 \%$ on average (up to $15 \%$ ).

2955 Computational modeling was used to show that the presence of limestone, ettringite is stabilized, 2956 and hemi-/monocarbonate forms instead of monosulfate. SCMs containing alumina can react with 2957 the limestone in PLCs to improved performance. The performance of PLC systems with respect to 2958 the heat released, porosity, quantity of limestone that reacts, Calcium Hydroxide consumed, the 2959 key phases that form (C-S-H, carboaluminates), and $\mathrm{pH}$ of pore solution is compared or improved 2960 when compared to the OPC counterparts. As a result, PLCs can be used as a direct replacement 2961 for OPC, and in OPC+SCM systems (for typical SCM replacement levels). Further, when PLCs 2962 are used with SCM there can be a synergistic behavior between the limestone and alumina that 2963 improves overall performance.

2964 The results indicate that PLCs can be used as a direct replacement for OPC. It should also be noted 2965 that PLC can replace OPC in systems containing SCM systems. Further, when PLCs are used with 2966 SCM, there can be a synergistic behavior between the limestone and reactive alumina in the SCMs 
2967 that improves overall performance. As such, we recommend that specifications that permit the use 2968 of OPC (ASTM C 150, AASHTO M 85) could also permit the use of PLC (ASTM C 595, 2969 AASHTO M 240). Specifications could also be developed to permit the use of up to $10 \%$ 2970 limestone with OPC; however, some details will be needed on the chemical and physical properties 2971 of the limestone to ensure its size (packing and reaction) and chemical purity.

2972 
1. Weiss, J.W., et al., Impact of Use of Portland-Limestone Cement on Concrete Performance as Plain or Reinforced Material, in California Department of Transportation - Research Proposal. 2017, Oregon State University: Corvallis.

2. Barrett, T.J., H. Sun, and W.J. Weiss, Performance of portland limestone cements: Cements designed to be more sustainable that include up to 15\% limestone addition. 2013.

3. Smartz, B.W., T.S. Laker, and T. Van Dam, Performance and Sustainability. Concrete international, 2013. 35(9): p. 39-43.

4. Hawkins, P., P.D. Tennis, and R.J. Detwiler, The use of limestone in Portland cement: a state-of-the-art review. 1996: Portland Cement Association.

5. Hooton, R., M. Nokken, and M. Thomas, Portland-limestone cement: state-of-the-art report and gap analysis for CSA A 3000. Cement Association of Canada. University of Toronto, 2007.

6. Tennis, P., M. Thomas, and W. Weiss, State-of-the-Art Report on Use of Limestone in Cements at Levels of up to 15\%. PCA R\&D SN3148, Portland Cement Association, Skokie, IL, 2011.

7. Chang, M.T., et al., Using X-ray fluorescence to assess the chemical composition and resistivity of simulated cementitious pore solutions. International Journal of Advances in Engineering Sciences and Applied Mathematics, 2017. 9(3): p. 136-143.

8. Lothenbach, B., K. Scrivener, and R.D. Hooton, Supplementary cementitious materials. Cement and Concrete Research, 2011. 41(12): p. 1244-1256.

9. Thomas, M., Supplementary cementing materials in concrete. 2013: CRC press.

10. Shaker, F., A. Rashad, and M. Allam, Properties of concrete incorporating locally produced Portland limestone cement. Ain Shams Engineering Journal, 2018. 9(4): p. 23012309.

11. Dhir, R., et al., Evaluation of Portland limestone cements for use in concrete construction. Materials and structures, 2007. 40(5): p. 459-473.

12. Chen, J., A. Kwan, and Y. Jiang, Adding limestone fines as cement paste replacement to reduce water permeability and sorptivity of concrete. Construction and Building Materials, 2014. 56: p. 87-93.

13. Meddah, M.S., M.C. Lmbachiya, and R.K. Dhir, Potential use of binary and composite limestone cements in concrete production. Construction and Building Materials, 2014. 58 : p. 193-205.

14. De Weerdt, K., et al., Synergy between fly ash and limestone powder in ternary cements. Cement and concrete composites, 2011. 33(1): p. 30-38.

15. Alunno-Rosetti, V. and F. Curcio. A contribution to the knowledge of the properties of Portland-Limestone Cement concretes, with respect to the requirements of European and Italian design code. in Proceedings of the 10th International Congress on the Chemistry of Cement. 1997. H. Justnes.

16. Bonavetti, V., et al., Influence of initial curing on the properties of concrete containing limestone blended cement. Cement and Concrete Research, 2000. 30(5): p. 703-708.

17. Irassar, E., et al., Mechanical properties and durability of concrete made with portland limestone cement. ACI SPECIAL PUBLICATIONS, 2001. 202: p. 431-450. 
3017

3018

3019

3020

3021

3022

3023

3024

3025

3026

3027

3028

3029

3030

3031

3032

3033

3034

3035

3036

3037

3038

3039

3040

3041

3042

3043

3044

3045

3046

3047

3048

3049

3050

3051

3052

3053

3054

3055

3056

3057

3058

3059

3060

3061

18. Ghrici, M., S. Kenai, and M. Said-Mansour, Mechanical properties and durability of mortar and concrete containing natural pozzolana and limestone blended cements. Cement and Concrete Composites, 2007. 29(7): p. 542-549.

19. Li, W., et al., Effects of nano-silica and nano-limestone on flowability and mechanical properties of ultra-high-performance concrete matrix. Construction and Building Materials, 2015. 95: p. 366-374.

20. Matschei, T., et al. Relationships of Cement Paste Mineralogy to Porosity and Mechanical Properties. in International Conference on Modelling of Heterogeneous Materials with Applications in Construction and Biomedical Engineering. 2007.

21. Hawkins, P., P.D. Tennis, and R.J. Detwiler, The use of limestone in Portland cement: a state-of-the-art review. ENGINEERING BULLETIN 227 ed. 2005: Portland Cement Association. 41.

22. Lothenbach, B., et al., Influence of limestone on the hydration of Portland cements. Cement and Concrete Research, 2008. 38(6): p. 848-860.

23. Vuk, T., et al., The effects of limestone addition, clinker type and fineness on properties of Portland cement. Cement and concrete Research, 2001. 31(1): p. 135-139.

24. Voglis, N., et al., Portland-limestone cements. Their properties and hydration compared to those of other composite cements. Cement and Concrete Composites, 2005. 27(2): p. 191-196.

25. Schmidt, M., Cement with interground additives--capabilities and environmental relief: I. ZKG International, Edition B, 1992. 45(2): p. 64-9.

26. Schmidt, M., Cement with interground additives--capabilities and environmental relief: II. ZKG International, Edition B, 1992. 45(6): p. 296-301.

27. Seyedalhosseini Natanzi, A.S., Improving Durability of Different Limestone Cement and Effects of it on Reinforcement. 2013.

28. Menéndez, G., V. Bonavetti, and E. Irassar, Strength development of ternary blended cement with limestone filler and blast-furnace slag. Cement and Concrete Composites, 2003. 25(1): p. 61-67.

29. Turker, P. and K. Erdogdu. Effects of Limestone Addition on Microstructure and Hydration of Cements. in PROCEEDINGS OF THE INTERNATIONAL CONFERENCE ON CEMENT MICROSCOPY. 2000. INTERNATIONAL CEMENT MICROSCOPY ASSOCIATION.

30. Ezziane, K., et al., Effect of mineral additives on the setting of blended cement by the maturity method. Materials and structures, 2010. 43(3): p. 393-401.

31. Choudhary, A., V. Shah, and S. Bishnoi, Effect of low cost fillers on cement hydration. Construction and Building Materials, 2016. 124: p. 533-543.

32. Sun, H., et al., Jet mill grinding of portland cement, limestone, and fly ash: Impact on particle size, hydration rate, and strength. Cement and Concrete Composites, 2013. 44: $\mathrm{p}$. 41-49.

33. Bentz, D.P., et al., Relating compressive strength to heat release in mortars. Advances in Civil Engineering Materials, 2012. 1(1): p. 1-14.

34. Choudhary, A., et al., Examining Factors that Influence the Measurement of Pozzolanic Reactivity of Supplementary Cementitious Materials. In Review, 2021.

35. Glosser, D., et al., Investigation of the Reactivity of Fly Ash and its Effect on Mixture Properties. ACI Materials Journal, 2019. In Press. 
3062

3063

3064

3065

3066

3067

3068

3069

3070

3071

3072

3073

3074

3075

3076

3077

3078

3079

3080

3081

3082

3083

3084

3085

3086

3087

3088

3089

3090

3091

3092

3093

3094

3095

3096

3097

3098

3099

3100

3101

3102

3103

3104

3105

3106

36. Moradllo, M.K., et al., Use of borosilicate glass powder in cementitious materials: Pozzolanic reactivity and neutron shielding properties. Cement and Concrete Composites, 2020. 112: p. 103640.

37. Suraneni, P. and J. Weiss, Examining the pozzolanicity of supplementary cementitious materials using isothermal calorimetry and thermogravimetric analysis. Cement and Concrete Composites, 2017. 83: p. 273-278.

38. ASTM, Standard Test Methods for Chemical Analysis of Hydraulic Cement, in C114-18. 2018, ASTM International: West Conshohocken.

39. Alarcon-Ruiz, L., et al., The use of thermal analysis in assessing the effect of temperature on a cement paste. Cement and Concrete research, 2005. 35(3): p. 609-613.

40. Pane, I. and W. Hansen, Investigation of blended cement hydration by isothermal calorimetry and thermal analysis. Cement and concrete research, 2005. 35(6): p. 11551164.

41. Villain, G., M. Thiery, and G. Platret, Measurement methods of carbonation profiles in concrete: Thermogravimetry, chemical analysis and gammadensimetry. Cement and concrete research, 2007. 37(8): p. 1182-1192.

42. McConnell, D., et al. Cement-aggregate reaction in concrete. in Journal Proceedings. 1947.

43. Kim, T. and J. Olek, Effects of sample preparation and interpretation of thermogravimetric curves on calcium hydroxide in hydrated pastes and mortars. Transportation research record, 2012. 2290(1): p. 10-18.

44. Lothenbach, B. and F. Winnefeld, Thermodynamic modelling of the hydration of Portland cement. Cement and Concrete Research, 2006. 36(2): p. 209-226.

45. Lothenbach, B., et al., Effect of temperature on the pore solution, microstructure and hydration products of Portland cement pastes. Cement and Concrete Research, 2007. 37(4): p. 483-491.

46. Azad, V.J., et al., A COMSOL-GEMS interface for modeling coupled reactive-transport geochemical processes. Computers \& Geosciences, 2016. 92: p. 79-89.

47. Guo, Y., et al., Internal Curing for Concrete Bridge Decks: Integration of a Social Cost Analysis in Evaluation of Long-Term Benefit. Transportation Research Record, 2016. 2577(1): p. 25-34.

48. Lothenbach, B., et al., Cemdata 18: A chemical thermodynamic database for hydrated Portland cements and alkali-activated materials. Cement and Concrete Research, 2019. 115: p. 472-506.

49. Stanton, T.E., Expansion of concrete through reaction between cement and aggregate. Transactions of the American Society of Civil Engineers, 1942. 107(1): p. 54-84.

50. Rajabipour, F., et al., Alkali-silica reaction: Current understanding of the reaction mechanisms and the knowledge gaps. Cement and Concrete Research, 2015. 76: p. 130146.

51. Thomas, M., The effect of supplementary cementing materials on alkali-silica reaction: A review. Cement and concrete research, 2011. 41(12): p. 1224-1231.

52. Bhatty, M. and N. Greening. Interaction of alkalies with hydrating and hydrated calcium silicates. in Proceedings. 1978.

53. Hong, S.-Y. and F. Glasser, Alkali binding in cement pastes: Part I. The CSH phase. Cement and Concrete Research, 1999. 29(12): p. 1893-1903. 
54. Chappex, T. and K. Scrivener, Alkali fixation of $C-S-H$ in blended cement pastes and its relation to alkali silica reaction. Cement and Concrete Research, 2012. 42(8): p. 10491054.

55. Shi, Z., B. Ma, and B. Lothenbach, Effect of Al on the formation and structure of alkalisilica reaction products. Cement and Concrete Research, 2021. 140: p. 106311.

56. Folliard, K.J., et al., Preventing Alkali-Silica Reaction and Delayed Ettringite Formation in New Concrete. Project Summary report 0-4085, Center for Transportation Research, University of Texas, Austin, 2006.

57. Ramezanianpour, A.A., et al., Influence of various amounts of limestone powder on performance of Portland limestone cement concretes. Cement and Concrete Composites, 2009. 31(10): p. 715-720.

58. ASTM, Standard Test Method for Determining the Potential Alkali-Silica Reactivity of Combinations of Cementitious Materials and Aggregate (Accelerated Mortar-Bar Method), in C1567-13. 2013, ASTM International: West Conshohocken.

59. ASTM, Standard Test Method for Determination of Length Change of Concrete Due to Alkali-Silica Reaction, in C1293-20a. 2020, ASTM International: West Conshohocken.

60. Thomas, M., et al., Test methods for evaluating preventive measures for controlling expansion due to alkali-silica reaction in concrete. Cement and Concrete Research, 2006. 36(10): p. 1842-1856.

61. AASHTO, Standard Method of Test for Potential Alkali Reactivity of Aggregates and Effectiveness of ASR Mitigation Measures (Miniature Concrete Prism Test, MCPT), in T 380-19. 2019, American Association of State Highway and Transportation Officials: Washington DC.

62. Chopperla, K.S.T. and e. al., Bench-marking the miniature concrete prism test and the concrete cylinder tests to field exposure blocks for mitigation of alkali-silica reaction, in 15th International Congreess on the Chemistry of Cement. 2019: Prague, Czech Republic.

63. Tanesi, J., et al., Divergence between Performance in the Field and Laboratory Test Results for Alkali-Silica Reaction. Transportation Research Record, 2020. 2674(5): p. 120134.

64. Laker, T. and B. Smartz, Evaluation of Portland limestone performance cements in Colorado and Utah transportation projects: 2007 to present, in Transportation Research Board. 2012.

65. Thomas, M., et al. Equivalent performance with half the clinker content using PLC and SCM. in Proc., NRMCA Concrete Sustainability Conference. 2010.

66. Hooton, R., A. Ramezanianpour, and U. Schutz. Decreasing the clinker component in cementing materials: performance of Portland-limestone cements in concrete in combination with supplementary cementing materials. in 2010 Concrete Sustainability Conference, NRMCA. 2010. Arizona State University Tempe, AZ.

67. ASTM, Standard Test Method for Effectiveness of Pozzolans or Ground Blast-Furnace Slag in Preventing Excessive Expansion of Concrete Due to the Alkali-Silica Reaction1, in C441-17. 2017, ASTM International: West Conshohocken.

68. ASTM, Standard Guide for Reducing the Risk of Deleterious Alkali-Aggregate Reaction in Concrete, in C1778-20. 2020, ASTM International: West Conshohocken.

69. Schlorholtz, S.M., Alkali Content of Fly Ash-Measuring and Testing Strategies for Compliance. 2015. 
70. Thomas, M. and M. Shehata. Use of blended cements to control expansion of concrete due to alkali-silica reaction. in Proc. 8 th CANMET/ACI Int. Conf. on Fly Ash, Silica Fume, Slag and Natural Pozzolans in Concrete. 2004.

71. Latifee, E.R. and P.R. Rangaraju, Miniature concrete prism test: rapid test method for evaluating alkali-silica reactivity of aggregates. Journal of Materials in Civil Engineering, 2015. 27(7): p. 04014215.

72. Thomson, W., 4. On the equilibrium of vapour at a curved surface of liquid. Proceedings of the Royal Society of Edinburgh, 1872. 7: p. 63-68.

73. Bucher, B., A. Radlinska, and J. Weiss. Preliminary comments on shrinkage and shrinkage cracking behavior of cement systems that contain limestone. in Concrete Technology Forum. Focus on Sustainable DevelopmentNational Ready Mixed Concrete Association. 2008.

74. Bucher, B.E., Shrinkage and shrinkage cracking behavior of cement systems containing ground limestone, fly ash, and lightweight synthetic particles. 2009.

75. Stubstad, R., D. Glauz, and D. Rufino, Use of Raw Limestone in Portland Cement. 2008, California Department of Transportation. p. 1-20.

76. Barrett, T., et al., Early-age shrinkage behavior of Portland limestone cement. Concrete international, 2014. 36(2).

77. Adams, L.D. and R.M. Race, Effect of limestone additions upon drying shrinkage of Portland cement mortar, in Carbonate additions to cement. 1990, ASTM International.

78. Cost, T. The Advantages of Portland Limestone Cement. 2014; Available from: https://www.concreteconstruction.net/concrete-production-precast/the-advantages-ofportland-limestone-cement_o.

79. Piasta, W. and H. Sikora, Effect of air entrainment on shrinkage of blended cements concretes. Construction and Building Materials, 2015. 99: p. 298-307.

80. Bentz, D.P., et al. Sorptivity-based service life predictions for concrete pavements. in Proceedings of the 7th International Conference on Concrete pavements. 2001. Citeseer.

81. ASTM, Standard Test Method for Length Change of Hardened Hydraulic-Cement Mortar and Concrete, in C157-17. 2017, ASTM International: West Conshohocken.

82. Pickett, G. Effect of aggregate on shrinkage of concrete and a hypothesis concerning shrinkage. in Journal Proceedings. 1956.

83. De Weerdt, K., et al., Hydration mechanisms of ternary Portland cements containing limestone powder and fly ash. Cement and Concrete Research, 2011. 41(3): p. 279-291.

84. Bentz, D.P., et al., Fine limestone additions to regulate setting in high volume fly ash mixtures. Cement and Concrete Composites, 2012. 34(1): p. 11-17.

85. Börger, A., P. Supancic, and R. Danzer, The ball on three balls test for strength testing of brittle discs: stress distribution in the disc. Journal of the European Ceramic Society, 2002. 22(9-10): p. 1425-1436.

86. Börger, A., P. Supancic, and R. Danzer, The ball on three balls test for strength testing of brittle discs: Part II: analysis of possible errors in the strength determination. Journal of the European Ceramic Society, 2004. 24(10-11): p. 2917-2928.

87. Borges, J.U.A., et al., Length effect on ductility of concrete in uniaxial and flexural compression. Structural Journal, 2004. 101(6): p. 765-772.

88. Danzer, R., et al., The ball on three balls test-Strength and failure analysis of different materials. Journal of the European ceramic society, 2007. 27(2-3): p. 1481-1485. 
89. Fu, T. and W.J. Weiss, The Ball-on-Three-Ball (B3B) Test-Application to Cement Paste and Mortar. Advances in Civil Engineering Materials, 2020. 9(1): p. 128-142.

90. AASHTO, Standard Method of Test for Determining the Total Pore Volume in Hardened Concrete Using Vacuum Saturation, in AASHTO TP 135-20. 2020, American Association of State Highway and Transportation Officials: Washington DC.

91. AASHTO, Standard Method of Test for Electrical Resistivity of a Concrete Cylinder Tested in a Uniaxial Resistance Test, in TP 119-20. 2020, American Association of State Highway and Transportation Officials: Washington DC.

92. Coyle, A.T., et al., Comparison of linear temperature corrections and activation energy temperature corrections for electrical resistivity measurements of concrete. Advances in Civil Engineering Materials, 2018. 7(1): p. 174-187.

93. Ramachandran, V.S., R. Seeley, and G. Polomark, Free and combined chloride in hydrating cement and cement components. Matériaux et Construction, 1984. 17(4): p. 285289.

94. Diamond, S., Chloride concentrations in concrete pore solutions resulting from calcium and sodium chloride admixtures. Cement, concrete and aggregates, 1986. 8(2): p. 97-102.

95. Beaudoin, J.J., V.S. Ramachandran, and R.F. Feldman, Interaction of chloride and $C \quad S$ H. Cement and Concrete Research, 1990. 20(6): p. 875-883.

96. Zibara, H., Binding of external chlorides by cement pastes. 2001.

97. Hussain, S.E. and S. Al-Saadoun, Effect of tricalcium aluminate content of cement on chloride binding corrosion of reinforcing steel in concrete. Materials Journal, 1993. 89(1): p. 3-12.

98. Thomas, M.D. and R.D. Hooton, The durability of concrete produced with portlandlimestone cement: Canadian studies. PCA R\&D SN3142, Portland Cement Association, Skokie, IL, 2010: p. 28.

99. Jafari Azad, V., et al., Relating the formation factor and chloride binding parameters to the apparent chloride diffusion coefficient of concrete. Journal of Materials in Civil Engineering, 2019. 31(2): p. 04018392.

100. Justnes, H., A review of chloride binding in cementitious systems. Nordic Concrete Research-Publications-, 1998. 21: p. 48-63.

101. Balonis, M., et al., Impact of chloride on the mineralogy of hydrated Portland cement systems. Cement and Concrete Research, 2010. 40(7): p. 1009-1022.

102. Luping, T. and L.-O. Nilsson, Chloride binding capacity and binding isotherms of OPC pastes and mortars. Cement and concrete research, 1993. 23(2): p. 247-253.

103. Csizmadia, J., G. Balázs, and F.D. Tamás, Chloride ion binding capacity of tetracalcium aluminoferrite. Periodica Polytechnica Civil Engineering, 2000. 44(2): p. 135-150.

104. Marty, N.C., et al., Dissolution kinetics of hydrated calcium aluminates (AFm-Cl) as a function of $\mathrm{pH}$ and at room temperature. Mineralogical Magazine, 2017. 81(5): p. 12451259.

105. Birnin-Yauri, U. and F. Glasser, Friedel's salt, $\mathrm{Ca} 2 \mathrm{Al}(\mathrm{OH}) 6(\mathrm{Cl}, \mathrm{OH}) \cdot 2 \mathrm{H} 2 \mathrm{O}$ : its solid solutions and their role in chloride binding. Cement and Concrete Research, 1998. 28(12): p. 1713-1723.

106. Sui, S., et al., Quantification methods for chloride binding in Portland cement and limestone systems. Cement and Concrete Research, 2019. 125: p. 105864. 
107. Glasser, F., A. Kindness, and S. Stronach, Stability and solubility relationships in AFm phases: Part I. Chloride, sulfate and hydroxide. Cement and Concrete Research, 1999. 29(6): p. 861-866.

108. Yoon, S., et al., Phase changes of monosulfoaluminate in $\mathrm{NaCl}$ aqueous solution. Materials, 2016. 9(5): p. 401.

109. Ipavec, A., et al., Chloride binding into hydrated blended cements: The influence of limestone and alkalinity. Cement and Concrete Research, 2013. 48: p. 74-85.

110. Ben-Yair, M., The effect of chlorides on concrete in hot and arid regions. Cement and Concrete Research, 1974. 4(3): p. 405-416.

111. Yuan, Q., et al., Chloride binding of cement-based materials subjected to external chloride environment-a review. Construction and building materials, 2009. 23(1): p. 1-13.

112. Qiao, C., P. Suraneni, and J. Weiss, Phase Diagram and Volume Change of the Ca (OH) 2 - $\mathrm{CaCl} 2-\mathrm{H} 2 \mathrm{O}$ System for Varying $\mathrm{Ca}(\mathrm{OH}) 2 / \mathrm{CaCl} 2$ Molar Ratios. Journal of Materials in Civil Engineering, 2018. 30(2): p. 04017281.

113. Qiao, C., et al., Chloride binding of cement pastes with fly ash exposed to CaCl2 solutions at 5 and $23^{\circ} \mathrm{C}$. Cement and Concrete Composites, 2019. 97: p. 43-53.

114. De Weerdt, K., et al., Impact of the associated cation on chloride binding of Portland cement paste. Cement and Concrete Research, 2015. 68: p. 196-202.

115. De Weerdt, K., D. Orsáková, and M.R. Geiker, The impact of sulphate and magnesium on chloride binding in Portland cement paste. Cement and Concrete Research, 2014. 65: p. 30-40.

116. $\mathrm{Bu}, \mathrm{Y} ., \mathrm{R}$. Spragg, and $\mathrm{W}$. Weiss, Comparison of the pore volume in concrete as determined using ASTM C642 and vacuum saturation. Advances in Civil Engineering Materials, 2014. 3(1): p. 308-315.

117. Jensen, O.M., et al., Chloride ingress in cement paste and mortar. Cement and Concrete Research, 1999. 29(9): p. 1497-1504.

118. Kalinichev, A.G. and R.J. Kirkpatrick, Molecular dynamics modeling of chloride binding to the surfaces of calcium hydroxide, hydrated calcium aluminate, and calcium silicate phases. Chemistry of Materials, 2002. 14(8): p. 3539-3549.

119. Delagrave, A., et al., Chloride binding capacity of various hydrated cement paste systems. Advanced Cement based materials, 1997. 6(1): p. 28-35.

120. $\mathrm{Bu}, \mathrm{Y}$. and $\mathrm{J}$. Weiss, The influence of alkali content on the electrical resistivity and transport properties of cementitious materials. Cement and Concrete Composites, 2014. 51: p. 49-58.

121. Thomas, J.J., A.J. Allen, and H.M. Jennings, Density and water content of nanoscale solid $C-S-H$ formed in alkali-activated slag (AAS) paste and implications for chemical shrinkage. Cement and Concrete Research, 2012. 42(2): p. 377-383.

122. Tabachnick, B.G. and L.S. Fidell, Experimental designs using ANOVA. 2007: Thomson/Brooks/Cole Belmont, CA.

123. Azad, V.J., et al., Interpreting the pore structure of hydrating cement phases through a synergistic use of the Powers-Brownyard model, hydration kinetics, and thermodynamic calculations. Advances in Civil Engineering Materials, 2017. 6(1): p. 1-16.

124. Shi, Z., et al., Role of calcium on chloride binding in hydrated Portland cementmetakaolin-limestone blends. Cement and Concrete Research, 2017. 95: p. 205-216. 
125. Ding, Z., et al., Cementing mechanism of potassium phosphate based magnesium phosphate cement. Ceramics International, 2012. 38(8): p. 6281-6288.

126. Qiao, C., et al., Prediction of chloride ingress in saturated concrete using formation factor and chloride binding isotherm. Advances in Civil Engineering Materials, 2018. 7(1): p. 206-220.

127. ASTM, Standard Test Method for Determining the Apparent Chloride Diffusion Coefficient of Cementitious Mixtures by Bulk Diffusion, in C1556-11a. 2016, ASTM International: West Conshohocken.

128. ASTM, Standard Test Method for Acid-Soluble Chloride in Mortar and Concrete, in C1152-20. 2020, ASTM International: West Conshohocken.

129. Trejo, D., C. Halmen, and K. Reinschmidt, Corrosion performance tests for reinforcing steel in concrete: technical report. 2009, Texas Transportation Institute.

130. Trejo, D., P.N. Vaddey, and C. Halmen, Quantifying the corrosion threshold of steel in cementitious systems. ACI Mater J, 2021. In Review.

131. Vaddey, P.N. and D. Trejo, Optimizing Test parameters for quantifying critical chloride threshold. ACI Mater J, 2021. In Review.

132. Andrade, C. and C. Alonso, On-site measurements of corrosion rate of reinforcements. Construction and building materials, 2001. 15(2-3): p. 141-145.

133. Arya, C., N. Buenfeld, and J. Newman, Factors influencing chloride-binding in concrete. Cement and Concrete research, 1990. 20(2): p. 291-300.

134. Diab, A.M., A.A. Aliabdo, and I.A. Mohamed, Corrosion behaviour of reinforced steel in concrete with ground limestone partial cement replacement. Magazine of Concrete Research, 2015. 67(14): p. 747-761.

135. Elgalhud, A.A., R.K. Dhir, and G. Ghataora, Chloride ingress in concrete: limestone addition effects. Magazine of Concrete Research, 2018. 70(6): p. 292-313.

136. Evgenia, Z., et al., Effect of corrosion inhibitors in limestone cement. Materials Sciences and Applications, 2013. 2013.

137. Shannon, J., I.L. Howard, and V.T. Cost, Benefits of portland-limestone cement for concrete with rounded gravel aggregates and higher fly ash replacement rates. 2015.

138. Thomas, M., et al., The effect of supplementary cementitious materials on chloride binding in hardened cement paste. Cement and Concrete Research, 2012. 42(1): p. 1-7.

139. Halmen, C. and D. Trejo, Accelerating Standard Test Method for Assessing Corrosion of Steel in Concrete. ACI Materials Journal, 2012. 109(4).

140. Mazarei, V., et al., Synergistic effects of ASR and fly ash on the corrosion characteristics of RC systems. Construction and Building Materials, 2017. 153: p. 647-655.

141. ACI, ACI Committee 201: Guide to Druable Concrete, in ACI 201.02R-08. 2008, American Concrete Institute.

142. Scherer, G.W., Crystallization in pores. Cement and Concrete research, 1999. 29(8): p. 1347-1358.

143. Scherer, G.W., Stress from crystallization of salt. Cement and concrete research, 2004. 34(9): p. 1613-1624.

144. Müllauer, W., R.E. Beddoe, and D. Heinz, Sulfate attack expansion mechanisms. Cement and concrete research, 2013. 52: p. 208-215. 
145. Santhanam, M., M.D. Cohen, and J. Olek, Effects of gypsum formation on the performance of cement mortars during external sulfate attack. Cement and concrete research, 2003. 33(3): p. 325-332.

146. Eglinton, M., Resistance of Concrete to Destructive. Lea's chemistry of cement and concrete, 2003: p. 299.

147. Hobbs, D., Thaumasite sulfate attack in field and laboratory concretes: implications for specifications. Cement and Concrete Composites, 2003. 25(8): p. 1195-1202.

148. Barcelo, L., et al., A modified ASTM C1012 procedure for qualifying blended cements containing limestone and SCMs for use in sulfate-rich environments. Cement and concrete research, 2014. 63: p. 75-88.

149. Rahman, M. and M. Bassuoni, Thaumasite sulfate attack on concrete: Mechanisms, influential factors and mitigation. Construction and Building Materials, 2014. 73: p. 652662.

150. Hooton, R. and M. Thomas, Sulfate Resistance of Mortar and Concrete Produced with Portland-Limestone Cement and Supplementary Cementing Materials: Recommendation for ASTM C595/AASHTO M 240. 2016, Portland Cement Association, Skokie, IL, USA, Report SN3285a.

151. Monteiro, P.J. and K.E. Kurtis, Time to failure for concrete exposed to severe sulfate attack. Cement and Concrete research, 2003. 33(7): p. 987-993.

152. Cyr, M., P. Lawrence, and E. Ringot, Efficiency of mineral admixtures in mortars: Quantification of the physical and chemical effects of fine admixtures in relation with compressive strength. Cement and concrete research, 2006. 36(2): p. 264-277.

153. Irassar, E., Sulfate attack on cementitious materials containing limestone filler-A review. Cement and Concrete Research, 2009. 39(3): p. 241-254.

154. Pipilikaki, P., M. Katsioti, and J. Gallias, Performance of limestone cement mortars in a high sulfates environment. Construction and Building Materials, 2009. 23(2): p. 10421049.

155. Schmidt, T., et al., Physical and microstructural aspects of sulfate attack on ordinary and limestone blended Portland cements. Cement and Concrete Research, 2009. 39(12): p. 1111-1121.

156. Lee, S.T., et al., Effect of limestone filler on the deterioration of mortars and pastes exposed to sulfate solutions at ambient temperature. Cement and concrete research, 2008. 38(1): p. 68-76.

157. Ramezanianpour, A.M. and R.D. Hooton, $A$ study on hydration, compressive strength, and porosity of Portland-limestone cement mixes containing SCMs. Cement and Concrete Composites, 2014. 51: p. 1-13.

158. Ryou, J., et al., Durability of cement mortars incorporating limestone filler exposed to sodium sulfate solution. KSCE Journal of Civil Engineering, 2015. 19(5): p. 1347-1358.

159. Thomas, M.D., et al., Field trials of concrete produced with Portland limestone cement. Concrete international, 2010. 32(1): p. 35-41.

160. Hossack, A.M. and M.D. Thomas, Varying fly ash and slag contents in Portland limestone cement mortars exposed to external sulfates. Construction and Building Materials, 2015. 78: p. 333-341.

161. Sezer, G.İ., Compressive strength and sulfate resistance of limestone and/or silica fume mortars. Construction and Building Materials, 2012. 26(1): p. 613-618. 
3373

3374

3375

3376

3377

3378

3379

3380

3381

3382

3383

3384

3385

3386

3387

3388

3389

3390

3391

3392

3393

3394

3395

3396

3397

3398

3399

3400

3401

3402

3403

3404

3405

3406

3407

3408

3409

3410

3411

3412

3413

3414

3415

3416

3417

162. Ramezanianpour, A.A., Cement replacement materials. Springer, Berlin. doi, 2014. 10: p. 978-3.

163. Tiburzi, N.B., et al., Sulfate resistance of portland-limestone cement systems containing greater than 15\% limestone. Cement and Concrete Composites, 2019. 100: p. 60-73.

164. ASTM C1753/C1753M-15el Standard Practice for Evaluating Early Hydration of Hydraulic Cementitious Mixtures Using Thermal Measurements. 2015, ASTM International: West Conshohocken, PA. p. 19.

165. Sandberg, J. and S. Liberman, Monitoring and evaluation of cement hydration by semiadiabatic field calorimetry. Special Publication, 2007. 241: p. 13-24.

166. Kosmatka, S.H., B. Kerkhoff, and W.C. Panarese, Design and control of concrete mixtures. Vol. 5420. 2002: Portland Cement Association Skokie, IL.

167. Environment, U., et al., Eco-efficient cements: Potential economically viable solutions for a low-CO2 cement-based materials industry. Cement and Concrete Research, 2018. 114: p. 2-26.

168. Tennis, P.D., J.W. Weiss and J.H. Ideker, Editors. 2021, CALTRANS Report Comments: Corvallis.

169. Gartner, E., Industrially interesting approaches to "low-CO2" cements. Cement and Concrete research, 2004. 34(9): p. 1489-1498.

170. Purnell, P. and L. Black, Embodied carbon dioxide in concrete: Variation with common mix design parameters. Cement and Concrete Research, 2012. 42(6): p. 874-877.

171. Ellis, G., Are there any practical alternatives to the manufacture of Portland cement clinker? Journal of The Chinese Ceramic Society, 2012. 40(1): p. 61-68.

172. Juenger, M., et al., Advances in alternative cementitious binders. Cement and concrete research, 2011. 41(12): p. 1232-1243.

173. EN, B.S., 206-1 Concrete-Part 1: Specification, performance, production and conformity. British Standards Institution, 2000.

174. Scrivener, K., et al., Calcined clay limestone cements (LC3). Cement and Concrete Research, 2018. 114: p. 49-56.

175. Miller, S.A., et al., Carbon dioxide reduction potential in the global cement industry by 2050. Cement and Concrete Research, 2018. 114: p. 115-124.

176. Miller, S.A., Life Cycle Inventory Data for Caltrans TO27. 2020: Davis, California.

177. Miller, S.A., Personal Communication about tool for LCI, J.W. Weiss, et al., Editors. 2021.

178. Damidot, D., et al., Thermodynamic investigation of the $\mathrm{CaO}$ Al2O3 $\mathrm{CaCO} 3 \mathrm{H} 2 \mathrm{O}$ closed system at $25^{\circ}$ C and the influence of Na2O. Cement and Concrete Research, 1994. 24(3): p. 563-572.

179. Matschei, T. and F.P. Glasser, Temperature dependence, 0 to $40 \mathrm{C}$, of the mineralogy of Portland cement paste in the presence of calcium carbonate. Cement and Concrete Research, 2010. 40(5): p. 763-777.

180. Bharadwaj, K., B.O. Isgor, and J.W. Weiss, Supplementary Cementitious Materials and Portland Limestone Cements. ACI Materials Journal, 2021. In Review.

181. Antoni, M., et al., Cement substitution by a combination of metakaolin and limestone. Cement and Concrete Research, 2012. 42(12): p. 1579-1589.

182. Lothenbach, B., et al., Thermodynamic modelling of the effect of temperature on the hydration and porosity of Portland cement. Cement and Concrete Research, 2008. 38(1): p. 1-18. 
183. Damidot, D., et al., Thermodynamics and cement science. Cement and Concrete Research, 2011. 41(7): p. 679-695.

184. Matschei, T., B. Lothenbach, and F.P. Glasser, The role of calcium carbonate in cement hydration. Cement and concrete research, 2007. 37(4): p. 551-558.

185. Matschei, T., B. Lothenbach, and F.P. Glasser, Thermodynamic properties of Portland cement hydrates in the system $\mathrm{CaO}-\mathrm{Al2O} 3-\mathrm{SiO} 2-\mathrm{CaSO} 4-\mathrm{CaCO}-\mathrm{H} 2 \mathrm{O}$. Cement and Concrete Research, 2007. 37(10): p. 1379-1410.

186. Powers, T.C., Structure and physical properties of hardened Portland cement paste. Journal of the American Ceramic Society, 1958. 41(1): p. 1-6.

187. Powers, T.C. and T.L. Brownyard. Studies of the physical properties of hardened Portland cement paste. in Journal Proceedings. 1946.

188. Glosser, D., et al., An extension of the Powers-Brownyard model to pastes containing SCM. ACI Materials Journal, 2019. in Press.

189. Bharadwaj, K., et al., Predicting Pore Volume, Compressive Strength, Pore Connectivity, and Formation Factor in Cementitious Pastes Containing Fly Ash. Cement and Concrete Composites, 2021. In Press.

190. Bharadwaj, K., et al., Toward the Prediction of Pore Volumes and Freeze-Thaw Performance of Concrete Using Thermodynamic Modelling. Cement and Concrete Research, 2019. 124: p. 105820.

191. Kulik, D.A., et al., GEM-Selektor geochemical modeling package: revised algorithm and GEMS3K numerical kernel for coupled simulation codes. Computational Geosciences, 2013. 17(1): p. 1-24.

192. Deschner, F., et al., Effect of temperature on the hydration of Portland cement blended with siliceous fly ash. Cement and Concrete Research, 2013. 52: p. 169-181.

193. Dilnesa, B.Z., et al., Synthesis and characterization of hydrogarnet Ca3 (AlxFe1-x) 2 (SiO4) y (OH) 4 (3-y). Cement and Concrete Research, 2014. 59: p. 96-111.

194. Parrot, L.J. Prediction of cement hydration. in Proceedings of the British Ceramic Society. 1984.

195. Glosser, D., et al., Estimating reaction kinetics of cementitious pastes containing fly ash. Cement and Concrete Composites, 2020: p. 103655.

196. Glosser, D.B., Equilibrium and Non-equilibrium Thermodynamic Modeling of Cement Pastes Containing Supplementary Cementitious Materials, in Civil Engineering. 2020, Oregon State University: Corvallis OR.

197. Taylor, H.F., Cement chemistry. Vol. 2. 1997: Thomas Telford London.

198. Choudhary, A., et al., Influence of limestone cement on the electrical and transport properties of cement mortar ASTM, 2021. In Preparation.

199. Zajac, M., et al., Influence of limestone and anhydrite on the hydration of Portland cements. Cement and Concrete Composites, 2014. 46: p. 99-108.

200. Schöler, A., et al., Hydration of quaternary Portland cement blends containing blastfurnace slag, siliceous fly ash and limestone powder. Cement and Concrete Composites, 2015. 55: p. 374-382.

201. Zajac, M., W. Dienemann, and G. Bolte. Comparative experimental and virtual investigations of the influence of calcium and magnesium carbonate on reacting cement. in Proceedings of the 13th international congress on the chemistry of cements, Madrid. 2011. 
3463 202. Zajac, M., et al., Influence of calcium and magnesium carbonates on hydration kinetics, $3464 \quad$ hydrate assemblage and microstructural development of metakaolin containing composite 3465 cements. Cement and Concrete Research, 2018. 106: p. 91-102.

3466 203. Schöler, A., et al., Early hydration of SCM-blended Portland cements: A pore solution and 3467

3468 isothermal calorimetry study. Cement and Concrete Research, 2017. 93: p. 71-82.

204. Tennis, P., Chemical and Physical Characteristics of US Hydraulic Cements: 2014. Portland Cement Association, Skokie, Ill, 2016. 


\section{APPENDIX A - Determination of ultimate Degree of Reactivity of SCM}

$3476 \quad 19.1$ Scope

3477 This test method covers the procedure for quantitative determination of the ultimate Degree of 3478 reactivity of an SCM using experimental methods and thermodynamic simulations. The values stated in SI units are to be regarded as the standard. This standard does not purport to address all of the safety problems, if any, associated with its use. It is the responsibility of the user of this standard to establish appropriate safety and health practices and determine the applicability of regulatory limitations prior to use.

\subsection{Terminology}

DOR of a supplementary cementitious material (SCM) at a given time - the amount of SCM (in $\%$ ) that has reacted with pure calcium hydroxide in an alkaline environment up to that time. DOR* of a supplementary cementitious material (SCM) - the amount of SCM (in \%) that has reacted with pure calcium hydroxide in an alkaline environment at an 'infinite' time. This can be considered as the maximum amount (in \%) of a SCM that is available for the pozzolanic reaction in cementitious system.

\subsection{Summary of Test Method}

The test method uses a combination of experimentally determined calcium hydroxide consumption and heat release values to predict DOR of SCM. The DOR* of an SCM is estimated by interpolating between the pure $\mathrm{SiO}_{2}$ and $\mathrm{Al}_{2} \mathrm{O}_{3}$ reaction reference lines. The reference lines are theoretically determined by reacting pure $\mathrm{SiO}_{2}$ and $\mathrm{Al}_{2} \mathrm{O}_{3}$ systems reacted from $0 \%$ to $100 \%$ in thermodynamic simulations.

The SCM is dry mixed with reagent grade calcium hydroxide $(\mathrm{CH})$ such that the mass ratio of $\mathrm{CH}: \mathrm{SCM}$ is $3: 1$. The dry blend is thoroughly mixed with $0.5 \mathrm{M}$ potassium hydroxide $(\mathrm{KOH})$ solution (liquid to powder $(\mathrm{CH}+\mathrm{SCM})$ mass ratio of 0.9$)$. The wet paste is immediately transferred to an isothermal calorimeter (IC) preconditioned at $50^{\circ} \mathrm{C} \pm 2^{\circ} \mathrm{C}$ and the heat release values are recorded for a period of 240 hours from mixing. The cumulative heat released value at the end of 240 hours are extrapolated to get the heat released after infinite reaction time. The reacted paste from the IC is subsequently tested for $\mathrm{CH}$ consumption using a Thermo-Gravimetric Analyzer (TGA). Further details about the test procedure, results, and analysis are presented in Section 10. 
3506 This specific standard is written for the reaction between a SCM and $\mathrm{CH}$ at a 3:1 mass ratio in an 3507 alkaline pore solution composed of $0.5 \mathrm{M}$ potassium hydroxide $(\mathrm{KOH})$ solution. The effect of 3508 varying the mass ratio of reacting species and introduction of different ions in the pore solution 3509 are still being studied.

\subsection{Significance and Use}

The test method is designed to allow for complete reaction of the reactive portion of the supplementary cementitious material (SCM) being tested by providing an excess of calcium hydroxide in a highly alkaline environment (to simulate the alkaline conditions in OPC- and OPC$\mathrm{SCM}$ systems) at an elevated temperature of $50^{\circ} \mathrm{C}$ for 240 hours. The underlying principle of the reactivity test is that if the main reactive phases of $\mathrm{SCM}$ (amorphous $\mathrm{SiO}_{2}$ and amorphous $\mathrm{Al}_{2} \mathrm{O}_{3}$ ) are thermodynamically simulated at different degrees of reaction, these values can serve as reference values against which to measure the reactivity of commercial SCMs such as fly ash. The test provides a methodology for measuring the amount of the SCM that is reactive. It should be noted that the methodology discussed in this report is designed to test SCMs which primarily react in a pozzolanic manner (where $\mathrm{SiO} 2$ and $\mathrm{Al} 2 \mathrm{O} 3$ in the $\mathrm{SCM}$ react with calcium hydroxide in the OPC-SCM system). While this approach can be extended to SCMs that also react hydraulically (like Slag) as there is no other test method that is currently available to determine the reactivity of these materials, work is ongoing on determining a more appropriate test method to determine the reactivity of such SCMs.

\subsection{Apparatus}

- Paste mixer - A mixer that can be used to mix cementitious paste

- Balance-Analytical balance, Class A, conforming to the requirements of M 231 to weigh the paste. The balance shall be accurate to $0.1 \mathrm{mg}$.

- Funnel - Used to pour reactive paste into the glass ampoules to ensure that all the paste directly goes at the bottom of the ampoule (with minimum splatter on the sides and the top of the ampoules) from where the heat is measured.

- IC ampoule assembly - These are glass ampoules (20 ml volume) with aluminum lids which can be used to measure the heat released from the reacting paste mixture inside them in an Isothermal Calorimeter (IC).

- Clamping tool - A mechanical or electrical clamping tool to seal the lids on the glass ampoules to prevent leakage during the test

- Isothermal calorimeter - The IC shall conform to ASTM C1679 and should be able to operate in a temperature of $50^{\circ} \mathrm{C} \pm 2^{\circ} \mathrm{C}$

- Decapper tool - used to remove the sealed cap from the IC ampoules 
- Spatula - used to scoop out (around $20 \mathrm{mg}$ ) of reacted paste from the IC ampoule

- TGA pan - This is platinum pan with a hanger assembly which is inert to the reacted paste sample and whose shape and structural integrity can withstand temperatures within the TGA machine.

- Thermo-gravimetric Analyzer (TGA) - The TGA shall conform to ASTM E1131 and shall be able to operate in a temperature range of $25^{\circ} \mathrm{C}$ to $1000^{\circ} \mathrm{C}$.

\subsection{Materials}

- Reagents-Reagent grade chemicals shall be used in all tests. Unless otherwise indicated, all reagents shall conform to the specifications of the Committee on Analytical Reagents of the American Chemical Society. For this standard, reagent grade calcium hydroxide $\left(\mathrm{Ca}(\mathrm{OH})_{2}\right)$ and potassium hydroxide $(\mathrm{KOH})$ is used to prepare paste mixture.

- Distilled Water - Unless otherwise indicated, water used shall be distilled water

- Pore Solution Preparation - in a small graduated cylinder, dissolve $2.805 \mathrm{~g}$ of anhydrous $\mathrm{KOH}$, in $50 \mathrm{~mL}$ of distilled water. Slowly, add distilled water with constant stirring to make the volume of the solution equal to $100 \mathrm{~mL}$. Store the solution in a sealed plastic container with secondary containment at a temperature of $23 \pm 1^{\circ} \mathrm{C}$. Use this solution within 7 days of its preparation. If any precipitates are noted in the solution at any time, discard the solution and prepare a fresh one.

- Note: The dissolution of KOH in water is highly exothermic, so precautions should be taken to prevent burning when large amounts of solution are to be prepared (over $30 \mathrm{~g}$ ).

\subsection{Sample Preparation}

Reactive Paste Preparation: For each test, gently dry mix the SCM and calcium hydroxide in a 1:3 mass ratio by hand for uniform distribution of SCM throughout the powder system. Prepare the paste mixture by thoroughly mixing the simulated pore solution with dry powder in 0.9:1 mass ratio.

\subsection{Testing Procedure}

Heat of reaction $(Q)$ measurement: Immediately after mixing, pour approximately $7 \mathrm{~g}$ of the wet paste into a glass ampoule using a funnel and then seal them. Transfer the ampoules to an IC that had been preconditioned at $50^{\circ} \mathrm{C} \pm 2^{\circ} \mathrm{C}$ for 24 hours. Allow signal stabilization (time varies depending of the instrument) and then record the heat flow for a total of 240 hours. Note down the cumulative heat at the end test period. 
3572 Calcium hydroxide measurement: After 240 hours of IC testing, remove the ampoules from the 3573 calorimeter. Take approximately $20 \mathrm{mg}$ of the reacted paste from the ampoules and put it on the 3574 TGA pan. Run the following TGA method on the sample. Equilibrate the pan at $25^{\circ} \mathrm{C}$. Heat the 3575 pan from $23^{\circ} \mathrm{C}$ to $1000^{\circ} \mathrm{C}$ in a nitrogen purged atmosphere at a rate of $10^{\circ} \mathrm{C} / \mathrm{min}$. (Note: Perform 3576 TGA within 12 hours of removing the samples from the isothermal calorimeter.)

\subsection{Calculation and Interpretation of Results}

3578 Reference $\mathrm{SiO}_{2}$ and $\mathrm{Al}_{2} \mathrm{O}_{3}$ reaction lines: Thermodynamic modelling can be used to provide reference reactivity range of pure silicate phases and aluminate phases. The modelling simulations calculate the heat release and calcium hydroxide consumed due to the pozzolanic reaction of pure amorphous silica and pure amorphous alumina. The simulations are run at a $\mathrm{CH}: \mathrm{SCM}$ ratio of $3: 1$, liquid to $\mathrm{CH}+\mathrm{SCM}$ ratio of 0.9 , and at a temperature of $50^{\circ} \mathrm{C}$ at varying degrees of reactivity $(0 \%$ to $100 \%$ ) of $\mathrm{SiO}_{2}$ and $\mathrm{Al}_{2} \mathrm{O}_{3}$. The calculated $\mathrm{Q} \infty$ vs $\mathrm{CH}$ consumption values for $\mathrm{SiO}_{2}$ and $\mathrm{Al}_{2} \mathrm{O}_{3}$ of varying degrees of reactivity is shown in Figure A-1.

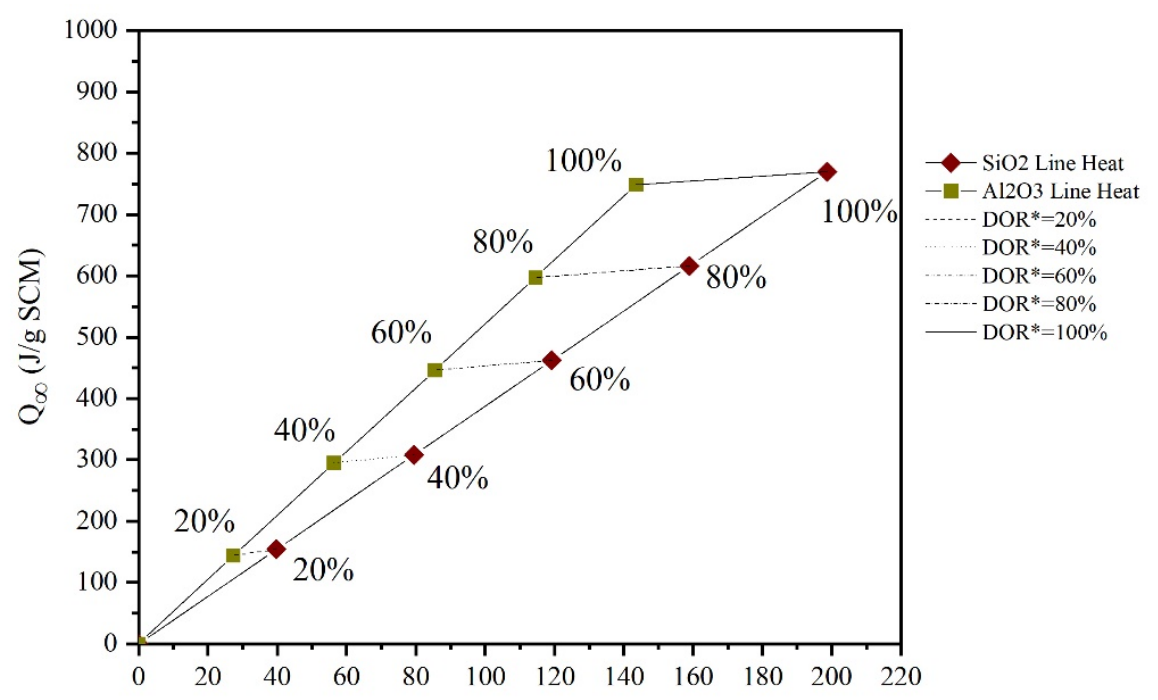

Figure A-19-1. Reference reaction lines of pure silica and pure alumina under simulated experimental condition

3590 Note: The thermodynamic modelling is performed using GEMS3K software and the CemData 3591 v.18 database 


\subsubsection{Ultimate heat of hydration calculation}

3594 Normalize the heat release values by the amount of SCM in the reacted paste, which was tested in 3595 the IC. Plot the normalized heat release curves vs reaction time. If it is still evident from the plot 3596 that the reaction would continue beyond the measured 240 hours, correct them mathematically for 3597 Q $\infty$ using either of the following two approaches mentioned in the next paragraph.

3598 Correction for ultimate heat released $(\mathrm{Q} \infty)$ - The heat released due to the pozzolanic reaction which takes place in the test setup starts off very rapidly in the beginning but slow down with time as the concentration of the reactants in the system start to decrease. It can be shown that a system reacting at 240 hours ( 10 days) at $50{ }^{\circ} \mathrm{C}$ is equivalent to a system reacting for 50 days at $23{ }^{\circ} \mathrm{C}$. For most SCMs, this amount of time is sufficient to allow the SCM to react to completion, and the value of heat released at 240 hours of experiment is the ultimate heat released from the system $(\mathrm{Q} \infty)$. However, in some cases, the SCM may not have reacted completely, and a correction may need to be done to estimate the ultimate value of heat released. One of the following corrections is used to provide the heat released values at reaction completion $(\mathrm{Q} \infty)$. The comparison of the $\mathrm{Q} \infty$ value from the two different correction methods are presented in Table A.1.

Table A.1. Comparison of different heat released correction methods

\begin{tabular}{|l|l|l|l|l|}
\hline & $\begin{array}{l}\text { Heat released in } \\
240 \text { hours }\end{array}$ & $\begin{array}{l}\text { Curve fitting } \\
\text { approach }\end{array}$ & \multicolumn{2}{|c|}{ Inverse heat and time approach } \\
\hline & Q $(\mathrm{J} / \mathrm{g}$ SCM $)$ & $\mathrm{Q}_{\infty}(\mathrm{J} / \mathrm{g}$ SCM $)$ & y-intercept & $\mathrm{Q}_{\infty}(\mathrm{J} / \mathrm{g} \mathrm{SCM})$ \\
\hline SCM1 & 223.44 & 223.44 & 0.0043 & 232.56 \\
\hline SCM2 & 399.73 & 457.6275 & 0.0021 & 476.19 \\
\hline
\end{tabular}

\subsubsection{Curve fitting approach}

3611 Fit the experimental data with a logarithmic function and extrapolate the curves till the completion 3612 of the reaction (defined as the point where instantaneous heat change per unit time approaches 0 ) 3613 is achieved. An example of the fitting model has been shown in Figure A-19-2. Figure A-19-2 3614 shows that the heat release curve of SCM1 has plateaued in 240 hours (duration of experiment) 3615 implying that the reaction between SCM1 and $\mathrm{CH}$ is completed. On the other hand, the heat release 3616 curve of SCM2 still has a positive slope at the end of 240 hours. In order to estimate the heat 3617 released at complete reaction of SCM2, a logarithmic model was used to fit the curve and the 3618 curve was extrapolated till slope almost became zero to get to the Qo value for SCM2. 


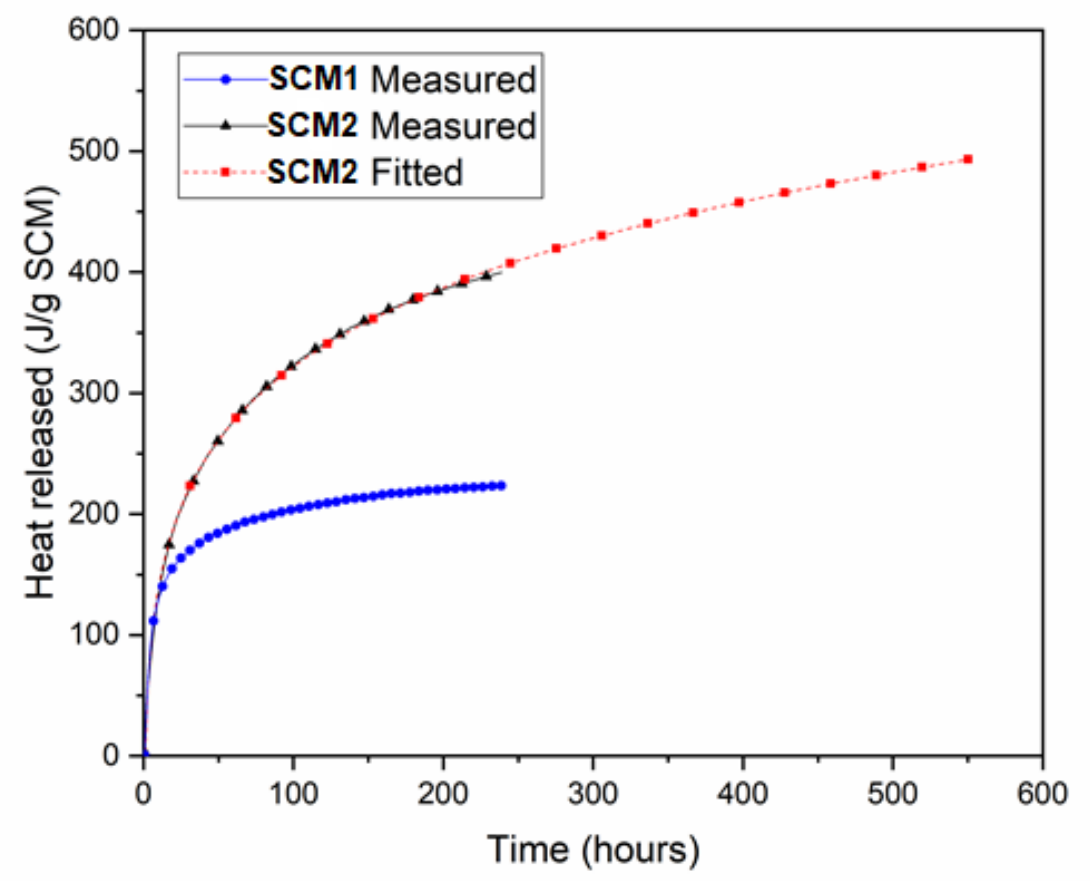

3620 Figure A-19-2. Heat release curves determined by isothermal calorimetry for SCM1 and 3621 SCM2. The heat release data for SCM2 data was extrapolated to equilibrium to calculate the heat release correction. SCM1 did not require any extrapolation.

\section{$3623 \quad 19.9 .3$ Inverse heat and time approach}

3624 Over the course of experiment, the heat release values start to plateau. This plateau is defined by 3625 the reaction rate $(\partial \mathrm{Q} / \partial \mathrm{t})$ being lesser than or equal to 0.05 . For SCMs that still have some reactivity 3626 potential left over after 240 hours $(\partial \mathrm{Q} / \partial \mathrm{t} \geq 0.05)$, the inverse of heat released values $(1 / \mathrm{Q})$ can be 3627 plotted against the inverse of time taken $(1 / t)$ for data range with $\partial \mathrm{Q} / \partial \mathrm{t} \leq 1$. The trend of this curve 3628 (mostly linear) is then extrapolated to intersect the y-axis. The y-intercept of 1/Q vs 1/t plot will 3629 represent 1/ Q $\infty$ for that SCM. An example of the fitting model using SCM1 and SCM2 is shown 3630 in Figure A-19-3. The figure shows 1/Q vs 1/t curve of SCM1 and SCM2. The y- intercept of the 3631 linear fit trend line on the data gives the $\mathrm{Q}_{\infty}$ value. 


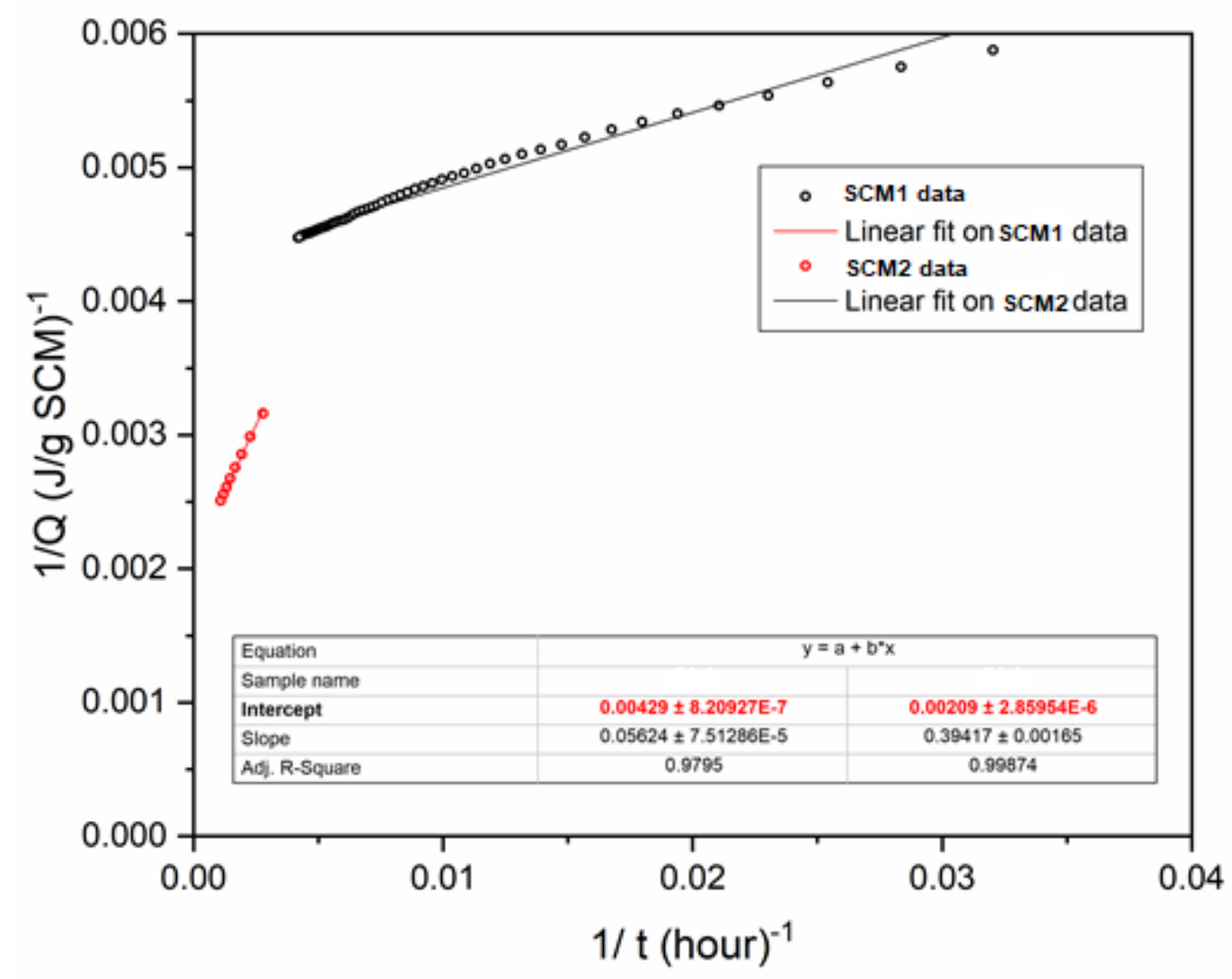

3634

3635

3636

3637

3638

3639

3640

3641

Figure A-19-3. 1/Q vs 1/t curve for SCM1 and SCM2 with their corresponding linear trendline

Calcium hydroxide consumed calculation: TGA data is reported as change in sample mass with temperature. the characteristic peak of the decomposition of certain compounds is used in quantitative analysis. In the case of $\mathrm{Ca}(\mathrm{OH})_{2}$, decomposition typically occurs between $350-450^{\circ} \mathrm{C}$. The mass loss in the sample between $350^{\circ} \mathrm{C}$ to $450^{\circ} \mathrm{C}$ corresponds to loss of water due the decomposition of $\mathrm{Ca}(\mathrm{OH})_{2}$ present in the system as shown in Figure A-19-4. The amount of $\mathrm{Ca}(\mathrm{OH})_{2}$ reacted (normalized per $100 \mathrm{~g}$ of SCM) is calculated using Equation A-1 and A-2:

$$
\begin{gathered}
\mathrm{Ca}(\mathrm{OH})_{2 \text { final }}=\left(\mathrm{\Delta m} \times \frac{74.09}{18.01}\right) \times 100 \\
\mathrm{Ca}(\mathrm{OH})_{2 \text { consumed }}=\left(\mathrm{Ca}(\mathrm{OH})_{2 \text { initial }}-\mathrm{Ca}(\mathrm{OH})_{2 \text { final }}\right) \times \frac{100}{13.16}
\end{gathered}
$$

3644 where: 

$\Delta m=$ weight reduction due loss of water detected in TGA between $350{ }^{\circ} \mathrm{C}$ to $450{ }^{\circ} \mathrm{C}$, $\mathrm{Ca}(\mathrm{OH})_{2 \text { initial }}=$ mass of $\mathrm{Ca}(\mathrm{OH})_{2}$ in the original paste per $100 \mathrm{~g}$ of paste, known to be 39.47 $3647 \mathrm{~g} / 100 \mathrm{~g}_{\text {paste }}$ from the initial mixture design,

$3648 \mathrm{Ca}(\mathrm{OH})_{2 \text { final }}=$ mass of $\mathrm{Ca}(\mathrm{OH})_{2}$ in the remaining in the reacted paste $\left(\right.$ in $\left.\mathrm{g} / \operatorname{lo0g}_{\text {paste }}\right)$,

$3649 \quad 100 / 13.16$ is the conversion factor ( $100 \mathrm{~g}$ paste contains $\sim 13.16 \mathrm{~g} \mathrm{SCM})$, and,

$3650 \mathrm{Ca}(\mathrm{OH})_{2 \text { consumed }}=$ mass of $\mathrm{Ca}(\mathrm{OH})_{2}$ consumed due to the pozzolanic reaction (in $\left.3651 \mathrm{~g} / 100 \mathrm{~g}_{\mathrm{SCM}}\right)$.

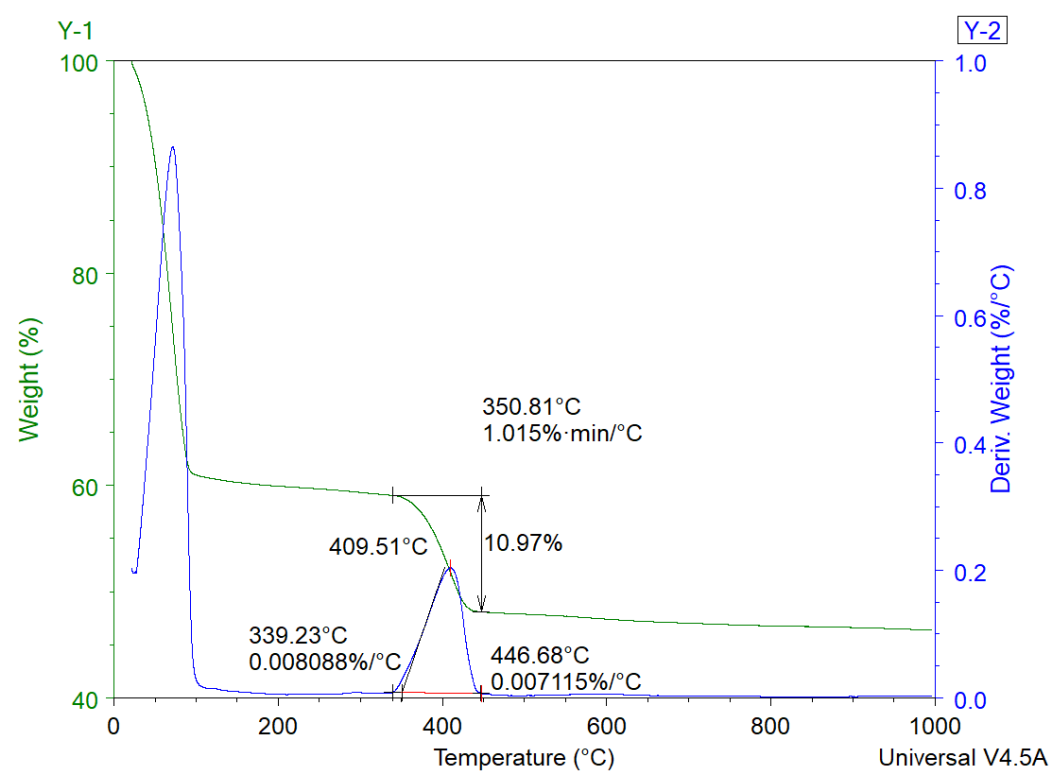

\subsection{Reporting}

3656 Plot the $\mathrm{Ca}(\mathrm{OH})_{2}$ consumption vs. heat release data results in comparison with the reference lines 3657 shown in Figure A-19-1. The reactivity of the SCM is read from the plot with respect to the 3658 reference lines. This method allows for the determination of the equilibrium degree of reactivity 3659 (DOR*) of a SCM. 


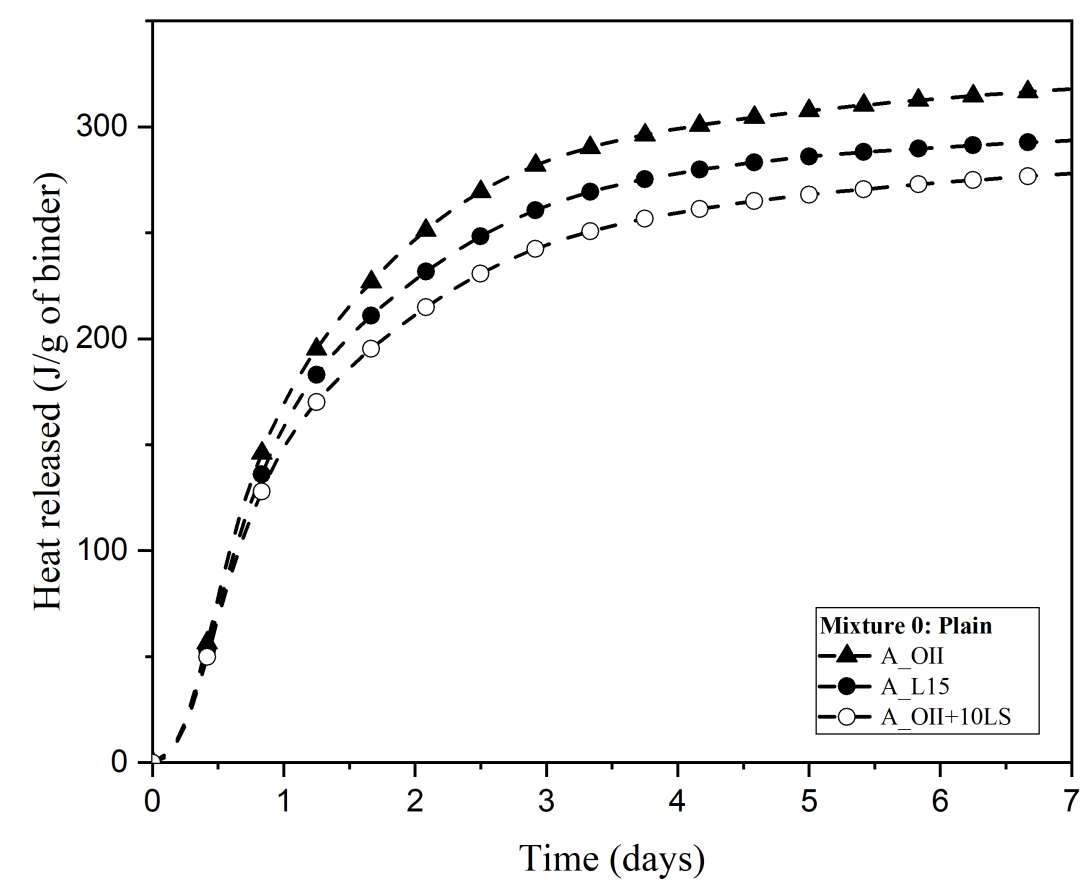

3661

3662

Figure B-20-1. Heat of Hydration of Cement A - MO

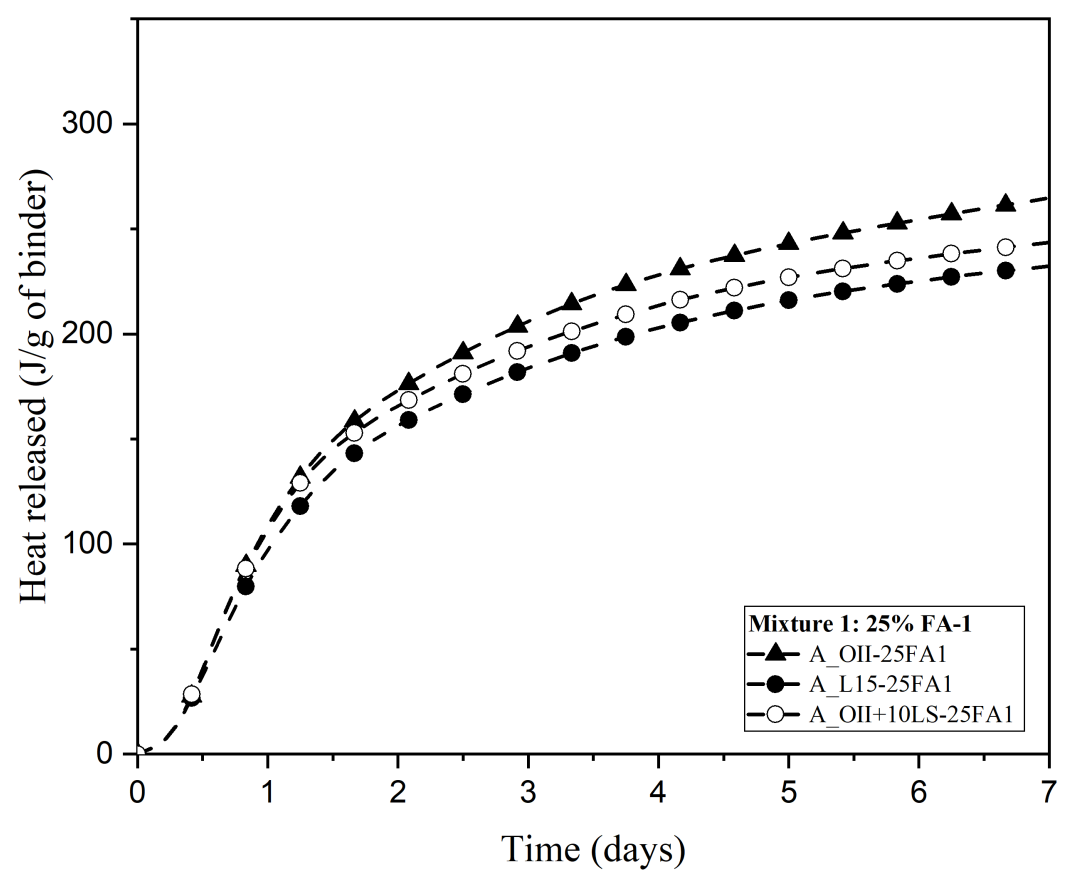

3663

3664

Figure B-20-2. Heat of Hydration of Cement A - M1 


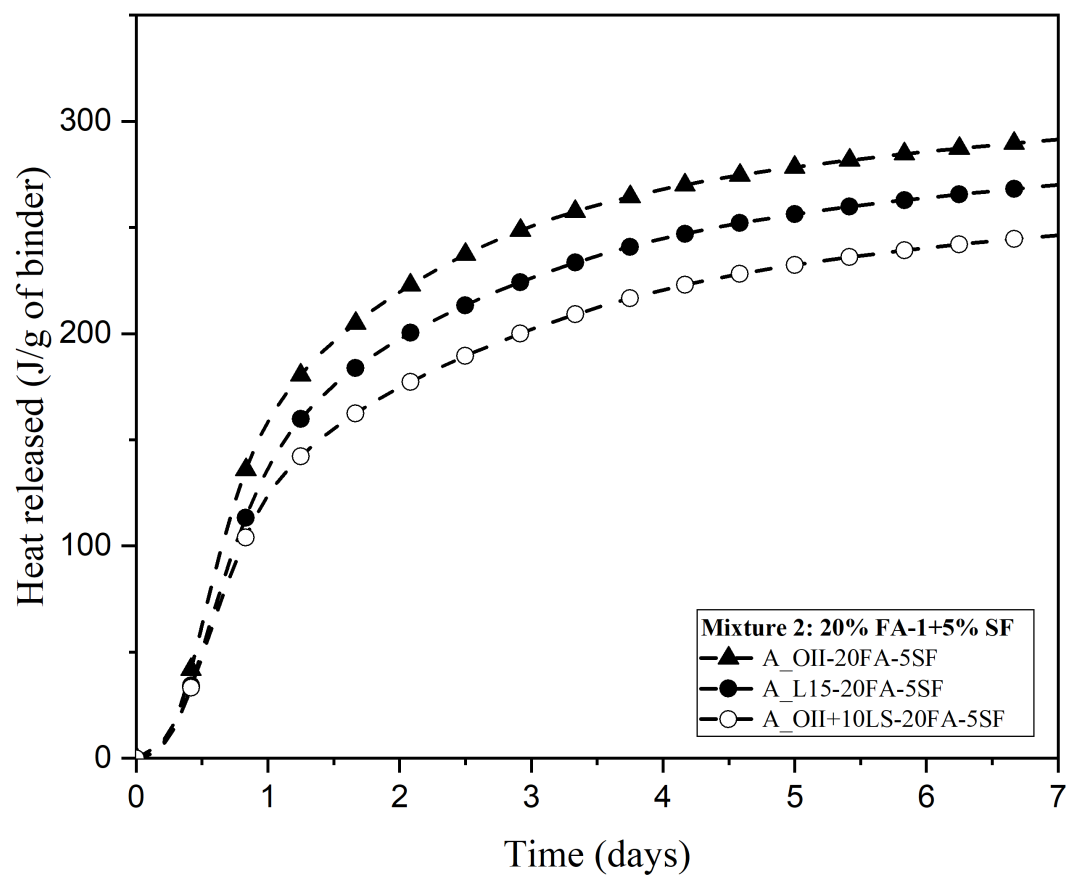

3665

3666

Figure B-20-3. Heat of Hydration of Cement A - M2

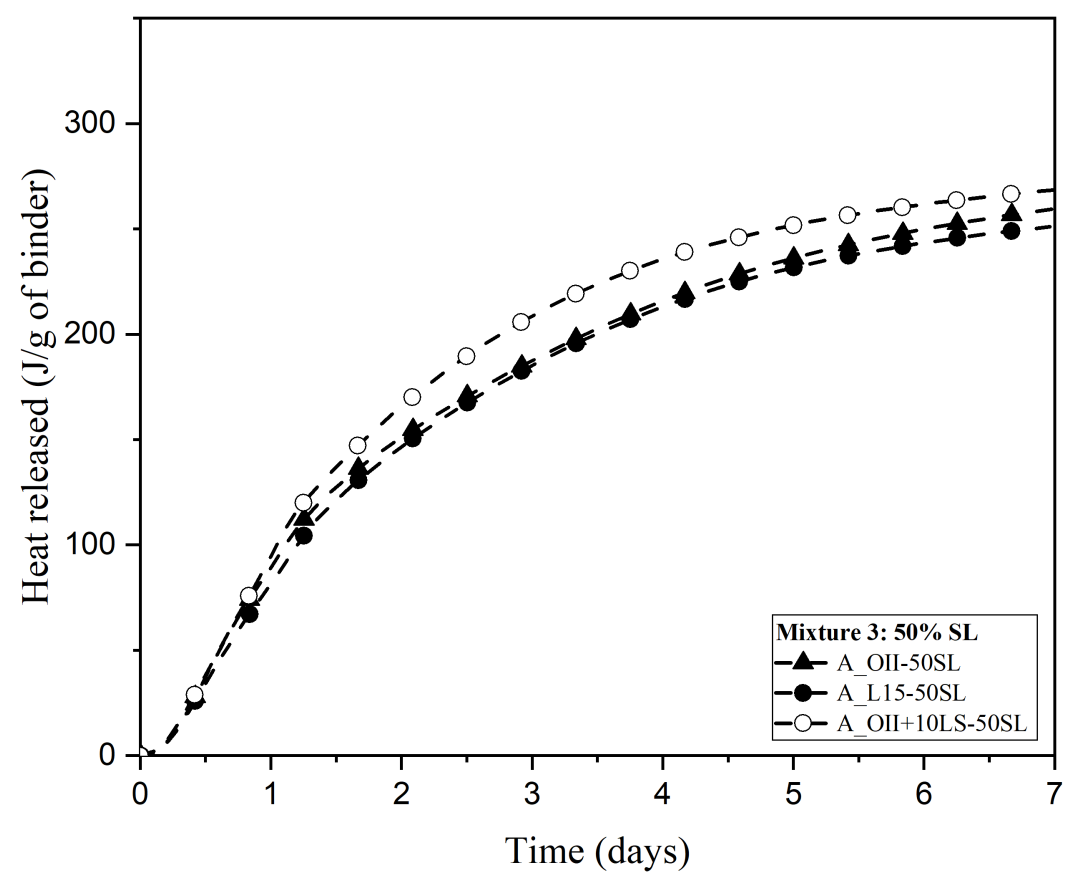

3667

3668

Figure B-20-4. Heat of Hydration of Cement A - M3

3669 


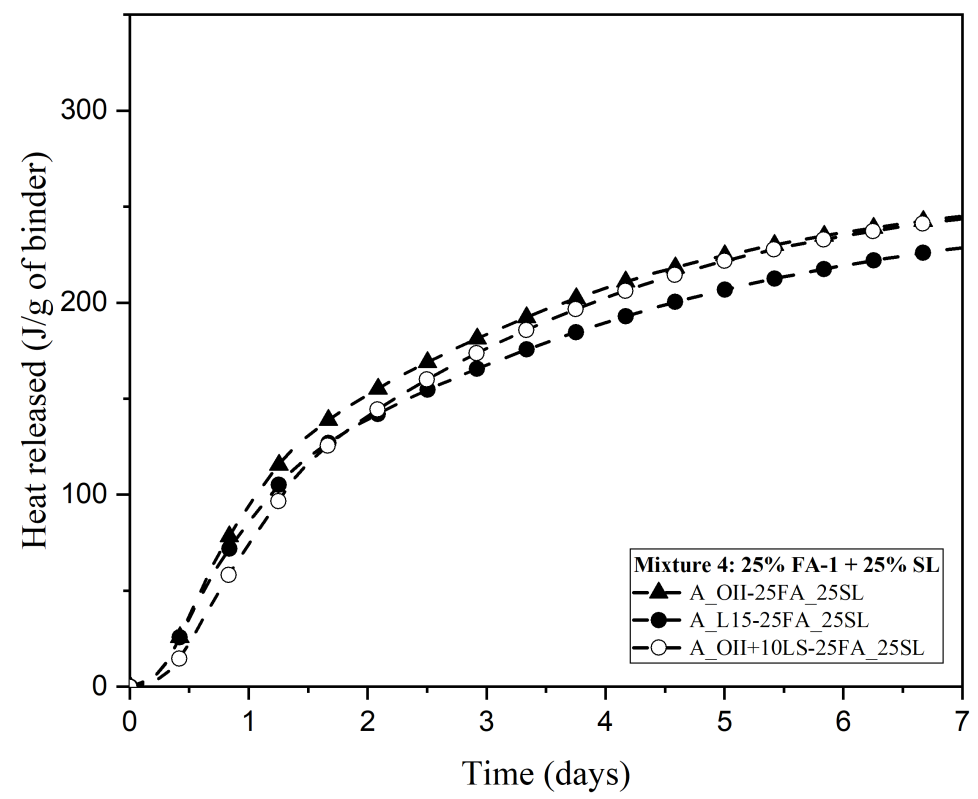

3670

3671

Figure B-20-5. Heat of Hydration of Cement A - M4

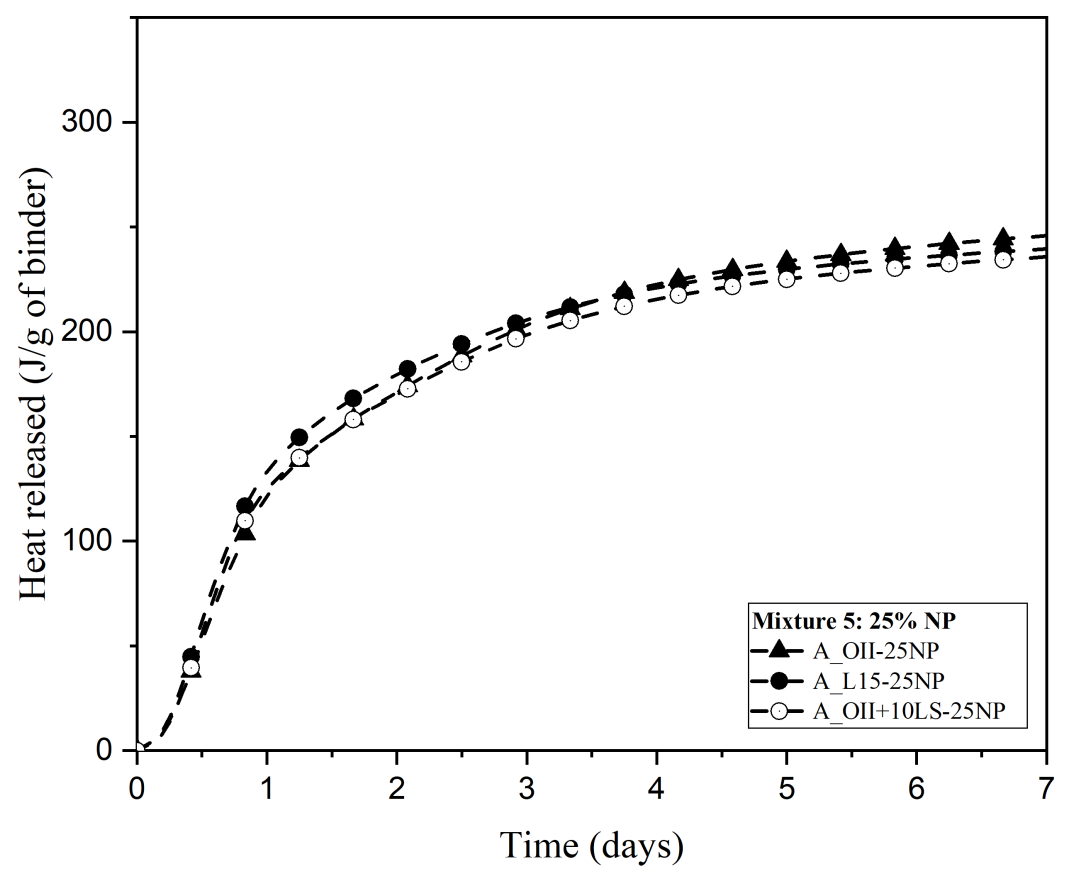

3672 


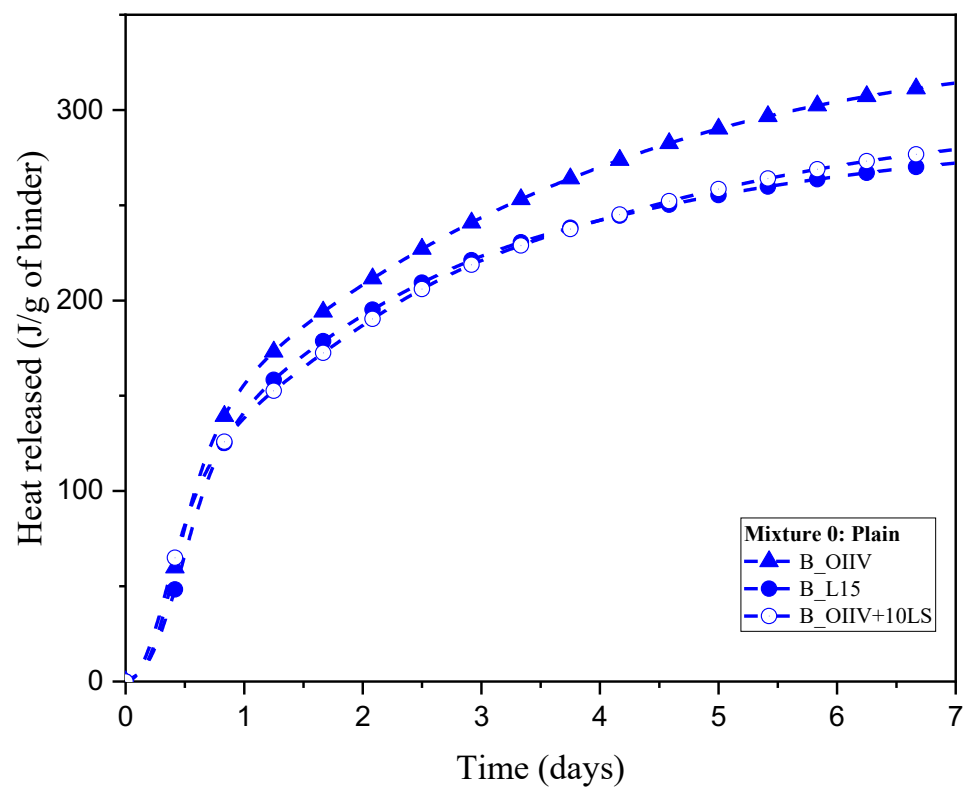

3675

3676

Figure B-20-7. Heat of Hydration of Cement B - M0

3677

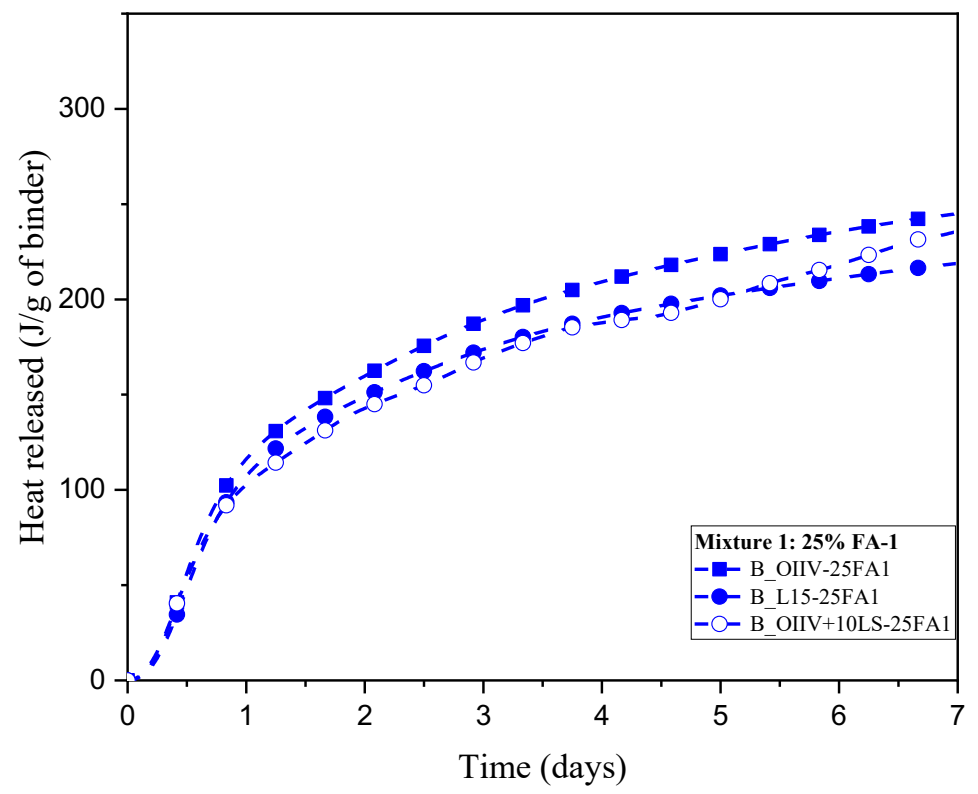

3678 


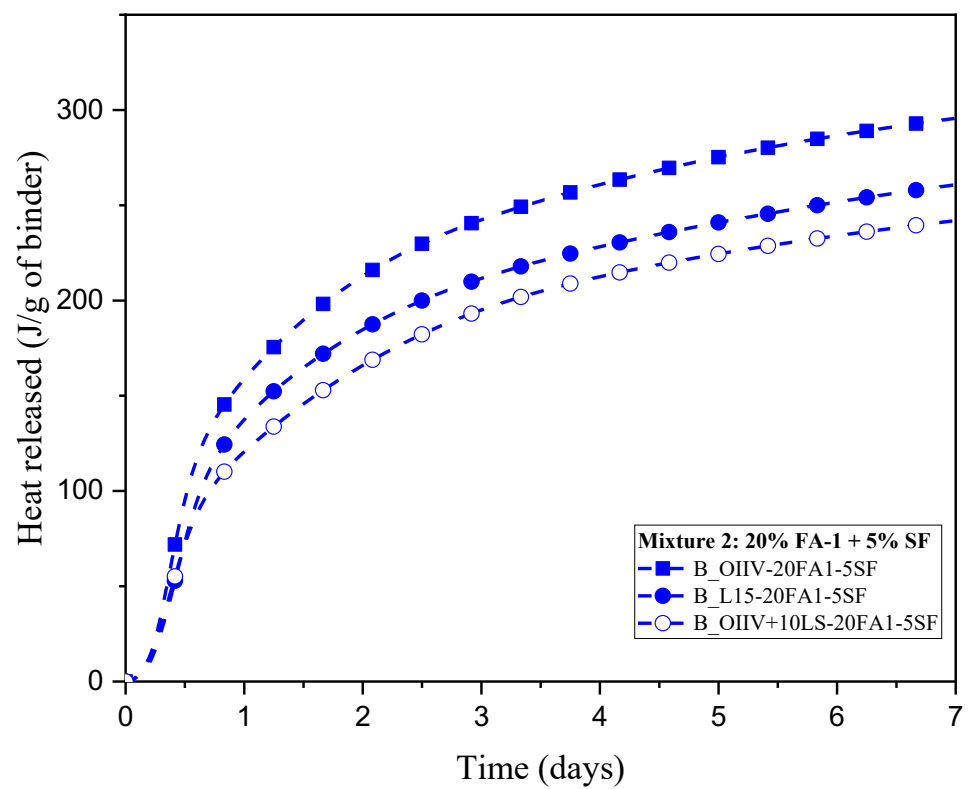

3680

3681

Figure B-20-9. Heat of Hydration of Cement B - M2

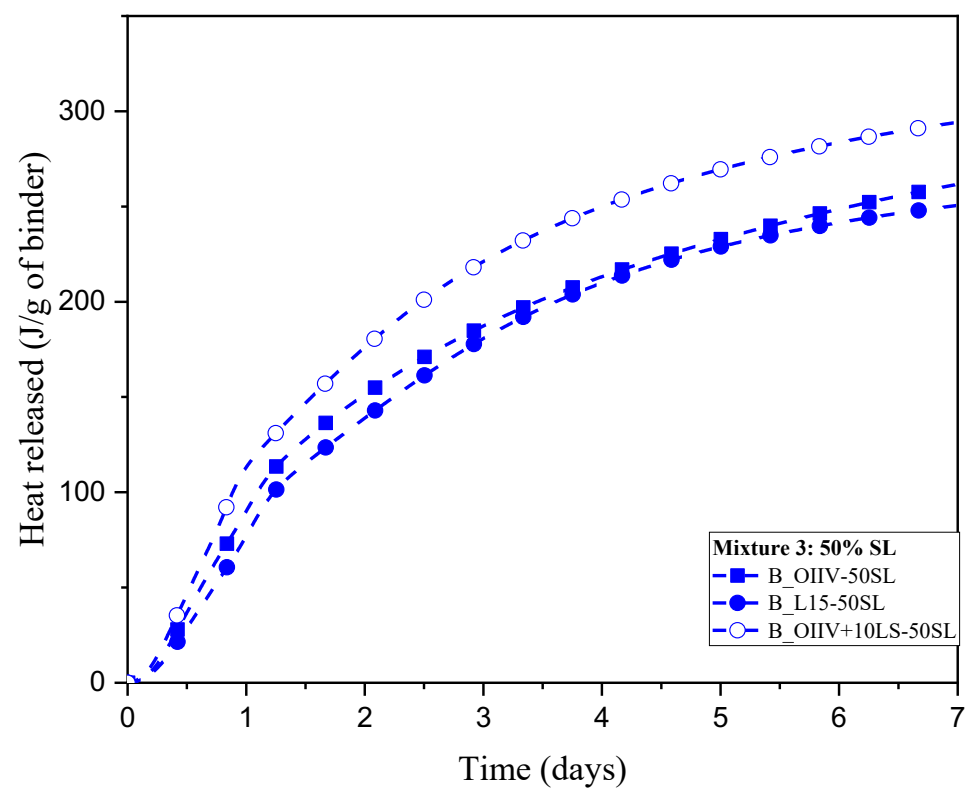

3682

Figure B-20-10. Heat of Hydration of Cement B - M3 


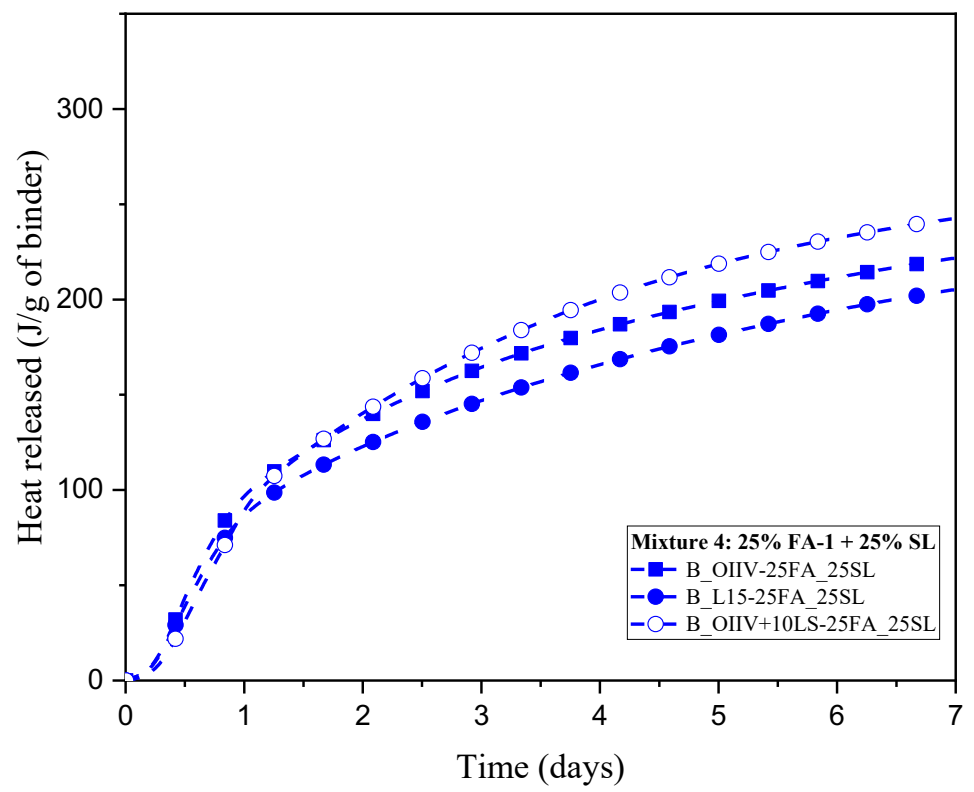

3685

3686

Figure B-20-11. Heat of Hydration of Cement B - M4

3687

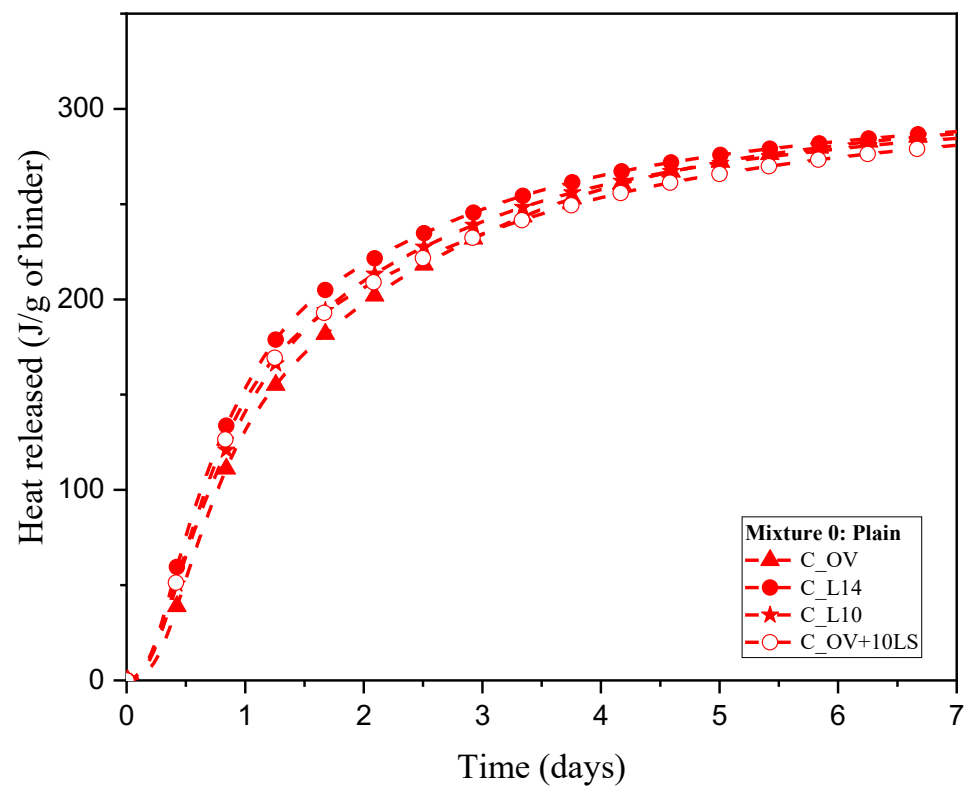

3688

Figure B-20-12. Heat of Hydration of Cement C - M0 


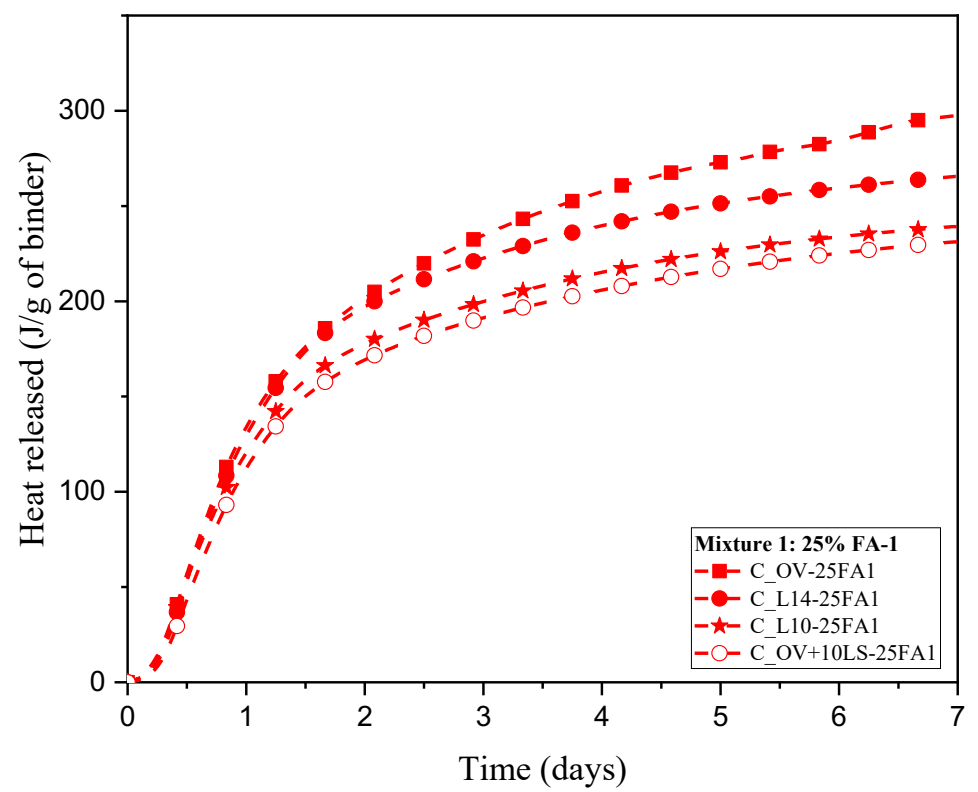

3691

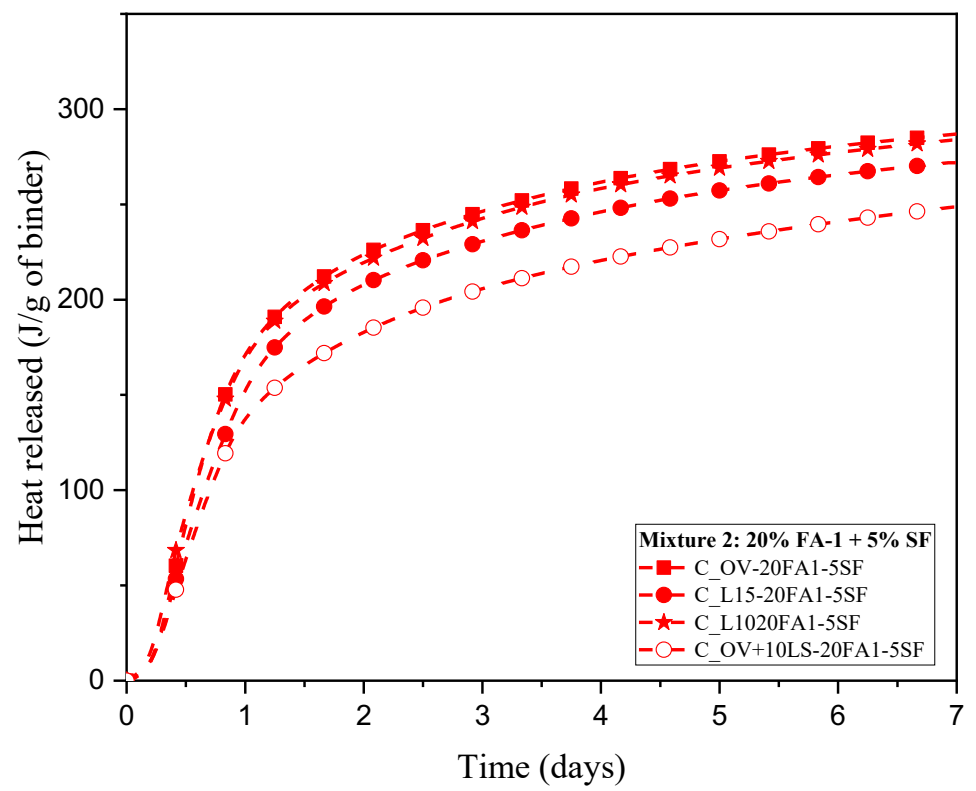




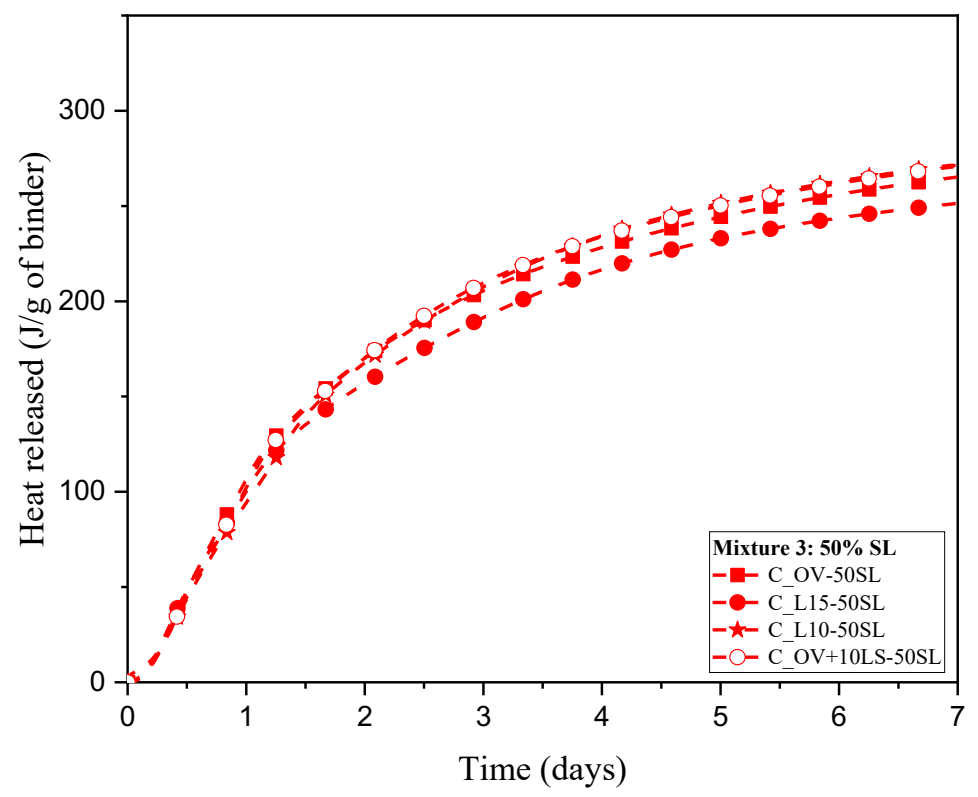

3696

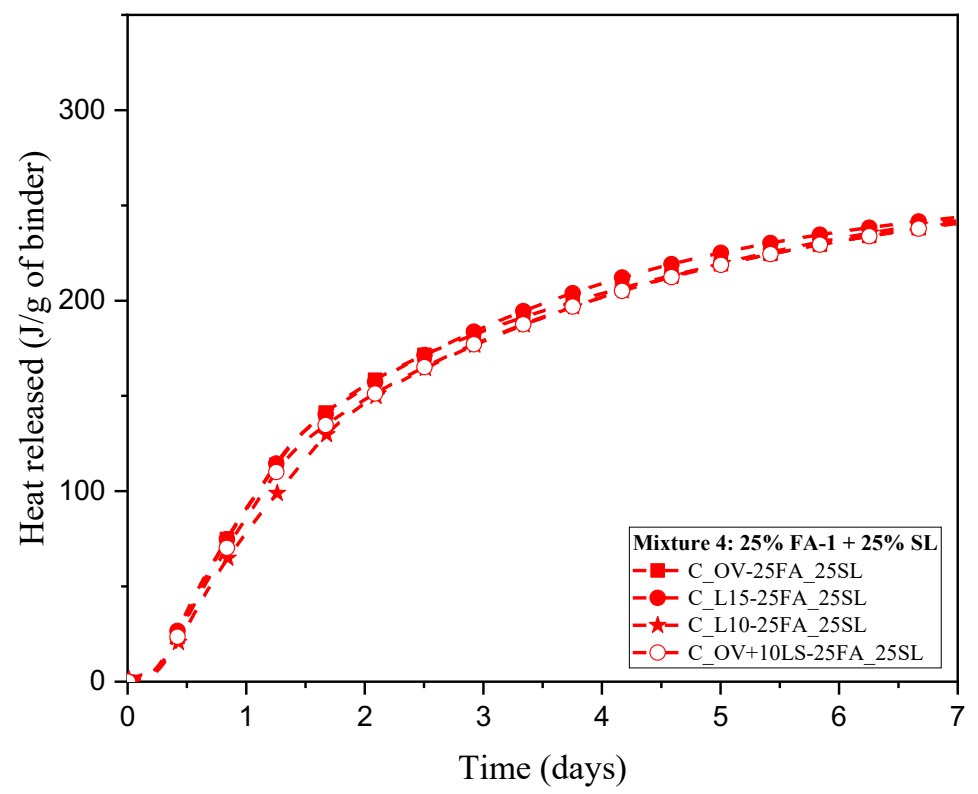




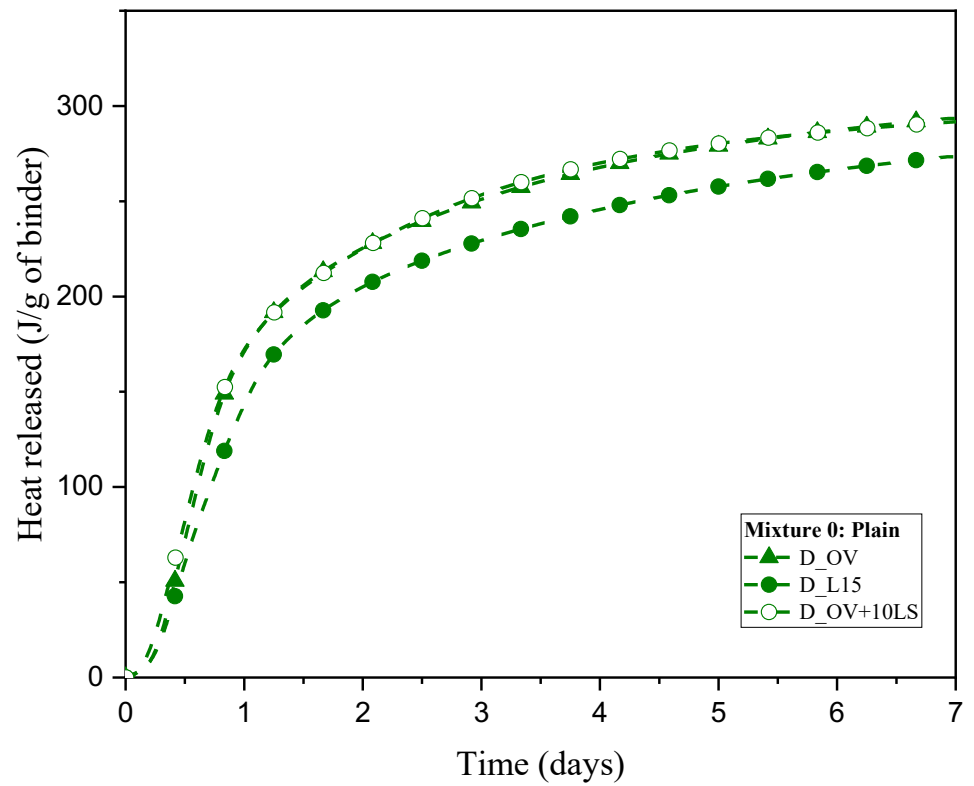

3700

3701

Figure B-20-17. Heat of Hydration of Cement D - M0

3702

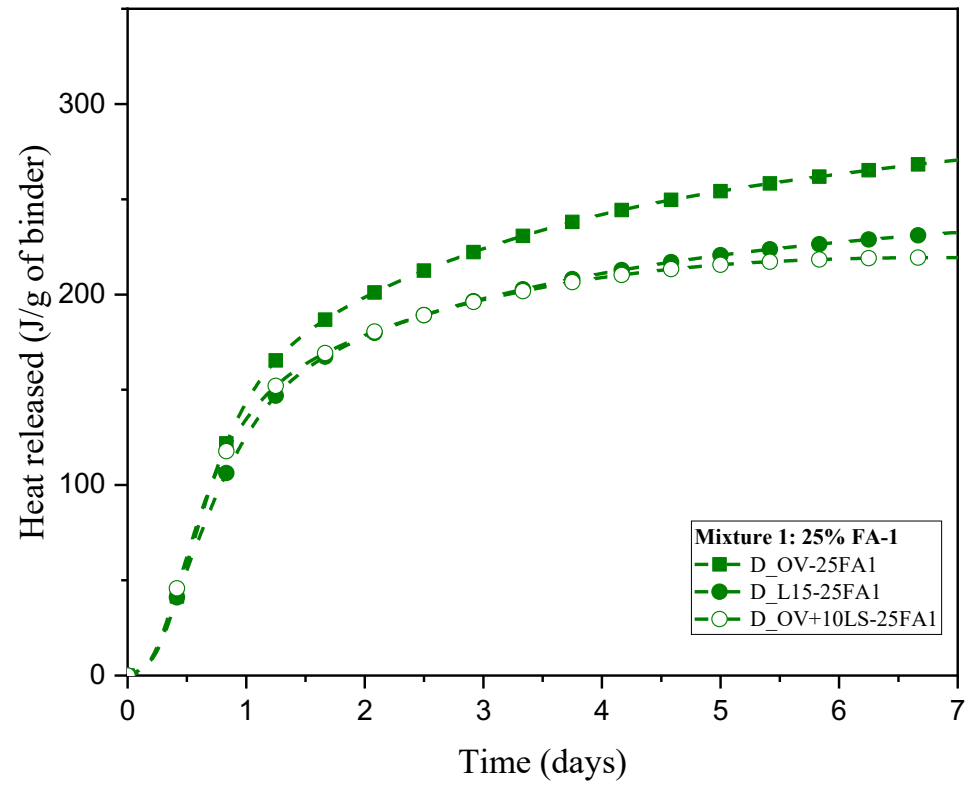

3703

3704

Figure B-20-18. Heat of Hydration of Cement D - M1 


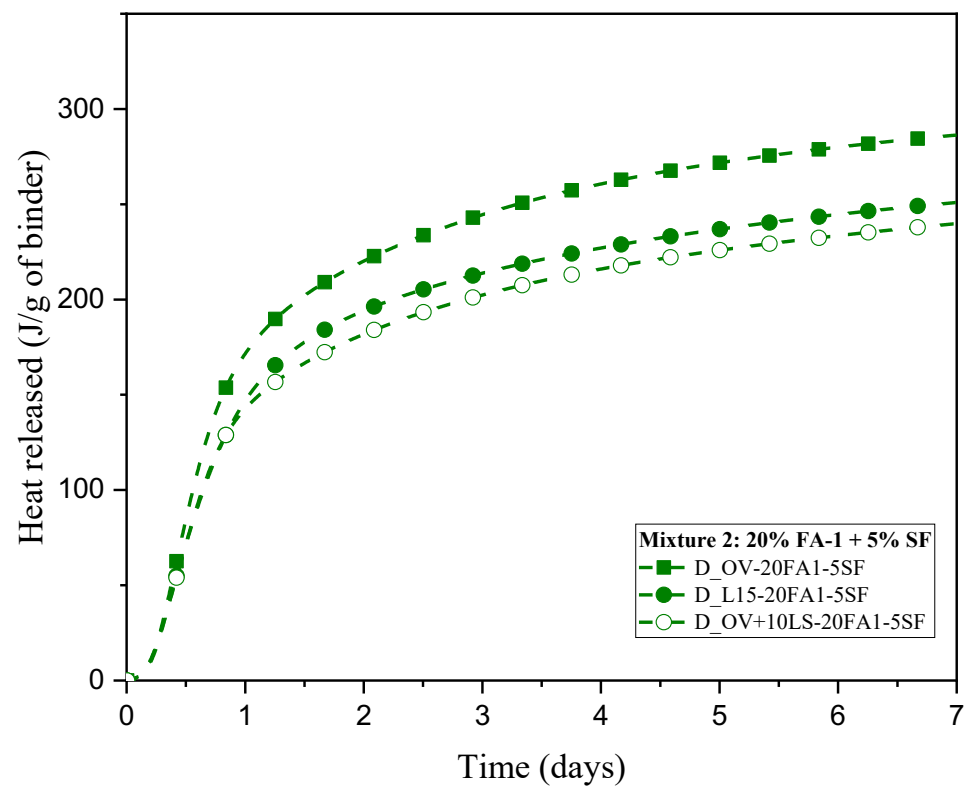

3705

3706

Figure B-20-19. Heat of Hydration of Cement D - M2

3707

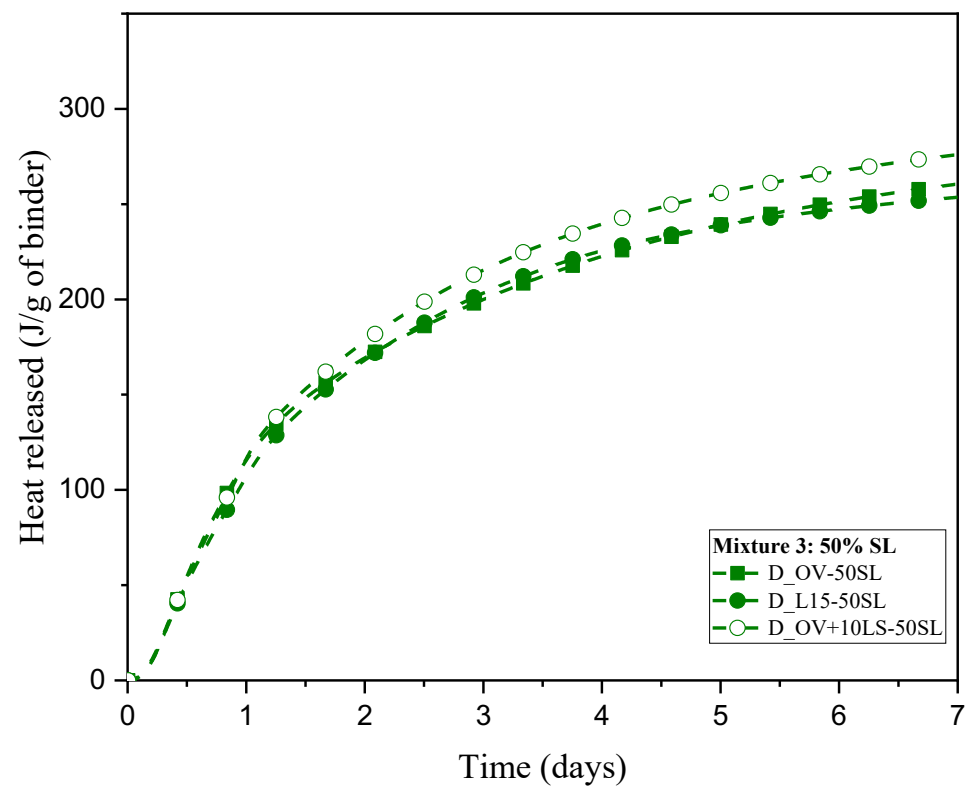

3708

Figure B-20-20. Heat of Hydration of Cement D - M3 


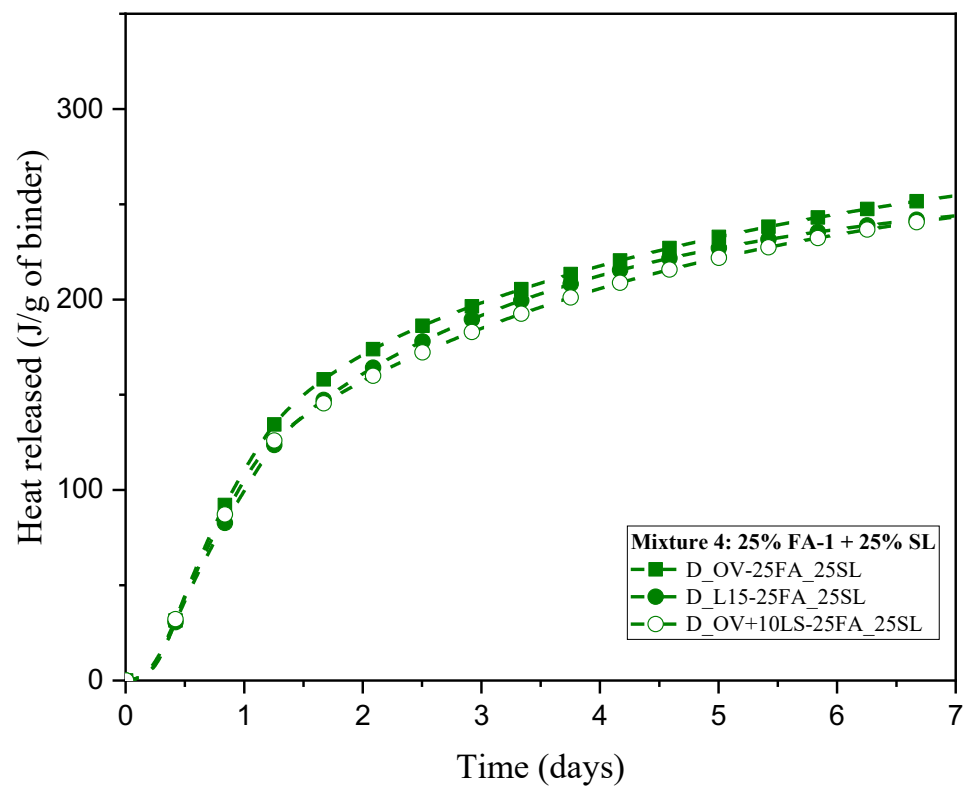

3710

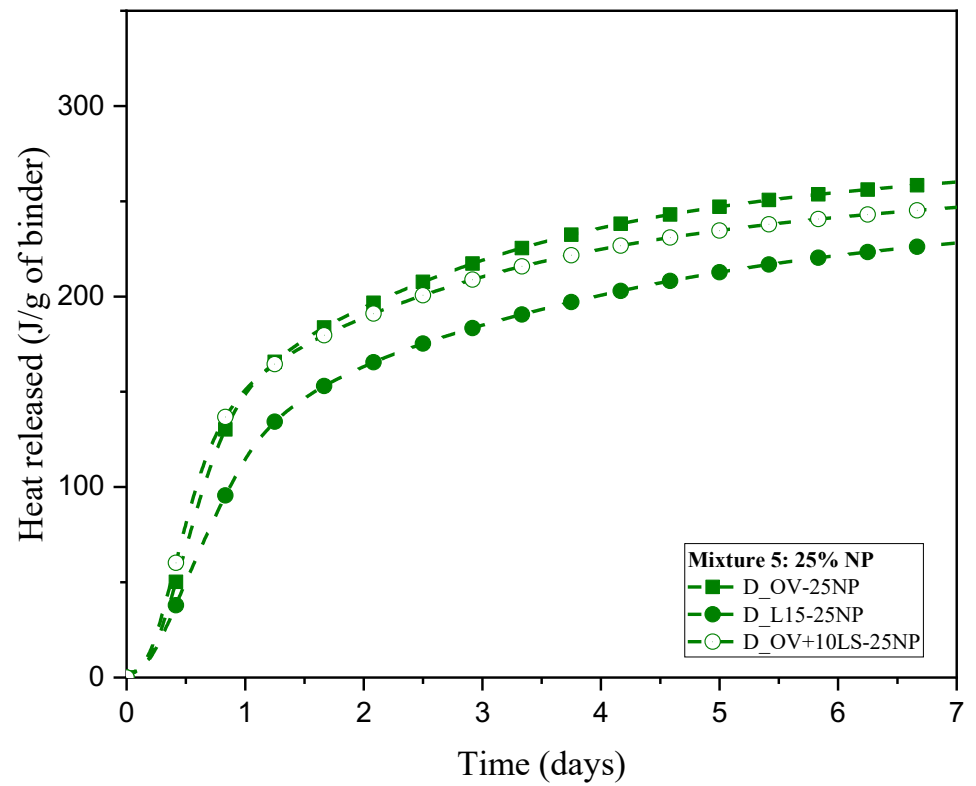




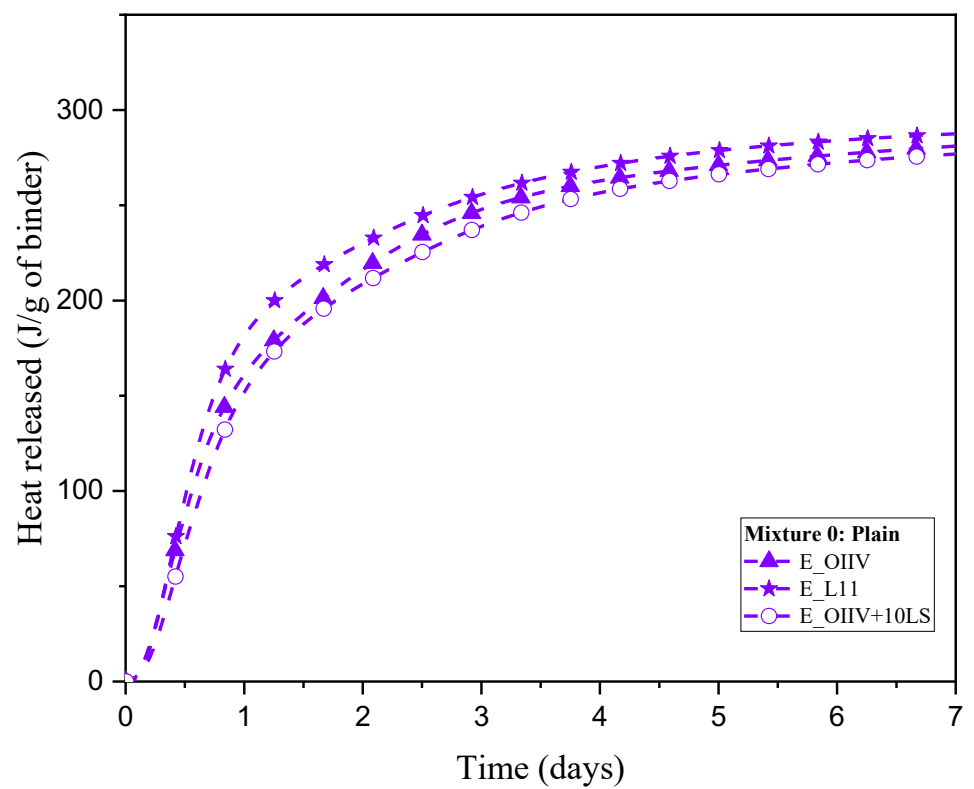

3715

Figure B-20-23. Heat of Hydration of Cement E - M0

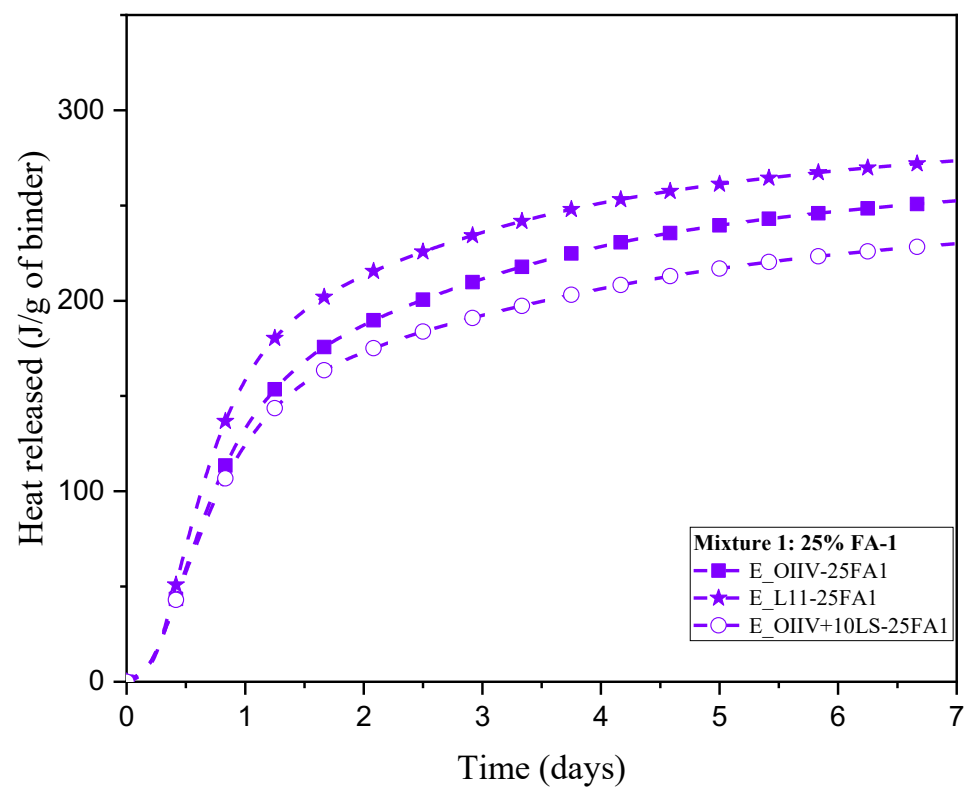




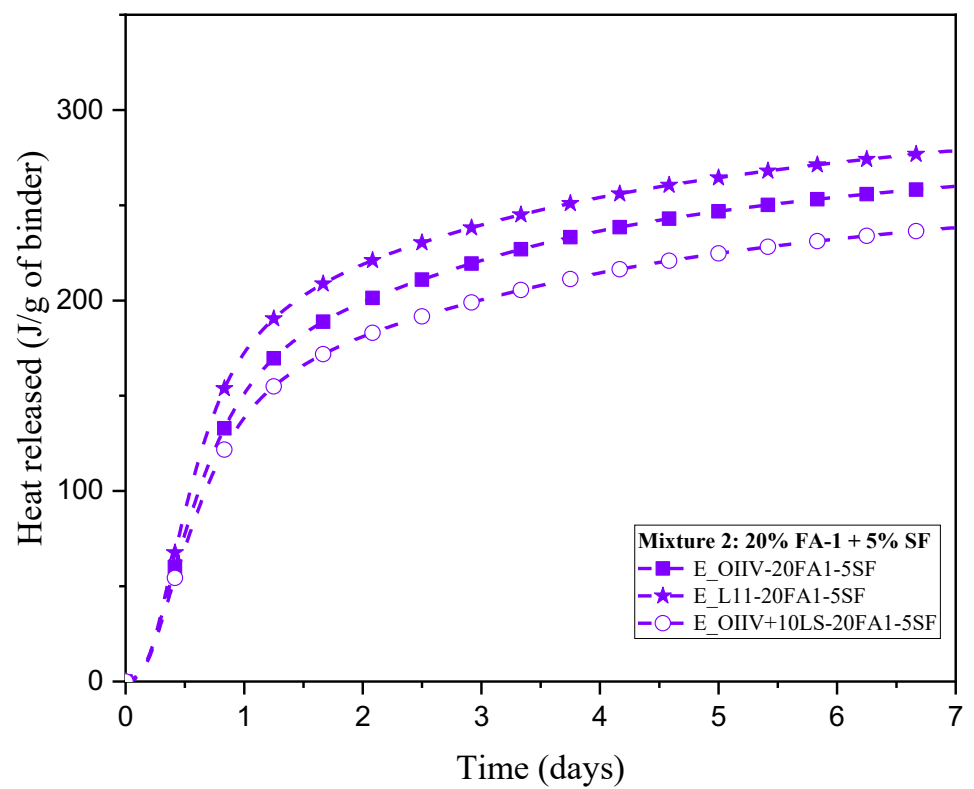

3720

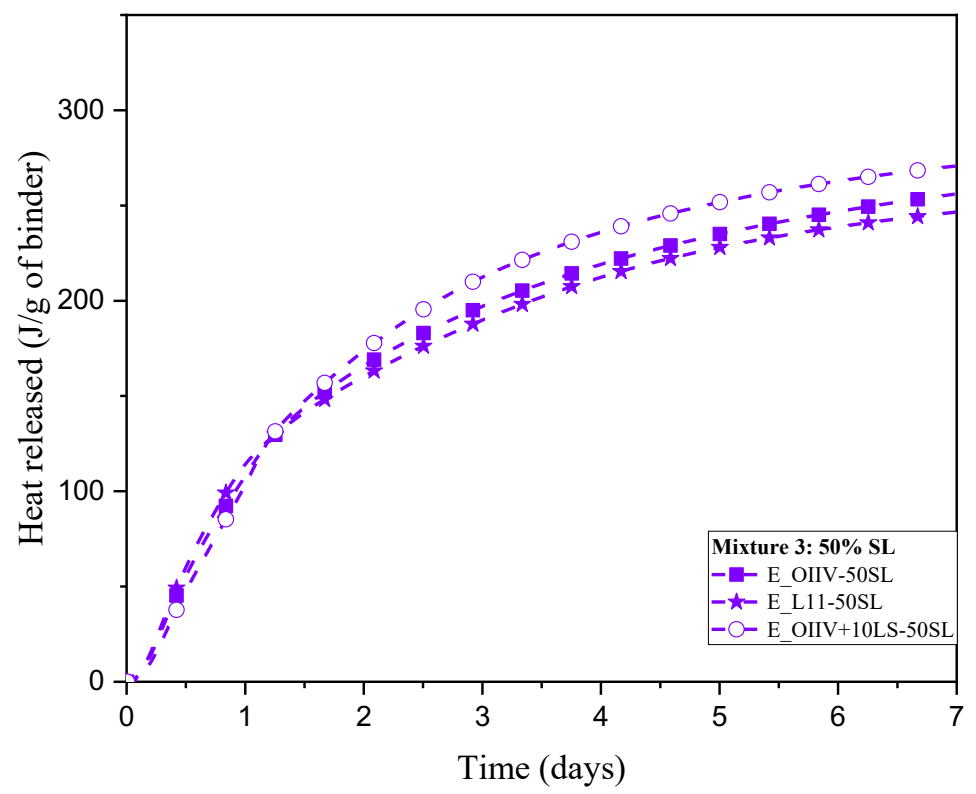




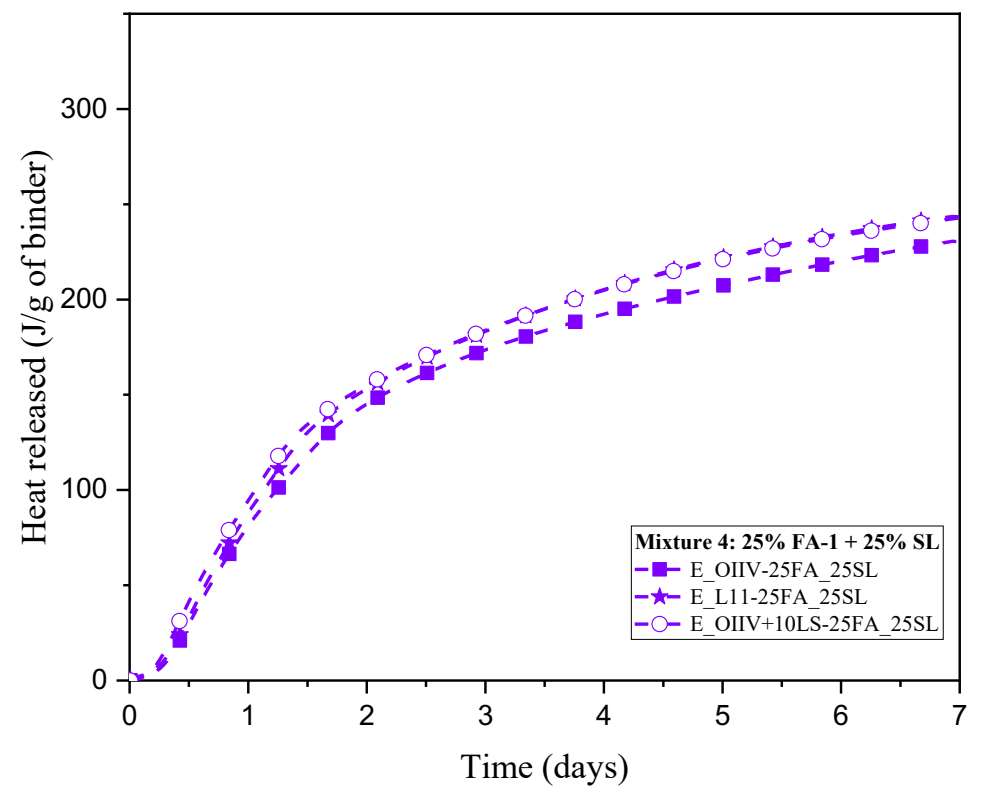

3725

3726

Figure B-20-27. Heat of Hydration of Cement E - M4

3727

3728

3729 
373021 APPENDIX C - Drying Shrinkage Results

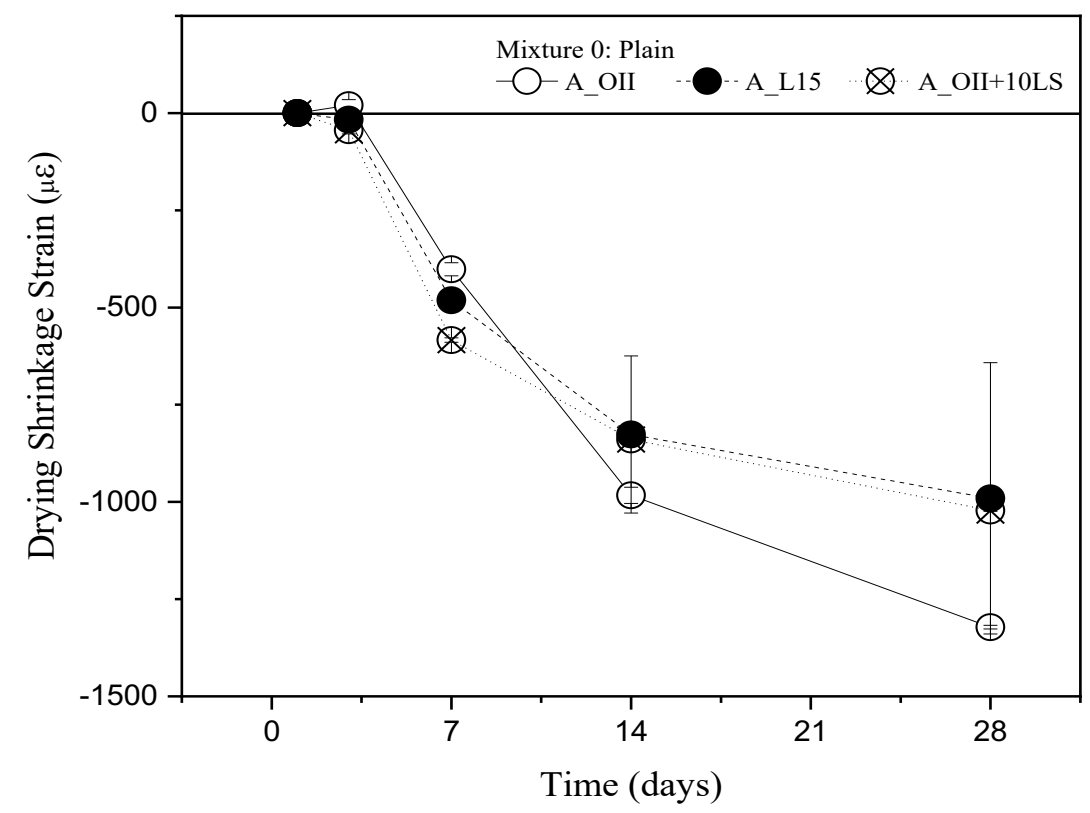

3731

3732

Figure C-21-1. Drying Shrinkage of Cement A - M0

3733 


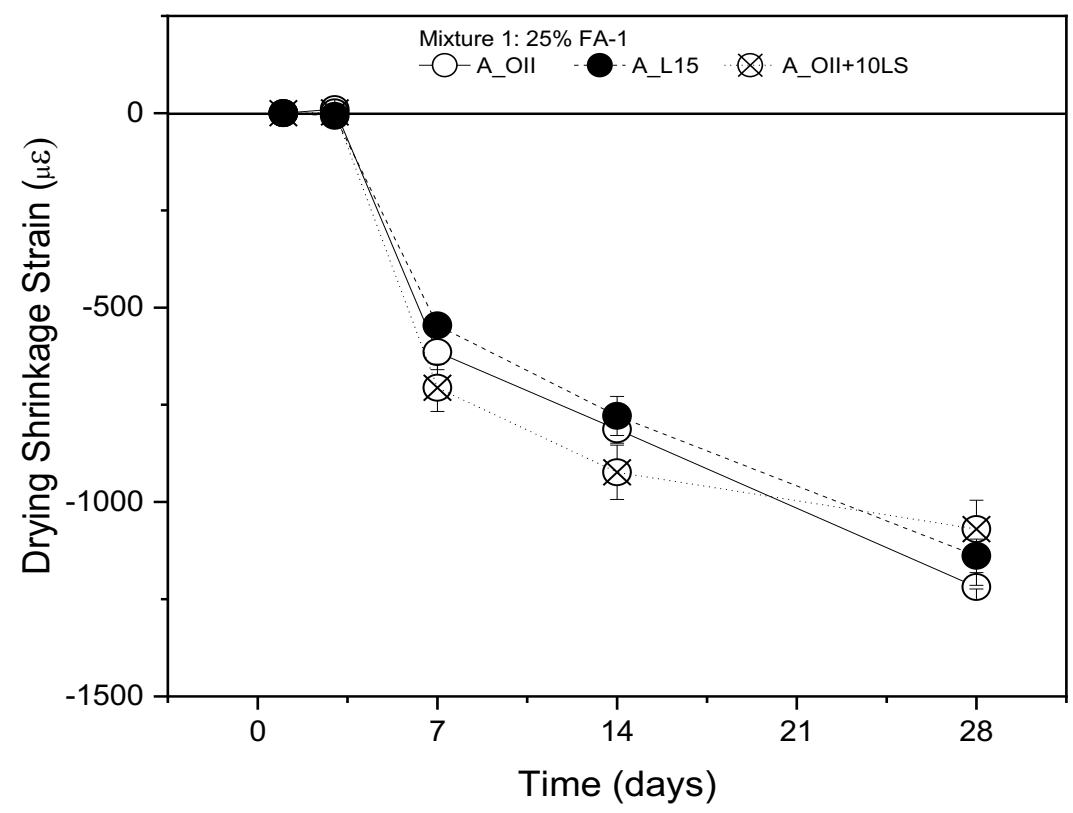

3734

3735

Figure C-21-2. Drying Shrinkage of Cement A - M1

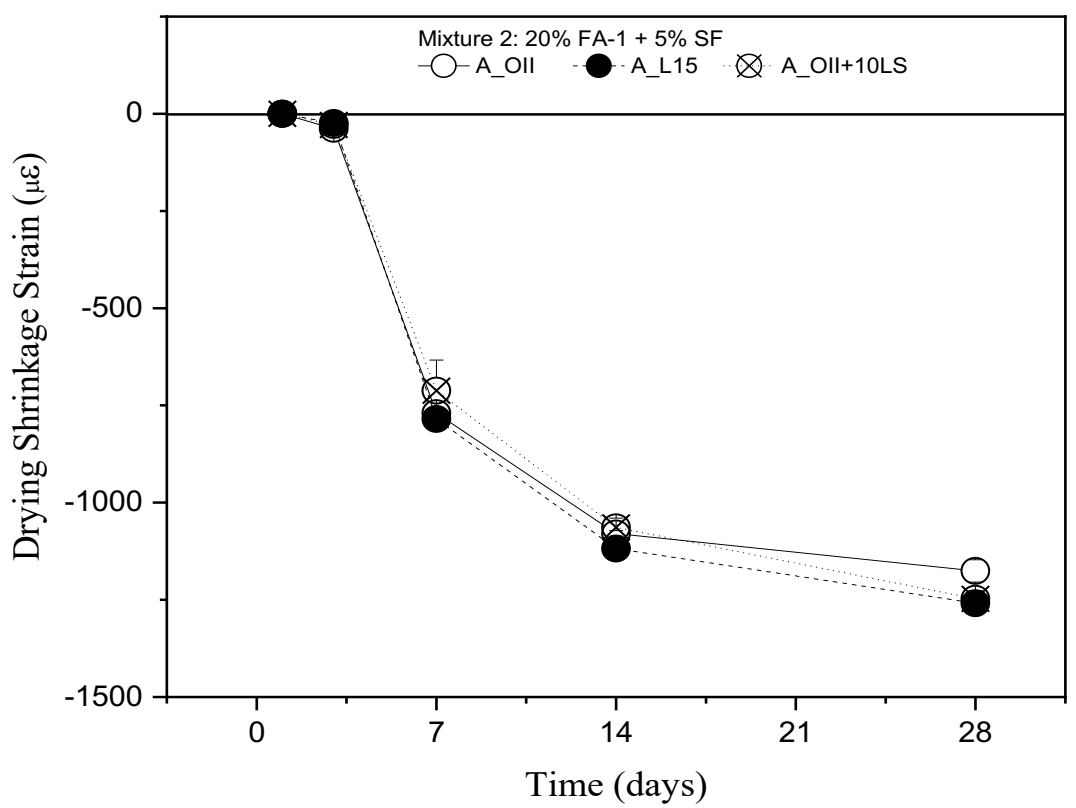

3736

3737

Figure C-21-3. Drying Shrinkage of Cement A - M2 


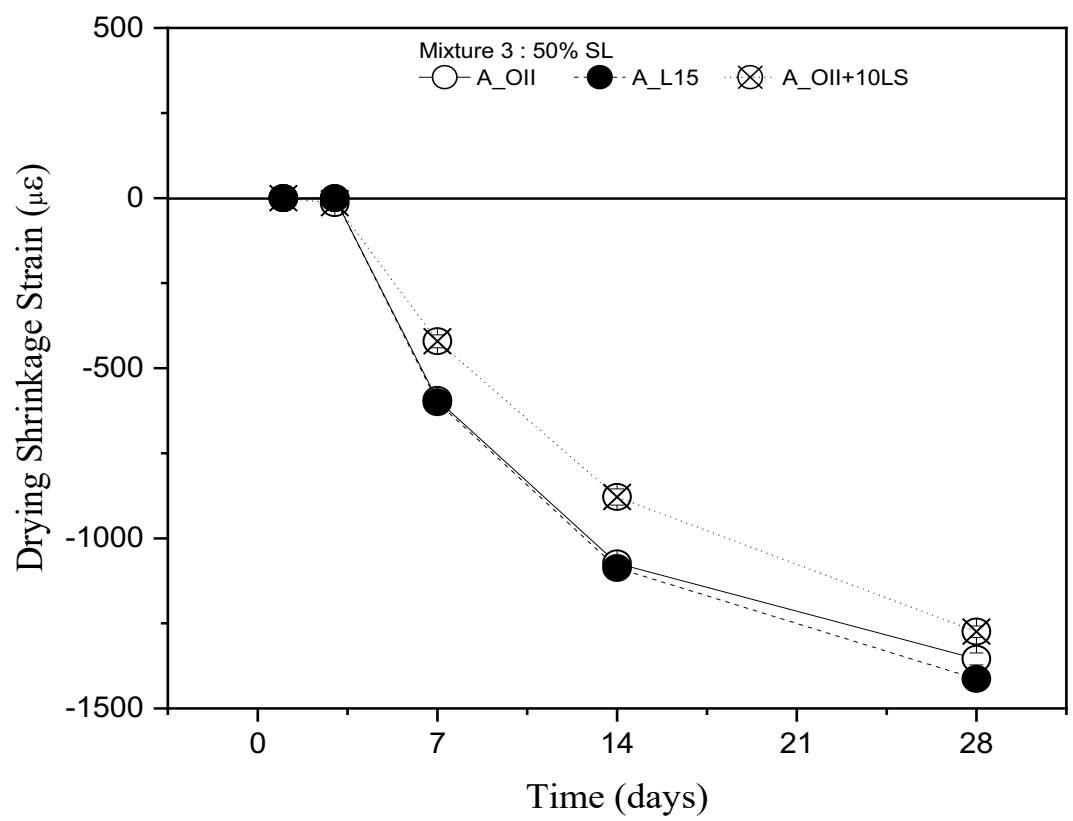

3738

Figure C-21-4. Drying Shrinkage of Cement A - M3

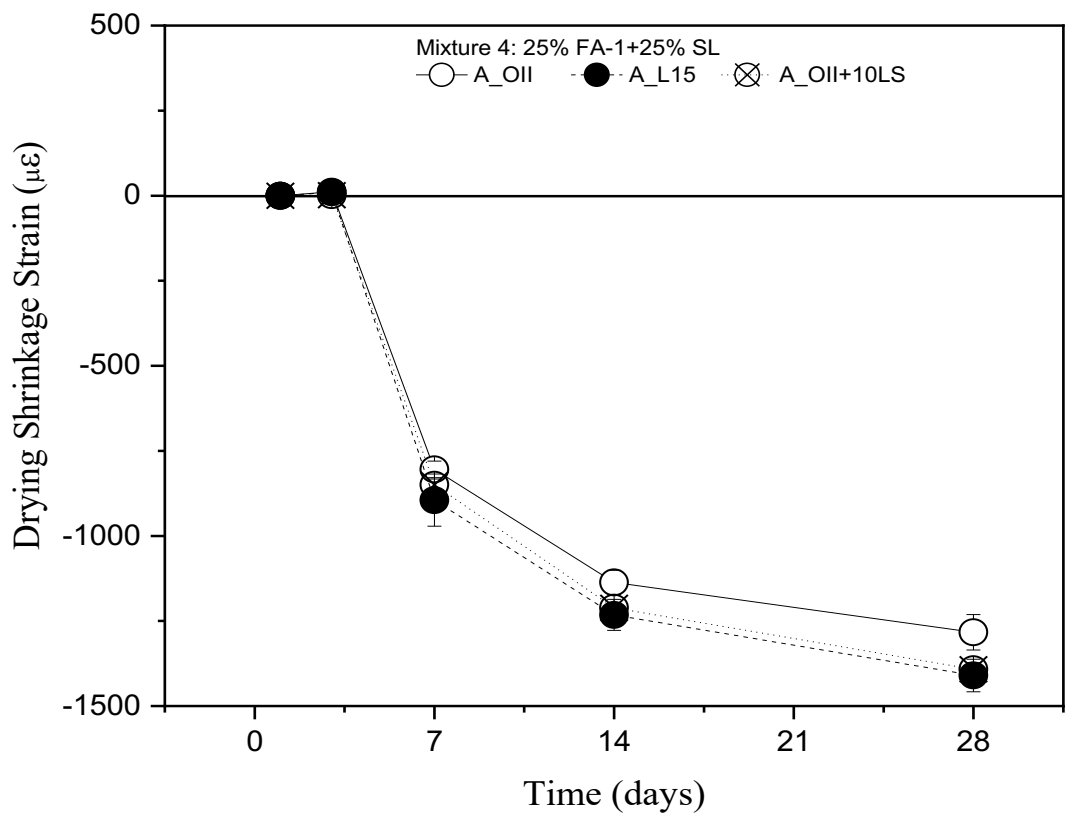

Figure C21-5. Drying Shrinkage of Cement A - M4 


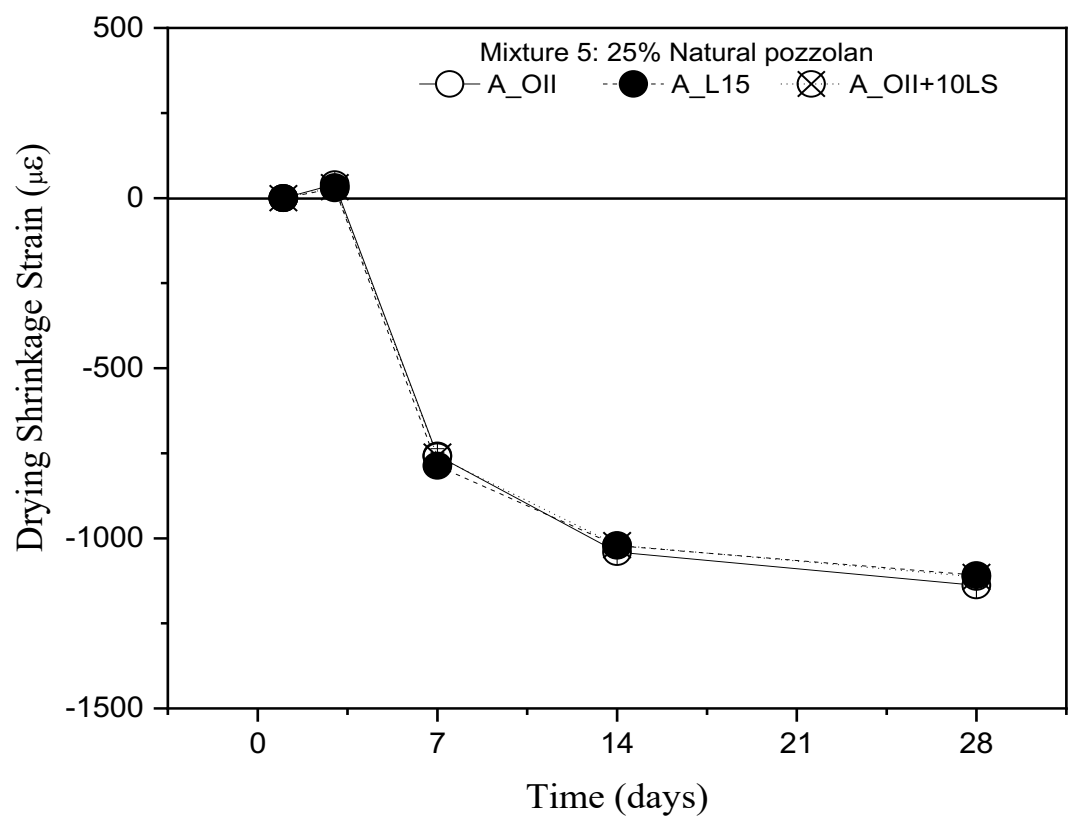

3742

Figure C-21-6. Drying Shrinkage of Cement A - M5

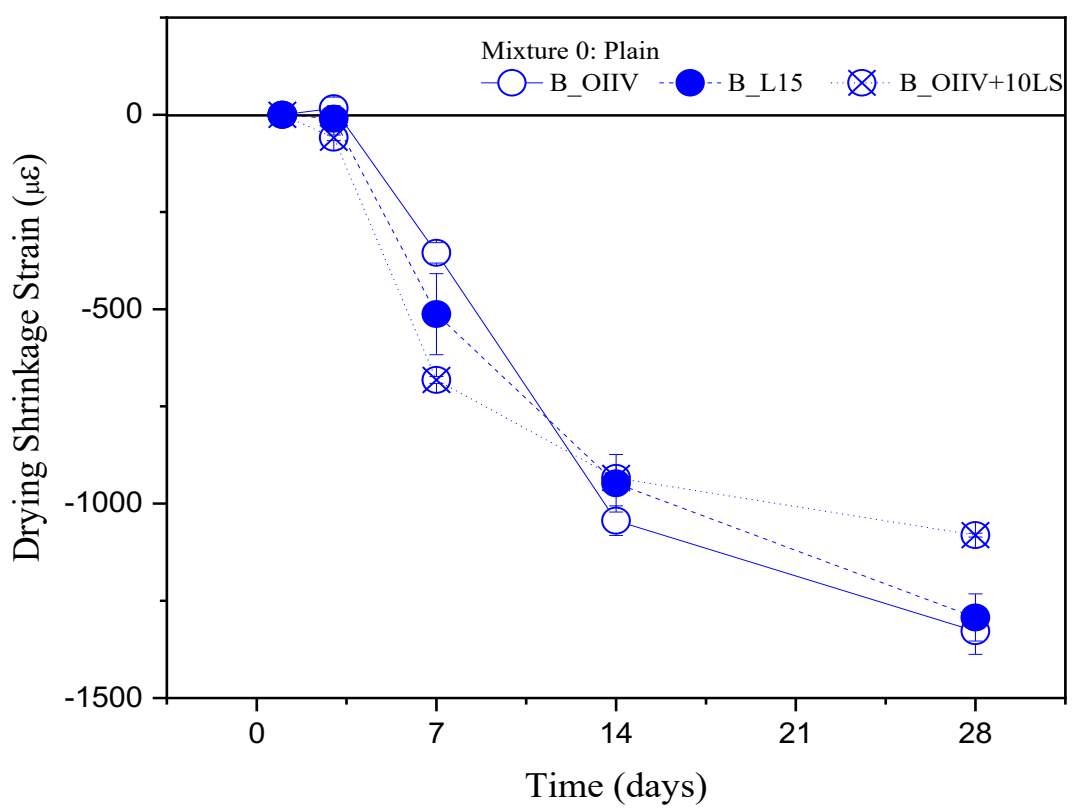




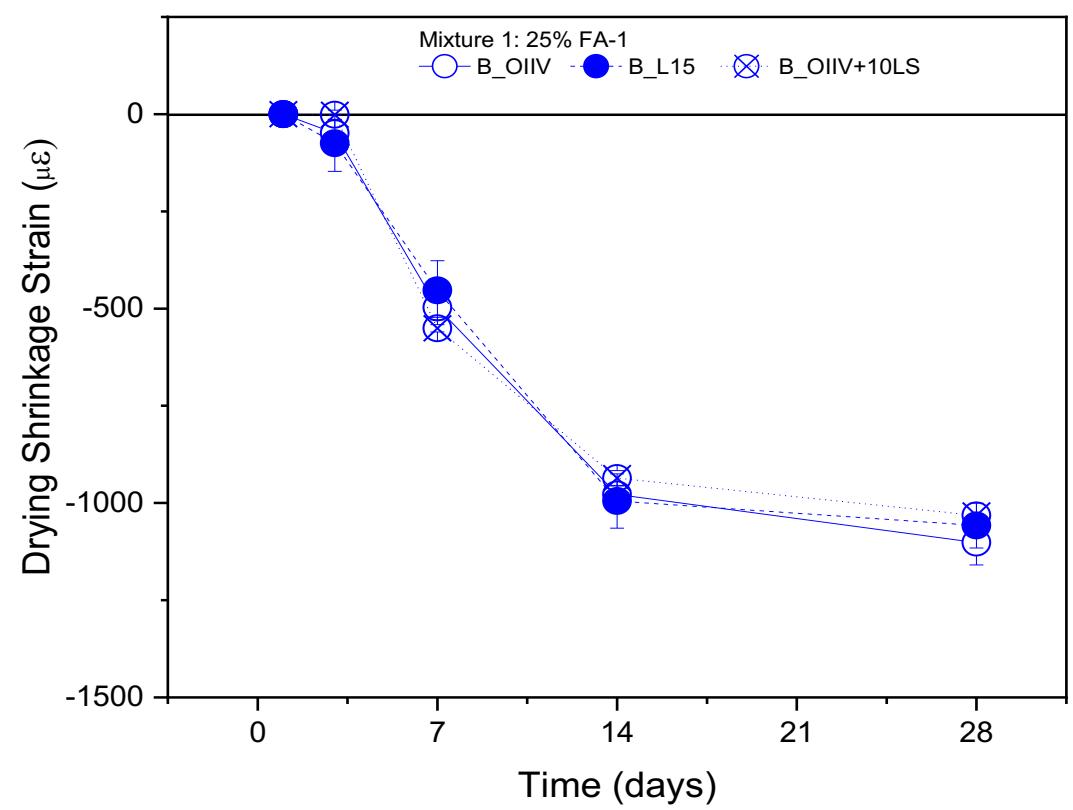

Figure C-21-8. Drying Shrinkage of Cement B - M1

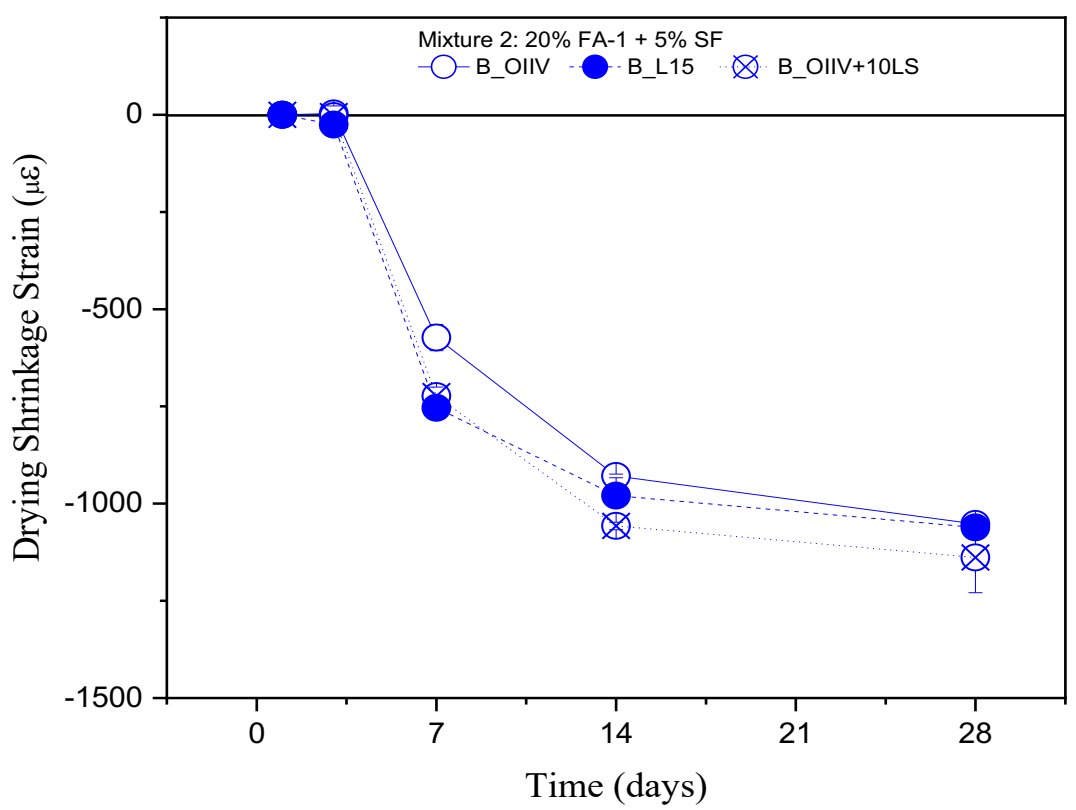




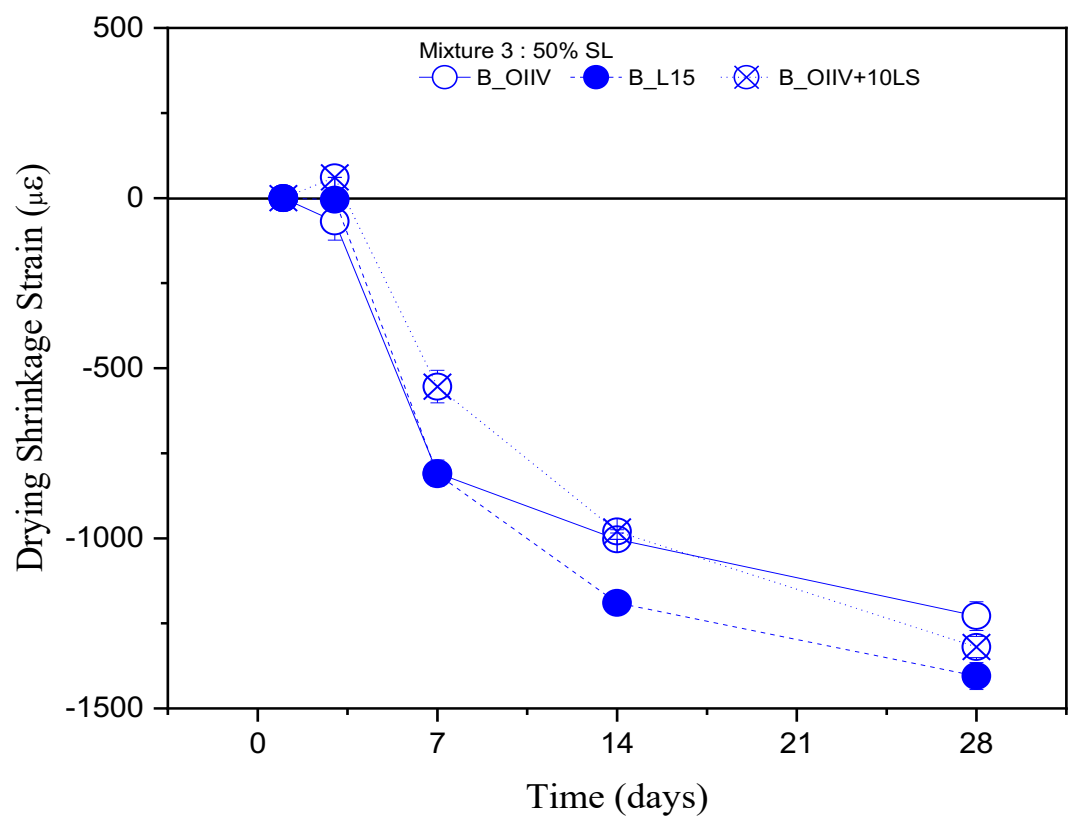

3750

Figure C-21-10. Drying Shrinkage of Cement B - M3

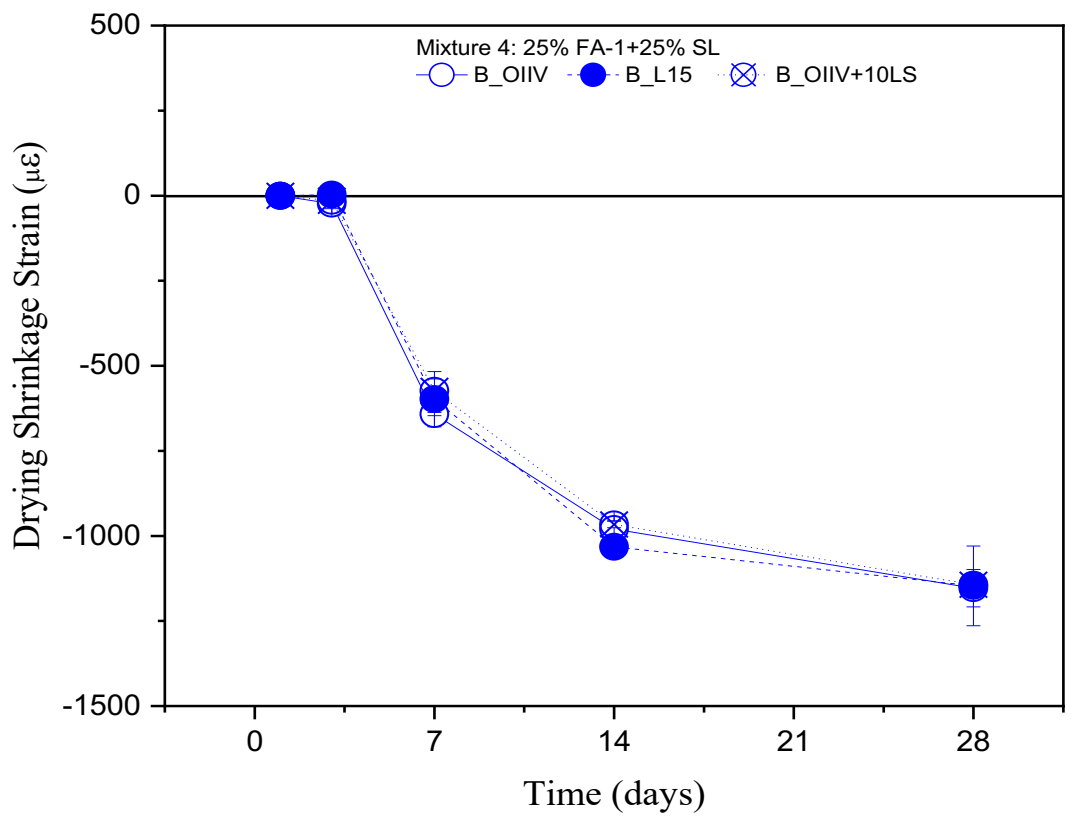




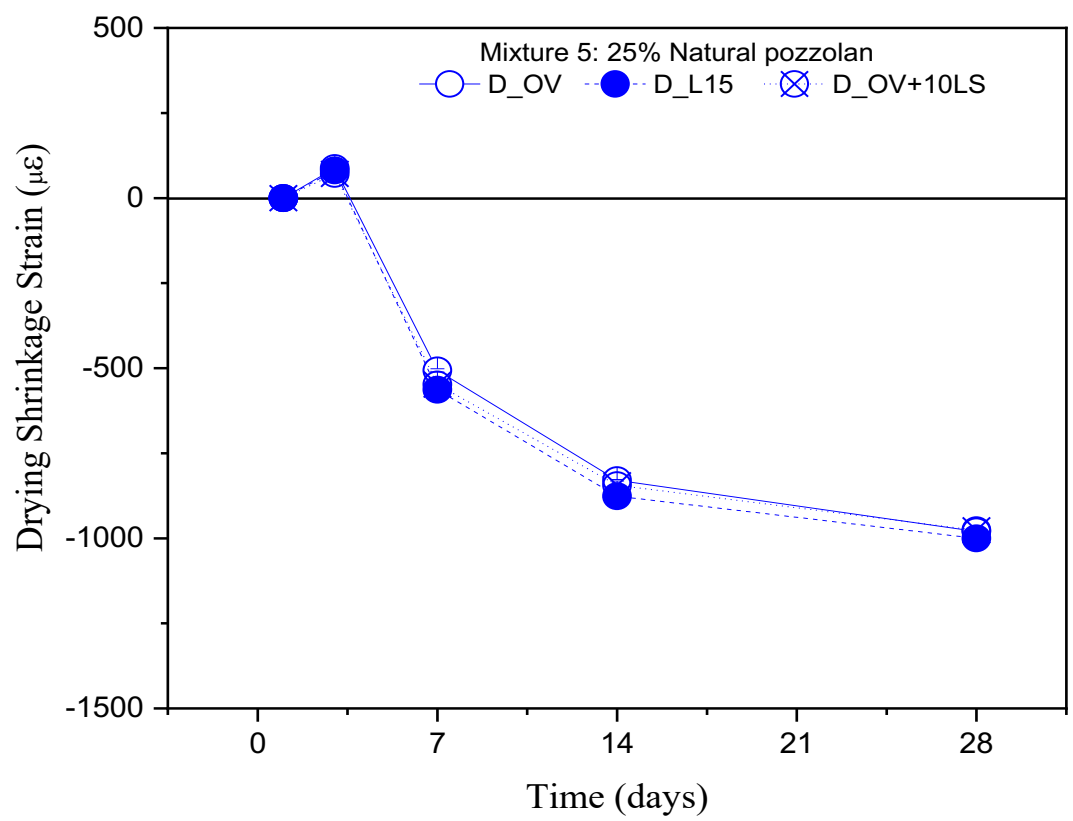

3754

Figure C-21-12. Drying Shrinkage of Cement B - M5

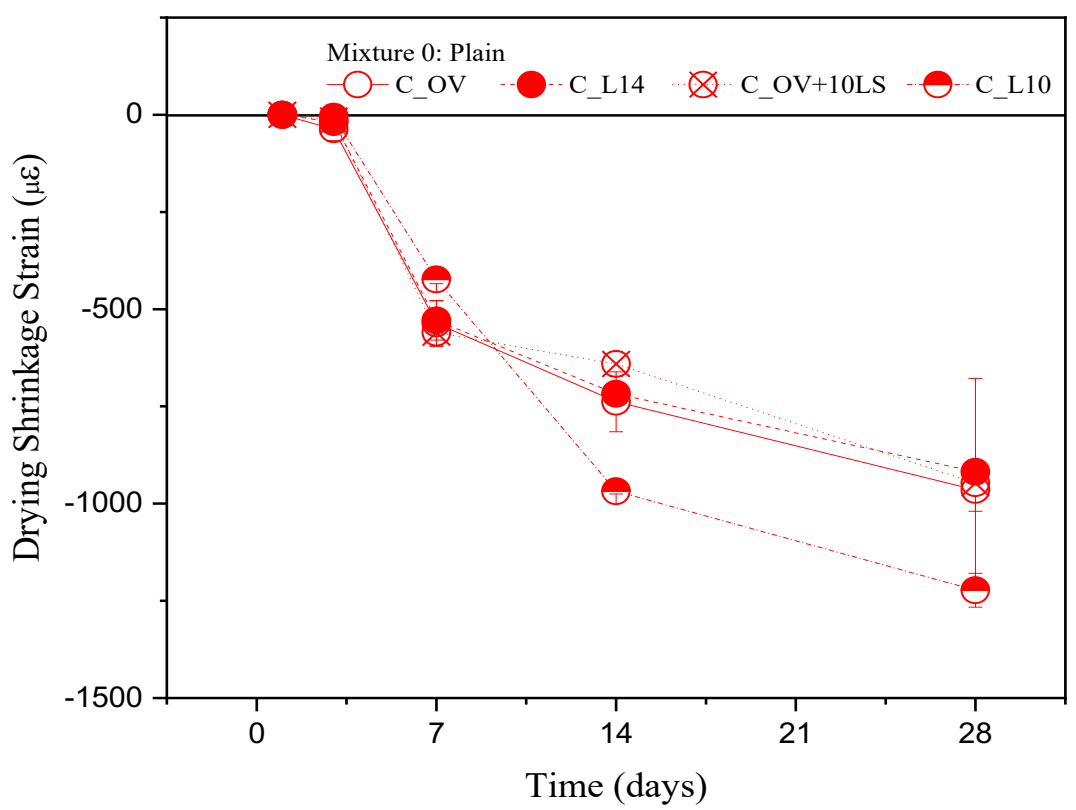




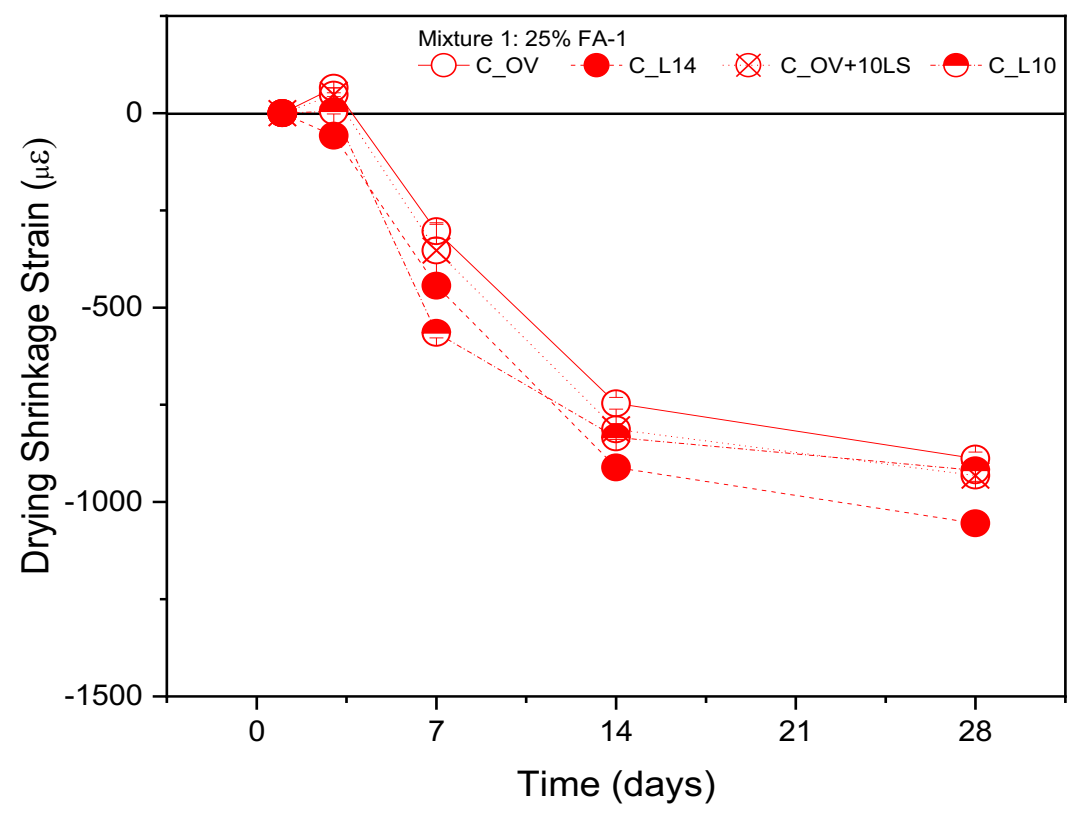

3758

3759

Figure C-21-14. Drying Shrinkage of Cement C - M1

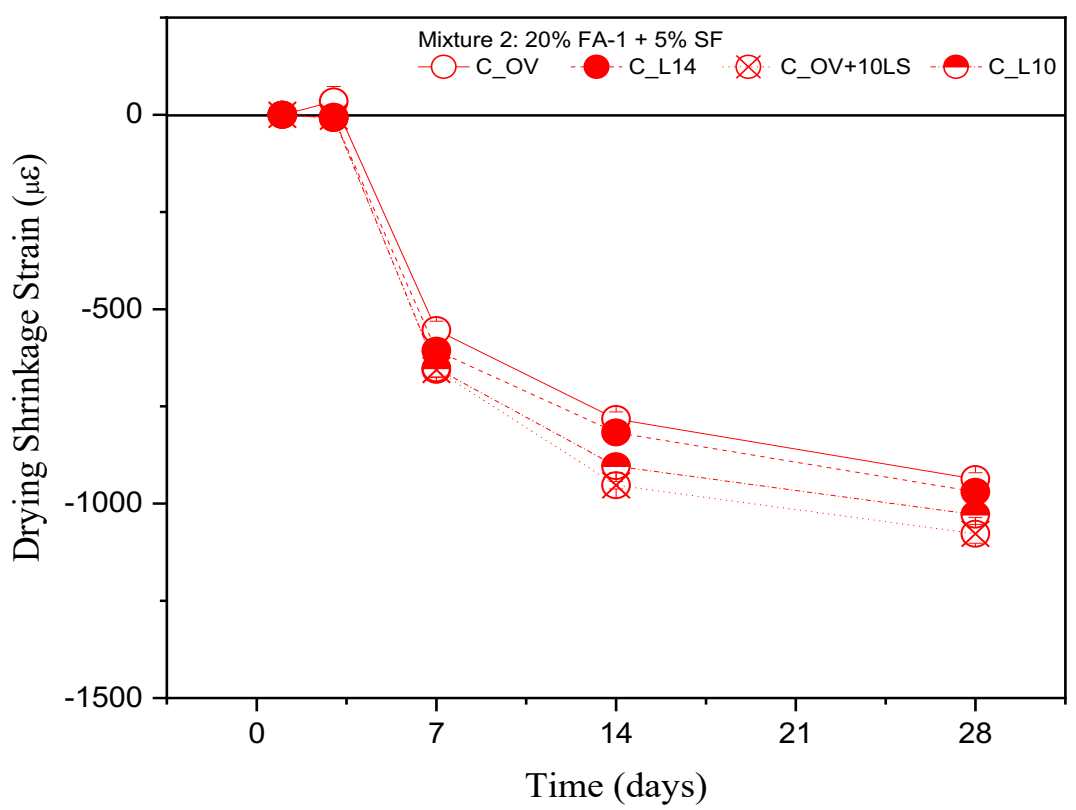

Figure C-21-15. Drying Shrinkage of Cement C - M2 


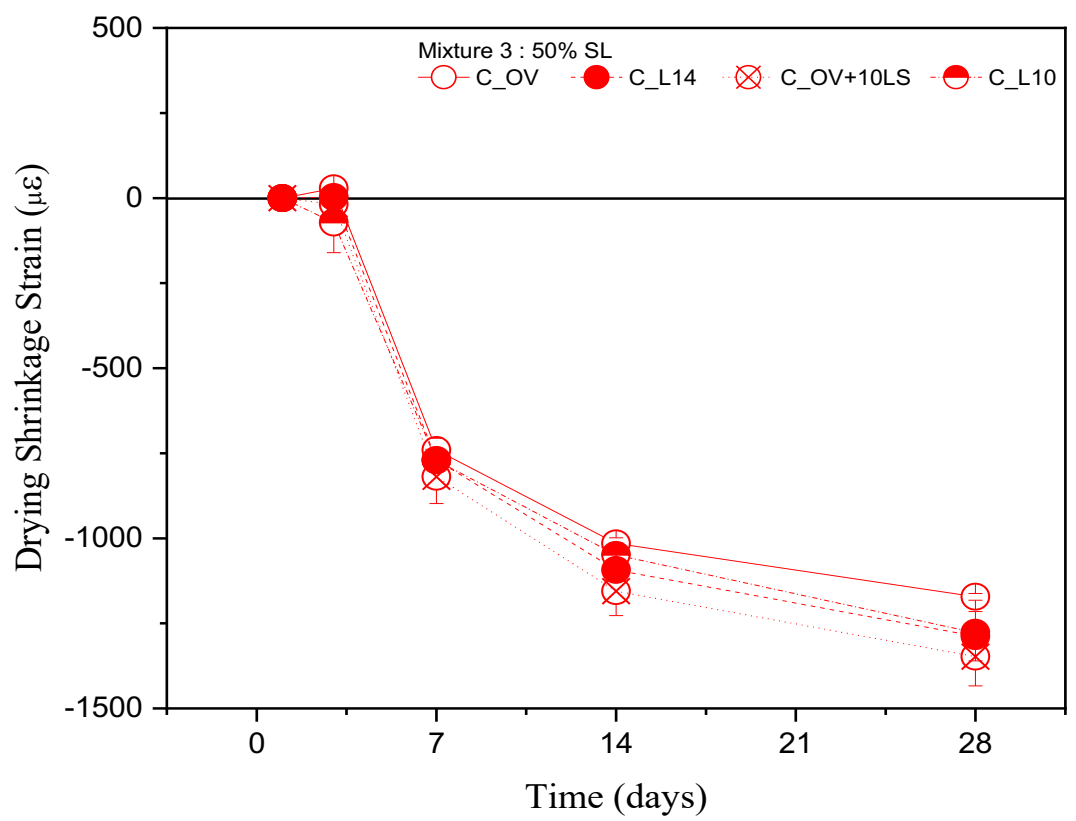

3762

3763

Figure C-21-16. Drying Shrinkage of Cement C - M3

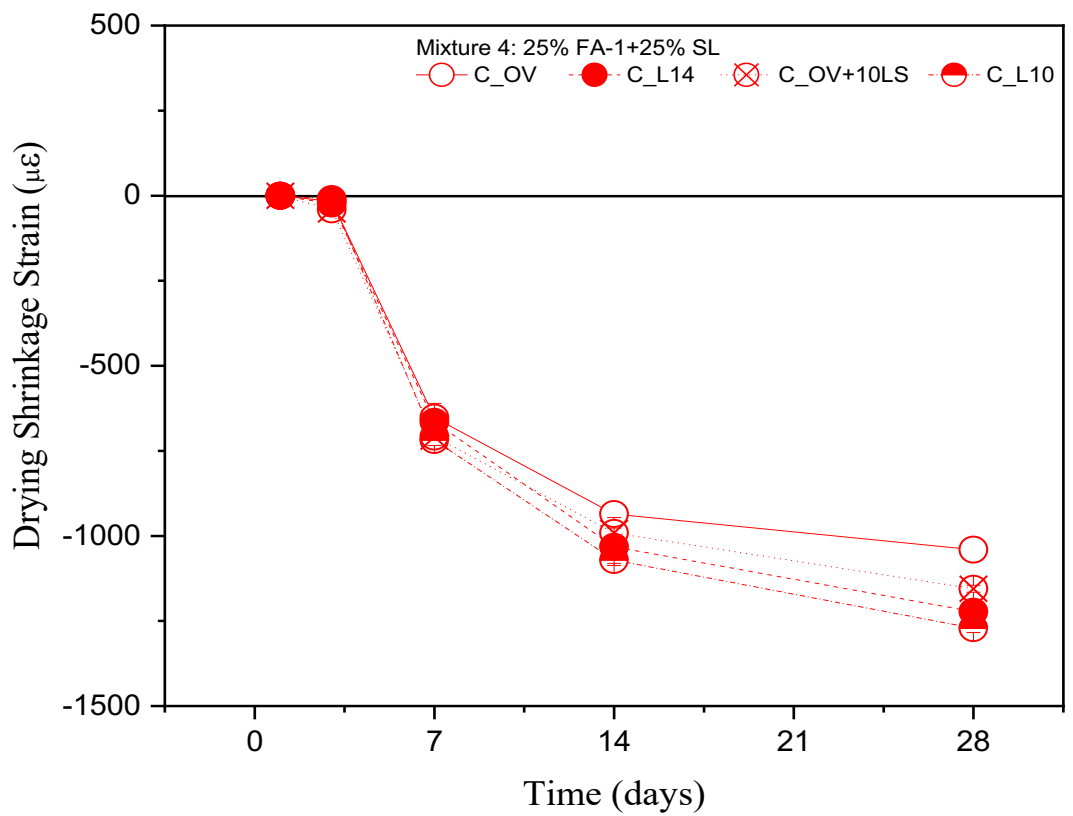

Figure C-21-17. Drying Shrinkage of Cement C - M4 


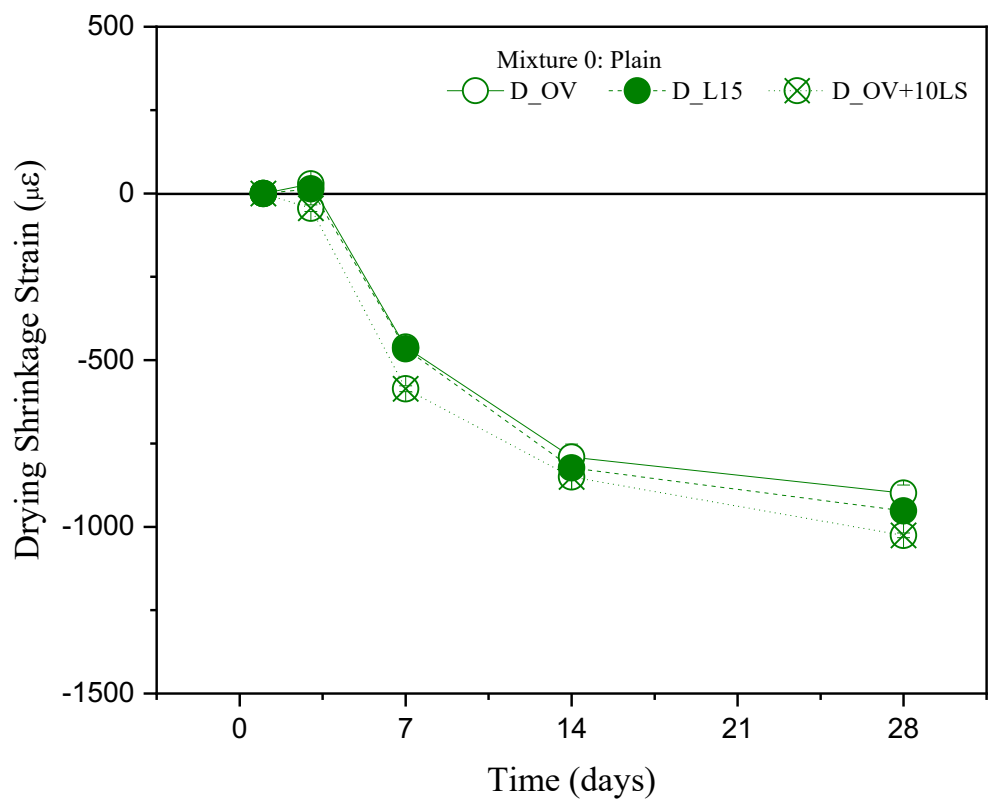

3766

Figure C-21-18. Drying Shrinkage of Cement D - M0

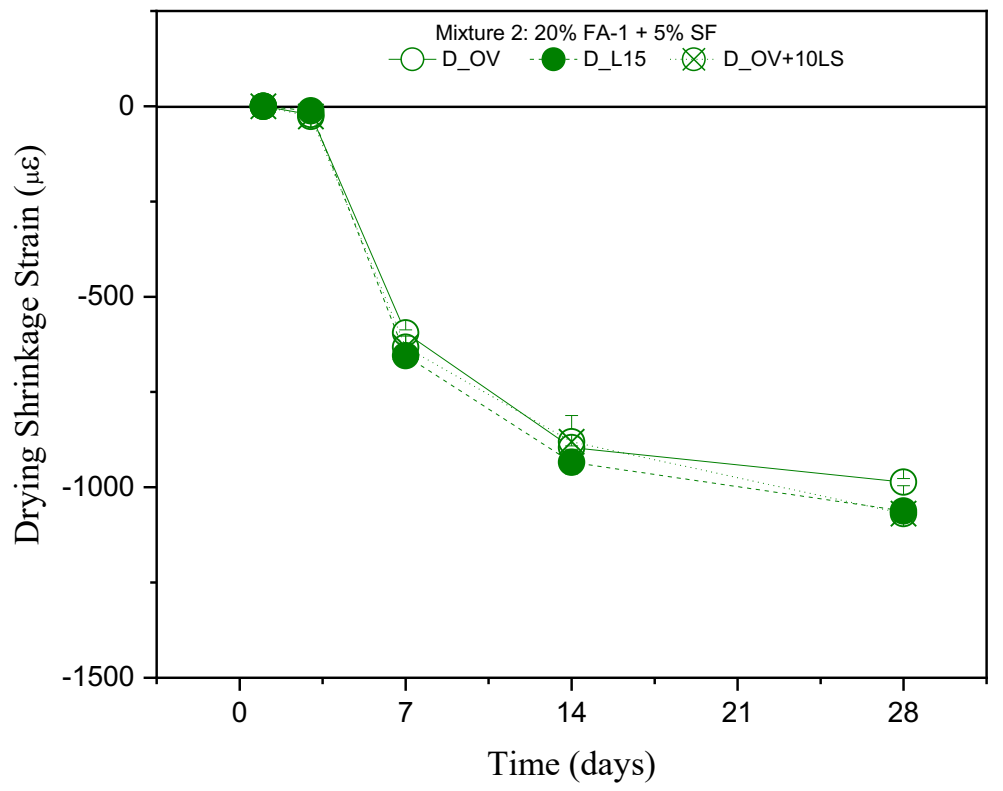

3768

Figure C-21-19. Drying Shrinkage of Cement D - M1 


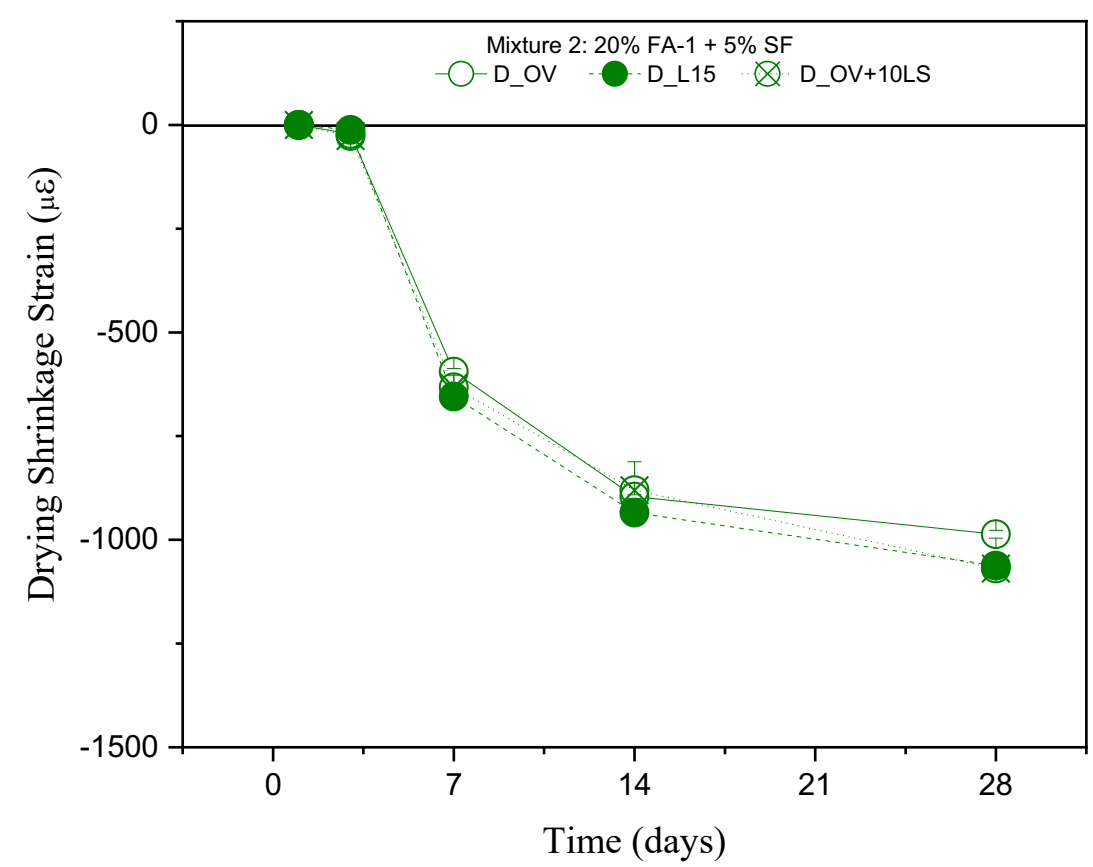

3770

Figure C-21-20. Drying Shrinkage of Cement D - M2

3772

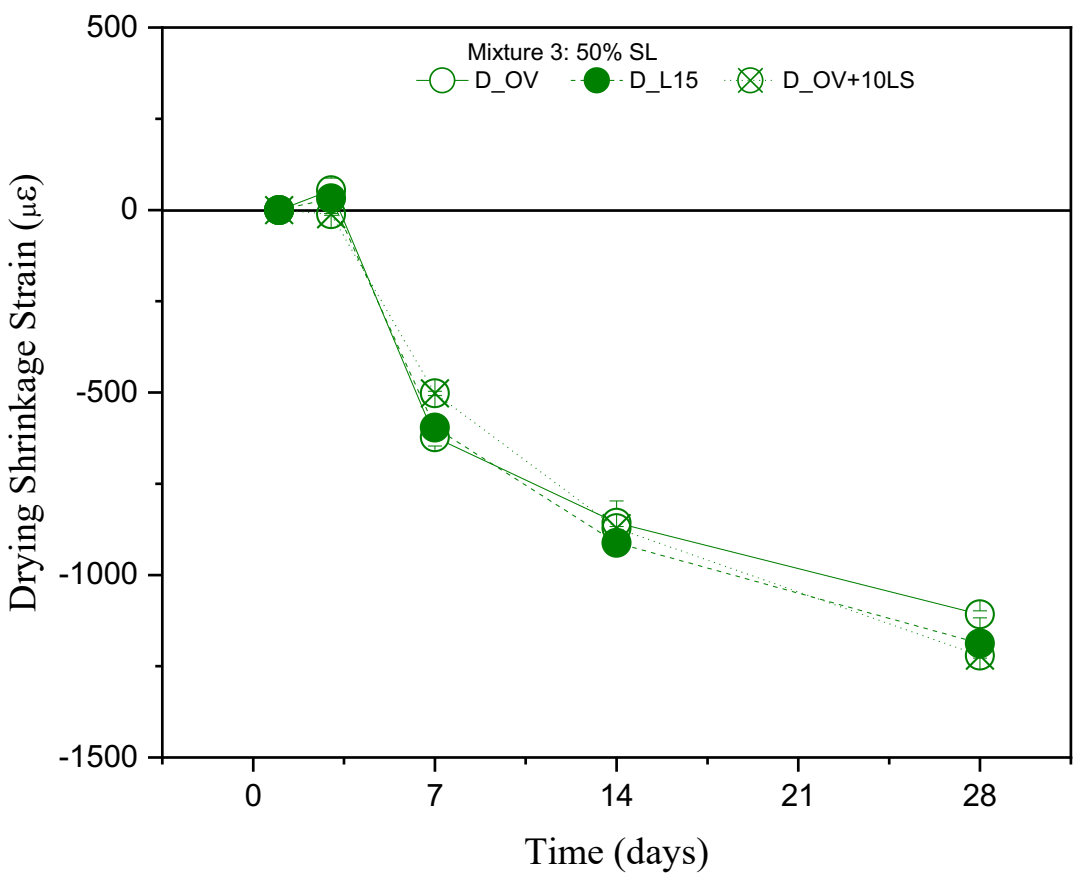




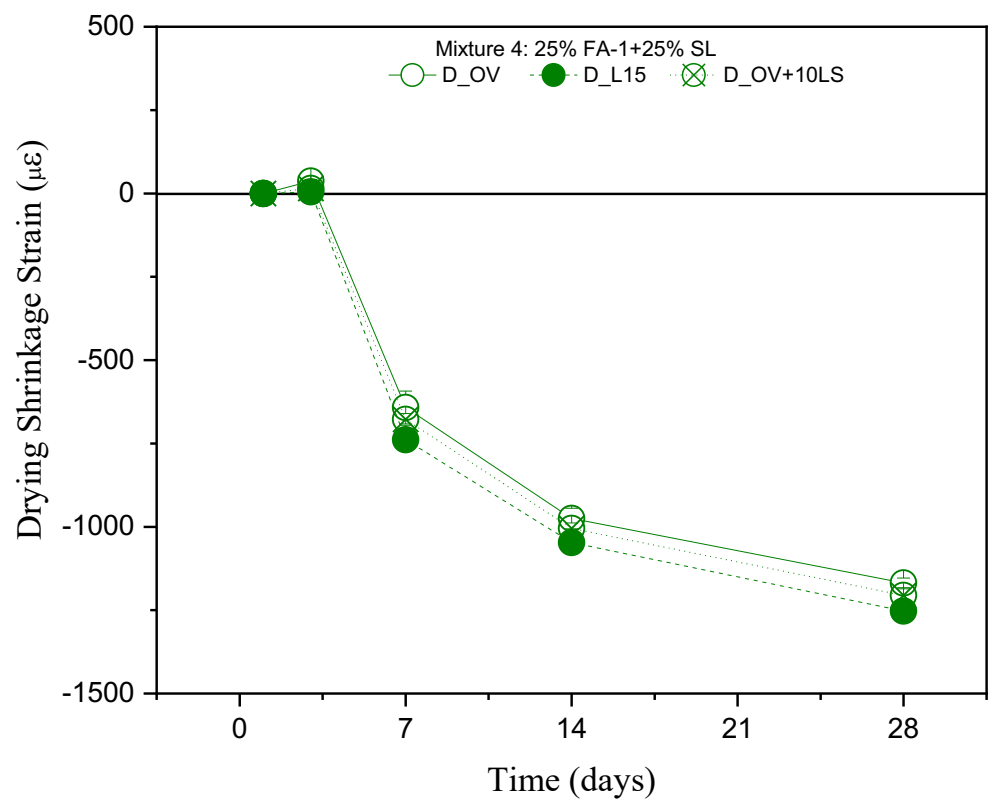

3775

3776

Figure C-21-22. Drying Shrinkage of Cement D - M4

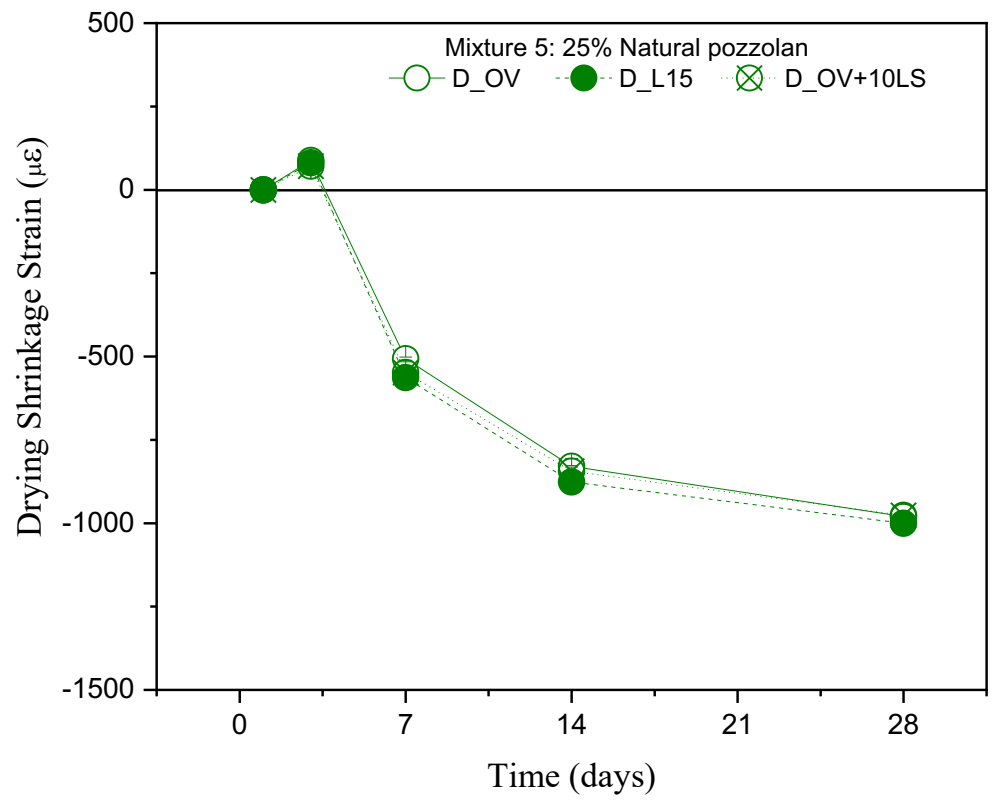




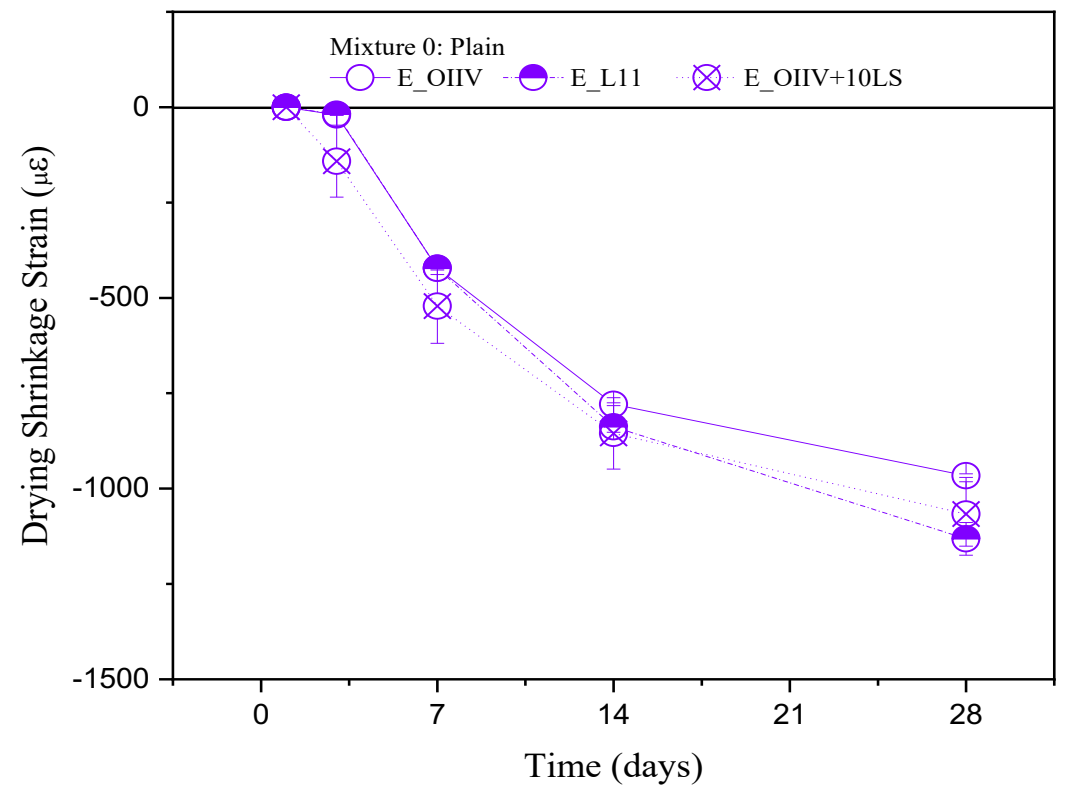

3779

3780

Figure C-21-24. Drying Shrinkage of Cement E - M0

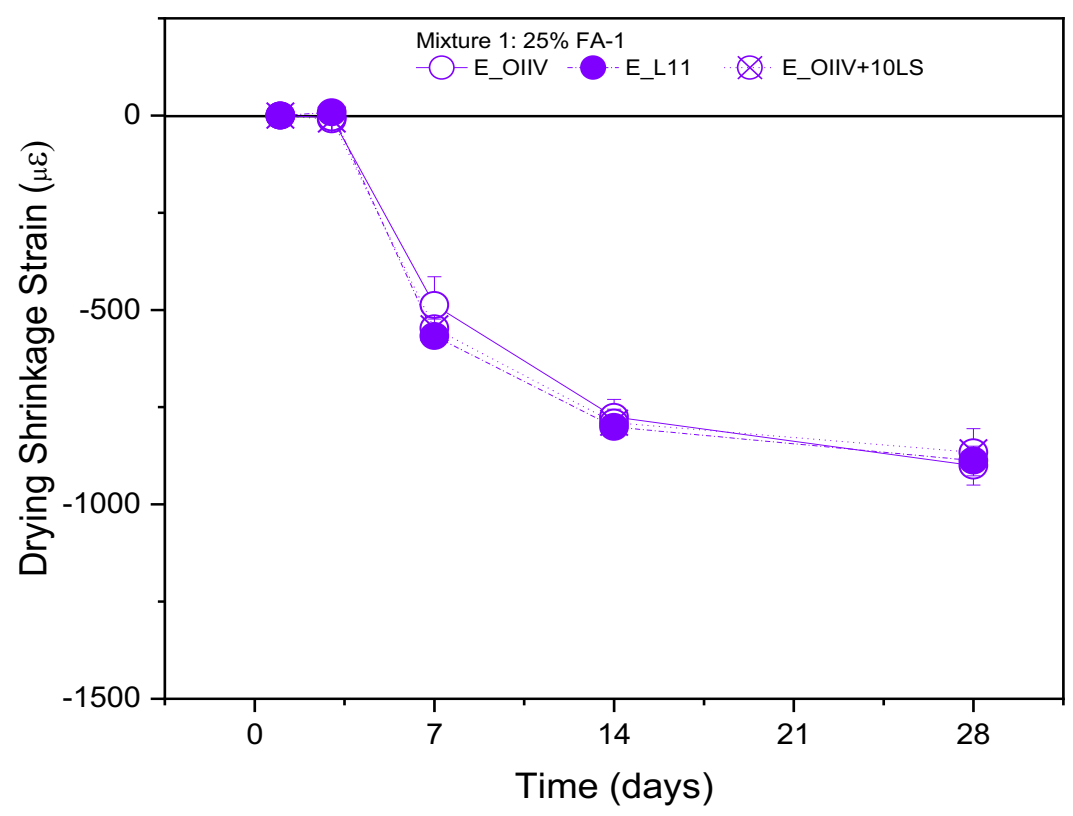

3781

3782

Figure C-21-25. Drying Shrinkage of Cement E - M1 


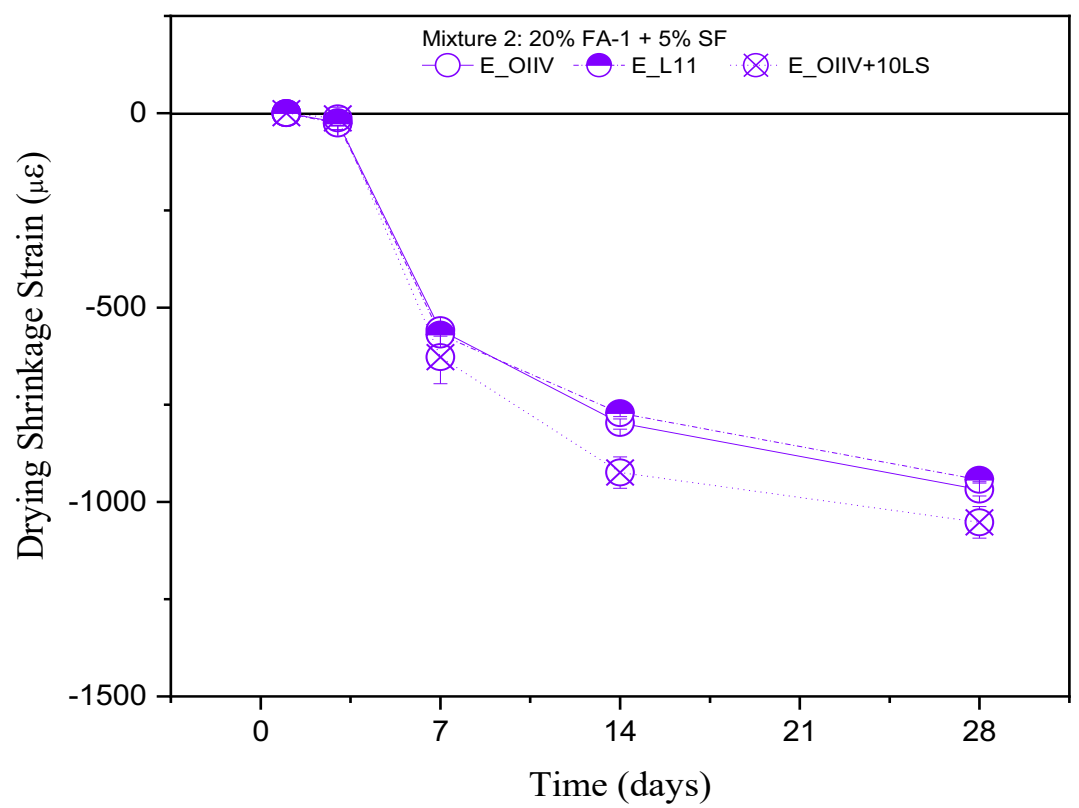

3783

3784

Figure C-21-26. Drying Shrinkage of Cement E - M2

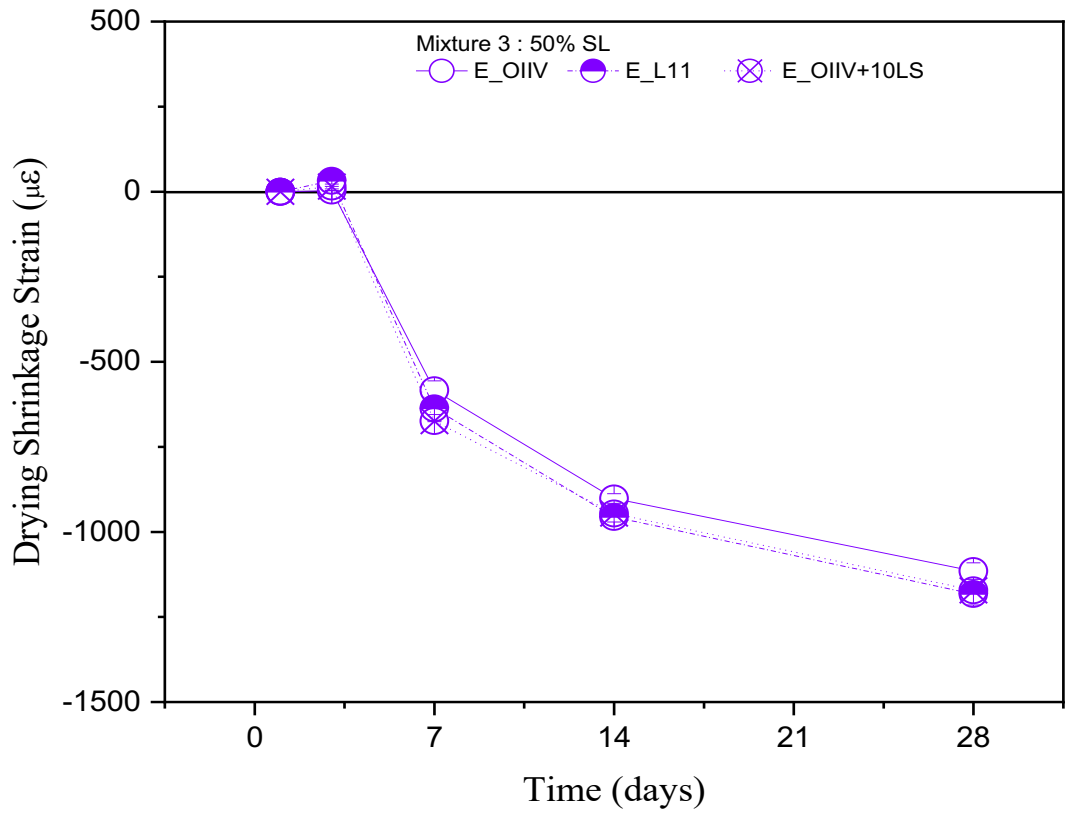

3785

Figure C-21-27. Drying Shrinkage of Cement E - M3 


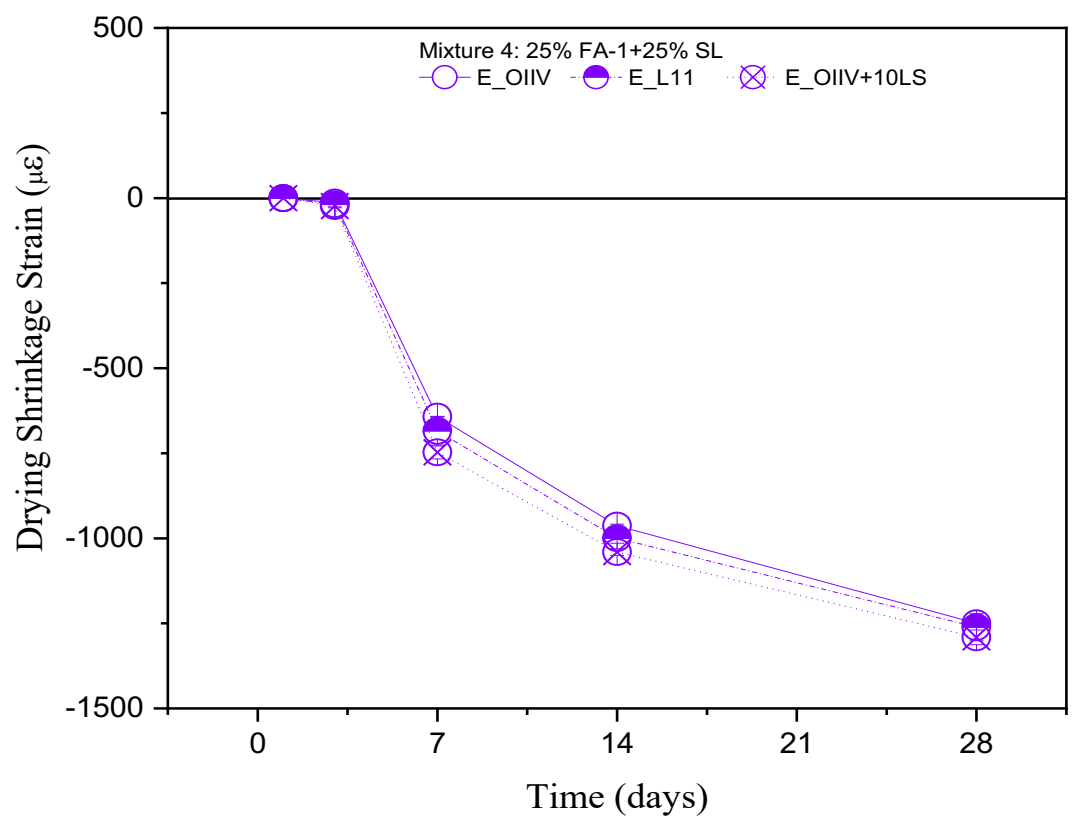

3787

3788

Figure C-21-28. Drying Shrinkage of Cement E - M4 


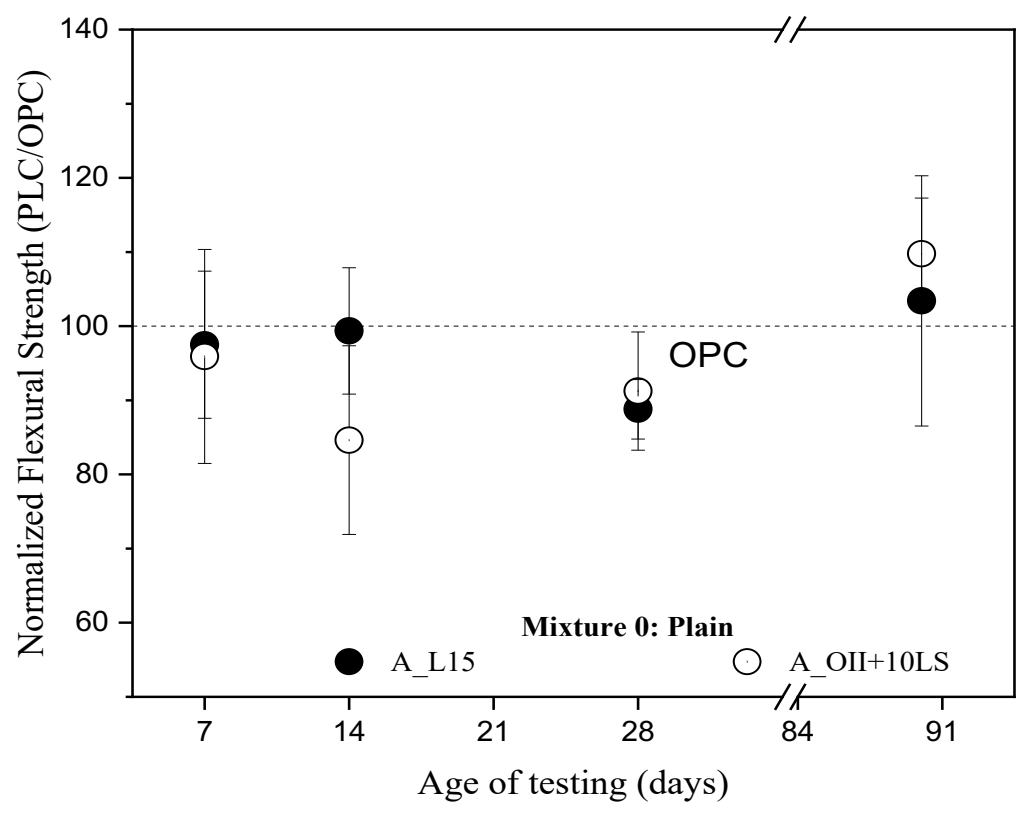

3790

3791

Figure D-22-1. Flexural Strength of Cement A - Mo

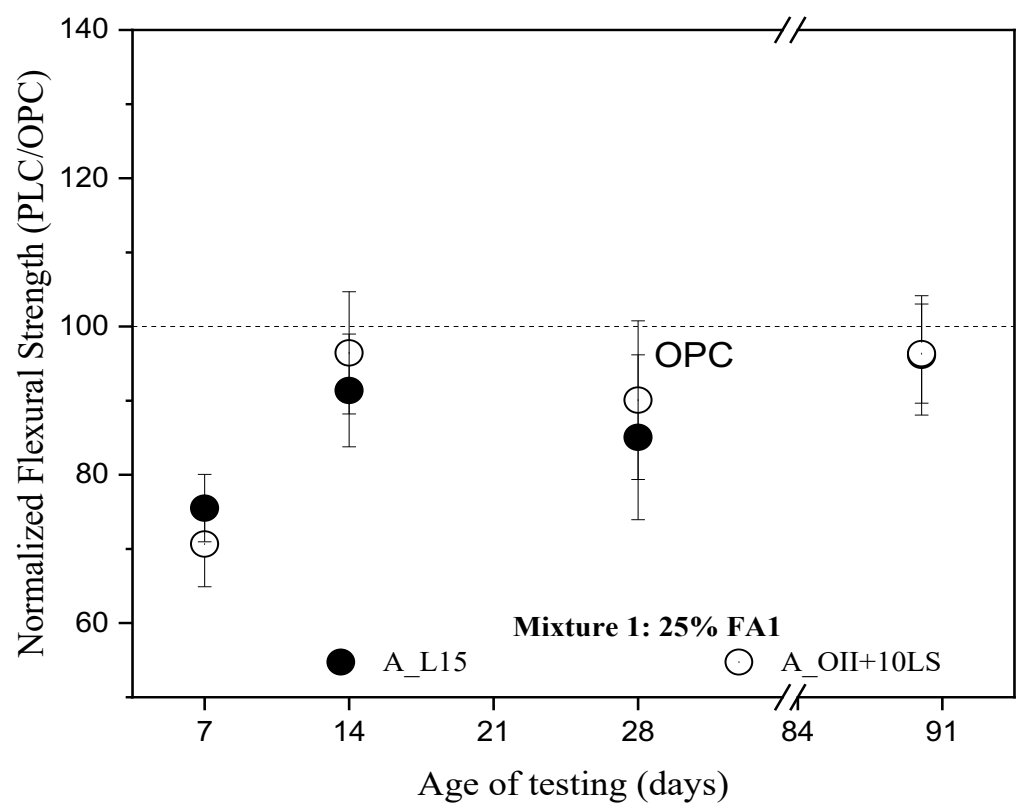




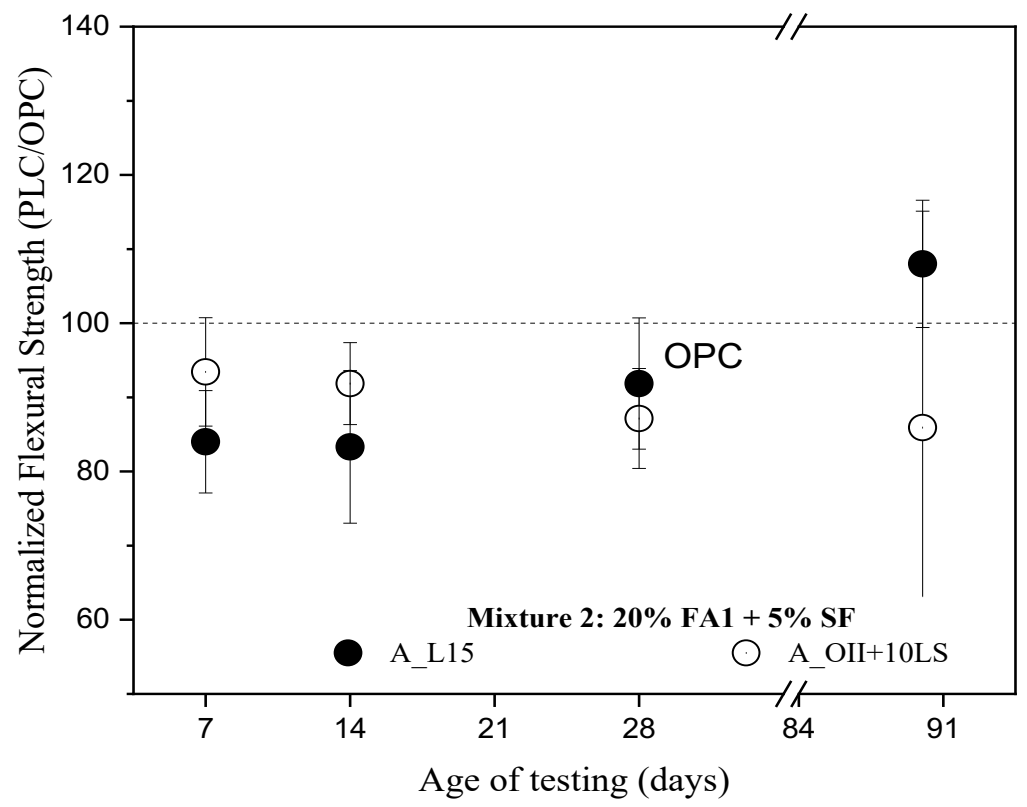

3794

3795

Figure D-22-3. Flexural Strength of Cement A - M2

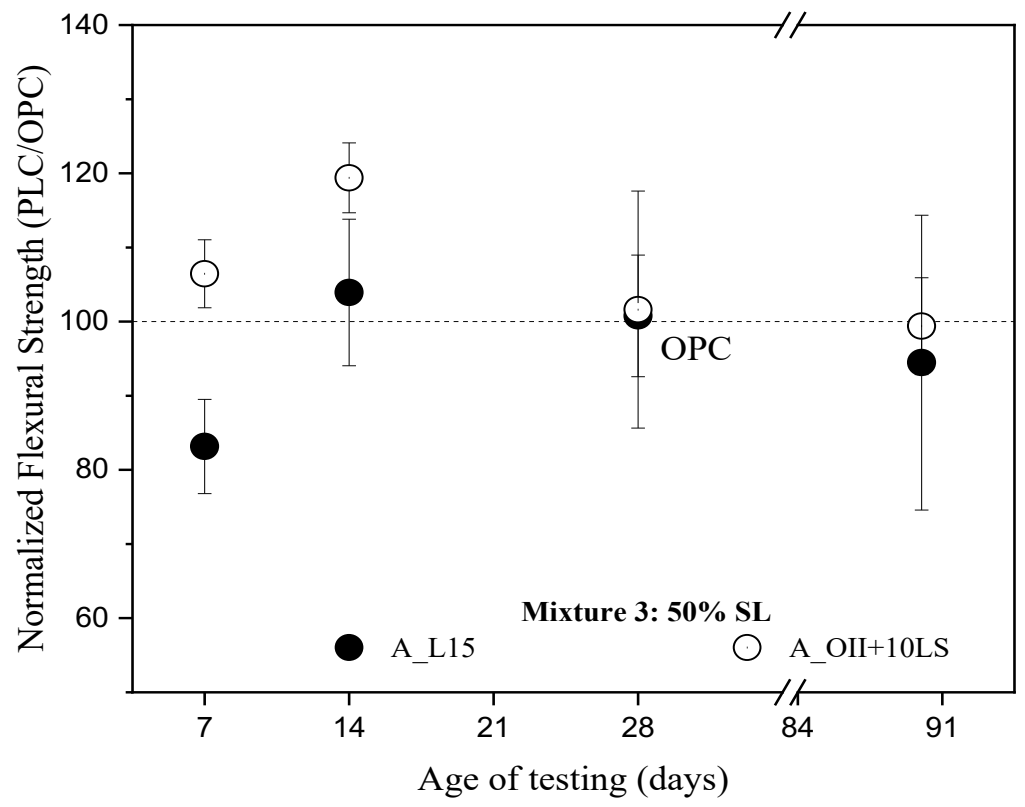




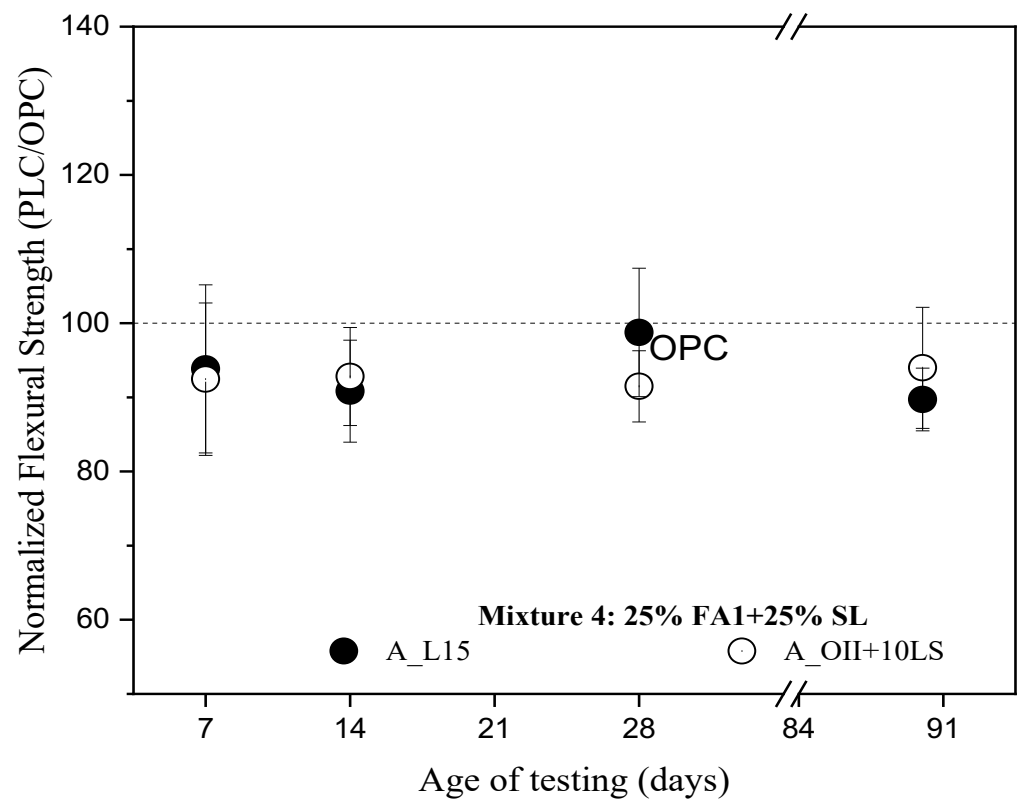

3798

3799

Figure D-22-5. Flexural Strength of Cement A - M4

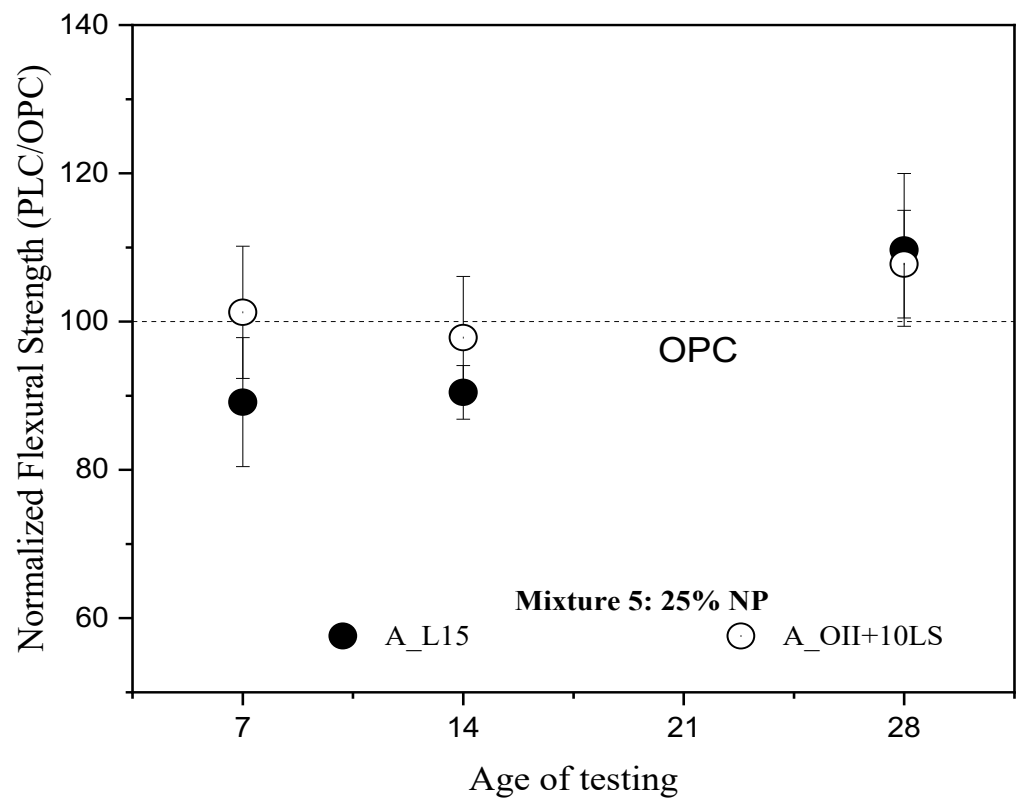

3800

Figure D-22-6. Flexural Strength of Cement A - M5 


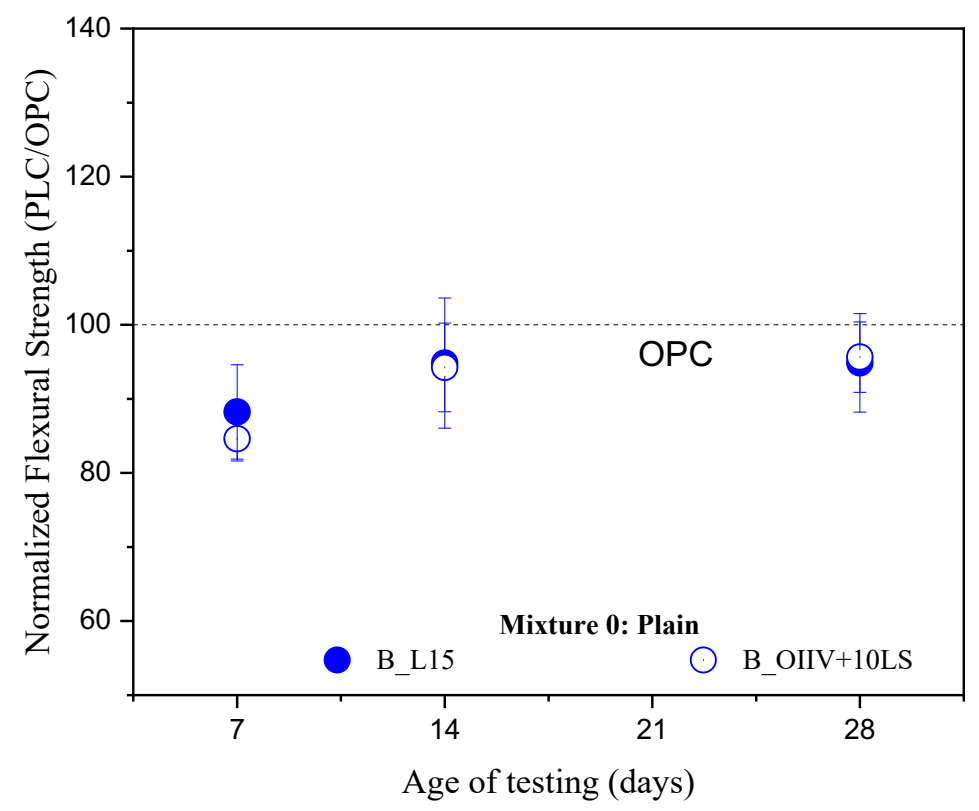

3802

Figure D-22-7. Flexural Strength of Cement B - M0

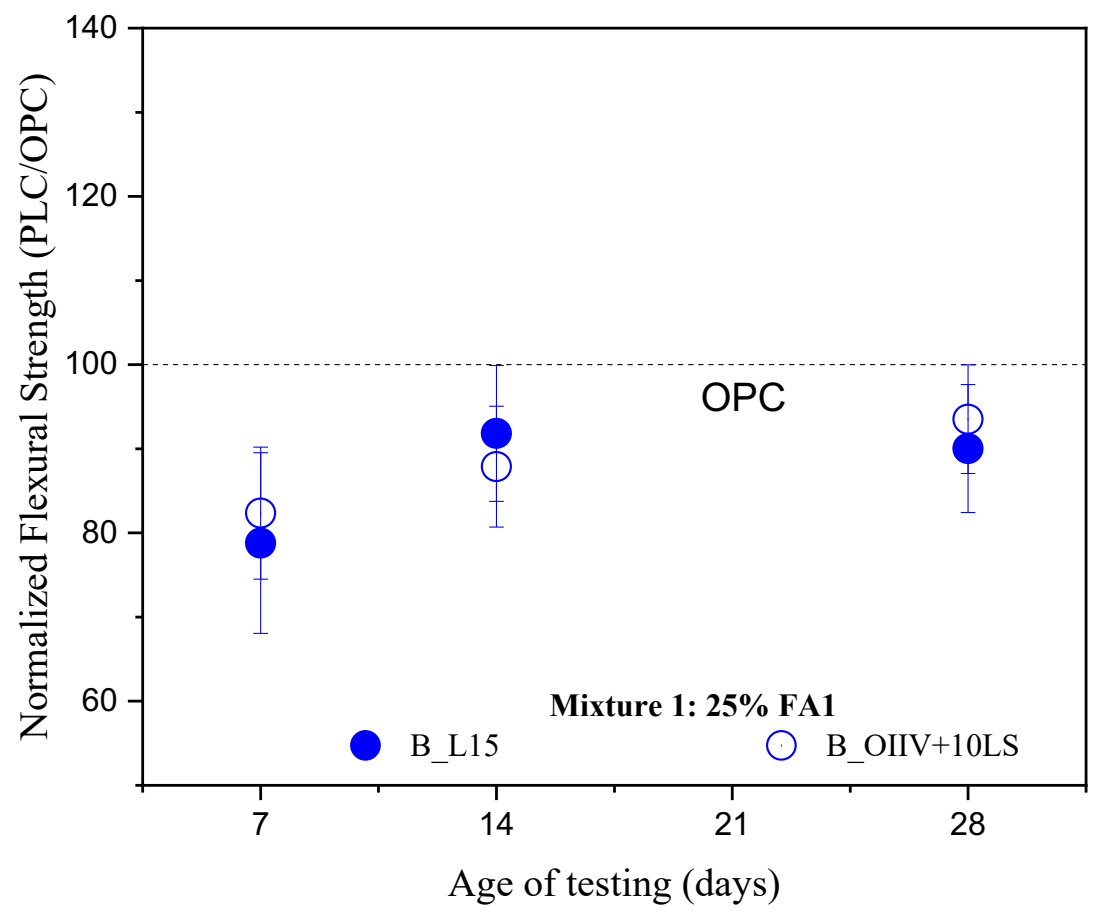

Figure D-22-8. Flexural Strength of Cement B - M1 


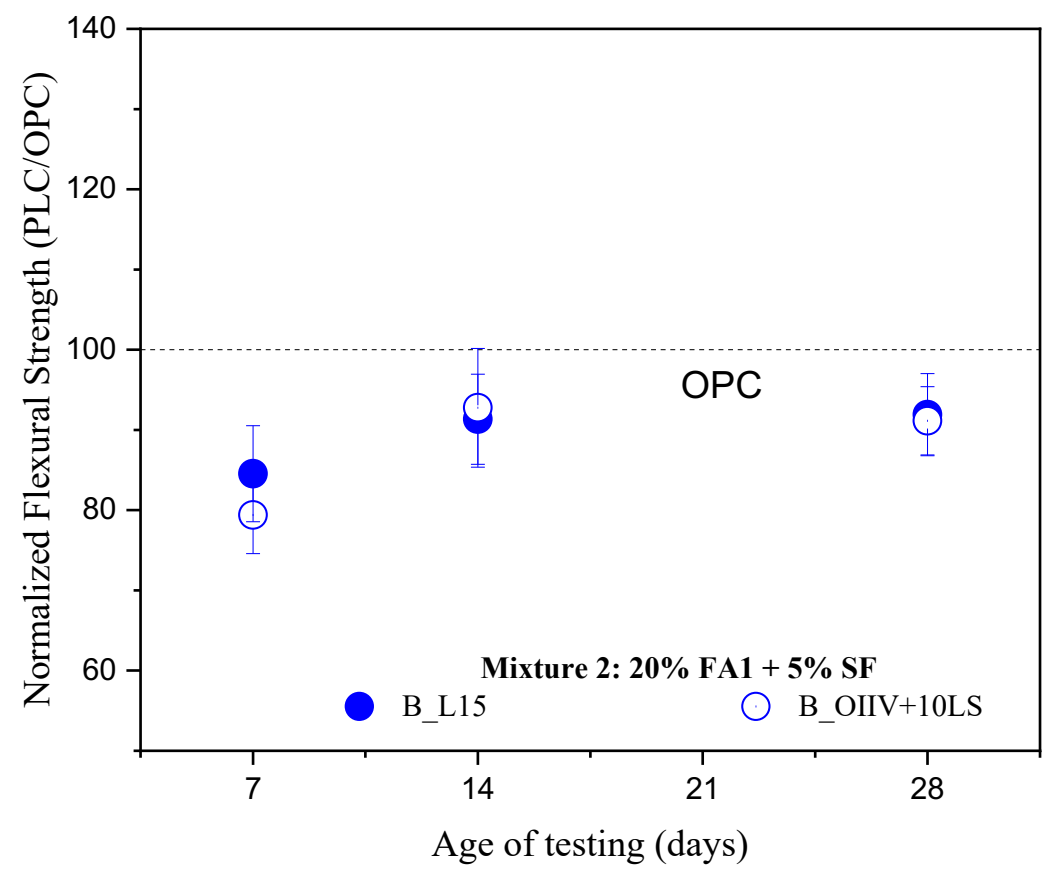

3806

Figure D-22-9. Flexural Strength of Cement B - M2

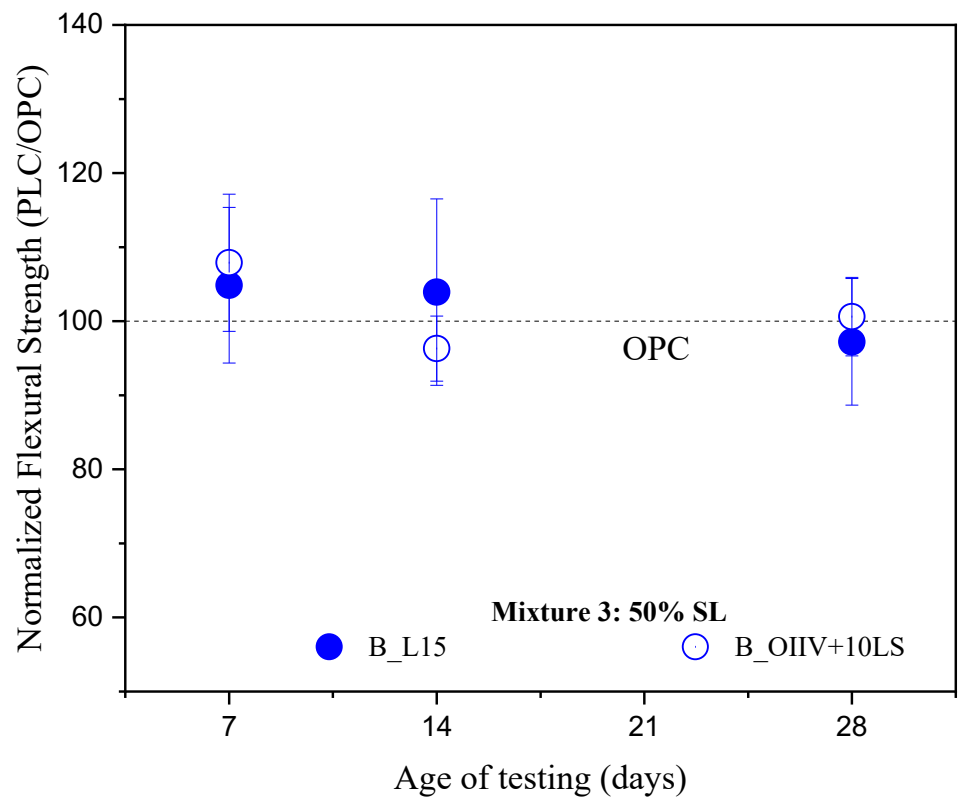




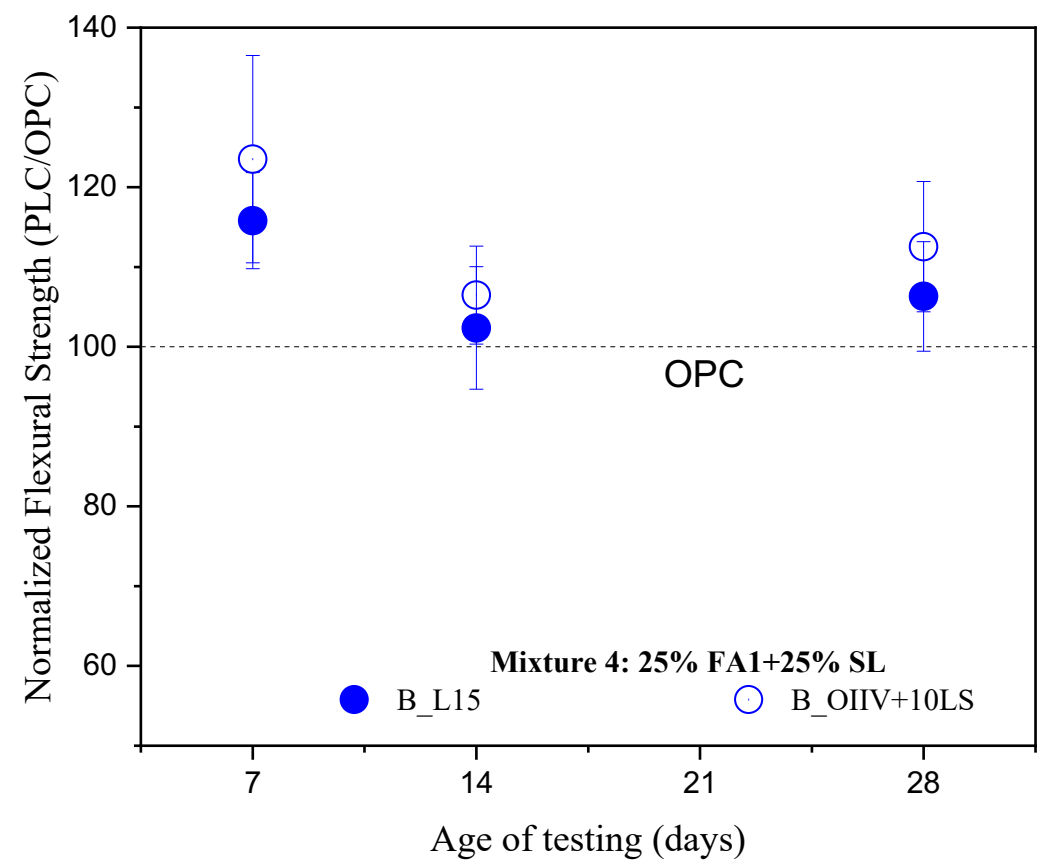

3810

3811

Figure D-22-11. Flexural Strength of Cement B - M4

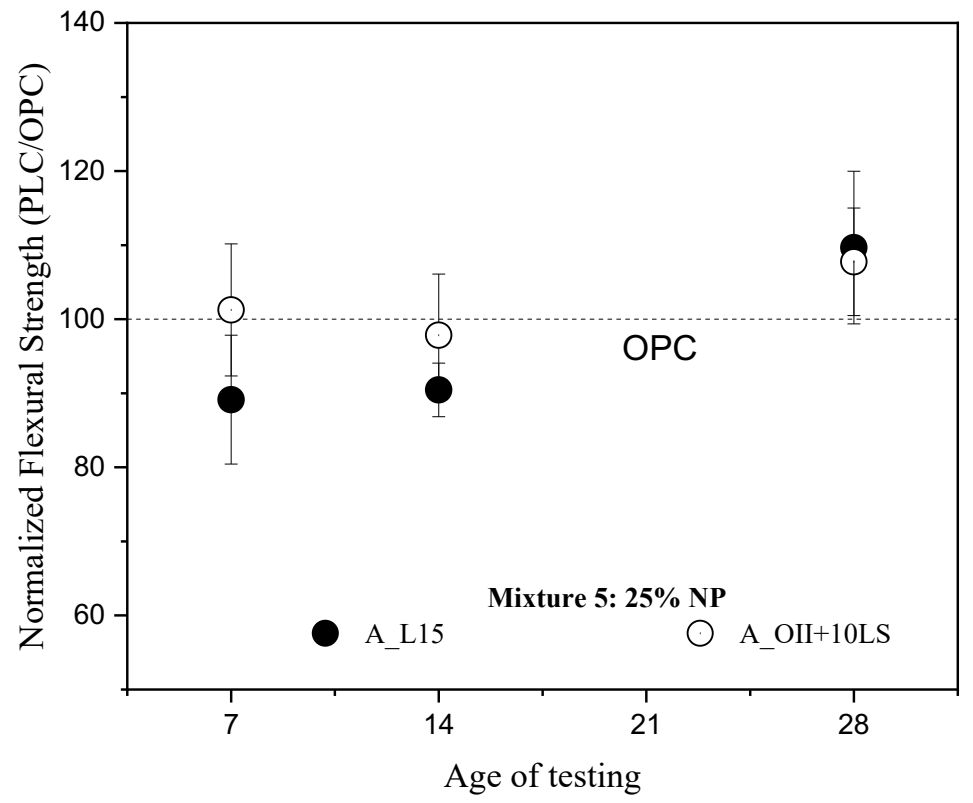

3812

3813

Figure D-22-12. Flexural Strength of Cement B - M5 


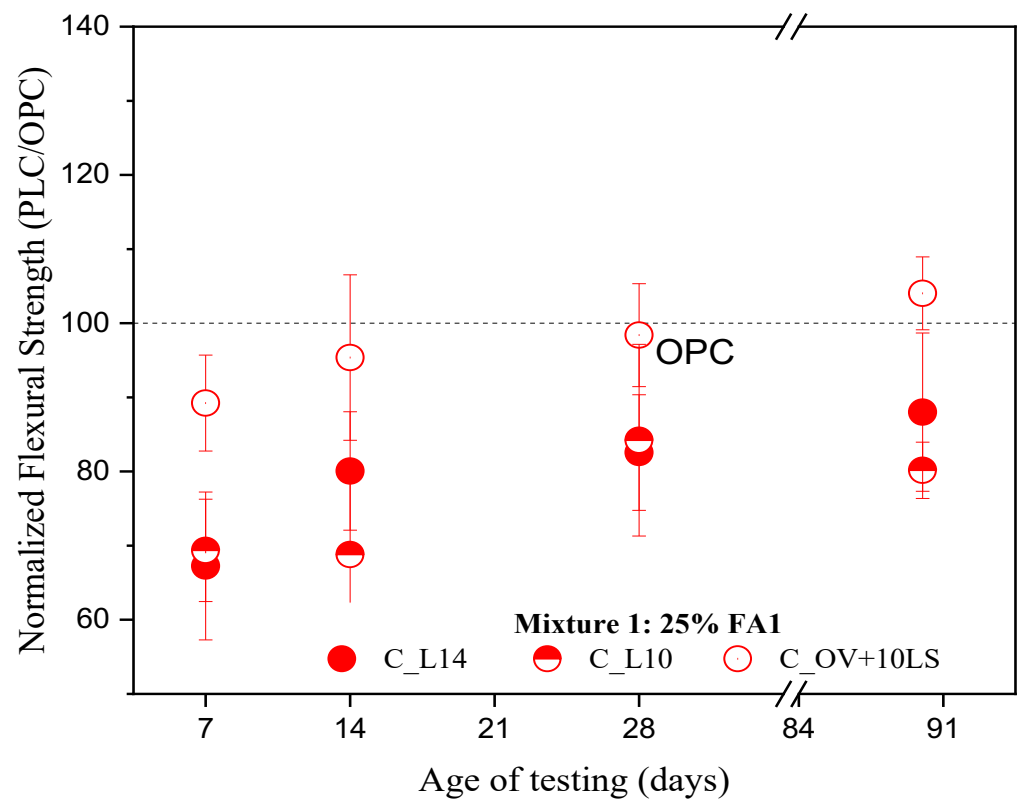

3814

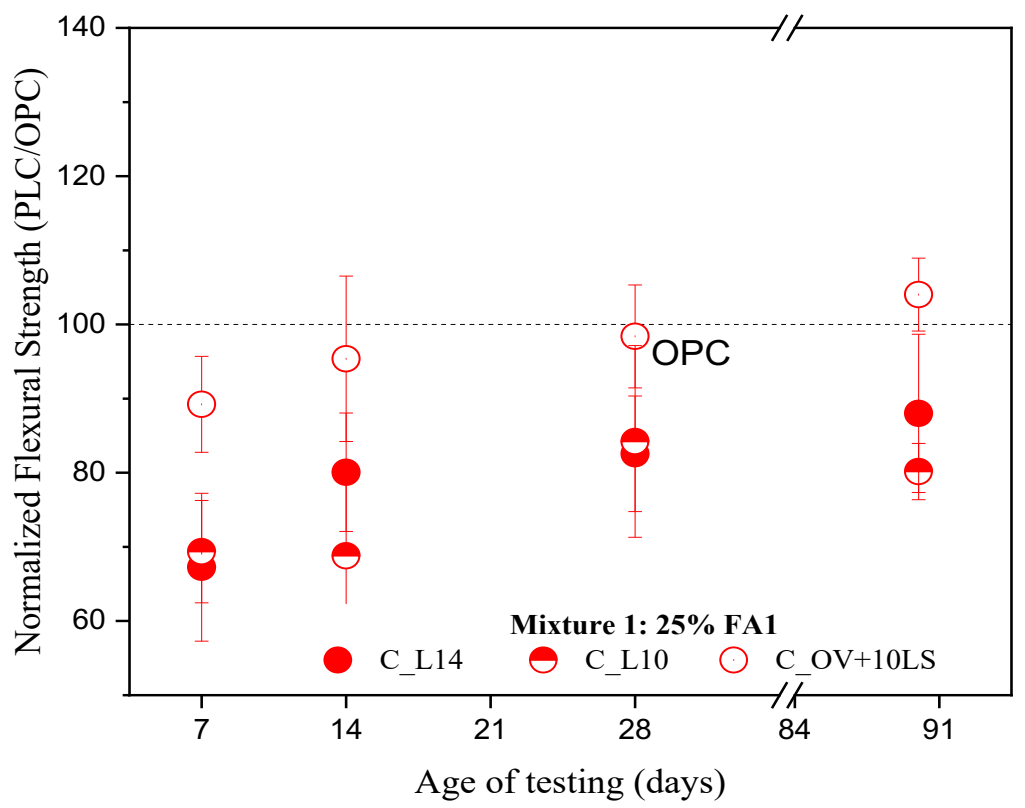




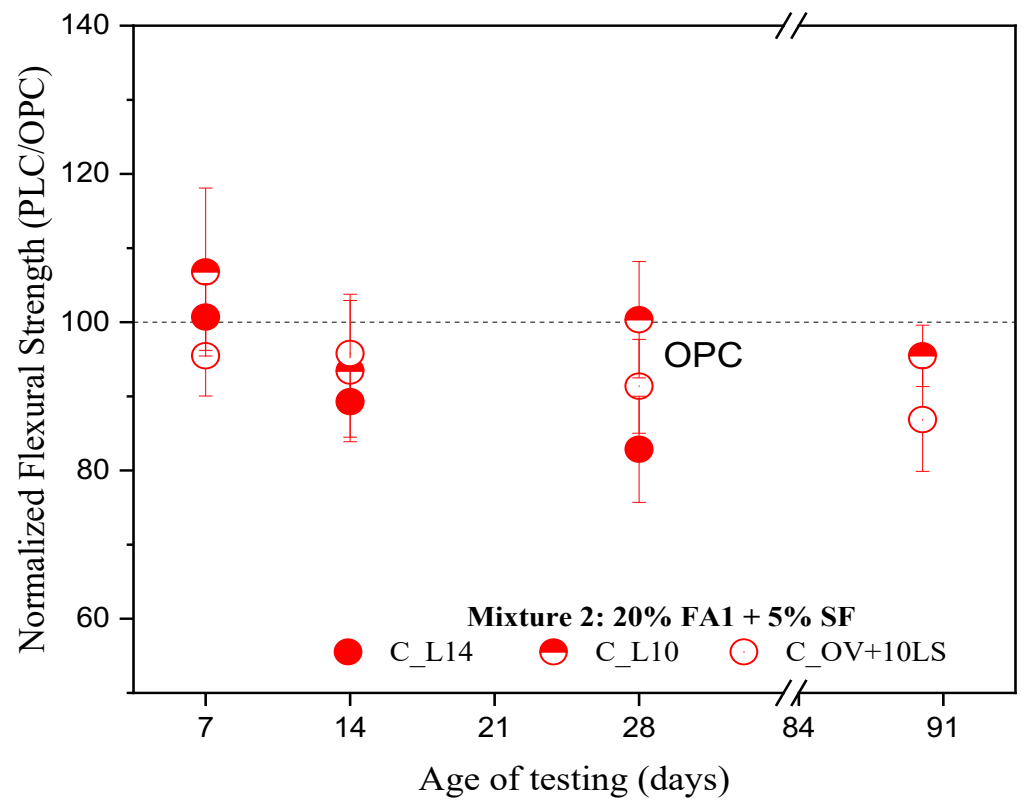

3818

Figure D-22-15. Flexural Strength of Cement C - M2

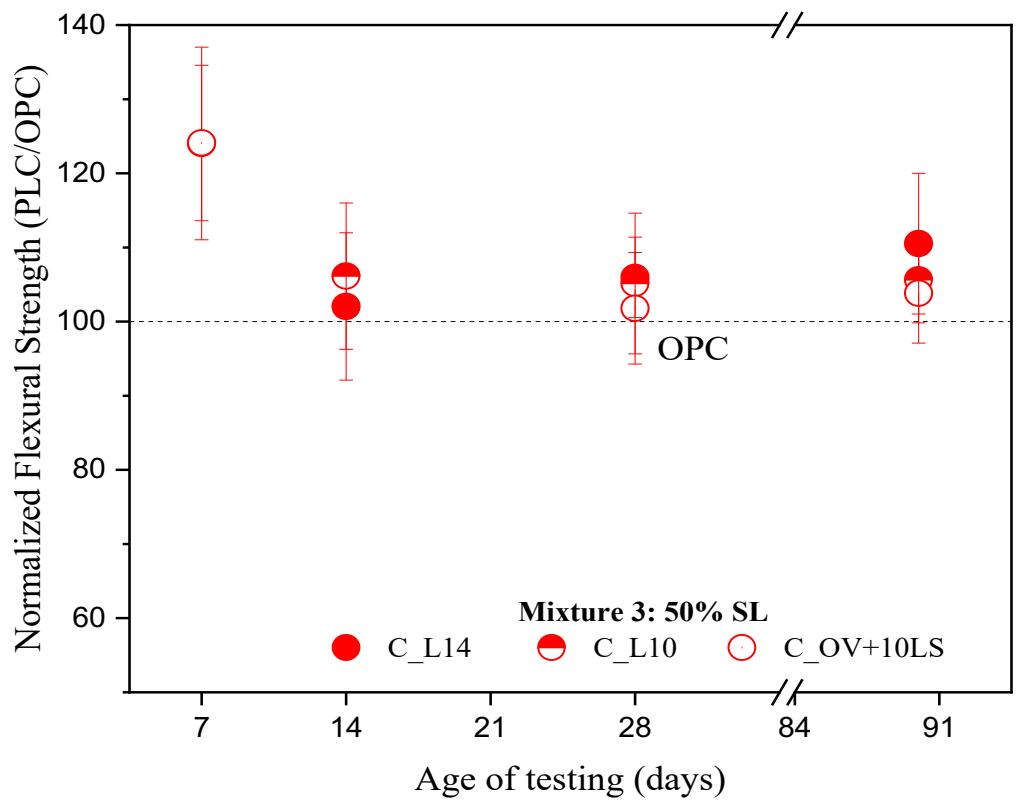




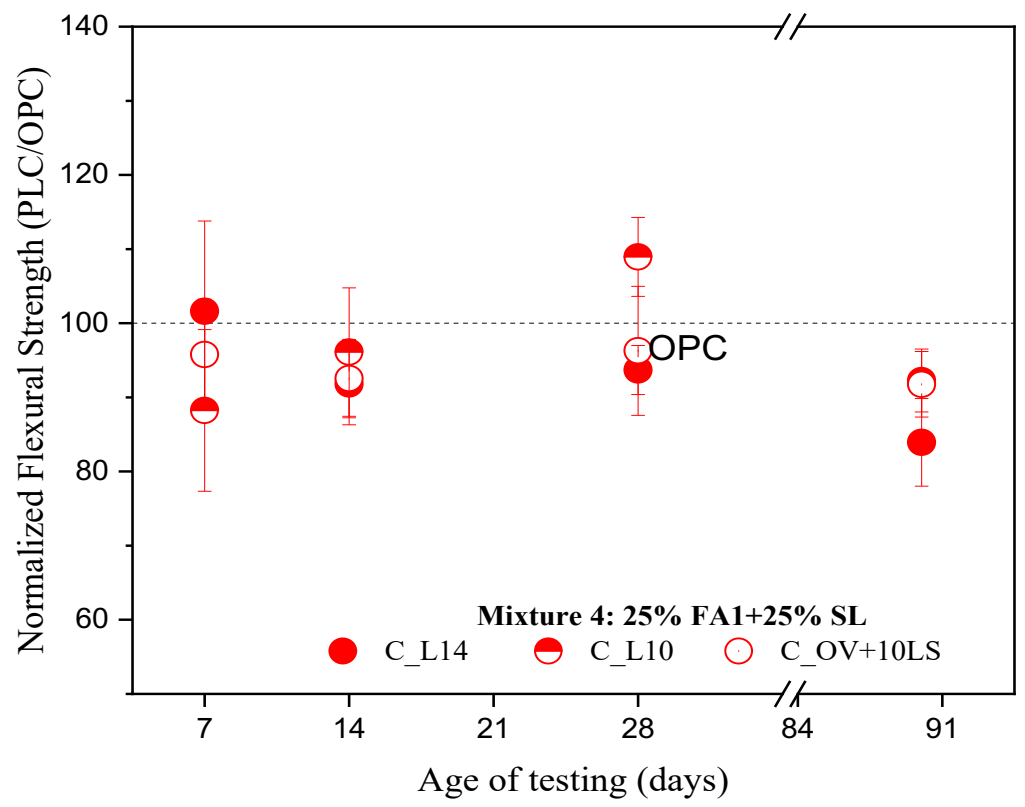

3822

Figure D-22-17. Flexural Strength of Cement C - M4

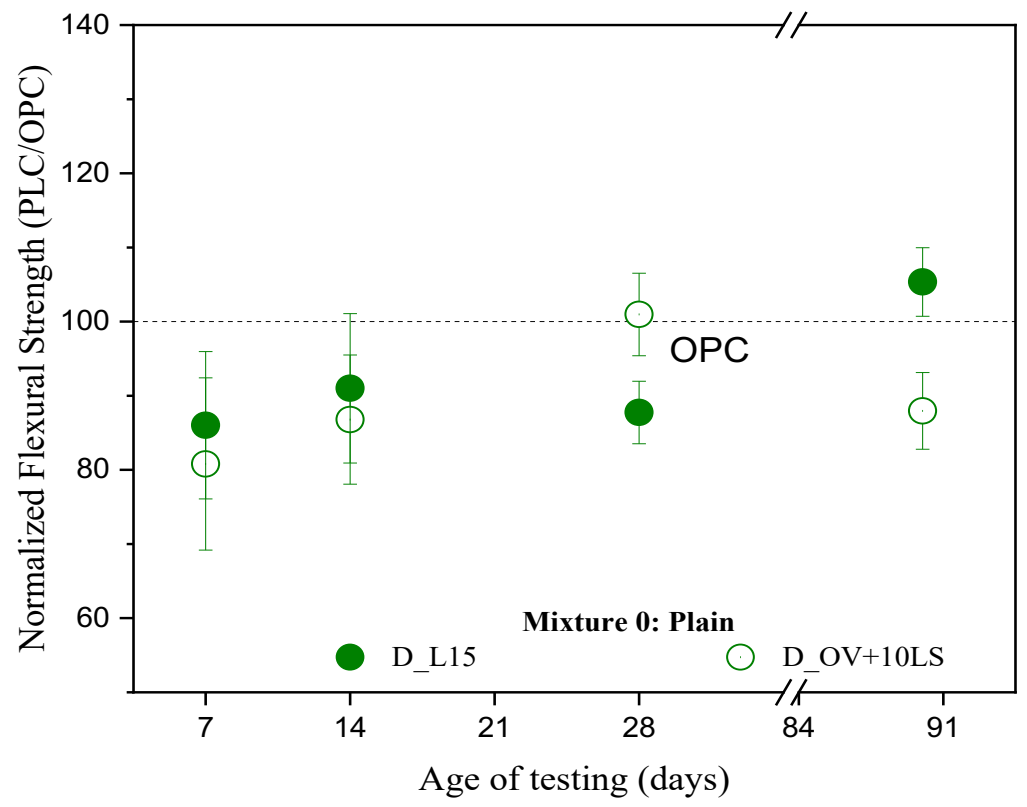




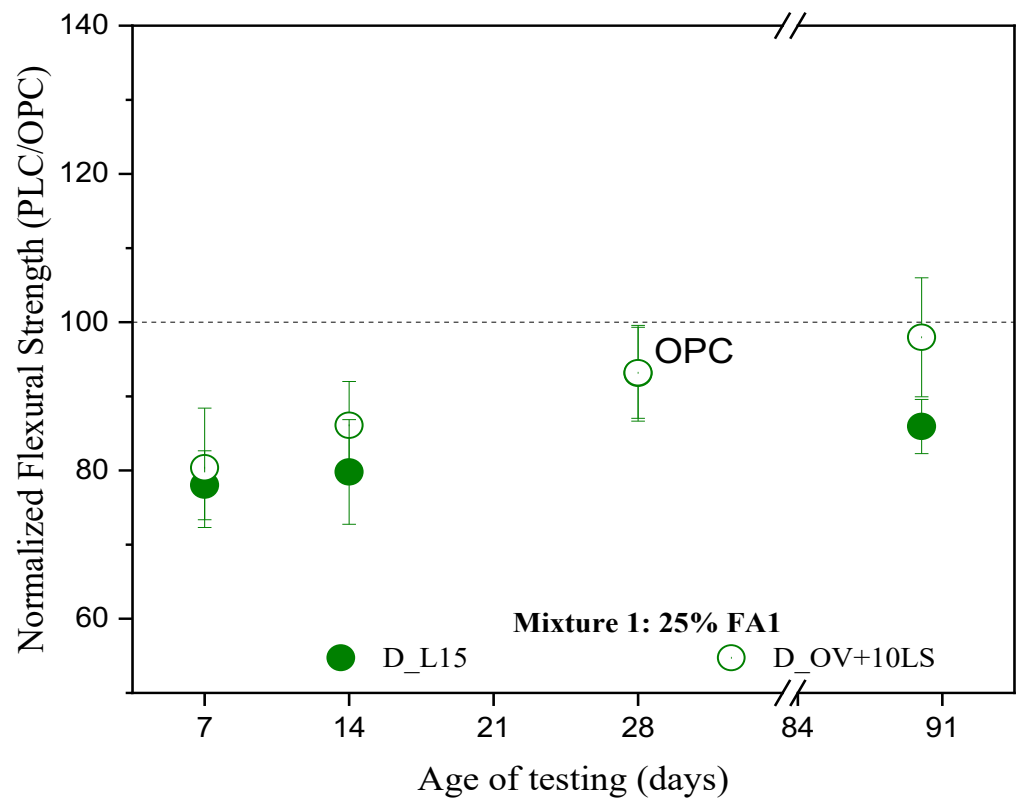

3826

Figure D-22-19. Flexural Strength of Cement D - M1

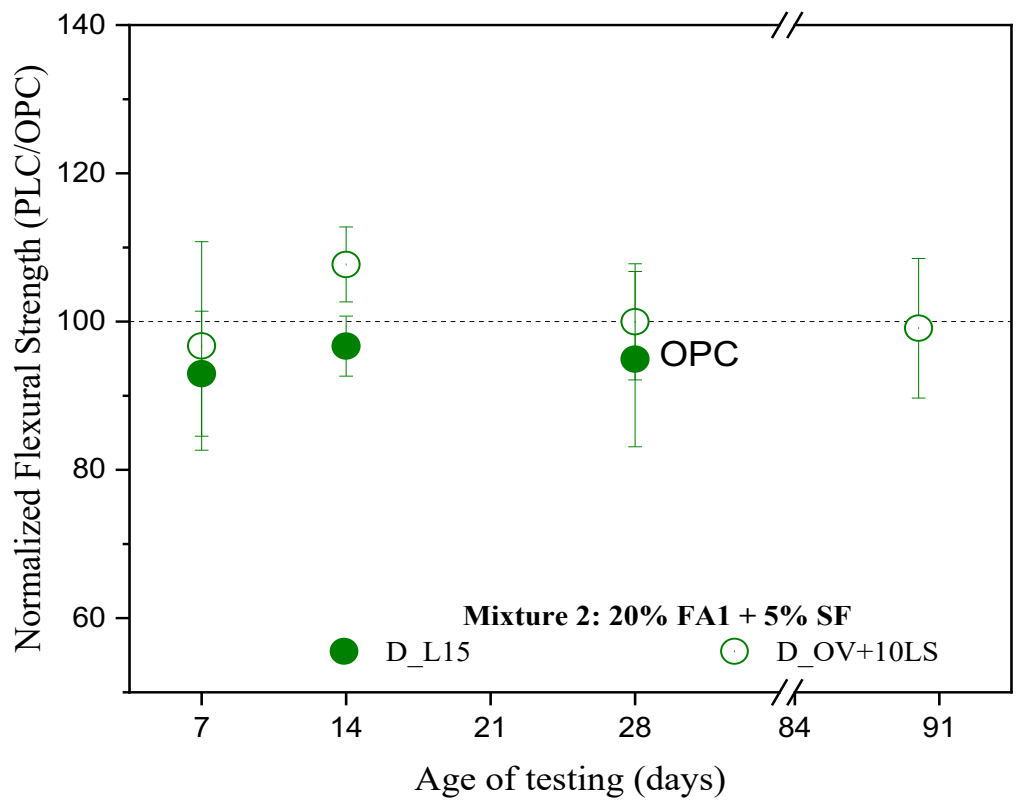




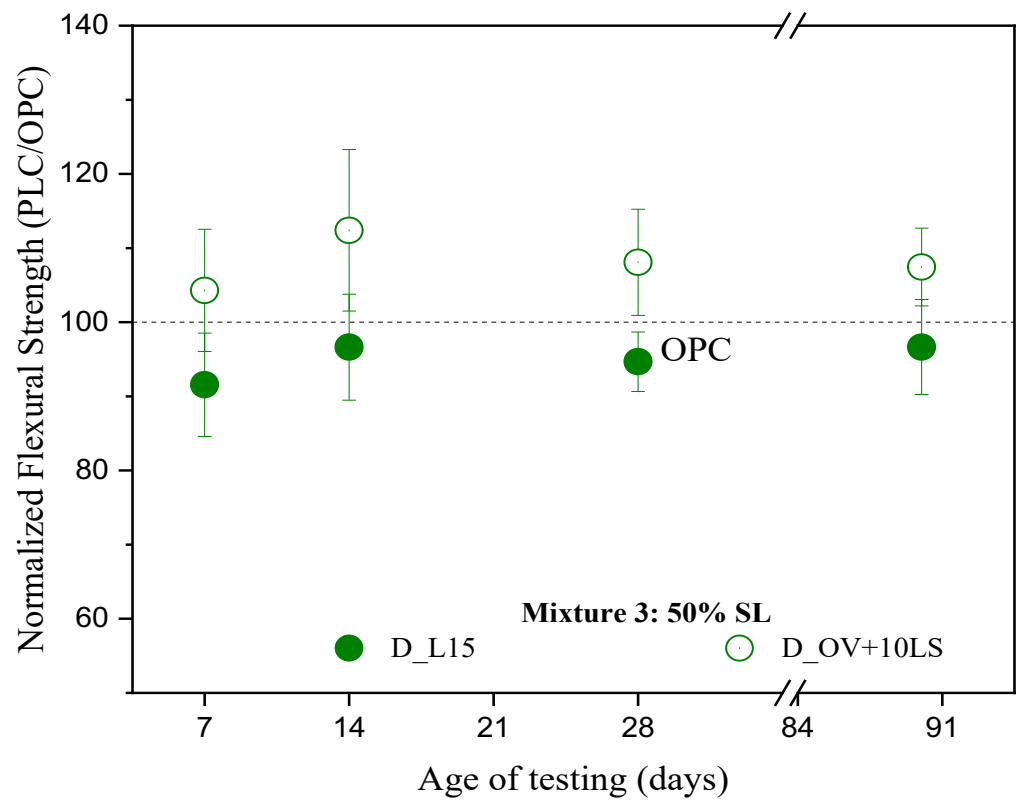

3830

Figure D-22-21. Flexural Strength of Cement D - M3

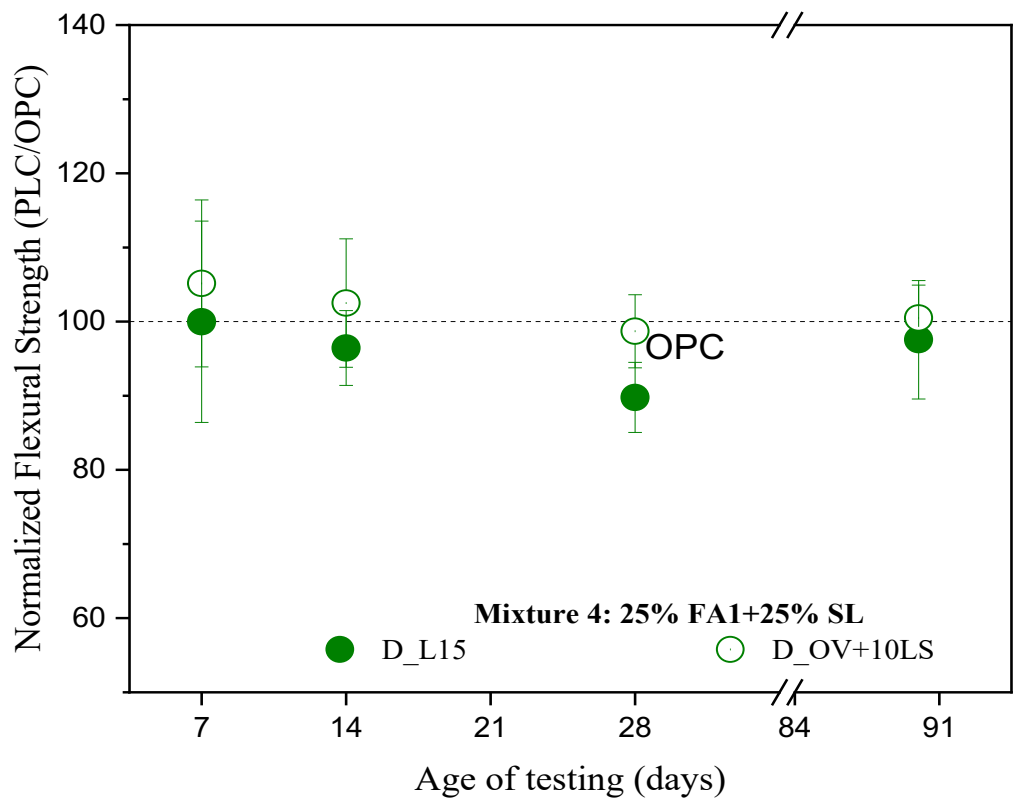




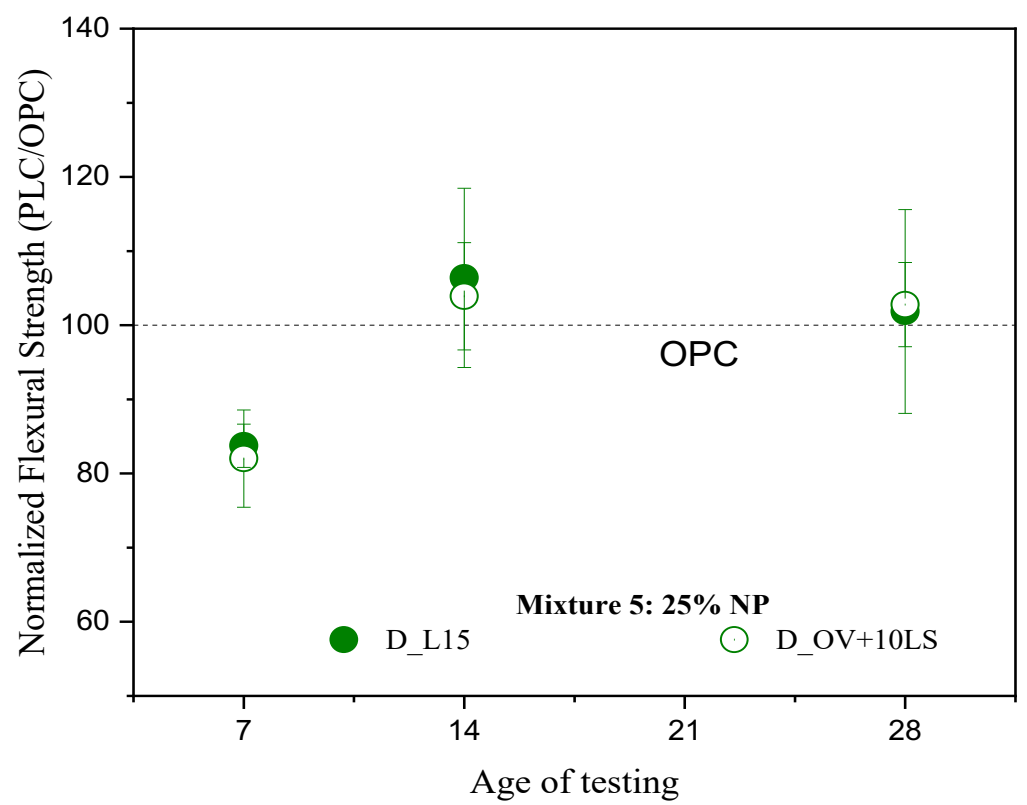

3834

Figure D-22-23. Flexural Strength of Cement D - M5

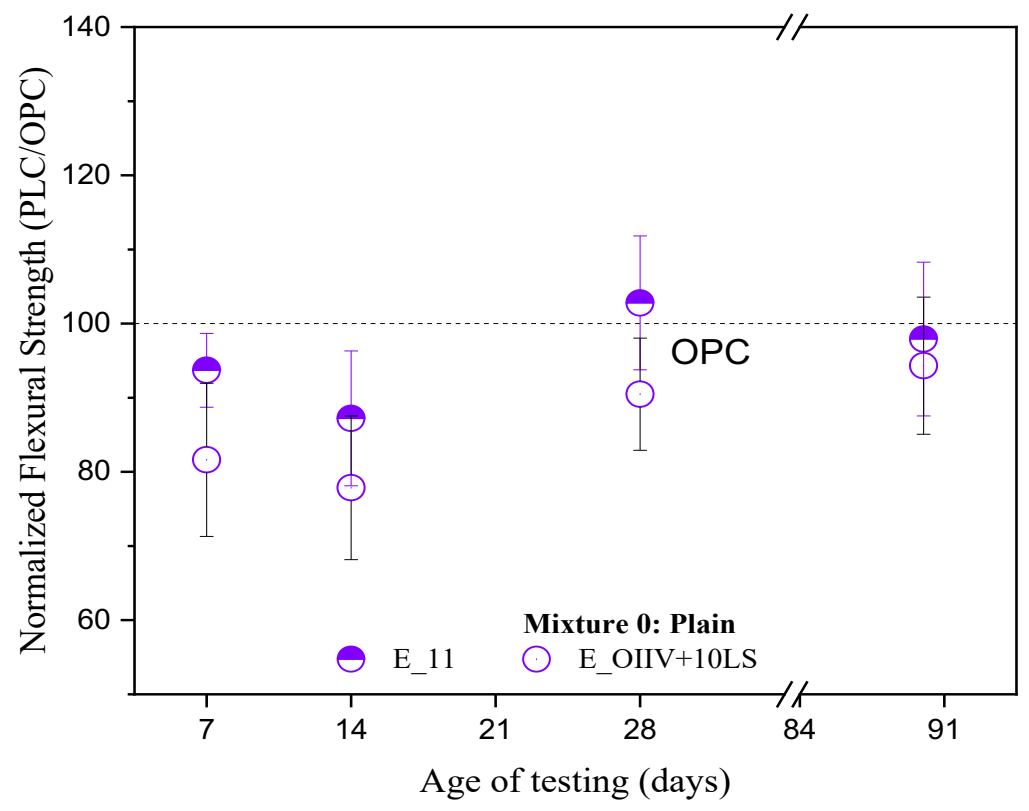




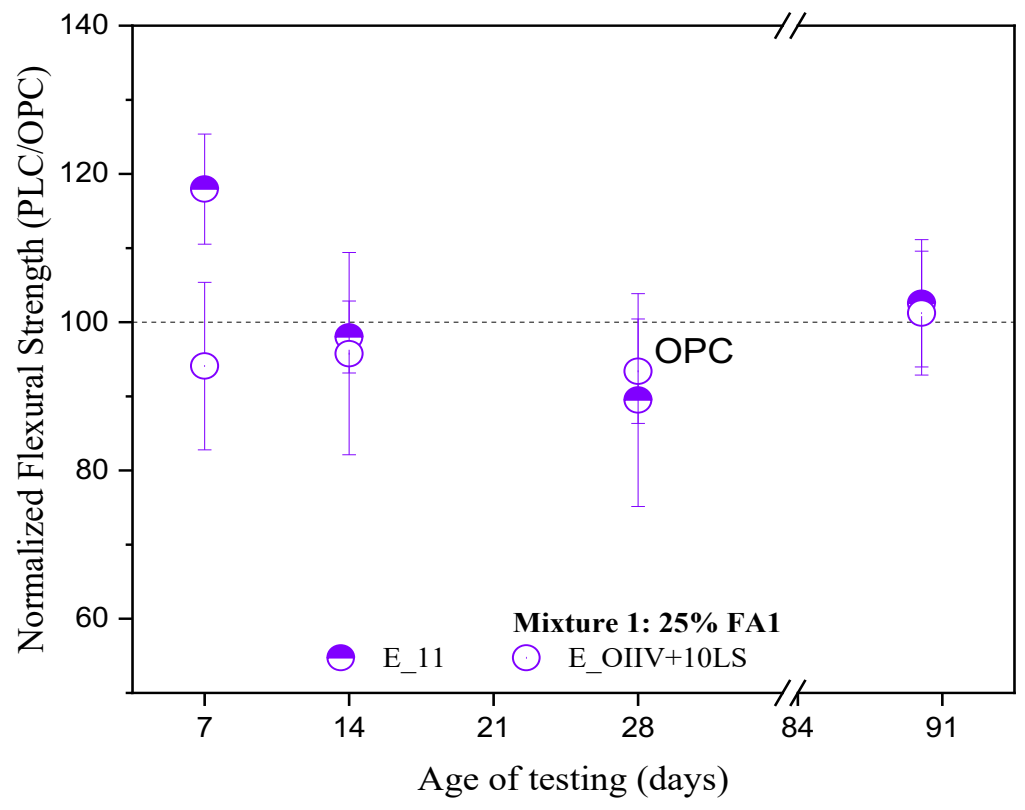

3838

3839

Figure D-22-25. Flexural Strength of Cement E - M1

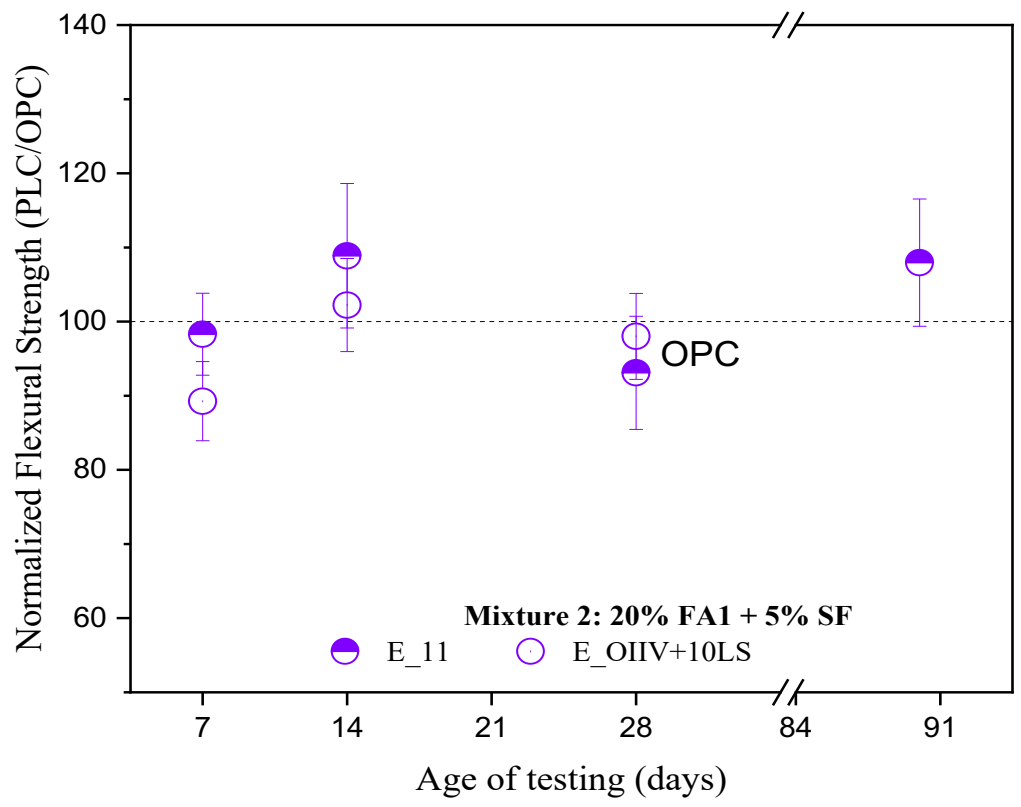

3840 


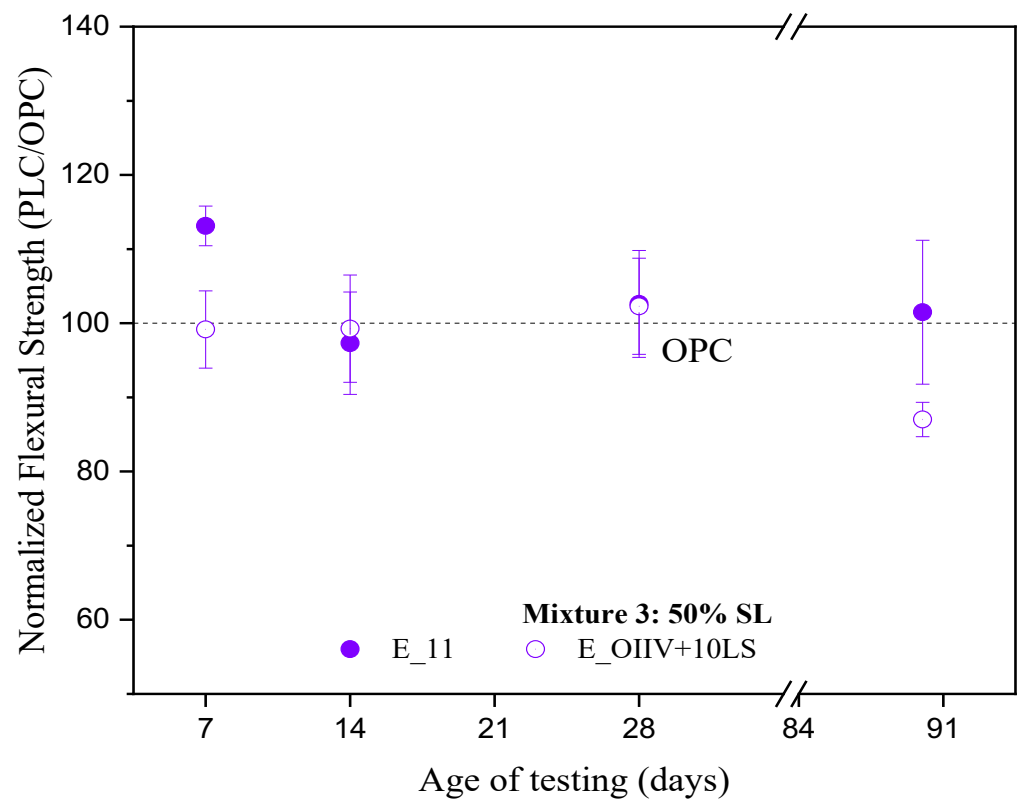

3842

3843

Figure D-22-27. Flexural Strength of Cement E - M3

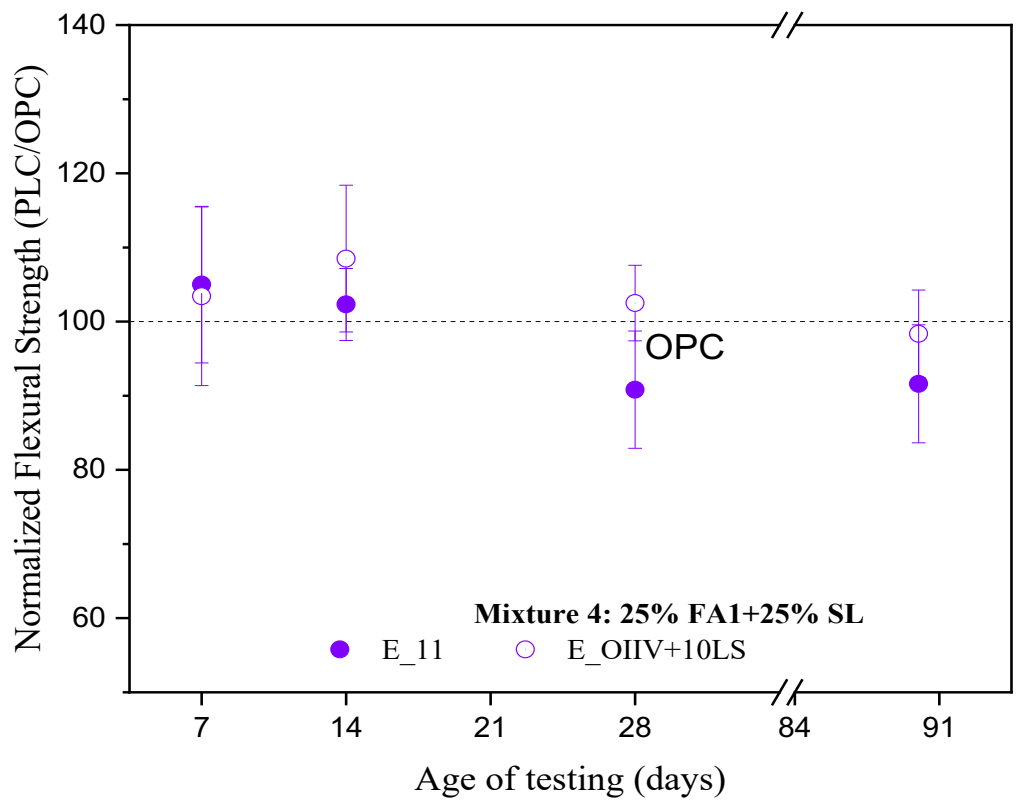

3844 
384723 APPENDIX E - Transport Properties Results

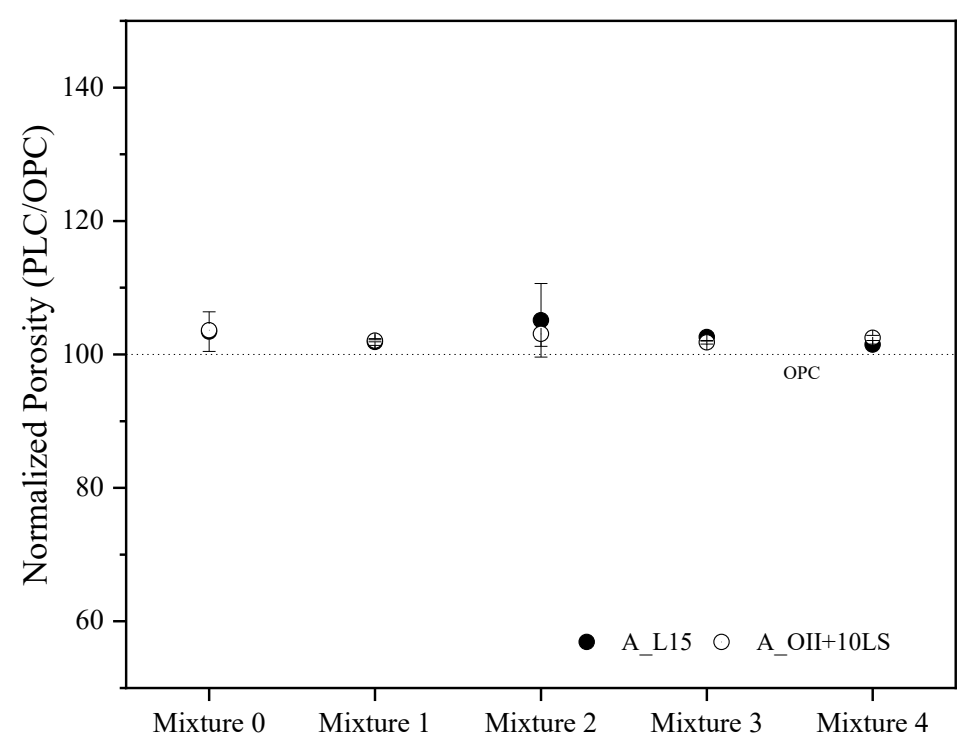

3848

Figure E-23-1. Porosity of Cement A

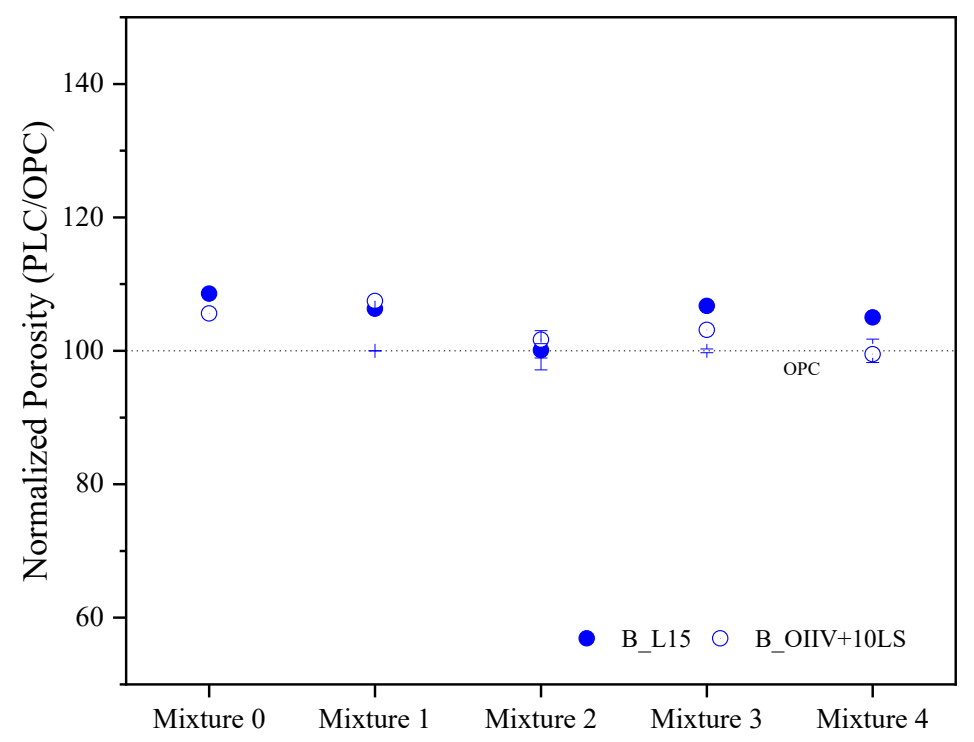




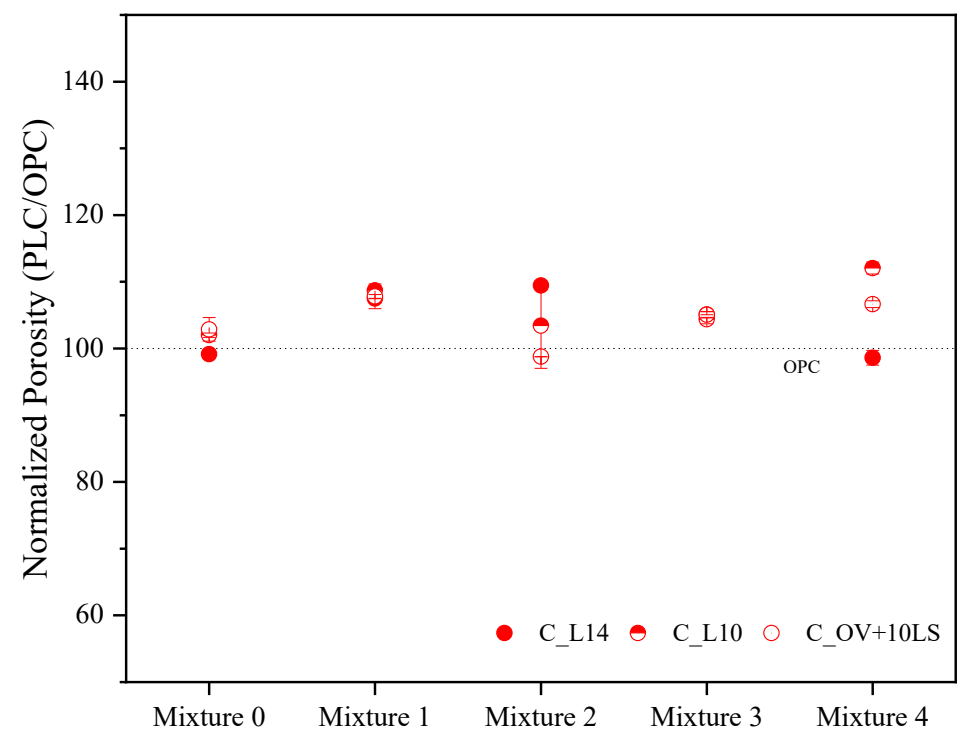

3852

Figure E-23-3. Porosity of Cement C

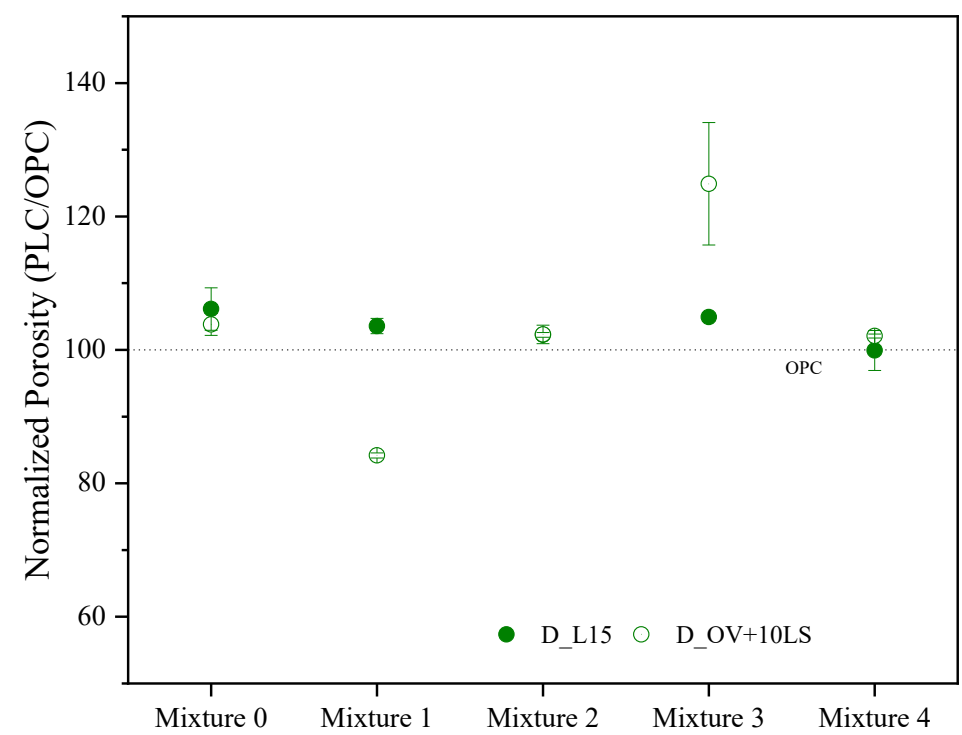



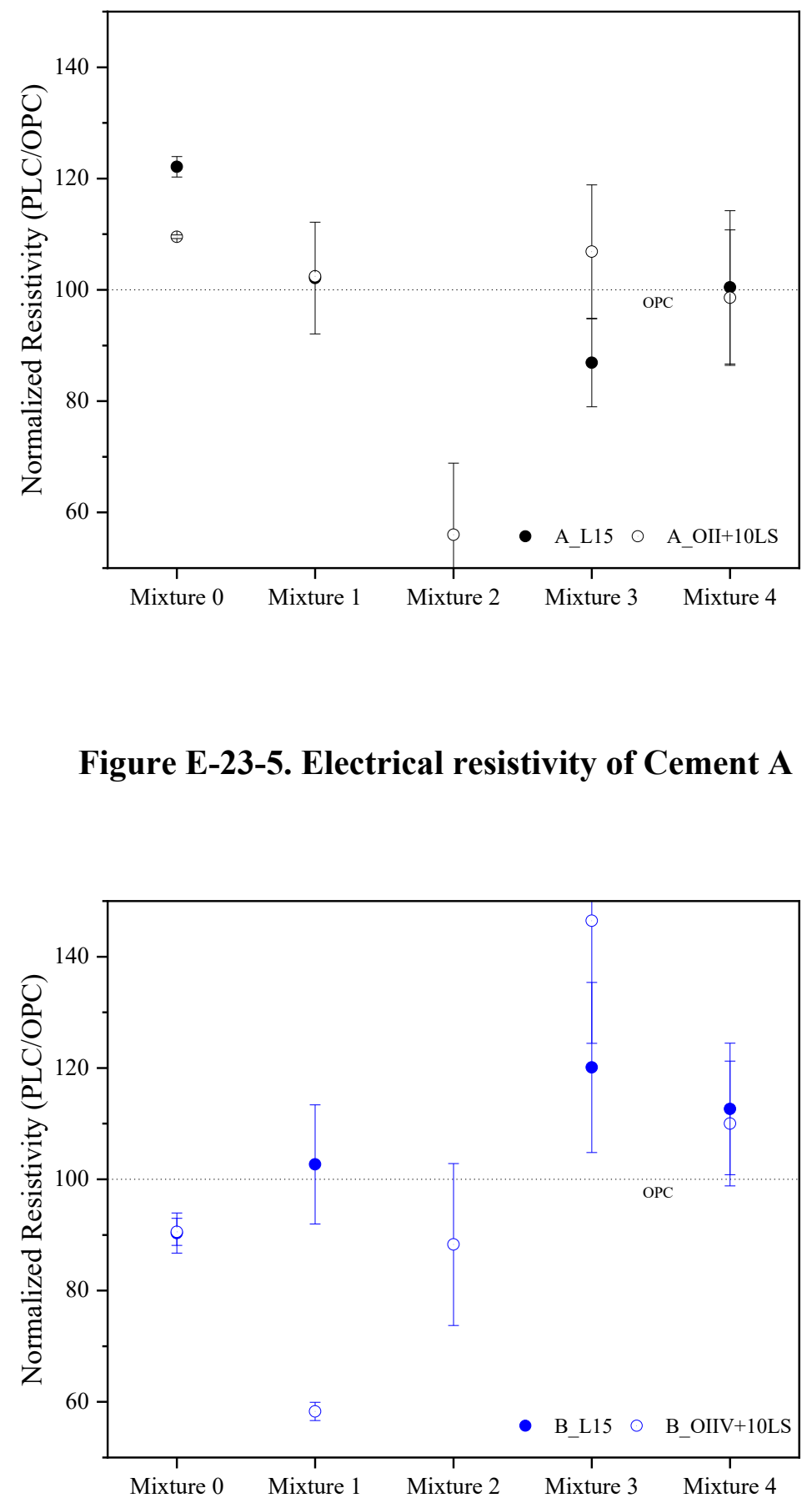


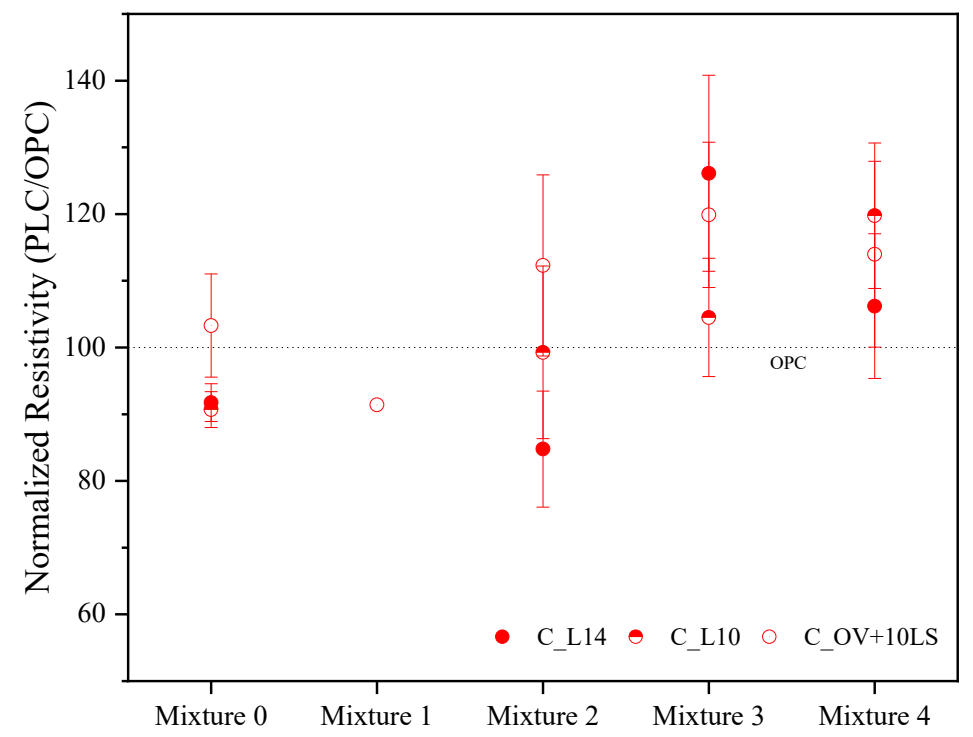

3862

3863

Figure E-23-7. Electrical resistivity of Cement C

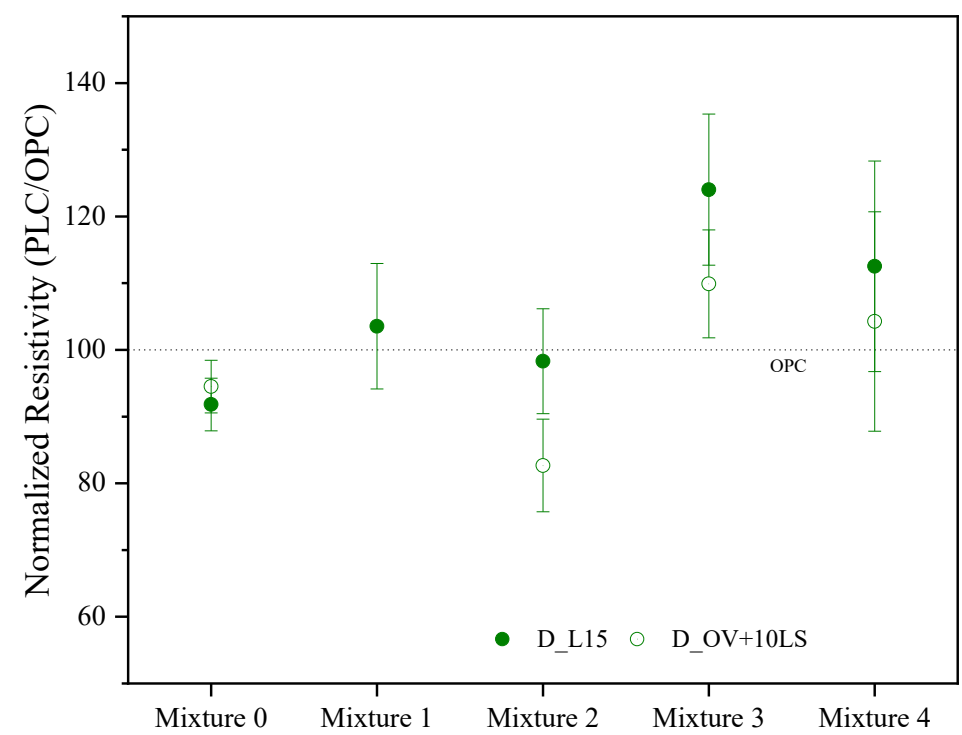



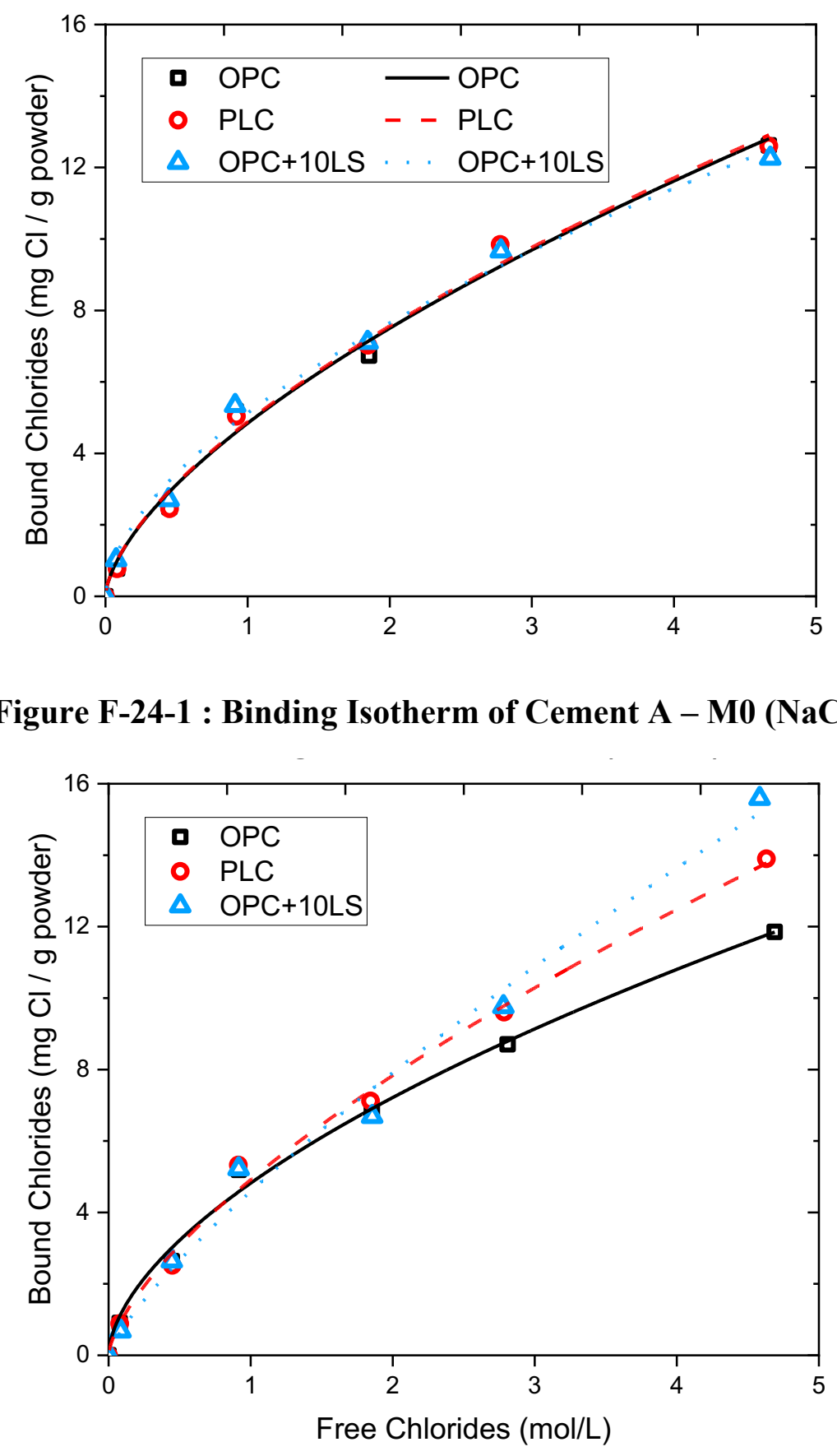

Figure F-24-2. Binding Isotherm of Cement $\mathrm{A}-\mathrm{M1}$ (NaCl) 
3873

3874

3875

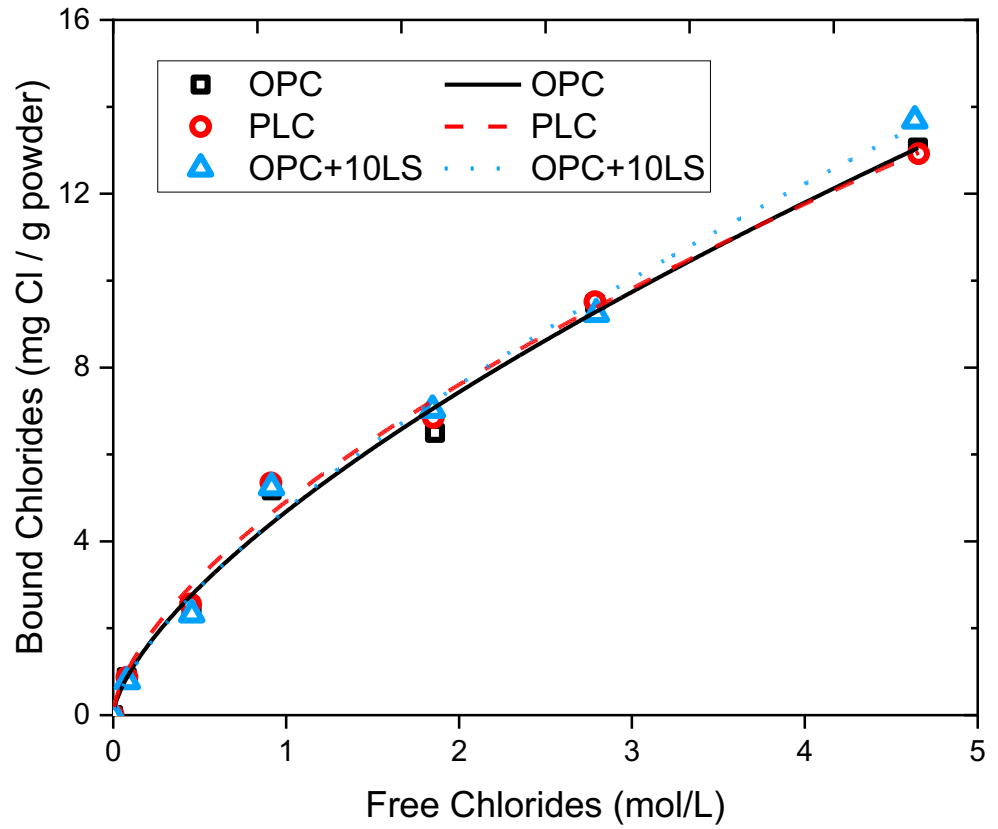

Figure F-24-3. Binding Isotherm of Cement $\mathrm{A}-\mathrm{M} 2(\mathrm{NaCl})$

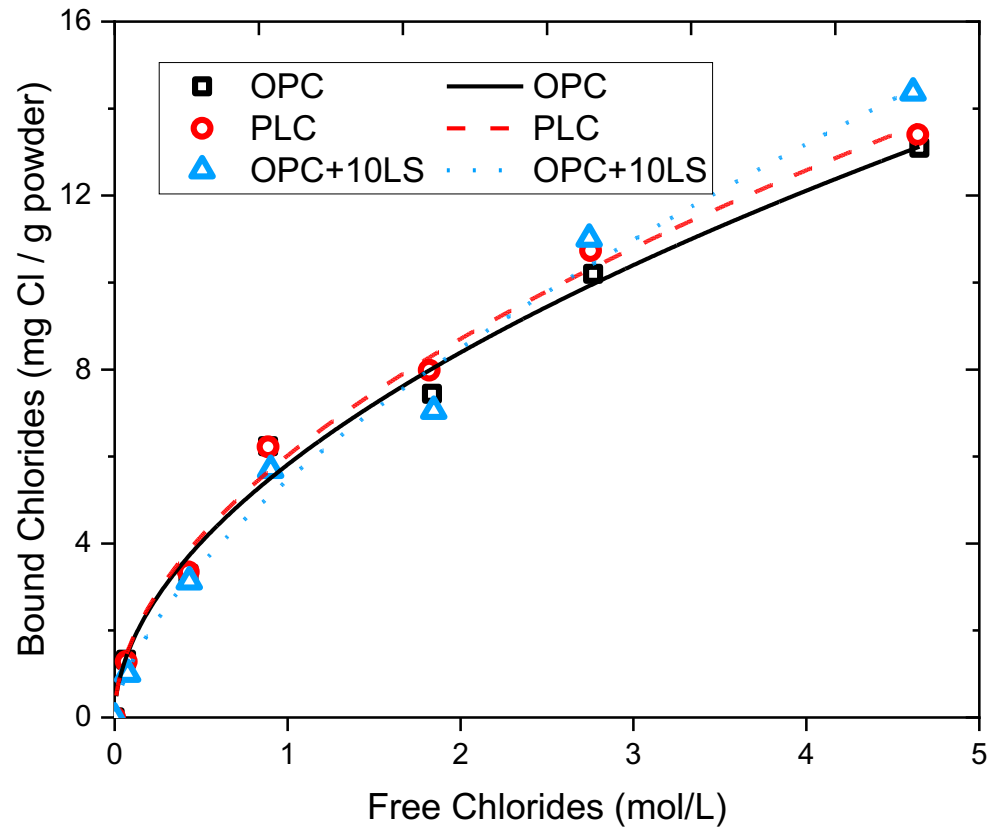

Figure F-24-4. Binding Isotherm of Cement $\mathrm{A}$ - M3 (NaCl) 

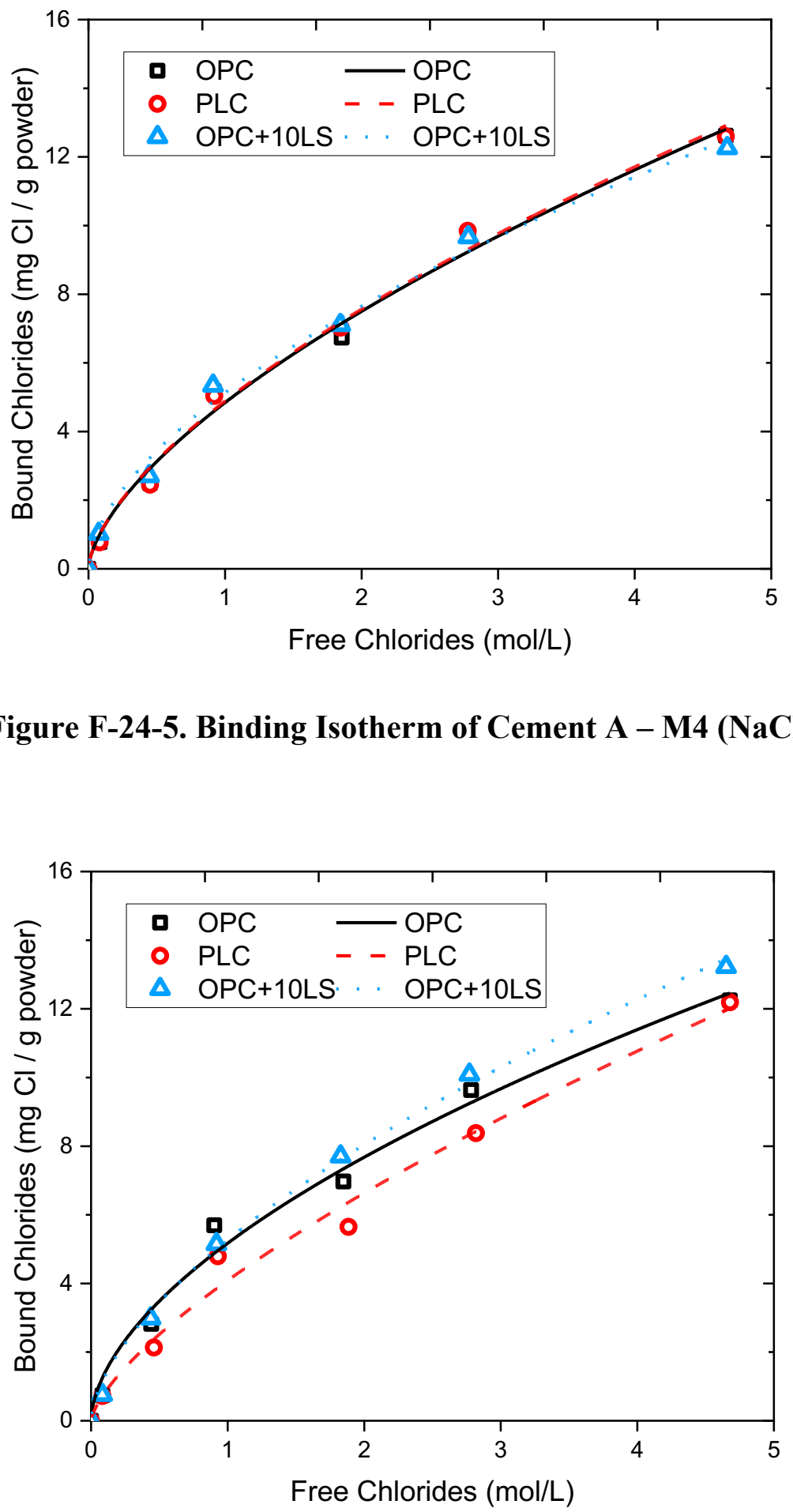


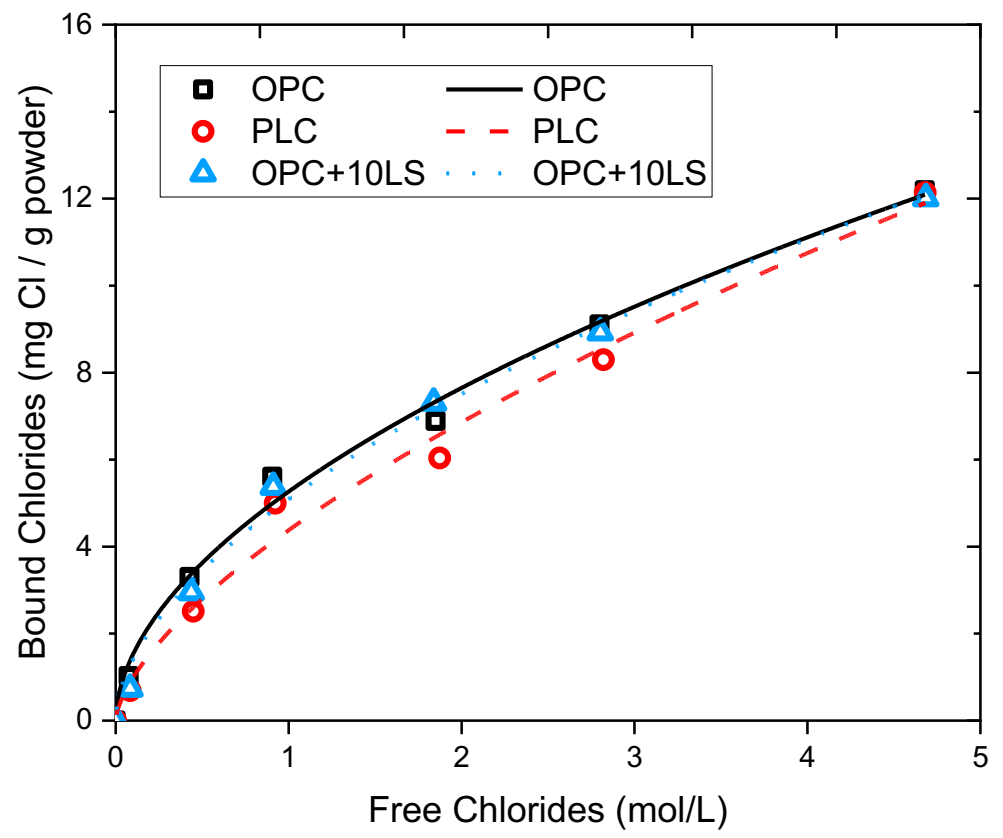

3884

3885

Figure F-24-7. Binding Isotherm of Cement B - M0 (NaCl)

3886

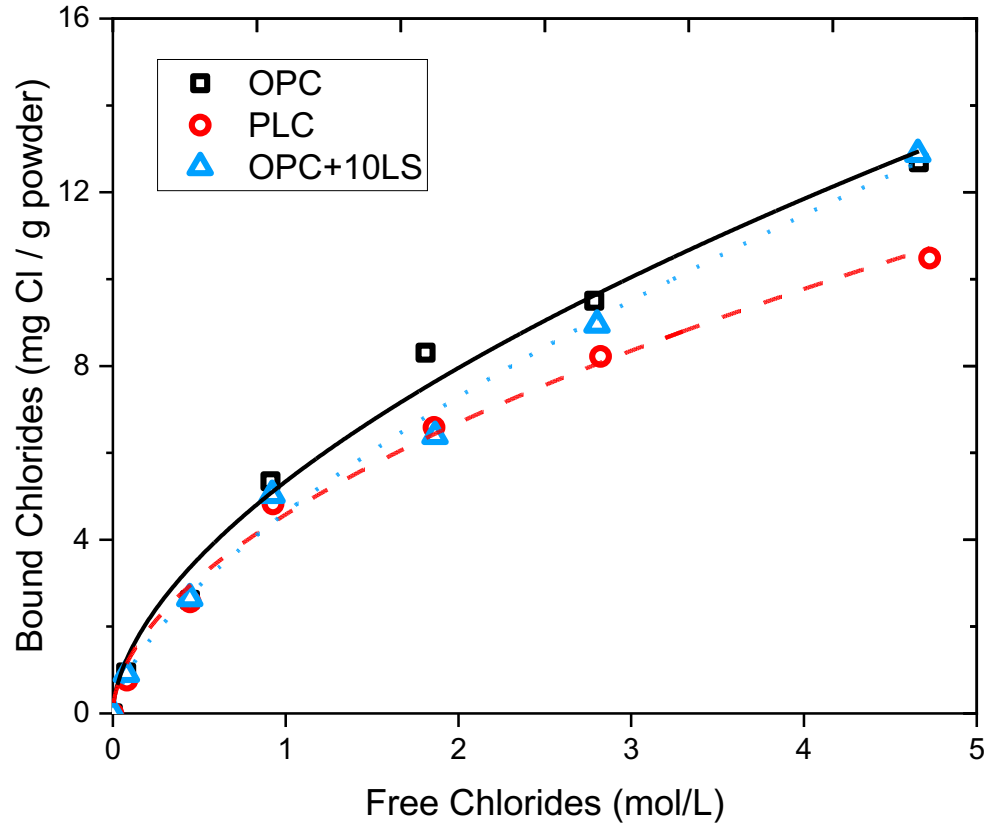

3888

Figure F-24-8. Binding Isotherm of Cement B - M1 (NaCl)

3889 


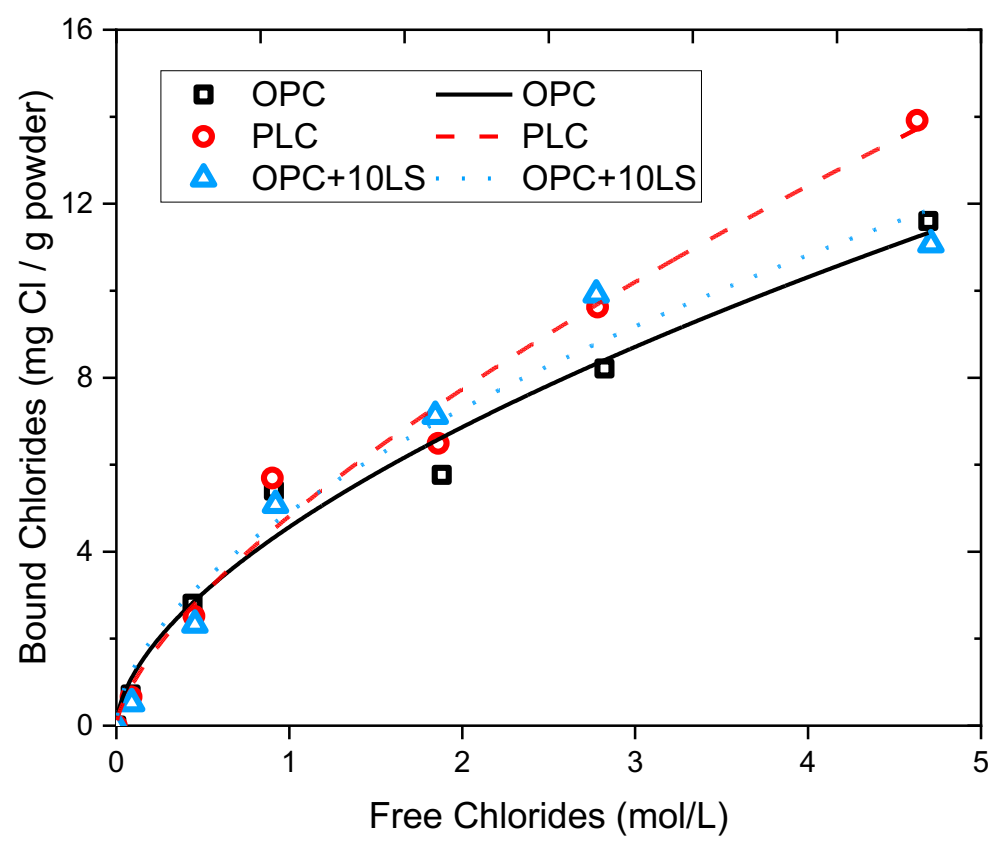

Figure F-24-9. Binding Isotherm of Cement B - M2 (NaCl)

3892

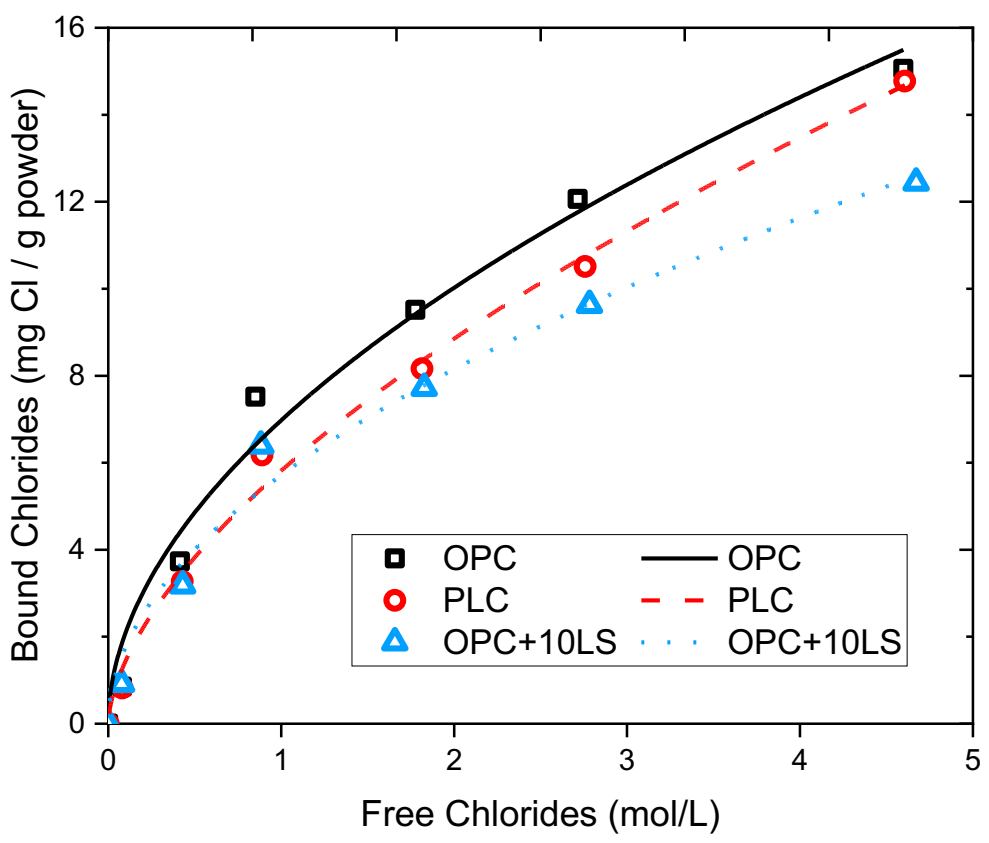




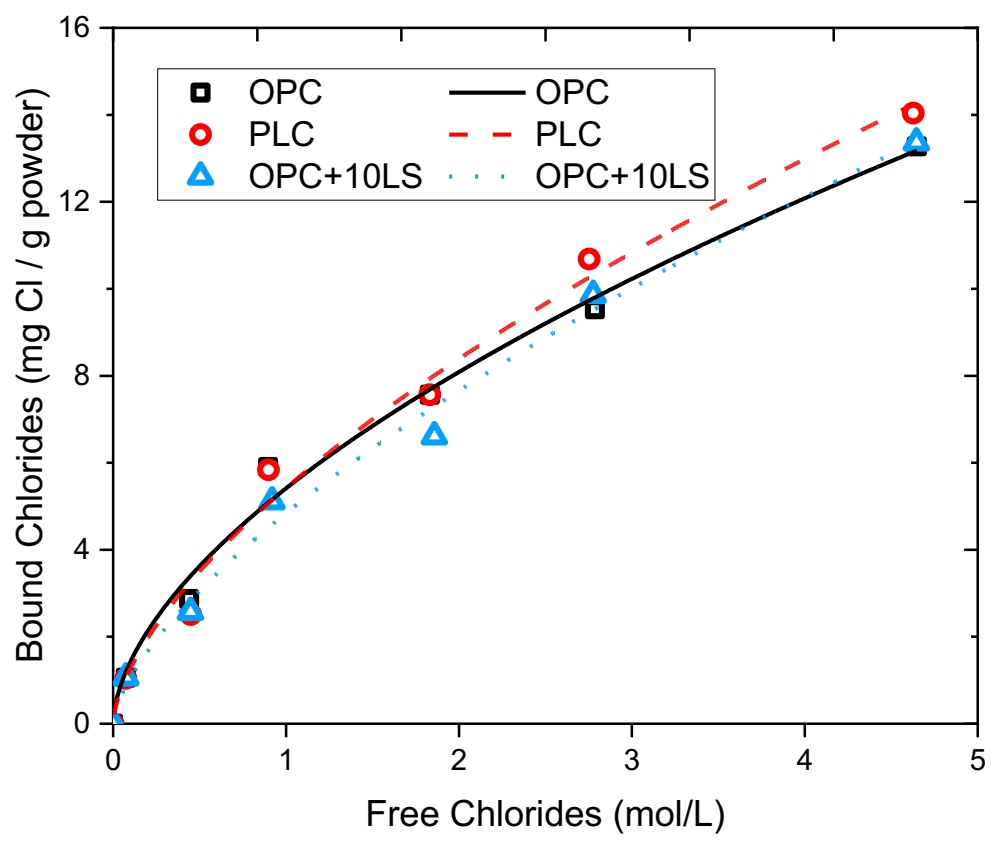

Figure F-24-11. Binding Isotherm of Cement B - M4 (NaCl)

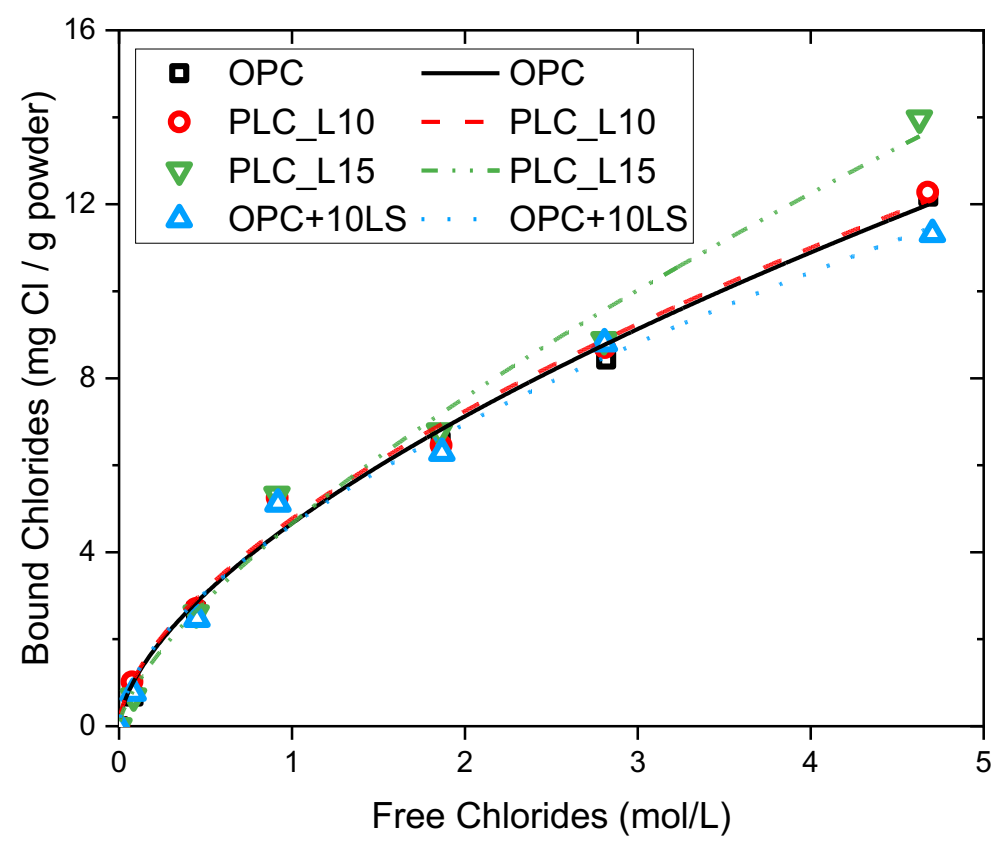




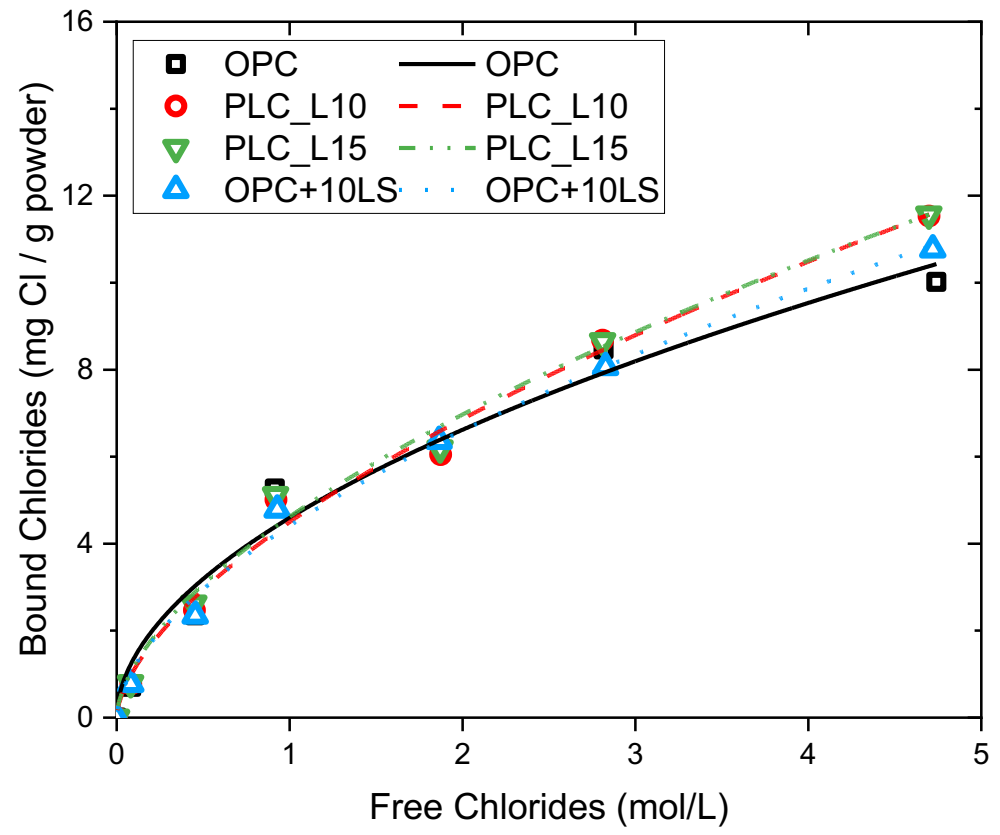

Figure F-24-13. Binding Isotherm of Cement C - M1 (NaCl)

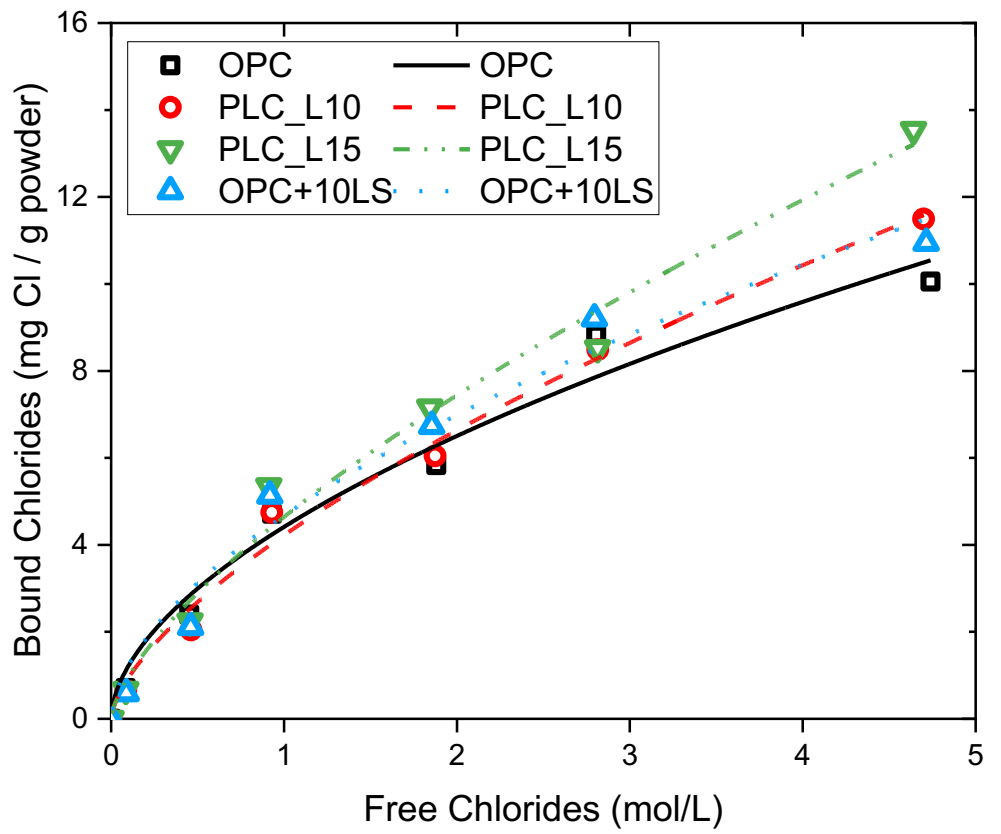

Figure F-24-14. Binding Isotherm of Cement C - M2 (NaCl) 


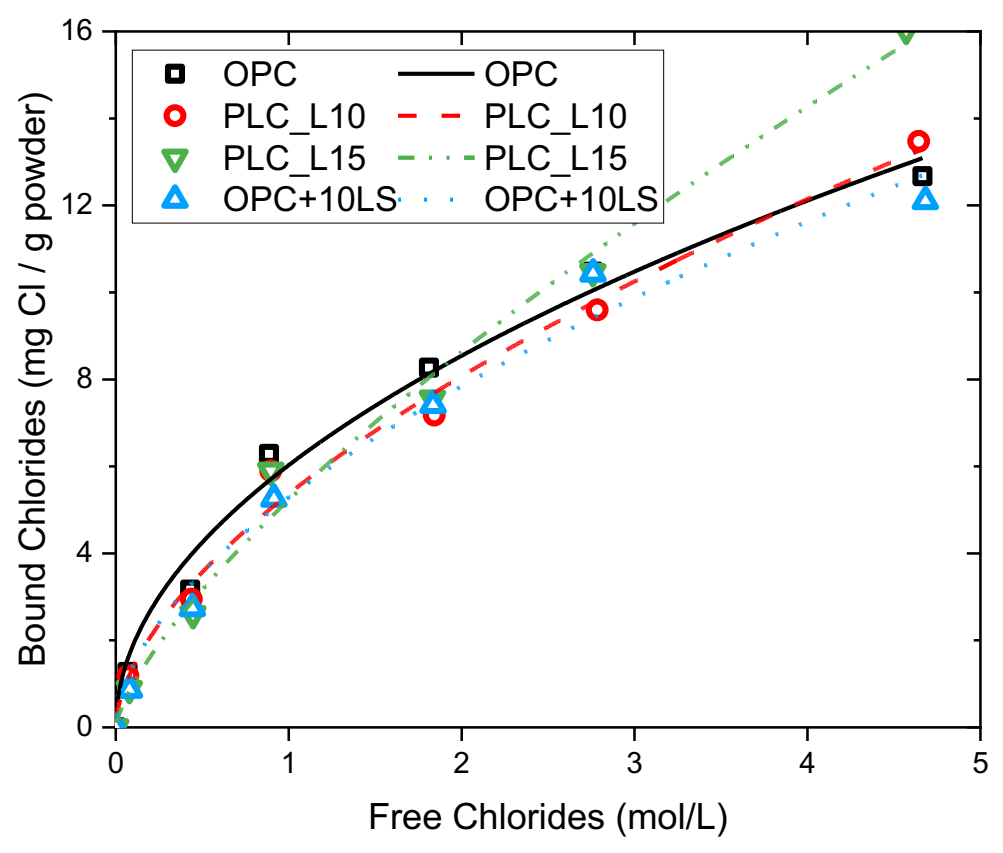

3906

3907

3908

3909

3910

3911
Figure F-24-15. Binding Isotherm of Cement C - M3 (NaCl)

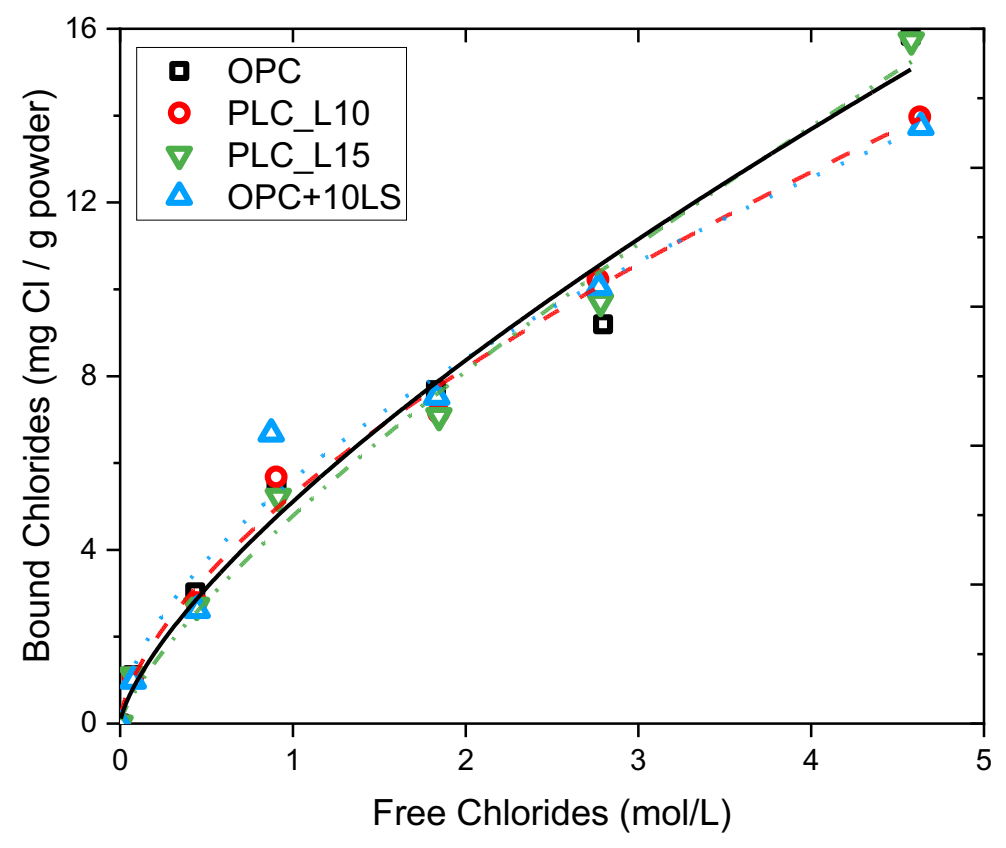

Figure F-24-16. Binding Isotherm of Cement C - M4 (NaCl) 


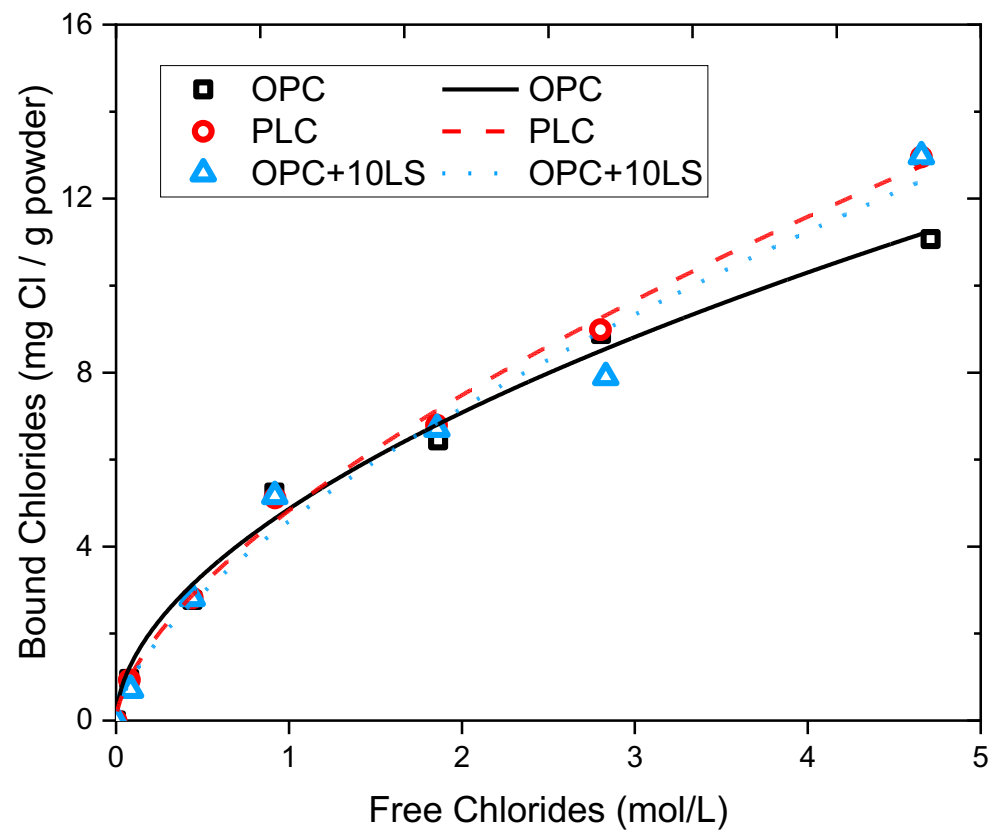

Figure F-24-17. Binding Isotherm of Cement D - M0 (NaCl)

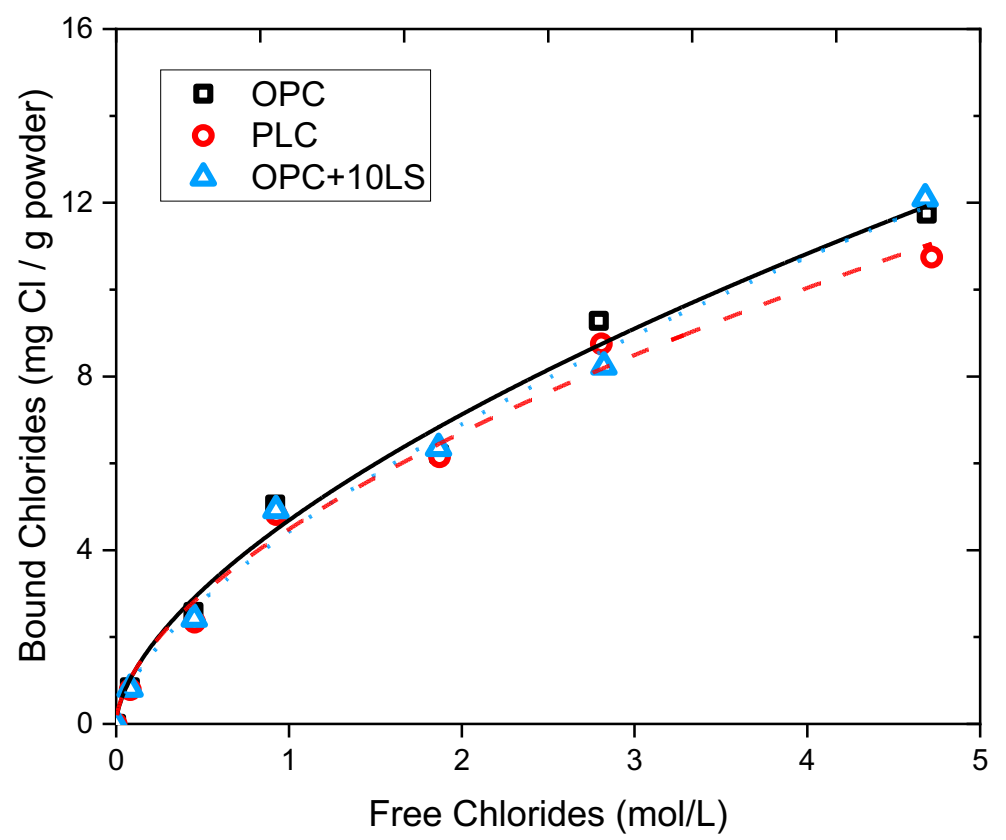

3914 


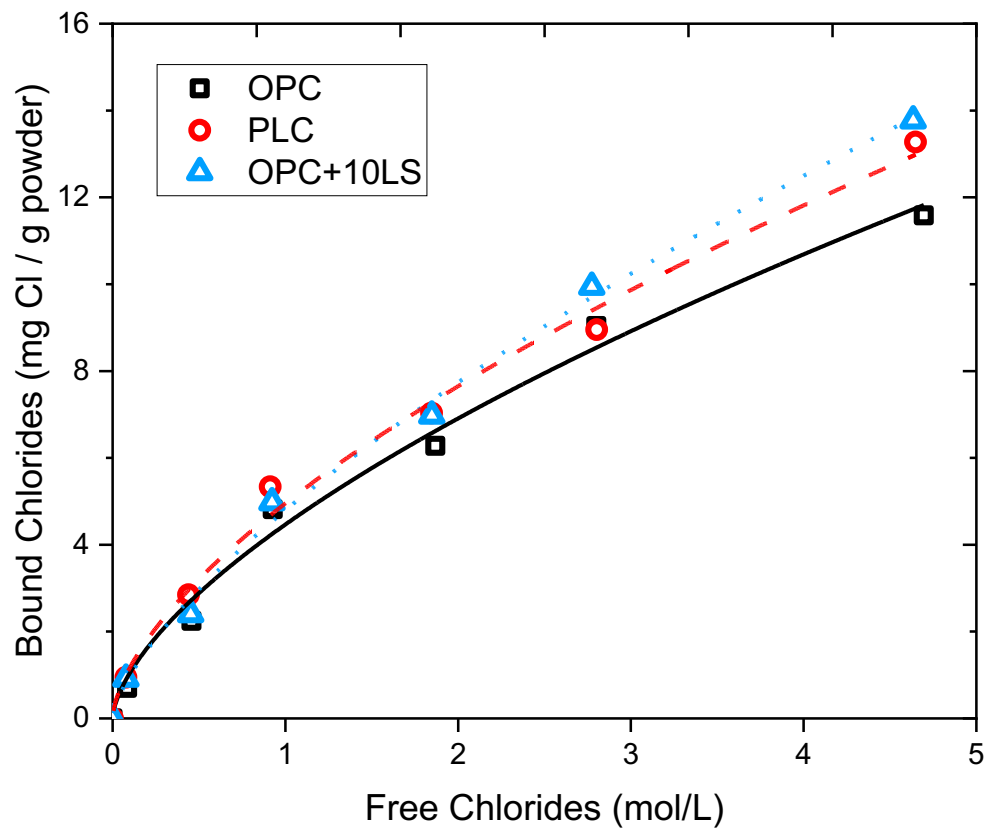

Figure F-24-19. Binding Isotherm of Cement D - M2 (NaCl)

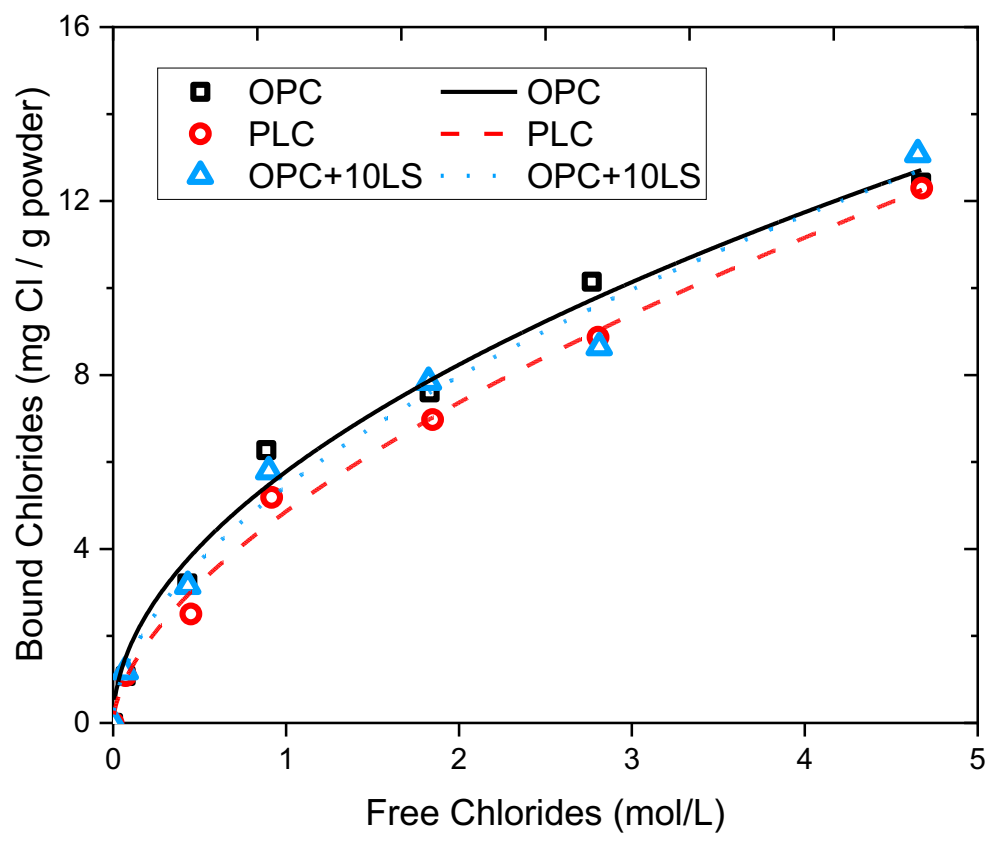




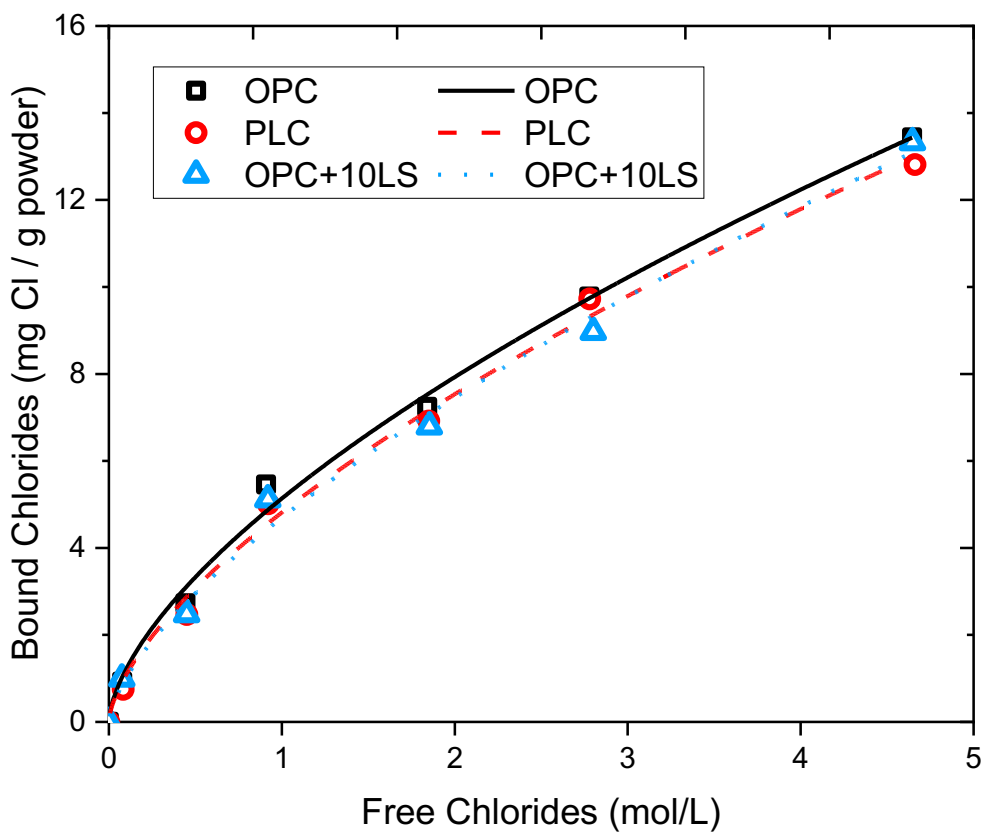

3923

Figure F-24-21. Binding Isotherm of Cement D - M4 (NaCl)

3924

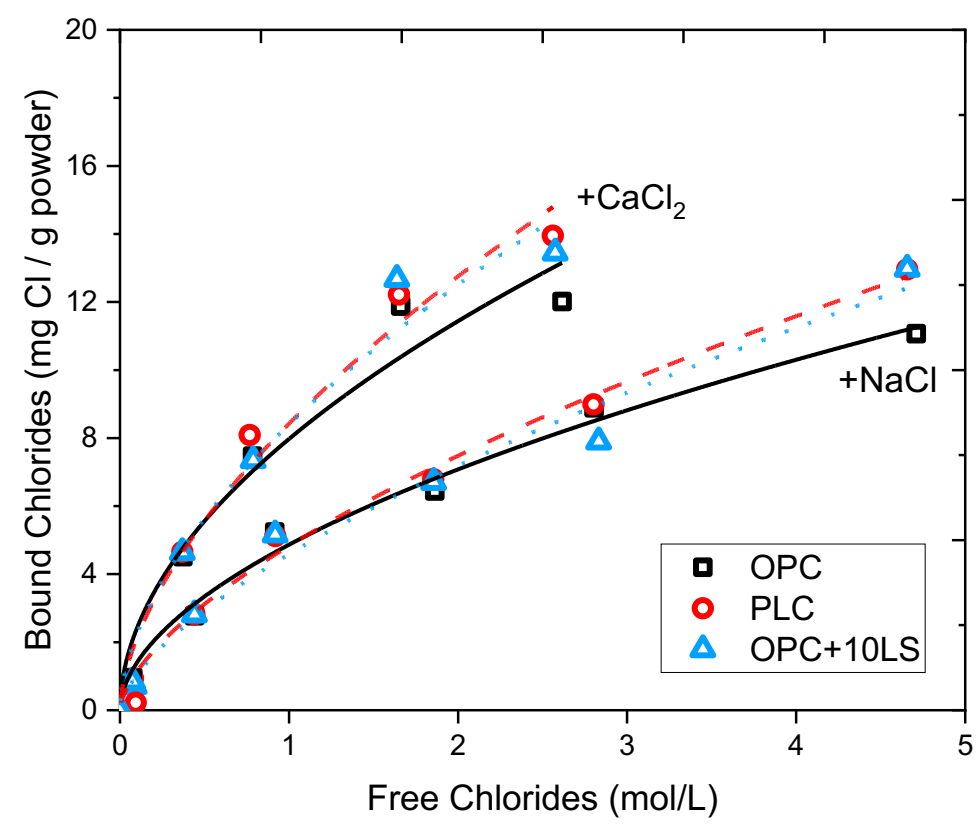




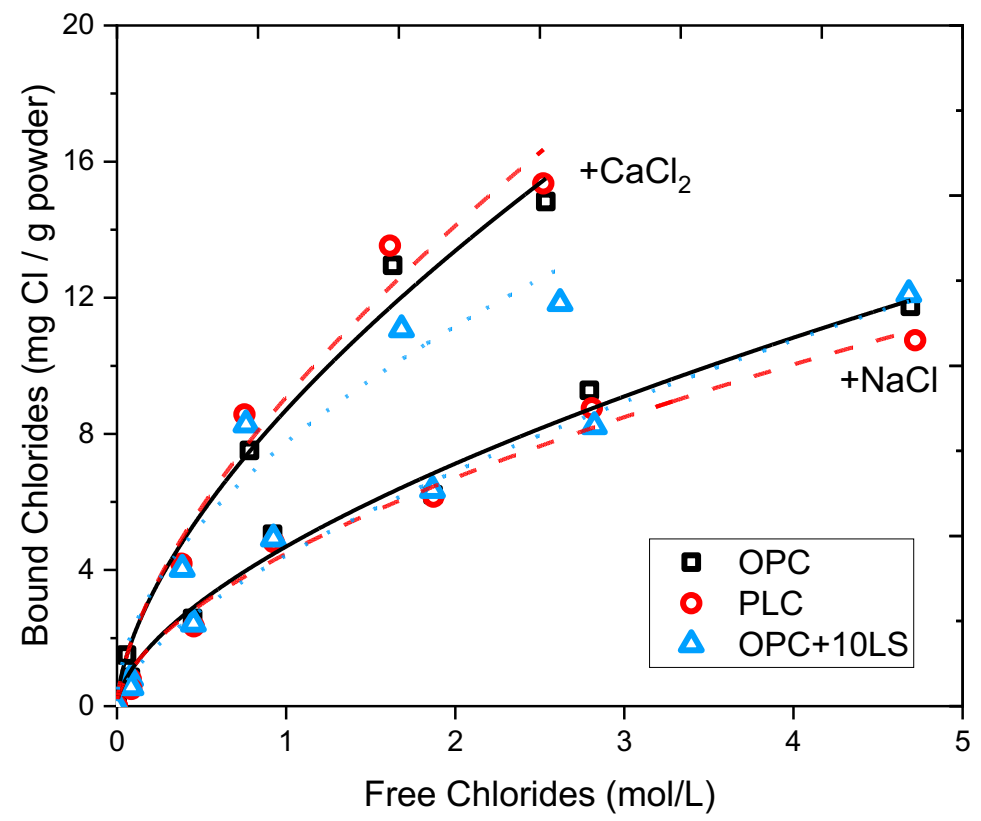

Figure F-24-23. Binding Isotherm of Cement D - M1 ( $\left.\mathrm{NaCl}, \mathrm{CaCl}_{2}\right)$

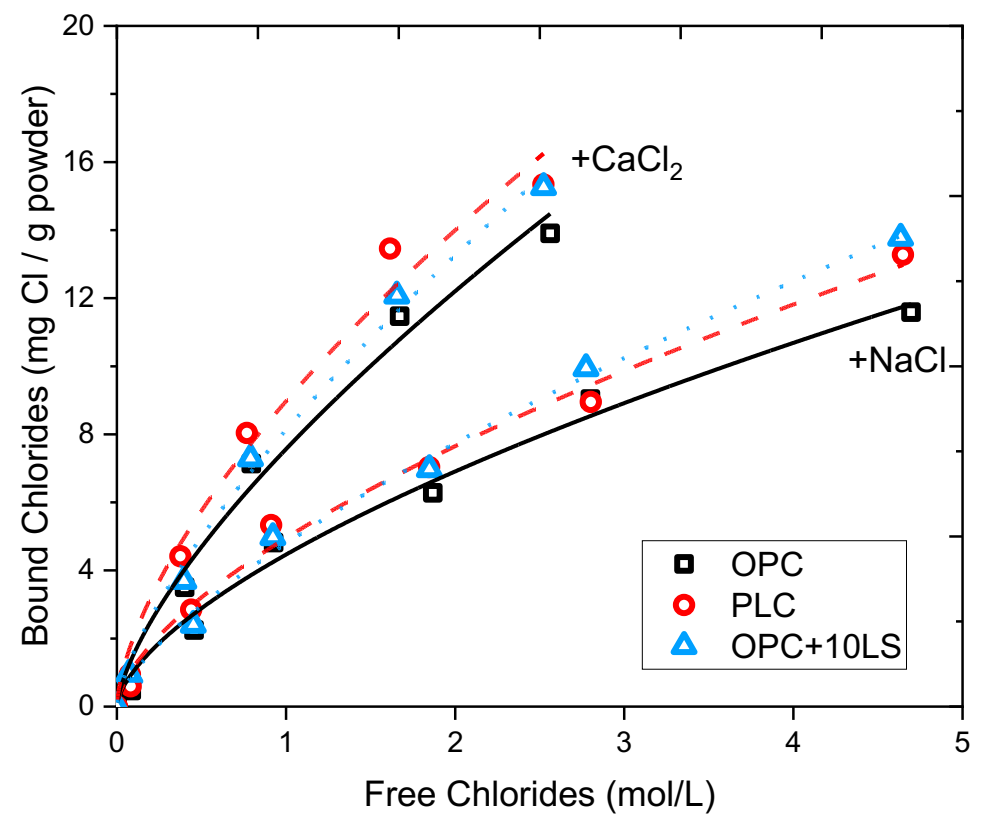




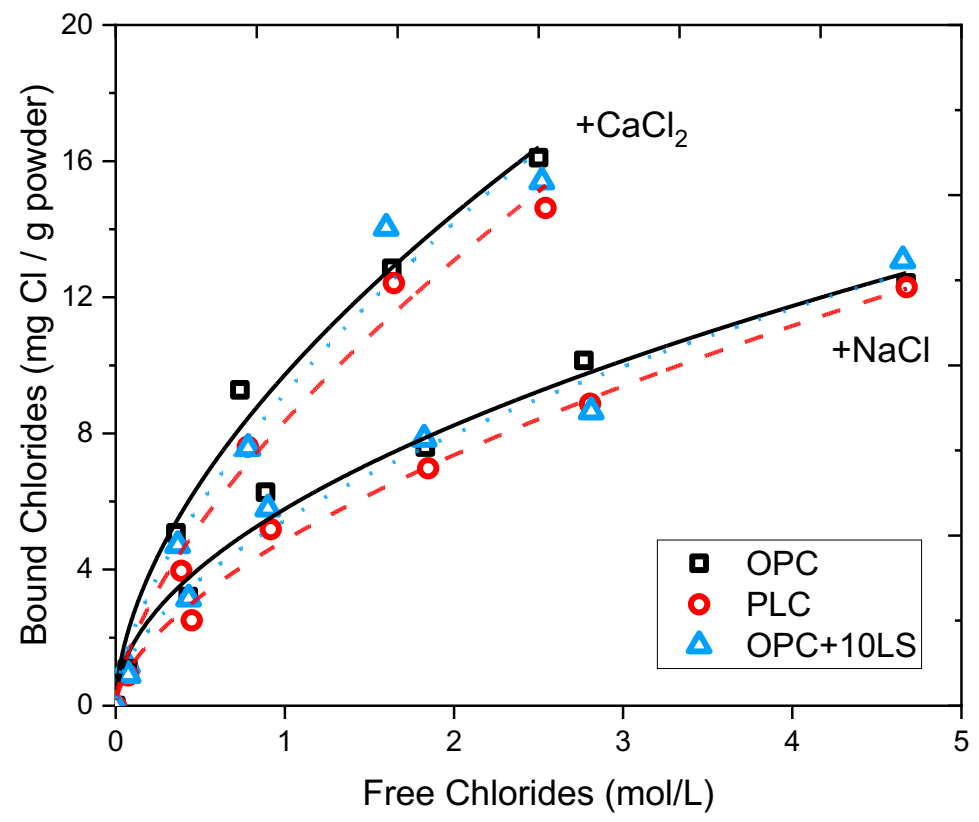

Figure F-24-25. Binding Isotherm of Cement D - M3 ( $\left.\mathrm{NaCl}, \mathrm{CaCl}_{2}\right)$

3934

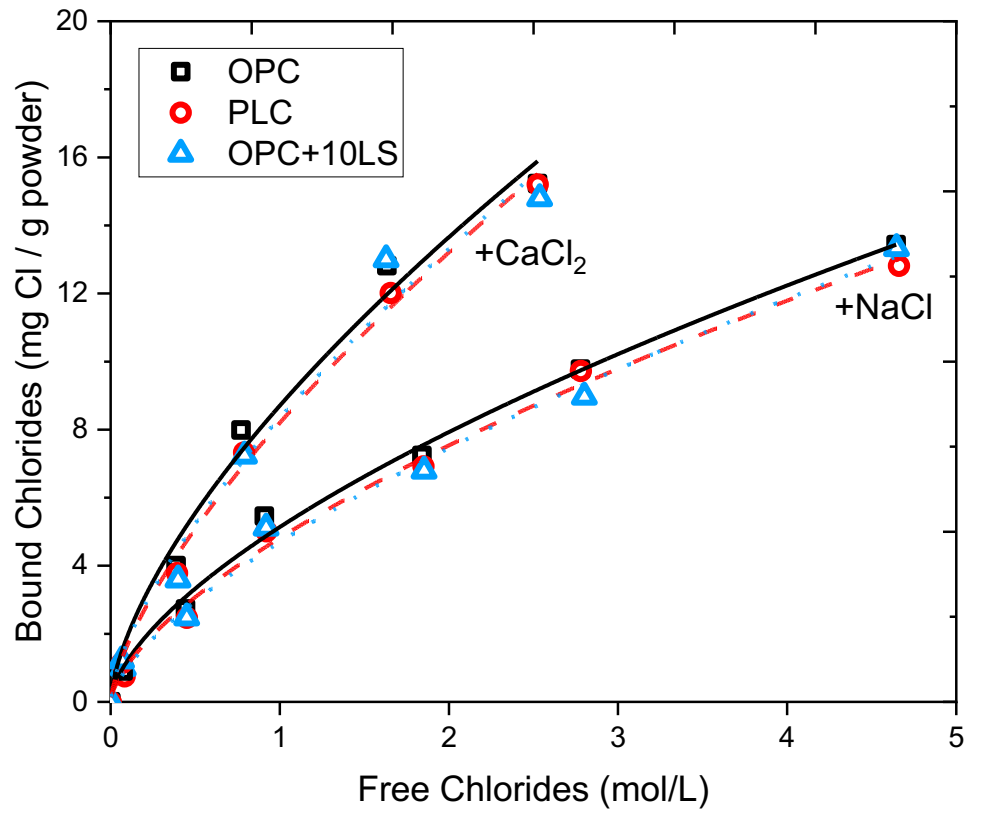

Figure F-24-26. Binding Isotherm of Cement D - M4 ( $\left.\mathrm{NaCl}, \mathrm{CaCl}_{2}\right)$ 


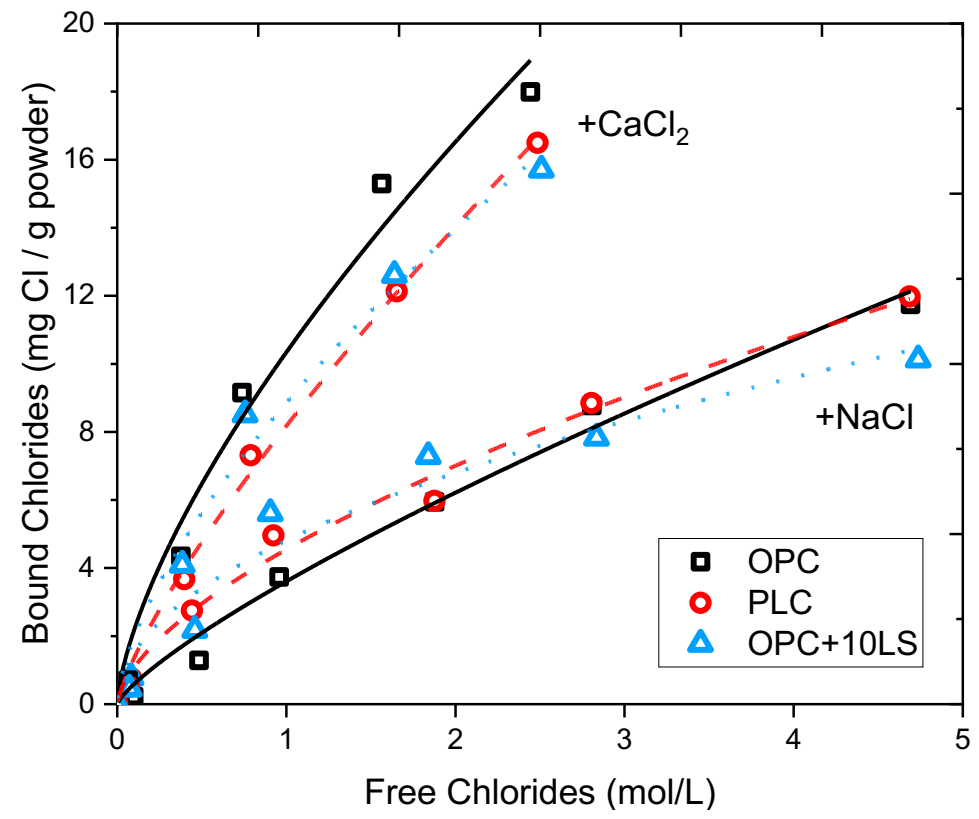

Figure F-24-27. Binding Isotherm of Cement D - M5 ( $\left.\mathrm{NaCl}, \mathrm{CaCl}_{2}\right)$

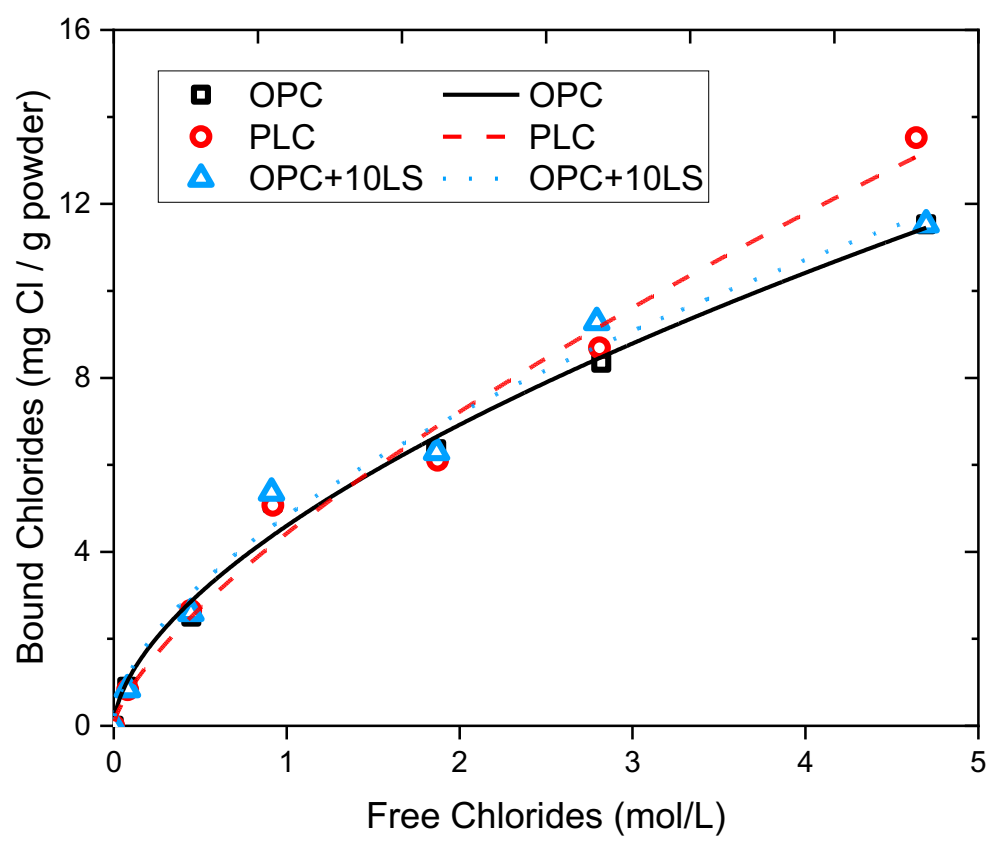




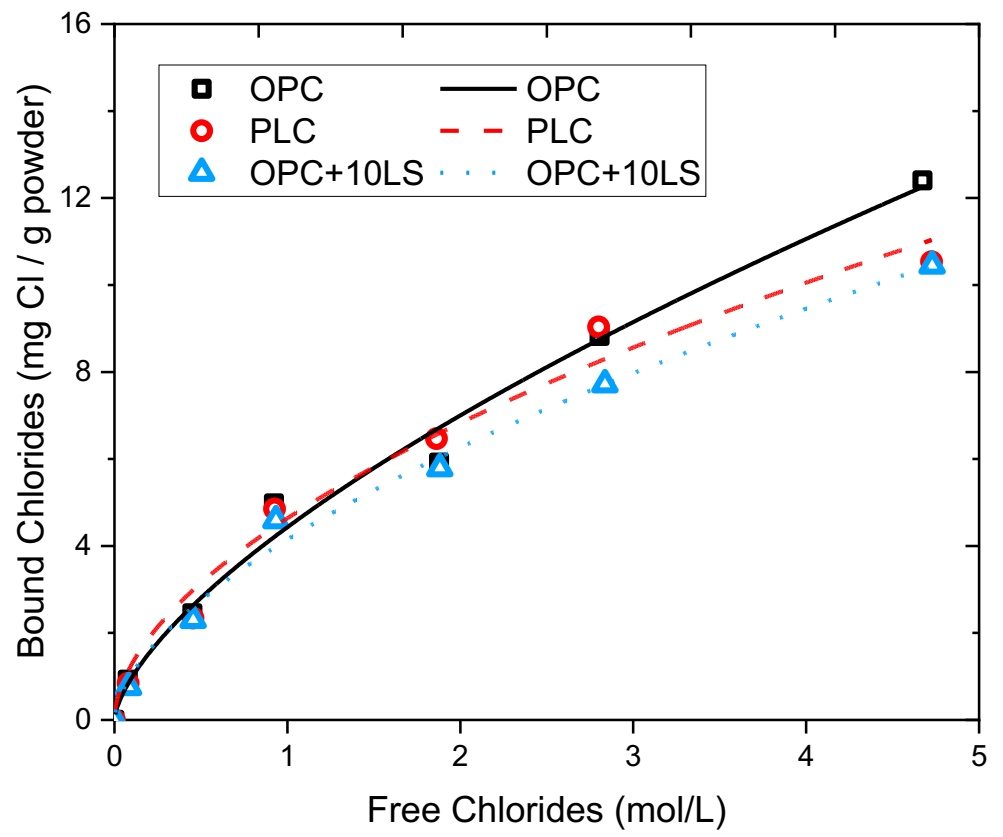

Figure F-24-29. Binding Isotherm of Cement E - M1 (NaCl)

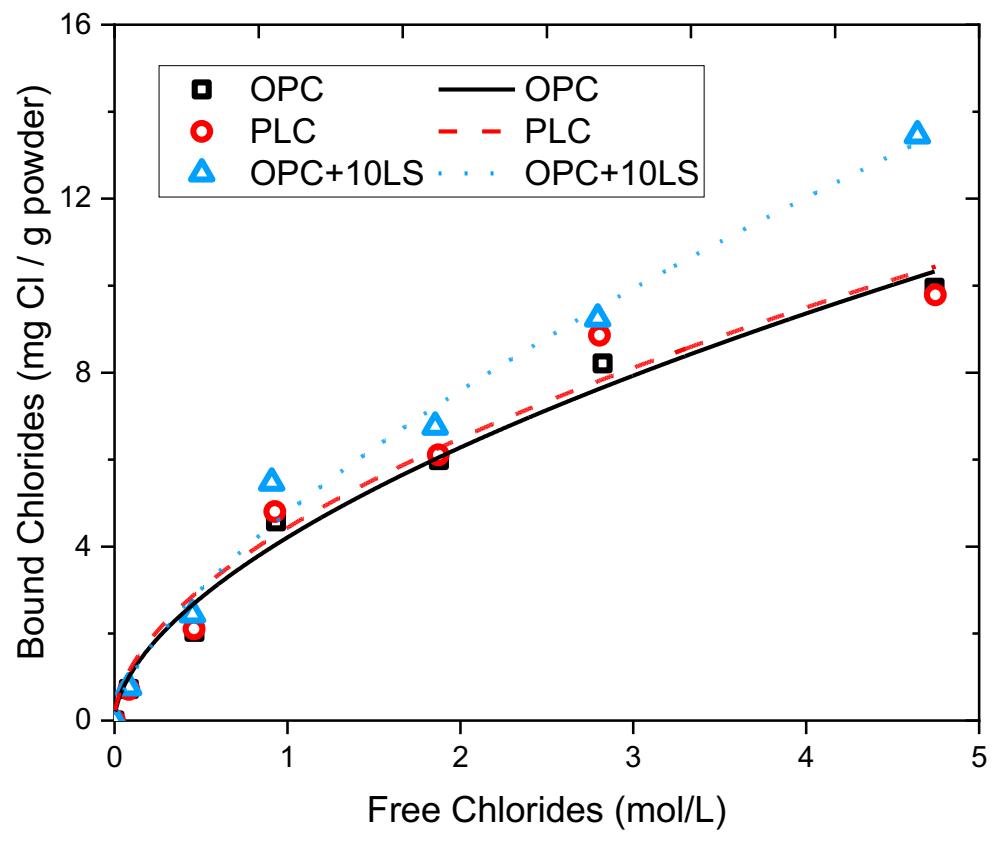



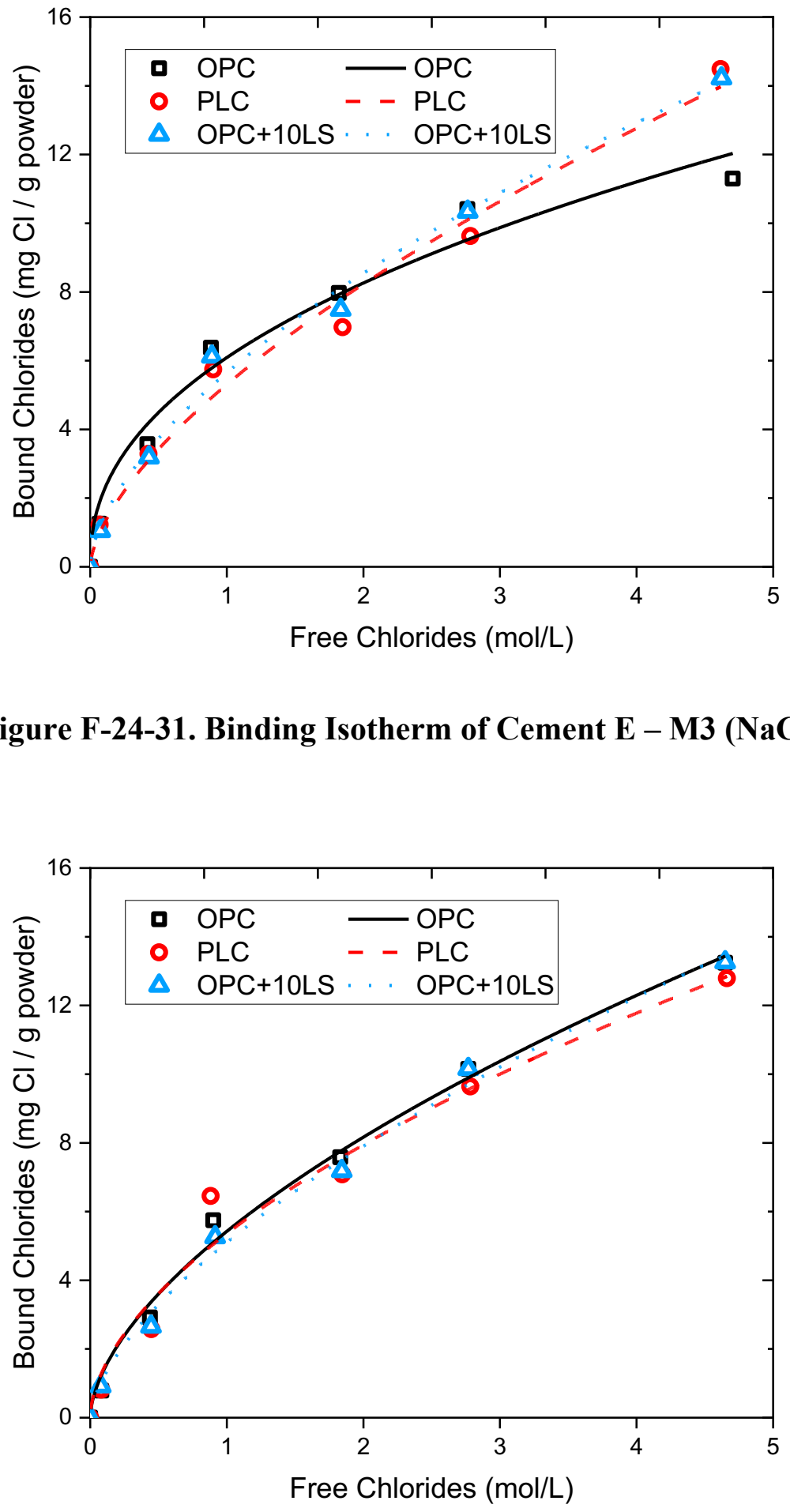
Table F.1. Binding Parameters of Cement A - Mixtures 0 to 5 (NaCl)

\begin{tabular}{|c|c|c|c|c|c|c|}
\hline \multicolumn{7}{|c|}{ A OPC } \\
\hline Sample & M0 & M1 & M2 & M3 & M4 & M5 \\
\hline $\boldsymbol{\alpha}$ & 5.29 & 4.82 & 4.68 & 5.81 & 4.84 & 5.17 \\
\hline $\boldsymbol{\beta}$ & 0.60 & 0.58 & 0.67 & 0.53 & 0.63 & 0.57 \\
\hline $\mathbf{R}^{2}$ & 0.992 & 0.991 & 0.986 & 0.985 & 0.987 & 0.980 \\
\hline
\end{tabular}

\begin{tabular}{|c|c|c|c|c|c|c|}
\hline \multicolumn{7}{|c|}{ A PLC_L15 } \\
\hline Sample & M0 & M1 & M2 & M3 & M4 & M5 \\
\hline $\boldsymbol{\alpha}$ & 5.11 & 4.91 & 4.92 & 6.02 & 4.89 & 4.09 \\
\hline $\boldsymbol{\beta}$ & 0.65 & 0.67 & 0.63 & 0.53 & 0.63 & 0.70 \\
\hline $\mathbf{R}^{2}$ & 0.982 & 0.992 & 0.989 & 0.989 & 0.989 & 0.980 \\
\hline
\end{tabular}

3954

\begin{tabular}{|c|c|c|c|c|c|c|}
\hline \multicolumn{7}{|c|}{ A OPC+10LS } \\
\hline Sample & M0 & M1 & M2 & M3 & M4 & M5 \\
\hline $\boldsymbol{\alpha}$ & 5.00 & 4.79 & 4.73 & 5.46 & 5.13 & 5.27 \\
\hline $\boldsymbol{\beta}$ & 0.64 & 0.69 & 0.69 & 0.64 & 0.58 & 0.61 \\
\hline $\mathbf{R}^{2}$ & 0.984 & 0.989 & 0.989 & 0.982 & 0.989 & 0.996 \\
\hline
\end{tabular}

3955 
Table F.2. Binding Parameters of Cement B - Mixtures 0 to 4 (NaCl) *M5 was not cast for Cement B

\begin{tabular}{|c|c|c|c|c|c|c|}
\hline \multicolumn{7}{|c|}{ B OPC } \\
\hline Sample & M0 & M1 & M2 & M3 & M4 & M5* \\
\hline $\boldsymbol{\alpha}$ & 5.26 & 5.28 & 4.57 & 6.97 & 5.41 & $\sim$ \\
\hline $\boldsymbol{\beta}$ & 0.54 & 0.55 & 0.59 & 0.52 & 0.58 & $\sim$ \\
\hline $\mathbf{R}^{2}$ & 0.990 & 0.975 & 0.964 & 0.973 & 0.987 & $\sim$ \\
\hline
\end{tabular}

\begin{tabular}{|c|c|c|c|c|c|c|}
\hline \multicolumn{7}{|c|}{ B PLC_L15 } \\
\hline Sample & M0 & M1 & M2 & M3 & M4 & M5* \\
\hline $\boldsymbol{\alpha}$ & 4.38 & 4.58 & 4.82 & 5.82 & 5.42 & $\sim$ \\
\hline $\boldsymbol{\beta}$ & 0.65 & 0.55 & 0.68 & 0.61 & 0.63 & $\sim$ \\
\hline $\mathbf{R}^{2}$ & 0.983 & 0.989 & 0.974 & 0.991 & 0.984 & $\sim$ \\
\hline
\end{tabular}

\begin{tabular}{|c|c|c|c|c|c|c|}
\hline \multicolumn{7}{|c|}{ B OPC+10LS } \\
\hline Sample & M0 & M1 & M2 & M3 & M4 & M5* $^{*}$ \\
\hline $\boldsymbol{\alpha}$ & 5.09 & 4.63 & 4.91 & 5.71 & 4.84 & $\sim$ \\
\hline $\boldsymbol{\beta}$ & 0.56 & 0.66 & 0.57 & 0.51 & 0.66 & $\sim$ \\
\hline $\mathbf{R}^{2}$ & 0.990 & 0.989 & 0.953 & 0.975 & 0.988 & $\sim$ \\
\hline
\end{tabular}



*M5 was not cast for Cement $C$

\begin{tabular}{|c|c|c|c|c|c|c|}
\hline \multicolumn{7}{|c|}{ C OPC } \\
\hline Sample & M0 & M1 & M2 & M3 & M4 & M5* \\
\hline $\boldsymbol{\alpha}$ & 4.66 & 4.59 & 4.41 & 6.03 & 5.39 & $\sim$ \\
\hline $\boldsymbol{\beta}$ & 0.61 & 0.53 & 0.56 & 0.50 & 0.54 & $\sim$ \\
\hline $\mathbf{R}^{2}$ & 0.984 & 0.960 & 0.962 & 0.981 & 0.992 & $\sim$ \\
\hline
\end{tabular}

\begin{tabular}{|c|c|c|c|c|c|c|}
\hline \multicolumn{7}{|c|}{ C PLC_L10 } \\
\hline Sample & M0 & M1 & M2 & M3 & M4 & M5* \\
\hline $\boldsymbol{\alpha}$ & 4.77 & 4.50 & 4.22 & 5.37 & 5.28 & $\sim$ \\
\hline $\boldsymbol{\beta}$ & 0.60 & 0.61 & 0.65 & 0.59 & 0.63 & $\sim$ \\
\hline $\mathbf{R}^{2}$ & 0.987 & 0.984 & 0.985 & 0.985 & 0.988 & $\sim$ \\
\hline
\end{tabular}

\begin{tabular}{|c|c|c|c|c|c|c|}
\hline \multicolumn{7}{|c|}{ C PLC_L14 } \\
\hline Sample & M0 & M1 & M2 & M3 & M4 & M5* \\
\hline $\boldsymbol{\alpha}$ & 4.66 & 4.62 & 4.64 & 5.23 & 4.78 & $\sim$ \\
\hline $\boldsymbol{\beta}$ & 0.70 & 0.59 & 0.68 & 0.72 & 0.76 & $\sim$ \\
\hline $\mathbf{R}^{2}$ & 0.981 & 0.985 & 0.975 & 0.985 & 0.984 & $\sim$ \\
\hline
\end{tabular}

\begin{tabular}{|c|c|c|c|c|c|c|}
\hline \multicolumn{7}{|c|}{ C OPC+10LS } \\
\hline Sample & M0 & M1 & M2 & M3 & M4 & M5* \\
\hline $\boldsymbol{\alpha}$ & 4.63 & 4.41 & 4.67 & 5.29 & 5.62 & $\sim$ \\
\hline $\boldsymbol{\beta}$ & 0.59 & 0.58 & 0.58 & 0.57 & 0.58 & $\sim$ \\
\hline $\mathbf{R}^{2}$ & 0.983 & 0.989 & 0.963 & 0.974 & 0.963 & $\sim$ \\
\hline
\end{tabular}


Table F.4. Binding Parameters of Cement D - Mixtures 0 to 5 (NaCl)

\begin{tabular}{|c|c|c|c|c|c|c|}
\hline \multicolumn{7}{|c|}{ D OPC } \\
\hline Sample & M0 & M1 & M2 & M3 & M4 & M5 \\
\hline $\boldsymbol{\alpha}$ & 4.87 & 4.69 & 4.47 & 5.78 & 5.10 & 3.61 \\
\hline $\boldsymbol{\beta}$ & 0.54 & 0.60 & 0.63 & 0.51 & 0.63 & 0.78 \\
\hline $\mathbf{R}^{2}$ & 0.985 & 0.982 & 0.985 & 0.979 & 0.993 & 0.983 \\
\hline
\end{tabular}

\begin{tabular}{|c|c|c|c|c|c|c|}
\hline \multicolumn{7}{|c|}{ D PLC_L15 } \\
\hline Sample & M0 & M1 & M2 & M3 & M4 & M5 \\
\hline $\boldsymbol{\alpha}$ & 4.83 & 4.95 & 4.48 & 4.86 & 4.81 & 4.55 \\
\hline $\boldsymbol{\beta}$ & 0.63 & 0.63 & 0.58 & 0.60 & 0.65 & 0.62 \\
\hline $\mathbf{R}^{2}$ & 0.993 & 0.989 & 0.981 & 0.991 & 0.992 & 0.984 \\
\hline
\end{tabular}

\begin{tabular}{|c|c|c|c|c|c|c|}
\hline \multicolumn{7}{|c|}{ D OPC+10LS } \\
\hline Sample & M0 & M1 & M2 & M3 & M4 & M5 \\
\hline $\boldsymbol{\alpha}$ & 4.59 & 4.43 & 4.79 & 5.45 & 4.69 & 4.81 \\
\hline $\boldsymbol{\beta}$ & 0.65 & 0.64 & 0.69 & 0.55 & 0.67 & 0.50 \\
\hline $\mathbf{R}^{2}$ & 0.969 & 0.988 & 0.994 & 0.977 & 0.989 & 0.934 \\
\hline
\end{tabular}

3965 


\begin{tabular}{|c|c|c|c|c|c|c|}
\hline \multicolumn{7}{|c|}{ D OPC } \\
\hline Sample & M0 & M1 & M2 & M3 & M4 & M5 \\
\hline $\boldsymbol{\alpha}$ & 7.97 & 8.70 & 7.57 & 9.72 & 8.70 & 10.34 \\
\hline $\boldsymbol{\beta}$ & 0.52 & 0.62 & 0.69 & 0.57 & 0.65 & 0.68 \\
\hline $\mathbf{R}^{2}$ & 0.926 & 0.976 & 0.974 & 0.977 & 0.976 & 0.968 \\
\hline
\end{tabular}

\begin{tabular}{|c|c|c|c|c|c|c|}
\hline \multicolumn{7}{|c|}{ D PLC_L15 } \\
\hline Sample & M0 & M1 & M2 & M3 & M4 & M5 \\
\hline $\boldsymbol{\alpha}$ & 8.42 & 9.08 & 8.97 & 8.36 & 8.20 & 8.18 \\
\hline $\boldsymbol{\beta}$ & 0.74 & 0.64 & 0.64 & 0.65 & 0.69 & 0.78 \\
\hline $\mathbf{R}^{2}$ & 0.941 & 0.949 & 0.963 & 0.977 & 0.993 & 0.995 \\
\hline
\end{tabular}

\begin{tabular}{|c|c|c|c|c|c|c|}
\hline \multicolumn{7}{|c|}{ D OPC+10LS } \\
\hline Sample & M0 & M1 & M2 & M3 & M4 & M5 \\
\hline $\boldsymbol{\alpha}$ & 8.40 & 7.74 & 8.13 & 9.17 & 8.35 & 8.86 \\
\hline $\boldsymbol{\beta}$ & 0.57 & 0.53 & 0.71 & 0.63 & 0.67 & 0.66 \\
\hline $\mathbf{R}^{2}$ & 0.948 & 0.897 & 0.991 & 0.957 & 0.966 & 0.971 \\
\hline
\end{tabular}

3968 
Table F.6. Binding Parameters of Cement $\mathrm{E}$ - Mixtures 0 to 4 (NaCl) *M5 was not cast for Cement $E$

\begin{tabular}{|c|c|c|c|c|c|c|}
\hline \multicolumn{7}{|c|}{ E OPC } \\
\hline Sample & M0 & M1 & M2 & M3 & M4 & M5 \\
\hline $\boldsymbol{\alpha}$ & 4.60 & 4.43 & 4.21 & 6.09 & 5.42 & $\sim$ \\
\hline $\boldsymbol{\beta}$ & 0.59 & 0.66 & 0.58 & 0.44 & 0.59 & $\sim$ \\
\hline $\mathbf{R}^{2}$ & 0.987 & 0.982 & 0.975 & 0.960 & 0.989 & $\sim$ \\
\hline
\end{tabular}

\begin{tabular}{|c|c|c|c|c|c|c|}
\hline \multicolumn{7}{|c|}{ E PLC_L11 } \\
\hline Sample & M0 & M1 & M2 & M3 & M4 & M5 \\
\hline $\boldsymbol{\alpha}$ & 4.42 & 4.64 & 4.43 & 5.31 & 5.36 & $\sim$ \\
\hline $\boldsymbol{\beta}$ & 0.71 & 0.56 & 0.55 & 0.63 & 0.57 & $\sim$ \\
\hline $\mathbf{R}^{2}$ & 0.977 & 0.972 & 0.949 & 0.978 & 0.958 & $\sim$ \\
\hline
\end{tabular}

\begin{tabular}{|c|c|c|c|c|c|c|}
\hline \multicolumn{7}{|c|}{ E OPC+10LS } \\
\hline Sample & M0 & M1 & M2 & M3 & M4 & M5 \\
\hline $\boldsymbol{\alpha}$ & 4.83 & 4.15 & 4.79 & 5.65 & 5.10 & $\sim$ \\
\hline $\boldsymbol{\beta}$ & 0.57 & 0.59 & 0.66 & 0.60 & 0.63 & $\sim$ \\
\hline $\mathbf{R}^{2}$ & 0.974 & 0.989 & 0.983 & 0.987 & 0.992 & $\sim$ \\
\hline
\end{tabular}

3972

3973 
3974 3975 3976

Table F.7. Bound chloride content for OPCs immersed in $\mathrm{NaCl}$ - Cements A to E, Mixtures 0 to $5(\mathrm{NaCl})$ *M5 was cast only for cements $A$ and $D$

\begin{tabular}{|c|c|c|c|c|c|c|}
\hline \multicolumn{7}{|c|}{ A OPC } \\
\hline Sample & M0 & M1 & M2 & M3 & M4 & M5 \\
\hline $\mathbf{0 . 1 M}$ & 0.86 & 0.90 & 0.88 & 1.33 & 0.77 & 0.74 \\
\hline $\mathbf{0 . 5 M}$ & 2.97 & 2.64 & 2.37 & 3.30 & 2.49 & 2.82 \\
\hline $\mathbf{1 M}$ & 5.53 & 5.19 & 5.17 & 6.23 & 5.18 & 5.69 \\
\hline $\mathbf{2 M}$ & 7.27 & 6.80 & 6.50 & 7.44 & 6.73 & 6.97 \\
\hline $\mathbf{3 M}$ & 10.02 & 8.71 & 9.47 & 10.20 & 9.70 & 9.63 \\
\hline $\mathbf{5 M}$ & 13.17 & 11.85 & 13.06 & 13.10 & 12.62 & 12.24 \\
\hline
\end{tabular}

\begin{tabular}{|c|c|c|c|c|c|c|}
\hline \multicolumn{7}{|c|}{ D OPC } \\
\hline Sample & M0 & M1 & M2 & M3 & M4 & M5 \\
\hline $\mathbf{0 . 1 M}$ & 0.96 & 0.84 & 0.70 & 1.08 & 0.93 & 0.22 \\
\hline $\mathbf{0 . 5 M}$ & 2.77 & 2.58 & 2.25 & 3.21 & 2.73 & 1.28 \\
\hline $\mathbf{1 M}$ & 5.24 & 5.05 & 4.82 & 6.27 & 5.46 & 3.74 \\
\hline $\mathbf{2 M}$ & 6.44 & 6.21 & 6.28 & 7.60 & 7.24 & 5.94 \\
\hline $\mathbf{3 M}$ & 8.89 & 9.28 & 9.03 & 10.14 & 9.78 & 8.77 \\
\hline $\mathbf{5 M}$ & 11.07 & 11.75 & 11.59 & 12.42 & 13.44 & 11.75 \\
\hline
\end{tabular}

\begin{tabular}{|c|c|c|c|c|c|c|}
\hline \multicolumn{7}{|c|}{ B OPC } \\
\hline Sample & M0 & M1 & M2 & M3 & M4 & M5 \\
\hline $\mathbf{0 . 1 M}$ & 1.03 & 0.94 & 0.72 & 0.85 & 1.05 & $\sim$ \\
\hline $\mathbf{0 . 5 M}$ & 3.30 & 2.61 & 2.80 & 3.73 & 2.87 & $\sim$ \\
\hline $\mathbf{1 M}$ & 5.61 & 5.34 & 5.40 & 7.52 & 5.90 & $\sim$ \\
\hline $\mathbf{2 M}$ & 6.89 & 8.30 & 5.77 & 9.51 & 7.56 & $\sim$ \\
\hline $\mathbf{3 M}$ & 9.11 & 9.50 & 8.21 & 12.07 & 9.53 & $\sim$ \\
\hline $\mathbf{5 M}$ & 12.19 & 12.69 & 11.60 & 15.06 & 13.28 & $\sim$ \\
\hline
\end{tabular}

\begin{tabular}{|c|c|c|c|c|c|c|}
\hline \multicolumn{7}{|c|}{ E OPC } \\
\hline Sample & M0 & M1 & M2 & M3 & M4 & M5 \\
\hline $\mathbf{0 . 1 M}$ & 0.90 & 0.92 & 0.73 & 1.25 & 0.79 & $\sim$ \\
\hline $\mathbf{0 . 5 M}$ & 2.51 & 2.45 & 2.04 & 3.57 & 2.91 & $\sim$ \\
\hline $\mathbf{1 M}$ & 5.11 & 4.98 & 4.58 & 6.38 & 5.75 & $\sim$ \\
\hline $\mathbf{2 M}$ & 6.36 & 5.91 & 5.99 & 7.97 & 7.58 & $\sim$ \\
\hline $\mathbf{3 M}$ & 8.35 & 8.83 & 8.21 & 10.41 & 10.16 & $\sim$ \\
\hline $\mathbf{5 M}$ & 11.53 & 12.40 & 9.95 & 11.30 & 13.24 & $\sim$ \\
\hline
\end{tabular}

\begin{tabular}{|c|c|c|c|c|c|c|}
\hline \multicolumn{7}{|c|}{ C OPC } \\
\hline Sample & M0 & M1 & M2 & M3 & M4 & M5 \\
\hline $\mathbf{0 . 1 M}$ & 0.68 & 0.71 & 0.70 & 1.27 & 1.11 & $\sim$ \\
\hline $\mathbf{0 . 5 M}$ & 2.66 & 2.35 & 2.40 & 3.17 & 3.02 & $\sim$ \\
\hline $\mathbf{1 M}$ & 5.23 & 5.27 & 4.72 & 6.29 & 5.58 & $\sim$ \\
\hline $\mathbf{2 M}$ & 6.55 & 6.20 & 5.83 & 8.27 & 7.68 & $\sim$ \\
\hline $\mathbf{3 M}$ & 8.43 & 8.46 & 8.83 & 10.48 & 9.19 & $\sim$ \\
\hline $\mathbf{5 M}$ & 12.17 & 10.02 & 10.05 & 12.67 & 15.84 & $\sim$ \\
\hline
\end{tabular}


3979

3980

3981

3982

3983

\begin{tabular}{|c|c|c|c|c|c|c|}
\hline \multicolumn{7}{|c|}{ A PLC_L15 } \\
\hline Sample & M0 & M1 & M2 & M3 & M4 & M5 \\
\hline $\mathbf{0 . 1 M}$ & 0.97 & 0.89 & 0.87 & 1.28 & 0.77 & 0.72 \\
\hline $\mathbf{0 . 5 M}$ & 2.85 & 2.52 & 2.56 & 3.35 & 2.45 & 2.13 \\
\hline $\mathbf{1 M}$ & 5.71 & 5.33 & 5.34 & 6.22 & 5.04 & 4.79 \\
\hline $\mathbf{2 M}$ & 7.12 & 7.12 & 6.85 & 7.98 & 7.02 & 5.65 \\
\hline $\mathbf{3 M}$ & 9.38 & 9.60 & 9.51 & 10.73 & 9.85 & 8.38 \\
\hline $\mathbf{5 M}$ & 14.17 & 13.90 & 12.92 & 13.40 & 12.60 & 12.19 \\
\hline
\end{tabular}

\begin{tabular}{|c|c|c|c|c|c|c|}
\hline \multicolumn{7}{|c|}{ B PLC_L15 } \\
\hline Sample & M0 & M1 & M2 & M3 & M4 & M5 \\
\hline $\mathbf{0 . 1 M}$ & 0.69 & 0.77 & 0.66 & 0.82 & 1.04 & $\sim$ \\
\hline $\mathbf{0 . 5 M}$ & 2.51 & 2.57 & 2.52 & 3.27 & 2.51 & $\sim$ \\
\hline $\mathbf{1 M}$ & 4.99 & 4.82 & 5.69 & 6.18 & 5.84 & $\sim$ \\
\hline $\mathbf{2 M}$ & 6.04 & 6.58 & 6.49 & 8.16 & 7.56 & $\sim$ \\
\hline $\mathbf{3 M}$ & 8.30 & 8.22 & 9.62 & 10.51 & 10.68 & $\sim$ \\
\hline $\mathbf{5 M}$ & 12.14 & 10.48 & 13.92 & 14.77 & 14.04 & $\sim$ \\
\hline
\end{tabular}

\begin{tabular}{|c|c|c|c|c|c|c|}
\hline \multicolumn{7}{|c|}{ C PLC_L10 } \\
\hline Sample & M0 & M1 & M2 & M3 & M4 & M5 \\
\hline $\mathbf{0 . 1 M}$ & 1.02 & 0.77 & 0.86 & 1.19 & 1.07 & $\sim$ \\
\hline $\mathbf{0 . 5 M}$ & 2.70 & 2.47 & 2.44 & 2.95 & 2.79 & $\sim$ \\
\hline $\mathbf{1 M}$ & 5.25 & 5.01 & 4.99 & 5.90 & 5.68 & $\sim$ \\
\hline $\mathbf{2 M}$ & 6.46 & 6.04 & 6.53 & 7.18 & 7.15 & $\sim$ \\
\hline $\mathbf{3 M}$ & 8.70 & 8.68 & 8.58 & 9.60 & 10.23 & $\sim$ \\
\hline $\mathbf{5 M}$ & 12.28 & 11.53 & 11.50 & 13.47 & 13.97 & $\sim$ \\
\hline
\end{tabular}

\begin{tabular}{|c|c|c|c|c|c|c|}
\hline \multicolumn{7}{|c|}{ C PLC_L14 } \\
\hline Sample & M0 & M1 & M2 & M3 & M4 & M5 \\
\hline $\mathbf{0 . 1 M}$ & 0.68 & 0.79 & 0.67 & 0.88 & 1.11 & $\sim$ \\
\hline $\mathbf{0 . 5 M}$ & 2.59 & 2.62 & 2.24 & 2.60 & 2.71 & $\sim$ \\
\hline $\mathbf{1 M}$ & 5.32 & 5.12 & 5.35 & 5.90 & 5.22 & $\sim$ \\
\hline $\mathbf{2 M}$ & 6.78 & 6.19 & 7.16 & 7.56 & 7.08 & $\sim$ \\
\hline $\mathbf{3 M}$ & 8.87 & 8.65 & 8.52 & 10.46 & 9.71 & $\sim$ \\
\hline $\mathbf{5 M}$ & 13.97 & 11.57 & 13.54 & 16.03 & 15.73 & $\sim$ \\
\hline
\end{tabular}

\begin{tabular}{|c|c|c|c|c|c|c|}
\hline \multicolumn{7}{|c|}{ D PLC_L15 } \\
\hline Sample & M0 & M1 & M2 & M3 & M4 & M5 \\
\hline $\mathbf{0 . 1 M}$ & 0.93 & 0.79 & 0.95 & 1.09 & 0.75 & 0.77 \\
\hline $\mathbf{0 . 5 M}$ & 2.82 & 2.34 & 2.85 & 2.51 & 2.47 & 2.75 \\
\hline $\mathbf{1 M}$ & 5.12 & 4.82 & 5.33 & 5.18 & 5.01 & 4.96 \\
\hline $\mathbf{2 M}$ & 6.79 & 6.15 & 7.04 & 6.98 & 6.92 & 5.97 \\
\hline $\mathbf{3 M}$ & 8.99 & 8.75 & 8.95 & 8.87 & 9.72 & 8.85 \\
\hline $\mathbf{5 M}$ & 12.96 & 10.75 & 13.28 & 12.30 & 12.81 & 11.98 \\
\hline
\end{tabular}

\begin{tabular}{|c|c|c|c|c|c|c|}
\hline \multicolumn{7}{|c|}{ E PLC_L11 } \\
\hline Sample & M0 & M1 & M2 & M3 & M4 & M5 \\
\hline $\mathbf{0 . 1 M}$ & 0.83 & 0.84 & 0.72 & 1.23 & 0.81 & $\sim$ \\
\hline $\mathbf{0 . 5 M}$ & 2.65 & 2.34 & 2.11 & 3.29 & 2.58 & $\sim$ \\
\hline $\mathbf{1 M}$ & 5.07 & 4.85 & 4.80 & 5.75 & 6.45 & $\sim$ \\
\hline $\mathbf{2 M}$ & 6.10 & 6.47 & 6.11 & 6.98 & 7.08 & $\sim$ \\
\hline $\mathbf{3 M}$ & 8.69 & 9.03 & 8.86 & 9.63 & 9.64 & $\sim$ \\
\hline $\mathbf{5 M}$ & 13.52 & 10.54 & 9.79 & 14.49 & 12.80 & $\sim$ \\
\hline
\end{tabular}


3986 3987 3988 3989

\begin{tabular}{|c|c|c|c|c|c|c|}
\hline \multicolumn{7}{|c|}{ A OPC+10LS } \\
\hline Sample & M0 & M1 & M2 & M3 & M4 & M5 \\
\hline $\mathbf{0 . 1 M}$ & 0.86 & 0.68 & 0.78 & 1.01 & 1.02 & 0.76 \\
\hline $\mathbf{0 . 5 M}$ & 2.81 & 2.64 & 2.32 & 3.13 & 2.70 & 2.98 \\
\hline $\mathbf{1 M}$ & 5.52 & 5.22 & 5.24 & 5.69 & 5.34 & 5.15 \\
\hline $\mathbf{2 M}$ & 6.66 & 6.69 & 7.03 & 7.05 & 7.11 & 7.71 \\
\hline $\mathbf{3 M}$ & 9.75 & 9.76 & 9.24 & 11.01 & 9.66 & 10.08 \\
\hline $\mathbf{5 M}$ & 13.49 & 15.59 & 13.69 & 14.37 & 12.26 & 13.22 \\
\hline
\end{tabular}

\begin{tabular}{|c|c|c|c|c|c|c|}
\hline \multicolumn{7}{|c|}{ B OPC+10LS } \\
\hline Sample & M0 & M1 & M2 & M3 & M4 & M5 \\
\hline $\mathbf{0 . 1 M}$ & 0.73 & 0.91 & 0.52 & 0.91 & 1.06 & $\sim$ \\
\hline $\mathbf{0 . 5 M}$ & 2.95 & 2.65 & 2.32 & 3.18 & 2.57 & $\sim$ \\
\hline $\mathbf{1 M}$ & 5.37 & 5.03 & 5.08 & 6.39 & 5.11 & $\sim$ \\
\hline $\mathbf{2 M}$ & 7.31 & 6.39 & 7.12 & 7.72 & 6.61 & $\sim$ \\
\hline $\mathbf{3 M}$ & 8.93 & 8.95 & 9.91 & 9.63 & 9.86 & $\sim$ \\
\hline $\mathbf{5 M}$ & 12.01 & 12.88 & 11.07 & 12.44 & 13.36 & $\sim$ \\
\hline
\end{tabular}

\section{OPC+10LS}

\begin{tabular}{|c|c|c|c|c|c|c|}
\hline \multicolumn{7}{|c|}{ C OPC+10LS } \\
\hline Sample & M0 & M1 & M2 & M3 & M4 & M5 \\
\hline $\mathbf{0 . 1 M}$ & 0.77 & 0.77 & 0.59 & 0.87 & 0.99 & $\sim$ \\
\hline $\mathbf{0 . 5 M}$ & 2.47 & 2.35 & 2.11 & 2.74 & 2.62 & $\sim$ \\
\hline $\mathbf{1 M}$ & 5.12 & 4.78 & 5.13 & 5.26 & 6.68 & $\sim$ \\
\hline $\mathbf{2 M}$ & 6.29 & 6.37 & 6.73 & 7.41 & 7.53 & $\sim$ \\
\hline $\mathbf{3 M}$ & 8.80 & 8.06 & 9.21 & 10.43 & 10.05 & $\sim$ \\
\hline $\mathbf{5 M}$ & 11.32 & 10.77 & 10.94 & 12.10 & 13.74 & $\sim$ \\
\hline
\end{tabular}

\section{OPC+10LS}

\begin{tabular}{|c|c|c|c|c|c|c|}
\hline Sample & M0 & M1 & M2 & M3 & M4 & M5 \\
\hline $\mathbf{0 . 1 M}$ & 0.71 & 0.81 & 0.91 & 1.19 & 1.00 & 0.79 \\
\hline $\mathbf{0 . 5 M}$ & 2.82 & 2.42 & 2.40 & 3.15 & 2.48 & 2.18 \\
\hline $\mathbf{1 M}$ & 5.16 & 4.92 & 4.98 & 5.79 & 5.12 & 5.61 \\
\hline $\mathbf{2 M}$ & 6.71 & 6.35 & 6.98 & 7.84 & 6.79 & 7.28 \\
\hline $\mathbf{3 M}$ & 7.90 & 8.23 & 9.94 & 8.64 & 8.97 & 7.83 \\
\hline $\mathbf{5 M}$ & 12.97 & 12.10 & 13.77 & 13.08 & 13.33 & 10.13 \\
\hline
\end{tabular}

\section{E OPC+10LS}

\begin{tabular}{|c|c|c|c|c|c|c|}
\hline Sample & M0 & M1 & M2 & M3 & M4 & M5 \\
\hline $\mathbf{0 . 1 M}$ & 0.84 & 0.75 & 0.77 & 1.04 & 0.91 & $\sim$ \\
\hline $\mathbf{0 . 5 M}$ & 2.59 & 2.30 & 2.44 & 3.19 & 2.65 & $\sim$ \\
\hline $\mathbf{1 M}$ & 5.36 & 4.59 & 5.47 & 6.12 & 5.26 & $\sim$ \\
\hline $\mathbf{2 M}$ & 6.29 & 5.79 & 6.76 & 7.49 & 7.18 & $\sim$ \\
\hline $\mathbf{3 M}$ & 9.28 & 7.72 & 9.24 & 10.34 & 10.16 & $\sim$ \\
\hline $\mathbf{5 M}$ & 11.53 & 10.45 & 13.45 & 14.22 & 13.26 & $\sim$ \\
\hline
\end{tabular}


3993 3994
Table F.10. Bound chloride content for Cement $\mathbf{D}$ mixtures immersed in $\mathbf{C a C l}_{2}-$ Mixtures 0 to $5\left(\mathrm{CaCl}_{2}\right)$

\begin{tabular}{|c|c|c|c|c|c|c|}
\hline \multicolumn{7}{|c|}{ Cement D+ $\mathbf{C a C l}_{\mathbf{2}}$} \\
\hline \multicolumn{7}{|c|}{ OPC } \\
\hline Sample & M0 & M1 & M2 & M3 & M4 & M5 \\
\hline $\mathbf{0 . 1 M}$ & 0.91 & 1.50 & 0.47 & 1.08 & 0.90 & 0.70 \\
\hline $\mathbf{0 . 5 M}$ & 4.51 & 4.10 & 3.51 & 5.08 & 4.00 & 4.34 \\
\hline $\mathbf{1 M}$ & 7.47 & 7.51 & 7.14 & 9.28 & 7.99 & 9.15 \\
\hline $\mathbf{2 M}$ & 11.88 & 12.95 & 11.47 & 12.85 & 12.82 & 15.30 \\
\hline $\mathbf{3 M}$ & 12.01 & 14.82 & 13.90 & 16.10 & 15.23 & 17.99 \\
\hline
\end{tabular}

\begin{tabular}{|c|c|c|c|c|c|c|}
\hline \multicolumn{7}{|c|}{ PLC_L15 } \\
\hline Sample & M0 & M1 & M2 & M3 & M4 & M5 \\
\hline $\mathbf{0 . 1 M}$ & 0.22 & 0.50 & 0.59 & 0.89 & 1.14 & 0.51 \\
\hline $\mathbf{0 . 5 M}$ & 4.66 & 4.18 & 4.42 & 3.96 & 3.78 & 3.67 \\
\hline $\mathbf{1 M}$ & 8.09 & 8.57 & 8.04 & 7.61 & 7.33 & 7.31 \\
\hline $\mathbf{2 M}$ & 12.21 & 13.53 & 13.45 & 12.42 & 12.01 & 12.13 \\
\hline 3M & 13.95 & 15.36 & 15.34 & 14.62 & 15.20 & 16.50 \\
\hline
\end{tabular}

3995

\begin{tabular}{|c|c|c|c|c|c|c|}
\hline \multicolumn{7}{|c|}{ OPC+10LS } \\
\hline Sample & M0 & M1 & M2 & M3 & M4 & M5 \\
\hline $\mathbf{0 . 1 M}$ & 0.84 & 0.56 & 0.96 & 0.90 & 1.22 & 0.46 \\
\hline $\mathbf{0 . 5 M}$ & 4.64 & 4.03 & 3.69 & 4.72 & 3.59 & 4.09 \\
\hline $\mathbf{1 M}$ & 7.35 & 8.28 & 7.29 & 7.57 & 7.24 & 8.51 \\
\hline $\mathbf{2 M}$ & 12.68 & 11.07 & 12.08 & 14.04 & 13.00 & 12.61 \\
\hline $\mathbf{3 M}$ & 13.45 & 11.84 & 15.26 & 15.41 & 14.80 & 15.70 \\
\hline
\end{tabular}

3996 
399725 APPENDIX G - Transport Properties of Concrete

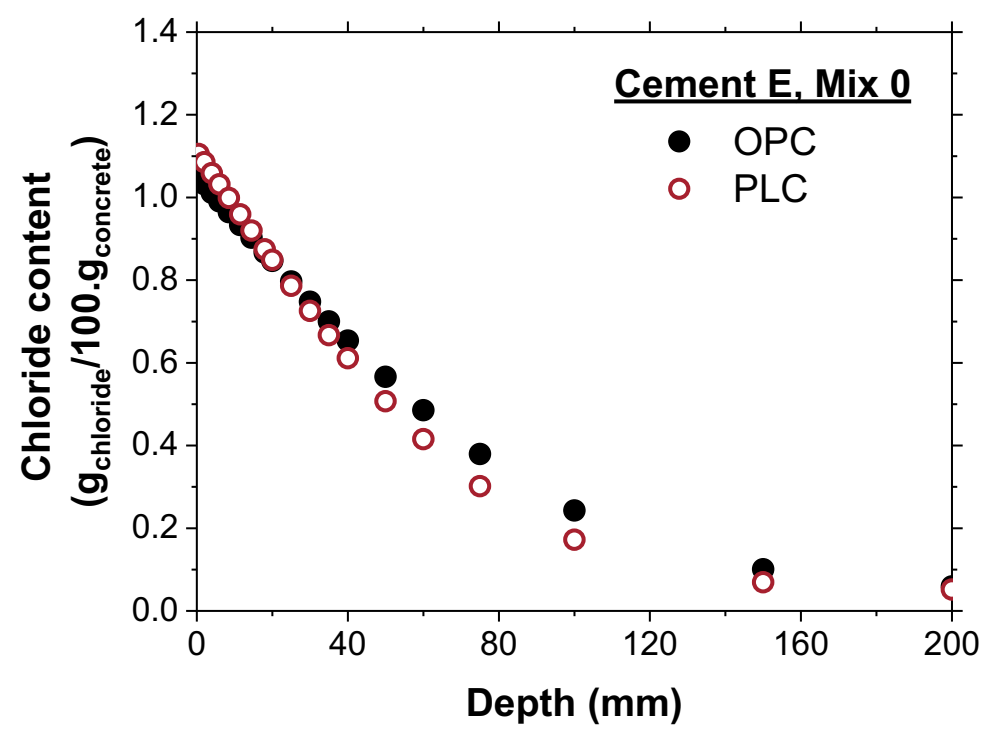

(a)

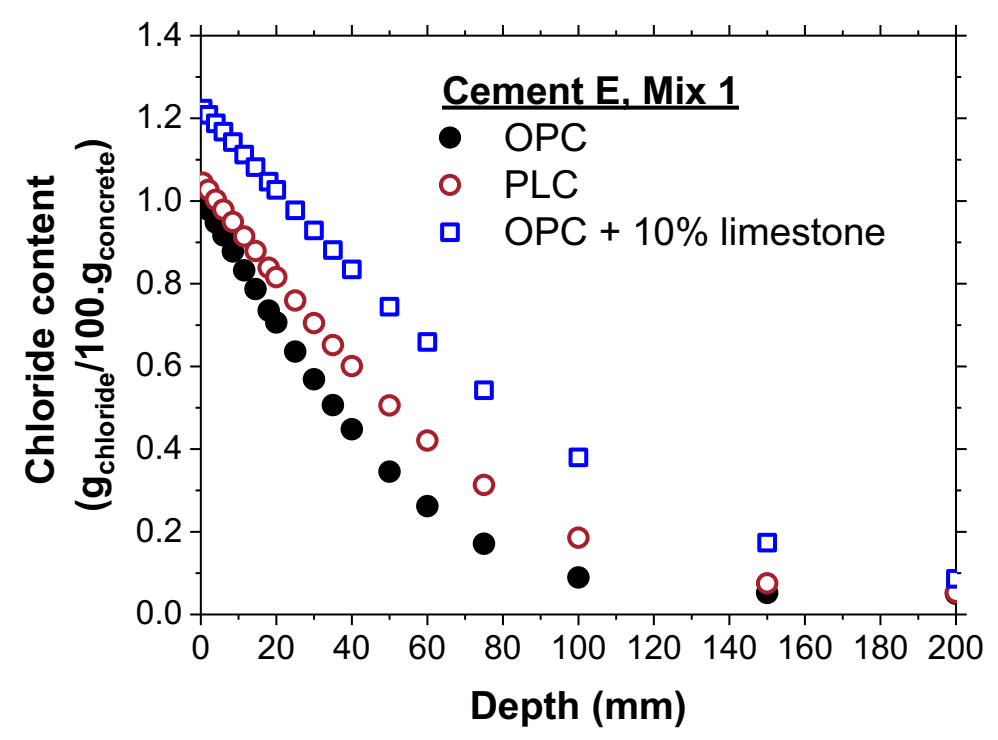

(b) 


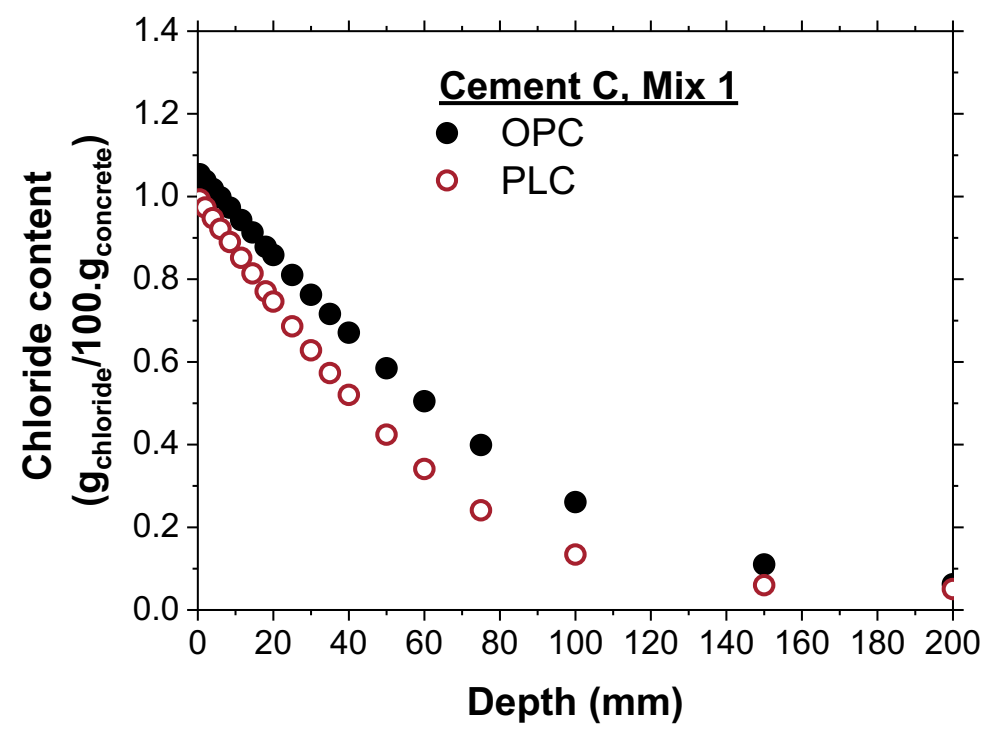

(c)

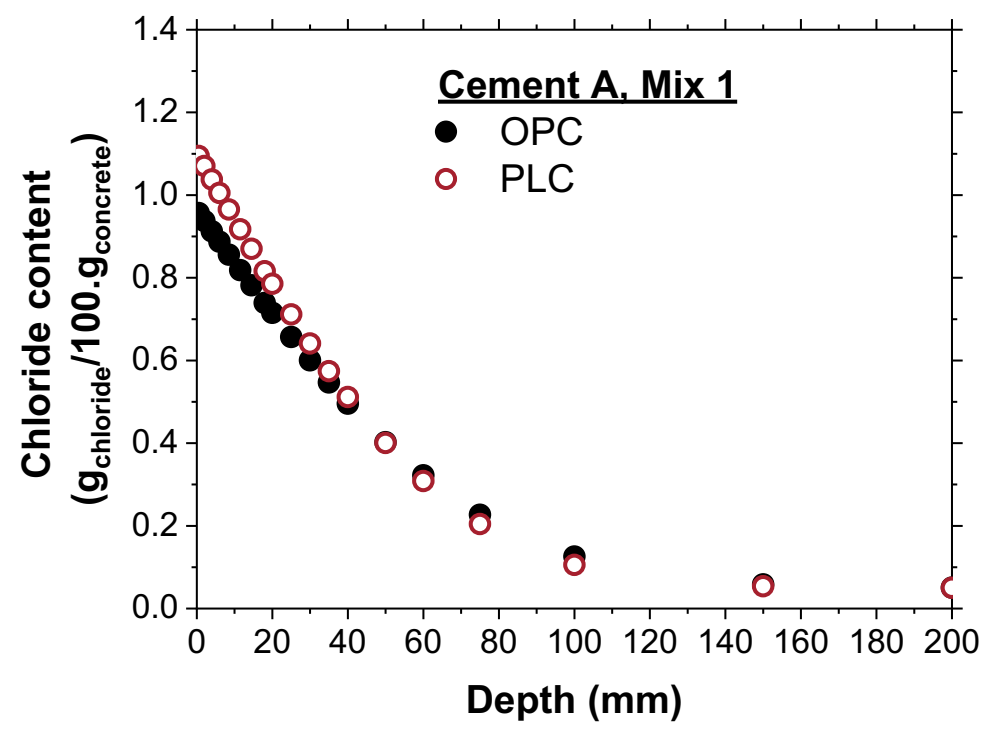

(d) 


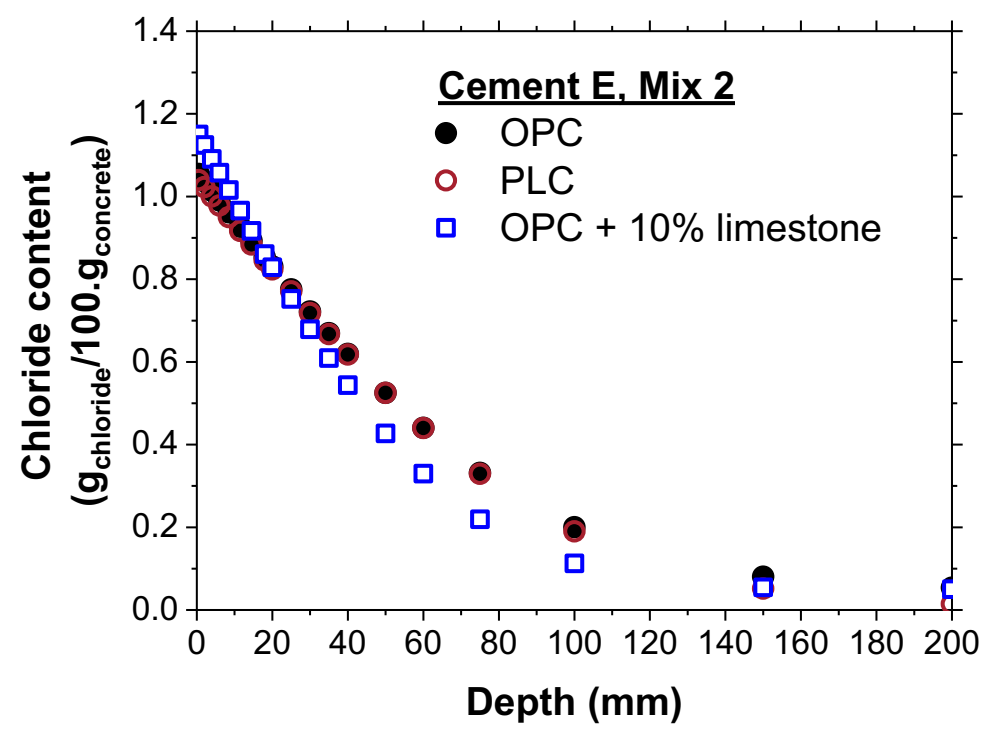

(e)

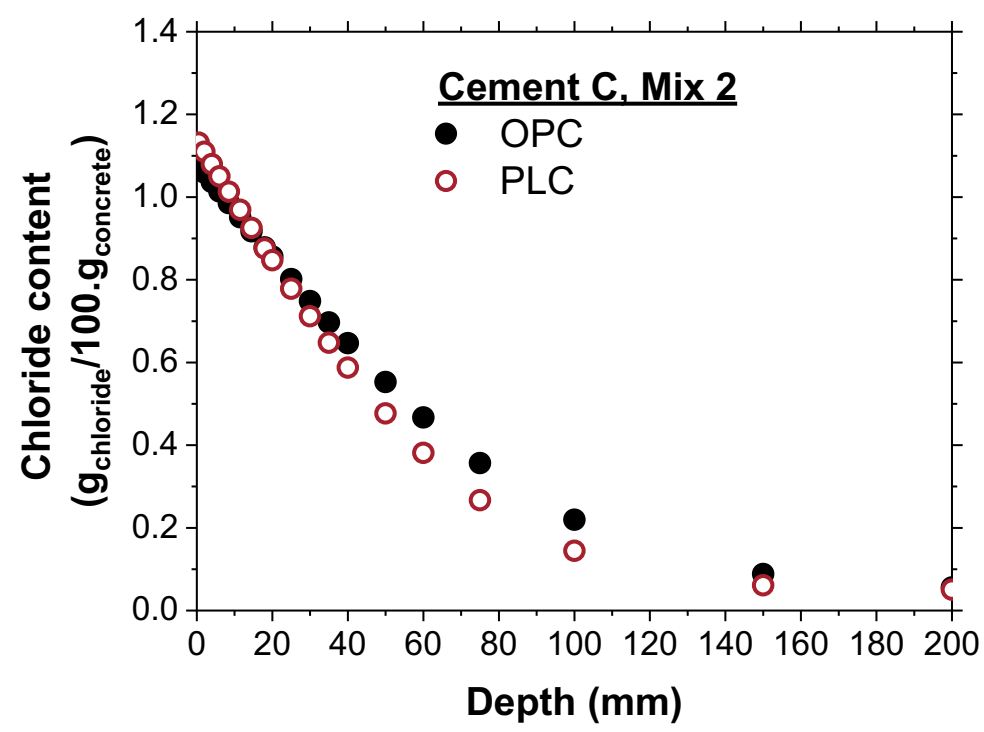

(f) 


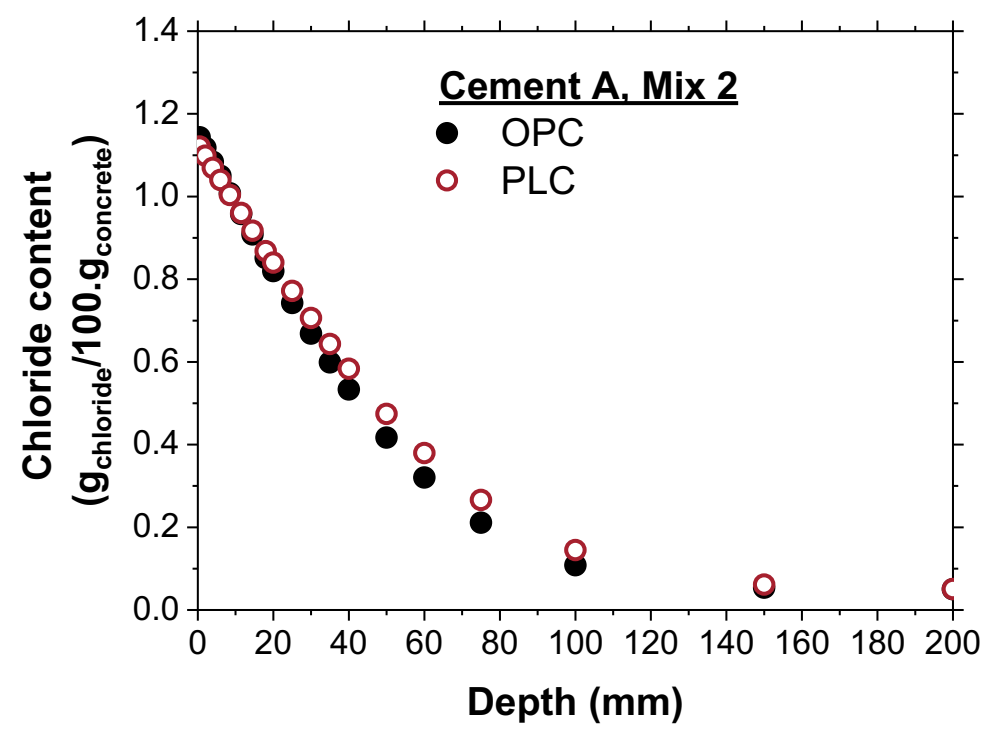

(g)

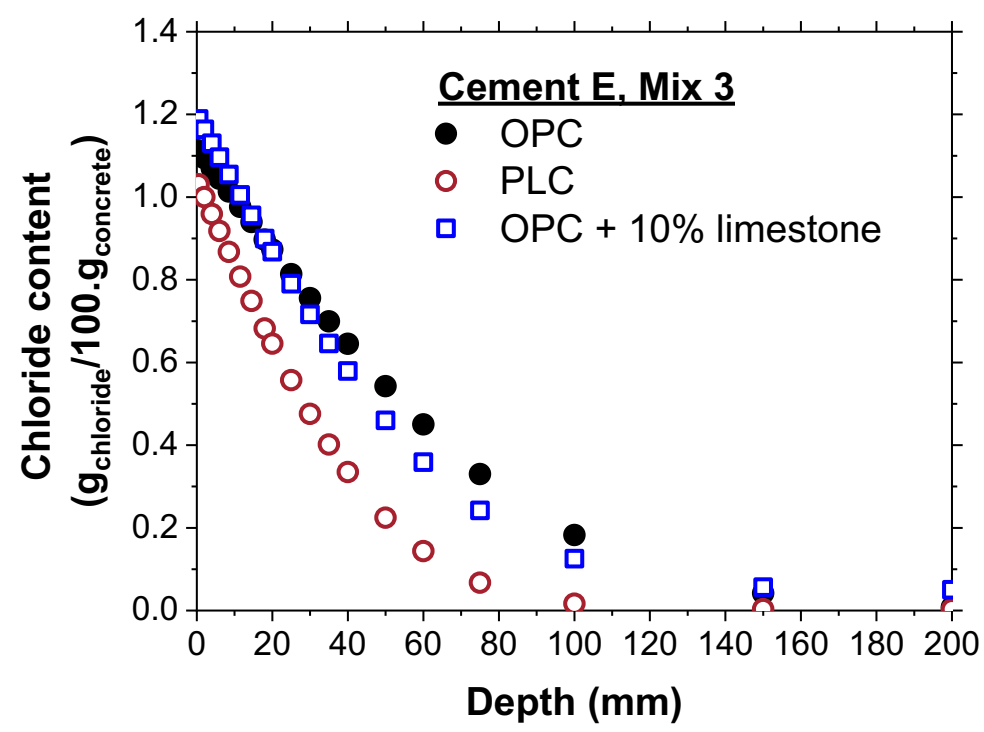

(h) 


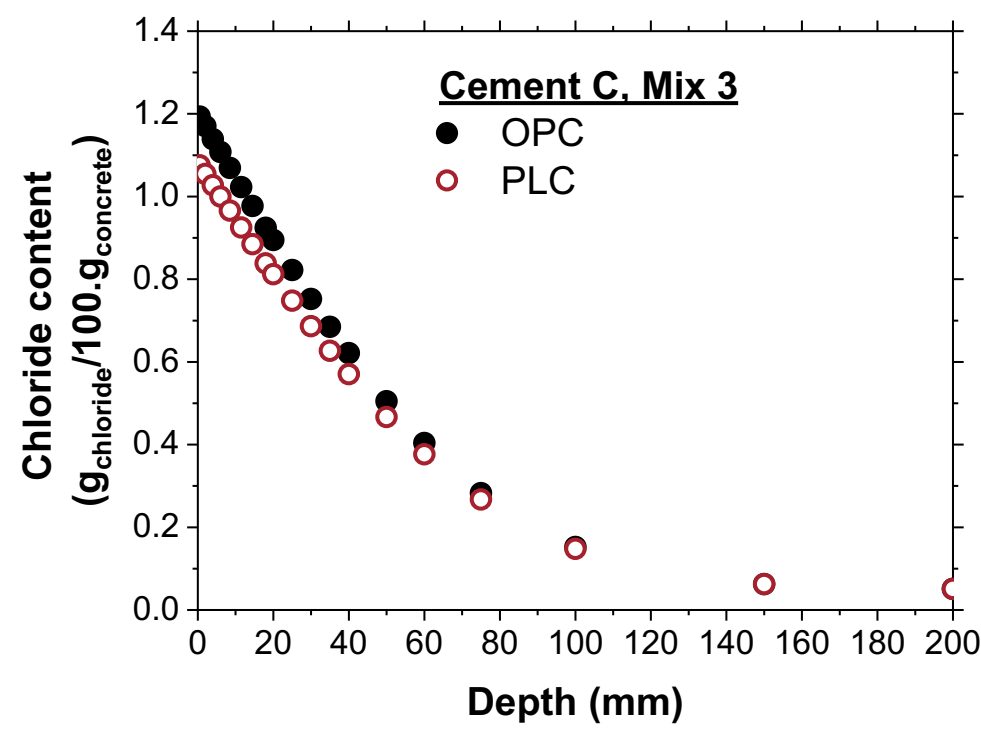

(i)

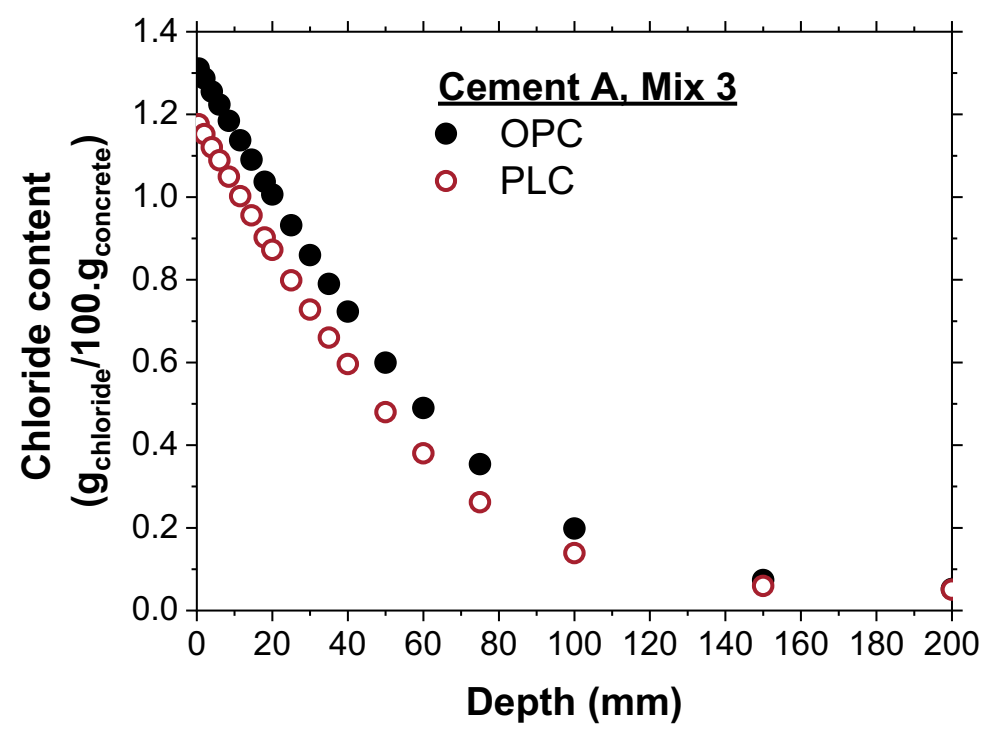

(j) 


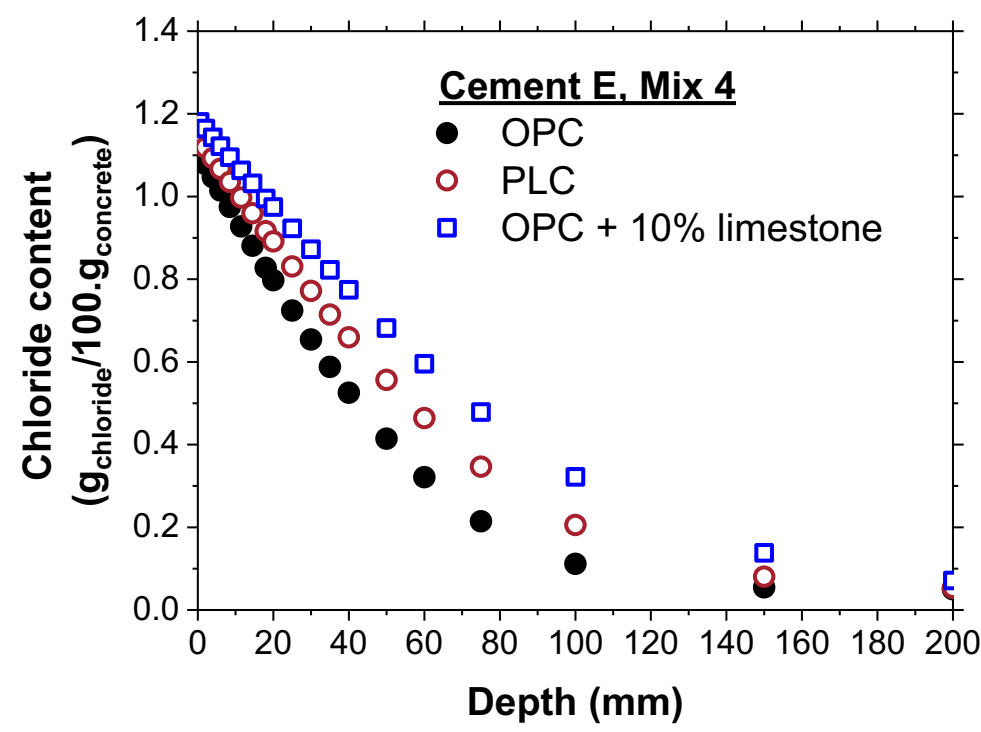

(k)

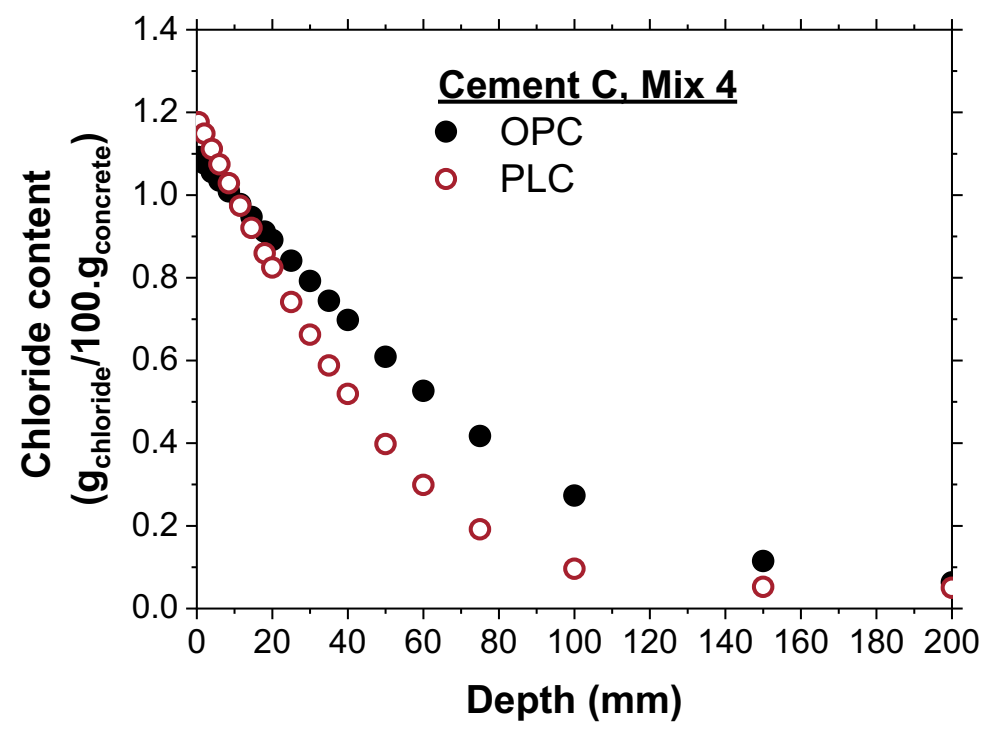

(1) 


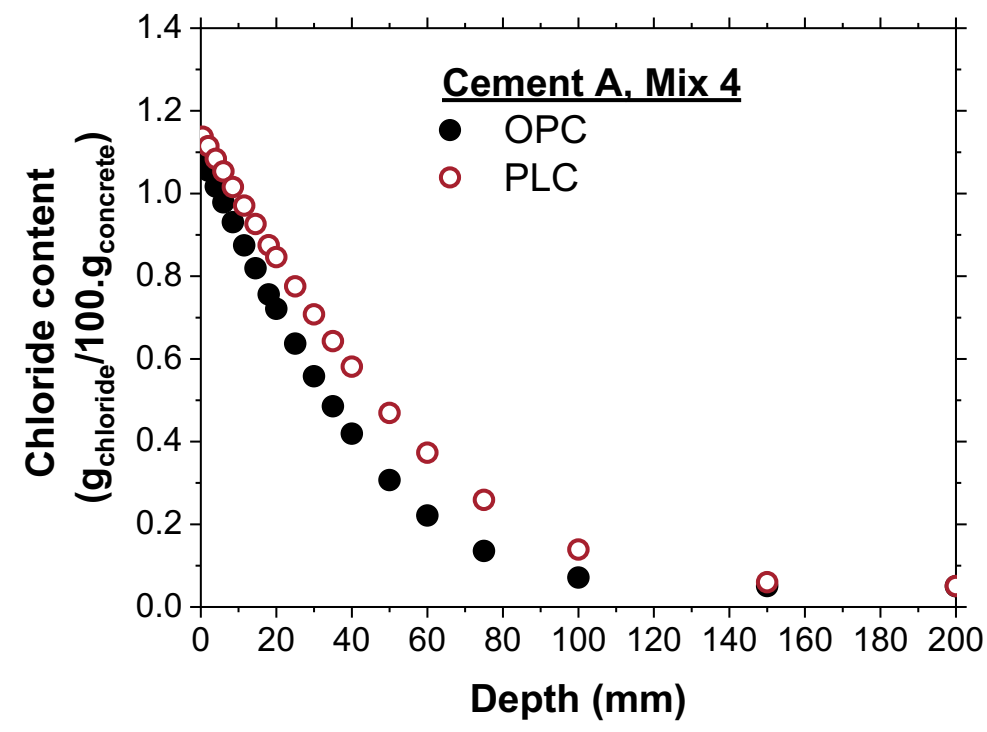

(m)

4004

4005
Figure Appendix G- Acid soluble profiles for all mixtures determined using the calculated values of $D_{a}$, and $C_{s-a}$ (from formation factor and porosity) for an exposure time of 20 years: (a) cement $\mathrm{E}$, Mix 0; (b) cement $\mathrm{E}$, Mix 1; (c) cement C, Mix 1; (d) Cement A, Mix 1; (e) cement E, Mix 2; (f) cement C, Mix 2; (g) Cement A, Mix 2; (h) cement E, Mix 3; (i) cement $C$, Mix3; (j) cement $A$, Mix3; (k) cement $E$, Mix 4; (l) cement $C$, Mix4; (m) cement A, Mix4. 


\section{H.1 Porosity Data}

\section{H.1.1 Total Porosity}

4009 The porosity of the paste as determined from thermodynamic modeling and the PPM is shown 4010 below in Table H.1.

Table H.1. Total porosity of paste from thermodynamic modeling

\begin{tabular}{|c|c|c|c|c|c|c|c|}
\hline \multirow[t]{2}{*}{$\begin{array}{l}\text { Paste Porosity } \\
\text { (in \%) }\end{array}$} & $\begin{array}{l}\text { Mix. } \\
\text { Long } \\
\text { Name }\end{array}$ & Control & $\begin{array}{c}\text { PLC + } \\
25 \% \\
\text { FA }\end{array}$ & $\begin{array}{c}\text { PLC + } \\
20 \% \text { FA } \\
+5 \% \\
\text { SF }\end{array}$ & $\begin{array}{c}\text { PLC + } \\
\mathbf{5 0 \%} \\
\text { SL }\end{array}$ & $\begin{array}{c}\text { PLC + } \\
25 \% \\
\text { SL + } \\
25 \% \\
\text { FA }\end{array}$ & $\begin{array}{c}\text { PLC + } \\
25 \% \\
\text { NP }\end{array}$ \\
\hline & $\begin{array}{l}\text { Mix } \\
\text { No. }\end{array}$ & M0 & M1 & M2 & M3 & M4 & M5 \\
\hline \multirow{5}{*}{$\begin{array}{c}\text { OPC }(<5.99 \% \\
\text { Ls) }\end{array}$} & A & $31 \%$ & $32 \%$ & $32 \%$ & $31 \%$ & $32 \%$ & $33 \%$ \\
\hline & B & $31 \%$ & $34 \%$ & $31 \%$ & $35 \%$ & $37 \%$ & $32 \%$ \\
\hline & C & $32 \%$ & $33 \%$ & $33 \%$ & $31 \%$ & $32 \%$ & $34 \%$ \\
\hline & D & $31 \%$ & $32 \%$ & $32 \%$ & $33 \%$ & $35 \%$ & $33 \%$ \\
\hline & $\mathbf{E}$ & $32 \%$ & $33 \%$ & $33 \%$ & $32 \%$ & $33 \%$ & $34 \%$ \\
\hline \multirow{5}{*}{$\begin{array}{c}\text { PLC (13-15\% } \\
\text { Ls) }\end{array}$} & $\mathbf{A}$ & $34 \%$ & $35 \%$ & $35 \%$ & $33 \%$ & $35 \%$ & $36 \%$ \\
\hline & B & $34 \%$ & $35 \%$ & $35 \%$ & $33 \%$ & $35 \%$ & $35 \%$ \\
\hline & $\mathrm{C}$ & $35 \%$ & $36 \%$ & $36 \%$ & $34 \%$ & $36 \%$ & $36 \%$ \\
\hline & D & $34 \%$ & $35 \%$ & $35 \%$ & $33 \%$ & $35 \%$ & $35 \%$ \\
\hline & $\mathbf{E}$ & $34 \%$ & $35 \%$ & $35 \%$ & $34 \%$ & $35 \%$ & $36 \%$ \\
\hline
\end{tabular}

4013 The total porosity of mortar consisting of $50 \%$ paste, $45 \%$ fine aggregates (of $3.7 \%$ absorption 4014 capacity), and 5\% entrapped air is shown in Table H.2. 
Table H.2. Total porosity of mortar calculated from the PPMC

\begin{tabular}{|c|c|c|c|c|c|c|c|}
\hline \multirow{2}{*}{$\begin{array}{c}\text { Mortar } \\
\text { Porosity (in } \\
\% \text { ) }\end{array}$} & $\begin{array}{l}\text { Mix. } \\
\text { Long } \\
\text { Name }\end{array}$ & Control & $\begin{array}{c}\mathrm{PLC}+ \\
25 \% \mathrm{FA}\end{array}$ & $\begin{array}{c}\text { PLC }+ \\
20 \% \mathrm{FA} \\
+5 \% \\
\mathrm{SF} \\
\end{array}$ & $\begin{array}{c}\mathrm{PLC}+ \\
50 \% \mathrm{SL}\end{array}$ & $\begin{array}{c}\text { PLC + } \\
25 \% \mathrm{SL} \\
+25 \% \\
\text { FA }\end{array}$ & $\begin{array}{c}\mathrm{PLC}+ \\
25 \% \mathrm{NP}\end{array}$ \\
\hline & $\begin{array}{l}\text { Mix } \\
\text { No. }\end{array}$ & M0 & M1 & M2 & M3 & M4 & M5 \\
\hline \multirow{5}{*}{$\begin{array}{c}\text { OPC }(<5.99 \% \\
\text { Ls })\end{array}$} & $\mathbf{A}$ & $22.1 \%$ & $22.8 \%$ & $22.8 \%$ & $21.9 \%$ & $22.6 \%$ & $23.2 \%$ \\
\hline & B & $22.0 \%$ & $23.7 \%$ & $22.3 \%$ & $24.3 \%$ & $25.2 \%$ & $22.8 \%$ \\
\hline & $\mathrm{C}$ & $22.6 \%$ & $23.3 \%$ & $23.2 \%$ & $22.2 \%$ & $22.9 \%$ & $23.7 \%$ \\
\hline & D & $22.0 \%$ & $22.5 \%$ & $22.6 \%$ & $23.2 \%$ & $24.4 \%$ & $23.2 \%$ \\
\hline & $\mathbf{E}$ & $22.5 \%$ & $23.2 \%$ & $23.2 \%$ & $22.5 \%$ & $23.1 \%$ & $23.6 \%$ \\
\hline \multirow{5}{*}{$\begin{array}{c}\text { PLC (13-15\% } \\
\text { Ls) }\end{array}$} & A & $23.7 \%$ & $24.1 \%$ & $24.0 \%$ & $23.4 \%$ & $24.2 \%$ & $24.4 \%$ \\
\hline & B & $23.6 \%$ & $24.0 \%$ & $24.0 \%$ & $23.3 \%$ & $24.2 \%$ & $24.4 \%$ \\
\hline & C & $24.2 \%$ & $24.5 \%$ & $24.7 \%$ & $23.6 \%$ & $24.5 \%$ & $24.8 \%$ \\
\hline & D & $23.6 \%$ & $24.0 \%$ & $24.0 \%$ & $23.3 \%$ & $24.2 \%$ & $24.4 \%$ \\
\hline & $\mathbf{E}$ & $23.8 \%$ & $24.1 \%$ & $24.1 \%$ & $23.4 \%$ & $24.2 \%$ & $24.5 \%$ \\
\hline
\end{tabular}

4016

4017 H.1.2 Phase Volumes (including Pore Volume Distribution)

4018 Table H.3. Phase volumes determined from thermodynamic modeling (output of the PPM)

\begin{tabular}{|c|c|c|c|c|c|c|c|c|}
\hline \multirow[t]{2}{*}{$\begin{array}{c}\text { Phase } \\
\text { Volumes in } \\
\text { Paste }\end{array}$} & $\begin{array}{l}\text { Mix. } \\
\text { Long } \\
\text { Name }\end{array}$ & & Control & $\begin{array}{c}\mathrm{PLC}+ \\
25 \% \mathrm{FA}\end{array}$ & $\begin{array}{c}\text { PLC }+ \\
20 \% \mathrm{FA} \\
+5 \% \\
\mathrm{SF} \\
\end{array}$ & $\begin{array}{c}\mathrm{PLC}+ \\
50 \% \mathrm{SL}\end{array}$ & $\begin{array}{c}\text { PLC + } \\
25 \% \text { SL } \\
+25 \% \\
\text { FA }\end{array}$ & $\begin{array}{c}\text { PLC + } \\
25 \% \text { NP }\end{array}$ \\
\hline & & $\begin{array}{l}\text { Mix } \\
\text { No. }\end{array}$ & MO & M1 & M2 & M3 & M4 & M5 \\
\hline \multirow{14}{*}{$\begin{array}{c}\text { OPC }(<5.99 \% \\
\text { Ls })\end{array}$} & \multirow{5}{*}{$\mathbf{A}$} & v_ub & $12 \%$ & $20 \%$ & $19 \%$ & $19 \%$ & $22 \%$ & $20 \%$ \\
\hline & & v_gs & $57 \%$ & $48 \%$ & $49 \%$ & $51 \%$ & $46 \%$ & $47 \%$ \\
\hline & & v_gw & $19 \%$ & $16 \%$ & $17 \%$ & $15 \%$ & $14 \%$ & $16 \%$ \\
\hline & & v_cw & $4 \%$ & $9 \%$ & $7 \%$ & $8 \%$ & $9 \%$ & $10 \%$ \\
\hline & & v_cs & $7 \%$ & $8 \%$ & $8 \%$ & $8 \%$ & $8 \%$ & $7 \%$ \\
\hline & \multirow{5}{*}{ B } & v_ub & $14 \%$ & $21 \%$ & $20 \%$ & $19 \%$ & $22 \%$ & $21 \%$ \\
\hline & & v_gs & $55 \%$ & $45 \%$ & $49 \%$ & $45 \%$ & $40 \%$ & $47 \%$ \\
\hline & & v_gw & $17 \%$ & $15 \%$ & $16 \%$ & $16 \%$ & $15 \%$ & $15 \%$ \\
\hline & & V_cw & $6 \%$ & $12 \%$ & $8 \%$ & $12 \%$ & $14 \%$ & $11 \%$ \\
\hline & & v_cs & $7 \%$ & $7 \%$ & $8 \%$ & $8 \%$ & $8 \%$ & $7 \%$ \\
\hline & \multirow{4}{*}{ C } & v_ub & $13 \%$ & $20 \%$ & $19 \%$ & $19 \%$ & $22 \%$ & $20 \%$ \\
\hline & & v_gs & $55 \%$ & $47 \%$ & $48 \%$ & $50 \%$ & $46 \%$ & $46 \%$ \\
\hline & & v_gw & $19 \%$ & $16 \%$ & $17 \%$ & $15 \%$ & $15 \%$ & $16 \%$ \\
\hline & & V_cw & $6 \%$ & $10 \%$ & $8 \%$ & $8 \%$ & $9 \%$ & $11 \%$ \\
\hline
\end{tabular}




\begin{tabular}{|c|c|c|c|c|c|c|c|c|}
\hline & & v_cs & $7 \%$ & $8 \%$ & $8 \%$ & $8 \%$ & $8 \%$ & $7 \%$ \\
\hline & \multirow{5}{*}{ D } & v_ub & $13 \%$ & $20 \%$ & $19 \%$ & $19 \%$ & $22 \%$ & $21 \%$ \\
\hline & & v_gs & $56 \%$ & $48 \%$ & $49 \%$ & $48 \%$ & $43 \%$ & $46 \%$ \\
\hline & & v_gw & $17 \%$ & $14 \%$ & $16 \%$ & $15 \%$ & $15 \%$ & $15 \%$ \\
\hline & & v_cw & $6 \%$ & $10 \%$ & $8 \%$ & $10 \%$ & $12 \%$ & $11 \%$ \\
\hline & & V_cs & $7 \%$ & $8 \%$ & $8 \%$ & $8 \%$ & $8 \%$ & $7 \%$ \\
\hline & \multirow{5}{*}{$\mathbf{E}$} & v_ub & $12 \%$ & $19 \%$ & $19 \%$ & $18 \%$ & $22 \%$ & $20 \%$ \\
\hline & & v_gs & $56 \%$ & $47 \%$ & $48 \%$ & $50 \%$ & $45 \%$ & $46 \%$ \\
\hline & & v_gw & $19 \%$ & $16 \%$ & $17 \%$ & $15 \%$ & $15 \%$ & $16 \%$ \\
\hline & & V_cw & $6 \%$ & $9 \%$ & $8 \%$ & $8 \%$ & $10 \%$ & $11 \%$ \\
\hline & & v_cs & $7 \%$ & $8 \%$ & $8 \%$ & $8 \%$ & $9 \%$ & $7 \%$ \\
\hline \multirow{25}{*}{$\begin{array}{c}\text { PLC }(13-15 \% \\
\text { Ls) }\end{array}$} & \multirow{5}{*}{$\mathbf{A}$} & v_ub & $13 \%$ & $20 \%$ & $19 \%$ & $19 \%$ & $22 \%$ & $20 \%$ \\
\hline & & v_gs & $53 \%$ & $45 \%$ & $46 \%$ & $48 \%$ & $43 \%$ & $44 \%$ \\
\hline & & v_gw & $17 \%$ & $14 \%$ & $15 \%$ & $14 \%$ & $14 \%$ & $15 \%$ \\
\hline & & V_cw & $12 \%$ & $14 \%$ & $12 \%$ & $11 \%$ & $13 \%$ & $15 \%$ \\
\hline & & v_cs & $5 \%$ & $7 \%$ & $7 \%$ & $8 \%$ & $9 \%$ & $6 \%$ \\
\hline & \multirow{5}{*}{ B } & v_ub & $13 \%$ & $20 \%$ & $20 \%$ & $19 \%$ & $22 \%$ & $21 \%$ \\
\hline & & v_gs & $53 \%$ & $45 \%$ & $46 \%$ & $48 \%$ & $43 \%$ & $44 \%$ \\
\hline & & v_gw & $15 \%$ & $13 \%$ & $14 \%$ & $13 \%$ & $13 \%$ & $13 \%$ \\
\hline & & v_cw & $13 \%$ & $15 \%$ & $13 \%$ & $12 \%$ & $13 \%$ & $16 \%$ \\
\hline & & V_cs & $6 \%$ & $7 \%$ & $7 \%$ & $8 \%$ & $9 \%$ & $6 \%$ \\
\hline & \multirow{5}{*}{$\mathbf{C}$} & v_ub & $13 \%$ & $20 \%$ & $19 \%$ & $19 \%$ & $22 \%$ & $20 \%$ \\
\hline & & v_gs & $52 \%$ & $45 \%$ & $45 \%$ & $48 \%$ & $43 \%$ & $43 \%$ \\
\hline & & v_gw & $18 \%$ & $15 \%$ & $19 \%$ & $15 \%$ & $14 \%$ & $15 \%$ \\
\hline & & v_cw & $12 \%$ & $14 \%$ & $10 \%$ & $11 \%$ & $13 \%$ & $15 \%$ \\
\hline & & v_cs & $5 \%$ & $7 \%$ & $6 \%$ & $8 \%$ & $9 \%$ & $6 \%$ \\
\hline & \multirow{5}{*}{ D } & v_ub & $13 \%$ & $20 \%$ & $19 \%$ & $19 \%$ & $22 \%$ & $20 \%$ \\
\hline & & v_gs & $53 \%$ & $45 \%$ & $46 \%$ & $48 \%$ & $43 \%$ & $44 \%$ \\
\hline & & v_gw & $15 \%$ & $13 \%$ & $14 \%$ & $13 \%$ & $13 \%$ & $13 \%$ \\
\hline & & V_cw & $13 \%$ & $15 \%$ & $13 \%$ & $12 \%$ & $13 \%$ & $16 \%$ \\
\hline & & v_cs & $6 \%$ & $7 \%$ & $7 \%$ & $8 \%$ & $9 \%$ & $6 \%$ \\
\hline & \multirow{5}{*}{$\mathbf{E}$} & v_ub & $13 \%$ & $20 \%$ & $19 \%$ & $19 \%$ & $22 \%$ & $20 \%$ \\
\hline & & v_gs & $53 \%$ & $45 \%$ & $46 \%$ & $48 \%$ & $43 \%$ & $44 \%$ \\
\hline & & $\mathbf{v \_ g w}$ & $18 \%$ & $15 \%$ & $16 \%$ & $15 \%$ & $14 \%$ & $15 \%$ \\
\hline & & V_cw & $10 \%$ & $13 \%$ & $12 \%$ & $11 \%$ & $12 \%$ & $14 \%$ \\
\hline & & v_cs & $6 \%$ & $7 \%$ & $7 \%$ & $8 \%$ & $9 \%$ & $6 \%$ \\
\hline
\end{tabular}


H.2 Reacted and Unreacted Limestone Data

4021

4022

Table H.4. Reacted calcite (limestone) in the systems as determined from thermodynamic modeling

\begin{tabular}{|c|c|c|c|c|c|c|c|}
\hline \multirow[t]{2}{*}{$\begin{array}{c}\text { Reacted } \\
\text { calcite (in } \\
\text { g/100gbinder) }\end{array}$} & $\begin{array}{l}\text { Mix. } \\
\text { Long } \\
\text { Name }\end{array}$ & Control & $\begin{array}{c}\text { PLC + } \\
25 \% \\
\text { FA }\end{array}$ & $\begin{array}{c}\text { PLC + } \\
20 \% \text { FA } \\
+5 \% \\
\text { SF }\end{array}$ & $\begin{array}{l}\text { PLC }+ \\
50 \% \\
\text { SL }\end{array}$ & $\begin{array}{c}\text { PLC + } \\
25 \% \\
\text { SL + } \\
25 \% \\
\text { FA } \\
\end{array}$ & $\begin{array}{c}\text { PLC + } \\
25 \% \\
\text { NP }\end{array}$ \\
\hline & $\begin{array}{l}\text { Mix } \\
\text { No. }\end{array}$ & M0 & M1 & M2 & M3 & M4 & M5 \\
\hline \multirow{5}{*}{$\begin{array}{c}\text { OPC }(<5.99 \% \\
\text { Ls })\end{array}$} & $\mathbf{A}$ & 1.67 & 4.31 & 4.17 & 4.31 & 4.31 & 3.63 \\
\hline & B & 1.79 & 1.79 & 1.79 & 1.79 & 1.79 & 1.79 \\
\hline & $\mathbf{C}$ & 1.38 & 4.20 & 3.91 & 4.20 & 4.20 & 3.37 \\
\hline & D & 2.05 & 3.48 & 3.48 & 3.48 & 3.48 & 3.48 \\
\hline & $\mathbf{E}$ & 1.41 & 4.76 & 4.39 & 5.99 & 5.99 & 3.85 \\
\hline \multirow{5}{*}{$\begin{array}{c}\text { PLC }(13-15 \% \\
\text { Ls) }\end{array}$} & $\mathbf{A}$ & 1.29 & 6.52 & 6.14 & 10.75 & 11.14 & 5.60 \\
\hline & B & 1.68 & 6.73 & 6.34 & 10.79 & 11.17 & 5.81 \\
\hline & $\mathbf{C}$ & 0.95 & 6.41 & 5.46 & 10.89 & 11.27 & 5.50 \\
\hline & D & 1.68 & 6.88 & 6.49 & 11.09 & 11.47 & 5.96 \\
\hline & $\mathbf{E}$ & 1.13 & 5.83 & 5.45 & 9.53 & 9.92 & 4.92 \\
\hline
\end{tabular}

4023

Table H.5. Unreacted calcite (limestone) that remains in the systems as determined from thermodynamic modeling

\begin{tabular}{|c|c|c|c|c|c|c|c|}
\hline \multirow[t]{2}{*}{$\begin{array}{l}\text { Unreacted } \\
\text { calcite (in } \\
\text { g/100g } g_{\text {binder }} \text { ) }\end{array}$} & $\begin{array}{l}\text { Mix. } \\
\text { Long } \\
\text { Name }\end{array}$ & Control & $\begin{array}{c}\text { PLC + } \\
25 \% \\
\text { FA }\end{array}$ & $\begin{array}{c}\text { PLC }+ \\
20 \% \text { FA } \\
+5 \% \\
\text { SF }\end{array}$ & $\begin{array}{c}\text { PLC }+ \\
50 \% \\
\text { SL }\end{array}$ & $\begin{array}{c}\text { PLC + } \\
25 \% \\
\text { SL + } \\
25 \% \\
\text { FA }\end{array}$ & $\begin{array}{c}\text { PLC + } \\
25 \% \\
\text { NP }\end{array}$ \\
\hline & $\begin{array}{l}\text { Mix } \\
\text { No. }\end{array}$ & M0 & M1 & M2 & M3 & M4 & M5 \\
\hline \multirow{5}{*}{$\begin{array}{c}\text { OPC }(<5.99 \% \\
\text { Ls })\end{array}$} & $\mathbf{A}$ & 2.64 & 0.00 & 0.14 & 0.00 & 0.00 & 0.68 \\
\hline & B & 0.00 & 0.00 & 0.00 & 0.00 & 0.00 & 0.00 \\
\hline & $\mathrm{C}$ & 2.82 & 0.00 & 0.29 & 0.00 & 0.00 & 0.83 \\
\hline & D & 1.43 & 0.00 & 0.00 & 0.00 & 0.00 & 0.00 \\
\hline & $\mathbf{E}$ & 4.58 & 1.23 & 1.60 & 0.00 & 0.00 & 2.14 \\
\hline \multirow{5}{*}{$\begin{array}{c}\text { PLC (13-15\% } \\
\text { Ls) }\end{array}$} & $\mathbf{A}$ & 12.03 & 6.80 & 7.18 & 2.57 & 2.18 & 7.72 \\
\hline & B & 11.43 & 6.38 & 6.77 & 2.32 & 1.94 & 7.30 \\
\hline & $\mathrm{C}$ & 13.05 & 7.59 & 8.54 & 3.11 & 2.73 & 8.50 \\
\hline & D & 12.03 & 6.83 & 7.22 & 2.62 & 2.24 & 7.75 \\
\hline & $\mathbf{E}$ & 9.98 & 5.28 & 5.66 & 1.58 & 1.19 & 6.19 \\
\hline
\end{tabular}


4028 Table H.6. CH Consumed in the systems as determined from thermodynamic modeling

\begin{tabular}{|c|c|c|c|c|c|c|c|}
\hline \multirow[t]{2}{*}{$\begin{array}{c}\mathrm{CH} \\
\text { Consumed (in } \\
\mathrm{g} / \mathbf{1 0 0 g}_{\text {binder }} \text { ) }\end{array}$} & $\begin{array}{l}\text { Mix. } \\
\text { Long } \\
\text { Name }\end{array}$ & Control & $\begin{array}{c}\text { PLC + } \\
25 \% \\
\text { FA }\end{array}$ & $\begin{array}{c}\text { PLC + } \\
20 \% \text { FA } \\
+5 \% \\
\text { SF }\end{array}$ & $\begin{array}{c}\text { PLC + } \\
50 \% \\
\text { SL }\end{array}$ & $\begin{array}{c}\text { PLC + } \\
25 \% \\
\text { SL + } \\
25 \% \\
\text { FA }\end{array}$ & $\begin{array}{c}\text { PLC + } \\
25 \% \\
\text { NP }\end{array}$ \\
\hline & $\begin{array}{l}\text { Mix } \\
\text { No. }\end{array}$ & Mo & M1 & M2 & M3 & M4 & M5 \\
\hline \multirow{5}{*}{$\begin{array}{c}\text { OPC }(<5.99 \% \\
\text { Ls) }\end{array}$} & A & 0.00 & 7.14 & 2.23 & 6.34 & 8.34 & 9.70 \\
\hline & B & 0.00 & 8.43 & 2.55 & 8.02 & 10.29 & 9.99 \\
\hline & C & 0.00 & 7.39 & 2.72 & 6.83 & 8.95 & 10.32 \\
\hline & D & 0.00 & 8.37 & 2.67 & 7.28 & 9.81 & 10.38 \\
\hline & $\mathbf{E}$ & 0.00 & 7.28 & 1.99 & 6.78 & 8.86 & 9.68 \\
\hline \multirow{5}{*}{$\begin{array}{c}\text { PLC (13-15\% } \\
\text { Ls) }\end{array}$} & $\mathbf{A}$ & 1.61 & 8.24 & 1.85 & 7.63 & 9.66 & 9.20 \\
\hline & B & 0.80 & 8.23 & 2.19 & 7.79 & 10.00 & 9.39 \\
\hline & C & 1.55 & 8.56 & 2.38 & 8.09 & 11.46 & 9.31 \\
\hline & D & 1.20 & 8.53 & 2.40 & 8.09 & 10.30 & 9.60 \\
\hline & $\mathbf{E}$ & 1.53 & 8.49 & 2.16 & 7.99 & 10.11 & 9.57 \\
\hline
\end{tabular}

4029

4030 


\title{
I.1 Jointed Plain Concrete Mix Design
}

\author{
STATE OF CALIFORNIA-BUSINESS TRANSPORTATION AND HOUSING AGENCY \\ DEPARTMENT OF TRANSPORTATION \\ TRUCKEE CONSTRUCTION FIELD OFFICE \\ 12047 DONNER PASS ROAD, SUITE B-4 \\ TRUCKEE, CA 9616 \\ PHONE (530) 587-9827; FAX (530) 587-9829 \\ May 13, 2010 \\ Mr. Chris Fallbeck, Project Manager \\ Teichert Construction \\ P.O. Box 276830 \\ Sacramento, CA 95827-6830 \\ ARNOID SCHWARZENEGGGER, Governot \\ Dear Mr. Purvis: \\ Flex your power!
Be energy efficient! \\ 03-NeV-080-R14.6/24.9 (KP) \\ ACIM-080-4(184)E \\ DONNER 1 - REHAB. \& WIDENING \\ CT Ref. No. 088

\section{$\underline{\text { Jointed Plain Concrete Pavement }}$} \\ Reference Teichert Construction's letter No. DOT-101, "PCC Mix Design," dated April \\ 29,2010 . \\ JPCP mix design No. 07-0010-2 (R3) designed by Coffman Specialties has been reviewed \\ and is approved in accordance with Section 10-1.56, "Jointed Plain Concrete Pavement," of \\ the Special Provisions. \\ If there are any questions regarding this letter feel free to contact me at your convenience. \\ Sincerely,

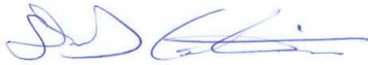 \\ DAVID F.CATANIA, P.E \\ Senior Resident Engineer \\ cc: NRCO, CT 5.3, 36.93.1 \\ Attachment: Yes
}




\begin{tabular}{|c|c|c|c|c|c|c|c|c|c|c|c|c|c|c|}
\hline Project Number: & $07-0010$ & \multicolumn{7}{|c|}{ CONCRETE MIX DESIGN } & & & Date & \multicolumn{2}{|c|}{$4 / 28 / 2010$} & \\
\hline Job: & \multicolumn{8}{|l|}{ Route 10} & \multicolumn{2}{|c|}{ Mix Design No.: } & \multicolumn{3}{|c|}{$07-0010-2(R 3)$} & \\
\hline Address: & \multicolumn{8}{|c|}{ Donner Lake UC to Trout Creek UC } & \multirow{2}{*}{\multicolumn{2}{|c|}{ Design Strenglh: }} & \multirow{2}{*}{\multicolumn{3}{|c|}{$\begin{array}{l}3.9 @ 28 \text { Days } \\
4.5 \text { (a) } 42 \text { Days }\end{array}$}} & \\
\hline Contractor: & \multicolumn{8}{|c|}{ Coffman Specialties, Inc } & & & & & & \\
\hline Mix Design use: & \multicolumn{8}{|c|}{ Slipform Pavement } & \multicolumn{2}{|c|}{ Spec. Slump (in.): } & \multicolumn{3}{|c|}{2.0} & \\
\hline Concrete Supplier: & \multicolumn{13}{|c|}{ Coffman Speciallies, Inc } & \\
\hline $\begin{array}{l}\text { Coarse Agg Source: } \\
\text { Fine Agg Source: }\end{array}$ & \multicolumn{8}{|c|}{ Teichert, Hallwood } & \multicolumn{6}{|c|}{ ADMIXTURES } \\
\hline Cement Source: & \multicolumn{8}{|c|}{ Cemex, Viclorville } & \multirow{4}{*}{$\begin{array}{l}\text { No. 1: } \\
\text { No. } 2: \\
\text { No. } 3 \text { : } \\
\text { No. } 4:\end{array}$} & \multicolumn{5}{|c|}{ Master Pave @ 22 oz/cu.yd. (851 m//m3) } \\
\hline Fly Ash Source: & \multicolumn{8}{|c|}{ N/A } & & \multicolumn{5}{|c|}{ PaveAir@ @8.4 oz/cu.yd. (325 ml/m3) } \\
\hline \multirow{2}{*}{\multicolumn{3}{|c|}{$\begin{array}{l}\text { Total Cementitious, Ib/yd^3 }\left(\mathrm{kg} / \mathrm{m}^{\wedge} 3\right) \text { : } \\
\text { Water / Cementitious Ratio (lb. } \mathrm{Ab} \text {. }) \text { : }\end{array}$}} & & 75 & $400 \mathrm{~kg} / \mathrm{h}$ & & & & & & & & & \\
\hline & & & & 43 & & & & & & & & & & \\
\hline \multicolumn{15}{|c|}{ GRADING ANALYSIS: PERCENT PASSING U.S. STANDARD SIEVE } \\
\hline SIEVE SIZE, $\mathrm{mm}$ & 50 & 37.5 & 25 & 19 & 12.5 & 9.5 & 4.75 & 2.36 & 1.18 & 0.600 & 0.300 & 0.150 & 0.075 & F.II. \\
\hline WC Sand & 100 & 100 & 100 & 100 & 100 & 100 & 96 & 81 & 66 & 49 & 27 & 8 & 1.8 & 2.73 \\
\hline & & & & & & & & & & & & & & \\
\hline $1^{\prime \prime} x \# 4$ & 100 & 100 & 100 & 91 & $\overline{44}$ & 28 & 4 & 1 & & & & & & \\
\hline $1-1 / 2^{\prime \prime} \times 3 / 4^{\prime \prime}$ & 100 & 97 & 38 & 7 & 2 & 1 & & & & & & & & \\
\hline & & & & & & & & & & & & & & \\
\hline CONBINED & 100 & 99 & 86 & 75 & 56 & 49 & 38 & 31 & 25 & T9 & 10 & 3 & 0.7 & \\
\hline " $x$ "Values & 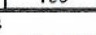 & & 35 & $\frac{15}{85}$ & & 25 & & & 68 & 46 & 25 & & & \\
\hline
\end{tabular}

DESIGN FOR ONE CUBIC YARD OF CONCRETE (SATURATRD AND SURFACE DRY') ABSOLUTE VOLUME OF AGGREGATE IN ONE CUBIC YARD. CUBIC FEET: SPECIEIC GRIVITY OF COMDINED NGGREGATES WEIGII OF AGGREGATES IN ONE CUBIC YARD BATCH. LDS.

\begin{tabular}{c}
17.70 \\
\hline 2.68 \\
\hline 2958.4 \\
\hline
\end{tabular}

\begin{tabular}{|c|c|c|c|c|c|}
\hline MATERIAL & PERCENT & BATCHLBS. & SP. GR. & ABSOLUTE VOLUME $(n 3)$ & SACK WEIGHTS (lbs) \\
\hline WC Sand & 38.0 & 1116 & 2.66 & 6.726 & \\
\hline & & & & & \\
\hline$j^{n} \times H 4(\# 57)$ & 39.0 & 1159 & 2.69 & 6.903 & \\
\hline $1-1 / 2^{11} \times 3 / 4^{n}(\# 4)$ & 23.0 & 683 & 2.69 & 4.071 & \\
\hline & & & & & \\
\hline WATER (gal) & & 290 & 1.00 & 4.651 & \\
\hline ELY'ASH (sks) & & & & & \\
\hline CEMENT (sks) & 100.0 & 675 & 3.15 & 3.434 & \\
\hline ESTINATED ENTRAINED AIR & 4.5 & & & 1.215 & \\
\hline TOTALS & & 3924 & & 27.00 & \\
\hline
\end{tabular}

DESIGN FOR ONE CUBIC METER OF CONCRETE (SATURATED AND SURFACE DRY') ABSOLUTE VOLUME OF AGGREGATE IN ONE CUBIC METER: SPECIFIC GRAVITY OF COMBINED AGGREGATES: WEIGHI OF AGGREGATES IN ONE CUBIC METER BATCH. Kg: $\frac{2.68}{1753.3}$

\begin{tabular}{|c|c|c|c|c|c|}
\hline MATERIAL. & PERCENT & BATCH $(\mathrm{kg})$ & SP. GR. & ABSOLUTE VOL.UNE $\left(\mathrm{m}^{\wedge} \mathrm{\jmath}\right)$ & SACK WEIGHTS (kg) \\
\hline WC Sand & 38.0 & 661.64 & 2.66 & 0.2493 & \\
\hline & & & & & \\
\hline $25 \mathrm{~mm} \times 4.75 \mathrm{~mm}$ & 39.0 & 686.71 & 2.69 & 0.2558 & \\
\hline $37.5 \mathrm{~mm} \times 19 \mathrm{~mm}$ & 23.0 & 404.98 & 2.69 & 0.1509 & \\
\hline & & & & & \\
\hline & & & 100 & 01720 & \\
\hline WATER (lit) & & 172.02 & 1.00 & & \\
\hline FLY ASII (hB) & & & & & \\
\hline CEMENT (kg) $\quad 400.05$ & 100.0 & 400.05 & 3.15 & 0.1270 & \\
\hline ESTIMATLD ENIRAINED AIR & 4.5 & & & 0.0450 & \\
\hline TOTALS & & 2325 & & 7.000 & \\
\hline
\end{tabular}

Calculated Wet Weight $\left(\mathrm{kg}_{\mathrm{m}} \mathrm{m}^{\wedge} 3\right): \quad 2325.4$

Light Weight Agg. C.F. L.oose Vo. At: pef.
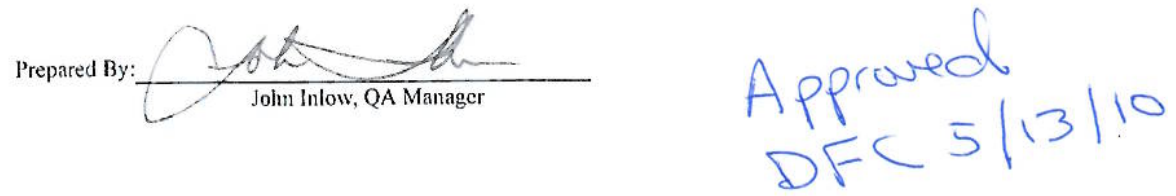


\section{I.2 Concrete Deck containing Slag - Mix Design}

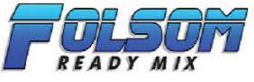

Concrete Mix Design Submittal

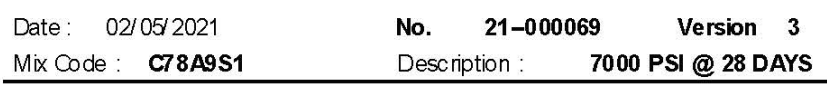

\begin{tabular}{|c|c|c|c|c|}
\hline & & & Design & Tolerance \\
\hline Customer & MYERS \& SONS CONSIRUCIION, LP & Air Content & 1.5 & \\
\hline Contact & BIANCA MEDINA & All cumlentil & 1.5 & \\
\hline Office Phone & $916-283-9950$ & Slump & 5 & $+/-1$ \\
\hline Project Name & \#111234 AB219 CDOT 03-0H4604 & Design Strength & 7000 & psi \\
\hline Project Contact & BROOKE & Unit Weight & 152.5 & $\mathrm{lb} / \mathrm{ft} 3$ \\
\hline Usage/ Placement & BRIDGE DEOK WITH FIBERS & W/C Ratio & 0.38 & \\
\hline
\end{tabular}

Admixtures may be adjusted to meet field conditions.

Please consult pump company prior to placement.

The following mix additions, if required, are to be requested by customer when placing order

SikaFiber Novomesh CAL-51 fibers with a 3 to 1 macro to micro blend added at 4 lbs per yard

\begin{tabular}{|c|c|c|c|c|c|c|}
\hline $\begin{array}{l}\text { Mate rial } \\
\text { Type }\end{array}$ & Description & Source Supplier & <ASTM> & Design Quantity & $\begin{array}{l}\text { Specific } \\
\text { Gravity }\end{array}$ & $\begin{array}{c}\text { Volume } \\
\text { (ft3) }\end{array}$ \\
\hline Coarse Agg regate & 1" ROCK & Western Aggregate-Ma rysville, CA & ASTM C33 & $1828 \mathrm{lb}$ & 2.77 & 10.58 \\
\hline Cement & CEMENT & Lehigh Southwest-Type II-V & ASTM C15C & $368 \mathrm{lb}$ & 3.15 & 1.87 \\
\hline Slag & SLAG & Cemex Victorville-Cemex Rizhao & ASTM C98S & $368 \mathrm{lb}$ & 2.92 & 2.02 \\
\hline Water & WATER & Municipal-Munic ipal & ASTM 1602 & $33.9 \mathrm{gal}$ & 1.00 & 4.53 \\
\hline Admixture & MASTERPCZZOLTH 322 & BASF Corporation-BASF USA & ASTM C494 & $44 \mathrm{lq}$ oz & - & - \\
\hline Admixture & MASTERPOLYHEED & BASF Corporation-BASF USA & ASTM C494 & $40 \mathrm{lq}$ oz & - & - \\
\hline \multirow{2}{*}{\multicolumn{3}{|c|}{ CONDITIONALLY }} & Air Content & $1.50 \%$ & - & 0.41 \\
\hline & & & Yield & $4117 \mathrm{lb}$ & - & 27.00 \\
\hline
\end{tabular}


Concrete Mix Design Submittal
Date : $\quad 02 / 052021$
No. 21-000069 Version 3
Description : $\quad 7000$ PSI @ 28DAYS

Mix Code : C78A9S1

\begin{tabular}{|c|c|c|c|}
\hline Sieve Sze & $\begin{array}{l}\text { 1ROCK } \\
\% \text { Passing }\end{array}$ & $\begin{array}{l}\text { SAND } \\
\% \text { Passing }\end{array}$ & $\begin{array}{l}\text { Combined } \\
\% \text { Passing }\end{array}$ \\
\hline $2^{\prime \prime}$ & 100.0 & 100.0 & 100.0 \\
\hline $1-1 / 2$ & 100.0 & 100.0 & 100.0 \\
\hline 1" & 99.0 & 100.0 & 99.4 \\
\hline $3 / 4^{\prime \prime}$ & 86.0 & 100.0 & 91.7 \\
\hline $1 / 2^{\prime \prime}$ & 42.0 & 100.0 & 65.8 \\
\hline $3 / 8^{\prime \prime}$ & 17.0 & 100.0 & 51.0 \\
\hline No. 4 & 2.0 & 96.0 & 40.5 \\
\hline No. 8 & 1.0 & 81.0 & 33.8 \\
\hline No. 16 & & 67.0 & 27.5 \\
\hline No. 30 & & 47.0 & 19.3 \\
\hline No. 50 & & 17.0 & 7.0 \\
\hline No. 100 & & 5.0 & 2.0 \\
\hline No. 200 & & 2.1 & 0.9 \\
\hline
\end{tabular}

\begin{tabular}{|c|c|c|c|}
\hline & 1ROCK & SAND & Combined \\
\hline DRUW Ib/ft3 & & & \\
\hline \% Agg & 59.0 & 41.0 & \\
\hline$\%$ Coarse Agg & 100.0 & & \\
\hline \% Fine Agg & & 100.0 & \\
\hline SG & 2.77 & 268 & \\
\hline FM & 8.53 & 3.85 & 6.61 \\
\hline
\end{tabular}

Prepared By:

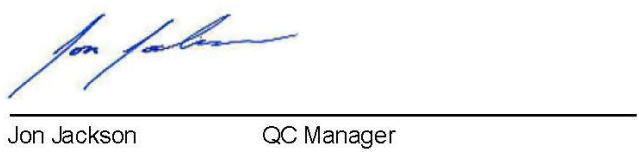




\section{I.3 Concrete Deck containing Fly Ash - Mix Design}

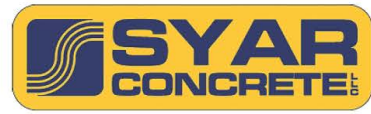

SYAR CONCRETE LLC

P.O. BOX 27 CO NAPA, CA 94558

(877) SYARMIX

Concrete Mix Design Submittal

DATE: $\quad 04 / 09 / 2020$

Mix Design Code: AE075CC5WA

Customer

Project Name

Usage/Placement

BRIDGE DECK

Design Strength 5000 PSI@42 DAYS

W/CM Ratio 0.38

$\begin{aligned} \text { Slump } 5.00^{\prime \prime} & +/-1 " \\ \text { Air Content } 6.00 & +/-1.5 \%\end{aligned}$

Unit Weight $142.15 \quad \mathrm{~b} / \mathrm{ft} 3$

SYAR CONCRETE LLC has no authority regarding the appropriate app lication of the mix design. It is the responsibility of the owner's representative and or contractor to insure that this mix design is appropriate for the intended use and environmental conditions for the intended application of the mix This concrete mix design will meet design strengths when tested in strict comp liance with current ASTM Standard and evaluated in accordance with ACI standard practices. Approval of the mix design carries the inclusion of SYAR CONCRETE LLC on the distribution list for all concrete test results.Cementitious content is expressed as a minimum and SYAR CONCRETE LLC reserves the right to increase the total cementitious content. Admixtures are dosed as per manufactures recommendations and may be adjusted to maintain mix design properties. Aggregate weights may be adjusted to maintain proper y ield and to comply with grading specifications.

It is the responsibility of the contractor to verify pumpability with the pumping contractor.

\begin{tabular}{|c|c|c|c|c|c|}
\hline $\begin{array}{l}\text { Mate rial } \\
\text { Type }\end{array}$ & \multicolumn{2}{|l|}{ De scription } & $\begin{array}{c}\text { Design Quantity } \\
\text { (S SD) }\end{array}$ & $\begin{array}{l}\text { Specific } \\
\text { Gravity }\end{array}$ & $\begin{array}{c}\text { Vol ume } \\
\text { (ft3) }\end{array}$ \\
\hline Cement & \multicolumn{2}{|l|}{ CEMENT TYPE II / VMODIFIED } & $562.5 \mathrm{lb}$ & 3.15 & 2.86 \\
\hline Coarse Aggregate & \multicolumn{2}{|l|}{ AST M C33 \#57 CONCRETE AGGREGATE } & $1650 \mathrm{lb}$ & 2.73 & 9.69 \\
\hline Fine Aggregate & \multicolumn{2}{|l|}{ AST M C33 CONCRETE SAND } & $1150 \mathrm{lb}$ & 2.67 & 6.91 \\
\hline Water & \multicolumn{2}{|l|}{ WATER } & $34.5 \mathrm{gal}$ & 1.00 & 4.61 \\
\hline Admixture & \multicolumn{2}{|l|}{ AE 200/AIR ENTRAINING ADMIXT URE } & $2 \mathrm{lq} \mathrm{oz}$ & - & - \\
\hline Admixture & \multicolumn{2}{|l|}{ MAST ERLIFE SRA 35 SHRINKAGE REDUCING ADM } & $128 \mathrm{lq} \mathrm{oz}$ & - & - \\
\hline & & AirC ontent & $6.00 \%$ & - & 1.62 \\
\hline & & Yield & $3838 \mathrm{lb}$ & - & 27.00 \\
\hline
\end{tabular}

Contingent on acceptable prequalification and shrinkage test results.

NOTES

BASF MASTERFIBER F70 (MICRO) FIBERS TO BE ADDED@1.0 LB/CYD BASF MASTERFIBER MACMATRIX (MACRO) FIBERS TO BE ADDED@ 3.0 LBS/CYI
AUTHORIZED

Pursuant to Section 5-1.23 of the Standard Specifications

\section{State of California \\ DEPARTMENT OF TRANSPORTATION \\ Division of Engineering Services \\ Offices of Structure Construction}

Prepared By :
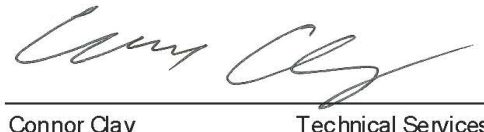

Signed

Structure Representative

Date 13 APRIL 2020 


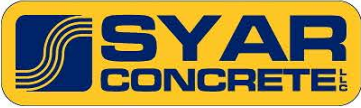

SYAR CONCRETE LLC

P.O. BOX 2700 NAPA, CA 94558

(877) SYARMIX

Concrete Mix Design Combined Aggregate Grading

Date: $04 / 09 / 2020$

Mix Code: AE075CC5WA

No. 042013 Version 1

\begin{tabular}{|c|c|c|c|c|c|}
\hline Sieve Size & $\begin{array}{c}\text { Coarse } \\
\mathbf{0 1 1 1 1 0} \\
\text { \% Passing }\end{array}$ & $\begin{array}{c}\text { Fine } \\
\mathbf{0 4 1 2 0 0} \\
\text { \% Passing }\end{array}$ & $\begin{array}{c}\text { Combined } \\
\text { \% Passing }\end{array}$ & $\begin{array}{c}\text { Caltrans 1" } \\
\text { Combined } \\
\text { Min } \\
\text { \% Passing }\end{array}$ & $\begin{array}{c}\text { Caltrans 1" } \\
\text { Combined } \\
\text { Max } \\
\text { \%assing }\end{array}$ \\
\hline $\mathbf{1 1 / 2}$ & 100.0 & 100.0 & 100.0 & 100.0 & 100.0 \\
\hline $\mathbf{1}$ & 100.0 & 100.0 & 100.0 & 90.0 & 100.0 \\
\hline $\mathbf{3 / 4}$ & 85.0 & 100.0 & 91.2 & 55.0 & 100.0 \\
\hline $\mathbf{1 / 2}$ & 50.0 & 100.0 & 70.5 & 50.0 & 88.0 \\
\hline $\mathbf{3 / 8}$ & 27.0 & 100.0 & 57.0 & 45.0 & 75.0 \\
\hline No. $\mathbf{4}$ & 2.0 & 100.0 & 42.3 & 35.0 & 60.0 \\
\hline No. $\mathbf{8}$ & 1.0 & 84.0 & 35.1 & 27.0 & 45.0 \\
\hline No. $\mathbf{1 6}$ & & 69.0 & 28.3 & 20.0 & 35.0 \\
\hline No. $\mathbf{3 0}$ & & 45.0 & 18.5 & 12.0 & 25.0 \\
\hline No. $\mathbf{5 0}$ & & 17.0 & 7.0 & 5.0 & 15.0 \\
\hline No. $\mathbf{1 0 0}$ & & 4.0 & 1.6 & 1.0 & 8.0 \\
\hline No. $\mathbf{2 0 0}$ & & 1.1 & 0.5 & 0.0 & 4.0 \\
\hline
\end{tabular}

\begin{tabular}{|c|c|c|c|}
\hline & $\begin{array}{c}\text { Coarse } \\
011110\end{array}$ & $\begin{array}{c}\text { Fine } \\
041200\end{array}$ & Combined \\
\hline DRUW lb/ft3 & & & \\
\hline$\%$ Agg & 58.9 & 41.1 & \\
\hline$\%$ Coarse Agg & 100.0 & & \\
\hline$\%$ Fine Agg & & 100.0 & \\
\hline SG & 2.73 & 2.67 & \\
\hline FM & 7.35 & 2.81 & 5.49 \\
\hline
\end{tabular}


4041 